\title{
Diversely Substituted Quinolines via Rhodium-Catalyzed Alkyne Hydroacylation
}

James D. Neuhaus, ${ }^{a}$ Sarah M. Morrow, ${ }^{a}$ Michael Brunavs, ${ }^{b}$ Michael. C. Willis ${ }^{a *}$ 


\section{Supporting Information}

I. General Considerations $\quad$ S3

$\begin{array}{lll}\text { II. Synthesis of hydroacylation adducts. } & \text { S4 }\end{array}$

General Procedure A - hydroacylation of 2-ethynyl aniline derivatives $\quad$ S4

III. Synthesis of quinolines.

General Procedure B - acidic cyclization of 2-aminochalcones to quinolines

$\mathrm{S} 30$

General Procedure C - thermal cyclization of 2-aminochalcones to quinolines

IV. Quinolines obtained via tandem hydroacylation - C-S functionalization

S55

General Procedure D - tandem hydroacylation - C-S functionalization reactions

V. Synthesis of quinoline $\mathrm{N}$-oxides via reductive cyclizations

S68

General Procedure E - reductive cyclization of 2-nitrochalcones to quinoline $N$-oxides

S68

VI. Synthesis of 4-substituted quinolines via intermediate functionalization.

General Procedure F - conjugate addition of Grignard reagents to chalcone intermediates

General Procedure $G$ - oxidative cyclisation of dihydrochalcones to

$\begin{array}{ll}\text { VII. Synthesis of starting materials } & \text { S76 }\end{array}$

General Procedure H - Boc protection of anilines using NaHMDS $\quad$ S76

General Procedure I - Boc protection of anilines via diBoc aniline $\quad$ S76

General Procedure $J$ - synthesis of TMS-protected alkynes via the $\begin{array}{ll}\text { Sonagashira reaction } & \text { S76 }\end{array}$

General Procedure K - TMS deprotection of terminal alkynes S76

$\begin{array}{lrr}\text { VIII. References } & \text { S108 }\end{array}$

IX. $\quad{ }^{\mathbf{1}} \mathrm{H}-\mathrm{NMR}$ and ${ }^{\mathbf{1 3}} \mathbf{C}-\mathrm{NMR}$ spectra $\quad$ S109 


\section{General Considerations:}

Reactions were performed under an inert atmosphere of nitrogen, using anhydrous solvent unless otherwise stated. All glassware was oven dried at $>80^{\circ} \mathrm{C}$, and allowed to cool to room temperature under a positive nitrogen pressure. Thin Layer Chromatography (TLC) analysis was carried out on Merck Kieselgel 60 PF254 precoated aluminium backed sheets and visualised either by UV fluorescence $(254 \mathrm{~nm})$ and/or by staining with potassium permanganate $\left(\mathrm{KMnO}_{4}\right)$ or Seebach's magic stain. Reagents were purchased from Sigma-Aldrich Chemical Co. Ltd., Fluorochem Ltd., Apollo Scientific Ltd., Acros Organics Ltd., Alfa Aesar Ltd., or Strem Chemicals Inc. and were used as supplied unless otherwise stated. Anhydrous acetonitrile, diethylether, dichloromethane, toluene and tetrahydrofuran were obtained by passing through anhydrous alumina columns using an Innovative Technology Inc.PS-400-7 solvent purification system. Acetone was distilled from Drierite ${ }^{\circledR}$. 'Petrol' refers to the fraction of light petroleum ether boiling in the range $40-60{ }^{\circ} \mathrm{C}$. Microwave heating was carried out using a CEM Discover-S unit. Flash column chromatography was performed using Apollo scientific silica gel 60 (particle size 0.040-0.063 nm) with the indicated eluents.

NMR spectra were recorded at ambient temperature on a Brüker AV400 (400 MHz) spectrometer. Chemical shifts $(\delta)$ are reported in parts per million (ppm) and referenced relative to the residual solvent peak(s) (as specified). Coupling constants $(J)$ are given in Hertz $(\mathrm{Hz})$ and rounded to the nearest $0.5 \mathrm{~Hz}$. Assignments were made on the basis of chemical shifts, coupling constants, COSY, HSQC and comparison with spectra of related compounds. Signal multiplicities are denoted as: $\mathrm{s}$,singlet; d, doublet; $\mathrm{t}$, triplet; q, quartet; m, multiplet; br., broad. Multiplicities are reported as observed. Low resolution ESI mass spectra were recorded on a Fisons Platform spectrometer. High resolution ESI mass spectrometry measurements were recorded on a Brucker Daltronics microTO (ESI) spectrometer by the internal service at the Department of Organic Chemistry, University of Oxford. Infra-red spectra were recorded as thin films on a Bruker Tensor 27 FTIR spectrometer. Melting points were determined using a Stuart Scientific Melting Point Apparatus SMP1. 


\section{General Procedure A - hydroacylation of 2-ethynyl aniline derivatives}

$\left[\mathrm{Rh}(\mathrm{nbd})_{2}\right] \mathrm{BF}_{4}(3.7 \mathrm{mg}, 0.01 \mathrm{mmol})$ and dcpm $(4.1 \mathrm{mg}, 0.01 \mathrm{mmol})$ were added to a microwave vial, which was then evacuated and backfilled with $\mathrm{N}_{2(\mathrm{~g})}$ three times. Then was added distilled and degassed acetone $(0.4 \mathrm{~mL})$, and the resulting suspension sonicated in order to solubilise the phosphine ligand. Once the solution was homogenous, $\mathrm{H}_{2(\mathrm{~g})}$ was bubbled through for 1 minute, followed by $\mathrm{N}_{2(\mathrm{~g})}$ until the volume was reduced to $0.1 \mathrm{~mL}$. This resulting solution was transferred by syringe into a microwave vial containing aldehyde $(0.24 \mathrm{mmol})$ and alkyne $(0.20 \mathrm{mmol})$. The resulting solution was stirred at the given temperature until full consumption of the starting alkyne was observed. This was often accompanied by product precipitation. No work up was necessary prior to purification.

\section{tert-Butyl (E)-(2-(3-(2-(methylthio)phenyl)-3-oxoprop-1-en-1- yl)phenyl)carbamate, $4 a$}<smiles>CS(=O)(=O)Nc1ccccc1/C=C/C(=O)c1ccccc1S(C)(=O)=O</smiles>

Compound 4a was prepared according to General Procedure A with alkyne 3a (43.5 $\mathrm{mg}, 0.20 \mathrm{mmol})$, aldehyde $\mathbf{2 a}(36.5 \mathrm{mg}, 0.24 \mathrm{mmol}),\left[\mathrm{Rh}(\mathrm{nbd})_{2}\right] \mathrm{BF}_{4}(3.7 \mathrm{mg}, 0.01$ $\mathrm{mmol})$, dcpm $(4.1 \mathrm{mg}, 0.01 \mathrm{mmol})$ and acetone $(0.1 \mathrm{~mL})$ for 3 hours. Flash column chromatography $\left(\mathrm{SiO}_{2}, 15-30 \% \mathrm{Et}_{2} \mathrm{O}\right.$ in petrol) afforded enone $\mathbf{4 a}$ as a yellow solid (70.8 mg, 96\% yield).

Alternatively:

Compound 4a was prepared according to General Procedure A with alkyne 3a (1.00 g, $4.60 \mathrm{mmol}$ ), aldehyde $\mathbf{2 a}$ (840 mg, $5.52 \mathrm{mmol})$, [Rh(nbd) ${ }_{2} \mathrm{BF}_{4}(33.7 \mathrm{mg}, 0.09 \mathrm{mmol})$, dcpm $(36.8 \mathrm{mg}, 0.09 \mathrm{mmol})$ and acetone $(2.8 \mathrm{~mL})$ for 16 hours at $40{ }^{\circ} \mathrm{C}$. Flash column chromatography $\left(\mathrm{SiO}_{2}, 15-20 \% \mathrm{Et}_{2} \mathrm{O}\right.$ in petrol) afforded enone $4 \mathbf{a}$ as a yellow solid (1.43 g, 84\% yield).

m.p.: 86-88 ${ }^{\circ} \mathrm{C}$ (crystalized from $\left.\mathrm{CHCl}_{3}\right) ;{ }^{1} \mathbf{H}-\mathbf{N M R}\left(400 \mathrm{MHz}, \mathrm{CDCl}_{3}\right)$ : $7.82(1 \mathrm{H}, \mathrm{d}$, $J$ 15.5, C(O)CHCH), $7.79(1 \mathrm{H}, \mathrm{d}, J 9.5, \mathrm{C}(8) H), 7.71(1 \mathrm{H}, \mathrm{dd}, J 7.5$ and 1.5, C(4)H), $7.54(1 \mathrm{H}, \mathrm{dd}, J 8.0$ and 1.5, C(5)H), $7.42(1 \mathrm{H}$, ddd, $J 8.0,7.5$ and 1.5, $\mathrm{C}(2) H), 7.34-$ $7.30(2 \mathrm{H}, \mathrm{m}, \mathrm{C}(1) H, \mathrm{C}(7) H), 7.25(1 \mathrm{H}, \mathrm{d}, J 15.5, \mathrm{C}(\mathrm{O}) \mathrm{CHCH}), 7.18(1 \mathrm{H}, \mathrm{td}, J 7.5$ and 1.0, C(3)H), $7.06(1 \mathrm{H}, \mathrm{t}, J 7.5, \mathrm{C}(6) H), 6.58\left(1 \mathrm{H}\right.$, br. s, NH), $2.41(3 \mathrm{H}, \mathrm{s}, \mathrm{SCH})_{3}, 1.45$ $\left(9 \mathrm{H}, \mathrm{s}, \mathrm{C}\left(\mathrm{CH}_{3}\right)_{3}\right) ;{ }^{13} \mathrm{C}-\mathrm{NMR}\left(100 \mathrm{MHz}, \mathrm{CDCl}_{3}\right): 191.8,152.9,139.5,137.2,136.6$, $131.9,131.1,129.8,129.4,128.9,127.3,126.3,126.0,124.23,124.16,122.7,81.0$, 28.3, 16.4; LRMS (ESI+, m/z): $392.2\left([\mathrm{M}+\mathrm{Na}]^{+}\right)$; HRMS (ESI+, $\left.\mathrm{m} / \mathrm{z}\right)$ : $\mathrm{C}_{21} \mathrm{H}_{23} \mathrm{NNaO}_{3} \mathrm{~S}\left([\mathrm{M}+\mathrm{Na}]^{+}\right)$requires 392.1291, found 392.1296; IR $v_{\max }($ film $) / \mathrm{cm}^{-1}$ : $3317,2978,1722,1655,1595,1515,1454,1367,1330,1239,1209,1158,1050$, 1026; 
<smiles>CSc1ccccc1C(=O)/C=C/c1c(F)cccc1NC(=O)c1ccccc1</smiles>

Compound $\mathbf{4 b}$ was prepared according to General Procedure A with alkyne 3b (47.1 $\mathrm{mg}, 0.20 \mathrm{mmol})$, aldehyde $2 \mathrm{a}(36.5 \mathrm{mg}, 0.24 \mathrm{mmol}),\left[\mathrm{Rh}(\mathrm{nbd})_{2}\right] \mathrm{BF}_{4}(3.7 \mathrm{mg}, 0.01$ mmol), dcpm $(4.1 \mathrm{mg}, 0.01 \mathrm{mmol})$ and acetone $(0.1 \mathrm{~mL})$ for 4 hours at $55{ }^{\circ} \mathrm{C}$. Flash column chromatography $\left(\mathrm{SiO}_{2}, 20-30 \% \mathrm{Et}_{2} \mathrm{O}\right.$ in petrol) afforded enone $4 \mathbf{b}$ as a yellow solid (62.9 $\mathrm{mg}, 81 \%$ yield).

m.p.: $127-129{ }^{\circ} \mathrm{C}$ (crystalized from $\left.\mathrm{CHCl}_{3}\right) ;{ }^{1} \mathbf{H}-\mathbf{N M R}\left(400 \mathrm{MHz}, \mathrm{CDCl}_{3}\right)$ : $7.81(1 \mathrm{H}$, $\mathrm{dd}, J 7.5$ and 1.5, C(4)H), $7.75(1 \mathrm{H}, \mathrm{d}, J 8.5, \mathrm{C}(7) H), 7.67(1 \mathrm{H}, \mathrm{d}, J 16.0, \mathrm{C}(\mathrm{O}) \mathrm{CHCH})$, $7.55(1 \mathrm{H}, \mathrm{dd}, J 16.0$ and 1.0, $\mathrm{C}(\mathrm{O}) \mathrm{CHCH}), 7.51(1 \mathrm{H}, \mathrm{ddd}, J 8.0,7.5$ and 1.5, $\mathrm{C}(2) H)$, $7.40(1 \mathrm{H}, \mathrm{d}, J 8.0, \mathrm{C}(1) H), 7.32(1 \mathrm{H}, \mathrm{ddd}, J 8.5,8.0$ and 6.5, C(6)H), $7.26(1 \mathrm{H}$, ddd, $J$ 7.5, 7.5 and 1.0, $\mathrm{C}(3) H), 6.88(1 \mathrm{H}, \mathrm{dd}, J 10.5$ and $8.5, \mathrm{C}(5) H), 6.79(1 \mathrm{H}, \mathrm{br} . \mathrm{s}, \mathrm{N} H)$, $2.49(3 \mathrm{H}, \mathrm{s}, \mathrm{SCH}), 1.55\left(9 \mathrm{H}, \mathrm{s}, \mathrm{C}\left(\mathrm{CH}_{3}\right)_{3}\right) ;{ }^{13} \mathbf{C}-\mathbf{N M R}\left(100 \mathrm{MHz}, \mathrm{CDCl}_{3}\right): 191.7$, 161.3 (d, $J 251.0), 152.6,141.7,138.6$ (d, J 6.0), 136.1, 133.0, 132.1, 131.0 (d, J 11.0), $130.11(\mathrm{~d}, J 11.0), 130.08,125.9,124.1,117.8(\mathrm{~d}, J 2.0), 114.7$ (d, $J 14.0), 111.0(\mathrm{~d}, J$ 22.5), 81.4, 28.3, 16.4; ${ }^{19}$ F-NMR $\left(376 \mathrm{MHz}, \mathrm{CDCl}_{3}\right)\left\{{ }^{1} \mathrm{H}\right\}:-110.5$; LRMS (ESI+, $\mathrm{m} / \mathrm{z}): 410.1\left([\mathrm{M}+\mathrm{Na}]^{+}, 100 \%\right) ; \quad$ HRMS $(\mathrm{ESI}+, \mathrm{m} / \mathrm{z}): \mathrm{C}_{21} \mathrm{H}_{22} \mathrm{FNNaO}_{3} \mathrm{~S}\left([\mathrm{M}+\mathrm{Na}]^{+}\right)$ requires 410.11966, found 410.11977; IR $v_{\max }\left(\right.$ film) $/ \mathrm{cm}^{-1}: 3318,2978,2925,1727$, $1657,1597,1583,1517,1472,1435,1392,1367,1320,1278,1247,1208,1156,1081$, 1048, 1014. 
<smiles>CSc1ccccc1C(=O)/C=C/c1ccc(Cl)cc1NC(=O)OCc1ccccc1</smiles>

Compound 4c was prepared according to General Procedure A with alkyne 3c (50.4 $\mathrm{mg}, 0.20 \mathrm{mmol})$, aldehyde $2 \mathrm{a}(36.5 \mathrm{mg}, 0.24 \mathrm{mmol}),\left[\mathrm{Rh}(\mathrm{nbd})_{2}\right] \mathrm{BF}_{4}(3.7 \mathrm{mg}, 0.01$ mmol), dcpm (4.1 mg, $0.01 \mathrm{mmol})$ and acetone $(0.1 \mathrm{~mL})$ for 2 hours at room temperature. Flash column chromatography $\left(\mathrm{SiO}_{2}, 15-30 \% \mathrm{Et}_{2} \mathrm{O}\right.$ in petrol) afforded enone $4 \mathbf{c}$ as a yellow oil (78.8 $\mathrm{mg}, 98 \%$ yield).

${ }^{1} \mathbf{H}-\mathbf{N M R}\left(400 \mathrm{MHz}, \mathrm{CDCl}_{3}\right): 7.93(1 \mathrm{H}$, br. s, C(7)H), $7.72(1 \mathrm{H}, \mathrm{d}, J$ 15.5, $\mathrm{C}(\mathrm{O}) \mathrm{CHCH}), 7.69(1 \mathrm{H}, \mathrm{dd}, J 6.5$ and 1.5, C(4)H), $7.42(1 \mathrm{H}, \mathrm{d}, J 8.5, \mathrm{C}(5) H), 7.41$ $(1 \mathrm{H}, \mathrm{td}, J 7.0$ and 1.5, C(3)H), $7.29(1 \mathrm{H}, \mathrm{d}, J 8.0, \mathrm{C}(1) H), 7.22(1 \mathrm{H}, \mathrm{d}, J 15.5$, $\mathrm{C}(\mathrm{O}) \mathrm{CHCH}), 7.16(1 \mathrm{H}, \mathrm{td}, J 7.5$ and $1.0, \mathrm{C}(2) H), 6.99(1 \mathrm{H}, \mathrm{dd}, J 8.5$ and $2.0, \mathrm{C}(6) H)$, $6.69(1 \mathrm{H}$, br. s, $\mathrm{NH}), 2.39(3 \mathrm{H}, \mathrm{s}, \mathrm{SCH}), 1.45\left(9 \mathrm{H}, \mathrm{s}, \mathrm{C}\left(\mathrm{CH}_{3}\right)_{3}\right) ;{ }^{13} \mathbf{C}-\mathbf{N M R}(100$ $\left.\mathrm{MHz}, \mathrm{CDCl}_{3}\right)$ : 191.4, 152.4, 141.4, 138.2, 138.0, 136.9, 136.4, 132.1, 129.9, 128.2, 126.5, 126.0, 124.2, 124.1, 123.9, 121.8, 81.6, 28.3, 16.4; LRMS (ESI+, m/z): 426.1 $\left(\left[\mathrm{M}\left({ }^{35} \mathrm{Cl}\right)+\mathrm{Na}\right]^{+}\right), 428.1\left(\left[\mathrm{M}\left({ }^{37} \mathrm{Cl}\right)+\mathrm{Na}\right]^{+}\right) ; \quad$ HRMS $(\mathrm{ESI}+, \mathrm{m} / \mathrm{z}): \mathrm{C}_{21} \mathrm{H}_{22} \mathrm{ClNNaO}_{3} \mathrm{~S}$ $\left(\left[\mathrm{M}\left({ }^{35} \mathrm{Cl}\right)+\mathrm{Na}\right]^{+}\right)$requires 426.0901 , found 426.0895, $\left(\left[\mathrm{M}\left({ }^{37} \mathrm{Cl}\right)+\mathrm{Na}\right]^{+}\right)$requires 428.0872, found 428.0865; IR $v_{\max }($ film $) / \mathrm{cm}^{-1}: 1725,1655,1590,1510,1462,1420$, 1392, 1367, 1329, 1235, 1209, 1155, 1050, 1013. 
<smiles>CC(C)(C)Nc1ccccc1C(=O)/C=C/c1cc(Br)ccc1NS(C)(=O)=O</smiles>

Compound 4d was prepared according to General Procedure A with alkyne 3d (59.2 $\mathrm{mg}, 0.20 \mathrm{mmol}$ ), aldehyde 3a (36.5 mg, $0.24 \mathrm{mmol}),\left[\mathrm{Rh}(\mathrm{nbd})_{2}\right] \mathrm{BF}_{4}(3.7 \mathrm{mg}, 0.01$ mmol), dcpm $(4.1 \mathrm{mg}, 0.01 \mathrm{mmol})$ and acetone $(0.1 \mathrm{~mL})$ for 20 minutes at room temperature. Flash column chromatography $\left(\mathrm{SiO}_{2}, 15-30 \% \mathrm{Et}_{2} \mathrm{O}\right.$ in petrol) afforded enone $\mathbf{4 d}$ as a yellow solid ( $88.7 \mathrm{mg}, 99 \%$ yield).

m.p.: $148-150{ }^{\circ} \mathrm{C}$ (crystalized from $\mathrm{CHCl}_{3}$ ). ${ }^{1} \mathbf{H}-\mathbf{N M R}\left(400 \mathrm{MHz}, \mathrm{CDCl}_{3}\right)$ : 7.73-7.69 (3H, m, C(O)CHCH, C(7)H, C(4)H), $7.62(1 \mathrm{H}, \mathrm{d}, J 2.0, \mathrm{C}(5) H), 7.42(1 \mathrm{H}, \mathrm{td}, J 7.5$, 1.5, C(6)H), $7.39(1 \mathrm{H}, \mathrm{dd}, J 9.0$ and 2.0, C(2)H), $7.30(1 \mathrm{H}, \mathrm{d}, J 8.0, \mathrm{C}(1) H), 7.24(1 \mathrm{H}$, $\mathrm{d}, J 15.5, \mathrm{C}(\mathrm{O}) \mathrm{CHCH}), 7.18(1 \mathrm{H}, \mathrm{td}, J 7.5$ and $0.5, \mathrm{C}(3) H), 6.58(1 \mathrm{H}$, br. s, $\mathrm{N} H), 2.40$ $\left.(3 \mathrm{H}, \mathrm{s}, \mathrm{SCH})_{3}\right), 1.44\left(9 \mathrm{H}, \mathrm{s}, \mathrm{C}\left(\mathrm{CH}_{3}\right)_{3}\right) ;{ }^{13} \mathbf{C}-\mathbf{N M R}\left(100 \mathrm{MHz}, \mathrm{CDCl}_{3}\right)$ : 191.1, 152.6, $141.7,137.5,136.2,136.1,133.6,132.2,130.0,129.7,127.8,127.1,125.9,124.2$,

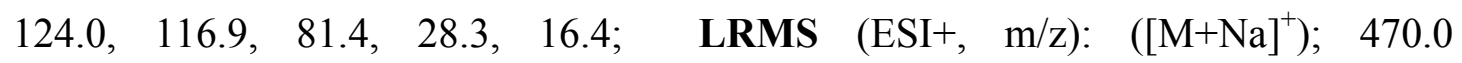
$\left(\left[\mathrm{M}\left({ }^{79} \mathrm{Br}\right)+\mathrm{Na}\right]^{+}, \quad 100 \%\right), 472.0\left(\left[\mathrm{M}\left({ }^{81} \mathrm{Br}\right)+\mathrm{Na}\right]^{+}, 100 \%\right) ; \quad$ HRMS $(\mathrm{ESI}+, \mathrm{m} / \mathrm{z})$ : $\mathrm{C}_{21} \mathrm{H}_{22} \mathrm{BrNNaO}_{3} \mathrm{~S}\left({ }^{79} \mathrm{Br},[\mathrm{M}+\mathrm{Na}]^{+}\right)$requires 470.0396, found 470.0388, $\left({ }^{81} \mathrm{Br}\right.$, $[\mathrm{M}+\mathrm{Na}]^{+}$) requires 472.0376, found 472.0365; IR $v_{\max }($ film $) / \mathrm{cm}^{-1}: 2979,2362,1703$, $1655,1598,1508,1404,1367,1327,1275,1209,1158,1051,1008$; 


\section{tert-Butyl (E)-(2-iodo-6-(3-(2-(methylthio)phenyl)-3-oxoprop-1-en-1- yl)phenyl)carbamate, $4 \mathrm{e}$}<smiles>CS(=O)(=O)Nc1ccccc1/C=C/C(=O)c1ccccc1S(C)(=O)=O</smiles>

Compound 4e was prepared according to General Procedure A with alkyne 3e (68.6 mg, $0.20 \mathrm{mmol})$, aldehyde 2a (36.5 mg, $0.24 \mathrm{mmol})$, [Rh(nbd) $\left.{ }_{2}\right] \mathrm{BF}_{4}(3.7 \mathrm{mg}, 0.01$ mmol), dcpm (4.1 mg, $0.01 \mathrm{mmol})$ and acetone $(0.1 \mathrm{~mL})$ for 3 hours at room temperature. Flash column chromatography $\left(\mathrm{SiO}_{2}, 20-40 \% \mathrm{Et}_{2} \mathrm{O}\right.$ in petrol) afforded enone $4 \mathbf{e}$ as a yellow solid (91.3 $\mathrm{mg}, 92 \%$ yield).

m.p.: 67-69 ${ }^{\circ} \mathrm{C}$ (crystalized from $\mathrm{Et}_{2} \mathrm{O} /$ petrol); ${ }^{1} \mathbf{H}-\mathbf{N M R}\left(400 \mathrm{MHz}, \mathrm{CDCl}_{3}\right.$ ): 7.89 $(1 \mathrm{H}, \mathrm{dd}, J 8.0$ and 1.5, C(7)H), $7.78(1 \mathrm{H}, \mathrm{d}, J 16.0, \mathrm{C}(\mathrm{O}) \mathrm{CHCH}), 7.73-7.70(2 \mathrm{H}, \mathrm{m}$, $\mathrm{C}(4) H, \mathrm{C}(5) H), 7.47(1 \mathrm{H}$, ddd, $J$ 8.0, 7.5 and 1.5, C(2)H), $7.38(1 \mathrm{H}, \mathrm{dd}, J 8.0$ and 1.0, $\mathrm{C}(1) H), 7.25(1 \mathrm{H}, \mathrm{d}, J 16.0, \mathrm{C}(\mathrm{O}) \mathrm{CHCH}), 7.23(1 \mathrm{H}, \mathrm{td}, J 7.5$ and 1.0, $\mathrm{C}(3) H), 7.03$ $(1 \mathrm{H}, \mathrm{t}, J$ 8.0, C(6)H), $6.26(1 \mathrm{H}$, br. s, $\mathrm{NH}), 2.46(3 \mathrm{H}, \mathrm{s}, \mathrm{SCH}), 1.48(9 \mathrm{H}$, br. s, $\left.\mathrm{C}\left(\mathrm{CH}_{3}\right)_{3}\right) ;{ }^{13} \mathbf{C}-\mathrm{NMR}\left(100 \mathrm{MHz}, \mathrm{CDCl}_{3}\right): 192.6,153.3,141.6,140.6(2 \times \mathrm{C}), 138.1$, 137.0, 134.3, 131.6, 129.6, 128.6, 127.4, 126.8, 126.3, 124.2, 100.6, 81.2, 28.3, 16.5; LRMS (ESI+, m/z): $518.0\left([\mathrm{M}+\mathrm{Na}]^{+}, 100 \%\right) ;$ HRMS (ESI+, m/z): $\mathrm{C}_{21} \mathrm{H}_{22} \mathrm{INNaO}_{3} \mathrm{~S}$ $\left([\mathrm{M}+\mathrm{Na}]^{+}\right)$requires 518.02573, found 518.02586; IR $v_{\max }($ film $) / \mathrm{cm}^{-1}: 3293,2976$, 2924, 1702, 1654, 1597, 1555, 1483, 1450, 1433, 1392, 1367, 1315, 1244, 1207, 1158, 1115, 1082, 1048, 1014. 


\section{tert-Butyl (E)-(2-(3-(2-(methylthio)phenyl)-3-oxoprop-1-en-1-yl)-4- \\ (trifluoromethyl)phenyl)carbamate, $4 \mathrm{f}$}<smiles>CS(=O)(=O)Nc1ccc(C(F)(F)F)cc1/C=C/C(=O)c1ccccc1S(C)(=O)=O</smiles>

Compound 4f was prepared according to General Procedure A with alkyne 3f (57.1 $\mathrm{mg}, 0.20 \mathrm{mmol})$, aldehyde $2 \mathrm{a}(36.5 \mathrm{mg}, 0.24 \mathrm{mmol}),\left[\mathrm{Rh}(\mathrm{nbd})_{2}\right] \mathrm{BF}_{4}(3.7 \mathrm{mg}, 0.01$ mmol), dcpm (4.1 mg, $0.01 \mathrm{mmol})$ and acetone $(0.1 \mathrm{~mL})$ for 20 minutes at room temperature. Flash column chromatography $\left(\mathrm{SiO}_{2}, 15-30 \% \mathrm{Et}_{2} \mathrm{O}\right.$ in petrol) afforded enone $\mathbf{4 f}$ as a yellow solid (80.6 mg, 92\% yield).

m.p.: $169-171{ }^{\circ} \mathrm{C}$ (crystalized from $\left.\mathrm{CHCl}_{3}\right) ;{ }^{1} \mathbf{H}-\mathbf{N M R}\left(400 \mathrm{MHz}, \mathrm{CDCl}_{3}\right): 8.08(1 \mathrm{H}$, $\mathrm{d}, J 8.5, \mathrm{C}(7) H), 7.79(1 \mathrm{H}, \mathrm{d}, J 15.5, \mathrm{C}(\mathrm{O}) \mathrm{CHCH}), 7.75(1 \mathrm{H}, \mathrm{d}, J 8.0$ and 1.0, C(4)H), $7.72(1 \mathrm{H}, \mathrm{d}, J 1.0, \mathrm{C}(5) H), 7.54(1 \mathrm{H}, \mathrm{dd}, J 8.5$ and $1.5, \mathrm{C}(6) H), 7.44(1 \mathrm{H}, \mathrm{td}, J 7.5$ and 1.5, C(2)H), $7.32(1 \mathrm{H}, \mathrm{d}, J 7.0, \mathrm{C}(1) H), 7.31(1 \mathrm{H}, \mathrm{d}, J 15.5, \mathrm{C}(\mathrm{O}) \mathrm{CHCH}), 7.20(1 \mathrm{H}$, td. $J 7.5$ and 1.0, $\mathrm{C}(3) H), 6.79(1 \mathrm{H}$, br. s, $\mathrm{NH}), 2.42(3 \mathrm{H}, \mathrm{s}, \mathrm{SCH}), 1.47(9 \mathrm{H}, \mathrm{s}$, $\left.\mathrm{C}\left(\mathrm{CH}_{3}\right)_{3}\right) ;{ }^{13} \mathrm{C}-\mathbf{N M R}\left(125 \mathrm{MHz}, \mathrm{CDCl}_{3}\right): 189.9,151.2,140.7,139.0,136.3,135.0$, $131.3,129.0,126.8,126.5$ (q, $J 3.5), 124.9,124.5$ (q, $J 33.0), 124.1,123.4$ (q, $J 3.5)$, 123.2, 122.9 (q, $J 272.0), 120.2,80.8,27.2,15.3 ;{ }^{19}$ F-NMR $\left(376 \mathrm{MHz}, \mathrm{CDCl}_{3}\right)\left\{{ }^{1} \mathrm{H}\right\}$ : -62.2; LRMS (ESI+, m/z): $460.1\left([\mathrm{M}+\mathrm{Na}]^{+}\right) ;$HRMS (ESI+, m/z): $\mathrm{C}_{22} \mathrm{H}_{23} \mathrm{NO}_{3} \mathrm{~F}_{3} \mathrm{~S}$ $\left([\mathrm{M}+\mathrm{H}]^{+}\right)$requires 438.1345 , found 438.1335 ; IR $v_{\max }($ film $) / \mathrm{cm}^{-1}: 1703,1655,1597$, 1519, 1485, 1323, 1271, 1153, 1108, 1006. 
<smiles>CC(C)(C)OC(=O)Nc1ccc(C=O)cc1/C=C/C(=O)c1ccccc1S(C)(=O)=O</smiles>

Compound 4g was prepared according to General Procedure A with alkyne 3g (49.1 $\mathrm{mg}, 0.20 \mathrm{mmol})$, aldehyde $\mathbf{2 a}(36.5 \mathrm{mg}, 0.24 \mathrm{mmol}),\left[\mathrm{Rh}(\mathrm{nbd})_{2}\right] \mathrm{BF}_{4}(3.7 \mathrm{mg}, 0.01$ mmol), dcpm (4.1 mg, $0.01 \mathrm{mmol})$ and acetone $(0.1 \mathrm{~mL})$ for 30 minutes at room temperature. Flash column chromatography $\left(\mathrm{SiO}_{2}, 30-60 \% \mathrm{Et}_{2} \mathrm{O}\right.$ in petrol) afforded enone $\mathbf{4 g}$ as a yellow solid (73.6 $\mathrm{mg}, 93 \%$ yield).

m.p.: $155-157{ }^{\circ} \mathrm{C}$ (crystalized from $\mathrm{Et}_{2} \mathrm{O} /$ petrol); ${ }^{1} \mathbf{H}-\mathbf{N M R}$ (400 MHz, $\mathrm{CDCl}_{3}$ ): 9.94 $(1 \mathrm{H}, \mathrm{s}, \mathrm{CHO}), 8.27(1 \mathrm{H}, \mathrm{d}, J 8.5, \mathrm{C}(7) H), 8.08(1 \mathrm{H}, \mathrm{d}, J 1.5, \mathrm{C}(5) H), 7.87(1 \mathrm{H}, \mathrm{d}, J$ 15.5, $\mathrm{C}(\mathrm{O}) \mathrm{CHCH}), 7.88-7.83(2 \mathrm{H}, \mathrm{m}, \mathrm{C}(4) H, \mathrm{C}(6) H), 7.51(1 \mathrm{H}, \mathrm{td}, J 7.5$ and 1.5, $\mathrm{C}(2) H), 7.45(1 \mathrm{H}, \mathrm{d}, J 15.5, \mathrm{C}(\mathrm{O}) \mathrm{CHCH}), 7.38(1 \mathrm{H}, \mathrm{d}, J 8.0, \mathrm{C}(1) H), 7.26(1 \mathrm{H}, \mathrm{td}, J$ 7.5 and 1.0, C(3)H), $7.11(1 \mathrm{H}$, br. s, $\mathrm{NH}), 2.48(3 \mathrm{H}, \mathrm{s}, \mathrm{SCH}), 1.55\left(9 \mathrm{H}, \mathrm{s}, \mathrm{C}\left(\mathrm{CH}_{3}\right)_{3}\right)$; ${ }^{13}$ C-NMR (100 MHz, $\left.\mathrm{CDCl}_{3}\right): 190.79,190.71,152.0,142.6,142.0,137.3,135.7$, 132.6, 132.3, 131.3, 130.1, 128.7, 127.7, 125.8, 125.0, 124.1, 120.5, 82.0, 28.2, 16.3; LRMS (ESI+, m/z): $452.1 \quad\left([\mathrm{M}+\mathrm{MeOH}+\mathrm{Na}]^{+}, 100 \%\right) ; \quad$ HRMS $(\mathrm{ESI}+, \mathrm{m} / \mathrm{z})$ : $\mathrm{C}_{23} \mathrm{H}_{27} \mathrm{NNaO}_{5} \mathrm{~S}\left([\mathrm{M}+\mathrm{MeOH}+\mathrm{Na}]^{+}\right)$requires 452.15021, found 452.14972; IR $v_{\max }$ (film) $/ \mathrm{cm}^{-1}: 3325,2979,2922,1730,1689,1655,1595,1576,1516,1477,1431,1368$, 1334, 1228, 1149, 1049, 1014. 
tert-Butyl (E)-(4-acetyl-2-(3-(2-(methylthio)phenyl)-3-oxoprop-1-en-1-

yl)phenyl)carbamate, $4 \mathrm{~h}$<smiles>CC(=O)Nc1ccc(C(C)=O)cc1/C=C/C(=O)c1ccccc1S(C)(=O)=O</smiles>

Compound 4h was prepared according to General Procedure A with alkyne 3h (51.9 $\mathrm{mg}, 0.20 \mathrm{mmol})$, aldehyde $\mathbf{2 a}(36.5 \mathrm{mg}, 0.24 \mathrm{mmol}),\left[\mathrm{Rh}(\mathrm{nbd})_{2}\right] \mathrm{BF}_{4}(3.7 \mathrm{mg}, 0.01$ mmol), dcpm (4.1 mg, $0.01 \mathrm{mmol})$ and acetone $(0.1 \mathrm{~mL})$ for 30 minutes at room temperature Flash column chromatography $\left(\mathrm{SiO}_{2}, 30-60 \% \mathrm{Et}_{2} \mathrm{O}\right.$ in petrol) afforded enone $4 \mathbf{h}$ as a yellow solid ( $80.6 \mathrm{mg}, 98 \%$ yield).

m.p.: $134-136{ }^{\circ} \mathrm{C}$ (crystalized from $\mathrm{Et}_{2} \mathrm{O} /$ petrol); ${ }^{1} \mathbf{H}-\mathbf{N M R}\left(400 \mathrm{MHz}, \mathrm{CDCl}_{3}\right): 8.16$ $(1 \mathrm{H}, \mathrm{s}, \mathrm{C}(5) H), 8.12(1 \mathrm{H}, \mathrm{d}, J 8.5, \mathrm{C}(7) H), 7.92(1 \mathrm{H}, \mathrm{d}, J 8.5, \mathrm{C}(6) H), 7.84(1 \mathrm{H}, \mathrm{d}, J$ 15.5, C(O)CHCH), $7.80(1 \mathrm{H}, \mathrm{d}, J 7.5, \mathrm{C}(4) H), 7.48(1 \mathrm{H}, \mathrm{t}, J 7.5, \mathrm{C}(2) H), 7.40(1 \mathrm{H}, \mathrm{d}$, $J$ 15.5, C(O)CHCH), $7.35(1 \mathrm{H}, \mathrm{d}, J 8.0, \mathrm{C}(1) H), 7.23(1 \mathrm{H}, \mathrm{t}, J 7.5, \mathrm{C}(3) H), 7.09(1 \mathrm{H}$, br. s, $\mathrm{NH}), 2.58\left(3 \mathrm{H}, \mathrm{s}, \mathrm{C}(\mathrm{O}) \mathrm{CH}_{3}\right), 2.45(3 \mathrm{H}, \mathrm{s}, \mathrm{SCH}), 1.53\left(9 \mathrm{H}, \mathrm{s}, \mathrm{C}\left(\mathrm{CH}_{3}\right)_{3}\right) ;{ }^{13} \mathrm{C}-$ NMR (100 MHz, $\left.\mathrm{CDCl}_{3}\right)$ : 196.7, 190.9, 152.2, 141.8, 141.4, 137.9, 135.8, 132.22, 132.03, 131.1, 130.1, 127.6, 127.3, 125.7, 124.7, 124.1, 120.3, 81.7, 28.2, 26.4, 16.3; LRMS (ESI+, m/z): $434.1\left([\mathrm{M}+\mathrm{Na}]^{+}, 100 \%\right) ;$ HRMS (ESI+, m/z): $\mathrm{C}_{23} \mathrm{H}_{25} \mathrm{NNaO}_{4} \mathrm{~S}$ $\left([\mathrm{M}+\mathrm{Na}]^{+}\right)$requires 434.13965, found 434.13934; IR $v_{\max }($ film $) / \mathrm{cm}^{-1}: 3313,2979$, 2921, 1727, 1675, 1594, 1574, 1517, 1476, 1433, 1367, 1333, 1284, 1234, 1150, 1048, 1013. 


\section{Methyl (E)-4-((tert-butoxycarbonyl)amino)-3-(3-(2-(methylthio)phenyl)-3- oxoprop-1-en-1-yl)benzoate, $4 \mathbf{i}$}<smiles>CCCCOC(=O)Nc1ccc(C(=O)OC)cc1/C=C/C(=O)c1ccccc1S(C)(=O)=O</smiles>

Compound 4i was prepared according to General Procedure A with alkyne 3i (55.1 mg, $0.20 \mathrm{mmol})$, aldehyde 2a (36.5 mg, $0.24 \mathrm{mmol}),\left[\mathrm{Rh}(\mathrm{nbd})_{2}\right] \mathrm{BF}_{4}(3.7 \mathrm{mg}, 0.01$ mmol), dcpm ( $4.1 \mathrm{mg}, 0.01 \mathrm{mmol})$ and DCE $(0.2 \mathrm{~mL})$ for 30 minutes at $55^{\circ} \mathrm{C}$. Flash column chromatography $\left(\mathrm{SiO}_{2}, 30-50 \% \mathrm{Et}_{2} \mathrm{O}\right.$ in petrol) afforded enone $4 \mathbf{i}$ as a yellow solid (80.3 mg, 94\% yield).

m.p.: $63-65{ }^{\circ} \mathrm{C}$ (crystalized from $\left.\mathrm{CHCl}_{3}\right) ;{ }^{1} \mathbf{H}-\mathbf{N M R}\left(400 \mathrm{MHz}, \mathrm{CDCl}_{3}\right): 8.18(1 \mathrm{H}, \mathrm{d}$, $J$ 2.0, C(5)H), $8.06(1 \mathrm{H}, \mathrm{d}, J 8.5, \mathrm{C}(7) H), 7.95(1 \mathrm{H}, \mathrm{dd}, J 8.5$ and 2.0, C(6)H), 7.89 $(1 \mathrm{H}, \mathrm{d}, J 15.5, \mathrm{C}(\mathrm{O}) \mathrm{CHCH}), 7.76(1 \mathrm{H}, \mathrm{dd}, J 8.5$ and $1.5, \mathrm{C}(4) H), 7.43(1 \mathrm{H}, \mathrm{td}, J 7.5$ and 1.5, C(2)H), $7.35(1 \mathrm{H}, \mathrm{d}, J 15.5, \mathrm{C}(\mathrm{O}) \mathrm{CHCH}), 7.31(1 \mathrm{H}, \mathrm{d}, J 8.0, \mathrm{C}(1) H), 7.19$ $\left(1 \mathrm{H}, \mathrm{td}, J 8.0\right.$ and 1.0, C(3)H), $6.87(1 \mathrm{H}$, br. s, $\mathrm{NH}), 3.84\left(3 \mathrm{H}, \mathrm{s}, \mathrm{CO}_{2} \mathrm{CH}_{3}\right), 2.41(3 \mathrm{H}, \mathrm{s}$, $\left.\mathrm{SCH}_{3}\right), 1.47\left(9 \mathrm{H}, \mathrm{s}, \mathrm{C}\left(\mathrm{CH}_{3}\right)_{3}\right) ;{ }^{13} \mathbf{C}-\mathbf{N M R}\left(100 \mathrm{MHz}, \mathrm{CDCl}_{3}\right): 191.1,166.3,152.2$, $141.8,141.2,137.7,136.1,132.2,132.1,130.1,129.0,127.3,125.9,125.0,124.5$, 124.1, 120.4 81.8, 52.2, 28.3, 16.4; LRMS (ESI+, m/z): 450.1 ([M+Na $\left.]^{+}\right) ;$HRMS $(\mathrm{ESI}+, \mathrm{m} / \mathrm{z}): \mathrm{C}_{23} \mathrm{H}_{25} \mathrm{NNaO}_{5} \mathrm{~S}\left([\mathrm{M}+\mathrm{Na}]^{+}\right)$requires 450.1346, found 450.1331; IR $v_{\max }$ (film) $/ \mathrm{cm}^{-1}:$ 2979, 1717, 1656, 1597, 1518, 1434, 1368, 1288, 1239, 1153, 1049, 1014 


\section{tert-Butyl (E)-(2-(3-(2-(methylthio)phenyl)-3-oxoprop-1-en-1-yl)-4- \\ nitrophenyl)carbamate, $4 \mathrm{j}$}<smiles>CC(C)(C)c1ccccc1C(=O)/C=C/c1cc([N+](=O)[O-])ccc1NS(C)(=O)=O</smiles>

Compound $\mathbf{4 j}$ was prepared according to General Procedure A with alkyne $\mathbf{3 j}$ (52.4 $\mathrm{mg}, 0.20 \mathrm{mmol})$, aldehyde $2 \mathrm{a}(36.5 \mathrm{mg}, 0.24 \mathrm{mmol}),\left[\mathrm{Rh}(\mathrm{nbd})_{2}\right] \mathrm{BF}_{4}(3.7 \mathrm{mg}, 0.01$ mmol), dcpm (4.1 mg, $0.01 \mathrm{mmol})$ and acetone $(0.4 \mathrm{~mL})$ for 20 minutes at room temperature. Flash column chromatography $\left(\mathrm{SiO}_{2}, 0-100 \% \mathrm{CH}_{2} \mathrm{Cl}_{2}\right.$ in petrol) afforded enone $\mathbf{4} \mathbf{j}$ as a yellow solid ( $81.2 \mathrm{mg}, 98 \%$ yield).

m.p.: $174-176{ }^{\circ} \mathrm{C}$ (crystalized from $\mathrm{CHCl}_{3}$ ); ${ }^{1} \mathbf{H}-\mathbf{N M R}\left(400 \mathrm{MHz}, \mathrm{CDCl}_{3}\right): 8.47(1 \mathrm{H}$, $\mathrm{d}, J 2.5, \mathrm{C}(5) H), 8.35(1 \mathrm{H}, \mathrm{d}, J 9.0, \mathrm{C}(7) H), 8.25(1 \mathrm{H}, \mathrm{dd}, J 9.0$ and 2.5, C(6)H), 7.88 $(1 \mathrm{H}, \mathrm{dd}, J 7.5$ and 1.5, C(4)H), $7.86(1 \mathrm{H}, \mathrm{dd}, J 15.5, \mathrm{C}(\mathrm{O}) \mathrm{CHCH}), 7.56(1 \mathrm{H}, \mathrm{ddd}, J$ 8.0, 7.5 and 1.5, $\mathrm{C}(2) H), 7.51(1 \mathrm{H}, \mathrm{d}, J 15.5, \mathrm{C}(\mathrm{O}) \mathrm{CHCH}), 7.43(1 \mathrm{H}, \mathrm{dd}, J$ 8.0, 1.0, $\mathrm{C}(1) H), 7.32(1 \mathrm{H}$, ddd, $J 7.5,7.5$ and 1.0, C(3)H), $7.10(1 \mathrm{H}$, br. s, $\mathrm{N} H), 2.53(3 \mathrm{H}, \mathrm{s}$, $\left.\mathrm{SCH}_{3}\right), 1.58\left(9 \mathrm{H}, \mathrm{s}, \mathrm{C}\left(\mathrm{CH}_{3}\right)_{3}\right)$; ${ }^{13} \mathbf{C}-\mathbf{N M R}\left(125 \mathrm{MHz}, \mathrm{CDCl}_{3}\right): 190.4,151.8,142.80$, 142.77, 142.1, 136.0, 135.6, 132.6, 130.2, 128.8, 125.92, 125.87, 124.7, 124.2, 123.0, 120.1, 82.5, 28.2, 16.4; LRMS (ESI+, m/z): $437.1\left([\mathrm{M}+\mathrm{Na}]^{+}, 100 \%\right) ;$ HRMS (ESI+, $\mathrm{m} / \mathrm{z}): \mathrm{C}_{21} \mathrm{H}_{23} \mathrm{~N}_{2} \mathrm{O}_{5} \mathrm{~S}\left([\mathrm{M}+\mathrm{H}]^{+}\right)$requires 415.13222, found 415.13226; IR $v_{\max }$ (film) $/ \mathrm{cm}^{-1}: 3369,2959,2922,1718,1656,1596,1580,1534,1502,1467,1340,1263$, 1232, 1216, 1157, 1087, 1050, 1009. 
tert-Butyl (E)-(4-cyano-2-(3-(2-(methylthio)phenyl)-3-oxoprop-1-en-1yl)phenyl)carbamate, $4 \mathrm{k}$<smiles>CC(C)(C)OC(=O)Nc1ccc(C#N)cc1/C=C/C(=O)c1ccccc1S(C)(=O)=O</smiles>

Compound 4k was prepared according to General Procedure A with alkyne 3k (48.4 $\mathrm{mg}, 0.20 \mathrm{mmol})$, aldehyde $2 \mathrm{a}(36.5 \mathrm{mg}, 0.24 \mathrm{mmol}),\left[\mathrm{Rh}(\mathrm{nbd})_{2}\right] \mathrm{BF}_{4}(3.7 \mathrm{mg}, 0.01$ mmol), dcpm (4.1 mg, $0.01 \mathrm{mmol})$ and acetone $(0.1 \mathrm{~mL})$ for 30 minutes at room temperature. Flash column chromatography $\left(\mathrm{SiO}_{2}, 0-20 \%\right.$ EtOAc in $\left.\mathrm{CH}_{2} \mathrm{Cl}_{2}\right)$ afforded enone $4 \mathbf{k}$ as a yellow solid $(73.5 \mathrm{mg}, 93 \%$ yield).

m.p.: $172-174{ }^{\circ} \mathrm{C}$ (crystalized from $\left.\mathrm{CHCl}_{3}\right) ;{ }^{1} \mathbf{H}-\mathbf{N M R}\left(400 \mathrm{MHz}, \mathrm{CDCl}_{3}\right): 8.26(1 \mathrm{H}$, $\mathrm{d}, J 8.5, \mathrm{C}(7) H), 7.86-7.83(2 \mathrm{H}, \mathrm{m}, \mathrm{C}(4) H, \mathrm{C}(5) H), 7.83(1 \mathrm{H}, \mathrm{d}, J 15.5, \mathrm{C}(\mathrm{O}) \mathrm{CHCH})$, $7.65(1 \mathrm{H}, \mathrm{dd}, J 8.5$ and 2.0, C(6)H), $7.55(1 \mathrm{H}$, ddd, $J$ 8.0, 7.5 and 1.5, C(2)H), 7.42 $(1 \mathrm{H}, \mathrm{dd}, J 8.0$ and 1.0, C(1)H), $7.41(1 \mathrm{H}, \mathrm{d}, J 15.5, \mathrm{C}(\mathrm{O}) \mathrm{CHCH}), 7.30(1 \mathrm{H}$, ddd, $J 7.5$, 7.5 and 1.0, $\mathrm{C}(3) H), 7.02(1 \mathrm{H}$, br. s, $\mathrm{NH}), 2.52(3 \mathrm{H}, \mathrm{s}, \mathrm{SCH}), 1.57\left(9 \mathrm{H}, \mathrm{s}, \mathrm{C}\left(\mathrm{CH}_{3}\right)_{3}\right)$; ${ }^{13}$ C-NMR (100 MHz, $\left.\mathrm{CDCl}_{3}\right): 190.6,151.9,141.9,141.1,136.2,135.7,134.1,132.5$, $131.4,130.2,128.5,125.9,125.3,124.3,120.8,118.5,106.6,82.3,28.2,16.4$; LRMS (ESI+, m/z): ([M+Na $\left.]^{+}, 100 \%\right) 417.1 ;$ HRMS (ESI+, m/z): $\mathrm{C}_{22} \mathrm{H}_{22} \mathrm{~N}_{2} \mathrm{NaO}_{3} \mathrm{~S}$ $\left([\mathrm{M}+\mathrm{Na}]^{+}\right)$requires 417.12433 , found 417.12417; IR $v_{\max }($ film $) / \mathrm{cm}^{-1}: 3375,2981$, 2924, 2229, 1715, 1655, 1598, 1571, 1508, 1474, 1392, 1368, 1333, 1300, 1273, 1235, 1213, 1160, 1081, 1051, 1011. 
<smiles>Cc1ccc(/C=C/C(=O)c2ccccc2S(C)(=O)=O)c(NC(C)(C)C)c1</smiles>

Compound 4l was prepared according to General Procedure A with alkyne 31 (46.2 $\mathrm{mg}, 0.20 \mathrm{mmol})$, aldehyde $\mathbf{2 a}(36.5 \mathrm{mg}, 0.24 \mathrm{mmol}),\left[\mathrm{Rh}(\mathrm{nbd})_{2}\right] \mathrm{BF}_{4}(3.7 \mathrm{mg}, 0.01$ mmol), dcpm ( $4.1 \mathrm{mg}, 0.01 \mathrm{mmol})$ and acetone $(0.1 \mathrm{~mL})$ for 3 hours at $55^{\circ} \mathrm{C}$. Flash column chromatography $\left(\mathrm{SiO}_{2}, 15-30 \% \mathrm{Et}_{2} \mathrm{O}\right.$ in petrol) afforded enone $\mathbf{4 l}$ as a yellow solid (54.6 mg, 71\% yield).

m.p.: $115-117{ }^{\circ} \mathrm{C}$ (crystalized from $\left.\mathrm{CHCl}_{3}\right) ;{ }^{1} \mathbf{H}$-NMR $\left(400 \mathrm{MHz}, \mathrm{CDCl}_{3}\right): 7.79(1 \mathrm{H}$, $\mathrm{d}, J 15.5, \mathrm{C}(\mathrm{O}) \mathrm{CHCH}), 7.69(1 \mathrm{H}, \mathrm{dd}, J 7.5$ and $1.5, \mathrm{C}(4) H), 7.63(1 \mathrm{H}$, br. s, C(7)H), $7.44(1 \mathrm{H}, \mathrm{d}, J 8.0, \mathrm{C}(5) H), 7.41(1 \mathrm{H}, \mathrm{td}, J 8.0$ and 1.5, $\mathrm{C}(2) H), 7.30(1 \mathrm{H}, \mathrm{d}, J 8.0$, $\mathrm{C}(1) H), 7.21(1 \mathrm{H}, \mathrm{d}, J 15.5, \mathrm{C}(\mathrm{O}) \mathrm{CHCH}), 7.16(1 \mathrm{H}, \mathrm{t}, J 7.0, \mathrm{C}(3) H), 6.87(1 \mathrm{H}, \mathrm{d}, J$ 8.0, C(6)H), $6.56\left(1 \mathrm{H}\right.$, br. s, NH), $2.40(3 \mathrm{H}, \mathrm{s}, \mathrm{SCH}), 2.29\left(3 \mathrm{H}, \mathrm{s}, \mathrm{ArCH}_{3}\right), 1.45(9 \mathrm{H}$, s, $\left.\mathrm{C}\left(\mathrm{CH}_{3}\right)_{3}\right) ;{ }^{13} \mathrm{C}-\mathbf{N M R}\left(100 \mathrm{MHz}, \mathrm{CDCl}_{3}\right): 190.9,151.9,141.0,140.0,138.4,136.1$, 135.8, 130.7, 128.7, 126.1, 124.9, 124.2, 124.1, 123.1, 122.3, 122.0, 79.9, 27.3, 20.7, 15.4; LRMS (ESI+, m/z): $406.1\left([\mathrm{M}+\mathrm{Na}]^{+}\right) ;$HRMS (ESI+, m/z): $\mathrm{C}_{22} \mathrm{H}_{25} \mathrm{NNaO}_{3} \mathrm{~S}$ $\left([\mathrm{M}+\mathrm{Na}]^{+}\right)$requires 406.1447, found 406.1440; IR $v_{\max }($ film $) / \mathrm{cm}^{-1}: 2922,2361,2342$, $1726,1653,1592,1523,1458,1367,1243,1158,1050$. 


\section{Di-tert-butyl (2-(3-(2-(methylthio)phenyl)-3-oxoprop-1-en-1-yl)-1,4- phenylene)(E)-dicarbamate, $4 \mathrm{~m}$}<smiles>CC(C)(C)OC(=O)Nc1ccc(NC(=O)OCc2ccccc2)c(/C=C/C(=O)c2ccccc2S(C)(=O)=O)c1</smiles>

Compound 4m was prepared according to General Procedure A with alkyne 3m (66.4 $\mathrm{mg}, 0.20 \mathrm{mmol})$, aldehyde $2 \mathrm{a}(36.5 \mathrm{mg}, 0.24 \mathrm{mmol}),\left[\mathrm{Rh}(\mathrm{nbd})_{2}\right] \mathrm{BF}_{4}(3.7 \mathrm{mg}, 0.01$ mmol), dcpm (4.1 mg, $0.01 \mathrm{mmol})$ and acetone $(0.1 \mathrm{~mL})$ for 7 hours at room temperature. Flash column chromatography $\left(\mathrm{SiO}_{2}, 20-30 \% \mathrm{Et}_{2} \mathrm{O}\right.$ in petrol) afforded enone $\mathbf{4 m}$ as a yellow solid (87.1 $\mathrm{mg}, 90 \%$ yield).

m.p.: $108-110{ }^{\circ} \mathrm{C}$ (crystalized from $\left.\mathrm{CHCl}_{3}\right) ;{ }^{1} \mathbf{H}-\mathbf{N M R}\left(400 \mathrm{MHz}, \mathrm{CDCl}_{3}\right): 7.82(1 \mathrm{H}$, d, $J$ 15.5, C(O)CHCH), $7.77(1 \mathrm{H}$, br. s, C $(5) H), 7.75(1 \mathrm{H}, \mathrm{dd}, J 7.5$ and 1.5, C(4)H), $7.60(1 \mathrm{H}, \mathrm{d}, J 7.5, \mathrm{C}(7) H), 7.47(1 \mathrm{H}$, ddd, $J 8.0,7.5$ and 1.5, C(2)H), $7.36(1 \mathrm{H}, \mathrm{dd}, J$ 8.0, 1.0, C(1)H), $7.27(1 \mathrm{H}, \mathrm{d}, J 15.5, \mathrm{C}(\mathrm{O}) \mathrm{CHCH}), 7.24-7.20(2 \mathrm{H}, \mathrm{m}, \mathrm{C}(6) H, \mathrm{C}(3) H)$, $6.85(1 \mathrm{H}$, br. s, $\mathrm{NH}), 6.64(1 \mathrm{H}$, br. s, $\mathrm{NH}), 2.47(3 \mathrm{H}, \mathrm{s}, \mathrm{SCH}), 1.52\left(9 \mathrm{H}, \mathrm{s}, \mathrm{C}\left(\mathrm{CH}_{3}\right)_{3}\right)$, $1.51\left(9 \mathrm{H}, \mathrm{s}, \mathrm{C}\left(\mathrm{CH}_{3}\right)_{3}\right) ;{ }^{13} \mathrm{C}-\mathrm{NMR}\left(100 \mathrm{MHz}, \mathrm{CDCl}_{3}\right)$ : 191.9, 153.4, 152.9, 141.1, $139.4,136.6,135.3,132.2,131.8,129.9,127.8,126.2,125.9,124.5,124.1,121.5$, 116.7, 80.8, 80.6, 28.4, 28.3, 16.4; LRMS (ESI+, m/z): $507.2\left([\mathrm{M}+\mathrm{Na}]^{+}, 100 \%\right)$; HRMS (ESI+, m/z): $\mathrm{C}_{26} \mathrm{H}_{32} \mathrm{~N}_{2} \mathrm{NaO}_{5} \mathrm{~S} \quad\left([\mathrm{M}+\mathrm{Na}]^{+}\right)$requires 507.19241, found 507.19227; IR $v_{\max }\left(\right.$ film) $/ \mathrm{cm}^{-1}: 3319,2978,2925,1696,1656,1588,1509,1433$, 1417, 1392, 1367, 1279, 1242, 1152, 1059, 1018. 
tert-Butyl (E)-(2-hydroxy-6-(3-(2-(methylthio)phenyl)-3-oxoprop-1-en-1yl)phenyl)carbamate, $4 \mathrm{n}$<smiles>CSc1ccccc1C(=O)/C=C/c1cccc(O)c1NC(=O)OC(C)(C)C</smiles>

Compound 4n was prepared according to General Procedure A with alkyne 3n (46.6 $\mathrm{mg}, 0.20 \mathrm{mmol})$, aldehyde $2 \mathrm{a}(36.5 \mathrm{mg}, 0.24 \mathrm{mmol}),\left[\mathrm{Rh}(\mathrm{nbd})_{2}\right] \mathrm{BF}_{4}(3.7 \mathrm{mg}, 0.01$ mmol), dcpm (4.1 mg, $0.01 \mathrm{mmol})$ and acetone $(0.1 \mathrm{~mL})$ for 16 hours at $55^{\circ} \mathrm{C}$. Flash column chromatography $\left(\mathrm{SiO}_{2}, 0-10 \%\right.$ EtOAc in $\left.\mathrm{CH}_{2} \mathrm{Cl}_{2}\right)$ afforded enone $4 \mathbf{n}$ as a yellow solid (69.0 mg, 89\% yield).

m.p.: $95-97{ }^{\circ} \mathrm{C}$ (crystalized from $\left.\mathrm{CHCl}_{3}\right)$; ${ }^{\mathbf{1}} \mathbf{H}$-NMR (400 MHz, $\left.\mathrm{CDCl}_{3}\right)$ : $8.25(1 \mathrm{H}, \mathrm{br}$. s, OH), $7.87(1 \mathrm{H}, \mathrm{d}, J 15.5, \mathrm{C}(\mathrm{O}) \mathrm{CHCH}), 7.76(1 \mathrm{H}, \mathrm{dd}, J 7.5$ and $1.5, \mathrm{C}(4) H), 7.49$ $(1 \mathrm{H}, \mathrm{ddd}, J 8.0,7.5$ and 1.5, C(2)H), $7.37(1 \mathrm{H}, \mathrm{dd}, J 8.0$ and 1.0, C(1)H), $7.29(1 \mathrm{H}, \mathrm{d}$, $J$ 15.5, C(O)CHCH), 7.26-7.22 (2H, m, C(3)H, C(5)H), $7.16(1 \mathrm{H}, \mathrm{t}, J$ 8.0, C(6)H), $7.09\left(1 \mathrm{H}, \mathrm{dd}, J 8.0\right.$ and 1.5, C(7)H), $6.98(1 \mathrm{H}$, br. s, $\mathrm{NH}), 2.47(3 \mathrm{H}, \mathrm{s}, \mathrm{SCH})_{3}, 1.54$ $\left(9 \mathrm{H}, \mathrm{s}, \mathrm{C}\left(\mathrm{CH}_{3}\right)_{3}\right) ;{ }^{13} \mathrm{C}-\mathrm{NMR}\left(100 \mathrm{MHz}, \mathrm{CDCl}_{3}\right)$ : 192.0, 155.5, 150.8, 141.2, 140.3, 136.4, 132.0, $129.9(2 \times \mathrm{C}), 127.1,126.6,125.9,124.6,124.2,121.7,119.6,82.8$, 28.2, 16.3; LRMS (ESI+, m/z): $408.1\left([\mathrm{M}+\mathrm{Na}]^{+}, 100 \%\right) ; \quad$ HRMS (ESI+, m/z): $\mathrm{C}_{21} \mathrm{H}_{23} \mathrm{NNaO}_{4} \mathrm{~S}\left([\mathrm{M}+\mathrm{Na}]^{+}\right)$requires 408.12400 , found $408.12404 ;$ IR $v_{\max }($ film $) / \mathrm{cm}^{-}$ ${ }^{1}: 3303,2978,2924,1682,1654,1580,1460,1368,1247,1154,1050,1020$. 
tert-Butyl (E)-(5-methoxy-2-(3-(2-(methylthio)phenyl)-3-oxoprop-1-en-1yl)phenyl)carbamate, 40<smiles>COC(=O)Nc1cc(OC)ccc1/C=C/C(=O)c1ccccc1S(C)(=O)=O</smiles>

Compound 4o was prepared according to General Procedure A with alkyne 3o (49.5 mg, $0.20 \mathrm{mmol})$, aldehyde $2 \mathrm{a}(36.5 \mathrm{mg}, 0.24 \mathrm{mmol})$, $\left[\mathrm{Rh}(\mathrm{nbd})_{2}\right] \mathrm{BF}_{4}(3.7 \mathrm{mg}, 0.01$ mmol), dcpm $(4.1 \mathrm{mg}, 0.01 \mathrm{mmol})$ and acetone $(0.1 \mathrm{~mL})$ for 16 hours at $55{ }^{\circ} \mathrm{C}$. Flash column chromatography $\left(\mathrm{SiO}_{2}, 30-60 \% \mathrm{Et}_{2} \mathrm{O}\right.$ in petrol) afforded enone 40 as a yellow oil (41.4 mg, 51\% yield).

${ }^{1}$ H-NMR (400 MHz, $\left.\mathrm{CDCl}_{3}\right): 7.87(1 \mathrm{H}, \mathrm{d}, J$ 15.5, C(O)CHCH), $7.78(1 \mathrm{H}, \mathrm{dd}, J 7.5$ and 1.5, C(4)H), $7.59(1 \mathrm{H}, \mathrm{d}, J 2.5, \mathrm{C}(7) H), 7.59(1 \mathrm{H}, \mathrm{d}, J 9.0, \mathrm{C}(5) H), 7.50(1 \mathrm{H}$, ddd, $J$ 8.0, 7.5 and 1.5, C(2)H), $7.40(1 \mathrm{H}$, ddd, $J$ 8.0, 1.0 and 0.5, C(1)H), $7.26(1 \mathrm{H}$, ddd, $J$ 7.5, 7.5 and 1.0, C(3)H), $7.26(1 \mathrm{H}, \mathrm{d}, J 15.5, \mathrm{C}(\mathrm{O}) \mathrm{CHCH}), 6.78(1 \mathrm{H}$, br. s, $\mathrm{NH}), 6.70$ $(1 \mathrm{H}$, ddd, $J$ 9.0, 2.5 and 0.5, C $(6) H), 3.88\left(3 \mathrm{H}, \mathrm{s}, \mathrm{OCH}_{3}\right), 2.50\left(3 \mathrm{H}, \mathrm{s}, \mathrm{SCH}_{3}\right), 1.56$ $\left(9 \mathrm{H}, \mathrm{s}, \mathrm{C}\left(\mathrm{CH}_{3}\right)_{3}\right) ;{ }^{13} \mathrm{C}-\mathrm{NMR}\left(100 \mathrm{MHz}, \mathrm{CDCl}_{3}\right): 191.8,162.3,152.6,140.9,139.05$, $138.85,137.1,131.7,129.6,128.5,125.9,124.1,123.5,117.8,111.2,106.1,81.1$, 55.5, 28.3, 16.4; LRMS (ESI+, m/z): ([M+Na $\left.]^{+}, 100 \%\right)$ 422.1; HRMS (ESI+, m/z): $\mathrm{C}_{22} \mathrm{H}_{25} \mathrm{NNaO}_{4} \mathrm{~S}\left([\mathrm{M}+\mathrm{Na}]^{+}\right)$requires 422.13965 , found $422.13953 ;$ IR $v_{\max }\left(\right.$ film) $/ \mathrm{cm}^{-}$ ': 3302, 2976, 2924, 1723, 1651, 1586, 1524, 1478, 1434, 1367, 1306, 1270, 1239, 1207, 1157, 1049, 1016. 


\section{Di-tert-butyl (3,6-bis((E)-3-(2-(methylthio)phenyl)-3-oxoprop-1-en-1-yl)-1,2- phenylene)dicarbamate, $4 p$}

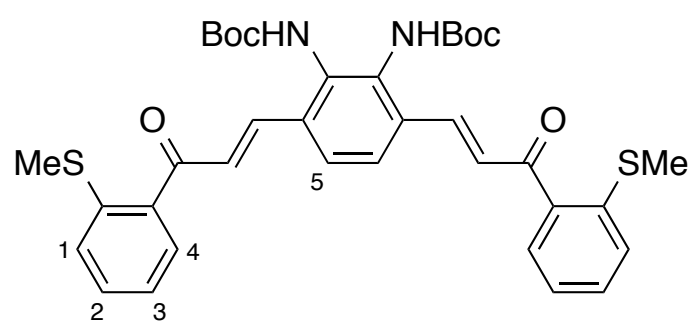

Compound 4p was prepared according to General Procedure A with dialkyne $\mathbf{3 p}$ (71.3 $\mathrm{mg}, 0.20 \mathrm{mmol})$, aldehyde $\mathbf{2 a}(73.1 \mathrm{mg}, 0.48 \mathrm{mmol}),\left[\mathrm{Rh}(\mathrm{nbd})_{2}\right] \mathrm{BF}_{4}(3.7 \mathrm{mg}, 0.01$ mmol), depm $(4.1 \mathrm{mg}, 0.01 \mathrm{mmol})$ and acetone $(0.1 \mathrm{~mL})$ for 15 minutes at $55^{\circ} \mathrm{C}$. Flash column chromatography $\left(\mathrm{SiO}_{2}, 0-100 \% \mathrm{Et}_{2} \mathrm{O}\right.$ in petrol) afforded enone $4 \mathbf{p}$ as a yellow solid (126.5 mg, 96\% yield).

m.p.: $158-160{ }^{\circ} \mathrm{C}$ (crystalized from $\left.\mathrm{CHCl}_{3}\right) ;{ }^{1} \mathbf{H}-\mathbf{N M R}\left(400 \mathrm{MHz}, \mathrm{CDCl}_{3}\right): 7.82(2 \mathrm{H}$, $\mathrm{d}, J$ 16.0, C(O)CHCH), $7.75(2 \mathrm{H}, \mathrm{dd}, J 7.5$ and 1.5, C(4)H), $7.67(2 \mathrm{H}, \mathrm{s}, \mathrm{C}(5) H), 7.49$ $(2 \mathrm{H}, \mathrm{td}, J 7.5$ and 1.5, C(2)H), $7.39(2 \mathrm{H}, \mathrm{d}, J 7.5, \mathrm{C}(1) H), 7.34(2 \mathrm{H}, \mathrm{d}, J$ 16.0, $\mathrm{C}(\mathrm{O}) \mathrm{CHCH}), 7.25(2 \mathrm{H}, \mathrm{td}, J 7.5$ and 1.0, C(3)H), $6.87(2 \mathrm{H}$, br. s, $\mathrm{NH}), 2.47(6 \mathrm{H}, \mathrm{s}$, $\left.\mathrm{SCH}_{3}\right), 1.50\left(18 \mathrm{H}\right.$, br. s, $\left.\mathrm{C}\left(\mathrm{CH}_{3}\right)_{3}\right) ;{ }^{13} \mathrm{C}-\mathrm{NMR}\left(100 \mathrm{MHz}, \mathrm{CDCl}_{3}\right): 192.3,153.9$, $140.9,139.8,136.7,134.5,133.4,131.7,129.7,127.1,126.1,125.3,124.2,81.2,28.2$, 16.4; LRMS (ESI+, m/z): ([M+Na $\left.]^{+}, 100 \%\right)$ 683.2; HRMS $(\mathrm{ESI}+, \mathrm{m} / \mathrm{z})$ : $\mathrm{C}_{36} \mathrm{H}_{40} \mathrm{~N}_{2} \mathrm{NaO}_{6} \mathrm{~S}_{2}\left([\mathrm{M}+\mathrm{Na}]^{+}\right)$requires 683.22200 , found 683.22168; IR $v_{\max }$ (film) $/ \mathrm{cm}^{-1}: 3270,2978,2922,1706,1655,1597,1559,1512,1468,1433,1367,1323$, $1253,1205,1167,1016$. 


\section{Di-tert-butyl (3,6-bis((E)-4-((ethylthio)methyl)-3-oxodec-1-en-1-yl)-1,2- phenylene)dicarbamate, $4 q$}

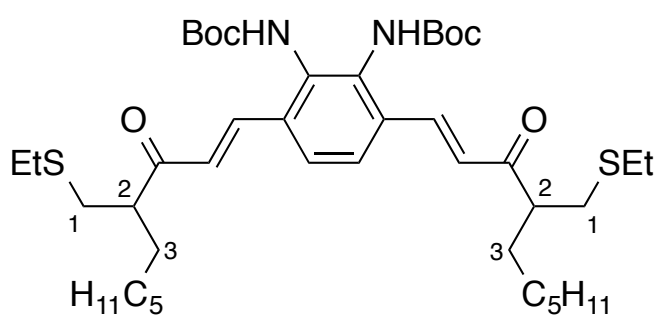

Compound $\mathbf{4 q}$ was prepared according to General Procedure A with dialkyne $\mathbf{3 p}$ (71.3 $\mathrm{mg}, 0.20 \mathrm{mmol})$, aldehyde $2 \mathbf{u}(103.9 \mathrm{mg}, 0.48 \mathrm{mmol}),\left[\mathrm{Rh}(\mathrm{nbd})_{2}\right] \mathrm{BF}_{4}(3.7 \mathrm{mg}, 0.01$ mmol), dcpm $(4.1 \mathrm{mg}, 0.01 \mathrm{mmol})$ and acetone $(0.3 \mathrm{~mL})$ for $1 \mathrm{hr}$ at $55{ }^{\circ} \mathrm{C}$. Flash column chromatography $\left(\mathrm{SiO}_{2}, 20-30 \% \mathrm{Et}_{2} \mathrm{O}\right.$ in petrol) afforded enone $\mathbf{4 q}$ as a yellow oil (138.7 mg, 91\% yield).

(isolated as an indistinguishable pair of diasteromers) ${ }^{\mathbf{1}} \mathbf{H}-\mathbf{N M R}\left(400 \mathrm{MHz}, \mathrm{CDCl}_{3}\right)$ : 7.79 (2H, d, J 16.0, C(O)CHCH), 7.60 (2H, s, ArH), 6.84 (2H, br. s, NH), $6.82(2 \mathrm{H}, \mathrm{d}$, $J$ 16.0, $\mathrm{C}(\mathrm{O}) \mathrm{CHCH}), 3.02(2 \mathrm{H}, \mathrm{tt}, J 8.0$ and 6.0, $\mathrm{C}(2) H), 2.85(2 \mathrm{H}, \mathrm{dd}, J 13.0$ and 8.0, $\left.\mathrm{C}(1) H_{\mathrm{A}} \mathrm{H}_{\mathrm{B}}\right), 2.65\left(2 \mathrm{H}, \mathrm{dd}, J 13.0\right.$ and 6.0, $\left.\mathrm{C}(1) \mathrm{H}_{\mathrm{A}} H_{B}\right), 2.53\left(4 \mathrm{H}, \mathrm{q}, J 7.5, \mathrm{SCH}_{2} \mathrm{CH}_{3}\right)$, 1.77-1.68 (2H, m, C(3) $\left.H_{\mathrm{A}} \mathrm{H}_{\mathrm{B}}\right), 1.64-1.57\left(2 \mathrm{H}, \mathrm{m}, \mathrm{C}(3) \mathrm{H}_{\mathrm{A}} H_{\mathrm{B}}\right), 1.50(18 \mathrm{H}$, br. s, $\left.\mathrm{C}\left(\mathrm{CH}_{3}\right)_{3}\right), 1.31-1.22\left(16 \mathrm{H}, \mathrm{m},\left(\mathrm{CH}_{2}\right)_{4} \mathrm{CH}_{3}\right), 1.24\left(6 \mathrm{H}, \mathrm{t}, J 7.5, \mathrm{SCH}_{2} \mathrm{CH}_{3}\right), 0.86(6 \mathrm{H}, \mathrm{t}$, $J$ 7.0, $\left.\left(\mathrm{CH}_{2}\right)_{4} \mathrm{CH}_{3}\right) ;{ }^{13} \mathbf{C}-\mathbf{N M R}\left(100 \mathrm{MHz}, \mathrm{CDCl}_{3}\right): 201.9,153.9,137.6,134.2,133.5$, $127.9,125.1,81.3,50.7,33.0,32.0,31.6,29.4,28.2,27.0,26.8,22.5,14.7,14.0$; MS: no molecular ion was observed in ESI, EI/FI or MALDI experiments; IR $v_{\max }$ (film) $/ \mathrm{cm}^{-1}: 3255,2958,2927,2856,1693,1604,1561,1521,1471,1456,1391,1367$, 1313, 1278, 1254, 1174, 1111, 1072. 
tert-Butyl (E)-(4-bromo-2-(3-(3-methoxy-2-(methylthio)phenyl)-3-oxoprop-1-en1-yl)phenyl)carbamate, $4 \mathrm{r}$<smiles>COc1cccc(C(=O)/C=C/c2cc(Br)ccc2NC(=O)OCc2ccccc2)c1S(C)(C)C</smiles>

Compound 4r was prepared according to General Procedure A with alkyne 3d (59.2 $\mathrm{mg}, 0.20 \mathrm{mmol})$, aldehyde $2 \mathrm{r}(43.2 \mathrm{mg}, 0.24 \mathrm{mmol}),\left[\mathrm{Rh}(\mathrm{nbd})_{2}\right] \mathrm{BF}_{4}(3.7 \mathrm{mg}, 0.01$ mmol), dcpm (4.1 mg, $0.01 \mathrm{mmol})$ and acetone $(0.1 \mathrm{~mL})$ for 3 hours at room temperature. Flash column chromatography $\left(\mathrm{SiO}_{2}, 40 \% \mathrm{Et}_{2} \mathrm{O}\right.$ in petrol) afforded enone $4 \mathrm{r}$ as a yellow oil (92.5 $\mathrm{mg}, 97 \%$ yield).

${ }^{1}$ H-NMR (400 MHz, CDCl $): 7.74(1 \mathrm{H}, \mathrm{d}, J$ 9.0, C(6)H), $7.66(1 \mathrm{H}, \mathrm{d}, J$ 2.0, C(4)H), $7.58(1 \mathrm{H}, \mathrm{d}, J 16.0, \mathrm{C}(\mathrm{O}) \mathrm{CHCH}), 7.46(1 \mathrm{H}, \mathrm{dd}, J 9.0$ and $2.0, \mathrm{C}(5) H), 7.40(1 \mathrm{H}, \mathrm{t}, J$ 8.0, C(2)H), $7.04(1 \mathrm{H}, \mathrm{d}, J 16.0, \mathrm{C}(\mathrm{O}) \mathrm{CHCH}), 7.03(2 \mathrm{H}$, ap. d, J 8.0, C(1)H, C(3)H), $6.62(1 \mathrm{H}$, br. s, $\mathrm{NH}), 3.98\left(3 \mathrm{H}, \mathrm{s}, \mathrm{OCH}_{3}\right), 2.40(3 \mathrm{H}, \mathrm{s}, \mathrm{SCH}), 1.52\left(9 \mathrm{H}, \mathrm{s}, \mathrm{C}\left(\mathrm{CH}_{3}\right)_{3}\right)$; ${ }^{13}$ C-NMR (100 MHz, $\mathrm{CDCl}_{3}$ ): 194.5, 160.1, 152.6, 145.9, 136.3, 136.0, 133.5, 130.2, 129.91, 129.78, 128.1, 124.1, 121.5, 119.8, 117.1, 112.7, 81.3, 56.1, 28.3, 18.8; LRMS (ESI+, m/z): $500.1\left(\left[\mathrm{M}\left({ }^{79} \mathrm{Br}\right)+\mathrm{Na}\right]^{+}, 100 \%\right), 502.0\left(\left[\mathrm{M}\left({ }^{81} \mathrm{Br}\right)+\mathrm{Na}\right]^{+}, 100 \%\right)$; HRMS $(\mathrm{ESI}+, \mathrm{m} / \mathrm{z}): \mathrm{C}_{22} \mathrm{H}_{24} \mathrm{BrNNaO}_{4} \mathrm{~S}\left(\left[\mathrm{M}\left({ }^{79} \mathrm{Br}\right)+\mathrm{Na}\right]^{+}\right)$requires 500.05016, found 500.05039, $\left(\left[\mathrm{M}\left({ }^{81} \mathrm{Br}\right)+\mathrm{Na}\right]^{+}\right)$requires 502.04812, found 502.04805; IR $v_{\max }$ (film) $/ \mathrm{cm}^{-1}:$ 3310, 3064, 2977, 2926, 2839, 2248, 1721, 1653, 1599, 1568, 1505, 1464, 1424, 1403, 1393, 1367, 1319, 1301, 1262, 1245, 1155, 1117, 1081, 1048, 1026. 
tert-Butyl (E)-(4-bromo-2-(3-(2-(methylthio)-5-(trifluoromethyl)phenyl)-3-

oxoprop-1-en-1-yl)phenyl)carbamate, $4 \mathrm{~s}$<smiles>CC(C)(C)OC(=O)c1ccc(Br)cc1/C=C/C(=O)c1cc(C(F)(F)F)ccc1S(C)(=O)=O</smiles>

Compound 4s was prepared according to General Procedure A with alkyne 3d (59.2 $\mathrm{mg}, 0.20 \mathrm{mmol})$, aldehyde $2 \mathrm{~s}(52.8 \mathrm{mg}, 0.24 \mathrm{mmol}),\left[\mathrm{Rh}(\mathrm{nbd})_{2}\right] \mathrm{BF}_{4}(3.7 \mathrm{mg}, 0.01$ mmol), dcpm (4.1 mg, $0.01 \mathrm{mmol})$ and acetone $(0.1 \mathrm{~mL})$ for 2 hours at room temperature. Flash column chromatography $\left(\mathrm{SiO}_{2}, 40-100 \% \mathrm{CH}_{2} \mathrm{Cl}_{2}\right.$ in petrol) afforded enone $\mathbf{4 s}$ as a yellow solid (92.8 $\mathrm{mg}, 90 \%$ yield).

m.p.: $169-171{ }^{\circ} \mathrm{C}$ (crystalized from $\left.\mathrm{CHCl}_{3}\right) ;{ }^{1} \mathbf{H}-\mathbf{N M R}\left(400 \mathrm{MHz}, \mathrm{CDCl}_{3}\right): 8.01(1 \mathrm{H}$, d, $J 1.5, \mathrm{C}(3) H), 7.87(1 \mathrm{H}, \mathrm{d}, J 15.5, \mathrm{C}(\mathrm{O}) \mathrm{CHCH}), 7.83(1 \mathrm{H}, \mathrm{d}, J 9.0, \mathrm{C}(6) H), 7.76$ $(1 \mathrm{H}, \mathrm{d}, J 2.5, \mathrm{C}(4) H), 7.75(1 \mathrm{H}, \mathrm{dd}, J 8.5$ and $1.5, \mathrm{C}(2) H), 7.53(1 \mathrm{H}, \mathrm{dd}, J 9.0$ and 2.5 , $\mathrm{C}(5) H), 7.50(1 \mathrm{H}, \mathrm{d}, J 8.5, \mathrm{C}(1) H), 7.32(1 \mathrm{H}, \mathrm{d}, J 15.5, \mathrm{C}(\mathrm{O}) \mathrm{CHCH}), 6.60(1 \mathrm{H}$, br. s, $\mathrm{NH}), 2.56\left(3 \mathrm{H}, \mathrm{s}, \mathrm{SCH} \mathrm{H}_{3}\right), 1.55\left(9 \mathrm{H}, \mathrm{s}, \mathrm{C}\left(\mathrm{CH}_{3}\right)_{3}\right) ;{ }^{13} \mathrm{C}-\mathrm{NMR}\left(125 \mathrm{MHz}, \mathrm{CDCl}_{3}\right): 190.1$, $152.5,146.7,138.8,136.4,135.9,134.0,129.8,128.3$ (q, $J 3.5), 127.5,126.4$ (q, $J$ 4.0), 126.3 (q, $J 33.5), 126.1,125.7,124.1,123.8$ (q, $J 272.0), 117.1,81.6,28.3,16.2$; ${ }^{19}$ F-NMR $\left(470 \mathrm{MHz}, \mathrm{CDCl}_{3}\right)\left\{{ }^{1} \mathrm{H}\right\}:-62.3$; LRMS (ESI+, m/z): $538.0\left(\mathrm{M}\left({ }^{79} \mathrm{Br}\right)+\mathrm{Na}\right.$, $100 \%), 540.0\left(\mathrm{M}\left({ }^{81} \mathrm{Br}\right)+\mathrm{Na}, 100 \%\right)$; HRMS (ESI+, $\left.\mathrm{m} / \mathrm{z}\right): \mathrm{C}_{22} \mathrm{H}_{21} \mathrm{BrF}_{3} \mathrm{NNaO}_{3} \mathrm{~S}$ $\left(\left[\mathrm{M}\left({ }^{79} \mathrm{Br}\right)+\mathrm{Na}\right]^{+}\right)$requires 538.02698, found 538.02710, $\left(\left[\mathrm{M}\left({ }^{81} \mathrm{Br}\right)+\mathrm{Na}\right]^{+}\right)$requires 540.02494 , found 540.02483; IR $v_{\max }($ film $) / \mathrm{cm}^{-1}: 3358,2977,2923,2852,1698$, $1657,1593,1566,1509,1467,1447,1404,1393,1359,1344,1314,1273,1241,1201$, $1175,1159,1116,1096,1069,1054,1027,1010$. 


\section{tert-Butyl (E)-(4-bromo-2-(3-(2-(methylthio)thiophen-3-yl)-3-oxoprop-1-en-1- yl)phenyl)carbamate, $4 \mathrm{t}$}<smiles>CC(C)(C)OC(=O)Nc1ccc(Br)cc1/C=C/C(=O)c1ccsc1S(C)(=O)=O</smiles>

Compound 4t was prepared according to General Procedure A with alkyne 3d (59.2 $\mathrm{mg}, 0.20 \mathrm{mmol})$, aldehyde $2 \mathrm{t}(37.9 \mathrm{mg}, 0.24 \mathrm{mmol}),\left[\mathrm{Rh}(\mathrm{nbd})_{2}\right] \mathrm{BF}_{4}(3.7 \mathrm{mg}, 0.01$ mmol), dcpm ( $4.1 \mathrm{mg}, 0.01 \mathrm{mmol})$ and acetone $(0.3 \mathrm{~mL})$ for 16 hours at $55^{\circ} \mathrm{C}$. Flash column chromatography $\left(\mathrm{SiO}_{2}, 40-60 \% \mathrm{Et}_{2} \mathrm{O}\right.$ in petrol) afforded enone $4 \mathbf{t}$ as a yellow solid (67.7 $\mathrm{mg}$, 74\% yield).

m.p.: $183-185{ }^{\circ} \mathrm{C}$ (crystalized from $\mathrm{Et}_{2} \mathrm{O} /$ petrol); ${ }^{\mathbf{1}} \mathbf{H}$-NMR (400 MHz, $\mathrm{CDCl}_{3}$ ): 7.89 $(1 \mathrm{H}, \mathrm{d}, J 15.0, \mathrm{C}(\mathrm{O}) \mathrm{CHCH}), 7.86(1 \mathrm{H}, \mathrm{d}, J 9.0, \mathrm{C}(5) H), 7.72(1 \mathrm{H}, \mathrm{d}, J 2.5, \mathrm{C}(3) H)$, $7.67(1 \mathrm{H}, \mathrm{d}, J 5.0, \mathrm{C}(2) H), 7.49(1 \mathrm{H}, \mathrm{dd}, J 9.0$ and $2.5, \mathrm{C}(4) H), 7.22(1 \mathrm{H}, \mathrm{d}, J 15.0$, $\mathrm{C}(\mathrm{O}) \mathrm{CHCH}), 7.12(1 \mathrm{H}, \mathrm{d}, J 5.0, \mathrm{C}(1) H), 6.64(1 \mathrm{H}$, br. s, $\mathrm{N} H), 2.61(3 \mathrm{H}, \mathrm{s}, \mathrm{SCH})_{3}$, $1.55\left(9 \mathrm{H}, \mathrm{s}, \mathrm{C}\left(\mathrm{CH}_{3}\right)_{3}\right) ;{ }^{13} \mathbf{C}-\mathbf{N M R}\left(100 \mathrm{MHz}, \mathrm{CDCl}_{3}\right): 180.5,152.5,148.1,136.3(2 \times$ C), 133.6, 131.8, 130.8, 129.6, 127.4, 126.6, 126.5, 123.7, 116.7, 81.4, 28.3, 16.8; LRMS $($ ESI,$+ \mathrm{m} / \mathrm{z}): 476.0\left(\left[\mathrm{M}\left({ }^{79} \mathrm{Br}\right)+\mathrm{Na}\right]^{+}\right), 478.0\left(\left[\mathrm{M}\left({ }^{81} \mathrm{Br}\right)+\mathrm{Na}\right]^{+}, 100 \%\right) ;$ HRMS $(\mathrm{ESI}+, \quad \mathrm{m} / \mathrm{z}): \quad \mathrm{C}_{19} \mathrm{H}_{20} \mathrm{BrNNaO}_{3} \mathrm{~S}_{2}\left(\left[\mathrm{M}\left({ }^{79} \mathrm{Br}\right)+\mathrm{Na}\right]^{+}\right)$requires 475.99602, found 475.99584, $\left(\left[\mathrm{M}\left({ }^{81} \mathrm{Br}\right)+\mathrm{Na}\right]^{+}\right)$requires 477.99397, found 477.99395; IR $v_{\max }$ (film) $/ \mathrm{cm}^{-1}: 3339,3078,2970,2920,2852,2101,1889,1696,1646,1591,1570,1510$, $1476,1447,1400,1366,1355,1316,1286,1273,1242$, 1198, 1181, 1161, 1117, 1099, 1080, 1053, 1033. 


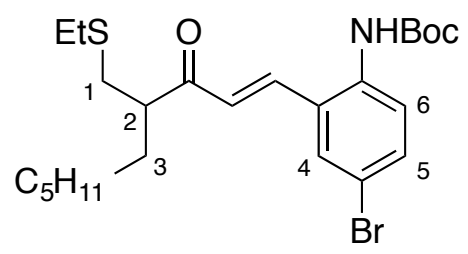

Compound 4u was prepared according to General Procedure A with alkyne 3d (59.2 $\mathrm{mg}, 0.20 \mathrm{mmol})$, aldehyde $2 \mathbf{u}(48.6 \mathrm{mg}, 0.24 \mathrm{mmol}),\left[\mathrm{Rh}(\mathrm{nbd})_{2}\right] \mathrm{BF}_{4}(3.7 \mathrm{mg}, 0.01$ mmol), dcpm (4.1 mg, $0.01 \mathrm{mmol})$ and acetone $(0.1 \mathrm{~mL})$ for 3 hours at $55^{\circ} \mathrm{C}$. Flash column chromatography $\left(\mathrm{SiO}_{2}, 10-20 \% \mathrm{Et}_{2} \mathrm{O}\right.$ in petrol) afforded enone $\mathbf{4} \mathbf{u}$ as a yellow oil (89.2 mg, 89\% yield).

${ }^{1}$ H-NMR (400 MHz, $\left.\mathrm{CDCl}_{3}\right): 7.77(1 \mathrm{H}, \mathrm{d}, J$ 9.0, C(6)H), $7.71(1 \mathrm{H}, \mathrm{d}, J$ 15.5, $\mathrm{C}(\mathrm{O}) \mathrm{CHCH}), 7.66(1 \mathrm{H}, \mathrm{d}, J 2.5, \mathrm{C}(4) H), 7.47(1 \mathrm{H}, \mathrm{dd}, J 9.0$ and 2.5, C(5)H), 6.78 $(1 \mathrm{H}, \mathrm{d}, J 15.5, \mathrm{C}(\mathrm{O}) \mathrm{CHCH}), 6.63(1 \mathrm{H}$, br. s, $\mathrm{NH}), 3.02-2.95(1 \mathrm{H}, \mathrm{m}, \mathrm{C}(2) H), 2.87(1 \mathrm{H}$, $\mathrm{dd}, J 13.0$ and 8.5, $\left.\mathrm{C}(1) H_{\mathrm{A}} \mathrm{H}_{\mathrm{B}}\right), 2.69\left(1 \mathrm{H}, \mathrm{dd}, J 13.0\right.$ and 5.5, $\left.\mathrm{C}(1) \mathrm{H}_{\mathrm{A}} H_{\mathrm{B}}\right), 2.56(2 \mathrm{H}, \mathrm{qd}$, $J 7.5$ and $\left.0.5, \mathrm{SCH}_{2} \mathrm{CH}_{3}\right), 1.78-1.58\left(2 \mathrm{H}, \mathrm{m}, \mathrm{C}(3) \mathrm{H}_{2}\right), 1.53\left(9 \mathrm{H}, \mathrm{s}, \mathrm{C}\left(\mathrm{CH}_{3}\right)_{3}\right), 1.32-$ $1.24\left(11 \mathrm{H}, \mathrm{m}, 4 \times \mathrm{CH}_{2}, \mathrm{SCH}_{2} \mathrm{CH}_{3}\right), 0.88\left(3 \mathrm{H}, \mathrm{t}, J 7.0,\left(\mathrm{CH}_{2}\right)_{4} \mathrm{CH}_{3}\right) ;{ }^{13} \mathbf{C}-\mathbf{N M R}(100$ $\left.\mathrm{MHz}, \mathrm{CDCl}_{3}\right)$ : 201.5, 152.6, 136.3, 135.8, 133.6, 129.6, 128.0, 127.6, 124.2, 117.0, 81.4, 51.5, 32.9, 32.0, 31.6, 29.3, 28.3, 27.1, 26.8, 22.6, 14.7, 14.1; LRMS (ESI+, $\mathrm{m} / \mathrm{z}): 520.2\left(\left[\mathrm{M}\left({ }^{79} \mathrm{Br}\right)+\mathrm{Na}\right]^{+}, 100 \%\right), 522.2\left(\left[\mathrm{M}\left({ }^{81} \mathrm{Br}\right)+\mathrm{Na}\right]^{+}, 100 \%\right) ; \quad$ HRMS (ESI+, $\mathrm{m} / \mathrm{z}): \mathrm{C}_{24} \mathrm{H}_{36} \mathrm{BrNNaO}_{3} \mathrm{~S}\left([\mathrm{M}+\mathrm{Na}]^{+}\right)$requires 520.14915, found 520.1493; IR $v_{\max }$ (film) $/ \mathrm{cm}^{-1}$ : 3308, 2957, 2926, 2855, 2361, 2342, 1729, 1692, 1603, 1571, 1507, 1456, 1404, 1392, 1367, 1311, 1287, 1234, 1154, 1117, 1083, 1049, 1024. 
tert-Butyl (E)-(4-bromo-2-(5-(ethylthio)-3-oxodec-1-en-1-yl)phenyl)carbamate, $4 \mathrm{v}$<smiles>CCCCCC(C)CC(=O)C=Cc1cc(Br)ccc1NC(=O)OCc1ccccc1</smiles>

Compound 4v was prepared according to General Procedure A with alkyne 3d (59.2 $\mathrm{mg}, 0.20 \mathrm{mmol})$, aldehyde $2 \mathrm{v}(45.2 \mathrm{mg}, 0.24 \mathrm{mmol}),\left[\mathrm{Rh}(\mathrm{nbd})_{2}\right] \mathrm{BF}_{4}(3.7 \mathrm{mg}, 0.01$ mmol), dcpm $(4.1 \mathrm{mg}, 0.01 \mathrm{mmol})$ and acetone $(0.1 \mathrm{~mL})$ for 3 hours at $55{ }^{\circ} \mathrm{C}$. Flash column chromatography $\left(\mathrm{SiO}_{2}, 10-20 \% \mathrm{Et}_{2} \mathrm{O}\right.$ in petrol) afforded enone $\mathbf{4 v}$ as a yellow oil (78.9 mg, $81 \%$ yield).

${ }^{1}$ H-NMR (400 MHz, $\left.\mathrm{CDCl}_{3}\right): 7.71(1 \mathrm{H}, \mathrm{d}, J$ 9.0, C(6)H), $7.66(1 \mathrm{H}, \mathrm{d}, J$ 15.5, $\mathrm{C}(\mathrm{O}) \mathrm{CHCH}), 7.65(1 \mathrm{H}, \mathrm{d}, J 2.5, \mathrm{C}(4) H), 7.46(1 \mathrm{H}, \mathrm{dd}, J 9.0$ and 2.5, $\mathrm{C}(5) H), 6.71$ $(1 \mathrm{H}, \mathrm{d}, J 15.5, \mathrm{C}(\mathrm{O}) \mathrm{CHCH}), 6.62(1 \mathrm{H}$, br. s, $\mathrm{N} H), 3.22(1 \mathrm{H}$, ap. quintet, $J 6.5, \mathrm{C}(2) H)$, $2.96\left(1 \mathrm{H}, \mathrm{dd}, J 16.5\right.$ and $\left.7.0, \mathrm{C}(3) H_{\mathrm{A}} \mathrm{H}_{\mathrm{B}}\right), 2.86\left(1 \mathrm{H}, \mathrm{dd}, J 16.5\right.$ and $\left.6.5, \mathrm{C}(3) \mathrm{H}_{\mathrm{A}} H_{\mathrm{B}}\right)$, $2.58\left(2 \mathrm{H}, \mathrm{q}, J\right.$ 7.5, $\left.\mathrm{SCH}_{2} \mathrm{CH}_{3}\right), 1.63-1.57\left(2 \mathrm{H}, \mathrm{m}, \mathrm{C}(1) \mathrm{H}_{2}\right), 1.53\left(9 \mathrm{H}, \mathrm{s}, \mathrm{C}\left(\mathrm{CH}_{3}\right)_{3}\right)$, 1.49-1.43 (2H, m, CH $), 1.37-1.29\left(4 \mathrm{H}, \mathrm{m}, 2 \times \mathrm{CH}_{2}\right), 1.26\left(3 \mathrm{H}, \mathrm{t}, J 7.5, \mathrm{SCH}_{2} \mathrm{CH}_{3}\right)$, $0.90\left(3 \mathrm{H}, \mathrm{t}, J\right.$ 7.0, $\left.\left(\mathrm{CH}_{2}\right)_{3} \mathrm{CH}_{3}\right) ;{ }^{13} \mathbf{C}-\mathbf{N M R}\left(100 \mathrm{MHz}, \mathrm{CDCl}_{3}\right): 197.9,152.7,136.2$, 135.8, 133.6, 129.6, 128.7, 127.9, 124.4, 117.2, 81.4, 48.2, 40.8, 35.4, 31.7, 28.3, 26.5, 25.1, 22.6, 14.9, 14.1; LRMS (ESI+, m/z): $506.2\left(\left[\mathrm{M}\left({ }^{79} \mathrm{Br}\right)+\mathrm{Na}\right]^{+}, 100 \%\right), 508.1$ $\left(\left[\mathrm{M}\left({ }^{81} \mathrm{Br}\right)+\mathrm{Na}\right]^{+}, \quad 100 \%\right) ; \quad$ HRMS $(\mathrm{ESI}+, \mathrm{m} / \mathrm{z}): \mathrm{C}_{23} \mathrm{H}_{34} \mathrm{BrNNaO}_{3} \mathrm{~S}\left(\left[\mathrm{M}\left({ }^{79} \mathrm{Br}\right) \mathrm{Na}\right]^{+}\right)$ requires 506.13350, found 506.13332, $\left(\left[\mathrm{M}\left({ }^{81} \mathrm{Br}\right) \mathrm{Na}\right]^{+}\right)$requires 506.13145 , found 506.13102; IR $v_{\max }($ film $) / \mathrm{cm}^{-1}: 3313,2959,2928,2858,1729,1695,1661,1606$, 1571, 1508, 1456, 1404, 1393, 1367, 1320, 1287, 1246, 1157, 1084, 1049, 1024. 
tert-Butyl (E)-(4-bromo-2-(3-(4-(methylthio)-5,6-dihydro-2H-pyran-3-yl)-3oxoprop-1-en-1-yl)phenyl)carbamate, $4 \mathrm{w}$<smiles>CC(C)(C)OC(=O)Nc1ccc(Br)cc1/C=C/C(=O)C1=C(S(C)(=O)=O)CCOC1</smiles>

Compound $\mathbf{4 w}$ was prepared according to General Procedure A with alkyne 3d (59.2 $\mathrm{mg}, 0.20 \mathrm{mmol})$, aldehyde $2 \mathrm{w}(38.0 \mathrm{mg}, 0.24 \mathrm{mmol})$, [Rh(nbd) $\left.{ }_{2}\right] \mathrm{BF}_{4}(3.7 \mathrm{mg}, 0.01$ mmol), dcpm $(4.1 \mathrm{mg}, 0.01 \mathrm{mmol})$ and acetone $(0.1 \mathrm{~mL})$ for 30 minutes at room temperature. Flash column chromatography $\left(\mathrm{SiO}_{2}, 40-70 \% \mathrm{Et}_{2} \mathrm{O}\right.$ in petrol) afforded enone $4 \mathbf{w}$ as a yellow solid (73.0 $\mathrm{mg}, 80 \%$ yield).

m.p.: $181-183{ }^{\circ} \mathrm{C}$ (crystalized from $\mathrm{Et}_{2} \mathrm{O} /$ petrol); ${ }^{1} \mathbf{H}-\mathbf{N M R}\left(400 \mathrm{MHz}, \mathrm{CDCl}_{3}\right): 7.84$ $(1 \mathrm{H}, \mathrm{d}, J$ 9.0, C(6)H), $7.76(1 \mathrm{H}, \mathrm{d}, J 15.0, \mathrm{C}(\mathrm{O}) \mathrm{CHC} H), 7.63(1 \mathrm{H}, \mathrm{d}, J 2.5, \mathrm{C}(4) H)$, $7.47(1 \mathrm{H}, \mathrm{dd}, J 9.0$ and $2.5, \mathrm{C}(5) H), 6.85(1 \mathrm{H}, \mathrm{d}, J 15.0, \mathrm{C}(\mathrm{O}) \mathrm{CHCH}), 6.65(1 \mathrm{H}$, br. s, $\mathrm{N} H), 4.62\left(2 \mathrm{H}, \mathrm{t}, J 2.0, \mathrm{C}(3) H_{2}\right), 3.90\left(2 \mathrm{H}, \mathrm{t}, J 5.5, \mathrm{C}(1) H_{2}\right), 2.64(2 \mathrm{H}, \mathrm{tt}, J 5.5$ and 2.0, $\left.\mathrm{C}(2) H_{2}\right), 2.36(3 \mathrm{H}, \mathrm{s}, \mathrm{SCH}), 1.54\left(9 \mathrm{H}, \mathrm{s}, \mathrm{C}\left(\mathrm{CH}_{3}\right)_{3}\right) ;{ }^{13} \mathbf{C}-\mathbf{N M R}\left(100 \mathrm{MHz}, \mathrm{CDCl}_{3}\right)$ : $186.7,152.5,151.0,136.2,136.0,133.4,129.5,128.0,127.7,126.2,123.6,116.6$, 81.4, 67.1, 64.1, 29.5, 28.3, 14.5; LRMS (ESI+, m/z): $476.0\left(\left[\mathrm{M}\left({ }^{79} \mathrm{Br}\right)+\mathrm{Na}\right]^{+}, 100 \%\right)$, $478.0 \quad\left(\left[\mathrm{M}\left({ }^{81} \mathrm{Br}\right)+\mathrm{Na}\right]^{+}, \quad 100 \%\right) ; \quad$ HRMS $\quad(\mathrm{ESI}+, \quad \mathrm{m} / \mathrm{z}): \quad \mathrm{C}_{20} \mathrm{H}_{24} \mathrm{BrNNaO}_{4} \mathrm{~S}$ $\left(\left[\mathrm{M}\left({ }^{79} \mathrm{Br}\right)+\mathrm{Na}\right]^{+}\right)$requires 476.05016 , found 476.05014, $\left(\left[\mathrm{M}\left({ }^{81} \mathrm{Br}\right)+\mathrm{Na}\right]^{+}\right)$requires 478.04812, found $478.04784\left({ }^{81} \mathrm{Br}\right) ;$ IR $v_{\max }($ film $) / \mathrm{cm}^{-1}: 3300,2976,2926,2856$, 2360, 2341, 1723, 1647, 1592, 1511, 1405, 1392, 1367, 1322, 1288, 1237, 1154, 1116 , 1070, 1049, 1020, 1007. 
<smiles>CNc1ccccc1C(=O)/C=C/c1cc(Br)ccc1NC(=O)OC(C)(C)C</smiles>

Compound $\mathbf{4 x}$ was prepared according to General Procedure A with alkyne 3d (59.2 $\mathrm{mg}, 0.20 \mathrm{mmol})$, aldehyde $2 \times(35.8 \mathrm{mg}, 0.24 \mathrm{mmol}),\left[\mathrm{Rh}(\mathrm{nbd})_{2}\right] \mathrm{BF}_{4}(3.7 \mathrm{mg}, 0.01$ mmol), dcpm $(4.1 \mathrm{mg}, 0.01 \mathrm{mmol})$ and acetone $(0.1 \mathrm{~mL})$ for $1 \mathrm{hr}$ at $55{ }^{\circ} \mathrm{C}$. Flash column chromatography $\left(\mathrm{SiO}_{2}, 20-30 \% \mathrm{Et}_{2} \mathrm{O}\right.$ in petrol) afforded enone $\mathbf{4 x}$ as a yellow-orange solid ( $82.3 \mathrm{mg}, 92 \%$ yield).

m.p.: $118-120{ }^{\circ} \mathrm{C}$ (crystalized from $\mathrm{Et}_{2} \mathrm{O} /$ petrol); ${ }^{\mathbf{1}} \mathbf{H}$-NMR $\left(400 \mathrm{MHz}, \mathrm{CDCl}_{3}\right): 7.83$ $(1 \mathrm{H}, \mathrm{d}, J 7.5, \mathrm{C}(7) H), 7.82(1 \mathrm{H}, \mathrm{d}, J 15.5, \mathrm{C}(\mathrm{O}) \mathrm{CHCH}), 7.66(1 \mathrm{H}, \mathrm{d}, J 2.5, \mathrm{C}(5) H)$, $7.58(1 \mathrm{H}, \mathrm{dd}, J 7.5$ and 1.5, C(6)H), 7.48-7.43 (2H, m, C(2)H, C(4)H), $7.34(1 \mathrm{H}, \mathrm{d}, J$ 15.5, C(O)CHCH), $7.08(1 \mathrm{H}, \mathrm{d}, J 8.0, \mathrm{C}(1) H), 7.02(1 \mathrm{H}, \mathrm{td}, J 7.5$ and $1.0, \mathrm{C}(3) H)$, $6.71\left(1 \mathrm{H}\right.$, br. s, NH), $2.86\left(6 \mathrm{H}, \mathrm{s}, \mathrm{N}\left(\mathrm{CH}_{3}\right)_{2}\right), 1.55\left(9 \mathrm{H}, \mathrm{s}, \mathrm{C}\left(\mathrm{CH}_{3}\right)_{3}\right) ;{ }^{13} \mathbf{C}-\mathbf{N M R}(100$ $\left.\mathrm{MHz}, \mathrm{CDCl}_{3}\right)$ : 194.0, 152.7, 152.6, 136.0, 134.5, 133.1, 132.5, 131.6, 130.6, 129.7, 129.3, 128.2 , 123.8, 120.7, 117.1, 116.8, 81.4, 44.7, 28.3; LRMS (ESI+, m/z): 425.1 $\left(\left[\mathrm{M}\left({ }^{79} \mathrm{Br}\right)+\mathrm{H}\right]^{+}, \quad 100 \%\right), \quad 427.1 \quad\left(\left[\mathrm{M}\left({ }^{81} \mathrm{Br}\right)+\mathrm{H}\right]^{+}, \quad 100 \%\right) ; \quad$ HRMS $(\mathrm{ESI}+, \mathrm{m} / \mathrm{z})$ : $\mathrm{C}_{22} \mathrm{H}_{26} \mathrm{BrN}_{2} \mathrm{O}_{3}\left(\left[\mathrm{M}\left({ }^{79} \mathrm{Br}\right)+\mathrm{H}\right]^{+}\right)$requires 445.11213, found 445.11227, $\left(\left[\mathrm{M}\left({ }^{81} \mathrm{Br}\right)+\mathrm{H}\right]^{+}\right)$ requires 447.11009, found 447.11026; IR $v_{\max }($ film $) / \mathrm{cm}^{-1}: 3304,3066,2978,2931$, 2868, 2841, 2794, 2250, 1726, 1657, 1599, 1571, 1505, 1454, 1433, 1402, 1393, 1367 , 1322, 1285 1236, 1155, 1103, 1081, 1050, 1021. 


\section{(E)-1-(2-(Methylthio)phenyl)-3-(2-nitrophenyl)prop-2-en-1-one, 4y}<smiles>CSc1ccccc1C(=O)/C=C/c1ccccc1[N+](=O)[O-]</smiles>

Compound $4 \mathbf{y}$ was prepared according to General Procedure A with alkyne $\mathbf{3 y}(2.50 \mathrm{~g}$, $17.0 \mathrm{mmol})$, aldehyde $2 \mathrm{a}(3.10 \mathrm{~g}, 20.4 \mathrm{mmol}),\left[\mathrm{Rh}(\mathrm{nbd})_{2}\right] \mathrm{BF}_{4}(127 \mathrm{mg}, 0.34 \mathrm{mmol})$, dcpm $(139 \mathrm{~g}, 0.34 \mathrm{mmol})$ and acetone $(11 \mathrm{~mL})$ for 15 minutes at $55^{\circ} \mathrm{C}$. Flash column chromatography $\left(\mathrm{SiO}_{2}, 20 \% \mathrm{Et}_{2} \mathrm{O}\right.$ in petrol, then $\left.\mathrm{CH}_{2} \mathrm{Cl}_{2}\right)$ afforded enone $4 \mathbf{y}$ as a yellow solid (4.92 g, 97\% yield).

m.p.: $96-98{ }^{\circ} \mathrm{C}$ (crystalized from $\mathrm{Et}_{2} \mathrm{O} /$ petrol); ${ }^{1} \mathbf{H}-\mathbf{N M R}$ (400 MHz, $\mathrm{CDCl}_{3}$ ): 8.07 $(1 \mathrm{H}, \mathrm{dd}, J 8.0$ and 1.0, C(8)H), $7.99(1 \mathrm{H}, \mathrm{dd}, J 16.0$ and $0.5, \mathrm{C}(\mathrm{O}) \mathrm{CHCH}), 7.78-7.73$ $(2 \mathrm{H}, \mathrm{m}, \mathrm{C}(4) H, \mathrm{C}(5) H), 7.70(1 \mathrm{H}, \mathrm{tdd}, J 7.5,1.5$ and $0.5, \mathrm{C}(6) H), 7.57(1 \mathrm{H}$, ddd, $J$ 8.0, 7.0 and $1.5, \mathrm{C}(7) H), 7.51(1 \mathrm{H}$, ddd, $J 8.0,7.5$ and 1.5, $\mathrm{C}(2) H), 7.41(1 \mathrm{H}, \mathrm{dd}, J 8.0$ and 1.0, $\mathrm{C}(1) H), 7.27(1 \mathrm{H}, \mathrm{ddd}, J 7.5,7.5$ and 1.0, $\mathrm{C}(3) H), 7.18(1 \mathrm{H}, \mathrm{d}, J 16.0$, $\mathrm{C}(\mathrm{O}) \mathrm{CHCH}), 2.49$ (3H, s, $\left.\mathrm{SCH}_{3}\right) ;{ }^{13} \mathrm{C}-\mathrm{NMR}\left(100 \mathrm{MHz}, \mathrm{CDCl}_{3}\right)$ : 192.5, 148.4, 140.9, 140.4, 136.2, 133.6, 131.9, 131.2, 130.4, 129.9, 129.7, 129.2, 126.4, 125.0, 124.2, 16.5; LRMS (ESI+, m/z): 322.1 ([M+Na $\left.]^{+}, \quad 100 \%\right) ; \quad$ HRMS (ESI+, m/z): $\mathrm{C}_{16} \mathrm{H}_{14} \mathrm{NO}_{3} \mathrm{~S}\left([\mathrm{M}+\mathrm{H}]^{+}\right)$requires 300.06889 , found 300.06897 ; IR $v_{\max }(\mathrm{film}) / \mathrm{cm}^{-1}$ : 3065, 2982, 2920, 2855, 1654, 1602, 1569, 1519, 1462, 1433, 1341, 1297, 1277, 1208, 1141, 1081, 1047, 1009. 
<smiles>COc1ccc(/C=C/C(=O)c2ccccc2S(C)(=O)=O)c([N+](=O)[O-])c1</smiles>

Compound $\mathbf{4 z}$ was prepared according to General Procedure A with alkyne $\mathbf{3 z}$ (88.6 $\mathrm{mg}, 0.5 \mathrm{mmol})$, aldehyde $2 \mathrm{a}(91.3 \mathrm{mg}, 0.6 \mathrm{mmol}),\left[\mathrm{Rh}(\mathrm{nbd})_{2}\right] \mathrm{BF}_{4}(3.7 \mathrm{mg}, 0.01$ mmol), dcpm (4.1 mg, $0.01 \mathrm{mmol})$ and acetone $(0.4 \mathrm{~mL})$ for 20 minutes at $55{ }^{\circ} \mathrm{C}$. Flash column chromatography $\left(\mathrm{SiO}_{2}, 20 \% \mathrm{Et}_{2} \mathrm{O}\right.$ in petrol, followed by $100 \% \mathrm{CH}_{2} \mathrm{Cl}_{2}$ ) afforded enone $\mathbf{4 z}$ as a yellow solid (159.7 $\mathrm{mg}, 97 \%$ yield).

m.p.: 146-148 ${ }^{\circ} \mathrm{C}$ (crystalized from $\mathrm{Et}_{2} \mathrm{O} /$ petrol); ${ }^{1} \mathbf{H}-\mathbf{N M R}\left(400 \mathrm{MHz}, \mathrm{CDCl}_{3}\right.$ ): 7.92 $(1 \mathrm{H}, \mathrm{dt}, J 16.0$ and $0.5, \mathrm{C}(\mathrm{O}) \mathrm{CHCH}), 7.73(1 \mathrm{H}$, ddd, $J 7.5,1.5$ and $0.5, \mathrm{C}(4) H), 7.71$ $(1 \mathrm{H}, \mathrm{d}, J 8.5, \mathrm{C}(5) H), 7.54(1 \mathrm{H}, \mathrm{d}, J 2.5, \mathrm{C}(7) H), 7.50(1 \mathrm{H}$, ddd, $J$ 8.0, 7.5 and 1.5, $\mathrm{C}(2) H), 7.41(1 \mathrm{H}, \mathrm{dd}, J 8.0$ and 1.0, $\mathrm{C}(1) H), 7.28(1 \mathrm{H}$, ddd, $J 7.5,7.5$ and 1.0, C(3)H), $7.22(1 \mathrm{H}$, ddd, $J 8.5,2.5$ and $0.5, \mathrm{C}(6) H), 7.12(1 \mathrm{H}, \mathrm{d}, J 16.0, \mathrm{C}(\mathrm{O}) \mathrm{CHCH}), 3.94(3 \mathrm{H}$, s, $\left.\left.\mathrm{OCH}_{3}\right), 2.49(3 \mathrm{H}, \mathrm{s}, \mathrm{SCH})_{3}\right) ;{ }^{13} \mathbf{C}-\mathbf{N M R}\left(100 \mathrm{MHz}, \mathrm{CDCl}_{3}\right): 193.0,161.0,149.5$, $140.5,140.1,136.7,131.7,130.1,129.7,128.2,126.5,124.3,123.0,120.0,109.6$, 56.1, 16.6; LRMS (ESI+, m/z): $352.0\left([\mathrm{M}+\mathrm{Na}]^{+}, 100 \%\right) ; \quad$ HRMS $(\mathrm{ESI}+, \mathrm{m} / \mathrm{z})$ : $\mathrm{C}_{17} \mathrm{H}_{15} \mathrm{NNaO}_{4} \mathrm{~S}\left([\mathrm{M}+\mathrm{Na}]^{+}\right)$requires 352.06140 , found 352.06143; IR $v_{\max }\left(\right.$ film) $/ \mathrm{cm}^{-}$ ${ }^{1}:$ 2919, 2848, 1721, 1654, 1593, 1559, 1524, 1496, 1463, 1433, 1350, 1291, 1253, 1208, 1186, 1172, 1142, 1084, 1067, 1049, 1030, 1008. 
General Procedure B - acidic cyclization of 2-aminochalcones to quinolines

To a solution of enone (1.0 equiv) in $\mathrm{CH}_{2} \mathrm{Cl}_{2}(0.04 \mathrm{M})$ was added TFA (24.0 equiv), and stirred at ambient temperature for 16 hours. The reaction was quenched by the addition of $1.0 \mathrm{M} \mathrm{NaOH}_{(\mathrm{aq})}$, and extracted three times with $\mathrm{CH}_{2} \mathrm{Cl}_{2}$. The organic layer was then dried over $\mathrm{MgSO}_{4}$ and concentrated in vacuo to afford the crude quinoline.

\section{General Procedure C- thermal cyclization of 2-aminochalcones to quinolines}

To a microwave vial containing enone (1.0 equiv), was added EtOH (1.5 mL) and $\mathrm{H}_{2} \mathrm{O}(1.5 \mathrm{~mL})$. The resulting suspension was heated at $170{ }^{\circ} \mathrm{C}$ under microwave conditions for 3 hours. Once the solution had cooled to room temperature, the reaction mixture was partitioned between $\mathrm{CH}_{2} \mathrm{Cl}_{2}$ and $1.0 \mathrm{M} \mathrm{NaOH}_{(\mathrm{aq})}$. The organic layer was then dried over $\mathrm{MgSO}_{4}$ and concentrated in vacuo to afford the crude quinoline. 


\section{2-(2-(Methylthio)phenyl)quinoline, 5a}<smiles>CS(=O)(=O)c1ccccc1-c1ccc2ccccc2n1</smiles>

Compound 5a was prepared according to General Procedure B with enone 4a (740 $\mathrm{mg}, 2.00 \mathrm{mmol})$, TFA $(3.67 \mathrm{~mL}, 48.0 \mathrm{mmol})$ and $\mathrm{CH}_{2} \mathrm{Cl}_{2}(50 \mathrm{~mL})$. Flash column chromatography $\left(\mathrm{SiO}_{2}, 20 \% \mathrm{Et}_{2} \mathrm{O}\right.$ in petrol) afforded quinoline 5a as a pale brown solid (466 mg, 93\% yield).

Alternatively:

To a solution of enone $4 \mathbf{y}(299 \mathrm{mg}, 1.0 \mathrm{mmol})$ in $\mathrm{EtOH}(15 \mathrm{~mL})$ was added concentrated $\mathrm{HCl}_{(\mathrm{aq})}(0.33 \mathrm{mmol}, 4.0 \mathrm{mmol})$ and $\mathrm{Sn}$ powder $(356 \mathrm{mg}, 3.0 \mathrm{mmol})$, and the resulting mixture refluxed for 20 hours. Once cooled to room temperature, the reaction was partitioned between $\mathrm{CH}_{2} \mathrm{Cl}_{2}(200 \mathrm{~mL})$ and $1.0 \mathrm{M} \mathrm{NaOH}_{(\mathrm{aq})}(200 \mathrm{~mL})$. The organic layer was then washed with brine $(200 \mathrm{~mL})$, dried over $\mathrm{MgSO}_{4}$ and concentrated in vacuo. Flash column chromatography $\left(\mathrm{SiO}_{2}, 10-20 \% \mathrm{Et}_{2} \mathrm{O}\right.$ in petrol) afforded quinoline 5a as an off white solid (177.8 $\mathrm{mg}, 70 \%$ yield).

\section{Alternatively:}

Compound 5a was prepared according to a combination of General Procedures A and C, involving $\mathrm{a} \mathrm{SiO}_{2}$ filtration between the steps, with alkyne 3a (43.5 mg, 0.20 mmol), aldehyde 2a (36.5 mg, $0.24 \mathrm{mmol})$, [Rh(nbd) $)_{2} \mathrm{BF}_{4}(3.7 \mathrm{mg}, 0.01 \mathrm{mmol}$ ), dcpm $(4.1 \mathrm{mg}, 0.01 \mathrm{mmol})$ and acetone $(0.1 \mathrm{~mL})$ for 3 hours at $55^{\circ} \mathrm{C}$, then $\mathrm{EtOH}(1.5$ $\mathrm{mL}), \mathrm{H}_{2} \mathrm{O}(1.5 \mathrm{~mL})$. Flash column chromatography $\left(\mathrm{SiO}_{2}, 10-20 \% \mathrm{Et}_{2} \mathrm{O}\right.$ in petrol) afforded quinoline $\mathbf{5 a}$ as an off white solid (40.1 $\mathrm{mg}, 80 \%$ yield).

m.p.: $69-71^{\circ} \mathrm{C}$ (crystalized from $\left.\mathrm{CHCl}_{3}\right) ;{ }^{1} \mathbf{H}-\mathbf{N M R}\left(400 \mathrm{MHz}, \mathrm{CDCl}_{3}\right): 8.12(1 \mathrm{H}, \mathrm{d}$, $J$ 8.0, C(6)H), $8.11(1 \mathrm{H}, \mathrm{d}, J 8.0, \mathrm{C}(10) H), 7.77(1 \mathrm{H}, \mathrm{dd}, J 8.0$ and 1.0, $\mathrm{C}(8) H), 7.65$ $(1 \mathrm{H}, \mathrm{td}, J 7.5$ and $1.5, \mathrm{C}(9) H), 7.61(1 \mathrm{H}, \mathrm{d}, J 8.5, \mathrm{C}(5) H), 7.49-7.45(2 \mathrm{H}, \mathrm{m}, \mathrm{C}(7) H$, $\mathrm{C}(4) H), 7.35-7.30(2 \mathrm{H}, \mathrm{m}, \mathrm{C}(1) H, \mathrm{C}(2) H), 7.20(1 \mathrm{H}, \mathrm{td}, \mathrm{C}(3) H), 2.30\left(3 \mathrm{H}, \mathrm{s}, \mathrm{SC} H_{3}\right)$; ${ }^{13}$ C-NMR (100 MHz, $\left.\mathrm{CDCl}_{3}\right): 158.7,147.8,140.1,137.8,136.0,130.1,129.7,129.6$, 129.1, 127.6, 127.0, 126.6, 126.5, 125.2, 122.2, 16.7; LRMS (ESI+, m/z): 252.0 $\left([\mathrm{M}+\mathrm{H}]^{+}\right)$.

Data is consistent with the literature. ${ }^{1}$ 


\section{5-Fluoro-2-(2-(methylthio)phenyl)quinoline, $5 \mathrm{~b}$}<smiles>CSc1ccccc1-c1ccc2c(F)cccc2n1</smiles>

Compound 5b was prepared according to General Procedure $\mathbf{B}$ with enone $\mathbf{4 b}(80.5$ $\mathrm{mg}, 0.208 \mathrm{mmol})$, TFA $(0.38 \mathrm{~mL}, 4.98 \mathrm{mmol})$ and $\mathrm{CH}_{2} \mathrm{Cl}_{2}(5 \mathrm{~mL})$. Flash column chromatography $\left(\mathrm{SiO}_{2}, 20-30 \% \mathrm{Et}_{2} \mathrm{O}\right.$ in petrol) afforded quinoline $\mathbf{5 b}$ as a yellow oil (47.3 mg, 85\% yield).

${ }^{1}$ H-NMR $\left(400 \mathrm{MHz}, \mathrm{CDCl}_{3}\right): 8.51(1 \mathrm{H}, \mathrm{dd}, J 8.5$ and 1.0, C(5)H), $8.03(1 \mathrm{H}, \mathrm{d}, J 8.5$, $\mathrm{C}(9) H), 7.79(1 \mathrm{H}, \mathrm{d}, J 8.5, \mathrm{C}(6) H), 7.69$ (1H, ddd, $J$ 8.5, 8.0 and 6.0, C(8)H), 7.59 $(1 \mathrm{H}$, ddd, $J 7.5,1.0$ and $0.5, \mathrm{C}(4) H), 7.48-7.42(2 \mathrm{H}, \mathrm{m}, \mathrm{C}(1) H, \mathrm{C}(2) H), 7.33$ (1H, ddd, $J$ 7.5, 6.5 and 2.5, C(3)H), $7.25(1 \mathrm{H}$, ddd, $J 9.5,8.0$ and 1.0, $\mathrm{C}(7) H), 2.43(3 \mathrm{H}, \mathrm{s}$, $\left.\mathrm{SCH}_{3}\right) ;{ }^{13} \mathrm{C}-\mathrm{NMR}\left(100 \mathrm{MHz}, \mathrm{CDCl}_{3}\right): 159.5,158.2$ (d, $J$ 254.5), 148.5 (d, $J$ 3.0), $139.5,137.8,130.1,129.4,129.3$ (d, $J 4.5), 129.2$ (d, $J$ 8.0), 126.6, 125.4 (d, $J 3.0)$, 125.2, 122.4, 117.7 (d, $J$ 15.5), 110.2 (d, $J$ 19.0), 16.7; ${ }^{19}$ F-NMR $\left(376 \mathrm{MHz}, \mathrm{CDCl}_{3}\right)$ $\left\{{ }^{1} \mathrm{H}\right\}:-123.0 ; \quad$ LRMS $(\mathrm{ESI}+, \mathrm{m} / \mathrm{z}): 270.1\left([\mathrm{M}+\mathrm{H}]^{+}, 100 \%\right) ; \quad$ HRMS $(\mathrm{ESI}+, \mathrm{m} / \mathrm{z})$ : $\mathrm{C}_{16} \mathrm{H}_{13} \mathrm{FNS}\left([\mathrm{M}+\mathrm{H}]^{+}\right)$requires 270.07472 , found $270.07471 ;$ IR $v_{\max }(\mathrm{film}) / \mathrm{cm}^{-1}$ : 3062, 2982, 2920, 2858, 2358, 2234, 1632, 1599, 1565, 1555, 1506, 1470, 1434, 1408, 1376, 1350, 1315, 1273, 1235, 1208, 1157, 1129, 1094, 1059, 1050, 1010. 


\section{7-Chloro-2-(2-(methylthio)phenyl)quinoline, 5c}<smiles>CSc1ccccc1-c1ccc2ccc(Cl)cc2n1</smiles>

Compound 5c was prepared according to General Procedure $\mathbf{B}$ with enone $4 \mathbf{c}(60.0$ $\mathrm{mg}, 0.15 \mathrm{mmol})$, TFA $(0.27 \mathrm{~mL}, 3.57 \mathrm{mmol})$ and $\mathrm{CH}_{2} \mathrm{Cl}_{2}(5.0 \mathrm{~mL})$. No further purification was necessary, with quinoline $\mathbf{5 c}$ isolated as a pale yellow oil (40.3 $\mathrm{mg}$, 94\% yield).

${ }^{1}$ H-NMR $\left(400 \mathrm{MHz}, \mathrm{CDCl}_{3}\right): 8.13(1 \mathrm{H}$, br. s, C(9)H), $8.11(1 \mathrm{H}, \mathrm{d}, J$ 8.5, C(6)H), 7.70 $(1 \mathrm{H}, \mathrm{d}, J 8.5, \mathrm{C}(7) H), 7.62(1 \mathrm{H}, \mathrm{d}, J 8.5, \mathrm{C}(5) H), 7.47(1 \mathrm{H}, \mathrm{d}, J 7.0, \mathrm{C}(4) H), 7.43(1 \mathrm{H}$, $\mathrm{dd}, J 8.5$ and 2.0, C(8)H), 7.37-7.31 (2H, m, C(1)H, C(2)H), $7.21(1 \mathrm{H}, \mathrm{td}, J 7.0$ and 2.0, C(3)H), $2.32\left(3 \mathrm{H}, \mathrm{s}, \mathrm{SCH}_{3}\right) ;{ }^{13} \mathbf{C}-\mathbf{N M R}\left(100 \mathrm{MHz}, \mathrm{CDCl}_{3}\right)$ : 159.6, 147.9, 139.4, 137.9, 136.0, 135.6, 130.1, 129.4, 128.8, 128.5, 127.7, 126.6, 125.4, 125.2, 122.5, 16.7; LRMS (ESI+, m/z): $286.0\left({ }^{35} \mathrm{Cl},[\mathrm{M}+\mathrm{H}]^{+}\right), 288.0\left({ }^{37} \mathrm{Cl},[\mathrm{M}+\mathrm{H}]^{+}\right) ; \quad$ HRMS $(\mathrm{ESI}+, \mathrm{m} / \mathrm{z}): \mathrm{C}_{16} \mathrm{H}_{13} \mathrm{ClNS}\left({ }^{35} \mathrm{Cl},[\mathrm{M}+\mathrm{H}]^{+}\right)$requires 286.0452, found 286.0450, $\left({ }^{37} \mathrm{Cl}\right.$, $[\mathrm{M}+\mathrm{H}]^{+}$) requires 288.0422 , found $288.0419 ; \mathbf{I R} v_{\max }(\mathrm{film}) / \mathrm{cm}^{-1}: 2920,1610,1597$, 1497, 1468, 1435, 1405, 1331, 1247, 1134, 1088, 1067, 1046, 1034. 


\section{6-Bromo-2-(2-(methylthio)phenyl)quinoline, 5d}<smiles>CSc1ccccc1-c1ccc2cc(Br)ccc2n1</smiles>

Compound 5d was prepared according to General Procedure B with enone 4d (63.1 $\mathrm{mg}, 0.14 \mathrm{mmol})$, TFA $(0.26 \mathrm{~mL}, 3.38 \mathrm{mmol})$ and $\mathrm{CH}_{2} \mathrm{Cl}_{2}(5.0 \mathrm{~mL})$. Flash column chromatography $\left(\mathrm{SiO}_{2}, 30 \% \mathrm{Et}_{2} \mathrm{O}\right.$ in petrol) afforded quinoline $\mathbf{5 d}$ as a pale yellow solid (32.5 mg, 70\% yield).

m.p.: $131-134{ }^{\circ} \mathrm{C}$ (crystalized from $\mathrm{CHCl}_{3}$ ). ${ }^{1} \mathbf{H}-\mathbf{N M R}\left(400 \mathrm{MHz}, \mathrm{CDCl}_{3}\right): 8.05(1 \mathrm{H}$, d, $J 8.5, \mathrm{C}(6) H), 8.00(1 \mathrm{H}, \mathrm{d}, J 9.0, \mathrm{C}(9) H), 7.94(1 \mathrm{H}, \mathrm{d}, J 2.0, \mathrm{C}(7) H), 7.72(1 \mathrm{H}, \mathrm{dd}$, $J 9.0$ and 2.0, $\mathrm{C}(8) H), 7.65(1 \mathrm{H}, \mathrm{d}, J 8.5, \mathrm{C}(5) H), 7.48(1 \mathrm{H}, \mathrm{dd}, J 7.5$ and 1.0, $\mathrm{C}(4) H)$, 7.37-7.31 (2H, m, C(1)H, C(2)H), $7.22(1 \mathrm{H}, \mathrm{td}, J 7.0$ and 2.0, C(3)H), $2.32(3 \mathrm{H}, \mathrm{s}$, $\left.\mathrm{SCH}_{3}\right) ;{ }^{13} \mathrm{C}-\mathrm{NMR}\left(125 \mathrm{MHz}, \mathrm{CDCl}_{3}\right): 157.9,145.1,138.3,136.8,134.1,132.2$, 130.1, 129.0, 128.5, 128.4, 127.0, 125.6, 124.2, 122.1, 119.5, 15.6; LRMS (ESI+, $\mathrm{m} / \mathrm{z}): 330.0\left({ }^{81} \mathrm{Br},[\mathrm{M}+\mathrm{H}]^{+}\right) ; \quad$ HRMS $(\mathrm{ESI}+, \mathrm{m} / \mathrm{z}): \mathrm{C}_{16} \mathrm{H}_{13} \mathrm{BrNS}\left({ }^{79} \mathrm{Br},[\mathrm{M}+\mathrm{H}]^{+}\right)$ requires 329.9947, found 329.9940; $\left({ }^{81} \mathrm{Br},[\mathrm{M}+\mathrm{H}]^{+}\right)$requires 331.9926, found 329.9918; IR $v_{\max }($ film $) / \mathrm{cm}^{-1}: 2917,1592,1546,1490,1472,1434,1305,1286$, 1248, 1187, 1129, 1061, 1045. 


\section{8-Iodo-2-(2-(methylthio)phenyl)quinoline, 5e}<smiles>CS(=O)(=O)c1ccccc1-c1ccc2cccc(I)c2n1</smiles>

Compound 5e was prepared according to General Procedure B with enone 4e $(92.7$ $\mathrm{mg}, 0.187 \mathrm{mmol})$, TFA $(0.34 \mathrm{~mL}, 4.49 \mathrm{mmol})$, and $\mathrm{CH}_{2} \mathrm{Cl}_{2}(5.0 \mathrm{~mL})$. Flash column chromatography $\left(\mathrm{SiO}_{2}, 50 \% \mathrm{CH}_{2} \mathrm{Cl}_{2}\right.$ in petrol) afforded enone $\mathbf{5 e}$ as a pale yellow oil (59.3 mg, 84\% yield)

${ }^{1}$ H-NMR $\left(400 \mathrm{MHz}, \mathrm{CDCl}_{3}\right): 8.39(1 \mathrm{H}, \mathrm{dd}, J 7.5$ and $1.5, \mathrm{C}(7) H), 8.16(1 \mathrm{H}, \mathrm{d}, J 8.5$, $\mathrm{C}(6) H), 7.85(1 \mathrm{H}, \mathrm{dd}, J 8.0$ and 1.5, C(9)H), $7.83(1 \mathrm{H}, \mathrm{d}, J 8.5, \mathrm{C}(5) H), 7.73(1 \mathrm{H}, \mathrm{ddd}$, $J 7.5,1.5$ and 0.5, C(4)H), 7.49-7.44 (2H, m, C(1)H, C(2)H), 7.34 (1H, ddd, $J 7.5,6.5$ and $2.5, \mathrm{C}(3) H), 7.28(1 \mathrm{H}$, dd, $J 8.0$ and $7.5, \mathrm{C}(8) H), 2.47\left(3 \mathrm{H}, \mathrm{s}, \mathrm{SCH}_{3}\right) ;{ }^{13} \mathbf{C}-\mathbf{N M R}$ $\left(100 \mathrm{MHz}, \mathrm{CDCl}_{3}\right): 159.3,146.6,140.2,139.3,138.3,136.7,130.9,129.3,128.4$, 127.64, 127.51, 127.0, 125.1, 123.0, 103.9, 17.3; LRMS (ESI+, m/z): $378.0\left([\mathrm{M}+\mathrm{H}]^{+}\right.$, $100 \%) ; \quad$ HRMS $(\mathrm{ESI}+, \mathrm{m} / \mathrm{z}): \mathrm{C}_{16} \mathrm{H}_{12} \mathrm{INNaS}\left([\mathrm{M}+\mathrm{Na}]^{+}\right)$requires 377.98079 , found $377.98080 ;$ IR $v_{\max }($ film $) / \mathrm{cm}^{-1}: 3053,2919,2854,1595,1534,1497,1467,1435$, 1420, 1316, 1288, 1267, 1249, 1199, 1143, 1090, 1058, 1045, 1031. 


\section{2-(2-(Methylthio)phenyl)-6-(trifluoromethyl)quinoline, $5 f$}<smiles>CS(=O)(=O)c1ccccc1-c1ccc2cc(C(F)(F)F)ccc2n1</smiles>

Compound $5 \mathbf{f}$ was prepared according to General Procedure $\mathbf{B}$ with enone $4 \mathbf{f}$ (72.6 $\mathrm{mg}$, $0.17 \mathrm{mmol})$, TFA $(0.30 \mathrm{~mL}, 3.98 \mathrm{mmol})$ and $\mathrm{CH}_{2} \mathrm{Cl}_{2}(5 \mathrm{~mL})$. Flash column chromatography $\left(\mathrm{SiO}_{2}, 50 \% \mathrm{Et}_{2} \mathrm{O}\right.$ in petrol) afforded quinoline $\mathbf{5 f}$ as a pale yellow solid (41.5 mg, 76\% yield).

m.p.: $98-101{ }^{\circ} \mathrm{C}$ (crystalized from $\left.\mathrm{CHCl}_{3}\right)$. ${ }^{1} \mathbf{H}-\mathrm{NMR}\left(400 \mathrm{MHz}, \mathrm{CDCl}_{3}\right)$ : $8.24(1 \mathrm{H}$, $\mathrm{d}, J 8.5, \mathrm{C}(9) H), 8.23(1 \mathrm{H}, \mathrm{d}, J 8.0, \mathrm{C}(6) H), 8.10(1 \mathrm{H}, \mathrm{s}, \mathrm{C}(7) H), 7.84(1 \mathrm{H}, \mathrm{dd}, J 9.0$ and 2.0, $\mathrm{C}(8) H), 7.75(1 \mathrm{H}, \mathrm{d}, J 8.5, \mathrm{C}(5) H), 7.50(1 \mathrm{H}, \mathrm{dd}, J 7.5$ and 1.0, C(4)H), 7.39$7.33(2 \mathrm{H}, \mathrm{m}, \mathrm{C}(1) H, \mathrm{C}(2) H), 7.23(1 \mathrm{H}, \mathrm{td}, J 7.0$ and $2.0, \mathrm{C}(3) H), 2.33(3 \mathrm{H}, \mathrm{s}, \mathrm{SCH})_{3}$; ${ }^{13}$ C-NMR (125 MHz, $\left.\mathrm{CDCl}_{3}\right): 159.7,147.5,138.1,136.9,135.9,129.6,129.1,128.6$, 127.4 (q, $J 32.5), 125.7,124.9,125.6-125.4(2 \times \mathrm{C}, \mathrm{m}), 124.2,123.0(\mathrm{q}, J 272.5)$, 122.5, 15.7; ${ }^{19}$ F-NMR (376 MHz, $\left.\mathrm{CDCl}_{3}\right)\left\{{ }^{1} \mathrm{H}\right\}:-62.3$; LRMS (ESI+, m/z): 320.1 $\left([\mathrm{M}+\mathrm{H}]^{+}\right) ; \quad$ HRMS $(\mathrm{ESI}+, \mathrm{m} / \mathrm{z}): \mathrm{C}_{17} \mathrm{H}_{13} \mathrm{~F}_{3} \mathrm{NS}\left([\mathrm{M}+\mathrm{H}]^{+}\right)$requires 320.0715 , found 320.0708; IR $v_{\max }\left(\right.$ film) $/ \mathrm{cm}^{-1}: 2923,1633,1601,1490,1463,1436,1394,1320$, 1302, 1284, 1195, 1164, 1116, 1063. 


\section{2-(2-(Methylthio)phenyl)quinoline-6-carbaldehyde, 5g}<smiles>CSc1ccccc1-c1ccc2cc(C=O)ccc2n1</smiles>

Compound 5g was prepared according to General Procedure B with enone 4g (69.1 $\mathrm{mg}, 0.174 \mathrm{mmol})$, TFA $(0.32 \mathrm{~mL}, 4.18 \mathrm{mmol})$ and $\mathrm{CH}_{2} \mathrm{Cl}_{2}(4.4 \mathrm{~mL})$. Flash column chromatography $\left(\mathrm{SiO}_{2}, 40-60 \% \mathrm{Et}_{2} \mathrm{O}\right.$ in petrol) afforded quinoline $\mathbf{5 g}$ as a pale yellow solid (45.2 $\mathrm{mg}, 93 \%$ yield).

m.p.: $127-129{ }^{\circ} \mathrm{C}$ (crystalized from $\left.\mathrm{CHCl}_{3}\right) ;{ }^{1} \mathbf{H}-\mathbf{N M R}\left(400 \mathrm{MHz}, \mathrm{CDCl}_{3}\right)$ : $10.22(1 \mathrm{H}$, s, CHO $), 8.39(1 \mathrm{H}, \mathrm{s}, \mathrm{C}(7) H), 8.38(1 \mathrm{H}, \mathrm{d}, J 8.5, \mathrm{C}(6) H), 8.29(1 \mathrm{H}, \mathrm{d}, J 8.5, \mathrm{C}(9) H)$, $8.23(1 \mathrm{H}, \mathrm{dd}, J 8.5$ and 1.5, C(8)H), $7.84(1 \mathrm{H}, \mathrm{d}, J 8.5, \mathrm{C}(5) H), 7.61(1 \mathrm{H}, \mathrm{d}, J 7.0$, $\mathrm{C}(4) H), 7.49-7.43(2 \mathrm{H}, \mathrm{m}, \mathrm{C}(1) H, \mathrm{C}(2) H), 7.33(1 \mathrm{H}$, ddd, $J$ 7.5, 7.0 and 2.0, C(3)H), $2.43(3 \mathrm{H}, \mathrm{s}, \mathrm{SCH}) ;{ }^{13} \mathbf{C}-\mathbf{N M R}\left(100 \mathrm{MHz}, \mathrm{CDCl}_{3}\right): 191.6,161.5,150.4,139.2,138.0$, $137.3,134.3,133.3,130.8,130.1,129.6,127.0,126.7,126.4,125.2,123.4,16.7$; LRMS (ESI+, m/z): $280.1\left([\mathrm{M}+\mathrm{H}]^{+}, 100 \%\right) ; \quad$ HRMS (ESI+, m/z): $\mathrm{C}_{17} \mathrm{H}_{14} \mathrm{NOS}$ $\left([\mathrm{M}+\mathrm{H}]^{+}\right)$requires 280.07906, found 280.07907; IR $v_{\max }($ film $) / \mathrm{cm}^{-1}: 3059,2919$, 2830, 1696, 1621, 1596, 1495, 1459, 1435, 1226, 1154, 1045. 


\section{1-(2-(2-(Methylthio)phenyl)quinolin-6-yl)ethan-1-one, $5 \mathrm{~h}$}<smiles>CC(=O)c1ccc2nc(-c3ccccc3S(C)(=O)=O)ccc2c1</smiles>

Compound 5h was prepared according to General Procedure B with enone 4h (78.0 $\mathrm{mg}, 0.190 \mathrm{mmol})$, TFA $(0.35 \mathrm{~mL}, 4.56 \mathrm{mmol})$ and $\mathrm{CH}_{2} \mathrm{Cl}_{2}(4.8 \mathrm{~mL})$. Flash column chromatography $\left(\mathrm{SiO}_{2}, 40-60 \% \mathrm{Et}_{2} \mathrm{O}\right.$ in petrol) afforded quinoline $\mathbf{5 h}$ as a pale yellow solid (54.5 mg, 98\% yield).

m.p.: $130-132{ }^{\circ} \mathrm{C}$ (crystalized from $\left.\mathrm{CHCl}_{3}\right) ;{ }^{1} \mathbf{H}-\mathbf{N M R}\left(400 \mathrm{MHz}, \mathrm{CDCl}_{3}\right): 8.49(1 \mathrm{H}$, $\mathrm{d}, J 2.0, \mathrm{C}(7) H), 8.32(1 \mathrm{H}, \mathrm{d}, J 8.5, \mathrm{C}(6) H), 8.30(1 \mathrm{H}, \mathrm{dd}, J 9.0$ and 2.0, $\mathrm{C}(8) H), 8.24$ $(1 \mathrm{H}, \mathrm{d}, J 9.0, \mathrm{C}(9) H), 7.79(1 \mathrm{H}, \mathrm{d}, J 8.5, \mathrm{C}(5) H), 7.59(1 \mathrm{H}, \mathrm{dd}, J 7.5$ and 1.0, C(4)H), 7.47-7.41 (2H, m, C(1)H, C(2)H), $7.31(1 \mathrm{H}, \mathrm{ddd}, J 7.5,6.5$ and 2.0, C(3)H), $2.76(3 \mathrm{H}$, s, $\left.\mathrm{C}(\mathrm{O}) \mathrm{CH}_{3}\right), 2.41(3 \mathrm{H}, \mathrm{s}, \mathrm{SCH}) ;{ }^{13} \mathrm{C}-\mathbf{N M R}\left(100 \mathrm{MHz}, \mathrm{CDCl}_{3}\right): 197.5,160.9,149.6$, 139.3, 138.0, 137.4, 134.8, 130.12, 130.09, 129.58, 129.53, 128.0, 126.6, 126.1, 125.2, 123.1, 26.8, 16.7; LRMS (ESI+, m/z): $294.1\left([\mathrm{M}+\mathrm{H}]^{+}, 100 \%\right) ;$ HRMS (ESI+, m/z): $\mathrm{C}_{18} \mathrm{H}_{16} \mathrm{NOS}\left([\mathrm{M}+\mathrm{H}]^{+}\right)$requires 294.09471, found 294.09468; IR $v_{\max }($ film $) / \mathrm{cm}^{-1}$ : 3059, 2919, 2850, 1679, 1617, 1595, 1490, 1458, 1434, 1393, 1356, 1291, 1257, 1186, 1046. 


\section{Methyl 2-(2-(methylthio)phenyl)quinoline-6-carboxylate, 5i}<smiles>CC(=O)c1ccc2nc(-c3ccccc3S(C)(=O)=O)ccc2c1</smiles>

Compound 5i was prepared according to General Procedure $\mathbf{B}$ with enone 4i $(42.5 \mathrm{mg}$, $0.10 \mathrm{mmol})$, TFA $(0.18 \mathrm{~mL}, 2.39 \mathrm{mmol})$ and $\mathrm{CH}_{2} \mathrm{Cl}_{2}(5 \mathrm{~mL})$. Flash column chromatography $\left(\mathrm{SiO}_{2}, 50 \% \mathrm{Et}_{2} \mathrm{O}\right.$ in petrol) afforded quinoline $\mathbf{5 i}$ as a pale yellow oil (28.8 mg, 93\% yield).

${ }^{1}$ H-NMR (400 MHz, $\left.\mathrm{CDCl}_{3}\right): 8.55(1 \mathrm{H}, \mathrm{d}, J 1.5, \mathrm{C}(7) H), 8.25(1 \mathrm{H}, \mathrm{dd}, J 9.0$ and 2.0, $\mathrm{C}(8) H), 8.24(1 \mathrm{H}, \mathrm{d}, J 8.5, \mathrm{C}(6) H), 8.16(1 \mathrm{H}, \mathrm{d}, J 9.0, \mathrm{C}(9) H), 7.71(1 \mathrm{H}, \mathrm{d}, J 8.5$, $\mathrm{C}(5) H), 7.51(1 \mathrm{H}, \mathrm{d}, J 7.5, \mathrm{C}(4) H), 7.38-7.32(2 \mathrm{H}, \mathrm{m}, \mathrm{C}(1) H, \mathrm{C}(2) H), 7.23(1 \mathrm{H}, \mathrm{td}, J$ 7.0 and 2.0, $\mathrm{C}(3) H), 3.93\left(3 \mathrm{H}, \mathrm{s}, \mathrm{CO}_{2} \mathrm{CH}_{3}\right), 2.33\left(3 \mathrm{H}, \mathrm{s}, \mathrm{SCH}_{3}\right) ;{ }^{13} \mathbf{C}-\mathbf{N M R}(100$ $\left.\mathrm{MHz}, \mathrm{CDCl}_{3}\right): 165.6,159.7,148.4,138.2,136.9,136.6,129.7,129.1,128.7,128.5$, 128.2, 127.0, 125.6, 125.1, 124.2, 122.0, 51.4, 15.7; LRMS (ESI+, m/z): 310.1 $\left([\mathrm{M}+\mathrm{H}]^{+}\right) ; \quad$ HRMS $(\mathrm{ESI}+, \mathrm{m} / \mathrm{z}): \mathrm{C}_{18} \mathrm{H}_{16} \mathrm{NO}_{2} \mathrm{~S}\left([\mathrm{M}+\mathrm{H}]^{+}\right)$requires 310.0896, found 310.0890; IR $v_{\max }($ film $) / \mathrm{cm}^{-1}: 1720,1623,1597,1459,1435,1292,1265,1195$, 1096. 


\section{2-(2-(Methylthio)phenyl)-6-nitroquinoline, 5j}

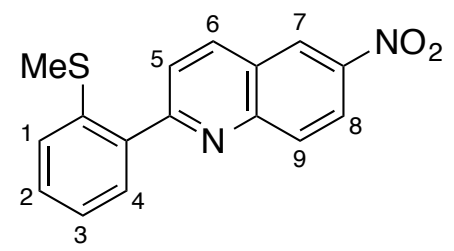

Compound 5j was prepared according to General Procedure $\mathbf{B}$ with enone $\mathbf{4 j}$ ( $78.6 \mathrm{mg}$, $0.190 \mathrm{mmol})$, TFA $(0.35 \mathrm{~mL}, 4.56 \mathrm{mmol})$, and $\mathrm{CH}_{2} \mathrm{Cl}_{2}(5.0 \mathrm{~mL})$. Flash column chromatography $\left(\mathrm{SiO}_{2}, \mathrm{CH}_{2} \mathrm{Cl}_{2}\right)$ afforded enone $\mathbf{5} \mathbf{j}$ as a yellow solid $(48.4 \mathrm{mg}, 86 \%$ yield).

m.p.: $185-187{ }^{\circ} \mathrm{C}$ (crystalized from $\left.\mathrm{CH}_{2} \mathrm{Cl}_{2}\right)$; ${ }^{\mathbf{1}} \mathbf{H}-\mathbf{N M R}\left(400 \mathrm{MHz}, \mathrm{CDCl}_{3}\right): 8.86(1 \mathrm{H}$, $\mathrm{d}, J 2.5, \mathrm{C}(7) H), 8.53(1 \mathrm{H}, \mathrm{dd}, J 9.0$ and $2.5, \mathrm{C}(8) H), 8.43(1 \mathrm{H}, \mathrm{d}, J 8.5, \mathrm{C}(6) H), 8.35$ $(1 \mathrm{H}, \mathrm{d}, J 9.0, \mathrm{C}(9) H), 7.93(1 \mathrm{H}, \mathrm{d}, J 8.5, \mathrm{C}(5) H), 7.63(1 \mathrm{H}$, ddd, $J 7.5,1.5$ and 0.5, $\mathrm{C}(4) H), 7.50(1 \mathrm{H}$, ddd, $J 8.0,7.0$ and $1.5, \mathrm{C}(2) H), 7.46(1 \mathrm{H}, \mathrm{dd}, J 8.0$ and 1.5, C(1)H), $7.36\left(1 \mathrm{H}\right.$, ddd, $J 7.5,7.0$ and 1.5, C(3)H), $2.46(3 \mathrm{H}, \mathrm{s}, \mathrm{SCH}) ;{ }^{13} \mathbf{C}-\mathbf{N M R}(100 \mathrm{MHz}$, $\left.\mathrm{CDCl}_{3}\right)$ : 162.1, 149.7, 145.5, 138.7, 138.1, 137.7, 131.2, 130.2, 130.0, 126.8, 125.7, 125.3, 124.34, 124.15, 123.2, 16.7; LRMS (ESI+, m/z): $297.1\left([\mathrm{M}+\mathrm{H}]^{+}, 100 \%\right)$; HRMS $\mathrm{C}_{16} \mathrm{H}_{13} \mathrm{~N}_{2} \mathrm{O}_{2} \mathrm{~S}(\mathrm{ESI}+, \mathrm{m} / \mathrm{z}):\left([\mathrm{M}+\mathrm{H}]^{+}\right)$requires 297.06922, found 297.06924; IR $v_{\max }(\mathrm{film}) / \mathrm{cm}^{-1}: 3084,3056,2925,2855,1619,1606,1587,1564,1553,1524$, $1487,1453,1428,1346,1334,1307,1286,1248,1236,1205,1154,1131,1080,1045$, 1034. 


\section{2-(2-(Methylthio)phenyl)quinoline-6-carbonitrile, 5k}<smiles>CSc1ccccc1-c1ccc2cc(C#N)ccc2n1</smiles>

Compound 5k was prepared according to General Procedure $\mathbf{B}$ with enone 4k (73.3 $\mathrm{mg}, 0.186 \mathrm{mmol})$, TFA $(0.34 \mathrm{~mL}, 4.46 \mathrm{mmol})$, and $\mathrm{CH}_{2} \mathrm{Cl}_{2}(5.0 \mathrm{~mL})$. Flash column chromatography $\left(\mathrm{SiO}_{2}, 50-100 \% \mathrm{CH}_{2} \mathrm{Cl}_{2}\right.$ in petrol) afforded quinoline $\mathbf{5 k}$ as an off white solid (43.3 $\mathrm{mg}, 85 \%$ yield).

m.p.: $145-147{ }^{\circ} \mathrm{C}$ (crystalized from $\mathrm{CHCl}_{3}$ ); ${ }^{\mathbf{1}} \mathbf{H}-\mathbf{N M R}$ (400 MHz, $\mathrm{CDCl}_{3}$ ): 8.29-8.26 $(3 \mathrm{H}, \mathrm{m}, \mathrm{C}(6) H, \mathrm{C}(7) H, \mathrm{C}(9) H), 7.89$ (1H, dd, $J 9.0,2.0, \mathrm{C}(8) H), 7.87(1 \mathrm{H}, \mathrm{d}, J 8.5$, $\mathrm{C}(5) H) 7.60(1 \mathrm{H}$, ddd, $J 7.5,1.5$ and $0.5, \mathrm{C}(4) H), 7.48(1 \mathrm{H}$, ddd, $J$ 8.0, 7.0, 1.5, $\mathrm{C}(2) H), 7.45(1 \mathrm{H}, \mathrm{dd}, J 8.0$ and $1.5, \mathrm{C}(1) H), 7.34(1 \mathrm{H}$, ddd, $J 7.5,7.0$ and $1.5, \mathrm{C}(3) H)$, $2.44(3 \mathrm{H}, \mathrm{s}, \mathrm{SCH}) ;{ }^{13} \mathbf{C}-\mathbf{N M R}\left(100 \mathrm{MHz}, \mathrm{CDCl}_{3}\right): 161.6,148.6,138.9,138.0,136.2$, $133.8,131.0,130.4,130.1,129.8,126.7,126.3,125.3,123.9,118.7,110.1,16.7$; LRMS (ESI+, m/z): $277.1\left([\mathrm{M}+\mathrm{H}]^{+}, 100 \%\right) ; \quad$ HRMS (ESI+, m/z): $\mathrm{C}_{17} \mathrm{H}_{13} \mathrm{~N}_{2} \mathrm{~S}$ $\left([\mathrm{M}+\mathrm{H}]^{+}\right)$requires 277.07940 , found 277.07934; IR $v_{\max }($ film $) / \mathrm{cm}^{-1}: 3060,2920$, 2851, 2227, 1619, 1595, 1567, 1556, 1490, 1457, 1433, 1393, 1341, 1310, 1291, 1242, 1160, 1138, 1086, 1045, 1033. 
7-Methyl-2-(2-(methylthio)phenyl)quinoline, 51<smiles>CSc1ccccc1-c1ccc2ccc(C)cc2n1</smiles>

Compound 5l was prepared according to General Procedure B with enone $4 \mathbf{l}(31.2 \mathrm{mg}$, $0.08 \mathrm{mmol})$, TFA $(0.15 \mathrm{~mL}, 1.95 \mathrm{mmol})$ and $\mathrm{CH}_{2} \mathrm{Cl}_{2}(5.0 \mathrm{~mL})$. Flash column chromatography $\left(\mathrm{SiO}_{2}, 30 \% \mathrm{Et}_{2} \mathrm{O}\right.$ in petrol) afforded quinoline $\mathbf{5} \mathbf{l}$ as a pale yellow oil (20.0 mg, 94\% yield).

${ }^{1}$ H-NMR (400 MHz, $\left.\mathrm{CDCl}_{3}\right): 8.09(1 \mathrm{H}, \mathrm{d}, J 8.5, \mathrm{C}(6) H), 7.91(1 \mathrm{H}$, br. s, C(9)H), 7.67 $(1 \mathrm{H}, \mathrm{d}, J 8.5, \mathrm{C}(7) H), 7.55(1 \mathrm{H}, \mathrm{d}, J 8.5, \mathrm{C}(5) H), 7.48(1 \mathrm{H}, \mathrm{d}, J 7.5, \mathrm{C}(4) H), 7.36-7.31$ $(3 \mathrm{H}, \mathrm{m}, \mathrm{C}(1) H, \mathrm{C}(2) H, \mathrm{C}(8) H), 7.21(1 \mathrm{H}, \mathrm{td}, J 6.5$ and $3.0, \mathrm{C}(3) H), 2.50(3 \mathrm{H}, \mathrm{s}$, $\left.\mathrm{ArCH}_{3}\right), 2.31(3 \mathrm{H}, \mathrm{s}, \mathrm{SCH}) ;{ }^{13} \mathbf{C}-\mathbf{N M R}\left(125 \mathrm{MHz}, \mathrm{CDCl}_{3}\right): 158.6,147.9,140.0$, $137.8,135.8,130.0,129.1,128.9$, 128.6, 127.2, 126.5, 125.5, 125.09, 125.06, 121.4, 22.0, 16.7; LRMS (ESI+, m/z): $266.1\left([\mathrm{M}+\mathrm{H}]^{+}\right) ;$HRMS (ESI+, m/z): $\mathrm{C}_{17} \mathrm{H}_{16} \mathrm{NS}$ $\left([\mathrm{M}+\mathrm{H}]^{+}\right)$requires 266.0998, found 266.0996; IR $v_{\max }($ film $) / \mathrm{cm}^{-1}: 2989,2361,1625$, 1598, 1508, 1471, 1436, 1311, 1240, 1146, 1086, 1046. 


\section{2-(2-(Methylthio)phenyl)quinolin-6-amine, $5 \mathrm{~m}$}<smiles>CS(=O)(=O)c1ccccc1-c1ccc2cc(N)ccc2n1</smiles>

Compound 5m was prepared according to General Procedure B with enone 4m $(87.0$ $\mathrm{mg}, 0.180 \mathrm{mmol})$, TFA $(0.33 \mathrm{~mL}, 4.30 \mathrm{mmol})$, and $\mathrm{CH}_{2} \mathrm{Cl}_{2}(5.0 \mathrm{~mL})$. Flash column chromatography $\left(\mathrm{SiO}_{2}, 20 \%\right.$ EtOAc in $\left.\mathrm{CH}_{2} \mathrm{Cl}_{2}\right)$ afforded enone $\mathbf{5 m}$ as a brown oil (45.3 mg, 95\% yield).

${ }^{1}$ H-NMR $\left(400 \mathrm{MHz}, \mathrm{CDCl}_{3}\right): 8.01(1 \mathrm{H}, \mathrm{dt}, J 9.0$ and $0.5, \mathrm{C}(9) H), 7.96(1 \mathrm{H}$, ddd, $J$ 8.5, 1.0 and 0.5, $\mathrm{C}(6) H), 7.59(1 \mathrm{H}, \mathrm{d}, J 8.5, \mathrm{C}(5) H), 7.57-7.55(1 \mathrm{H}$, ddd, $J 7.5,1.5$ and $0.5, \mathrm{C}(4) H), 7.43-7.39(2 \mathrm{H}, \mathrm{m}, \mathrm{C}(1) H, \mathrm{C}(2) H), 7.29(1 \mathrm{H}$, ddd, $J 7.5,5.5$ and 3.0, $\mathrm{C}(3) H), 7.17(1 \mathrm{H}, \mathrm{dd}, J 9.0$ and 2.5, C(8)H), $6.93(1 \mathrm{H}, \mathrm{d}, J 2.5, \mathrm{C}(7) H), 4.01(2 \mathrm{H}, \mathrm{br}$. s, $\left.\mathrm{NH}_{2}\right), 2.41\left(3 \mathrm{H}, \mathrm{s}, \mathrm{SCH}_{3}\right) ;{ }^{13} \mathbf{C}-\mathbf{N M R}\left(100 \mathrm{MHz}, \mathrm{CDCl}_{3}\right): 155.0,144.8,142.8$, $140.3,137.7,133.8,130.7,130.0,128.7,128.5,126.3,125.0,122.4,121.7,107.3$, 16.6; LRMS (ESI+, m/z): $267.1\left([\mathrm{M}+\mathrm{H}]^{+}, 100 \%\right) ;$ HRMS (ESI+, m/z): $\mathrm{C}_{16} \mathrm{H}_{15} \mathrm{~N}_{2} \mathrm{~S}$ $\left([\mathrm{M}+\mathrm{H}]^{+}\right)$requires 267.09505, found 267.09504; IR $v_{\max }($ film $) / \mathrm{cm}^{-1}: 3453,3333$, 3210, 3052, 2920, 2854, 1627, 1595, 1556, 1500, 1461, 1434, 1384, 1350, 1317, 1268, 1247, 1175, 1127, 1087, 1045, 1034. 


\section{2-(2-(Methylthio)phenyl)quinolin-8-ol, 5n}<smiles>CS(=O)(=O)c1ccccc1-c1ccc2cccc(O)c2n1</smiles>

Compound 5n was prepared according to General Procedure $\mathbf{B}$ with enone 4n (69.0 $\mathrm{mg}, 0.179 \mathrm{mmol})$, TFA $(0.33 \mathrm{~mL}, 4.30 \mathrm{mmol})$, and $\mathrm{CH}_{2} \mathrm{Cl}_{2}(5.0 \mathrm{~mL})$. Flash column chromatography $\left(\mathrm{SiO}_{2}, 20 \%\right.$ EtOAc in $\left.\mathrm{CH}_{2} \mathrm{Cl}_{2}\right)$ afforded quinoline $\mathbf{5 n}$ as a cream solid (42.5 mg, 89\% yield).

m.p.: $108-110{ }^{\circ} \mathrm{C}$ (crystalized from $\mathrm{CHCl}_{3}$ ); ${ }^{1} \mathbf{H}-\mathbf{N M R}\left(400 \mathrm{MHz}, \mathrm{CDCl}_{3}\right): 8.44(1 \mathrm{H}$, br. s, OH), $8.25(1 \mathrm{H}, \mathrm{d}, J 8.5, \mathrm{C}(6) H), 7.81(1 \mathrm{H}, \mathrm{d}, J 8.5, \mathrm{C}(5) H), 7.70(1 \mathrm{H}, \mathrm{ddd}, J 7.5$, 1.5 and $0.5, \mathrm{C}(4) H), 7.52-7.47(3 \mathrm{H}, \mathrm{m}, \mathrm{C}(1) H, \mathrm{C}(2) H, \mathrm{C}(8) H), 7.39(1 \mathrm{H}, \mathrm{dd}, J 8.0$ and 1.0, C(7)H), $7.34(1 \mathrm{H}$, ddd, $J 7.5,6.0$ and 3.0, C(3)H), $7.25(1 \mathrm{H}, \mathrm{dd}, J 7.5$ and 1.0, $\mathrm{C}(9) H), 2.48\left(3 \mathrm{H}, \mathrm{s},\left(\mathrm{SCH}_{3}\right) ;{ }^{13} \mathbf{C}-\mathbf{N M R}\left(100 \mathrm{MHz}, \mathrm{CDCl}_{3}\right)\right.$ : 155.7, 152.5, 138.5, $138.1,137.3,136.4,130.1,129.4,127.9,127.1,126.1,124.8,122.1,117.6,110.3$, 16.7; LRMS (ESI+, m/z): $268.1\left([\mathrm{M}+\mathrm{H}]^{+}, 100 \%\right) ; \quad$ HRMS $(\mathrm{ESI}+, \mathrm{m} / \mathrm{z})$ : $\mathrm{C}_{16} \mathrm{H}_{13} \mathrm{NNaOS}\left([\mathrm{M}+\mathrm{Na}]^{+}\right)$requires 268.07906, found 268.07897; IR $v_{\max }\left(\right.$ film) $/ \mathrm{cm}^{-1}$ : 3379, 3053, 2919, 2851, 1597, 1573, 1557, 1504, 1471, 1434, 1401, 1366, 1327, 1294 , $1275,1245,1204,1163,1085,1051$. 


\section{7-Methoxy-2-(2-(methylthio)phenyl)quinoline, 50}

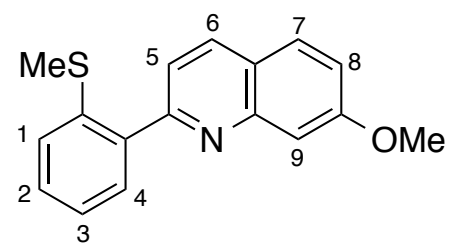

Compound 5o was prepared according to General Procedure B with enone 4o (41.4 $\mathrm{mg}$, mmol), TFA $(0.19 \mathrm{~mL}, 2.48 \mathrm{mmol})$ and $\mathrm{CH}_{2} \mathrm{Cl}_{2}(2.5 \mathrm{~mL})$. Flash column chromatography $\left(\mathrm{SiO}_{2}, 30-40 \% \mathrm{Et}_{2} \mathrm{O}\right.$ in petrol) afforded quinoline $\mathbf{5 0}$ as a cream solid (22.7 mg, $71 \%$ yield).

Alternatively:

Under a $\mathrm{N}_{2(\mathrm{~g})}$ atmosphere, to a solution of enone $4 \mathbf{z}(83.0 \mathrm{mg}, 0.25 \mathrm{mmol})$ in $\mathrm{MeOH}$ $(1.5 \mathrm{~mL})$ was added sat. $\mathrm{NH}_{4} \mathrm{Cl}_{(\mathrm{aq})}(0.5 \mathrm{~mL})$ and $\mathrm{In}$ powder $(144 \mathrm{mg}, 1.25 \mathrm{mmol})$, and the resulting mixture refluxed for 4 hours. Once cooled to room temperature, the reaction was partitioned between EtOAc $(50 \mathrm{~mL})$ and $1.0 \mathrm{M} \mathrm{NaOH}_{(\mathrm{aq})}(50 \mathrm{~mL})$. The organic layer was then washed with brine $(50 \mathrm{~mL})$, dried over $\mathrm{MgSO}_{4}$ and concentrated in vacuo. Flash column chromatography $\left(\mathrm{SiO}_{2}, 30-40 \% \mathrm{Et}_{2} \mathrm{O}\right.$ in petrol) afforded quinoline $\mathbf{5 0}$ as a cream solid (53.9 $\mathrm{mg}, 77 \%$ yield)

m.p.: $180-182{ }^{\circ} \mathrm{C}$ (crystalized from $\left.\mathrm{CHCl}_{3}\right) ;{ }^{1} \mathbf{H}-\mathbf{N M R}\left(400 \mathrm{MHz}, \mathrm{CDCl}_{3}\right): 8.16(1 \mathrm{H}$, $\mathrm{d}, J 8.5, \mathrm{C}(6) H), 7.76(1 \mathrm{H}, \mathrm{d}, J 9.0, \mathrm{C}(7) H), 7.58(1 \mathrm{H}, \mathrm{d}, J 8.5, \mathrm{C}(5) H), 7.56(1 \mathrm{H}, \mathrm{dd}$, $J 7.5$ and 1.5, C(4)H), $7.54(1 \mathrm{H}, \mathrm{d}, J 2.5, \mathrm{C}(9) H), 7.46-7.40(2 \mathrm{H}, \mathrm{m}, \mathrm{C}(1) H, \mathrm{C}(2) H)$, $7.31(1 \mathrm{H}$, ddd, $J 7.5,6.5$ and 2.0, C(3)H), $7.24(1 \mathrm{H}, \mathrm{dd}, J 9.0$ and 2.5, C(8)H), 3.99 $\left(3 \mathrm{H}, \mathrm{s}, \mathrm{OCH}_{3}\right), 2.43\left(3 \mathrm{H}, \mathrm{s}, \mathrm{SCH} H_{3}\right) ;{ }^{13} \mathbf{C}-\mathbf{N M R}\left(100 \mathrm{MHz}, \mathrm{CDCl}_{3}\right): 160.9,158.9$, $149.4,140.1,137.7,135.7,129.9,129.0,128.5,126.3,125.0,122.2,119.99,119.85$, 107.5, 55.6, 16.6; LRMS (ESI+, m/z): $282.1\left([\mathrm{M}+\mathrm{H}]^{+}, 100 \%\right) ;$ HRMS (ESI+, m/z): $\mathrm{C}_{17} \mathrm{H}_{16} \mathrm{NOS}\left([\mathrm{M}+\mathrm{H}]^{+}\right)$requires 282.09471 , found $282.09470 ; \quad$ IR $v_{\max }($ film $) / \mathrm{cm}^{-1}$ : $3058,2961,2919,2835,1621,1599,1509,1455,1434,1384,1336,1274,1258,1216$, $1174,1138,1121,1028$. 


\section{2,9-Bis(2-(methylthio)phenyl)-1,10-phenanthroline, 5p}

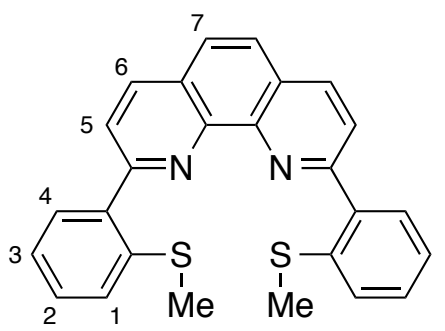

Compound 5p was prepared according to General Procedure B with enone 4p (112 $\mathrm{mg}$, mmol), TFA $(0.31 \mathrm{~mL}, 4.06 \mathrm{mmol})$ and $\mathrm{CH}_{2} \mathrm{Cl}_{2}(4.2 \mathrm{~mL})$. Flash column chromatography $\left(\mathrm{SiO}_{2}, 30-40 \%\right.$ EtOAc in petrol) afforded phenanthroline $\mathbf{5 p}$ as a pale brown solid (63.7 $\mathrm{mg}, 89 \%$ yield).

m.p.: $154-156{ }^{\circ} \mathrm{C}$ (crystalized from $\left.\mathrm{CHCl}_{3}\right) ;{ }^{1} \mathbf{H}-\mathbf{N M R}\left(400 \mathrm{MHz}, \mathrm{CDCl}_{3}\right): 8.28(2 \mathrm{H}$, $\mathrm{d}, J 8.5, \mathrm{C}(6) H), 8.05(2 \mathrm{H}, \mathrm{d}, J 8.5, \mathrm{C}(5) H), 7.88(2 \mathrm{H}, \mathrm{dd}, J 7.5$ and 1.5, C(4)H), 7.81 $(2 \mathrm{H}, \mathrm{s}, \mathrm{C}(7) H), 7.46(2 \mathrm{H}, \mathrm{dd}, J 8.0$ and $1.5, \mathrm{C}(1) H), 7.42(2 \mathrm{H}, \mathrm{ddd}, J 8.0,7.0$ and 1.5, $\mathrm{C}(2) H), 7.33(2 \mathrm{H}$, ddd, $J 7.5,7.0$ and $1.5, \mathrm{C}(3) H), 2.47\left(6 \mathrm{H}, \mathrm{s}, \mathrm{SCH}_{3}\right) ;{ }^{13} \mathbf{C}-\mathbf{N M R}$ $\left(100 \mathrm{MHz}, \mathrm{CDCl}_{3}\right): 158.2,145.9,140.4,138.1,135.7,131.1,129.0,127.6,127.1$, $126.3,125.1,123.8,17.3 ; \quad$ LRMS $(\mathrm{ESI}+, \mathrm{m} / \mathrm{z}): 425.1\left([\mathrm{M}+\mathrm{H}]^{+}, 100 \%\right) ; \quad$ HRMS $(\mathrm{ESI}+, \mathrm{m} / \mathrm{z}): \mathrm{C}_{26} \mathrm{H}_{21} \mathrm{~N}_{2} \mathrm{~S}_{2}\left([\mathrm{M}+\mathrm{H}]^{+}\right)$requires 425.11407 , found $425.11386 ;$ IR $v_{\max }$ (film) $/ \mathrm{cm}^{-1}: 3054,2981,2918,2856,1619,1605,1585,1562,1541,1495,1466,1435$, 1419, 1357, 1305, 1269, 1245, 1232, 1163, 1144, 1102, 1080, 1042, 1030. 


\section{2,9-Bis(1-(ethylthio)octan-2-yl)-1,10-phenanthroline, 5q}

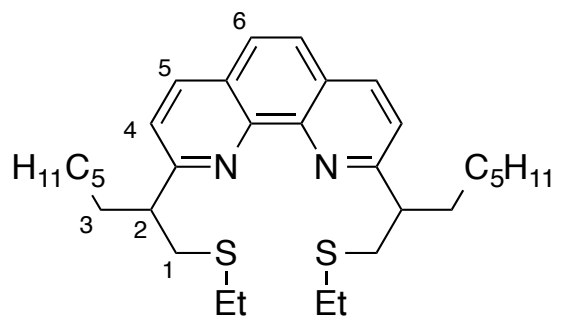

Compound 5q was prepared according to General Procedure B with enone 4q (138.7 $\mathrm{mg}, 0.175 \mathrm{mmol})$, TFA $(0.32 \mathrm{~mL}, 4.20 \mathrm{mmol})$ and $\mathrm{CH}_{2} \mathrm{Cl}_{2}(4.4 \mathrm{~mL})$. Flash column chromatography $\left(\mathrm{SiO}_{2}, 5-10 \% \mathrm{NEt}_{3}\right.$ in petrol) afforded phenanthroline $\mathbf{5 q}$ as a yellow oil (69.1 mg, 83\% yield).

(isolated as a 1:1 mixture of diastereoisomers) ${ }^{1} \mathbf{H}-\mathbf{N M R}\left(400 \mathrm{MHz}, \mathrm{CDCl}_{3}\right): 8.16(2 \mathrm{H}$, $\mathrm{d}, J$ 8.0, C(5)H), $7.73(2 \mathrm{H}, \mathrm{s}, \mathrm{C}(6) H), 7.51(2 \mathrm{H}, \mathrm{dd}, J 8.0$ and 1.0, C(4)H), 3.40-3.26 $\left(4 \mathrm{H}, \mathrm{m}, \mathrm{C}(1) H_{\mathrm{A}} \mathrm{H}_{\mathrm{B}}, \mathrm{C}(2) H\right), 3.09\left(2 \mathrm{H}, \mathrm{dd}, J 12.5\right.$ and $\left.6.0, \mathrm{C}(1) \mathrm{H}_{\mathrm{A}} H_{\mathrm{B}}\right), 2.53(4 \mathrm{H}, \mathrm{q}, J$ 7.5, $\mathrm{SCH}_{2} \mathrm{CH}_{3}$ (diast)), 2.52 (4H, q, $J$ 7.5, $\mathrm{SCH}_{2} \mathrm{CH}_{3}$ (diast)), 2.05-1.98 (4H, m, $\left.\mathrm{C}(3) H_{2}\right), 1.41-1.20\left(16 \mathrm{H}, \mathrm{m}, 8 \times \mathrm{CH}_{2}\right), 1.24\left(6 \mathrm{H}, \mathrm{t}, J 7.5, \mathrm{SCH}_{2} \mathrm{CH}_{3}\right.$ (diast)), $1.23(6 \mathrm{H}$, t, $J$ 7.5, $\mathrm{SCH}_{2} \mathrm{CH}_{3}$ (diast)), $0.86\left(6 \mathrm{H}, \mathrm{t}, J 7.0,\left(\mathrm{CH}_{2}\right)_{4} \mathrm{CH}_{3}\right) ;{ }^{13} \mathbf{C}-\mathbf{N M R}(100 \mathrm{MHz}$, $\mathrm{CDCl}_{3}$ ): 163.8, 145.8, 135.8, 127.4, 125.6, 122.44 (diast), 122.37 (diast), 48.6 (diast), 48.5 (diast), 37.1 (diast), 37.0 (diast), 35.0 (diast), 34.8 (diast), 31.8, 29.50 (diast), 29.48 (diast), 27.5, 26.65 (diast), 26.61 (diast), 22.7, 14.9 (diast), 14.1 (diast); LRMS $(\mathrm{ESI}+, \mathrm{m} / \mathrm{z}): 525.3\left([\mathrm{M}+\mathrm{H}]^{+}, 100 \%\right) ; \quad$ HRMS $(\mathrm{ESI}+, \mathrm{m} / \mathrm{z}): \mathrm{C}_{32} \mathrm{H}_{49} \mathrm{~N}_{2} \mathrm{~S}_{2}\left([\mathrm{M}+\mathrm{H}]^{+}\right)$ requires 525.33317, found 525.33279; IR $v_{\max }\left(\right.$ film) $/ \mathrm{cm}^{-1}: 3040,2956,2926,2855$, 1618, 1608, 1590, 1548, 1495, 1421, 1376, 1299, 1264, 1188, 1144, 1091, 1053. 


\section{6-Bromo-2-(3-methoxy-2-(methylthio)phenyl)quinoline, 5 r}<smiles>COc1cccc(-c2ccc3cc(Br)ccc3n2)c1S(C)(=O)=O</smiles>

Compound 5r was prepared according to General Procedure $\mathbf{B}$ with enone 4r $(63.0$ $\mathrm{mg}, 0.132 \mathrm{mmol})$, TFA $(0.24 \mathrm{~mL}, 3.17 \mathrm{mmol})$ and $\mathrm{CH}_{2} \mathrm{Cl}_{2}(3.3 \mathrm{~mL})$ at $55^{\circ} \mathrm{C}$. Flash column chromatography $\left(\mathrm{SiO}_{2}, 20 \% \mathrm{Et}_{2} \mathrm{O}\right.$ in petrol) afforded quinoline $\mathbf{5 r}$ as an off white solid (41.6 mg, $88 \%$ yield).

m.p.: $136-138{ }^{\circ} \mathrm{C}$ (crystalized from $\left.\mathrm{CHCl}_{3}\right) ;{ }^{1} \mathbf{H}-\mathbf{N M R}\left(400 \mathrm{MHz}, \mathrm{CDCl}_{3}\right): 8.10(1 \mathrm{H}$, $\mathrm{d}, J 8.5, \mathrm{C}(5) H), 8.05(1 \mathrm{H}, \mathrm{d}, J 9.0, \mathrm{C}(8) H), 8.04(1 \mathrm{H}, \mathrm{d}, J 2.5, \mathrm{C}(6) H), 7.81(1 \mathrm{H}, \mathrm{dd}$, $J 9.0$ and 2.5, C(7)H), $7.68(1 \mathrm{H}, \mathrm{d}, J 8.5, \mathrm{C}(4) H), 7.43(1 \mathrm{H}, \mathrm{dd}, J 8.5$ and $7.5, \mathrm{C}(2) H)$, $7.22(1 \mathrm{H}, \mathrm{dd}, J 7.5$ and 1.5, C(3)H), $7.03(1 \mathrm{H}, \mathrm{dd}, J 8.5$ and 1.5, C(1)H), $4.01(3 \mathrm{H}, \mathrm{s}$, $\left.\mathrm{OCH}_{3}\right), 2.24(3 \mathrm{H}, \mathrm{s}, \mathrm{SCH}) ;{ }^{13} \mathbf{C}-\mathbf{N M R}\left(100 \mathrm{MHz}, \mathrm{CDCl}_{3}\right): 160.2,160.0,146.3$, 146.0, 134.0, 132.9, 131.3, 129.6, 129.3, 128.2, 124.3, 122.9, 122.6, 120.3, 111.4, 56.2, 18.6; LRMS (ESI+, m/z): $360.0\left(\left[\mathrm{M}\left({ }^{79} \mathrm{Br}\right)+\mathrm{H}\right]^{+}, 100 \%\right), 362.0\left(\left[\mathrm{M}\left({ }^{81} \mathrm{Br}\right)+\mathrm{H}\right]^{+}\right.$, $100 \%)$ HRMS $(\mathrm{ESI}+, \mathrm{m} / \mathrm{z}): \mathrm{C}_{17} \mathrm{H}_{15} \mathrm{BrNOS}\left(\left[\mathrm{M}\left({ }^{79} \mathrm{Br}\right)+\mathrm{H}\right]^{+}\right)$requires 360.00522, found 360.00516, $\left(\left[\mathrm{M}\left({ }^{81} \mathrm{Br}\right)+\mathrm{H}\right]^{+}\right)$requires 362.00318, found 362.00288 ; IR $v_{\max }$ (film) $/ \mathrm{cm}^{-1}: 3061,3001,2959,2922,2835,1595,1583,1567,1546,1489,1470,1457$, $1437,1423,1374,1334,1303,1259,1186,1040$. 


\section{6-Bromo-2-(2-(methylthio)-5-(trifluoromethyl)phenyl)quinoline, $5 \mathrm{~s}$}<smiles>CSc1ccc(C(F)(F)F)cc1-c1ccc2cc(Br)ccc2n1</smiles>

Compound 5s was prepared according to General Procedure B with enone 4s (70.3 $\mathrm{mg}, 0.136 \mathrm{mmol})$, TFA $(0.25 \mathrm{~mL}, 3.26 \mathrm{mmol})$ and $\mathrm{CH}_{2} \mathrm{Cl}_{2}(3.4 \mathrm{~mL})$. Flash column chromatography $\left(\mathrm{SiO}_{2}, 20 \% \mathrm{Et}_{2} \mathrm{O}\right.$ in petrol) afforded quinoline $\mathbf{5 s}$ as a white solid (50.5 mg, 93\% yield).

m.p.: $102-104{ }^{\circ} \mathrm{C}$ (crystalized from $\left.\mathrm{CHCl}_{3}\right) ;{ }^{1} \mathbf{H}-\mathbf{N M R}\left(400 \mathrm{MHz}, \mathrm{CDCl}_{3}\right): 8.18(1 \mathrm{H}$, $\mathrm{d}, J 8.5, \mathrm{C}(5) H), 8.07(1 \mathrm{H}, \mathrm{d}, J 9.0, \mathrm{C}(8) H), 8.06(1 \mathrm{H}, \mathrm{d}, J 2.0, \mathrm{C}(6) H), 7.85(1 \mathrm{H}, \mathrm{dd}$, $J 9.0$ and 2.0, C(7)H), $7.82(1 \mathrm{H}, \mathrm{d}, J 2.0, \mathrm{C}(3) H), 7.75(1 \mathrm{H}, \mathrm{d}, J 8.5, \mathrm{C}(4) H), 7.68(1 \mathrm{H}$, $\mathrm{dd}, J 8.5$ and 2.0, $\mathrm{C}(2) H), 7.47(1 \mathrm{H}, \mathrm{d}, J 8.5, \mathrm{C}(1) H), 2.48(3 \mathrm{H}, \mathrm{s}, \mathrm{SCH}) ;{ }^{13} \mathbf{C}-\mathbf{N M R}$ $\left(125 \mathrm{MHz}, \mathrm{CDCl}_{3}\right): 157.5,146.3,143.3,139.1,135.5,133.4,131.3,129.6,128.2$, 127.0 (q, $J 33.0), 126.7$ (q, $J 4.0), 125.8$ (q, $J 3.5), 125.5,124.1$ (q, $J 272.0), 122.6$, 121.0, 16.1; ${ }^{19}$ F-NMR $\left(470 \mathrm{MHz}, \mathrm{CDCl}_{3}\right)\left\{{ }^{1} \mathrm{H}\right\}:-62.2$; LRMS (ESI+, m/z): 398.0 $\left(\left[\mathrm{M}\left({ }^{79} \mathrm{Br}\right)+\mathrm{H}\right]^{+}, \quad 100 \%\right), \quad 400.0\left(\left[\mathrm{M}\left({ }^{81} \mathrm{Br}\right)+\mathrm{H}\right]^{+}, \quad 100 \%\right) ; \quad$ HRMS $(\mathrm{ESI}+, \quad \mathrm{m} / \mathrm{z})$ : $\left(\mathrm{C}_{17} \mathrm{H}_{11} \mathrm{BrF}_{3} \mathrm{NNaS} \quad\left[\mathrm{M}\left({ }^{79} \mathrm{Br}\right)+\mathrm{Na}\right]^{+}\right)$requires 397.98204, found 397.98200, $\left(\left[\mathrm{M}\left({ }^{81} \mathrm{Br}\right)+\mathrm{Na}\right]^{+}\right)$requires 399.98000 , found 399.97974; IR $v_{\max }($ film $) / \mathrm{cm}^{-1}: 2922$, 2855, 1610, 1591, 1548, 1490, 1409, 1378, 1339, 1326, 1263, 1235, 1173, 1144, 1121, 1099, 1067, 1060, 1046. 


\section{6-Bromo-2-(3-(methylthio)thiophen-2-yl)quinoline, 5t}<smiles>CSc1ccsc1-c1ccc2cc(Br)ccc2n1</smiles>

Compound 5t was prepared according to General Procedure $\mathbf{B}$ with enone $\mathbf{4 t}$ (67.7 $\mathrm{mg}$, $0.149 \mathrm{mmol})$, TFA $(0.27 \mathrm{~mL}, 3.58 \mathrm{mmol})$ and $\mathrm{CH}_{2} \mathrm{Cl}_{2}(3.8 \mathrm{~mL})$. Flash column chromatography $\left(\mathrm{SiO}_{2}, 10-20 \% \mathrm{Et}_{2} \mathrm{O}\right.$ in petrol) afforded quinoline $\mathbf{5 t}$ as a cream solid (42.6 mg, 85\% yield).

Compound 5t was prepared according to General Procedure $\mathbf{C}$ with enone 4t $(41.3$ $\mathrm{mg}, 0.087 \mathrm{mmol})$, EtOH $(1.5 \mathrm{~mL})$ and $\mathrm{H}_{2} \mathrm{O}(1.5 \mathrm{~mL})$. Flash column chromatography $\left(\mathrm{SiO}_{2}, 10-20 \% \mathrm{Et}_{2} \mathrm{O}\right.$ in petrol) afforded quinoline $\mathbf{5 t}$ as a cream solid $(27.7 \mathrm{mg}, 91 \%$ yield).

m.p.: $142-144{ }^{\circ} \mathrm{C}$ (crystalized from $\left.\mathrm{CHCl}_{3}\right) ;{ }^{1} \mathbf{H}-\mathbf{N M R}\left(400 \mathrm{MHz}, \mathrm{CDCl}_{3}\right)$ : $8.12(1 \mathrm{H}$, $\mathrm{d}, J 9.0, \mathrm{C}(4) H), 8.07(1 \mathrm{H}, \mathrm{d}, J 9.0, \mathrm{C}(3) H), 8.01(1 \mathrm{H}, \mathrm{d}, J 9.0, \mathrm{C}(7) H), 7.95(1 \mathrm{H}, \mathrm{d}, J$ 2.0, $\mathrm{C}(5) H), 7.78(1 \mathrm{H}, \mathrm{dd}, J 9.0$ and 2.0, C(6)H), $7.48(1 \mathrm{H}, \mathrm{d}, J 5.5, \mathrm{C}(1) H), 7.14(1 \mathrm{H}$, $\mathrm{d}, J 5.5, \mathrm{C}(2) H), 2.58\left(3 \mathrm{H}, \mathrm{s}, \mathrm{SCH}{ }_{3}\right) ;{ }^{13} \mathbf{C}-\mathbf{N M R}\left(100 \mathrm{MHz}, \mathrm{CDCl}_{3}\right): 152.9,146.3$, $136.0,135.4,134.7,133.2,130.8,129.5,128.9$, 127.6, 127.3, 120.5, 119.9, 18.1; LRMS $(\mathrm{ESI}+, \mathrm{m} / \mathrm{z}): 336.0\left(\left[\mathrm{M}\left({ }^{79} \mathrm{Br}\right)+\mathrm{H}\right]^{+}, 100 \%\right), 338.0\left(\left[\mathrm{M}\left({ }^{81} \mathrm{Br}\right)+\mathrm{H}\right]^{+}, 100 \%\right)$; HRMS (ESI+, m/z): $\mathrm{C}_{14} \mathrm{H}_{11} \mathrm{BrNS}_{2}\left(\left[\mathrm{M}\left({ }^{79} \mathrm{Br}\right)+\mathrm{H}\right]^{+}\right)$requires 335.95108, found 335.95120, $\left(\left[\mathrm{M}\left({ }^{81} \mathrm{Br}\right)+\mathrm{H}\right]^{+}\right)$requires 337.94903, found 337.94882; IR $v_{\max }($ film $) / \mathrm{cm}^{-}$ ${ }^{1}: 3082,3058,2985,2918,2855,1589,1541,1501,1488,1458,1439,1413,1382$, 1351, 1317, 1266, 1236, 1192, 1164, 1128, 1095, 1058. 


\section{6-bromo-2-(1-(ethylthio)octan-2-yl)quinoline, 5u}<smiles>CCSCCC(CC)c1ccc2cc(Br)ccc2n1</smiles>

Compound 5u was prepared according to General Procedure B with enone $4 \mathbf{u}(62.5$ $\mathrm{mg}, 0.125 \mathrm{mmol})$, TFA $(0.23 \mathrm{~mL}, 3.0 \mathrm{mmol})$ and $\mathrm{CH}_{2} \mathrm{Cl}_{2}(3.2 \mathrm{~mL})$. Flash column chromatography $\left(\mathrm{SiO}_{2}, 10 \% \mathrm{Et}_{2} \mathrm{O}\right.$ in petrol) afforded quinoline $\mathbf{5 u}$ as a pale yellow oil (34.0 mg, 71\% yield).

${ }^{1}$ H-NMR $\left(400 \mathrm{MHz}, \mathrm{CDCl}_{3}\right): 8.01(1 \mathrm{H}, \mathrm{d}, J$ 8.5, C(5)H), 7.97-7.94 (2H, m, C(6)H, $\mathrm{C}(8) H), 7.76(1 \mathrm{H}$, dd, $J 9.0$ and 2.0, C(7)H), $7.33(1 \mathrm{H}, \mathrm{d}, J 8.5, \mathrm{C}(4) H), 3.17(1 \mathrm{H}$, ap. tt, $J 8.0$ and 6.0, C(2)H), $3.07\left(1 \mathrm{H}, \mathrm{dd}, J 12.5\right.$ and 8.0, $\left.\mathrm{C}(1) H_{\mathrm{A}} \mathrm{H}_{\mathrm{B}}\right), 2.95(1 \mathrm{H}, \mathrm{dd}, J$ 12.5 and 6.0, $\left.\mathrm{C}(1) \mathrm{H}_{\mathrm{A}} H_{\mathrm{B}}\right), 2.47\left(2 \mathrm{H}, \mathrm{qd}, J 7.5\right.$ and $\left.1.0, \mathrm{SCH}_{2} \mathrm{CH}_{3}\right), 1.95-1.82(2 \mathrm{H}, \mathrm{m}$, $\left.\mathrm{C}(3) H_{2}\right), 1.31-1.19\left(8 \mathrm{H}, \mathrm{m}, 4 \times \mathrm{CH}_{2}\right), 1.21\left(1 \mathrm{H}, \mathrm{d}, J 7.5, \mathrm{SCH}_{2} \mathrm{CH}_{3}\right), 0.85(3 \mathrm{H}, \mathrm{t}, J 7.0$, $\left.\left(\mathrm{CH}_{2}\right)_{4} \mathrm{CH}_{3}\right) ;{ }^{13} \mathrm{C}-\mathbf{N M R}\left(100 \mathrm{MHz}, \mathrm{CDCl}_{3}\right): 164.8,146.6,135.0,132.6,131.0,129.5$, $128.2,121.9,119.5,48.8,37.0,35.0,31.7,29.3,27.4,26.6,22.6,14.7,14.0$; LRMS $(\mathrm{ESI}+, \mathrm{m} / \mathrm{z}): 380.1\left(\left[\mathrm{M}\left({ }^{79} \mathrm{Br}\right)+\mathrm{H}\right]^{+}, 100 \%\right), 382.1\left(\left[\mathrm{M}\left({ }^{81} \mathrm{Br}\right)+\mathrm{H}\right]^{+}, 100 \%\right) ; \quad$ HRMS $(\mathrm{ESI}+, \mathrm{m} / \mathrm{z}): \mathrm{C}_{19} \mathrm{H}_{27} \mathrm{BrNS}\left(\left[\mathrm{M}\left({ }^{79} \mathrm{Br}\right)+\mathrm{H}\right]^{+}\right)$requires 380.10421 , found 380.10435, $\left(\left[\mathrm{M}\left({ }^{81} \mathrm{Br}\right)+\mathrm{H}\right]^{+}\right)$requires 382.10216 , found 382.10212; IR $v_{\max }($ film $) / \mathrm{cm}^{-1}: 3053$, 2955, 2925, 2854, 2361, 2341, 1912, 1653, 1595, 1555, 1488, 1456, 1388, 1375, 1297 , 1264, 1188, 1117, 1059. 


\section{6-Bromo-2-(2-(ethylthio)heptyl)quinoline, 5v}<smiles>CSC(CS)Cc1ccc2cc(Br)ccc2n1</smiles>

Compound 5v was prepared according to General Procedure B with enone 4v (78.5 $\mathrm{mg}, 0.16 \mathrm{mmol})$, TFA $(0.30 \mathrm{~mL}, 3.84 \mathrm{mmol})$ and $\mathrm{CH}_{2} \mathrm{Cl}_{2}(4.0 \mathrm{~mL})$. Flash column chromatography $\left(\mathrm{SiO}_{2}, 0-10 \% \mathrm{Et}_{2} \mathrm{O}\right.$ in petrol) afforded quinoline $\mathbf{5 v}$ as a pale brown oil (47.5 mg, 80\% yield).

${ }^{1}$ H-NMR (400 MHz, $\left.\mathrm{CDCl}_{3}\right): 8.00(1 \mathrm{H}, \mathrm{d}, J 8.5, \mathrm{C}(4) H), 7.96(1 \mathrm{H}, \mathrm{d}, J 2.0, \mathrm{C}(5) H)$, $7.93(1 \mathrm{H}, \mathrm{d}, J 9.0, \mathrm{C}(7) H), 7.76(1 \mathrm{H}, \mathrm{dd}, J 9.0$ and $2.0, \mathrm{C}(6) H), 7.38(1 \mathrm{H}, \mathrm{d}, J 8.5$, $\mathrm{C}(3) H), 3.30-3.16\left(3 \mathrm{H}, \mathrm{m}, \mathrm{C}(1) \mathrm{H}, \mathrm{C}(2) \mathrm{H}_{2}\right), 2.50-2.41\left(2 \mathrm{H}, \mathrm{m}, \mathrm{SCH}_{2} \mathrm{CH}_{3}\right), 1.71-1.42$ $\left(4 \mathrm{H}, \mathrm{m}, 2 \times \mathrm{CH}_{2}\right), 1.34-1.24\left(4 \mathrm{H}, \mathrm{m}, 2 \times \mathrm{CH}_{2}\right), 1.17\left(3 \mathrm{H}, \mathrm{t}, J 7.5, \mathrm{SCH}_{2} \mathrm{CH}_{3}\right), 0.89$ (3H, t, $J$ 7.0, $\left.\left(\mathrm{CH}_{2}\right)_{4} \mathrm{CH}_{3}\right) ;{ }^{13} \mathbf{C}-\mathbf{N M R}\left(100 \mathrm{MHz}, \mathrm{CDCl}_{3}\right): 161.1,146.5,134.9,132.8$, 130.7, 129.5, 128.0, 123.3, 119.6, 45.6, 45.2, 35.1, 31.7, 26.4, 24.8, 22.6, 14.9, 14.1; LRMS (ESI+, m/z): $366.1\left(\left[\mathrm{M}\left({ }^{79} \mathrm{Br}\right)+\mathrm{Na}\right]^{+}, 100 \%\right), 368.1\left(\left[\mathrm{M}\left({ }^{81} \mathrm{Br}\right)+\mathrm{Na}\right]^{+}, 100 \%\right)$; HRMS $(\mathrm{ESI}+, \mathrm{m} / \mathrm{z}): \mathrm{C}_{18} \mathrm{H}_{24} \mathrm{BrNNaS}\left(\left[\mathrm{M}\left({ }^{79} \mathrm{Br}\right)+\mathrm{Na}\right]^{+}\right)$requires 366.08856, found 366.08857, $\left(\left[\mathrm{M}\left({ }^{81} \mathrm{Br}\right)+\mathrm{Na}\right]^{+}\right)$requires 368.08651 , found 368.08634 ; IR $v_{\max }$ (film) $/ \mathrm{cm}^{-1}: 2956,2927,2856,1650,1594,1556,1487,1457,1377,1303,1263,1186$, 1119, 1059. 


\section{6-Bromo-2-(4-(methylthio)-5,6-dihydro-2H-pyran-3-yl)quinoline, 5w}<smiles>CSC1=C(c2ccc3cc(Br)ccc3n2)COCC1</smiles>

Compound 5w was prepared according to General Procedure $\mathbf{C}$ with enone $\mathbf{4 w}$ (45.4 $\mathrm{mg}, 0.1 \mathrm{mmol})$, EtOH $(1.5 \mathrm{~mL})$ and $\mathrm{H}_{2} \mathrm{O}(1.5 \mathrm{~mL})$. Flash column chromatography $\left(\mathrm{SiO}_{2}, 20 \%\right.$ EtOAc in petrol) afforded quinoline $\mathbf{5 w}$ as a pale brown solid (28.0 $\mathrm{mg}$, $83 \%$ yield).

m.p.: $117-119{ }^{\circ} \mathrm{C}$ (crystalized from $\mathrm{Et}_{2} \mathrm{O} /$ petrol); ${ }^{\mathbf{1}} \mathbf{H}$-NMR $\left(400 \mathrm{MHz}, \mathrm{CDCl}_{3}\right): 8.05$ $(1 \mathrm{H}, \mathrm{d}, J 8.5, \mathrm{C}(5) H), 7.97(1 \mathrm{H}, \mathrm{d}, J 2.0, \mathrm{C}(6) H), 7.97(1 \mathrm{H}, \mathrm{d}, J 9.0, \mathrm{C}(8) H), 7.77(1 \mathrm{H}$, dd, $J 9.0$ and 2.0, C(7)H), $7.61(1 \mathrm{H}, \mathrm{d}, J 8.5, \mathrm{C}(4) H), 4.66\left(2 \mathrm{H}, \mathrm{t}, J 2.5, \mathrm{C}(3) H_{2}\right), 4.02$ $\left(2 \mathrm{H}, \mathrm{t}, J 5.5, \mathrm{C}(1) H_{2}\right), 2.59\left(2 \mathrm{H}, \mathrm{tt}, J 5.5\right.$ and $\left.2.5, \mathrm{C}(2) H_{2}\right), 2.23\left(3 \mathrm{H}, \mathrm{s}, \mathrm{SCH}_{3}\right) ;{ }^{13} \mathrm{C}-$ NMR (100 MHz, $\left.\mathrm{CDCl}_{3}\right): 157.0,146.2,134.7,133.6,132.9,131.6,131.0,129.5$, 128.0, 122.7, 120.3， 68.7， 64.8， 29.2， 14.3; LRMS (ESI+, m/z): 336.0 $\left(\left[\mathrm{M}\left({ }^{79} \mathrm{Br}\right)+\mathrm{Na}\right]^{+}, \quad 100 \%\right), 338.0\left(\left[\mathrm{M}\left({ }^{81} \mathrm{Br}\right)+\mathrm{Na}\right]^{+}, 100 \%\right) ; \quad$ HRMS $(\mathrm{ESI}+, \mathrm{m} / \mathrm{z})$ : $\mathrm{C}_{15} \mathrm{H}_{15} \mathrm{BrNOS}\left(\left[\mathrm{M}\left({ }^{79} \mathrm{Br}\right)+\mathrm{H}\right]^{+}\right)$requires 336.00522, found 336.00532, $\left(\left[\mathrm{M}\left({ }^{81} \mathrm{Br}\right)+\mathrm{H}\right]^{+}\right)$ requires 338.00318, found 338.00310; IR $v_{\max }\left(\right.$ film) $/ \mathrm{cm}^{-1}: 2959,2922,2851,1589$, 1555, 1539, 1486, 1421, 1380, 1319, 1301, 1240, 1188, 1139, 1121, 1102, 1059, 1017. 


\section{2-(6-Bromoquinolin-2-yl)- $N, N$-dimethylaniline, $5 x$}<smiles>CN(C)c1ccccc1-c1ccc2cc(Br)ccc2n1</smiles>

Compound 5x was prepared according to General Procedure $\mathbf{B}$ with enone $4 \mathbf{x}(81.2$ $\mathrm{mg}, 0.182 \mathrm{mmol})$, TFA $(0.34 \mathrm{~mL}, 4.38 \mathrm{mmol})$ and $\mathrm{CH}_{2} \mathrm{Cl}_{2}(4.6 \mathrm{~mL})$. Flash column chromatography $\left(\mathrm{SiO}_{2}, 20 \% \mathrm{Et}_{2} \mathrm{O}\right.$ in petrol) afforded quinoline $\mathbf{5 x}$ as a pale brown solid (51.2 $\mathrm{mg}, 86 \%$ yield).

Compound 5x was prepared according to General Procedure $\mathbf{C}$ with enone $4 \mathbf{x}$ (46.3 $\mathrm{mg}, 0.104 \mathrm{mmol})$, EtOH $(1.5 \mathrm{~mL})$ and $\mathrm{H}_{2} \mathrm{O}(1.5 \mathrm{~mL})$. Flash column chromatography $\left(\mathrm{SiO}_{2}, 20 \% \mathrm{Et}_{2} \mathrm{O}\right.$ in petrol) afforded quinoline $\mathbf{5 x}$ as a pale brown solid $(31.0 \mathrm{mg}, 91 \%$ yield).

m.p.: $92-94{ }^{\circ} \mathrm{C}$ (crystalized from $\left.\mathrm{CHCl}_{3}\right) ;{ }^{1} \mathbf{H}-\mathbf{N M R}\left(400 \mathrm{MHz}, \mathrm{CDCl}_{3}\right): 8.06(1 \mathrm{H}, \mathrm{d}$, $J$ 9.0, C(9)H), $8.05(1 \mathrm{H}, \mathrm{d}, J 8.5, \mathrm{C}(6) H), 8.03(1 \mathrm{H}, \mathrm{d}, J 8.5, \mathrm{C}(5) H), 8.00(1 \mathrm{H}, \mathrm{d}, J$ 2.0, $\mathrm{C}(7) H), 7.79(1 \mathrm{H}, \mathrm{dd}, J 9.0$ and 2.0, $\mathrm{C}(8) H), 7.72(1 \mathrm{H}, \mathrm{dd}, J 7.5$ and 1.5, C(4)H), $7.40(1 \mathrm{H}$, ddd, 8.0, 7.5, 1.5, C(2)H), 7.16-7.12 (2H, m, C(1)H, C(2)H), $2.63(6 \mathrm{H}, \mathrm{s}$, $\left.\mathrm{N}\left(\mathrm{CH}_{3}\right)_{2}\right) ;{ }^{13} \mathbf{C}-\mathrm{NMR}\left(100 \mathrm{MHz}, \mathrm{CDCl}_{3}\right): 160.7,151.6,147.1,134.3,132.5,132.4$, 131.9, 131.2, 129.9, 129.4, 128.1, 123.5, 122.1, 119.9, 117.6, 44.0; LRMS (ESI+, $\mathrm{m} / \mathrm{z}): 327.0\left(\left[\mathrm{M}\left({ }^{79} \mathrm{Br}\right)+\mathrm{H}\right]^{+}, 100 \%\right), 329.0\left(\left[\mathrm{M}\left({ }^{81} \mathrm{Br}\right)+\mathrm{H}\right]^{+}, 100 \%\right) ; \quad$ HRMS (ESI+, $\mathrm{m} / \mathrm{z}): \quad \mathrm{C}_{17} \mathrm{H}_{16} \mathrm{BrN}_{2}\left(\left[\mathrm{M}\left({ }^{79} \mathrm{Br}\right)+\mathrm{H}\right]^{+}\right) \quad$ requires 327.04914, found 327.04916, $\left(\left[\mathrm{M}\left({ }^{79} \mathrm{Br}\right)+\mathrm{H}\right]^{+}\right)$requires 329.04709 , found $329.04705 ;$ IR $v_{\max }($ film $) / \mathrm{cm}^{-1}: 3058$, 2984, 2941, 2864, 2833, 2785, 1595, 1572, 1546, 1485, 1453, 1432, 1379, 1305, 1188, $1158,1142,1126,1105,1060,1034$. 
General Procedure D - tandem hydroacylation - C-S functionalisation reactions

$\left[\mathrm{Rh}(\mathrm{nbd})_{2}\right] \mathrm{BF}_{4}(3.7 \mathrm{mg}, 0.01 \mathrm{mmol})$ and dcpm $(4.1 \mathrm{mg}, 0.01 \mathrm{mmol})$ were added to a microwave vial, which was then evacuated and backfilled with $\mathrm{N}_{2(\mathrm{~g})}$ three times. Then was added distilled and degassed acetone $(0.4 \mathrm{~mL})$, and the resulting suspension sonicated in order to solubilise the phosphine ligand. Once the solution was homogenous, $\mathrm{H}_{2(\mathrm{~g})}$ was bubbled through for 1 minute, followed by $\mathrm{N}_{2(\mathrm{~g})}$ until the volume was reduced to $0.1 \mathrm{~mL}$. This resulting solution was transferred by syringe into a microwave vial containing aldehyde $(0.24 \mathrm{mmol})$ and alkyne $(0.20 \mathrm{mmol})$. The resulting solution was stirred at the given temperature until full consumption of the starting alkyne was observed. Upon consumption of the starting alkyne the resulting mixture was transferred by syringe into a microwave vial containing $\mathrm{Ag}_{2} \mathrm{CO}_{3}$ (55.2 $\mathrm{mg}, 0.20 \mathrm{mmol})$ and boronic acid $(0.30 \mathrm{mmol})$ with the aid of DCE $(0.5 \mathrm{~mL})$. The resulting solution was stirred at $55{ }^{\circ} \mathrm{C}$ for 16 hours. The reaction mixture was filtered through a pad of $\mathrm{SiO}_{2}$ (washed with $\mathrm{Et}_{2} \mathrm{O}$ ), and concentrated in vacuo to afford the crude hyroacylation-Suzuki product. 


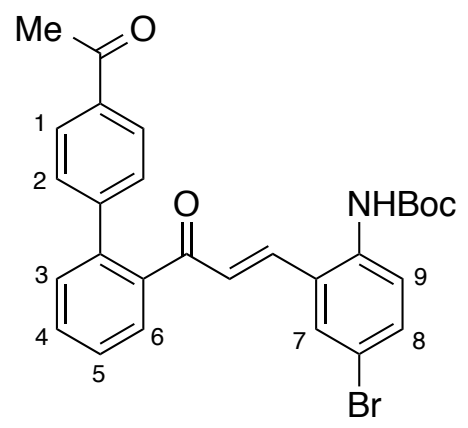

Compound 9a was prepared according to General Procedure D with $\left[\mathrm{Rh}(\mathrm{nbd})_{2}\right] \mathrm{BF}_{4}$ (3.7 mg, $0.01 \mathrm{mmol})$, dcpm (4.1 mg, $0.01 \mathrm{mmol}$ ), aldehyde $\mathbf{2 a}(36.5 \mathrm{mg}, 0.24 \mathrm{mmol})$, alkyne 3d $(59.2 \mathrm{mg}, 0.20 \mathrm{mmol})$ and acetone $(0.1 \mathrm{~mL})$, 4-acetylphenylboronic acid (49.2 mg, $0.30 \mathrm{mmol}), \mathrm{Ag}_{2} \mathrm{CO}_{3}(55.2 \mathrm{mg}, 0.20 \mathrm{mmol})$ and $\mathrm{DCE}(0.5 \mathrm{~mL})$ at $55^{\circ} \mathrm{C}$ for the first step. Flash column chromatography $\left(\mathrm{SiO}_{2}, 20-40 \% \mathrm{Et}_{2} \mathrm{O}\right.$ in petrol) afforded enone $9 \mathrm{a}$ as a pale brown oil (92.2 $\mathrm{mg}, 90 \%$ yield).

${ }^{1}$ H-NMR $\left(400 \mathrm{MHz}, \mathrm{CDCl}_{3}\right): 8.04(2 \mathrm{H}, \mathrm{d}, J$ 8.5, C(1)H), $7.75(1 \mathrm{H}, \mathrm{dd}, J 7.5$ and 1.0, $\mathrm{C}(6) H), 7.65(1 \mathrm{H}, \mathrm{d}, J 9.0, \mathrm{C}(9) H), 7.63(2 \mathrm{H}, \mathrm{td}, J 7.5$ and $1.5, \mathrm{C}(4) H), 7.56-7.46(5 \mathrm{H}$, m, C(2)H, C(O)CHCH, C(3)H, C(5)H), $7.37(1 \mathrm{H}, \mathrm{dd}, J 9.0$ and 2.5, C(8)H), $6.84(1 \mathrm{H}$, $\mathrm{d}, J 2.5, \mathrm{C}(7) H), 6.45(1 \mathrm{H}$, br. s, NH), $6.45(1 \mathrm{H}, \mathrm{d}, J 15.5, \mathrm{C}(\mathrm{O}) \mathrm{CHCH}), 2.64(3 \mathrm{H}, \mathrm{s}$, $\left.\mathrm{C}(\mathrm{O}) \mathrm{CH}_{3}\right), 1.52\left(9 \mathrm{H}, \mathrm{s}, \mathrm{C}\left(\mathrm{CH}_{3}\right)_{3}\right) ;{ }^{13} \mathbf{C}-\mathbf{N M R}\left(100 \mathrm{MHz}, \mathrm{CDCl}_{3}\right)$ : 197.3, 194.3, 152.5, $145.3,140.0,139.3,136.5,136.2,136.0,133.5,131.5,130.2,129.6,129.5,129.4$, 129.3, 128.8, 128.4, 127.8, 124.0, 116.8, 81.4, 28.3, 26.8; LRMS (ESI+, m/z): 542.1 $\left(\left[\mathrm{M}\left({ }^{79} \mathrm{Br}\right)+\mathrm{Na}\right]^{+}, \quad 100 \%\right), \quad 544.1\left(\left[\mathrm{M}\left({ }^{81} \mathrm{Br}\right)+\mathrm{Na}\right]^{+}, \quad 100 \%\right) ; \quad$ HRMS $(\mathrm{ESI}+, \mathrm{m} / \mathrm{z})$ : $\mathrm{C}_{28} \mathrm{H}_{26} \mathrm{BrNNaO}_{4} \quad\left(\left[\mathrm{M}\left({ }^{79} \mathrm{Br}\right)+\mathrm{Na}\right]^{+}\right)$requires 542.09374, found 542.09373, $\left(\left[\mathrm{M}\left({ }^{81} \mathrm{Br}\right)+\mathrm{Na}\right]^{+}\right)$requires 544.09170, found 544.09171; IR $v_{\max }($ film $) / \mathrm{cm}^{-1}: 3317$, 3062, 2977, 2931, 1724, 1681, 1603, 1509, 1403, 1367, 1324, 1266, 1156, 1050, 1023. 
<smiles>CCCCNc1ccc(Br)cc1/C=C/C(=O)c1ccccc1-c1ccc(C)cc1</smiles>

Compound 9b was prepared according to General Procedure $\mathbf{D}$ with $\left[\mathrm{Rh}(\mathrm{nbd})_{2}\right] \mathrm{BF}_{4}$ (3.7 mg, $0.01 \mathrm{mmol})$, dcpm (4.1 mg, $0.01 \mathrm{mmol})$, aldehyde $2 \mathrm{a}(36.5 \mathrm{mg}, 0.24 \mathrm{mmol}$ ), alkyne $3 \mathbf{d}(59.2 \mathrm{mg}, 0.20 \mathrm{mmol})$ and acetone $(0.1 \mathrm{~mL})$, 4-methylphenylboronic acid (40.8 mg, $0.30 \mathrm{mmol}), \mathrm{Ag}_{2} \mathrm{CO}_{3}(55.2 \mathrm{mg}, 0.20 \mathrm{mmol})$ and $\mathrm{DCE}(0.5 \mathrm{~mL})$ at $55^{\circ} \mathrm{C}$ for the first step. Flash column chromatography $\left(\mathrm{SiO}_{2}, 10-30 \% \mathrm{Et}_{2} \mathrm{O}\right.$ in petrol) afforded enone $\mathbf{9 b}$ as a pale yellow powder (90.4 $\mathrm{mg}, 92 \%$ yield).

m.p.: $192-194{ }^{\circ} \mathrm{C}$ (crystalized from $\mathrm{Et}_{2} \mathrm{O} /$ petrol); ${ }^{1} \mathbf{H}-\mathbf{N M R}\left(400 \mathrm{MHz}, \mathrm{CDCl}_{3}\right.$ ): 7.74 $(1 \mathrm{H}, \mathrm{d}, J 7.5, \mathrm{C}(6) H), 7.71(1 \mathrm{H}, \mathrm{d}, J 9.0, \mathrm{C}(9) H), 7.61(1 \mathrm{H}$, ddd, $J$ 8.0, 7.0 and 1.5, $\mathrm{C}(4) H), 7.51-7.46(2 \mathrm{H}, \mathrm{m}, \mathrm{C}(3) H, \mathrm{C}(5) H), 7.44(1 \mathrm{H}, \mathrm{d}, J 15.5, \mathrm{C}(\mathrm{O}) \mathrm{CHCH}), 7.37(1 \mathrm{H}$, $\mathrm{dd}, J 9.0$ and $2.5, \mathrm{C}(8) H), 7.33-7.28(4 \mathrm{H}, \mathrm{m}, \mathrm{C}(1) H, \mathrm{C}(2) H), 6.74(1 \mathrm{H}, \mathrm{d}, J 2.5$, $\mathrm{C}(7) H), 6.42\left(1 \mathrm{H}\right.$, br. s, NH), $6.32(1 \mathrm{H}, \mathrm{d}, J 15.5, \mathrm{C}(\mathrm{O}) \mathrm{CHCH}), 2.44\left(3 \mathrm{H}, \mathrm{s}, \mathrm{CH}_{3}\right)$, $1.55\left(9 \mathrm{H}, \mathrm{s}, \mathrm{C}\left(\mathrm{CH}_{3}\right)_{3}\right) ;{ }^{13} \mathrm{C}-\mathrm{NMR}\left(100 \mathrm{MHz}, \mathrm{CDCl}_{3}\right): 195.0,152.5,141.3,139.4$, $138.5,137.6,135.8,134.6,133.1,131.4,130.3,130.1,129.8,129.7,129.4,129.2$, 127.9, 127.5, 123.4, 116.5, 81.4, 28.3, 21.4; LRMS (ESI+, m/z): 514.1 $\left(\left[\mathrm{M}\left({ }^{79} \mathrm{Br}\right)+\mathrm{Na}\right]^{+}, \quad 100 \%\right), \quad 516.1\left(\left[\mathrm{M}\left({ }^{81} \mathrm{Br}\right)+\mathrm{Na}\right]^{+}, 100 \%\right) ; \quad$ HRMS $(\mathrm{ESI}+, \mathrm{m} / \mathrm{z})$ : $\mathrm{C}_{27} \mathrm{H}_{26} \mathrm{BrNNaO}_{3} \quad\left(\left[\mathrm{M}\left({ }^{79} \mathrm{Br}\right)+\mathrm{Na}\right]^{+}\right)$requires 514.09883, found 514.09876, $\left(\left[\mathrm{M}\left({ }^{81} \mathrm{Br}\right)+\mathrm{Na}\right]^{+}\right)$requires 516.09678, found 516.09679; IR $v_{\max }($ film $) / \mathrm{cm}^{-1}: 3317$, 2977, 2927, 1725, 1663, 1602, 1507, 1401, 1393, 1367, 1324, 1288, 1234, 1206, 1154, $1049,1020$. 
<smiles>CCCCNc1ccc(Br)cc1/C=C/C(=O)c1ccccc1-c1ccc(OC)cc1</smiles>

Compound 9c was prepared according to General Procedure D with $\left[\mathrm{Rh}(\mathrm{nbd})_{2}\right] \mathrm{BF}_{4}$ (3.7 mg, $0.01 \mathrm{mmol})$, dcpm (4.1 mg, $0.01 \mathrm{mmol})$, aldehyde $2 \mathrm{a}(36.5 \mathrm{mg}, 0.24 \mathrm{mmol}$ ), alkyne $3 \mathbf{d}(59.2 \mathrm{mg}, 0.20 \mathrm{mmol})$ and acetone $(0.1 \mathrm{~mL})$, 4-methoxyphenylboronic acid (45.6 mg, $0.30 \mathrm{mmol}), \mathrm{Ag}_{2} \mathrm{CO}_{3}(55.2 \mathrm{mg}, 0.20 \mathrm{mmol})$ and DCE $(0.5 \mathrm{~mL})$ at $55^{\circ} \mathrm{C}$ for the first step. Flash column chromatography $\left(\mathrm{SiO}_{2}, 20-40 \% \mathrm{Et}_{2} \mathrm{O}\right.$ in petrol) afforded enone $9 \mathrm{c}$ as a fluffy off white solid (94.2 $\mathrm{mg}, 93 \%$ yield).

m.p.: $156-158{ }^{\circ} \mathrm{C}$ (crystalized from $\mathrm{Et}_{2} \mathrm{O} /$ petrol); ${ }^{1} \mathbf{H}-\mathbf{N M R}\left(400 \mathrm{MHz}, \mathrm{CDCl}_{3}\right)$ : 7.71 $(1 \mathrm{H}, \mathrm{d}, \mathrm{J} 7.5, \mathrm{C}(6) H), 7.70(1 \mathrm{H}, \mathrm{d}, J 9.0, \mathrm{C}(9) H) 7.60(1 \mathrm{H}$, ddd, $J$ 8.0, 7.0 and 1.5, $\mathrm{C}(4) H)$ 7.49-7.45 (2H, m, C(3)H, C(5)H), $7.44(1 \mathrm{H}, \mathrm{d}, J 15.6, \mathrm{C}(\mathrm{O}) \mathrm{CHCH}), 7.37(1 \mathrm{H}$, $\mathrm{dd}, J 9.0$ and 2.5, C(8)H), $7.34(2 \mathrm{H}, \mathrm{d}, J 9.0, \mathrm{C}(2) H), 7.00(2 \mathrm{H}, \mathrm{d}, J 9.0, \mathrm{C}(1) H), 6.87$ $(1 \mathrm{H}, \mathrm{d}, J 2.5, \mathrm{C}(7) H), 6.43(1 \mathrm{H}$, br. s, $\mathrm{NH}), 6.36(1 \mathrm{H}, \mathrm{d}, J 15.5, \mathrm{C}(\mathrm{O}) \mathrm{CHCH}), 3.85$ $\left(3 \mathrm{H}, \mathrm{s}, \mathrm{OCH}_{3}\right), 1.55\left(9 \mathrm{H}, \mathrm{s}, \mathrm{C}\left(\mathrm{CH}_{3}\right)_{3}\right) ;{ }^{13} \mathbf{C}-\mathbf{N M R}\left(100 \mathrm{MHz}, \mathrm{CDCl}_{3}\right): 195.2,159.8$, $152.5,140.9$, 139.3, 135.8, 134.8, 133.2, 132.7, 131.4, 130.6, 130.1, 130.0, 129.7, 129.2, 127.9, 127.3, 123.5, 116.6, 114.3, 81.4, 55.4, 28.3; LRMS (ESI+, m/z): 530.1 $\left(\left[\mathrm{M}\left({ }^{79} \mathrm{Br}\right)+\mathrm{Na}\right]^{+}, \quad 100 \%\right), \quad 532.1\left(\left[\mathrm{M}\left({ }^{81} \mathrm{Br}\right)+\mathrm{Na}\right]^{+}, 100 \%\right) ; \quad$ HRMS (ESI+, $\left.\mathrm{m} / \mathrm{z}\right)$ : $\mathrm{C}_{27} \mathrm{H}_{26} \mathrm{BrNNaO}_{4} \quad\left(\left[\mathrm{M}\left({ }^{79} \mathrm{Br}\right)+\mathrm{Na}\right]^{+}\right)$requires 530.09374, found 530.09382, $\left(\left[\mathrm{M}\left({ }^{81} \mathrm{Br}\right)+\mathrm{Na}\right]^{+}\right)$requires 532.09170, found 532.09177; IR $v_{\max }($ film $) / \mathrm{cm}^{-1}: 3319$, 3008, 2976, 2933, 2837, 1722, 1662, 1604, 1509, 1474, 1401, 1367, 1323, 1289, 1245, 1153, 1035, 1020. 
<smiles>CC(=O)OCCC(C)(C)Oc1ccc(Br)cc1/C=C/C(=O)C1=C(c2ccc(C(C)=O)cc2)CCOC1</smiles>

Compound 9d was prepared according to General Procedure $\mathbf{D}$ with $\left[\mathrm{Rh}(\mathrm{nbd})_{2}\right] \mathrm{BF}_{4}$ (3.7 mg, $0.01 \mathrm{mmol})$, dcpm ( $4.1 \mathrm{mg}, 0.01 \mathrm{mmol})$, aldehyde $2 \mathrm{w}(38.0 \mathrm{mg}, 0.24 \mathrm{mmol})$, alkyne 3d $(59.2 \mathrm{mg}, 0.20 \mathrm{mmol})$ and acetone $(0.1 \mathrm{~mL})$, 4-acetylphenylboronic acid (49.2 mg, $0.30 \mathrm{mmol}), \mathrm{Ag}_{2} \mathrm{CO}_{3}(55.2 \mathrm{mg}, 0.20 \mathrm{mmol})$ and DCE $(0.5 \mathrm{~mL})$ at r.t. for the first step. Flash column chromatography $\left(\mathrm{SiO}_{2}, 10-40 \%\right.$ EtOAc in petrol) afforded enone $9 d$ as a pale brown oil ( $91.3 \mathrm{mg}, 87 \%$ yield).

${ }^{1}$ H-NMR (400 MHz, CDCl $): 8.01(2 \mathrm{H}, \mathrm{d}, J$ 8.5, C(1)H), $7.62(1 \mathrm{H}, \mathrm{d}, J$ 8.5, C(8)H), $7.40(1 \mathrm{H}, \mathrm{d}, J 15.5, \mathrm{C}(\mathrm{O}) \mathrm{CHCH}), 7.39(2 \mathrm{H}, \mathrm{d}, J 8.5, \mathrm{C}(2) H), 7.31(1 \mathrm{H}, \mathrm{dd}, J 8.5$ and 2.5, C(7)H), $6.49(1 \mathrm{H}$, br. s, NH), $6.47(1 \mathrm{H}, \mathrm{d}, J 2.5, \mathrm{C}(6) H), 6.04(1 \mathrm{H}, \mathrm{d}, J$ 15.5, $\mathrm{C}(\mathrm{O}) \mathrm{CHCH}), 4.54\left(2 \mathrm{H}, \mathrm{t}, J 2.5, \mathrm{C}(5) \mathrm{H}_{2}\right), 3.97\left(2 \mathrm{H}, \mathrm{t}, J 5.5, \mathrm{C}(3) \mathrm{H}_{2}\right), 2.65(2 \mathrm{H}, \mathrm{tt}, J$ 5.5 and $\left.2.5, \mathrm{C}(4) \mathrm{H}_{2}\right), 2.61\left(3 \mathrm{H}, \mathrm{s}, \mathrm{C}(\mathrm{O}) \mathrm{CH}_{3}\right), 1.51\left(9 \mathrm{H}, \mathrm{s}, \mathrm{C}\left(\mathrm{CH}_{3}\right)_{3}\right) ;{ }^{13} \mathbf{C}-\mathbf{N M R}(100$ $\left.\mathrm{MHz}, \mathrm{CDCl}_{3}\right)$ : 197.0, 192.0, 152.5, 145.2, 143.4, 137.3, 137.2, 135.9, 134.2, 133.2, 129.2, 129.1, 128.9, 128.6, 127.8, 123.9, 116.6, 81.4, 66.0, 64.1, 31.0, 28.2, 26.8; LRMS (ESI+, m/z): $548.1\left(\left[\mathrm{M}\left({ }^{79} \mathrm{Br}\right)+\mathrm{Na}\right]^{+}, 100 \%\right), 550.1\left(\left[\mathrm{M}\left({ }^{81} \mathrm{Br}\right)+\mathrm{Na}\right]^{+}, 100 \%\right)$; HRMS $(\mathrm{ESI}+, \mathrm{m} / \mathrm{z}): \mathrm{C}_{27} \mathrm{H}_{28} \mathrm{BrNNaO}_{5}\left(\left[\mathrm{M}\left({ }^{79} \mathrm{Br}\right)+\mathrm{Na}\right]^{+}\right)$requires 548.10431, found 548.10433, $\left(\left[\mathrm{M}\left({ }^{81} \mathrm{Br}\right)+\mathrm{Na}\right]^{+}\right)$requires 550.10226, found 550.10245; IR $v_{\max }$ (film) $/ \mathrm{cm}^{-1}: 3306,2975,2929,2855,1721,1684,1650,1596,1507,1402,1365,1321$, 1261, 1233, 1206, 1153, 1049, 1011. 
<smiles>CCCCNc1ccc(Br)cc1/C=C/C(=O)C1=C(c2ccc(C)cc2)CCOC1</smiles>

Compound 9e was prepared according to General Procedure D with $\left[\mathrm{Rh}(\mathrm{nbd})_{2}\right] \mathrm{BF}_{4}$ (3.7 mg, $0.01 \mathrm{mmol})$, dcpm (4.1 mg, $0.01 \mathrm{mmol}$ ), aldehyde $2 \mathrm{w}$ (38.0 mg, $0.24 \mathrm{mmol}$ ), alkyne 3d $(59.2 \mathrm{mg}, 0.20 \mathrm{mmol})$ and acetone $(0.1 \mathrm{~mL})$, 4-methylphenylboronic acid (40.8 mg, $0.30 \mathrm{mmol}), \mathrm{Ag}_{2} \mathrm{CO}_{3}(55.2 \mathrm{mg}, 0.20 \mathrm{mmol})$ and DCE $(0.5 \mathrm{~mL})$ at r.t. for the first step. Flash column chromatography $\left(\mathrm{SiO}_{2}, 20-40 \%\right.$ EtOAc in petrol) afforded enone $9 \mathrm{e}$ as a pale yellow solid ( $83.6 \mathrm{mg}, 84 \%$ yield).

m.p.: $194-196{ }^{\circ} \mathrm{C}$ (crystalized from $\mathrm{Et}_{2} \mathrm{O} /$ petrol); ${ }^{1} \mathbf{H}-\mathbf{N M R}\left(400 \mathrm{MHz}, \mathrm{CDCl}_{3}\right)$ : 7.68 $(1 \mathrm{H}, \mathrm{d}, J$ 9.0, C(8)H), $7.37(1 \mathrm{H}, \mathrm{d}, J 15.5, \mathrm{C}(\mathrm{O}) \mathrm{CHCH}), 7.34(1 \mathrm{H}, \mathrm{dd}, J 9.0$ and 2.5, $\mathrm{C}(7) H), 7.25(2 \mathrm{H}, \mathrm{d}, J 8.0, \mathrm{C}(2) H), 7.19(2 \mathrm{H}, \mathrm{d}, J 8.0, \mathrm{C}(1) H), 6.54(1 \mathrm{H}, \mathrm{d}, J 2.5$, $\mathrm{C}(6) H), 6.48(1 \mathrm{H}$, br. s, NH), $6.04(1 \mathrm{H}, \mathrm{d}, J 15.5, \mathrm{C}(\mathrm{O}) \mathrm{CHCH}), 4.55(2 \mathrm{H}, \mathrm{t}, J 2.5$, $\left.\mathrm{C}(5) H_{2}\right), 3.96\left(2 \mathrm{H}, \mathrm{t}, J 5.5, \mathrm{C}(3) H_{2}\right), 2.66\left(2 \mathrm{H}, \mathrm{tt}, J 5.5\right.$ and 2.5, C(4) $\left.H_{2}\right), 2.44(3 \mathrm{H}, \mathrm{s}$, $\left.\mathrm{CH}_{3}\right), 1.53\left(9 \mathrm{H}, \mathrm{s}, \mathrm{C}\left(\mathrm{CH}_{3}\right)_{3}\right) ;{ }^{13} \mathrm{C}-\mathrm{NMR}\left(100 \mathrm{MHz}, \mathrm{CDCl}_{3}\right): 192.4,152.6,145.9$, $139.8,137.6,135.9,135.8,132.9,132.6,130.0,129.7,129.4,128.4,128.0,123.5$, 116.4, 81.3, 66.1, 64.3, 31.3, 28.3, 21.5; LRMS (ESI+, m/z): $520.1\left(\left[\mathrm{M}\left({ }^{79} \mathrm{Br}\right)+\mathrm{Na}\right]^{+}\right.$, $100 \%), 522.1\left(\left[\mathrm{M}\left({ }^{81} \mathrm{Br}\right)+\mathrm{Na}\right]^{+}, 100 \%\right) ; \quad$ HRMS (ESI+, m/z): $\mathrm{C}_{26} \mathrm{H}_{28} \mathrm{BrNNaO}_{4}$ $\left(\left[\mathrm{M}\left({ }^{79} \mathrm{Br}\right)+\mathrm{Na}\right]^{+}\right)$requires 520.10939 , found 520.10934, $\left(\left[\mathrm{M}\left({ }^{81} \mathrm{Br}\right)+\mathrm{Na}\right]^{+}\right)$requires 522.10735, found 522.10713; IR $v_{\max }\left(\right.$ film) $/ \mathrm{cm}^{-1}: 3306,2976,2924,2851,1726$, 1649, 1594, 1509, 1402, 1366, 1321, 1233, 1156, 1049. 
<smiles>CCCCOC(=O)Nc1ccc(Br)cc1/C=C/C(=O)C1=C(c2ccc(OC)cc2)CCOC1</smiles>

Compound 9f was prepared according to General Procedure D with $\left[\mathrm{Rh}(\mathrm{nbd})_{2}\right] \mathrm{BF}_{4}$ (3.7 mg, $0.01 \mathrm{mmol})$, dcpm (4.1 mg, $0.01 \mathrm{mmol}$ ), aldehyde $2 \mathrm{w}$ (38.0 mg, $0.24 \mathrm{mmol}$ ), alkyne 3d $(59.2 \mathrm{mg}, 0.20 \mathrm{mmol})$ and acetone $(0.1 \mathrm{~mL})$, 4-methoxyphenylboronic acid (45.6 mg, $0.30 \mathrm{mmol}), \mathrm{Ag}_{2} \mathrm{CO}_{3}(55.2 \mathrm{mg}, 0.20 \mathrm{mmol})$ and DCE $(0.5 \mathrm{~mL})$ at r.t. for the first step. Flash column chromatography $\left(\mathrm{SiO}_{2}, 20-30 \%\right.$ EtOAc in petrol) afforded enone $9 f$ as a fluffy white powder $(83.7 \mathrm{mg}, 81 \%$ yield $)$.

m.p.: $160-162{ }^{\circ} \mathrm{C}$ (crystalized from $\mathrm{Et}_{2} \mathrm{O} /$ petrol); ${ }^{1} \mathbf{H}-\mathbf{N M R}\left(400 \mathrm{MHz}, \mathrm{CDCl}_{3}\right.$ ): 7.69 $(1 \mathrm{H}, \mathrm{d}, J 9.0, \mathrm{C}(8) H), 7.38(1 \mathrm{H}, \mathrm{d}, J 15.5, \mathrm{C}(\mathrm{O}) \mathrm{CHCH}), 7.35(1 \mathrm{H}, \mathrm{dd}, J 9.0$ and 2.5, $\mathrm{C}(7) H), 7.22(2 \mathrm{H}, \mathrm{d}, J 9.0, \mathrm{C}(1) H), 6.95(2 \mathrm{H}, \mathrm{d}, J 9.0, \mathrm{C}(2) H), 6.65(1 \mathrm{H}, \mathrm{d}, J 2.5$, $\mathrm{C}(6) H), 6.47(1 \mathrm{H}$, br. s, NH), $6.08(1 \mathrm{H}, \mathrm{d}, J 15.5, \mathrm{C}(\mathrm{O}) \mathrm{CHCH}), 4.55(2 \mathrm{H}, \mathrm{t}, J 2.5$, $\left.\mathrm{C}(5) H_{2}\right), 3.96\left(2 \mathrm{H}, \mathrm{t}, J 5.5, \mathrm{C}(3) H_{2}\right), 3.84\left(3 \mathrm{H}, \mathrm{s}, \mathrm{OCH}_{3}\right), 2.66(2 \mathrm{H}, \mathrm{tt}, J 5.5$ and 2.5, $\left.\mathrm{C}(4) H_{2}\right), 1.53\left(9 \mathrm{H}, \mathrm{s}, \mathrm{C}\left(\mathrm{CH}_{3}\right)_{3}\right) ;{ }^{13} \mathbf{C}-\mathbf{N M R}\left(100 \mathrm{MHz}, \mathrm{CDCl}_{3}\right): 192.5,160.6,152.5$, $145.4,135.7,135.6,132.9,132.6,130.0,129.9(2 \times \mathrm{C}), 129.4,128.0,123.5,116.5$, 114.3, 81.3, 66.2, 64.3, 55.4, 31.3, 28.3; LRMS (ESI+, m/z): $536.1\left(\left[\mathrm{M}\left({ }^{79} \mathrm{Br}\right)+\mathrm{Na}\right]^{+}\right.$, $100 \%), 538.1\left(\left[\mathrm{M}\left({ }^{81} \mathrm{Br}\right)+\mathrm{Na}\right]^{+}, \quad 100 \%\right)$; HRMS (ESI+, m/z): $\mathrm{C}_{26} \mathrm{H}_{28} \mathrm{BrNNaO}_{5}$ $\left(\left[\mathrm{M}\left({ }^{79} \mathrm{Br}\right)+\mathrm{Na}\right]^{+}\right)$requires 536.10431, found 536.10424, $\left(\left[\mathrm{M}\left({ }^{81} \mathrm{Br}\right)+\mathrm{Na}\right]^{+}\right)$requires 538.10226, found 538.10193; IR $v_{\max }(\mathrm{film}) / \mathrm{cm}^{-1}: 3296,2976,2931,2838,1723$, 1648, 1593, 1508, 1463, 1392, 1366, 1320, 1292, 1248, 1206, 1154, 1030. 


\section{1-(2'-(6-Bromoquinolin-2-yl)-[1,1'-biphenyl]-4-yl)ethan-1-one, 6a}<smiles>CC(=O)c1ccc(-c2ccccc2-c2ccc3cc(Br)ccc3n2)cc1</smiles>

Compound 6a was prepared according to General Procedure $\mathbf{C}$ with enone 9a (59.7 $\mathrm{mg}, 0.114 \mathrm{mmol})$, EtOH $(1.5 \mathrm{~mL})$ and $\mathrm{H}_{2} \mathrm{O}(1.5 \mathrm{~mL})$. Flash column chromatography $\left(\mathrm{SiO}_{2}, 20-30 \% \mathrm{Et}_{2} \mathrm{O}\right.$ in petrol) afforded quinoline $6 \mathbf{a}$ as a colourless oil (43.2 mg, $94 \%$ yield).

${ }^{1}$ H-NMR $\left(400 \mathrm{MHz}, \mathrm{CDCl}_{3}\right): 8.01(1 \mathrm{H}, \mathrm{d}, J$ 9.0, C(11)H), $7.92(1 \mathrm{H}, \mathrm{d}, J 2.0, \mathrm{C}(9) H)$, 7.87-7.78 (4H, m, C(1)H, C(6)H, C(10)H), $7.77(1 \mathrm{H}, \mathrm{d}, J$ 8.5, C $(8) H), 7.60-7.56(2 \mathrm{H}$, m, C(4)H, C(5)H), 7.52-7.50 (1H, m, C(3)H), $7.30(2 \mathrm{H}, \mathrm{d}, J 8.5, \mathrm{C}(2) H), 6.99(1 \mathrm{H}, \mathrm{d}$, $J$ 8.5, C(7)H), $2.57\left(3 \mathrm{H}, \mathrm{s}, \mathrm{C}(\mathrm{O}) \mathrm{CH}_{3}\right) ;{ }^{13} \mathbf{C}-\mathbf{N M R}\left(100 \mathrm{MHz}, \mathrm{CDCl}_{3}\right)$ : 197.7, 159.7, $146.7,146.0,139.6,139.5,135.5,134.2,133.0,131.2,130.9,130.4,130.0,129.5$, 129.2, 128.6, 128.3， 127.7， 124.1，120.4， 26.6; LRMS (ESI+， m/z): 402.0 $\left(\left[\mathrm{M}\left({ }^{79} \mathrm{Br}\right)+\mathrm{H}\right]^{+}, \quad 100 \%\right), 404.0\left(\left[\mathrm{M}\left({ }^{81} \mathrm{Br}\right)+\mathrm{H}\right]^{+}, \quad 100 \%\right) ; \quad$ HRMS (ESI+, $\left.\mathrm{m} / \mathrm{z}\right)$ : $\mathrm{C}_{23} \mathrm{H}_{16} \mathrm{BrNNaO} \quad\left(\left[\mathrm{M}\left({ }^{79} \mathrm{Br}\right)+\mathrm{Na}\right]^{+}\right)$requires 402.04990, found 402.04878, $\left(\left[\mathrm{M}\left({ }^{81} \mathrm{Br}\right)+\mathrm{Na}\right]^{+}\right)$requires 404.04676 , found 404.04670; IR $v_{\max }($ film $) / \mathrm{cm}^{-1}: 3060$, 2923, 2853, 1682, 1603, 1548, 1482, 1403, 1357, 1267, 1185, 1060, 1033, 1005. 


\section{6-Bromo-2-(4'-methyl-[1,1'-biphenyl]-2-yl)quinoline, 6b}<smiles>COc1ccc(-c2ccccc2-c2ccc3cc(Br)ccc3n2)cc1</smiles>

Compound $\mathbf{6 b}$ was prepared according to General Procedure $\mathbf{C}$ with enone $9 \mathbf{b}$ (80.9 $\mathrm{mg}, 0.164 \mathrm{mmol})$, EtOH $(1.5 \mathrm{~mL})$ and $\mathrm{H}_{2} \mathrm{O}(1.5 \mathrm{~mL})$. Flash column chromatography $\left(\mathrm{SiO}_{2}, 10-20 \% \mathrm{Et}_{2} \mathrm{O}\right.$ in petrol) afforded quinoline $\mathbf{6 b}$ as a colourless oil $(57.8 \mathrm{mg}, 94 \%$ yield).

${ }^{1}$ H-NMR (400 MHz, $\left.\mathrm{CDCl}_{3}\right): 8.07(1 \mathrm{H}, \mathrm{d}, J$ 9.0, C(11)H), $7.92(1 \mathrm{H}, \mathrm{d}, J 2.0, \mathrm{C}(9) H)$, 7.87-7.85 $(1 \mathrm{H}, \mathrm{m}, \mathrm{C}(6) H), 7.81(1 \mathrm{H}, \mathrm{dd}, J 9.0$ and 2.0, $\mathrm{C}(10) H), 7.73(1 \mathrm{H}, \mathrm{d}, J 8.5$, $\mathrm{C}(8) H), 7.55-7.50(3 \mathrm{H}, \mathrm{m}, \mathrm{C}(3) H, \mathrm{C}(4) H, \mathrm{C}(5) H), 7.11(2 \mathrm{H}, \mathrm{d}, J 8.0, \mathrm{C}(2) H), 7.05$ $\left(2 \mathrm{H}, \mathrm{d}, J\right.$ 8.0, C(1)H), $6.99(1 \mathrm{H}, \mathrm{d}, J 8.5, \mathrm{C}(7) H), 2.34\left(3 \mathrm{H}, \mathrm{s}, \mathrm{CH}_{3}\right) ;{ }^{13} \mathbf{C}-\mathbf{N M R}(100$ $\left.\mathrm{MHz}, \mathrm{CDCl}_{3}\right)$ : 160.5, 146.8, 140.7, 139.3, 138.0, 136.7, 133.7, 132.7, 131.3, 130.7, 130.6, 129.7, 129.5, 129.1, 129.0, $127.7(2 \times \mathrm{C}), 124.5,120.1,21.1$; LRMS (ESI+, $\mathrm{m} / \mathrm{z}): 374.1\left(\left[\mathrm{M}\left({ }^{79} \mathrm{Br}\right)+\mathrm{H}\right]^{+}, 100 \%\right), 376.1\left(\left[\mathrm{M}\left({ }^{81} \mathrm{Br}\right)+\mathrm{H}\right]^{+}, 100 \%\right) ; \quad$ HRMS (ESI+, $\mathrm{m} / \mathrm{z}): \quad \mathrm{C}_{22} \mathrm{H}_{17} \mathrm{BrN} \quad\left(\left[\mathrm{M}\left({ }^{79} \mathrm{Br}\right)+\mathrm{H}\right]^{+}\right) \quad$ requires 374.05389 , found 374.05412, $\left(\left[\mathrm{M}\left({ }^{81} \mathrm{Br}\right)+\mathrm{H}\right]^{+}\right)$requires 376.05184 , found 376.05202 ; IR $v_{\max }($ film $) / \mathrm{cm}^{-1}: 3056$, 3023, 2921, 2864, 1594, 1547, 1517, 1482, 1442, 1378, 1332, 1307, 1292, 1187, 1127 , 1059, 1034, 1006. 


\section{6-Bromo-2-(4'-methoxy-[1,1'-biphenyl]-2-yl)quinoline, $6 c$}<smiles>COc1ccc(-c2ccccc2-c2ccc3cc(Br)ccc3n2)cc1</smiles>

Compound 6c was prepared according to General Procedure $\mathbf{C}$ with enone 9c $(88.3$ $\mathrm{mg}, 0.174 \mathrm{mmol})$, EtOH $(1.5 \mathrm{~mL})$ and $\mathrm{H}_{2} \mathrm{O}(1.5 \mathrm{~mL})$. Flash column chromatography $\left(\mathrm{SiO}_{2}, 10-20 \% \mathrm{Et}_{2} \mathrm{O}\right.$ in petrol) afforded quinoline $\mathbf{6 c}$ as a colourless oil $(61.6 \mathrm{mg}, 91 \%$ yield).

${ }^{1}$ H-NMR $\left(400 \mathrm{MHz}, \mathrm{CDCl}_{3}\right): 8.07(1 \mathrm{H}, \mathrm{d}, J$ 9.0, C(11)H), $7.92(1 \mathrm{H}, \mathrm{d}, J 2.0, \mathrm{C}(9) H)$, 7.86-7.84 $(1 \mathrm{H}, \mathrm{m}, \mathrm{C}(6) H), 7.80(1 \mathrm{H}, \mathrm{dd}, J 9.0$ and 2.0, $\mathrm{C}(10) H), 7.74(1 \mathrm{H}, \mathrm{d}, J 8.5$, $\mathrm{C}(8) H)$, 7.54-7.48 (3H, m, C(3)H, C(4)H, C(5)H), $7.12(2 \mathrm{H}, \mathrm{d}, J 9.0, \mathrm{C}(2) H), 6.99$ $(1 \mathrm{H}, \mathrm{d}, J 8.5, \mathrm{C}(7) H), 6.78(2 \mathrm{H}, \mathrm{d}, J 9.0, \mathrm{C}(1) H), 3.79\left(3 \mathrm{H}, \mathrm{s}, \mathrm{OCH}_{3}\right) ;{ }^{13} \mathbf{C}-\mathbf{N M R}(100$ $\left.\mathrm{MHz}, \mathrm{CDCl}_{3}\right)$ : 160.6, 158.8, 146.8, 140.4, 139.2, 133.7, 133.3, 132.7, 131.3, 130.9, $130.8,130.5,129.5,129.1,127.7,127.6,124.5,120.1,113.7,55.2 ;$ LRMS (ESI+, $\mathrm{m} / \mathrm{z}): 390.0\left(\left[\mathrm{M}\left({ }^{79} \mathrm{Br}\right)+\mathrm{H}\right]^{+}, 100 \%\right), 392.0\left(\left[\mathrm{M}\left({ }^{81} \mathrm{Br}\right)+\mathrm{H}\right]^{+}, 100 \%\right) ; \quad$ HRMS (ESI+, $\mathrm{m} / \mathrm{z}): \mathrm{C}_{22} \mathrm{H}_{17} \mathrm{BrNO}\left(\left[\mathrm{M}\left({ }^{79} \mathrm{Br}\right)+\mathrm{H}\right]^{+}\right)$requires 390.04880, found 390.04931, $\left(\left[\mathrm{M}\left({ }^{81} \mathrm{Br}\right)+\mathrm{H}\right]^{+}\right)$requires 392.04676 , found 392.04717; IR $v_{\max }($ film $) / \mathrm{cm}^{-1}: 3059$, 2955, 2933, 2835, 1610, 1594, 1547, 1516, 1482, 1440, 1298, 1246, 1178, 1060, 1038. 
6d<smiles>CC(=O)c1ccc(C2=C(c3ccc4cc(Br)ccc4n3)COCC2)cc1</smiles>

Compound 6d was prepared according to General Procedure $\mathbf{C}$ with enone 9d (90.6 $\mathrm{mg}, 0.172 \mathrm{mmol})$, EtOH $(1.5 \mathrm{~mL})$ and $\mathrm{H}_{2} \mathrm{O}(1.5 \mathrm{~mL})$. Flash column chromatography $\left(\mathrm{SiO}_{2}, 50-70 \% \mathrm{Et}_{2} \mathrm{O}\right.$ in petrol) afforded quinoline $\mathbf{6 d}$ as a fluffy white powder $(52.0$ $\mathrm{mg}, 74 \%$ yield).

m.p.: $149-151{ }^{\circ} \mathrm{C}$ (crystalized from $\mathrm{Et}_{2} \mathrm{O} /$ petrol); ${ }^{\mathbf{1}} \mathbf{H}-\mathbf{N M R}\left(400 \mathrm{MHz}, \mathrm{CDCl}_{3}\right.$ ): 7.91 $(1 \mathrm{H}, \mathrm{d}, J$ 9.0, C(10)H), $7.85(1 \mathrm{H}, \mathrm{d}, J 2.0, \mathrm{C}(8) H), 7.78(2 \mathrm{H}, \mathrm{d}, J 8.5, \mathrm{C}(1) H), 7.75$ $(1 \mathrm{H}, \mathrm{dd}, J 9.0$ and 2.0, C(9)H), $7.65(1 \mathrm{H}, \mathrm{d}, J 8.5, \mathrm{C}(7) H), 7.20(2 \mathrm{H}, \mathrm{d}, J 8.5, \mathrm{C}(2) H)$, $6.80(1 \mathrm{H}, \mathrm{d}, J 8.5, \mathrm{C}(6) H), 4.81\left(2 \mathrm{H}, \mathrm{t}, J 2.5, \mathrm{C}(5) H_{2}\right), 4.09\left(2 \mathrm{H}, \mathrm{t}, J 5.5, \mathrm{C}(3) H_{2}\right)$, $2.67\left(2 \mathrm{H}, \mathrm{tt}, J 5.5\right.$ and 2.5, C(4) $\left.\mathrm{H}_{2}\right), 2.54\left(3 \mathrm{H}, \mathrm{s}, \mathrm{CH}_{3}\right) ;{ }^{13} \mathbf{C}-\mathbf{N M R}\left(100 \mathrm{MHz}, \mathrm{CDCl}_{3}\right)$ : $197.5,157.8,146.3,146.0,136.1,135.9,135.3,134.2,132.9,131.0,129.5,128.9$, 128.4, 127.8, 123.9, 120.4, 68.1, 64.6, 30.6, 26.6; LRMS (ESI+, m/z): 408.1 $\left(\left[\mathrm{M}\left({ }^{79} \mathrm{Br}\right)+\mathrm{H}\right]^{+}, \quad 100 \%\right), \quad 410.1 \quad\left(\left[\mathrm{M}\left({ }^{79} \mathrm{Br}\right)+\mathrm{H}\right]^{+}, \quad 100 \%\right) ; \quad$ HRMS $(\mathrm{ESI}+, \quad \mathrm{m} / \mathrm{z})$ : $\mathrm{C}_{22} \mathrm{H}_{19} \mathrm{BrNO}_{2}\left(\left[\mathrm{M}\left({ }^{79} \mathrm{Br}\right)+\mathrm{H}\right]^{+}\right)$requires 408.05937 , found 408.05912, $\left(\left[\mathrm{M}\left({ }^{81} \mathrm{Br}\right)+\mathrm{H}\right]^{+}\right)$ requires 410.05732, found 410.05706; IR $v_{\max }\left(\right.$ film) $/ \mathrm{cm}^{-1}: 3059,2961,2923,2853$, 1681, 1602, 1591, 1558, 1544, 1486, 1357, 1303, 1267, 1186, 1141, 1117, 1096, 1060, 1014. 


\section{6-Bromo-2-(4-(p-tolyl)-5,6-dihydro-2H-pyran-3-yl)quinoline, 6e}<smiles>Cc1ccc(C2=C(c3ccc4cc(Br)ccc4n3)COCC2)cc1</smiles>

Compound 6e was prepared according to General Procedure $\mathbf{C}$ with enone 9e (69.1 $\mathrm{mg}, 0.139 \mathrm{mmol}), \mathrm{EtOH}(1.5 \mathrm{~mL})$ and $\mathrm{H}_{2} \mathrm{O}(1.5 \mathrm{~mL})$. Flash column chromatography $\left(\mathrm{SiO}_{2}, 20-30 \% \mathrm{Et}_{2} \mathrm{O}\right.$ in petrol) afforded quinoline $6 \mathrm{e}$ as a grey solid $(28.1 \mathrm{mg}, 53 \%$ yield).

m.p.: $157-159{ }^{\circ} \mathrm{C}$ (crystalized from $\mathrm{Et}_{2} \mathrm{O} /$ petrol); ${ }^{1} \mathbf{H}-\mathbf{N M R}\left(400 \mathrm{MHz}, \mathrm{CDCl}_{3}\right): 7.93$ $(1 \mathrm{H}, \mathrm{d}, J 9.0, \mathrm{C}(10) H), 7.84(1 \mathrm{H}, \mathrm{d}, J 2.0, \mathrm{C}(8) H), 7.75(1 \mathrm{H}, \mathrm{dd}, J 9.0$ and 2.0, C(9)H), $7.63(1 \mathrm{H}, \mathrm{d}, J 8.5, \mathrm{C}(7) H), 7.01(4 \mathrm{H}, \mathrm{s}, \mathrm{C}(1) H, \mathrm{C}(2) H), 6.84(1 \mathrm{H}, \mathrm{d}, J 8.5, \mathrm{C}(6) H)$, $4.82\left(2 \mathrm{H}, \mathrm{t}, J 2.5, \mathrm{C}(5) H_{2}\right), 4.08\left(2 \mathrm{H}, \mathrm{t}, J 5.5, \mathrm{C}(3) H_{2}\right), 2.66(2 \mathrm{H}, \mathrm{tt}, J 5.5$ and 2.5, $\left.\mathrm{C}(4) H_{2}\right), 2.31\left(3 \mathrm{H}, \mathrm{s}, \mathrm{CH}_{3}\right) ;{ }^{13} \mathbf{C}-\mathbf{N M R}\left(100 \mathrm{MHz}, \mathrm{CDCl}_{3}\right): 158.7,146.3,138.0,137.2$, 136.5, 134.0, 133.7, 132.6, 131.0, 129.4, 129.1, 128.5, 127.7, 124.3, 120.0, 68.1, 64.8, 31.1, 21.2; LRMS (ESI+, m/z): $380.1\left(\left[\mathrm{M}\left({ }^{79} \mathrm{Br}\right)+\mathrm{Na}\right]^{+}, 100 \%\right), 382.1\left(\left[\mathrm{M}\left({ }^{81} \mathrm{Br}\right)+\mathrm{Na}\right]^{+}\right.$, $100 \%)$; HRMS $(\mathrm{ESI}+, \mathrm{m} / \mathrm{z}): \mathrm{C}_{21} \mathrm{H}_{19} \mathrm{BrNO}\left(\left[\mathrm{M}\left({ }^{79} \mathrm{Br}\right)+\mathrm{H}\right]^{+}\right)$requires 380.06445 , found 380.06507, $\left(\left[\mathrm{M}\left({ }^{81} \mathrm{Br}\right)+\mathrm{H}\right]^{+}\right)$requires 382.06241, found 382.06290; IR $v_{\max }($ film $) / \mathrm{cm}^{-}$ ': 3023, 2959, 2921, 2851, 1641, 1592, 1543, 1511, 1485, 1445, 1382, 1302, 1230, 1187, 1140, 1115, 1096, 1059, 1014. 
<smiles>COc1ccc(C2=C(c3ccc4cc(Br)ccc4n3)COCC2)cc1</smiles>

Compound 6f was prepared according to General Procedure $\mathbf{C}$ with enone 9f (70.2 $\mathrm{mg}, 0.136 \mathrm{mmol})$, EtOH $(1.5 \mathrm{~mL})$ and $\mathrm{H}_{2} \mathrm{O}(1.5 \mathrm{~mL})$. Flash column chromatography $\left(\mathrm{SiO}_{2}, 30-40 \% \mathrm{Et}_{2} \mathrm{O}\right.$ in petrol) afforded quinoline $\mathbf{6 f}$ as off white needles (45.8 $\mathrm{mg}$, $85 \%$ yield).

m.p.: 142-144 ${ }^{\circ} \mathrm{C}$ (crystalized from $\mathrm{Et}_{2} \mathrm{O} /$ petrol); ${ }^{1} \mathbf{H}-\mathbf{N M R}\left(400 \mathrm{MHz}, \mathrm{CDCl}_{3}\right): 7.92$ $(1 \mathrm{H}, \mathrm{d}, J 9.0, \mathrm{C}(10) H), 7.84(1 \mathrm{H}, \mathrm{d}, J 2.0, \mathrm{C}(8) H), 7.75(1 \mathrm{H}, \mathrm{dd}, J 9.0$ and 2.0, C(9)H), $7.64(1 \mathrm{H}, \mathrm{d}, J 8.5, \mathrm{C}(7) H), 7.04(2 \mathrm{H}, \mathrm{d}, J 9.0, \mathrm{C}(2) H), 6.84(1 \mathrm{H}, \mathrm{d}, J 8.5, \mathrm{C}(6) H), 6.74$ $(2 \mathrm{H}, \mathrm{d}, J 9.0, \mathrm{C}(1) H), 4.81\left(2 \mathrm{H}, \mathrm{t}, J 2.5, \mathrm{C}(5) H_{2}\right), 4.08\left(2 \mathrm{H}, \mathrm{t}, J 5.5, \mathrm{C}(3) H_{2}\right), 3.77(3 \mathrm{H}$, s, $\left.\mathrm{OCH}_{3}\right), 2.65\left(2 \mathrm{H}, \mathrm{tt}, J 5.5\right.$ and $\left.2.5, \mathrm{C}(4) \mathrm{H}_{2}\right) ;{ }^{13} \mathbf{C}-\mathbf{N M R}\left(100 \mathrm{MHz}, \mathrm{CDCl}_{3}\right): 158.9$, $158.8,146.3,136.1,133.8,133.6,133.2$, 132.6, 131.0, 129.8, 129.4, 127.7, 124.4, 120.0, 113.8, 68.1, 64.8, 55.2, 31.0; LRMS (ESI+, m/z): $396.1\left(\left[\mathrm{M}\left({ }^{79} \mathrm{Br}\right)+\mathrm{H}\right]^{+}\right.$, $100 \%) \quad 398.1 \quad\left(\left[\mathrm{M}\left({ }^{81} \mathrm{Br}\right)+\mathrm{H}\right]^{+}, \quad 100 \%\right) ; \quad$ HRMS $\quad(\mathrm{ESI}+, \quad \mathrm{m} / \mathrm{z}): \mathrm{C}_{21} \mathrm{H}_{19} \mathrm{BrNO}_{2}$ $\left(\left[\mathrm{M}\left({ }^{79} \mathrm{Br}\right)+\mathrm{H}\right]^{+}\right)$requires 396.05937, found 396.05952, $\left(\left[\mathrm{M}\left({ }^{81} \mathrm{Br}\right)+\mathrm{H}\right]^{+}\right)$requires 398.05732, found 398.05739; IR $v_{\max }\left(\right.$ film) $/ \mathrm{cm}^{-1}: 2957,2928,2835,1607,1591$, 1510, 1485, 1462, 1382, 1301, 1247, 1178, 1141, 1113, 1034, 1014. 
General Procedure E - reductive cyclization of 2-nitrochalcones to quinoline $\mathrm{N}$ oxides

To a solution of enone (1.0 equiv) in a 1:1 mixture of EtOH and $\mathrm{H}_{2} \mathrm{O}(0.0125 \mathrm{M})$ was added $\mathrm{NH}_{4} \mathrm{Cl}$ (3.0 equiv) and $\mathrm{Sn}$ (3.0 equiv), and the resulting mixture refluxed for 20 hours. Once cooled to room temperature, the reaction was partitioned between $\mathrm{CH}_{2} \mathrm{Cl}_{2}$ and $\mathrm{H}_{2} \mathrm{O}$. The organic layer was then washed with brine, dried over $\mathrm{MgSO}_{4}$ and concentrated in vacuo to afford the crude quinoline $N$-oxide.

\section{2-(2-(Methylthio)phenyl)quinoline 1-oxide, 7a}<smiles>CSc1ccccc1-c1ccc2ccccc2[n+]1[O-]</smiles>

Compound 7a was prepared according to General Procedure $\mathbf{E}$ with enone $4 \mathbf{y}$ (150 $\mathrm{mg}, 0.5 \mathrm{mmol})$, Sn (178 mg, $1.5 \mathrm{mmol}), \mathrm{NH}_{4} \mathrm{Cl}(80 \mathrm{mg}, 1.5 \mathrm{mmol})$, EtOH (20 mL) and $\mathrm{H}_{2} \mathrm{O}(20 \mathrm{~mL})$. Flash column chromatography $\left(\mathrm{SiO}_{2}, 50-100 \%\right.$ EtOAc in petrol) afforded quinoline $N$-oxide $7 \mathbf{a}$ as a light brown solid ( $92.8 \mathrm{mg}, 69 \%$ yield).

m.p.: $136-138{ }^{\circ} \mathrm{C}$ (crystalized from $\left.\mathrm{CHCl}_{3}\right) ;{ }^{1} \mathbf{H}-\mathbf{N M R}\left(400 \mathrm{MHz}, \mathrm{CDCl}_{3}\right): 8.86(1 \mathrm{H}$, $\mathrm{d}, J 9.0, \mathrm{C}(10) H), 7.87(1 \mathrm{H}$, dd, $J 8.0$ and 1.5, C(7)H), $7.75(1 \mathrm{H}$, ddd, $J 9.0,7.0$ and 1.5, C(9)H), $7.73(1 \mathrm{H}, \mathrm{d}, J 8.5, \mathrm{C}(6) H), 7.63(1 \mathrm{H}, \mathrm{ddd}, J 8.0,7.0$ and 1.0, $\mathrm{C}(8) H)$, 7.47-7.38 (3H, m, C(1)H, C(2)H, C(4)H), 7.37 (1H, d, $J 8.5, \mathrm{C}(5) H), 7.30$ (1H, ddd, $J$ 7.5, 6.0 and 2.5, C(3)H), $2.40(3 \mathrm{H}, \mathrm{s}, \mathrm{SCH})_{3} ;{ }^{13} \mathbf{C}-\mathbf{N M R}\left(100 \mathrm{MHz}, \mathrm{CDCl}_{3}\right): 145.2$, 142.0, 138.9, 133.5, 130.3, 130.0, 129.80, 129.77, 128.6, 128.1, 126.7, 125.4, 124.6, 123.8, 120.3, 16.4; LRMS (ESI+, m/z): $268.1\left([\mathrm{M}+\mathrm{H}]^{+}, 100 \%\right) ; \quad$ HRMS (ESI+, $\mathrm{m} / \mathrm{z}): \mathrm{C}_{16} \mathrm{H}_{14} \mathrm{NOS}\left([\mathrm{M}+\mathrm{H}]^{+}\right)$requires 268.07906 , found 268.07892 ; IR $v_{\max }$ (film) $/ \mathrm{cm}^{-1}: 3424,3057,2920,2854,1560,1511,1472,1449,1435,1369,1340,1322$, $1260,1242,1215,1163,1135,1112,1080,1048,1032$. 


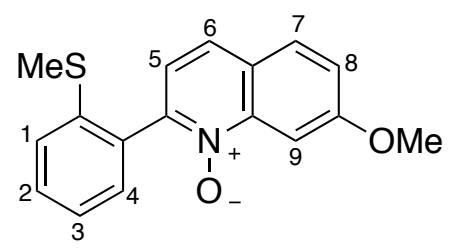

Compound $\mathbf{7 b}$ was prepared according to General Procedure $\mathbf{E}$ with enone $\mathbf{4 z}$ (70.0 $\mathrm{mg}, 0.21 \mathrm{mmol})$, Sn powder (75.0 mg, $0.64 \mathrm{mmol}), \mathrm{NH}_{4} \mathrm{Cl}$ (34.0 mg, mmol), EtOH (8 $\mathrm{mL})$ and $\mathrm{H}_{2} \mathrm{O}(8 \mathrm{~mL})$. Flash column chromatography $\left(\mathrm{SiO}_{2}, 50-100 \%\right.$ EtOAc in petrol) afforded quinoline $N$-oxide $7 \mathbf{b}$ as a white solid (44.5 $\mathrm{mg}, 70 \%$ yield).

m.p.: $172-174{ }^{\circ} \mathrm{C}$ (crystalized from EtOAc); ${ }^{1} \mathbf{H}-\mathbf{N M R}\left(400 \mathrm{MHz}, \mathrm{CDCl}_{3}\right): 8.24(1 \mathrm{H}$, $\mathrm{d}, J 2.5, \mathrm{C}(9) H), 7.78(1 \mathrm{H}, \mathrm{d}, J 9.0, \mathrm{C}(7) H), 7.71(1 \mathrm{H}, \mathrm{d}, J 8.5, \mathrm{C}(6) H), 7.49-7.42(2 \mathrm{H}$, $\mathrm{m}, \mathrm{C}(1) H, \mathrm{C}(2) H), 7.38(1 \mathrm{H}, \mathrm{dd}, J 7.5$ and 1.0, C(4)H), 7.33-7.28 (2H, m, C(3)H, $\mathrm{C}(8) H), 7.26(1 \mathrm{H}, \mathrm{d}, J 8.5, \mathrm{C}(5) H), 4.00\left(3 \mathrm{H}, \mathrm{s}, \mathrm{OCH}_{3}\right), 2.42\left(3 \mathrm{H}, \mathrm{s}, \mathrm{SCH}_{3}\right) ;{ }^{13} \mathrm{C}-$ NMR (100 MHz, $\left.\mathrm{CDCl}_{3}\right): 162.0,145.7,143.2,138.9,133.6,129.73,129.69,129.3$, 126.5, 125.26, 125.09, 124.7, 121.6, 121.2, 98.9, 55.9, 16.3; LRMS (ESI+, m/z): $298.1\left([\mathrm{M}+\mathrm{H}]^{+}, 100 \%\right) ; \quad$ HRMS $(\mathrm{ESI}+, \mathrm{m} / \mathrm{z}): \mathrm{C}_{17} \mathrm{H}_{16} \mathrm{NO}_{2} \mathrm{~S}\left([\mathrm{M}+\mathrm{H}]^{+}\right)$requires 298.08963, found 298.08950; IR $v_{\max }\left(\right.$ film) $/ \mathrm{cm}^{-1}: 3059,3007,2968,2920,2838$, 1624, 1597, 1561, 1522, 1474, 1434, 1382, 1346, 1325, 1272, 1218, 1165, 1021. 


\section{General Procedure F - conjugate addition of Grignard reagents to chalcone intermediates}

To a suspension of $\mathrm{CuI}(0.2$ equiv) in THF $(0.25 \mathrm{~mL}$ per mmol $)$ was added a Grignard reagent (3.3 equiv), and then stirred at $0{ }^{\circ} \mathrm{C}$ for 15 minutes. A solution of enone (1.0 equiv) in THF $(0.25 \mathrm{~mL}$ per $\mathrm{mmol})$ was then added, and the reaction mixture stirred for 2 hours at $0{ }^{\circ} \mathrm{C}$. The reaction was quenched with $\mathrm{MeOH}$ under $\mathrm{N}_{2(\mathrm{~g})}$ and immediately filtered through a $\mathrm{SiO}_{2}$ pad (washed with $\mathrm{Et}_{2} \mathrm{O}$ ) to remove the $\mathrm{Cu}$ residues. The mixture was concentrated in vacuo to afford the crude conjugate addition product.

tert-Butyl (2-(1-(2-(methylthio)phenyl)-1-oxopentan-3-yl)phenyl)carbamate, 10a<smiles>CCC(CC(=O)c1ccccc1S(C)(=O)=O)c1ccccc1NC(=O)OC</smiles>

Compound 10a was prepared according to General Procedure F with enone 4a (370 $\mathrm{mg}, 1.0 \mathrm{mmol}), \mathrm{EtMgCl}(2.2 \mathrm{~mL}, 1.47 \mathrm{M}$ solution in THF, $3.3 \mathrm{mmol}), \mathrm{CuI}(38.1 \mathrm{mg}$, $0.2 \mathrm{mmol})$ and THF $(6 \mathrm{~mL})$. Flash column chromatography $\left(\mathrm{SiO}_{2}, 20 \% \mathrm{Et}_{2} \mathrm{O}\right.$ in petrol) afforded ketone 10a as a pale yellow oil (347 mg, $87 \%$ yield).

${ }^{1}$ H-NMR $\left(400 \mathrm{MHz}, \mathrm{CDCl}_{3}\right): 7.74(1 \mathrm{H}, \mathrm{dd}, J 8.0$ and 1.5, C(4)H), $7.63(1 \mathrm{H}, \mathrm{d}, J 7.0$, $\mathrm{C}(8) H), 7.54(1 \mathrm{H}$, br. s, $\mathrm{N} H), 7.45(1 \mathrm{H}, \mathrm{ddd}, J 8.0,7.5$ and 1.0, $\mathrm{C}(2) H), 7.31(1 \mathrm{H}, \mathrm{d}, J$ 8.0, C(1)H), 7.20-7.15 (3H, m, C(3)H, C(5)H, C(7)H), $7.09(1 \mathrm{H}, \mathrm{td}, J 7.5$ and 1.0, $\mathrm{C}(6) H), 3.55-3.48(1 \mathrm{H}, \mathrm{m}, \mathrm{CH}(\mathrm{Ar}) \mathrm{Et}), 3.40\left(1 \mathrm{H}, \mathrm{dd}, J 17.5\right.$ and $\left.9.0, \mathrm{C}(\mathrm{O}) \mathrm{CH}_{\mathrm{A}} \mathrm{H}_{\mathrm{B}}\right)$, $3.26\left(1 \mathrm{H}, \mathrm{dd}, J 17.5\right.$ and $\left.\left.4.5, \mathrm{C}(\mathrm{O}) \mathrm{CH}_{\mathrm{A}} H_{\mathrm{B}}\right), 2.40(3 \mathrm{H}, \mathrm{s}, \mathrm{SCH})_{3}\right), 1.81-1.69(2 \mathrm{H}, \mathrm{m}$, $\left.\mathrm{CH}_{2} \mathrm{CH}_{3}\right), 1.56\left(9 \mathrm{H}, \mathrm{s}, \mathrm{C}\left(\mathrm{CH}_{3}\right)_{3}\right), 0.82\left(3 \mathrm{H}, \mathrm{t}, J 7.5, \mathrm{CH}_{2} \mathrm{CH}_{3}\right) ;{ }^{13} \mathbf{C}-\mathbf{N M R}(100 \mathrm{MHz}$, $\left.\mathrm{CDCl}_{3}\right):$ 201.1, 154.2, 142.1, 136.7, 136.3, 134.6, 132.2, 130.0, 126.4, 125.9, 125.3, 124.8, 124.6, 123.6, 79.8, 47.6, 35.1, 29.4, 28.5, 16.0, 12.1; LRMS (ESI+, m/z): $422.2\left([\mathrm{M}+\mathrm{Na}]^{+}, 100 \%\right) ; \quad$ HRMS $(\mathrm{ESI}+, \mathrm{m} / \mathrm{z}): \mathrm{C}_{23} \mathrm{H}_{29} \mathrm{NNaO}_{3} \mathrm{~S}\left([\mathrm{M}+\mathrm{Na}]^{+}\right)$requires 422.17604, found 422.17588; IR $v_{\max }\left(\right.$ film) $/ \mathrm{cm}^{-1}: 3361,2967,2930,2874,1721$, $1665,1587,1510,1450,1366,1232,1160,1048,1024$. 
<smiles>COc1ccccc1NC(=O)c1ccccc1C(CC(=O)c1ccccc1S(C)(=O)=O)c1ccc(C)cc1</smiles>

Compound 10b was prepared according to General Procedure $\mathbf{F}$ with enone 4a (111 $\mathrm{mg}, 0.3 \mathrm{mmol})$, $p$-tolylMgBr (1.22 mL, $0.82 \mathrm{M}$ solution in THF, $1.0 \mathrm{mmol}), \mathrm{CuI}$ (11.4 $\mathrm{mg}, 0.06 \mathrm{mmol})$ and THF $(1.8 \mathrm{~mL})$. Flash column chromatography $\left(\mathrm{SiO}_{2}, 15-30 \%\right.$ $\mathrm{Et}_{2} \mathrm{O}$ in petrol) afforded ketone $\mathbf{1 0 b}$ as a pale yellow solid (130.6 mg, 94\% yield).

m.p.: $137-139{ }^{\circ} \mathrm{C}$ (crystalized from $\mathrm{Et}_{2} \mathrm{O} /$ petrol); ${ }^{1} \mathbf{H}-\mathbf{N M R}\left(400 \mathrm{MHz}, \mathrm{CDCl}_{3}\right)$ : 7.79 $(1 \mathrm{H}, \mathrm{dd}, J 8.0$ and 1.5, C(4)H), $7.71(1 \mathrm{H}, \mathrm{d}, J 7.0, \mathrm{C}(8) H), 7.46(1 \mathrm{H}, \mathrm{td}, J 7.5$ and 1.0, $\mathrm{C}(2) H), 7.32(1 \mathrm{H}, \mathrm{d}, J$ 8.0, C(1)H), 7.26-7.18 (3H, m, C(3)H, C(5)H, C(7)H), 7.19 $(2 \mathrm{H}, \mathrm{d}, J 8.0, \mathrm{C}(9) H), 7.12(2 \mathrm{H}, \mathrm{d}, J 8.0, \mathrm{C}(10) H), 7.09(1 \mathrm{H}, \mathrm{td}, J 7.5$ and 1.0, C(6)H), $7.05(1 \mathrm{H}, \mathrm{s}, \mathrm{N} H), 4.98\left(1 \mathrm{H}, \mathrm{t}, J 7.0, \mathrm{C}(\mathrm{O}) \mathrm{CH}_{\mathrm{A}} \mathrm{H}_{\mathrm{B}} \mathrm{CH}(\mathrm{Ar})_{2}\right), 3.80(1 \mathrm{H}, \mathrm{dd}, J 17.0$ and 7.5, $\left.\mathrm{C}(\mathrm{O}) \mathrm{CH}_{\mathrm{A}} \mathrm{H}_{\mathrm{B}}\right), 3.74\left(1 \mathrm{H}, \mathrm{dd}, J 17.0\right.$ and $\left.6.5, \mathrm{C}(\mathrm{O}) \mathrm{CH}_{\mathrm{A}} H_{\mathrm{B}}\right), 2.40\left(3 \mathrm{H}, \mathrm{s}, \mathrm{SCH}_{3}\right)$, $2.32\left(3 \mathrm{H}, \mathrm{s}, \mathrm{ArCH}_{3}\right), 1.55\left(9 \mathrm{H}, \mathrm{s}, \mathrm{C}\left(\mathrm{CH}_{3}\right)_{3}\right) ;{ }^{13} \mathbf{C}-\mathbf{N M R}\left(100 \mathrm{MHz}, \mathrm{CDCl}_{3}\right): 199.6$, $153.7,142.3,140.1,136.2,135.8,135.6,134.7,132.3,130.0,129.5,127.7,127.4$, 127.0, 125.4, 124.6, 124.2, 123.6, 80.0, 46.0, 39.5, 28.4, 21.0, 16.0; LRMS (ESI+, $\mathrm{m} / \mathrm{z}): 484.2\left([\mathrm{M}+\mathrm{Na}]^{+}, 100 \%\right) ; \quad$ HRMS $(\mathrm{ESI}+, \mathrm{m} / \mathrm{z}): \mathrm{C}_{28} \mathrm{H}_{31} \mathrm{NNaO}_{3} \mathrm{~S}\left([\mathrm{M}+\mathrm{Na}]^{+}\right)$ requires 484.19169, found 484.19074; IR $v_{\max }(\mathrm{film}) / \mathrm{cm}^{-1}: 3399,2978$ 2922, 1720, 1669, 1587, 1510, 1449, 1433, 1366, 1297, 1230, 1155, 1048, 1023. 
<smiles>CCCCOC(=O)Nc1ccccc1C(CC(=O)c1ccccc1S(C)(=O)=O)C1CC1</smiles>

Compound 10c was prepared according to General Procedure F with enone 4a (73.9 $\mathrm{mg}, 0.2 \mathrm{mmol})$, cyclopropylMgBr $(1.05 \mathrm{~mL}, 0.76 \mathrm{M}$ solution in THF, $0.8 \mathrm{mmol})$, CuI (76.2 mg, $0.4 \mathrm{mmol})$ and THF $(1.2 \mathrm{~mL})$. Flash column chromatography $\left(\mathrm{SiO}_{2}, 20\right.$ $30 \% \mathrm{Et}_{2} \mathrm{O}$ in petrol) afforded ketone $10 \mathrm{c}$ as an off white solid (70.7 $\mathrm{mg}, 86 \%$ yield).

m.p.: $139-141{ }^{\circ} \mathrm{C}$ (crystalized from $\mathrm{Et}_{2} \mathrm{O} /$ petrol); ${ }^{1} \mathbf{H}-\mathbf{N M R}\left(400 \mathrm{MHz}, \mathrm{CDCl}_{3}\right): 7.80$ $(1 \mathrm{H}, \mathrm{dd}, J 8.0$ and 1.0, C(4)H), $7.65(1 \mathrm{H}, \mathrm{d}, J 6.5, \mathrm{C}(8) H), 7.51(1 \mathrm{H}$, br. s, NH), 7.45 $(1 \mathrm{H}, \mathrm{ddd}, J 8.0,7.5$ and 1.5, C(2)H), $7.34(1 \mathrm{H}, \mathrm{dd}, J 7.5$ and $1.5, \mathrm{C}(5) H), 7.30(1 \mathrm{H}, \mathrm{d}$, $J$ 8.0, C(1)H), 7.21-7.15 (2H, m, C(3)H, C(7)H), $7.11(1 \mathrm{H}, \mathrm{td}, J 7.5$ and 1.0, C(6)H), $3.54\left(1 \mathrm{H}, \mathrm{dd}, J 17.5\right.$ and 9.0, $\left.\mathrm{C}(\mathrm{O}) \mathrm{CH}_{\mathrm{A}} \mathrm{H}_{\mathrm{B}}\right), 3.45(1 \mathrm{H}, \mathrm{dd}, J 17.5$ and 4.5, $\left.\mathrm{C}(\mathrm{O}) \mathrm{CH}_{\mathrm{A}} H_{\mathrm{B}}\right), 2.87\left(1 \mathrm{H}, \mathrm{td}, J 9.0\right.$ and $\left.4.5, \mathrm{C}(\mathrm{O}) \mathrm{CH}_{\mathrm{A}} \mathrm{H}_{\mathrm{B}} \mathrm{CH}\right), 2.39\left(3 \mathrm{H}, \mathrm{s}, \mathrm{SCH} H_{3}\right), 1.55$ $\left(9 \mathrm{H}, \mathrm{s}, \mathrm{C}\left(\mathrm{CH}_{3}\right)_{3}\right), 1.23-1.14(1 \mathrm{H}, \mathrm{m}, \mathrm{Cpr} H), 0.58(1 \mathrm{H}, \mathrm{tt}, J 9.0$ and 4.5, $\mathrm{Cpr} H), 0.43$ $(1 \mathrm{H}, \mathrm{tt}, J 9.0$ and 4.5, CprH), $0.26(1 \mathrm{H}, \mathrm{dq}, J 9.0$ and 4.5, CprH), $0.14(1 \mathrm{H}, \mathrm{dq}, J 9.0$ and 4.5, $\mathrm{Cpr} H) ;{ }^{13} \mathbf{C}-\mathbf{N M R}\left(100 \mathrm{MHz}, \mathrm{CDCl}_{3}\right): 200.9,154.1,142.4,136.7,135.7$, $134.3,132.3,130.2,126.7,126.5,125.2,124.7,124.4,123.5,79.8,47.5,38.9,28.4$, 17.3, 16.0, 5.4, 4.5; LRMS (ESI+, m/z): $434.2\left([\mathrm{M}+\mathrm{Na}]^{+}, 100 \%\right) ;$ HRMS (ESI+, $\mathrm{m} / \mathrm{z}): \mathrm{C}_{24} \mathrm{H}_{29} \mathrm{NNaO}_{3} \mathrm{~S}\left([\mathrm{M}+\mathrm{Na}]^{+}\right)$requires 434.17604, found 434.17503; IR $v_{\max }$ (film) $/ \mathrm{cm}^{-1}: 3356,2978,2920,1719,1662,1587,1509,1449,1433,1366,1232,1215$, 1156, 1047, 1022. 


\section{General Procedure G - oxidative cyclisation of dihydrochalcones to quinolines}

To a solution of enone (1.0 equiv) in $\mathrm{CH}_{2} \mathrm{Cl}_{2}(0.04 \mathrm{M})$ was added TFA (24.0 equiv) and the reaction stirred at $35^{\circ} \mathrm{C}$. After 30 minutes, DDQ (1.0 equiv) was added and the reaction stirred at $35{ }^{\circ} \mathrm{C}$ for a further 30 minutes. The reaction was quenched by the addition of $1.0 \mathrm{M} \mathrm{NaOH}_{(\mathrm{aq})}$, and extracted three times with $\mathrm{CH}_{2} \mathrm{Cl}_{2}$. The organic layer was then dried over $\mathrm{MgSO}_{4}$ and concentrated in vacuo to afford the crude quinoline.

\section{4-Ethyl-2-(2-(methylthio)phenyl)quinoline, 8a}<smiles>CCc1cc(-c2ccccc2S(C)(=O)=O)nc2ccccc12</smiles>

Compound 8a was prepared according to General Procedure $\mathbf{G}$ with ketone 10a (120 $\mathrm{mg}, 0.30 \mathrm{mmol})$, TFA $(0.55 \mathrm{~mL}, 7.20 \mathrm{mmol})$, DDQ $(68.1 \mathrm{mg}, 0.30 \mathrm{mmol})$ and $\mathrm{CH}_{2} \mathrm{Cl}_{2}(7.5 \mathrm{~mL})$. Flash column chromatography $\left(\mathrm{SiO}_{2}, 20-30 \% \mathrm{Et}_{2} \mathrm{O}\right.$ in petrol) afforded quinoline $8 \mathbf{a}$ as a pale yellow oil(76.5 mg, 92\% yield).

${ }^{1}$ H-NMR $\left(400 \mathrm{MHz}, \mathrm{CDCl}_{3}\right): 8.23(1 \mathrm{H}, \mathrm{dt}, J 8.5$ and $0.5, \mathrm{C}(6) H), 8.11(1 \mathrm{H}, \mathrm{dd}, J 8.5$ and 1.0, $\mathrm{C}(9) H), 7.75(1 \mathrm{H}$, ddd, $J 8.5,7.0$ and 1.5, C(7)H), 7.62-7.58 $(3 \mathrm{H}, \mathrm{m}, \mathrm{C}(4) H$, $\mathrm{C}(5) H, \mathrm{C}(8) H), 7.47-7.41$ (2H, m, C(1)H, C(2)H), $7.32(1 \mathrm{H}$, ddd, $J$ 7.5, 6.0 and 2.5, $\mathrm{C}(3) H), 3.21\left(2 \mathrm{H}, \mathrm{qd}, J 7.5\right.$ and $\left.0.5, \mathrm{CH}_{2} \mathrm{CH}_{3}\right), 2.43(3 \mathrm{H}, \mathrm{s}, \mathrm{SCH}), 1.47(3 \mathrm{H}, \mathrm{t}, J 7.5$, $\left.\mathrm{CH}_{2} \mathrm{CH}_{3}\right) ;{ }^{13} \mathrm{C}-\mathbf{N M R}\left(100 \mathrm{MHz}, \mathrm{CDCl}_{3}\right)$ : 158.5, 149.7, 147.8, 140.3, 137.8, 130.4, 130.0, 129.17, 129.01, $126.3(3 \times \mathrm{C}), 125.0,123.3,120.8,25.2,16.6,14.0$; LRMS $(\mathrm{ESI}+, \mathrm{m} / \mathrm{z}): 280.1\left([\mathrm{M}+\mathrm{H}]^{+}, 100 \%\right) ; \quad$ HRMS $(\mathrm{ESI}+, \mathrm{m} / \mathrm{z}): \mathrm{C}_{18} \mathrm{H}_{18} \mathrm{NS}\left([\mathrm{M}+\mathrm{H}]^{+}\right)$ requires 280.11545, found 280.11520; IR $v_{\max }\left(\right.$ film) $/ \mathrm{cm}^{-1}: 3060,2968,2919,2874$, 1597, 1566, 1552, 1506, 1434, 1351, 1054. 


\section{2-(2-(Methylthio)phenyl)-4-(p-tolyl)quinoline, $8 b$}<smiles>Cc1ccc(-c2cc(-c3ccccc3S(C)(=O)=O)nc3ccccc23)cc1</smiles>

Compound $\mathbf{8 b}$ was prepared according to General Procedure $\mathbf{G}$ with ketone 10b (118 $\mathrm{mg}, 0.256 \mathrm{mmol})$, TFA $(0.47 \mathrm{~mL}, 6.14 \mathrm{mmol})$, DDQ (58.1 $\mathrm{mg}, 0.256 \mathrm{mmol})$ and $\mathrm{CH}_{2} \mathrm{Cl}_{2}(6.4 \mathrm{~mL})$. Flash column chromatography $\left(\mathrm{SiO}_{2}, 20-30 \% \mathrm{Et}_{2} \mathrm{O}\right.$ in petrol) afforded quinoline $\mathbf{8 b}$ as a pale brown oil (84.2 $\mathrm{mg}, 96 \%$ yield).

${ }^{1}$ H-NMR $\left(400 \mathrm{MHz}, \mathrm{CDCl}_{3}\right): 8.31(1 \mathrm{H}, \mathrm{d}, J 8.5, \mathrm{C}(6) H), 8.05(1 \mathrm{H}, \mathrm{dd}, J 8.5$ and 1.0, $\mathrm{C}(9) H), 7.78(1 \mathrm{H}$, ddd, $J 8.5,7.0$ and $1.5, \mathrm{C}(7) H), 7.71(1 \mathrm{H}, \mathrm{s}, \mathrm{C}(5) H), 7.66(1 \mathrm{H}, \mathrm{d}, J$ 7.0, C(4)H), $7.55(1 \mathrm{H}$, ddd, $J$ 8.5, 7.0 and 1.5, C $(8) H), 7.53(2 \mathrm{H}, \mathrm{d}, J 8.0, \mathrm{C}(10) H)$, 7.46-7.43 (2H, m, C(1)H, C(2)H), $7.38(2 \mathrm{H}, \mathrm{d}, J$ 8.0, C(11)H), 7.33 (1H, ddd, J 7.5, 5.0 and 3.5, $\mathrm{C}(3) H), 2.50\left(3 \mathrm{H}, \mathrm{s}, \mathrm{ArCH}_{3}\right), 2.46(3 \mathrm{H}, \mathrm{s}, \mathrm{SCH}) ;{ }^{13} \mathrm{C}-\mathbf{N M R}(100 \mathrm{MHz}$, $\left.\mathrm{CDCl}_{3}\right)$ : 158.2, 148.40, 148.35, 139.9, 138.3, 137.9, 135.3, 130.1, 130.0, 129.6, 129.4, 129.3, 129.1, 126.6, 126.4, 125.8, 125.6, 125.1, 122.4, 21.4, 16.7; LRMS (ESI+, $\mathrm{m} / \mathrm{z}): 342.1\left([\mathrm{M}+\mathrm{H}]^{+}, 100 \%\right) ; \quad$ HRMS $(\mathrm{ESI}+, \mathrm{m} / \mathrm{z}): \mathrm{C}_{23} \mathrm{H}_{20} \mathrm{NS}\left([\mathrm{M}+\mathrm{H}]^{+}\right)$requires 342.13110, found 342.13087; IR $v_{\max }($ film $) / \mathrm{cm}^{-1}: 3058,2919,2859,1613,1591$, 1567, 1546, 1499, 1473, 1434, 1411, 1357, 1051, 1022. 


\section{4-Cyclopropyl-2-(2-(methylthio)phenyl)quinoline, 8c}

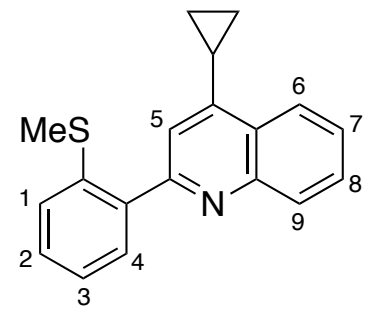

Compound 8c was prepared according to General Procedure $\mathbf{G}$ with ketone 10c (67.0 $\mathrm{mg}, 0.163 \mathrm{mmol})$, TFA $(0.30 \mathrm{~mL}, 3.91 \mathrm{mmol})$, DDQ (37.0 $\mathrm{mg}, 0.163 \mathrm{mmol})$ and $\mathrm{CH}_{2} \mathrm{Cl}_{2}(4.1 \mathrm{~mL})$. Flash column chromatography $\left(\mathrm{SiO}_{2}, 20-40 \% \mathrm{Et}_{2} \mathrm{O}\right.$ in petrol) afforded quinoline $8 \mathrm{c}$ as a pale brown oil (44.6 $\mathrm{mg}$, 94\% yield).

${ }^{1}$ H-NMR $\left(400 \mathrm{MHz}, \mathrm{CDCl}_{3}\right): 8.38(1 \mathrm{H}, \mathrm{d}, J$ 8.0, C(6)H), $8.23(1 \mathrm{H}, \mathrm{d}, J$ 8.5, C(9)H), $7.76(1 \mathrm{H}$, ddd, $J 8.5,7.0$ and 1.0, C(8)H), $7.62(1 \mathrm{H}$, ddd, $J$ 8.0, 7.0 and 1.0, C(7)H), $7.57(1 \mathrm{H}, \mathrm{d}, J 7.0, \mathrm{C}(4) H), 7.45-7.40(2 \mathrm{H}, \mathrm{m}, \mathrm{C}(1) H, \mathrm{C}(2) H), 7.37(1 \mathrm{H}, \mathrm{s}, \mathrm{C}(5) H)$, $7.30(1 \mathrm{H}$, ddd, $J$ 7.0, 7.0 and 2.0, C(3)H), $2.51(1 \mathrm{H}, \mathrm{tt}, J 9.0$ and 5.0, $\mathrm{CprH}), 2.41(3 \mathrm{H}$, s, $\left.\mathrm{SCH}_{3}\right), 1.23-1.19$ (2H, m, CprH), 0.97-0.93 (2H, m, CprH); ${ }^{13} \mathbf{C}-\mathbf{N M R}(100 \mathrm{MHz}$, $\left.\mathrm{CDCl}_{3}\right)$ : 158.5, 149.4, 147.7, 140.3, 137.7, 130.18, 129.99, 129.3, 129.0, 127.6, 126.31, 126.28, 125.1, 123.8, 118.3, 16.6, 12.3, 7.9; LRMS (ESI+, m/z): 292.1 $\left([\mathrm{M}+\mathrm{H}]^{+}, 100 \%\right) ; \quad$ HRMS $(\mathrm{ESI}+, \mathrm{m} / \mathrm{z}): \mathrm{C}_{19} \mathrm{H}_{18} \mathrm{NS}\left([\mathrm{M}+\mathrm{H}]^{+}\right)$requires 292.11545, found 292.11541; IR $v_{\max }($ film $) / \mathrm{cm}^{-1}: 3061,3005,2919,2850,1596,1551,1505$, 1434, 1376, 1321, 1266, 1050, 1026. 


\section{General Procedure H - Boc protection of anilines using NaHMDS}

Under a $\mathrm{N}_{2(\mathrm{~g})}$ atomsphere at $0{ }^{\circ} \mathrm{C}$, to a solution of aniline (1.0 equiv) in THF (1.0 M), was added NaHMDS (2.2 equiv, $2.0 \mathrm{M}$ solution in THF). The resulting solution was stirred at $0{ }^{\circ} \mathrm{C}$ for 30 minutes, before a solution of $\mathrm{Boc}_{2} \mathrm{O}(1.1$ equiv) in THF $(1.0 \mathrm{M})$ was added dropwise. The reaction was stirred for 15 minutes at $0{ }^{\circ} \mathrm{C}$, before allowing to warm to room temperature over 30 minutes. The reaction was then concentrated in vacuo, before being partitioned between EtOAc and $1.0 \mathrm{~N} \mathrm{HCl}_{(\mathrm{aq})}$. The organic layer was washed with brine, dried over $\mathrm{MgSO}_{4}$, and concentrated in vacuo to afford the crude $N$-Boc aniline.

\section{General procedure I - Boc protection of anilines via diBoc aniline}

To a solution of substituted 2-iodo aniline (1.0 equiv) in THF $(0.15 \mathrm{M})$ was added sequentially $\mathrm{Boc}_{2} \mathrm{O}$ (2.3 equiv) and DMAP ( 0.1 equiv). The reaction was then heated at reflux for 3 hours. Once cooled to room temperature the solvent was removed in vacuo, and the resulting residue partitioned between $1.0 \mathrm{~N} \mathrm{HCl}_{(\mathrm{aq})}$ and $\mathrm{Et}_{2} \mathrm{O}$. The organic layer was then washed with brine, dried over $\mathrm{MgSO}_{4}$ and concentrated in vacuo. The crude reaction mixture was then dissolved in $\mathrm{MeOH}(0.15 \mathrm{M}) . \mathrm{K}_{2} \mathrm{CO}_{3}$ was added, and the reaction was stirred at reflux until full consumption of the $N, N$ diBoc aniline was observed by TLC analysis ( 30 minutes- 5 hours). Once cooled to room temperature the solvent was removed in vacuo, and the resulting residue partitioned between $1.0 \mathrm{~N} \mathrm{HCl}_{(\mathrm{aq})}$ and $\mathrm{Et}_{2} \mathrm{O}$. The organic layer was then washed with brine, dried over $\mathrm{MgSO}_{4}$ and concentrated in vacuo to afford the crude $\mathrm{N}$-Boc aniline.

\section{General Procedure J - synthesis of TMS-protected alkynes via the Sonagashira reaction}

A flask containing aryl iodide (1.0 equiv), $\mathrm{Pd}\left(\mathrm{PPh}_{3}\right)_{2} \mathrm{Cl}_{2}$ (0.05 equiv.) and $\mathrm{CuI}(0.05$ equiv) was evacuated and back filled with $\mathrm{N}_{2(\mathrm{~g})}$ three times. Then was added sequentially, with stirring, DMF $(0.2 \mathrm{M}), \mathrm{NEt}_{3}$ (1.5 equiv) and trimethylsilyl acetylene (1.5 equiv). The resulting solution was stirred at room temperature for 16 hours, before quenching with sat. $\mathrm{NH}_{4} \mathrm{Cl}_{(\mathrm{aq})}$. The mixture was diluted with EtOAc, and washed three times with $5 \% \mathrm{LiCl}_{(\mathrm{aq})}$, then brine. The organic layers were dried over $\mathrm{MgSO}_{4}$ and concentrated in vacuo to afford the crude TMS-protected aryl alkyne.

\section{General Procedure K - TMS deprotection of terminal alkynes}

To a solution of TMS-protected aryl alkyne (1.0 equiv) in $\mathrm{MeOH}(0.05 \mathrm{M})$ was added $\mathrm{K}_{2} \mathrm{CO}_{3}$ (1.0 equiv), and the resulting mixture stirred at room temperature for 1 hour. The reaction mixture was diluted with $\mathrm{Et}_{2} \mathrm{O}$ and extracted with brine. The aqueous layer was then washed with $\mathrm{Et}_{2} \mathrm{O}$, the organic layers combined then dried over $\mathrm{MgSO}_{4}$, and concentrated in vacuo to afford the crude terminal aryl alkyne. 


\section{tert-Butyl (2-iodophenyl)carbamate, 11a}<smiles>CC(C)(C)Nc1ccccc1I</smiles>

Compound 11a was prepared according to General Procedure $\mathbf{H}$ with 2-iodoaniline (5.00 g, $22.8 \mathrm{mmol})$, NaHMDS (25.1 mL, 2.0 M solution in THF, $50.2 \mathrm{mmol}$ ), $\mathrm{Boc}_{2} \mathrm{O}$ $(5.48 \mathrm{~g}, 25.1 \mathrm{mmol})$ and THF $(50 \mathrm{~mL})$. No further purification was necessary and NBoc aniline 11a was isolated as a dark brown oil (7.06 g, 97\% yield).

${ }^{1}$ H-NMR (400 MHz, $\left.\mathrm{CDCl}_{3}\right): 7.98(1 \mathrm{H}, \mathrm{dd}, J 8.0$ and 1.0, C(4)H), $7.67(1 \mathrm{H}, \mathrm{dd}, J 8.0$ and 1.5, C(1)H), $7.24(1 \mathrm{H}, \mathrm{td}, J 8.0,1.0, \mathrm{C}(2) H), 6.75(1 \mathrm{H}, \mathrm{br} . \mathrm{s}, \mathrm{N} H), 6.69(1 \mathrm{H}, \mathrm{td}, J$ 7.5 and $1.5, \mathrm{C}(3) H), 1.46\left(9 \mathrm{H}, \mathrm{s}, \mathrm{C}\left(\mathrm{CH}_{3}\right)_{3}\right) ;{ }^{13} \mathrm{C}-\mathrm{NMR}\left(100 \mathrm{MHz}, \mathrm{CDCl}_{3}\right): 152.6$, 138.9, 138.8, 129.2, 124.7, 120.2, 88.8, 81.1, 28.3; LRMS (ESI+, m/z): 342.0 $\left([\mathrm{M}+\mathrm{Na}]^{+}\right)$.

Data is consistent with the literature. ${ }^{2}$

\section{tert-Butyl (2-((trimethylsilyl)ethynyl)phenyl)carbamate, 12a}

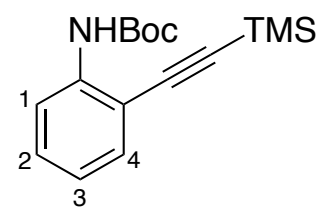

Compound 12a was prepared according to General Procedure $\mathbf{J}$ with aryl iodide 11a (882 mg, $2.76 \mathrm{mmol}), \mathrm{Pd}\left(\mathrm{PPh}_{3}\right)_{2} \mathrm{Cl}_{2}$ (97.0 mg, $\left.0.14 \mathrm{mmol}\right), \mathrm{CuI}$ (26.3 mg, $\left.0.14 \mathrm{mmol}\right)$, TMS-acetylene $(0.59 \mathrm{~mL}, 4.14 \mathrm{mmol}), \mathrm{NEt}_{3}(0.58 \mathrm{~mL}, 4.14 \mathrm{mmol})$ and DMF (37 mL). Flash column chromatography $\left(\mathrm{SiO}_{2}, 5 \% \mathrm{Et}_{2} \mathrm{O}\right.$ in petrol) afforded aryl alkyne 12a as an orange oil (722 $\mathrm{mg}, 90 \%$ yield).

${ }^{1}$ H-NMR $\left(400 \mathrm{MHz}, \mathrm{CDCl}_{3}\right): 7.96(1 \mathrm{H}, \mathrm{d}, J$ 8.5, C(1)H), $7.23(1 \mathrm{H}, \mathrm{dd}, J 7.5$ and 1.0, $\mathrm{C}(4) H), 7.23(1 \mathrm{H}$, br. s, $\mathrm{N} H), 7.16(1 \mathrm{H}, \mathrm{td}, J 8.0$ and $1.5, \mathrm{C}(2) H), 6.79(1 \mathrm{H}, \mathrm{td}, J 7.5$ and 1.0, $\mathrm{C}(3) H), 1.39\left(9 \mathrm{H}, \mathrm{s}, \mathrm{C}\left(\mathrm{CH}_{3}\right)_{3}\right), 0.15\left(9 \mathrm{H}, \mathrm{s}, \mathrm{Si}\left(\mathrm{CH}_{3}\right)_{3}\right) ;{ }^{13} \mathrm{C}-\mathrm{NMR}(100 \mathrm{MHz}$, $\left.\mathrm{CDCl}_{3}\right): 152.5,140.4,131.4,130.0,122.0,117.3,111.0,102.14,100.7,80.7,28.4$, $0.0 ; \operatorname{LRMS}(\mathrm{ESI}+, \mathrm{m} / \mathrm{z}): 312.1\left([\mathrm{M}+\mathrm{Na}]^{+}\right)$.

Data is consistent with the literature. ${ }^{3}$ 


\section{tert-Butyl (2-ethynylphenyl)carbamate, 3a}

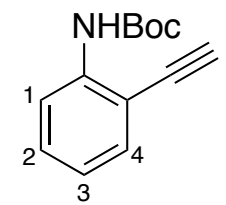

Compound 3a was prepared according to General Procedure K with alkyne 3a (130 $\mathrm{mg}, 0.45 \mathrm{mmol}), \mathrm{K}_{2} \mathrm{CO}_{3}(62.2 \mathrm{mg}, 0.45 \mathrm{mmol})$ and $\mathrm{MeOH}(9.0 \mathrm{~mL})$. Flash column chromatography $\left(\mathrm{SiO}_{2}, 5 \% \mathrm{Et}_{2} \mathrm{O}\right.$ in petrol) afforded terminal alkyne 3a as an off white solid (91 mg, 93\% yield).

m.p.: $42-44{ }^{\circ} \mathrm{C}$ (crystalized from $\mathrm{Et}_{2} \mathrm{O} /$ petrol); ${ }^{1} \mathbf{H}-\mathbf{N M R}\left(400 \mathrm{MHz}, \mathrm{CDCl}_{3}\right): 8.08$ $(1 \mathrm{H}, \mathrm{d}, J 8.5, \mathrm{C}(1) H), 7.34(1 \mathrm{H}, \mathrm{dd}, J 7.5$ and $1.5, \mathrm{C}(4) H), 7.25(1 \mathrm{H}, \mathrm{td}, J 8.0$ and 1.5, $\mathrm{C}(2) H), 7.20(1 \mathrm{H}$, br. s, $\mathrm{N} H), 6.88(1 \mathrm{H}, \mathrm{td}, J 7.5$ and $1.0, \mathrm{C}(3) H), 3.41(1 \mathrm{H}, \mathrm{s}, \mathrm{CCH})$, $1.46\left(9 \mathrm{H}, \mathrm{s}, \mathrm{C}\left(\mathrm{CH}_{3}\right)_{3}\right) ;{ }^{13} \mathrm{C}-\mathrm{NMR}\left(100 \mathrm{MHz}, \mathrm{CDCl}_{3}\right): 152.4,140.3,132.3,130.2$, 122.0, 117.6, 109.8, 84.1, 80.9, 79.4, 28.3; LRMS (ESI+, m/z): $240.1\left([\mathrm{M}+\mathrm{Na}]^{+}\right)$.

Data is consistent with the literature. ${ }^{4}$ 


\section{tert-Butyl (2-ethynyl-3-fluorophenyl)carbamate, $3 b$}

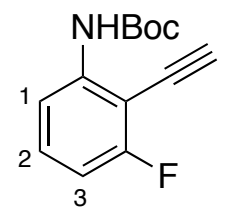

Compound $\mathbf{3 b}$ was prepared according to a combination of General Procedures $\mathbf{H}, \mathbf{J}$ and $\mathbf{K}$, involving a $\mathrm{SiO}_{2}$ filtration between $\mathbf{J}$ and $\mathbf{K}$, with $\mathbf{J}$ conducted at $70{ }^{\circ} \mathrm{C}$, using 3-fluoro-2-iodoaniline (1.0 g, $4.22 \mathrm{mmol}$ ), NaHMDS (4.64 mL, $2.0 \mathrm{M}$ solution in THF, $9.28 \mathrm{mmol})$, Boc $2 \mathrm{O}$ (1.01 mg, $4.64 \mathrm{mmol})$, THF (8 mL), $\mathrm{Pd}\left(\mathrm{PPh}_{3}\right)_{2} \mathrm{Cl}_{2}(147 \mathrm{mg}$, $0.21 \mathrm{mmol}), \mathrm{CuI}(40 \mathrm{mg}, 0.21 \mathrm{mmol})$, TMS-acetylene $\left(0.90 \mathrm{~mL}, 6.33 \mathrm{mmol}^{2}, \mathrm{NEt}_{3}\right.$ (0.88 mL, $6.33 \mathrm{mmol})$, DMF (12 mL), $\mathrm{K}_{2} \mathrm{CO}_{3}(583 \mathrm{mg}, 4.22 \mathrm{mmol})$ and $\mathrm{MeOH}(40$ $\mathrm{mL})$. Flash column chromatography $\left(\mathrm{SiO}_{2}, 0-10 \% \mathrm{Et}_{2} \mathrm{O}\right.$ in petrol) afforded terminal alkyne $\mathbf{3 b}$ as a pale brown solid (693 $\mathrm{mg}, 69 \%$ yield).

m.p.: $64-66{ }^{\circ} \mathrm{C}$ (crystalized from $\mathrm{Et}_{2} \mathrm{O} /$ petrol); ${ }^{1} \mathbf{H}-\mathbf{N M R}\left(400 \mathrm{MHz}, \mathrm{CDCl}_{3}\right.$ ): 8.00 $(1 \mathrm{H}, \mathrm{d}, J 8.5, \mathrm{C}(1) H), 7.31(1 \mathrm{H}, \mathrm{td}, J 8.5$ and 6.5, C(2)H), $7.28(1 \mathrm{H}$, br. s, NH), 6.77 $\left(1 \mathrm{H}, \mathrm{ddd}, J 9.0,8.5\right.$ and 1.0, C(3)H), $3.74(1 \mathrm{H}, \mathrm{d}, J 0.5, \mathrm{CCH}), 1.56\left(9 \mathrm{H}, \mathrm{s}, \mathrm{C}\left(\mathrm{CH}_{3}\right)_{3}\right)$; ${ }^{13}$ C-NMR (100 MHz, $\left.\mathrm{CDCl}_{3}\right): 163.5$ (d, J 251.0), 152.1, 141.8 (d, J 3.0), $130.8(2 \times \mathrm{C}$, ap. d, $J$ 9.5), 113.0 (d, $J$ 3.0), 108.8 (d, $J 20.0), 89.1$ (d, $J 4.5), 81.3,73.2$ (d, $J 3.0)$, 28.3; ${ }^{19}$ F-NMR (376 MHz, $\left.\mathrm{CDCl}_{3}\right)\left\{{ }^{1} \mathrm{H}\right\}:-109.0 ; \quad$ LRMS (ESI+, m/z): 258.1 $\left([\mathrm{M}+\mathrm{Na}]^{+}, \quad 100 \%\right) ; \quad$ HRMS $(\mathrm{ESI}+, \mathrm{m} / \mathrm{z}): \mathrm{C}_{13} \mathrm{H}_{14} \mathrm{FNNaO}_{2}\left([\mathrm{M}+\mathrm{Na}]^{+}\right)$requires 258.09008, found 258.09028; IR $v_{\max }(\mathrm{film}) / \mathrm{cm}^{-1}: 3407,3295,2980,2934,1733$, $1617,1580,1523,1474,1436,1394,1369,1313,1281,1252,1231,1153,1090,1065$, 1036. 
<smiles>O=C(Nc1ccc(Cl)cc1I)c1ccccc1</smiles>

Compound 11c was prepared according to General Procedure $\mathbf{H}$ with 5-chloro-2iodoaniline (1.00 g, $3.95 \mathrm{mmol})$, NaHMDS (3.95 mL, $2.0 \mathrm{M}$ solution in THF, 7.90 $\mathrm{mmol}), \mathrm{Boc}_{2} \mathrm{O}(1.03 \mathrm{~g}, 4.73 \mathrm{mmol})$ and THF (14 mL). Flash column chromatography $\left(\mathrm{SiO}_{2}, 5 \% \mathrm{Et}_{2} \mathrm{O}\right.$ in petrol) afforded $N$-Boc aniline 11c as an orange oil (548 mg, 39\% yield).

${ }^{1}$ H-NMR $\left(400 \mathrm{MHz}, \mathrm{CDCl}_{3}\right): 8.10(1 \mathrm{H}, \mathrm{d}, J 2.5, \mathrm{C}(1) H), 7.56(1 \mathrm{H}, \mathrm{d}, J$ 8.5, C(3)H), $6.77(1 \mathrm{H}$, br. s., $\mathrm{NH}), 6.70(1 \mathrm{H}, \mathrm{dd}, J 8.5$ and $2.5, \mathrm{C}(2) H), 1.47\left(9 \mathrm{H}, \mathrm{s}, \mathrm{C}\left(\mathrm{CH}_{3}\right)_{3}\right) ;{ }^{13} \mathrm{C}-$ NMR $\left(100 \mathrm{MHz}, \mathrm{CDCl}_{3}\right): 152.2,139.9,139.3,135.5,124.6,119.7,85.1,81.6,28.3$; LRMS $(\mathrm{ESI}+, \mathrm{m} / \mathrm{z}): 376.0\left(\left[\mathrm{M}\left({ }^{35} \mathrm{Cl}\right)+\mathrm{Na}\right]^{+}, 100 \%\right), 378.0\left(\left[\mathrm{M}\left({ }^{37} \mathrm{Cl}\right)+\mathrm{Na}\right]^{+}, 100 \%\right)$.

Data is consistent with the literature. ${ }^{5}$

tert-Butyl (5-chloro-2-((trimethylsilyl)ethynyl)phenyl)carbamate, 12c<smiles>CC(C)(C)C#Cc1ccc(Cl)cc1NC(C)(C)C</smiles>

Compound 12c was prepared according to General Procedure $\mathbf{J}$ with aryl iodide 11c (497 mg, $1.40 \mathrm{mmol}$ ), $\mathrm{Pd}\left(\mathrm{PPh}_{3}\right)_{2} \mathrm{Cl}_{2}$ (49.3 mg, $0.07 \mathrm{mmol}$ ), $\mathrm{CuI}$ (13.4 mg, $0.07 \mathrm{mmol}$ ), TMS-acetylene $(0.30 \mathrm{~mL}, 2.11 \mathrm{mmol}), \mathrm{NEt}_{3}(0.29 \mathrm{~mL}, 2.11 \mathrm{mmol})$ and DMF $(20 \mathrm{~mL})$. Flash column chromatography $\left(\mathrm{SiO}_{2}, 0-3 \% \mathrm{Et}_{2} \mathrm{O}\right.$ in petrol) afforded aryl alkyne 12c as an orange oil (310 $\mathrm{mg}, 68 \%$ yield).

${ }^{1}$ H-NMR (400 MHz, CDCl $)$ ): $8.02(1 \mathrm{H}, \mathrm{d}, J$ 2.0, C(1)H), $7.17(1 \mathrm{H}$, br. s, NH), 7.08 $(1 \mathrm{H}, \mathrm{d}, J 9.0, \mathrm{C}(3) H), 6.72(1 \mathrm{H}, \mathrm{dd}, J 9.0$ and $2.0, \mathrm{C}(2) H), 1.34\left(9 \mathrm{H}, \mathrm{s}, \mathrm{C}\left(\mathrm{CH}_{3}\right)_{3}\right), 0.10$ (9H, s, $\left.\mathrm{Si}\left(\mathrm{CH}_{3}\right)_{3}\right) ;{ }^{13} \mathrm{C}-\mathrm{NMR}\left(100 \mathrm{MHz}, \mathrm{CDCl}_{3}\right): 152.2,141.3,136.1,132.2,122.3$, 117.5, 109.4, 103.3, 99.7, 81.3, 28.4, 0.0; LRMS (ESI+, m/z): $346.1\left(\left[\mathrm{M}\left({ }^{35} \mathrm{Cl}\right)+\mathrm{Na}\right]^{+}\right.$ , 100\%), $348.1\left(\left[\mathrm{M}\left({ }^{37} \mathrm{Cl}\right)+\mathrm{Na}\right]^{+}, 100 \%\right) ; \quad$ HRMS $(\mathrm{ESI}+, \mathrm{m} / \mathrm{z}): \mathrm{C}_{16} \mathrm{H}_{22} \mathrm{ClNNaO}_{2} \mathrm{Si}$ $\left(\left[\mathrm{M}\left({ }^{35} \mathrm{Cl}\right)+\mathrm{Na}\right]^{+}\right)$requires 346.1001, found 346.0998; IR $v_{\max }($ film $) / \mathrm{cm}^{-1}: 2977,2152$, $1737,1570,1511,1452,1423,1368,1281,1251,1232,1152,1050$. 


\section{tert-Butyl (5-chloro-2-ethynylphenyl)carbamate, 3c}<smiles>C#Cc1ccc(Cl)cc1NC(=O)OCc1ccccc1</smiles>

Compound 3c was prepared according to General Procedure K with alkyne 12c (260 $\mathrm{mg}, 0.80 \mathrm{mmol}), \mathrm{K}_{2} \mathrm{CO}_{3}(111 \mathrm{mg}, 0.80 \mathrm{mmol})$ and $\mathrm{MeOH}(16 \mathrm{~mL})$. Flash column chromatography $\left(\mathrm{SiO}_{2}, 1 \% \mathrm{Et}_{2} \mathrm{O}\right.$ in petrol) afforded terminal alkyne $3 \mathbf{c}$ as an orange oil (189 mg, 75\% yield).

${ }^{1}$ H-NMR (400 MHz, $\left.\mathrm{CDCl}_{3}\right): 8.19(1 \mathrm{H}$, br. s, C(1)H), $7.26(1 \mathrm{H}, \mathrm{d}, J$ 8.5, C(3)H), $7.19(1 \mathrm{H}$, br. s, $\mathrm{NH}), 6.87(1 \mathrm{H}, \mathrm{dd}, J 8.5$ and $2.0, \mathrm{C}(2) H), 3.44(1 \mathrm{H}, \mathrm{s}, \mathrm{CC} H), 1.46$ $\left(9 \mathrm{H}, \mathrm{s}, \mathrm{C}\left(\mathrm{CH}_{3}\right)_{3}\right) ;{ }^{13} \mathrm{C}-\mathrm{NMR}\left(100 \mathrm{MHz}, \mathrm{CDCl}_{3}\right): 152.1,141.2,136.3,133.0,122.2$, 117.7, 108.1, 84.9, 81.5, 78.5, 28.3; LRMS (ESI+, m/z): $274.1\left(\left[\mathrm{M}\left({ }^{35} \mathrm{Cl}\right)+\mathrm{Na}\right]^{+}\right)$, $276.0\left(\left[\mathrm{M}\left({ }^{37} \mathrm{Cl}\right)+\mathrm{Na}\right]^{+}\right) ; \quad$ HRMS $(\mathrm{ESI}+, \mathrm{m} / \mathrm{z}): \mathrm{C}_{13} \mathrm{H}_{14} \mathrm{ClNNaO}_{2}\left(\left[\mathrm{M}\left({ }^{35} \mathrm{Cl}\right)+\mathrm{Na}\right]^{+}\right)$ requires 274.0605, found 274.0608, $\left(\left[\mathrm{M}\left({ }^{37} \mathrm{Cl}\right)+\mathrm{Na}\right]^{+}\right)$requires 276.0577 , found 276.0581 ; IR $v_{\max }($ film $) / \mathrm{cm}^{-1}: 3404,3296,2980,1733,1571,1510,1451,1418$, 1369, 1280, 1264, 1232, 1153, 1050. 


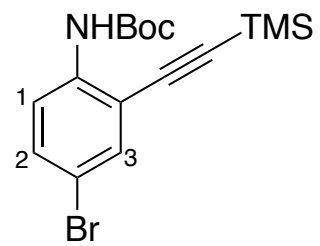

Compound 12d was prepared according to a combination of General Procedure $\mathbf{H}$ and J, with 4-bromo-2-iodo-aniline (4.875 g, $16.4 \mathrm{mmol}$ ), NaHMDS (18.5 mL, $2.0 \mathrm{M}$ solution in THF, $36.9 \mathrm{mmol}), \mathrm{Boc}_{2} \mathrm{O}(4.04 \mathrm{~g}, 18.5 \mathrm{mmol})$, THF (35 mL), $\mathrm{Pd}\left(\mathrm{PPh}_{3}\right)_{2} \mathrm{Cl}_{2}(590 \mathrm{mg}, 0.84 \mathrm{mmol}), \mathrm{CuI}(160 \mathrm{mg}, 0.84 \mathrm{mmol})$, TMS-acetylene (2.86 $\mathrm{mL}, 20.2 \mathrm{mmol}), \mathrm{NEt}_{3}(2.80 \mathrm{~mL}, 20.2 \mathrm{mmol})$ and DMF $(50 \mathrm{~mL})$. Flash column chromatography $\left(\mathrm{SiO}_{2}, 0-5 \% \mathrm{Et}_{2} \mathrm{O}\right.$ in petrol) afforded aryl alkyne 12d as an orange oil (4.79 g, 79\% yield over 2 steps).

m.p.: 66-68 ${ }^{\circ} \mathrm{C}$ (crystalized from $\left.\mathrm{CHCl}_{3}\right) ;{ }^{1} \mathbf{H}-\mathbf{N M R}\left(400 \mathrm{MHz}, \mathrm{CDCl}_{3}\right): 8.06(1 \mathrm{H}, \mathrm{d}$, $J$ 9.0, $\mathrm{C}(1) H), 7.52(1 \mathrm{H}, \mathrm{d}, J 2.5, \mathrm{C}(3) H), 7.42(1 \mathrm{H}, \mathrm{dd}, J 9.0$ and 2.5, $\mathrm{C}(2) H), 7.34$ $\left(1 \mathrm{H}\right.$, br. s, NH), $1.55\left(9 \mathrm{H}, \mathrm{s}, \mathrm{C}\left(\mathrm{CH}_{3}\right)_{3}\right), 0.32\left(9 \mathrm{H}, \mathrm{s}, \mathrm{Si}\left(\mathrm{CH}_{3}\right)_{3}\right) ;{ }^{13} \mathbf{C}-\mathbf{N M R}(100 \mathrm{MHz}$, $\left.\mathrm{CDCl}_{3}\right)$ : 152.4, 139.6, 133.9, 133.0, 118.9, 114.1, 113.0, 103.8, 99.2, 81.2, 28.5, 0.0; HRMS $(\mathrm{ESI}+, \mathrm{m} / \mathrm{z}): \mathrm{C}_{16} \mathrm{H}_{22} \mathrm{BrNNaO}_{2} \mathrm{Si}\left(\left[\mathrm{M}\left({ }^{79} \mathrm{Br}\right)+\mathrm{Na}\right]^{+}\right)$requires 390.0495 , found 390.0502; $\left(\left[\mathrm{M}\left({ }^{81} \mathrm{Br}\right)+\mathrm{Na}\right]^{+}\right)$requires 392.0476, found 392.0481.

Data is consistent with the literature. ${ }^{6}$

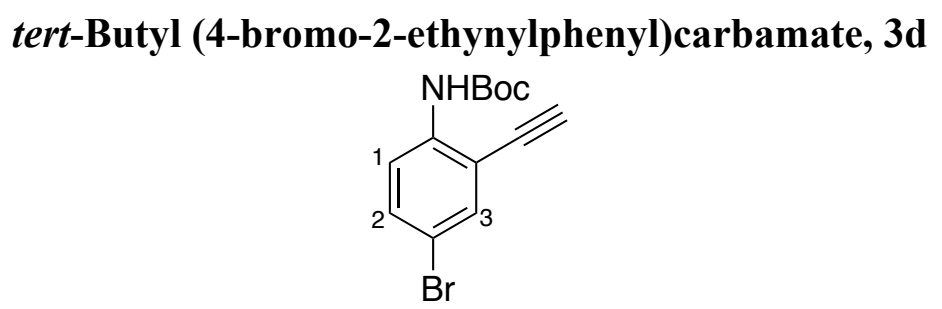

Compound 3d was prepared according to General Procedure K with alkyne 12d (508 $\mathrm{mg}, 1.38 \mathrm{mmol}), \mathrm{K}_{2} \mathrm{CO}_{3}(191 \mathrm{mg}, 1.38 \mathrm{mmol})$ and $\mathrm{MeOH}(30 \mathrm{~mL})$. Flash column chromatography $\left(\mathrm{SiO}_{2}, 5 \% \mathrm{Et}_{2} \mathrm{O}\right.$ in petrol) afforded terminal alkyne $\mathbf{3 d}$ as a pale orange solid (333 mg, 82\% yield);

m.p.: $62-64{ }^{\circ} \mathrm{C}$ (crystalized from $\mathrm{Et}_{2} \mathrm{O} /$ petrol); ${ }^{1} \mathbf{H}-\mathbf{N M R}\left(400 \mathrm{MHz}, \mathrm{CDCl}_{3}\right.$ ): 8.01 $(1 \mathrm{H}, \mathrm{d}, J 9.0, \mathrm{C}(1) H), 7.46(1 \mathrm{H}, \mathrm{d}, J 2.5, \mathrm{C}(3) H), 7.35(1 \mathrm{H}, \mathrm{dd}, J 9.0$ and $2.5, \mathrm{C}(2) H)$, $7.14(1 \mathrm{H}$, br. s, $\mathrm{NH}), 3.45(1 \mathrm{H}, \mathrm{s}, \mathrm{CCH}), 1.46\left(9 \mathrm{H}, \mathrm{s}, \mathrm{C}\left(\mathrm{CH}_{3}\right)_{3}\right) ;{ }^{13} \mathrm{C}-\mathrm{NMR}(100 \mathrm{MHz}$, $\left.\mathrm{CDCl}_{3}\right): 152.2,139.5,134.5,133.1,119.1,113.9,111.7,85.3,81.3,78.0,28.3$; LRMS (ESI+, m/z): $318.0\left(\left[\mathrm{M}\left({ }^{79} \mathrm{Br}\right)+\mathrm{Na}\right]^{+}, 100 \%\right), 320.0\left(\left[\mathrm{M}\left({ }^{81} \mathrm{Br}\right)+\mathrm{Na}\right]^{+}, 100 \%\right)$.

Data is consistent with the literature. ${ }^{6}$ 
tert-Butyl (2-iodo-6-((trimethylsilyl)ethynyl)phenyl)carbamate, 12e

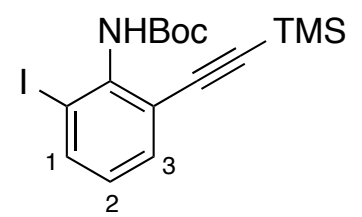

At $-78{ }^{\circ} \mathrm{C}$, to a solution of compound $12 \mathrm{a}(800 \mathrm{mg}, 2.76 \mathrm{mmol})$ in $\mathrm{Et}_{2} \mathrm{O}(6 \mathrm{~mL})$ was added dropwise ${ }^{t} \mathrm{BuLi}(1.7 \mathrm{M}$ in pentane, $3.58 \mathrm{~mL}, 6.08 \mathrm{mmol})$. The reaction mixture was warmed to $-20{ }^{\circ} \mathrm{C}$ and stirred for 3 hours. The reaction was then cooled to $-78{ }^{\circ} \mathrm{C}$ and a solution of $\mathrm{I}_{2}(1.05 \mathrm{~g}, 4.14 \mathrm{mmol})$ in $\mathrm{Et}_{2} \mathrm{O}(8 \mathrm{~mL})$ was added dropwise. The reaction was allowed to warm to room temperature over 16 hours, after which time the reaction was quenched with $\mathrm{H}_{2} \mathrm{O}(15 \mathrm{~mL})$, then extracted with $\mathrm{Et}_{2} \mathrm{O}(3 \times 15$ $\mathrm{mL})$. The combined organic fractions were washed with brine $(30 \mathrm{~mL})$, dried over $\mathrm{MgSO}_{4}$ and concentrated in vacuo. Flash column chromatography $\left(\mathrm{SiO}_{2}, 0-5 \%\right.$ EtOAc in petrol) afforded aryl iodide 12e as a light brown solid (745 mg, 64\% yield).

m.p.: $88-90{ }^{\circ} \mathrm{C}$ (crystalized from EtOAc/petrol); ${ }^{1}$ H-NMR (400 MHz, $\mathrm{CDCl}_{3}$ ): 7.81 $(1 \mathrm{H}, \mathrm{d}, J 8.0, \mathrm{C}(1) H), 7.45(1 \mathrm{H}, \mathrm{d}, J 8.0, \mathrm{C}(3) H), 6.88(1 \mathrm{H}, \mathrm{t}, J 8.0, \mathrm{C}(2) H), 6.37(1 \mathrm{H}$, br. s, $\mathrm{NH}), 1.51\left(9 \mathrm{H}\right.$, br. s, $\left.\mathrm{C}\left(\mathrm{CH}_{3}\right)_{3}\right), 0.25\left(9 \mathrm{H}, \mathrm{s}, \mathrm{Si}\left(\mathrm{CH}_{3}\right)_{3}\right) ;{ }^{13} \mathbf{C}-\mathbf{N M R}(100 \mathrm{MHz}$, $\left.\mathrm{CDCl}_{3}\right): 152.7,140.6,139.6,132.6,127.7,122.5,101.0,100.5,98.5,80.6,28.3,-0.1$; LRMS (ESI+, m/z): $438.0\left([\mathrm{M}+\mathrm{Na}]^{+}, 100 \%\right) ;$ HRMS (ESI+, m/z): $\mathrm{C}_{16} \mathrm{H}_{22} \mathrm{INNaO}_{2} \mathrm{Si}$ $\left([\mathrm{M}+\mathrm{Na}]^{+}\right)$requires 438.03567 , found 438.03524; IR $v_{\max }($ film $) / \mathrm{cm}^{-1}: 3403,2965$, 2899, 2162, 1707, 1481, 1447, 1367, 1247, 1158, 1049, 1023. 
tert-Butyl (2-ethynyl-6-iodophenyl)carbamate, $3 \mathrm{e}$<smiles>C#Cc1cccc(I)c1N</smiles>

Compound 3e was prepared according to General Procedure $\mathbf{K}$ with alkyne 12e (600 $\mathrm{mg}, 1.44 \mathrm{mmol}), \mathrm{K}_{2} \mathrm{CO}_{3}(200 \mathrm{mg}, 1.44 \mathrm{mmol})$, and $\mathrm{MeOH}(30 \mathrm{~mL})$. Flash column chromatography $\left(\mathrm{SiO}_{2}, 0-20 \%\right.$ EtOAc in petrol) afforded terminal alkyne $\mathbf{3 e}$ as an off white solid (477 mg, 96\% yield).

m.p.: $127-129{ }^{\circ} \mathrm{C}$ (crystalized from EtOAc/petrol); ${ }^{\mathbf{1}} \mathbf{H}-\mathbf{N M R}\left(400 \mathrm{MHz}, \mathrm{CDCl}_{3}\right)$ : $7.84(1 \mathrm{H}, \mathrm{dd}, J 8.0$ and 1.5, C(1)H), $7.50(1 \mathrm{H}, \mathrm{dd}, J 8.0$ and $1.5, \mathrm{C}(3) H), 6.91(1 \mathrm{H}, \mathrm{t}, J$ 8.0, C(2)H), $6.28\left(1 \mathrm{H}\right.$, br. s, NH), $3.31(1 \mathrm{H}, \mathrm{s}, \mathrm{CCH}), 1.51\left(9 \mathrm{H}, \mathrm{s}, \mathrm{C}\left(\mathrm{CH}_{3}\right)_{3}\right)$; ${ }^{13} \mathrm{C}-$ NMR $\left(100 \mathrm{MHz}, \mathrm{CDCl}_{3}\right): 152.8,140.7,139.8,133.3,127.8,121.8,98.7,82.5,81.0$, 80.1, 28.3; LRMS (ESI+, m/z): $709.0\left([2 \mathrm{M}+\mathrm{Na}]^{+}, 100 \%\right) ; \quad$ HRMS (ESI+, m/z): $\mathrm{C}_{13} \mathrm{H}_{14} \mathrm{INNaO}_{2}\left([\mathrm{M}+\mathrm{Na}]^{+}\right)$requires 365.99614 , found $365.99616 ;$ IR $v_{\max }(\mathrm{film}) / \mathrm{cm}^{-}$ ${ }^{1}:$ 3294, 2977, 2931, 1703, 1482, 1448, 1392, 1367, 1242, 1158, 1121, 1077, 1049, 1022. 
<smiles>CC(C)(C)OC(=O)Nc1ccc(C(F)(F)F)cc1I</smiles>

Compound 11f was prepared according to General Procedure I with 4-amino-3iodobenzotrifluoride $(1.50 \mathrm{~g}, 5.23 \mathrm{mmol}), \mathrm{Boc}_{2} \mathrm{O}$ (2.63 g, $\left.12.0 \mathrm{mmol}\right)$, DMAP (63.8 $\mathrm{mg}, 0.52 \mathrm{mmol})$, THF (35 mL), $\mathrm{K}_{2} \mathrm{CO}_{3}(2.17 \mathrm{~g}, 15.7 \mathrm{mmol})$ and $\mathrm{MeOH}(35 \mathrm{~mL})$ for 16 hours. Flash column chromatography $\left(\mathrm{SiO}_{2}, 2-3 \% \mathrm{Et}_{2} \mathrm{O}\right.$ in petrol) afforded $N$-Boc aniline 11f as a pale yellow solid (753 $\mathrm{mg}, 37 \%$ yield).

m.p.: $65-67^{\circ} \mathrm{C}$ (crystalized from $\left.\mathrm{CHCl}_{3}\right) ;{ }^{1} \mathbf{H}-\mathbf{N M R}\left(400 \mathrm{MHz}, \mathrm{CDCl}_{3}\right)$ : $8.16(1 \mathrm{H}, \mathrm{d}$, $J$ 8.5, C(1)H), $7.90(1 \mathrm{H}, \mathrm{d}, J 2.0, \mathrm{C}(3) H), 7.48(1 \mathrm{H}, \mathrm{dd}, J 8.5$ and 2.0, $\mathrm{C}(2) H), 6.93$ $(1 \mathrm{H}$, br. s, $\mathrm{NH}), 1.47$ (9H, s, $\left.\mathrm{C}\left(\mathrm{CH}_{3}\right)_{3}\right) ;{ }^{13} \mathrm{C}-\mathrm{NMR}\left(100 \mathrm{MHz}, \mathrm{CDCl}_{3}\right): 152.1,142.0$, 135.8 (q, $J 4.0), 126.4$ (q, $J 3.5), 126.0$ (q, $J 33.5), 123.1$ (q, $J 272.0), 118.8,87.0$, 81.9, 28.2; ${ }^{19}$ F-NMR $\left(376 \mathrm{MHz}, \mathrm{CDCl}_{3}\right)\left\{{ }^{1} \mathrm{H}\right\}:-62.0 ; \quad$ LRMS (ESI+, m/z): 410.0 $\left([\mathrm{M}+\mathrm{Na}]^{+}, 100 \%\right)$.

Data is consistent with the literature. ${ }^{1}$

tert-Butyl (4-(trifluoromethyl)-2-((trimethylsilyl)ethynyl)phenyl)carbamate, $12 \mathrm{f}$<smiles>CC(C)(C)C#Cc1cc(C(F)(F)F)ccc1NC(C)(C)C</smiles>

Compound 12f was prepared according to General Procedure $\mathbf{J}$ with aryl iodide 11f (740 mg, $1.91 \mathrm{mmol}), \mathrm{Pd}\left(\mathrm{PPh}_{3}\right)_{2} \mathrm{Cl}_{2}(67.1 \mathrm{mg}, 0.10 \mathrm{mmol}), \mathrm{CuI}(18.2 \mathrm{mg}, 0.10 \mathrm{mmol})$, TMS-acetylene $(0.41 \mathrm{~mL}, 2.87 \mathrm{mmol}), \mathrm{NEt}_{3}(0.40 \mathrm{~mL}, 2.87 \mathrm{mmol})$ and DMF $(25 \mathrm{~mL})$. Flash column chromatography $\left(\mathrm{SiO}_{2}, 5-10 \% \mathrm{Et}_{2} \mathrm{O}\right.$ in petrol) afforded aryl alkyne $\mathbf{1 2 f}$ as a pale yellow (677 $\mathrm{mg}, 99 \%$ yield).

m.p.: 76-78 ${ }^{\circ} \mathrm{C}$ (crystalized from $\left.\mathrm{CHCl}_{3}\right) ;{ }^{1} \mathbf{H}-\mathbf{N M R}\left(400 \mathrm{MHz}, \mathrm{CDCl}_{3}\right)$ : $8.09(1 \mathrm{H}, \mathrm{d}$, $J$ 9.0, C(1)H), $7.44(1 \mathrm{H}, \mathrm{d}, J 2.0, \mathrm{C}(3) H), 7.34(1 \mathrm{H}, \mathrm{dd}, J 9.0$ and 2.0, $\mathrm{C}(2) H), 7.32$ $(1 \mathrm{H}$, br. s, $\mathrm{NH}), 1.35\left(9 \mathrm{H}, \mathrm{s}, \mathrm{C}\left(\mathrm{CH}_{3}\right)_{3}\right), 0.12\left(9 \mathrm{H}, \mathrm{s}, \mathrm{Si}\left(\mathrm{CH}_{3}\right)_{3}\right) ;{ }^{13} \mathrm{C}-\mathbf{N M R}(100 \mathrm{MHz}$, $\mathrm{CDCl}_{3}$ ): 152.2, 143.3, 128.7 (q, $\left.J 4.0\right), 127.0$ (q, $\left.J 3.5\right), 124.2$ (q, $\left.J 33.0\right), 124.1$ (q, $J$ 271.5), 117.2, 111.4, 104.1, 99.2, 81.7, 28.5, 0.0; ${ }^{19}$ F-NMR $\left(376 \mathrm{MHz}, \mathrm{CDCl}_{3}\right)\left\{{ }^{1} \mathrm{H}\right\}$ : -62.2; LRMS (ESI+, m/z): $380.1\left([\mathrm{M}+\mathrm{Na}]^{+}, 100 \%\right) ; \quad$ HRMS (ESI+, m/z): $\mathrm{C}_{17} \mathrm{H}_{22} \mathrm{~F}_{3} \mathrm{NNaO}_{2} \mathrm{Si}\left([\mathrm{M}+\mathrm{Na}]^{+}\right)$requires 392.1264 , found $392.1275 ;$ IR $v_{\max }\left(\right.$ film) $/ \mathrm{cm}^{-}$ ${ }^{1}: 1742,1586,1525,1471,1421,1330,1311,1287,1250,1232,1150,1116,1075$, 1050, 1026; 
tert-Butyl (2-ethynyl-4-(trifluoromethyl)phenyl)carbamate, $3 f$<smiles>[R6]Nc1ccc(C(F)(F)F)cc1C#C</smiles>

Compound 3f was prepared according to General Procedure $\mathbf{K}$ with alkyne $\mathbf{1 2 f}$ (550 $\mathrm{mg}, 1.54 \mathrm{mmol}), \mathrm{K}_{2} \mathrm{CO}_{3}(213 \mathrm{mg}, 1.54 \mathrm{mmol})$ and $\mathrm{MeOH}(30 \mathrm{~mL})$. Flash column chromatography $\left(\mathrm{SiO}_{2}, 10 \% \mathrm{Et}_{2} \mathrm{O}\right.$ in petrol) afforded terminal alkyne $\mathbf{3 f}$ as a pale yellow solid (392 mg, 89\% yield).

m.p.: $97-99{ }^{\circ} \mathrm{C}$ (crystalized from $\left.\mathrm{CHCl}_{3}\right) ;{ }^{1} \mathbf{H}-\mathbf{N M R}\left(400 \mathrm{MHz}, \mathrm{CDCl}_{3}\right): 8.24(1 \mathrm{H}, \mathrm{d}$, $J$ 9.0, C(1)H), $7.59(1 \mathrm{H}, \mathrm{d}, J 2.0, \mathrm{C}(3) H), 7.47(1 \mathrm{H}, \mathrm{dd}, J 9.0$ and 2.0, C(2)H), 7.32 $\left(1 \mathrm{H}\right.$, br. s, NH), $3.47(1 \mathrm{H}, \mathrm{s}, \mathrm{CCH}), 1.46\left(9 \mathrm{H}, \mathrm{s}, \mathrm{C}\left(\mathrm{CH}_{3}\right)_{3}\right) ;{ }^{13} \mathrm{C}-\mathrm{NMR}(100 \mathrm{MHz}$, $\mathrm{CDCl}_{3}$ ): 152.0, 143.1, 129.3 (q, $\left.J 4.0\right), 127.0$ (q, $\left.J 3.5\right), 124.1$ (q, $\left.J 33.5\right), 123.7$ (q, $J$ 271.5), 117.3, 109.9, 85.4, 81.7, 77.9, 28.2; ${ }^{19}$ F-NMR (376 MHz, $\left.\mathrm{CDCl}_{3}\right)\left\{{ }^{1} \mathrm{H}\right\}:$ -62.3; HRMS (FI+, m/z): $\mathrm{C}_{14} \mathrm{H}_{14} \mathrm{~F}_{3} \mathrm{NNaO}_{2}\left([\mathrm{M}+\mathrm{Na}]^{+}\right)$requires 285.0970, found 285.0977; IR $v_{\max }($ film $) / \mathrm{cm}^{-1}: 1736,1589,1526,1475,1419,1370,1329,1253$, 1230, 1150, 1118, 1076, 1048, 1024; 


\section{tert-Butyl (2-ethynyl-4-formylphenyl)carbamate, $3 \mathrm{~g}$}

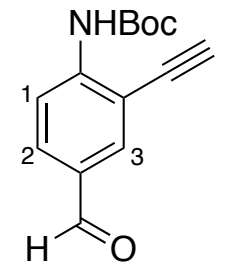

To a solution of ester 12i $(1.0 \mathrm{~g}, 2.88 \mathrm{mmol})$ and $\mathrm{MeOH}(0.23 \mathrm{~mL}, 5.76 \mathrm{mmol})$ in THF $(15 \mathrm{~mL})$ at $0{ }^{\circ} \mathrm{C}$, was slowly added $\mathrm{LiBH}_{4}(2.88 \mathrm{~mL}, 5.76 \mathrm{mmol}, 2.0 \mathrm{M}$ solution in THF). The reaction was stirred at $0{ }^{\circ} \mathrm{C}$ for 15 minutes, then allowed to warm to room temperature. After stirring for 16 hours, the reaction mixture was poured onto $5 \%$ citric $\operatorname{acid}_{(\mathrm{aq})}(100 \mathrm{~mL})$, then extracted with $\mathrm{Et}_{2} \mathrm{O}(100 \mathrm{~mL})$, washed with sat. $\mathrm{NaHCO}_{3 \text { (aq) }}(100 \mathrm{~mL})$, then brine $(100 \mathrm{~mL})$, before being dried over $\mathrm{MgSO}_{4}$ and concentrated in vacuo. The crude alcohol was then dissolved in dry $\mathrm{CH}_{2} \mathrm{Cl}_{2}(16 \mathrm{~mL})$. Dess Martin Periodinane (1.47 g, $3.46 \mathrm{mmol}$ ) and $\mathrm{NaHCO}_{3}(726 \mathrm{mg}, 8.64 \mathrm{mmol})$ were then added, and the reaction stirred at room temperature for 16 hours. The reaction was quenched with sat. $\mathrm{NaHSO}_{3(\mathrm{aq})}$, then partitioned between $\mathrm{H}_{2} \mathrm{O}(100 \mathrm{~mL})$ and $\mathrm{CH}_{2} \mathrm{Cl}_{2}(100 \mathrm{~mL})$. The organic layer was then washed with brine $(100 \mathrm{~mL})$, dried over $\mathrm{MgSO}_{4}$ and concentrated in vacuo. Flash column chromatography $\left(\mathrm{SiO}_{2}, 5-10 \%\right.$ $\mathrm{Et}_{2} \mathrm{O}$ in petrol) afforded $551 \mathrm{mg}$ of a mixture of the desired aldehyde and ester $\mathbf{3 i}$. This mixture was then dissolved in $\mathrm{MeOH}\left(35 \mathrm{~mL}\right.$ ), and $\mathrm{K}_{2} \mathrm{CO}_{3}$ added (240 mg, 1.74 mmol). The reaction was stirred at room temperature for $1 \mathrm{hr}$, and then quenched with $\mathrm{H}_{2} \mathrm{O}(200 \mathrm{~mL})$, before extracting with $\mathrm{Et}_{2} \mathrm{O}(3 \times 200 \mathrm{~mL})$. The combined organic layers were then washed with brine $(200 \mathrm{~mL})$, dried over $\mathrm{MgSO}_{4}$, and concentrated in vacuo. Flash column chromatography $\left(\mathrm{SiO}_{2}, 5-10 \% \mathrm{Et}_{2} \mathrm{O}\right.$ in petrol) afforded aldehyde $\mathbf{3 g}$ as a white solid (300 $\mathrm{mg}, 42 \%$ yield over 3 steps).

m.p.: 97-99 ${ }^{\circ} \mathrm{C}$ (crystalized from $\mathrm{Et}_{2} \mathrm{O} /$ petrol); ${ }^{\mathbf{1}} \mathbf{H}-\mathbf{N M R}\left(400 \mathrm{MHz}, \mathrm{CDCl}_{3}\right.$ ): 9.88 (1H, s, CHO), $8.41(1 \mathrm{H}, \mathrm{d}, J 8.5, \mathrm{C}(1) H), 7.98(1 \mathrm{H}, \mathrm{d}, J 2.0, \mathrm{C}(3) H), 7.87(1 \mathrm{H}, \mathrm{dd}, J$ 8.5 and 2.0, $\mathrm{C}(2) H), 7.55(1 \mathrm{H}$, br. s, $\mathrm{NH}), 3.60(1 \mathrm{H}, \mathrm{s}, \mathrm{CCH}), 1.57\left(9 \mathrm{H}, \mathrm{s}, \mathrm{C}\left(\mathrm{CH}_{3}\right)_{3}\right)$; ${ }^{13}$ C-NMR (100 MHz, $\mathrm{CDCl}_{3}$ ): 190.1, 151.8, 145.3, 134.1, 131.8, 130.4, 117.1, 110.2, 85.5, 82.0, 78.0, 28.2; LRMS (ESI+, m/z): 300.1 ([M+MeOH+Na $\left.]^{+}, 100 \%\right)$; HRMS $(\mathrm{ESI}+, \mathrm{m} / \mathrm{z}): \mathrm{C}_{15} \mathrm{H}_{19} \mathrm{NNaO}_{4}\left([\mathrm{M}+\mathrm{MeOH}+\mathrm{Na}]^{+}\right)$requires 300.12063, found 300.12073; IR $v_{\max }($ film $) / \mathrm{cm}^{-1}: 3399,3255,2979,2823,1736,1693,1577,1518,1465,1423$, 1369, 1310, 1233, 1204, 1150, 1049, 1024. 


\section{1-(4-Amino-3-iodophenyl)ethan-1-one, 13}<smiles>CC(=O)c1ccc(N)c(I)c1</smiles>

To a solution of 4'-aminoacetophenone $(2.03 \mathrm{~g}, 15.0 \mathrm{mmol})$ in $\mathrm{CH}_{2} \mathrm{Cl}_{2}(30 \mathrm{~mL})$ and $\mathrm{H}_{2} \mathrm{O}(6.0 \mathrm{~mL})$ was added sequentially $\mathrm{NaHCO}_{3}(1.51 \mathrm{~g}, 18.0 \mathrm{mmol})$ and $\mathrm{I}_{2}(3.81 \mathrm{~g}$, $15.0 \mathrm{mmol}$ ), and the reaction stirred at room temperature for 16 hours. The reaction was quenched with sat. $\mathrm{Na}_{2} \mathrm{~S}_{2} \mathrm{O}_{3(\mathrm{aq})}(10 \mathrm{~mL})$, and partitioned between $\mathrm{CH}_{2} \mathrm{Cl}_{2}(200$ $\mathrm{mL})$ and $\mathrm{H}_{2} \mathrm{O}(200 \mathrm{~mL})$. The aqueous layer was extracted with $\mathrm{CH}_{2} \mathrm{Cl}_{2}(2 \times 100 \mathrm{~mL})$, the combined organic layers washed with brine $(100 \mathrm{~mL})$, dried over $\mathrm{MgSO}_{4}$ and concentrated in vacuo. Flash column chromatography $\left(\mathrm{SiO}_{2}, \mathrm{CH}_{2} \mathrm{Cl}_{2}\right)$ afforded aryl iodide 13 as a pale brown oil (2.58 g, 66\% yield)

${ }^{1}$ H-NMR $\left(400 \mathrm{MHz}, \mathrm{CDCl}_{3}\right): 8.28(1 \mathrm{H}, \mathrm{d}, J 2.0, \mathrm{C}(3) H), 7.77(1 \mathrm{H}, \mathrm{dd}, J 8.5$ and 2.0, $\mathrm{C}(2) H), 6.72(1 \mathrm{H}, \mathrm{d}, J 8.5, \mathrm{C}(1) H), 4.68\left(2 \mathrm{H}\right.$, br. s, $\left.\mathrm{N} H_{2}\right), 2.50\left(3 \mathrm{H}, \mathrm{s}, \mathrm{CH}_{3}\right) ;{ }^{13} \mathrm{C}-$ NMR (100 MHz, $\left.\mathrm{CDCl}_{3}\right)$ : 195.2, 151.0, 140.3, 130.3, 129.2, 113.1, 82.6, 26.1; LRMS (ESI+, m/z): $284.0\left([\mathrm{M}+\mathrm{Na}]^{+}, 100 \%\right)$.

Data is consistent with the literature. ${ }^{7}$

\section{tert-Butyl (4-acetyl-2-iodophenyl)carbamate, $11 \mathrm{~h}$}<smiles>CC(=O)OCc1ccc(N)cc1I</smiles>

Compound 11h was prepared according to General Procedure $\mathbf{H}$ with aniline $\mathbf{1 3}$ (385 $\mathrm{mg}, 1.48 \mathrm{mmol})$, NaHMDS (2.43 mL, 2.0 M solution in THF, $4.86 \mathrm{mmol}$ ), $\mathrm{Boc}_{2} \mathrm{O}$ (354 mg, $1.62 \mathrm{mmol})$ and THF (3 mL). Flash column chromatography $\left(\mathrm{SiO}_{2}, 0-10 \%\right.$ $\mathrm{Et}_{2} \mathrm{O}$ in petrol) afforded $\mathrm{N}$-Boc aniline $11 \mathrm{~h}$ as a cream solid (275 $\mathrm{mg}, 52 \%$ yield).

m.p.: $42-44{ }^{\circ} \mathrm{C}$ (crystalized from $\mathrm{Et}_{2} \mathrm{O} /$ petrol); ${ }^{\mathbf{1}} \mathbf{H}-\mathbf{N M R}\left(400 \mathrm{MHz}, \mathrm{CDCl}_{3}\right.$ ): 8.38 $(1 \mathrm{H}, \mathrm{d}, J 2.0, \mathrm{C}(3) H), 8.24(1 \mathrm{H}, \mathrm{d}, J 8.5, \mathrm{C}(1) H), 7.91(1 \mathrm{H}, \mathrm{dd}, J 8.5$ and $2.0, \mathrm{C}(2) H)$, $7.12(1 \mathrm{H}$, br. s, $\mathrm{NH}), 2.57\left(3 \mathrm{H}, \mathrm{s}, \mathrm{C}(\mathrm{O}) \mathrm{CH}_{3}\right), 1.57\left(9 \mathrm{H}, \mathrm{s}, \mathrm{C}\left(\mathrm{CH}_{3}\right)_{3}\right) ;{ }^{13} \mathbf{C}-\mathbf{N M R}(100$ $\left.\mathrm{MHz}_{\mathrm{CDCl}}\right)$ : 195.6, 152.0, 142.9, 139.3, 133.0, 129.8, 118.1, 87.6, 81.9, 28.2, 26.3; LRMS $(E S I+, \mathrm{m} / \mathrm{z}): 384.0\left([\mathrm{M}+\mathrm{Na}]^{+}, 100 \%\right) ; \quad$ HRMS $(\mathrm{ESI}+, \mathrm{m} / \mathrm{z}): \mathrm{C}_{13} \mathrm{H}_{16} \mathrm{INO}_{3}$ $\left([\mathrm{M}+\mathrm{Na}]^{+}\right)$requires 384.00671 , found $384.00668 ;$ IR $v_{\max }($ film $) / \mathrm{cm}^{-1}: 3387,2978$, 2931, 1737, 1681, 1590, 1563, 1516, 1457, 1388, 1368, 1285, 1234, 1151, 1057, 1018. 


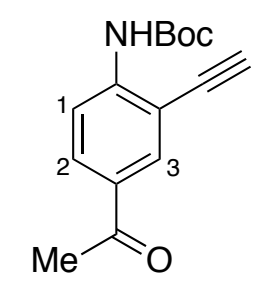

Compound $\mathbf{3 h}$ was prepared according to a combination of General Procedures $\mathbf{J}$ and $\mathbf{K}$, with a $\mathrm{SiO}_{2}$ filtration after step $\mathbf{J}$, using aryl iodide $\mathbf{1 1 h}(253 \mathrm{mg}, 0.7 \mathrm{mmol})$, $\mathrm{Pd}\left(\mathrm{PPh}_{3}\right)_{2} \mathrm{Cl}_{2}$ (24.6 mg, $\left.0.035 \mathrm{mmol}\right), \mathrm{CuI}(6.7 \mathrm{mg}, 0.035 \mathrm{mmol})$, TMS-acetylene $(0.150 \mathrm{~mL}, 1.05 \mathrm{mmol}), \mathrm{NEt}_{3}(0.146 \mathrm{~mL}, 1.05 \mathrm{mmol}), \mathrm{DMF}(3.5 \mathrm{~mL}), \mathrm{K}_{2} \mathrm{CO}_{3}(96.7$ $\mathrm{mg}, 0.7 \mathrm{mmol})$ and $\mathrm{MeOH}(14 \mathrm{~mL})$. Flash column chromatography $\left(\mathrm{SiO}_{2}, 0-10 \%\right.$ $\mathrm{Et}_{2} \mathrm{O}$ in petrol) afforded terminal alkyne $\mathbf{3 h}$ as a brown solid $(136.7 \mathrm{mg}, 75 \%$ yield over 2 steps).

m.p.: $95-97{ }^{\circ} \mathrm{C}$ (crystalized from $\left.\mathrm{CHCl}_{3}\right) ;{ }^{1} \mathbf{H}-\mathbf{N M R}\left(400 \mathrm{MHz}, \mathrm{CDCl}_{3}\right): 8.26(1 \mathrm{H}, \mathrm{d}$, $J$ 9.0, C(1)H), $8.02(1 \mathrm{H}, \mathrm{d}, J 2.0, \mathrm{C}(3) H), 7.90(1 \mathrm{H}, \mathrm{dd}, J 9.0$ and 2.0, $\mathrm{C}(2) H), 7.46$ $(1 \mathrm{H}$, br. s, $\mathrm{NH}), 3.56(1 \mathrm{H}, \mathrm{s}, \mathrm{CCH}), 2.53\left(3 \mathrm{H}, \mathrm{s}, \mathrm{C}(\mathrm{O}) \mathrm{CH}_{3}\right), 1.53\left(9 \mathrm{H}, \mathrm{s}, \mathrm{C}\left(\mathrm{CH}_{3}\right)_{3}\right)$; ${ }^{13}$ C-NMR (100 MHz, $\left.\mathrm{CDCl}_{3}\right)$ : 196.0, 151.8, 144.1, 132.8, 130.9, 130.5, 116.7, 109.6, 85.1, 81.7, 78.3, 28.2, 26.3; LRMS (ESI+, m/z): $282.1\left([\mathrm{M}+\mathrm{Na}]^{+}, 100 \%\right) ; \quad$ HRMS $(\mathrm{ESI}+, \mathrm{m} / \mathrm{z}): \mathrm{C}_{15} \mathrm{H}_{18} \mathrm{NO}_{3}\left([\mathrm{M}+\mathrm{H}]^{+}\right)$requires 260.12812, found 260.12829; IR $v_{\max }$ (film) $/ \mathrm{cm}^{-1}: 3401,3250,2979,1733,1678,1575,1517,1465,1368,1283,1236,1147$, $1047,1023$. 


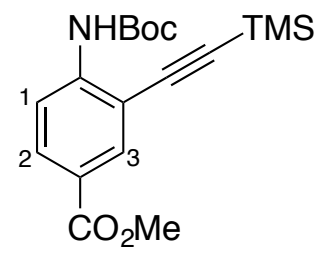

Compound 12i was prepared according to a combination of General Procedure $\mathbf{H}$ and J, with methyl-4-amino-3-iodobenzoate (2.0 g, 7.22 mmol), NaHMDS (7.94 mL, 2.0 $\mathrm{M}$ solution in THF, $15.9 \mathrm{mmol})$, $\mathrm{Boc}_{2} \mathrm{O}$ (1.73 g, $\left.7.94 \mathrm{mmol}\right)$, THF (15 mL), $\mathrm{Pd}\left(\mathrm{PPh}_{3}\right)_{2} \mathrm{Cl}_{2}(253 \mathrm{mg}, 0.36 \mathrm{mmol}), \mathrm{CuI}(69 \mathrm{mg}, 0.36 \mathrm{mmol}), \mathrm{TMS}-$ acetylene (1.54 $\mathrm{mL}, 10.8 \mathrm{mmol}), \mathrm{NEt}_{3}(1.51 \mathrm{~mL}, 10.8 \mathrm{mmol})$ and DMF $(35 \mathrm{~mL})$. Flash column chromatography $\left(\mathrm{SiO}_{2}, 5-10 \% \mathrm{Et}_{2} \mathrm{O}\right.$ in petrol) afforded aryl alkyne $\mathbf{1 2} \mathbf{i}$ as a pale yellow solid ( $2.14 \mathrm{~g}, 85 \%$ yield over 2 steps).

m.p.: 95-98 ${ }^{\circ} \mathrm{C}$ (crystalized from $\left.\mathrm{CHCl}_{3}\right) .{ }^{1} \mathbf{H}-\mathbf{N M R}\left(400 \mathrm{MHz}, \mathrm{CDCl}_{3}\right): 8.07(1 \mathrm{H}, \mathrm{d}$, $J$ 9.0, $\mathrm{C}(1) H), 7.93(1 \mathrm{H}, \mathrm{d}, J 2.0, \mathrm{C}(3) H), 7.82(1 \mathrm{H}, \mathrm{dd}, J 9.0$ and 2.0, $\mathrm{C}(2) H), 7.41$ $(1 \mathrm{H}$, br. s, $\mathrm{NH}), 3.74\left(3 \mathrm{H}, \mathrm{s}, \mathrm{CO}_{2} \mathrm{CH}_{3}\right), 1.39\left(9 \mathrm{H}, \mathrm{s}, \mathrm{C}\left(\mathrm{CH}_{3}\right)_{3}\right), 0.15\left(9 \mathrm{H}, \mathrm{s}, \mathrm{Si}\left(\mathrm{CH}_{3}\right)_{3}\right)$; ${ }^{13}$ C-NMR (100 MHz, $\left.\mathrm{CDCl}_{3}\right)$ : 166.3, 157.1, 144.1, 133.3, 131.6, 123.7, 116.5, 110.9, 103.3, 99.6, 81.5, 52.3, 28.4, 0.0; LRMS (ESI+, m/z): $370.2\left([\mathrm{M}+\mathrm{Na}]^{+}\right) ; \quad$ HRMS $(\mathrm{ESI}+, \mathrm{m} / \mathrm{z}): \mathrm{C}_{18} \mathrm{H}_{25} \mathrm{NNaO}_{4} \mathrm{Si}\left([\mathrm{M}+\mathrm{Na}]^{+}\right)$requires 370.1445 , found $370.1461 ;$ IR $v_{\max }$ (film) $/ \mathrm{cm}^{-1}: 3392,2957,2150,1740,1721,1580,1520,1466,1436,1413,1369$, $1289,1235,1151,1101,1049$.

\section{Methyl 4-((tert-butoxycarbonyl)amino)-3-ethynylbenzoate, 3i}<smiles>C#Cc1cc(C(=O)OC)ccc1NC(=O)OC</smiles>

Compound 3i was prepared according to General Procedure K with alkyne 12i (407 $\mathrm{mg}, 1.17 \mathrm{mmol}), \mathrm{KF}(68.0 \mathrm{mg}, 1.17 \mathrm{mmol})$ and $\mathrm{MeOH}(25 \mathrm{~mL})$. Flash column chromatography $\left(\mathrm{SiO}_{2}, 5-10 \% \mathrm{Et}_{2} \mathrm{O}\right.$ in petrol) afforded terminal alkyne $\mathbf{3 i}$ as a pale yellow solid (289 $\mathrm{mg}, 90 \%$ yield).

m.p.: $145-147{ }^{\circ} \mathrm{C}$ (crystalized from $\left.\mathrm{CHCl}_{3}\right) ;{ }^{1} \mathbf{H}-\mathbf{N M R}\left(400 \mathrm{MHz}, \mathrm{CDCl}_{3}\right): 8.20(1 \mathrm{H}$, $\mathrm{d}, J 9.0, \mathrm{C}(1) H), 8.05(1 \mathrm{H}, \mathrm{d}, J 2.0, \mathrm{C}(3) H), 7.93(1 \mathrm{H}, \mathrm{dd}, J 9.0$ and 2.0, $\mathrm{C}(2) H), 7.38$ $(1 \mathrm{H}$, br. s, $\mathrm{NH}), 3.82\left(3 \mathrm{H}, \mathrm{s}, \mathrm{CO}_{2} \mathrm{CH}_{3}\right), 3.46(1 \mathrm{H}, \mathrm{s}, \mathrm{CCH}), 1.47\left(9 \mathrm{H}, \mathrm{s}, \mathrm{C}\left(\mathrm{CH}_{3}\right)_{3}\right)$; ${ }^{13}$ C-NMR (100 MHz, $\mathrm{CDCl}_{3}$ ): 166.0, 151.9, 144.1, 134.0, 131.7, 123.7, 116.7, 109.6, 84.9, 81.7, 78.4, 52.1, 28.3; LRMS (ESI+, m/z): $298.1\left([\mathrm{M}+\mathrm{Na}]^{+}\right) ; \quad$ HRMS (ESI+, $\mathrm{m} / \mathrm{z}): \mathrm{C}_{15} \mathrm{H}_{17} \mathrm{NNaO}_{4}\left([\mathrm{M}+\mathrm{Na}]^{+}\right)$requires 298.1050, found 298.1046; IR $v_{\max }$ (film)/cm $\mathrm{cm}^{-1}$ : 1719, 1581, 1520, 1469, 1409, 1290, 1242, 1153, 1100, 1049. 


\section{tert-Butyl (2-iodo-4-nitro-phenyl)carbamate, 11j}<smiles>Nc1cccc([N+](=O)[O-])c1I</smiles>

Compound 11j was prepared according to General Procedure I with 2-iodo-4nitroaniline (2.00 g, $8.33 \mathrm{mmol}$ ), $\mathrm{Boc}_{2} \mathrm{O}$ (4.18 g, $19.2 \mathrm{mmol}$ ), DMAP (102 mg, 0.82 mmol), THF (55 mL), $\mathrm{K}_{2} \mathrm{CO}_{3}(3.46 \mathrm{~g}, 25.0 \mathrm{mmol})$ and $\mathrm{MeOH}(55 \mathrm{~mL})$ for 8 hours. Flash column chromatography $\left(\mathrm{SiO}_{2}, 0-10 \%\right.$ EtOAc in petrol) afforded $N$-Boc aniline $\mathbf{1 1} \mathbf{j}$ as a pale yellow solid (1.25 g, $45 \%$ yield).

m.p.: $109-111^{\circ} \mathrm{C}$ (crystalized from EtOAc/petrol); ${ }^{\mathbf{1}} \mathbf{H}-\mathbf{N M R}\left(400 \mathrm{MHz}, \mathrm{CDCl}_{3}\right)$ : $8.63(1 \mathrm{H}, \mathrm{d}, J 2.5, \mathrm{C}(3) H), 8.34(1 \mathrm{H}, \mathrm{d}, J 9.0, \mathrm{C}(1) H), 8.20(1 \mathrm{H}, \mathrm{dd}, J 9.0$ and 2.5, $\mathrm{C}(2) H), 7.19\left(1 \mathrm{H}\right.$, br. s, NH), $1.56\left(9 \mathrm{H}, \mathrm{s}, \mathrm{C}\left(\mathrm{CH}_{3}\right)_{3}\right) ;{ }^{13} \mathrm{C}-\mathbf{N M R}\left(100 \mathrm{MHz}, \mathrm{CDCl}_{3}\right)$ : 151.7, 144.7, 142.6, 134.4, 124.9, 117.7, 85.8, 82.6, 28.2; LRMS (ESI+, m/z): 387.0 $\left([\mathrm{M}+\mathrm{Na}]^{+}, \quad 100 \%\right) ; \quad$ HRMS $(\mathrm{ESI}+, \mathrm{m} / \mathrm{z}): \mathrm{C}_{11} \mathrm{H}_{13} \mathrm{IN}_{2} \mathrm{NaO}_{4}\left([\mathrm{M}+\mathrm{Na}]^{+}\right)$requires 386.98122, found 386.98120; IR $v_{\max }\left(\right.$ film) $/ \mathrm{cm}^{-1}: 3382,2979,2932,1737,1581$, 1527, 1504, 1452, 1391, 1369, 1337, 1307, 1246, 1220, 1145, 1116, 1056, 1034, 1019.

\section{tert-Butyl (4-nitro-2-((trimethylsilyl)ethynyl)phenyl)carbamate, $12 \mathrm{j}$}

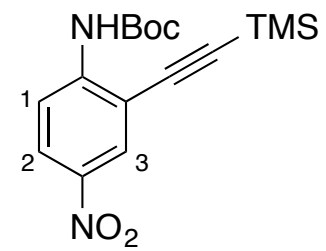

Compound 12j was prepared according to General Procedure $\mathrm{J}$ with aryl iodide $\mathbf{1 1} \mathbf{j}$ (1.00 g, $2.75 \mathrm{mmol}), \mathrm{Pd}\left(\mathrm{PPh}_{3}\right)_{2} \mathrm{Cl}_{2}(96 \mathrm{mg}, 0.14 \mathrm{mmol}), \mathrm{CuI}$ (26 mg, $\left.0.14 \mathrm{mmol}\right)$, TMS-acetylene (0.586 mL, $4.12 \mathrm{mmol}), \mathrm{NEt}_{3}(0.574 \mathrm{~mL}, 4.12 \mathrm{mmol})$ and DMF (40 $\mathrm{mL})$. Flash column chromatography $\left(\mathrm{SiO}_{2}, 0-10 \%\right.$ EtOAc in petrol) afforded aryl alkyne $\mathbf{1 2} \mathbf{j}$ as a cream solid $(0.837 \mathrm{~g}, 91 \%$ yield $)$.

m.p.: $147-149{ }^{\circ} \mathrm{C}$ (crystalized from EtOAc); ${ }^{\mathbf{1}} \mathbf{H}-\mathbf{N M R}\left(400 \mathrm{MHz}, \mathrm{CDCl}_{3}\right): 8.34(1 \mathrm{H}$, $\mathrm{d}, J 9.5, \mathrm{C}(1) H), 8.26(1 \mathrm{H}, \mathrm{d}, J 2.5, \mathrm{C}(3) H), 8.16(1 \mathrm{H}, \mathrm{dd}, J 9.5$ and $2.5, \mathrm{C}(2) H), 7.66$, $(1 \mathrm{H}$, br. s, $\mathrm{NH}), 1.55\left(9 \mathrm{H}, \mathrm{s}, \mathrm{C}\left(\mathrm{CH}_{3}\right)_{3}\right), 0.32\left(9 \mathrm{H}, \mathrm{s}, \mathrm{Si}\left(\mathrm{CH}_{3}\right)_{3}\right) ;{ }^{13} \mathbf{C}-\mathbf{N M R}(100 \mathrm{MHz}$, $\left.\mathrm{CDCl}_{3}\right): 151.9,145.8,141.9,127.3,125.7,116.8,111.6,105.4,98.2,82.4,28.5,0.0$; LRMS (ESI+, m/z): $357.1\left([\mathrm{M}+\mathrm{Na}]^{+}, 100 \%\right) ;$ HRMS (ESI+, m/z): $\mathrm{C}_{16} \mathrm{H}_{22} \mathrm{~N}_{2} \mathrm{NaO}_{4} \mathrm{Si}$ $\left([\mathrm{M}+\mathrm{Na}]^{+}\right)$requires 357.12410 , found $357.12400 ;$ IR $v_{\max }($ film $) / \mathrm{cm}^{-1}: 3381,2985$, 2959, 2150, 1745, 1739, 1581, 1536, 1513, 1478, 1458, 1421, 1394, 1369, 1343, 1319, 1290, 1252, 1234, 1188, 1151, 1088, 1052, 1027. 


\section{tert-Butyl (2-ethynyl-4-nitrophenyl)carbamate, $3 \mathbf{j}$}<smiles>[R6]Nc1ccc([N+](=O)[O-])cc1C#C</smiles>

Compound $\mathbf{3 j}$ was prepared according to General Procedure $\mathbf{K}$ with alkyne $\mathbf{1 2 \mathbf { j }}$ (460 $\mathrm{mg}, 1.38 \mathrm{mmol})$, KF (80 mg, $1.38 \mathrm{mmol})$, and $\mathrm{MeOH}(30 \mathrm{~mL})$. Flash column chromatography $\left(\mathrm{SiO}_{2}, 0-10 \%\right.$ EtOAc in petrol) afforded terminal alkyne $\mathbf{3} \mathbf{j}$ as a brown solid (318 mg, 88\% yield).

m.p.: $128-130{ }^{\circ} \mathrm{C}$ (crystalized from EtOAc/petrol); ${ }^{\mathbf{1}} \mathbf{H}-\mathbf{N M R}\left(400 \mathrm{MHz}, \mathrm{CDCl}_{3}\right)$ : $8.32(1 \mathrm{H}, \mathrm{d}, J 9.5, \mathrm{C}(1) H), 8.23(1 \mathrm{H}, \mathrm{d}, J 2.5, \mathrm{C}(3) H), 8.12(1 \mathrm{H}, \mathrm{dd}, J 9.5$ and 2.5 , $\mathrm{C}(2) H), 7.48\left(1 \mathrm{H}\right.$, br. s, NH), $3.55(1 \mathrm{H}, \mathrm{s}, \mathrm{CCH}), 1.48\left(9 \mathrm{H}, \mathrm{s}, \mathrm{C}\left(\mathrm{CH}_{3}\right)_{3}\right) ;{ }^{\mathbf{1 3}} \mathbf{C}-\mathbf{N M R}$ $\left(100 \mathrm{MHz}, \mathrm{CDCl}_{3}\right)$ : 150.6, 144.6, 140.6, 126.9, 124.7, 115.9, 109.0, 85.3, 81.4, 76.1, 27.1; LRMS (ESI+, m/z): $285.1\left([\mathrm{M}+\mathrm{Na}]^{+}, 100 \%\right) ; \quad$ HRMS (ESI+, $\left.\mathrm{m} / \mathrm{z}\right)$ : $\mathrm{C}_{13} \mathrm{H}_{14} \mathrm{~N}_{2} \mathrm{NaO}_{4}\left([\mathrm{M}+\mathrm{Na}]^{+}\right)$requires 285.08458 , found $285.08453 ;$ IR $v_{\max }($ film $) / \mathrm{cm}^{-}$ ${ }^{1}:$ 3402, 3294, 2986, 2936, 1736, 1614, 1580, 1543, 1505, 1471, 1416, 1395, 1370, $1356,1319,1253,1236,1179,1155,1089,1052,1027$. 
<smiles>N#Cc1ccc(N)c(I)c1</smiles>

Compound 11k was prepared according to General Procedure I with 4-amino-3iodobenzonitrile (2.00 g, $8.2 \mathrm{mmol})$, $\mathrm{Boc}_{2} \mathrm{O}$ (4.12 g, $\left.18.9 \mathrm{mmol}\right)$, DMAP (100 mg, $0.82 \mathrm{mmol}$ ), THF (55 mL), $\mathrm{K}_{2} \mathrm{CO}_{3}(3.40 \mathrm{~g}, 24.6 \mathrm{mmol})$ and $\mathrm{MeOH}$ (55 mL) for 16 hours. Flash column chromatography $\left(\mathrm{SiO}_{2}, 0-10 \%\right.$ EtOAc in petrol) afforded $N$-Boc aniline $11 \mathrm{k}$ as a white solid (1.73 $\mathrm{g}, 62 \%$ yield).

m.p.: $105-107{ }^{\circ} \mathrm{C}$ (crystalized from EtOAc/petrol); ${ }^{1} \mathbf{H}-\mathbf{N M R}\left(400 \mathrm{MHz}, \mathrm{CDCl}_{3}\right)$ : $8.28(1 \mathrm{H}, \mathrm{d}, J 9.0, \mathrm{C}(1) H), 8.02(1 \mathrm{H}, \mathrm{d}, J 2.0, \mathrm{C}(3) H), 7.59(1 \mathrm{H}, \mathrm{dd}, J 9.0$ and 2.0, $\mathrm{C}(2) H), 7.08(1 \mathrm{H}$, br.s, $\mathrm{NH}), 1.55\left(9 \mathrm{H}, \mathrm{s}, \mathrm{C}\left(\mathrm{CH}_{3}\right)_{3}\right)$; ${ }^{13} \mathrm{C}-\mathrm{NMR}\left(100 \mathrm{MHz}, \mathrm{CDCl}_{3}\right)$ : 151.8, 143.1, 142.2, 133.1, 118.6, 117.4, 107.3, 86.8, 82.4, 28.2; LRMS (ESI+, m/z): $367.0\left([\mathrm{M}+\mathrm{Na}]^{+}, 100 \%\right) ; \quad$ HRMS $(\mathrm{ESI}+, \mathrm{m} / \mathrm{z}): \mathrm{C}_{12} \mathrm{H}_{13} \mathrm{IN}_{2} \mathrm{NaO}_{2}\left([\mathrm{M}+\mathrm{Na}]^{+}\right)$requires 366.99139, found 366.99138; IR $v_{\max }(\mathrm{film}) / \mathrm{cm}^{-1}: 3386,2979,2933,2227,1736$, 1594, 1558, 1509, 1451, 1390, 1369, 1304, 1277, 1255, 1222, 1148, 1059, 1021.

tert-Butyl (4-cyano-2-((trimethylsilyl)ethynyl)phenyl)carbamate, 12k

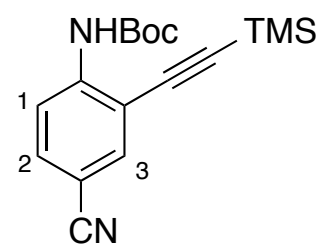

Compound 12k was prepared according to General Procedure $\mathbf{J}$ with aryl iodide $\mathbf{1 1 k}$ (1.00 g, $2.91 \mathrm{mmol}), \mathrm{Pd}\left(\mathrm{PPh}_{3}\right)_{2} \mathrm{Cl}_{2}(108 \mathrm{mg}, 0.15 \mathrm{mmol})$, CuI (29 mg, $\left.0.15 \mathrm{mmol}\right)$, TMS-acetylene $(0.620 \mathrm{~mL}, 4.36 \mathrm{mmol}), \mathrm{NEt}_{3}(0.607 \mathrm{~mL}, 4.36 \mathrm{mmol})$ and DMF (40 $\mathrm{mL})$. Flash column chromatography $\left(\mathrm{SiO}_{2}, 0-10 \%\right.$ EtOAc in petrol) afforded aryl alkyne $12 \mathbf{k}$ as a cream solid $(0.821 \mathrm{~g}, 90 \%$ yield $)$.

m.p.: $131-133{ }^{\circ} \mathrm{C}$ (crystalized from EtOAc/petrol); ${ }^{\mathbf{1}} \mathbf{H}-\mathbf{N M R}\left(400 \mathrm{MHz}, \mathrm{CDCl}_{3}\right)$ : $8.29(1 \mathrm{H}, \mathrm{d}, J 8.5, \mathrm{C}(1) H), 7.65(1 \mathrm{H}, \mathrm{d}, J 2.0, \mathrm{C}(3) H), 7.56(1 \mathrm{H}$, br. s, NH), $7.55(1 \mathrm{H}$, $\mathrm{dd}, J 8.5,2.0, \mathrm{C}(2) H), 1.54\left(9 \mathrm{H}, \mathrm{s}, \mathrm{C}\left(\mathrm{CH}_{3}\right)_{3}\right), 0.31\left(9 \mathrm{H}, \mathrm{s}, \mathrm{Si}\left(\mathrm{CH}_{3}\right)_{3}\right) ;{ }^{13} \mathbf{C}-\mathbf{N M R}(100$ $\left.\mathrm{MHz}, \mathrm{CDCl}_{3}\right)$ : 152.0, 144.1, 135.3, 133.7, 118.7, 117.5, 112.1, 105.4, 105.2, 98.2, 82.1, 28.5, 0.0; LRMS (ESI+, m/z): $337.1\left([\mathrm{M}+\mathrm{Na}]^{+}, 100 \%\right) ; \quad$ HRMS (ESI+, m/z): $\mathrm{C}_{17} \mathrm{H}_{22} \mathrm{~N}_{2} \mathrm{NaO}_{2} \mathrm{Si}\left([\mathrm{M}+\mathrm{Na}]^{+}\right)$requires 337.13428, found 337.13434; IR $v_{\max }$ (film) $/ \mathrm{cm}^{-1}:$ 3384, 2982, 2227, 2147, 1742, 1735, 1574, 1514, 1457, 1410, 1393, 1369, $1312,1278,1265,1239,1227,1151,1052,1027$. 
tert-Butyl (4-cyano-2-ethynylphenyl)carbamate, $3 \mathrm{k}$

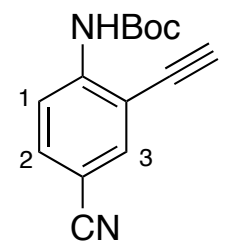

Compound 3k was prepared according to General Procedure $\mathbf{K}$ with alkyne 12k (700 $\mathrm{mg}, 2.23 \mathrm{mmol})$, KF (129 mg, $2.23 \mathrm{mmol})$, and $\mathrm{MeOH}(50 \mathrm{~mL})$. Flash column chromatography $\left(\mathrm{SiO}_{2}, 0-10 \%\right.$ EtOAc in petrol) afforded terminal alkyne $3 \mathbf{k}$ as a cream solid (510 $\mathrm{mg}, 95 \%$ yield).

m.p.: $120-122{ }^{\circ} \mathrm{C}$ (crystalized from EtOAc/petrol); ${ }^{\mathbf{1}} \mathbf{H}-\mathbf{N M R}\left(400 \mathrm{MHz}, \mathrm{CDCl}_{3}\right)$ : $8.34(1 \mathrm{H}, \mathrm{d}, J 9.0, \mathrm{C}(1) H), 7.69(1 \mathrm{H}, \mathrm{d}, J 2.0, \mathrm{C}(3) H), 7.59(1 \mathrm{H}, \mathrm{dd}, J 9.0$ and 2.0 , $\mathrm{C}(2) H), 7.46\left(1 \mathrm{H}\right.$, br. s, NH), $3.62(1 \mathrm{H}, \mathrm{s}, \mathrm{CCH}), 1.55\left(9 \mathrm{H}, \mathrm{s}, \mathrm{C}\left(\mathrm{CH}_{3}\right)_{3}\right) ;{ }^{13} \mathbf{C}-\mathbf{N M R}$ $\left(100 \mathrm{MHz}, \mathrm{CDCl}_{3}\right): 151.6,144.0,136.0,133.7,118.2,117.6,110.5,105.3,86.3,82.1$, 77.1, 28.2; LRMS (ESI+, m/z): $265.1\left([\mathrm{M}+\mathrm{Na}]^{+}, 100 \%\right) ; \quad$ HRMS $(\mathrm{ESI}+, \mathrm{m} / \mathrm{z})$ : $\mathrm{C}_{14} \mathrm{H}_{14} \mathrm{~N}_{2} \mathrm{NaO}_{2}\left([\mathrm{M}+\mathrm{Na}]^{+}\right)$requires 265.09475 , found $265.09461 ;$ IR $v_{\max }($ film $) / \mathrm{cm}^{-}$ 1: 3401, 3257, 2980, 2935, 2229, 1734, 1605, 1575, 1512, 1461, 1406, 1394, 1368, 1320, 1280, 1235, 1148, 1049, 1025. 
<smiles>Cc1ccc(N)c(Br)c1</smiles>

Compound 111 was prepared according to General Procedure $\mathbf{H}$ with 2-iodo-5methylaniline (500 mg, $2.15 \mathrm{mmol})$, NaHMDS (2.15 mL, 2.0 M solution in THF, 4.25 $\mathrm{mmol})$, Boc $\mathrm{O}(562 \mathrm{mg}, 2.57 \mathrm{mmol})$ and $\mathrm{THF}(14 \mathrm{~mL})$. Flash column chromatography $\left(\mathrm{SiO}_{2}, 25 \% \mathrm{CH}_{2} \mathrm{Cl}_{2}\right.$ in petrol) afforded $N$-Boc aniline $\mathbf{1 1 l}$ as a yellow oil (365 mg, 51\% yield).

${ }^{1}$ H-NMR (400 MHz, $\left.\mathrm{CDCl}_{3}\right): 7.84(1 \mathrm{H}, \mathrm{d}, J$ 1.0, C(1)H), $7.52(1 \mathrm{H}, \mathrm{d}, J$ 8.0, $\mathrm{C}(3) H), 6.72(1 \mathrm{H}$, br. s, $\mathrm{N} H), 6.54(1 \mathrm{H}$, ddd, $J 8.0,2.0$ and $0.5, \mathrm{C}(2) H), 2.24(3 \mathrm{H}$, $\left.\mathrm{s}, \mathrm{ArCCH}_{3}\right), 1.47\left(9 \mathrm{H}, \mathrm{s}, \mathrm{C}\left(\mathrm{CH}_{3}\right)_{3}\right) ;{ }^{13} \mathrm{C}-\mathbf{N M R}\left(100 \mathrm{MHz}, \mathrm{CDCl}_{3}\right): 152.7,139.5$, 138.5, 138.4, 125.8, 120.7, 84.7, 81.0, 28.3, 21.3; LRMS (ESI+, m/z): 356.0 $\left([\mathrm{M}+\mathrm{Na}]^{+}\right)$.

Data is consistent with the literature. ${ }^{6}$

tert-Butyl (5-methyl-2-((trimethylsilyl)ethynyl)phenyl)carbamate, 12 l<smiles>Cc1ccc(C#CC(C)(C)C)c(NC(C)(C)C)c1</smiles>

Compound 12l was prepared according to General Procedure $\mathbf{J}$ with aryl iodide 111 (435 mg, $1.04 \mathrm{mmol}), \mathrm{Pd}\left(\mathrm{PPh}_{3}\right)_{2} \mathrm{Cl}_{2}$ (36.3 mg, $\left.0.05 \mathrm{mmol}\right), \mathrm{CuI}(9.9 \mathrm{mg}, 0.05 \mathrm{mmol})$, TMS-acetylene (0.22 mL, $1.55 \mathrm{mmol}), \mathrm{NEt}_{3}(0.22 \mathrm{~mL}, 1.55 \mathrm{mmol})$ and DMF (14 mL). Flash column chromatography $\left(\mathrm{SiO}_{2}, 2-3 \% \mathrm{Et}_{2} \mathrm{O}\right.$ in petrol) afforded aryl alkyne $\mathbf{1 2 l}$ as an orange oil (158 $\mathrm{mg}, 50 \%$ yield).

${ }^{1}$ H-NMR (400 MHz, $\left.\mathrm{CDCl}_{3}\right): 7.83(1 \mathrm{H}$, br. s, C(1)H), $7.22(1 \mathrm{H}$, br. s, NH), 7.12 $(1 \mathrm{H}, \mathrm{d}, J 8.5, \mathrm{C}(3) H), 6.62(1 \mathrm{H}, \mathrm{ddd}, J 8.0,1.5$ and $0.5, \mathrm{C}(2) H), 2.21(3 \mathrm{H}, \mathrm{s}$, $\left.(\mathrm{Ar}) \mathrm{CCH}_{3}\right), 1.40\left(9 \mathrm{H}, \mathrm{s}, \mathrm{C}\left(\mathrm{CH}_{3}\right)_{3}\right), 0.15\left(9 \mathrm{H}, \mathrm{s}, \mathrm{Si}\left(\mathrm{CH}_{3}\right)_{3}\right) ;{ }^{13} \mathrm{C}-\mathrm{NMR}(100 \mathrm{MHz}$, $\left.\mathrm{CDCl}_{3}\right): 152.3,140.6,140.2,131.1,122.9,117.8,108.2,101.3,100.9,80.6,28.4$, 22.1, 0.0; LRMS (ESI+, m/z): $326.2\left([\mathrm{M}+\mathrm{Na}]^{+}\right)$.

Data is consistent with the literature. ${ }^{6}$ 


\section{tert-Butyl (2-ethynyl-5-methylphenyl)carbamate, 31}<smiles>C#Cc1ccc(C)cc1NC(=O)OCc1ccccc1</smiles>

Compound 31 was prepared according to General Procedure K with alkyne 12I (136 $\mathrm{mg}, 0.45 \mathrm{mmol}), \mathrm{K}_{2} \mathrm{CO}_{3}(61.8 \mathrm{mg}, 0.45 \mathrm{mmol})$ and $\mathrm{MeOH}(9.0 \mathrm{~mL})$. Flash column chromatography $\left(\mathrm{SiO}_{2}, 5 \% \mathrm{Et}_{2} \mathrm{O}\right.$ in petrol) afforded terminal alkyne $3 \mathrm{I}$ as an orange solid (104 mg, 99\% yield).

m.p.: $48-51{ }^{\circ} \mathrm{C}$ (crystalized from $\left.\mathrm{CHCl}_{3}\right) ;{ }^{1} \mathbf{H}-\mathbf{N M R}\left(400 \mathrm{MHz}, \mathrm{CDCl}_{3}\right)$ : $7.87(1 \mathrm{H}$, br. s, C(1)H), $7.16(1 \mathrm{H}, \mathrm{d}, J 8.0, \mathrm{C}(3) H), 7.10(1 \mathrm{H}$, br. s, NH), $6.64(1 \mathrm{H}, \mathrm{d}, J 8.0$, $\mathrm{C}(2) H), 3.30(1 \mathrm{H}, \mathrm{s}, \mathrm{CCH}), 2.21\left(3 \mathrm{H}, \mathrm{s}, \mathrm{ArCCH}_{3}\right), 1.39\left(9 \mathrm{H}, \mathrm{s}, \mathrm{C}\left(\mathrm{CH}_{3}\right)_{3}\right) ;{ }^{13} \mathrm{C}-$ NMR (100 MHz, $\left.\mathrm{CDCl}_{3}\right)$ : 152.5, 140.8, 140.2, 132.0, 123.0, 118.1, 107.0, 83.4, 80.8, 79.6, 29.7, 22.0; LRMS (ESI+, m/z): $254.1\left([\mathrm{M}+\mathrm{Na}]^{+}\right)$.

Data is consistent with the literature. ${ }^{6}$ 
tert-Butyl [4-(tert-butoxycarbonylamino)-2-iodo-phenyl]carbamate, 11m<smiles>CC(C)(C)OC(=O)Nc1cccc(N)c1I</smiles>

Compound $11 \mathrm{~m}$ was prepared according to General Procedure I with 2-iodo-1,4benzenediamine (2.00 g, $8.54 \mathrm{mmol}), \mathrm{Boc}_{2} \mathrm{O}(8.6 \mathrm{~g}, 39.3 \mathrm{mmol})$, DMAP (209 mg, $1.71 \mathrm{mmol})$, THF $(55 \mathrm{~mL}), \mathrm{K}_{2} \mathrm{CO}_{3}(4.70 \mathrm{~g}, 34.2 \mathrm{mmol})$ and $\mathrm{MeOH}(55 \mathrm{~mL})$ for 16 hours. Flash column chromatography $\left(\mathrm{SiO}_{2}, 0-30 \%\right.$ EtOAc in petrol) afforded $N$-Boc aniline $11 \mathrm{~m}$ as a pale pink solid (1.89 $\mathrm{g}, 51 \%$ yield).

m.p.: $161-163{ }^{\circ} \mathrm{C}$ (crystalized from EtOAc/petrol); ${ }^{1} \mathbf{H}-\mathbf{N M R}\left(400 \mathrm{MHz}, \mathrm{CDCl}_{3}\right)$ : $7.99(1 \mathrm{H}$, br. s, C(3)H), $7.87(1 \mathrm{H}, \mathrm{d}, J 9.0, \mathrm{C}(1) H), 7.14(1 \mathrm{H}, \mathrm{dd}, J 9.0$ and 2.5, $\mathrm{C}(2) H), 6.67(1 \mathrm{H}$, br. s, $\mathrm{NH}), 6.45(1 \mathrm{H}$, br. s, $\mathrm{NH}), 1.52\left(9 \mathrm{H}, \mathrm{s}, \mathrm{C}\left(\mathrm{CH}_{3}\right)_{3}\right), 1.51(9 \mathrm{H}, \mathrm{s}$, $\left.\mathrm{C}\left(\mathrm{CH}_{3}\right)_{3}\right) ;{ }^{13} \mathrm{C}-\mathrm{NMR}\left(100 \mathrm{MHz}, \mathrm{CDCl}_{3}\right): 152.8,152.6,134.7,134.3,128.5,120.6$, 119.4, 89.4, 80.9, 80.8, $28.3(2 \times \mathrm{C})$; LRMS $(\mathrm{ESI}+, \mathrm{m} / \mathrm{z}): 457.1\left([\mathrm{M}+\mathrm{Na}]^{+}, 100 \%\right)$; HRMS (ESI+, m/z): $\quad \mathrm{C}_{16} \mathrm{H}_{23} \mathrm{IN}_{2} \mathrm{NaO}_{4}\left([\mathrm{M}+\mathrm{Na}]^{+}\right)$requires 457.05947, found 457.05921; IR $v_{\max }\left(\right.$ film) $/ \mathrm{cm}^{-1}: 3328,2978,2932,1697,1586,1511,1477,1456$, 1389, 1293, 1237, 1148, 1062, 1021.

\section{Di-tert-butyl (2-((trimethylsilyl)ethynyl)-1,4-phenylene)dicarbamate, $12 \mathrm{~m}$}

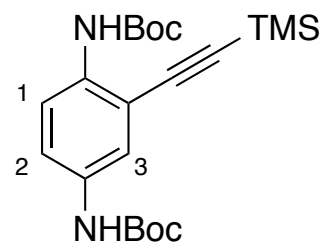

Compound $\mathbf{1 2} \mathbf{m}$ was prepared according to General Procedure $\mathbf{J}$ with aryl iodide $\mathbf{1 1} \mathbf{m}$ (1.50 g, $3.45 \mathrm{mmol}), \mathrm{Pd}\left(\mathrm{PPh}_{3}\right)_{2} \mathrm{Cl}_{2}(121 \mathrm{mg}, 0.17 \mathrm{mmol}), \mathrm{CuI}(33 \mathrm{mg}, 0.17 \mathrm{mmol})$, TMS-acetylene $(0.736 \mathrm{~mL}, 5.18 \mathrm{mmol}), \mathrm{NEt}_{3}(0.722 \mathrm{~mL}, 5.18 \mathrm{mmol})$ and DMF (50 $\mathrm{mL})$. Flash column chromatography $\left(\mathrm{SiO}_{2}, 0-10 \%\right.$ EtOAc in petrol) afforded aryl alkyne $\mathbf{1 2 m}$ as a pale brown solid (1.35 g, 97\% yield).

m.p.: $153-155{ }^{\circ} \mathrm{C}$ (crystalized from EtOAc/petrol); ${ }^{1} \mathbf{H}-\mathbf{N M R}\left(400 \mathrm{MHz}, \mathrm{CDCl}_{3}\right)$ : $8.00(1 \mathrm{H}, \mathrm{d}, J 9.0, \mathrm{C}(1) H), 7.60(1 \mathrm{H}$, br. s, NH), $7.24(1 \mathrm{H}$, br. s, C $(3) H), 7.12(1 \mathrm{H}, \mathrm{dd}$, $J 9.0$ and 2.5, $\mathrm{C}(2) H), 6.41(1 \mathrm{H}$, br. s, $\mathrm{NH}), 1.52\left(9 \mathrm{H}, \mathrm{s}, \mathrm{C}\left(\mathrm{CH}_{3}\right)_{3}\right), 1.50(9 \mathrm{H}, \mathrm{s}$, $\left(\mathrm{C}\left(\mathrm{CH}_{3}\right)_{3}\right), 0.27\left(9 \mathrm{H}, \mathrm{s}, \mathrm{Si}\left(\mathrm{CH}_{3}\right)_{3}\right) ;{ }^{13} \mathrm{C}-\mathbf{N M R}\left(100 \mathrm{MHz}, \mathrm{CDCl}_{3}\right): 152.8,152.4,135.8$, $132.5,121.3,120.3,117.9,111.6,102.0,100.2,80.56,80.48,28.33,28.28,-0.2$; LRMS (ESI+, m/z): $427.1\left([\mathrm{M}+\mathrm{Na}]^{+}, 100 \%\right) ;$ HRMS (ESI+, m/z): $\mathrm{C}_{21} \mathrm{H}_{32} \mathrm{~N}_{2} \mathrm{NaO}_{4} \mathrm{Si}$ $\left([\mathrm{M}+\mathrm{Na}]^{+}\right)$requires 427.20236, found 427.20236; IR $v_{\max }($ film $) / \mathrm{cm}^{-1}: 34.00,3338$, 2978, 2933, 2145, 1731, 1709, 1617, 1593, 1533, 1517, 1476, 1476, 1457, 1411, 1393, 1368, 1300, 1276, 1240, 1153, 1056, 1029. 


\section{Di-tert-butyl (2-ethynyl-1,4-phenylene)dicarbamate, 3m}<smiles>C#Cc1cc(NC(=O)OCc2ccccc2)ccc1NC(=O)OCc1ccccc1</smiles>

Compound 3m was prepared according to General Procedure $\mathbf{K}$ with alkyne $12 \mathbf{m}$ (1.00 g, $2.47 \mathrm{mmol}), \mathrm{K}_{2} \mathrm{CO}_{3}(342 \mathrm{mg}, 2.47 \mathrm{mmol})$, and $\mathrm{MeOH}(50 \mathrm{~mL})$. Flash column chromatography $\left(\mathrm{SiO}_{2}, 0-30 \%\right.$ EtOAc in petrol) afforded terminal alkyne $\mathbf{3 m}$ as an off white solid (759 $\mathrm{mg}, 92 \%$ yield).

m.p.: $163-165{ }^{\circ} \mathrm{C}$ (crystalized from EtOAc/petrol); ${ }^{1} \mathbf{H}-\mathbf{N M R}\left(400 \mathrm{MHz}, \mathrm{CDCl}_{3}\right)$ : $7.96(1 \mathrm{H}, \mathrm{d}, J 9.0, \mathrm{C}(1) H), 7.53(1 \mathrm{H}$, br. s, C(3)H), $7.12(1 \mathrm{H}, \mathrm{dd}, J 9.0$ and 2.5, $\mathrm{C}(2) H), 7.07(1 \mathrm{H}$, br. s, NH), $6.51(1 \mathrm{H}$, br. s, NH), $3.37(1 \mathrm{H}, \mathrm{s}, \mathrm{CC} H), 1.45(9 \mathrm{H}, \mathrm{s}$, $\left.\mathrm{C}\left(\mathrm{CH}_{3}\right)_{3}\right), 1.43\left(9 \mathrm{H}, \mathrm{s}, \mathrm{C}\left(\mathrm{CH}_{3}\right)_{3}\right) ;{ }^{13} \mathbf{C}-\mathbf{N M R}\left(100 \mathrm{MHz}, \mathrm{CDCl}_{3}\right): 151.8,151.5,134.8$, 131.7, 121.2, 119.7, 117.4, 109.5, 83.0, 79.8, 79.6, 78.1, $27.3(2 \times \mathrm{C})$; LRMS (ESI+, $\mathrm{m} / \mathrm{z}): 355.2\left([\mathrm{M}+\mathrm{Na}]^{+}, 100 \%\right) ; \quad$ HRMS $(\mathrm{ESI}+, \mathrm{m} / \mathrm{z}): \mathrm{C}_{18} \mathrm{H}_{24} \mathrm{~N}_{2} \mathrm{NaO}_{4}\left([\mathrm{M}+\mathrm{Na}]^{+}\right)$ requires 355.16283, found 355.16289; IR $v_{\max }\left(\right.$ film) $/ \mathrm{cm}^{-1}: 3409,3299,2979,2933$, 1704, 1617, 1593, 1516, 1477, 1407, 1394, 1368, 1297, 1275, 1241, 1151, 1057, 1028. 


\section{tert-Butyl (2-hydroxy-6-iodo-phenyl)carbamate, 11n}<smiles>O=C(O)Nc1c(O)cccc1I</smiles>

To a solution of 2-amino-3-iodophenol $(500 \mathrm{mg}, 2.13 \mathrm{mmol})$ and $\mathrm{NEt}_{3}(0.891 \mathrm{~mL}$, $6.39 \mathrm{mmol})$ in THF $(6 \mathrm{~mL})$, was added $\mathrm{Boc}_{2} \mathrm{O}(465 \mathrm{mg}, 2.13 \mathrm{mmol})$. The resulting solution was heated at reflux for 16 hours. Once cooled to $25{ }^{\circ} \mathrm{C}$ the solvent was removed in vacuo, and the resulting residue partitioned between $1.0 \mathrm{~N} \mathrm{HCl}_{(\mathrm{aq})}$ and $\mathrm{Et}_{2} \mathrm{O}$. The organic layer was then washed with brine, dried over $\mathrm{MgSO}_{4}$ and concentrated in vacuo. Flash column chromatography $\left(\mathrm{SiO}_{2}, 0-25 \%\right.$ EtOAc in petrol) afforded $N$-Boc aniline 11n as an orange oil (532 mg, 75\% yield).

${ }^{1}$ H-NMR (400 MHz, $\left.\mathrm{CDCl}_{3}\right): 8.67(1 \mathrm{H}$, br. s, OH), $7.36(1 \mathrm{H}, \mathrm{d}, J$ 8.0, C(3)H), 6.99 $(1 \mathrm{H}, \mathrm{d}, J 8.0, \mathrm{C}(1) H), 6.80(1 \mathrm{H}, \mathrm{t}, J 8.0, \mathrm{C}(2) H), 6.68(1 \mathrm{H}$, br. s, NH), $1.54(9 \mathrm{H}, \mathrm{s}$, $\left.\mathrm{C}\left(\mathrm{CH}_{3}\right)_{3}\right) ;{ }^{13} \mathrm{C}-\mathrm{NMR}\left(100 \mathrm{MHz}, \mathrm{CDCl}_{3}\right): 155.6,149.6,131.1,128.1,126.8,120.6$, 93.4, 83.1, 28.2; LRMS (ESI+, m/z): 358.0 ([M+Na $\left.]^{+}, 100 \%\right) ; H R M S(E S I+, ~ m / z):$ $\mathrm{C}_{11} \mathrm{H}_{14} \mathrm{INNaO}_{3}\left([\mathrm{M}+\mathrm{Na}]^{+}\right)$requires 357.99106, found 357.99094; IR $v_{\max }\left(\right.$ film) $/ \mathrm{cm}^{-}$ ${ }^{1}: 3371,2979,2932,1684,1571,1510,1463,1392,1367,1291,1249,1152,1077$, $1052,1026$.

tert-Butyl (2-hydroxy-6-((trimethylsilyl)ethynyl)phenyl)carbamate, 12n

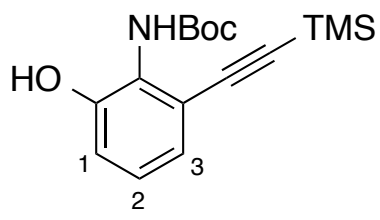

Compound 12n was prepared according to General Procedure $\mathbf{J}$ with aryl iodide $11 \mathbf{n}$ (443 mg, $1.32 \mathrm{mmol}$ ), $\mathrm{Pd}\left(\mathrm{PPh}_{3}\right)_{2} \mathrm{Cl}_{2}$ (46 mg, $0.07 \mathrm{mmol}$ ), CuI (13 mg, $0.07 \mathrm{mmol}$ ), TMS-acetylene $(0.280 \mathrm{~mL}, 1.98 \mathrm{mmol}), \mathrm{NEt}_{3}(0.276 \mathrm{~mL}, 1.98 \mathrm{mmol})$ and DMF (19 $\mathrm{mL})$. Flash column chromatography $\left(\mathrm{SiO}_{2}, 0-20 \%\right.$ EtOAc in petrol) afforded aryl alkyne $12 \mathrm{n}$ as a brown oil (340 $\mathrm{mg}, 84 \%$ yield).

${ }^{1}$ H-NMR $\left(400 \mathrm{MHz}, \mathrm{CDCl}_{3}\right): 9.50(1 \mathrm{H}$, br. s, OH), $7.30(1 \mathrm{H}$, br. s, NH), $6.98(3 \mathrm{H}, \mathrm{s}$, $\mathrm{ArH}), 1.54\left(9 \mathrm{H}, \mathrm{s}, \mathrm{C}\left(\mathrm{CH}_{3}\right)_{3}\right), 0.29\left(9 \mathrm{H}, \mathrm{s}, \mathrm{Si}\left(\mathrm{CH}_{3}\right)_{3}\right) ;{ }^{13} \mathrm{C}-\mathrm{NMR}\left(100 \mathrm{MHz}, \mathrm{CDCl}_{3}\right)$ : $155.9,148.1,127.3,125.5,123.5,121.2,115.2,102.2,100.8,83.0,28.3,0.0$; LRMS $(\mathrm{ESI}+, \mathrm{m} / \mathrm{z}):\left([\mathrm{M}+\mathrm{Na}]^{+}, 100 \%\right) \quad 328.1 ; \quad$ HRMS $(\mathrm{ESI}+, \mathrm{m} / \mathrm{z}): \mathrm{C}_{16} \mathrm{H}_{23} \mathrm{NNaO}_{3} \mathrm{Si}$ $\left([\mathrm{M}+\mathrm{Na}]^{+}\right)$requires 328.13394 , found 328.13399 ; IR $v_{\max }($ film $) / \mathrm{cm}^{-1}: 3376,3119$, 2979, 2148, 1690, 1601, 1524, 1474, 1394, 1370, 1305, 1250, 1207, 1153, 1056, 1005. 


\section{tert-Butyl (2-ethynyl-6-hydroxyphenyl)carbamate, 3n}

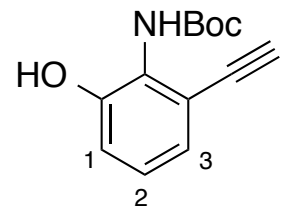

Compound 3n was prepared according to General Procedure K with alkyne 12n (297 $\mathrm{mg}, 0.97 \mathrm{mmol})$, KF (56.0 mg, $0.97 \mathrm{mmol})$, and $\mathrm{MeOH}(30 \mathrm{~mL})$. Flash column chromatography $\left(\mathrm{SiO}_{2}, 0-20 \%\right.$ EtOAc in petrol) afforded terminal alkyne $\mathbf{3 n}$ as an off white solid (190 mg, 84\% yield).

m.p.: $136-138{ }^{\circ} \mathrm{C}$ (crystalized from $\left.\mathrm{H}_{2} \mathrm{O} / \mathrm{MeCN}\right)$; ${ }^{1} \mathbf{H}-\mathbf{N M R}\left(400 \mathrm{MHz}, \mathrm{CDCl}_{3}\right.$ ): 9.35 $(1 \mathrm{H}$, br. s, $\mathrm{OH}), 7.20(1 \mathrm{H}$, br. s, $\mathrm{NH}), 7.06-7.00(3 \mathrm{H}, \mathrm{m}, 3 \times \mathrm{ArH}), 3.45(1 \mathrm{H}, \mathrm{s}, \mathrm{CCH})$, $1.54\left(9 \mathrm{H}, \mathrm{s}, \mathrm{C}\left(\mathrm{CH}_{3}\right)_{3}\right) ;{ }^{13} \mathrm{C}-\mathrm{NMR}\left(100 \mathrm{MHz}, \mathrm{CDCl}_{3}\right): 155.7,148.3,127.1,125.6$, 124.5, 121.5, 114.2, 84.0, 83.1, 79.5, 28.2; LRMS (ESI+, m/z): $256.1\left([\mathrm{M}+\mathrm{Na}]^{+}\right.$, $100 \%) ; \quad H R M S(E S I+, m / z): \mathrm{C}_{13} \mathrm{H}_{15} \mathrm{NNaO}_{3}\left([\mathrm{M}+\mathrm{Na}]^{+}\right)$requires 256.09441, found 256.09427; IR $v_{\max }\left(\right.$ film) $/ \mathrm{cm}^{-1}: 3319,3260,2976,2931,2855,1674,1598,1533$, 1464, 1393, 1369, 1303, 1275, 1254, 1212, 1156, 1087, 1052, 1031. 
<smiles>C#Cc1ccc(OC)cc1NC(=O)OC</smiles>

Compound 3o was prepared according to a combination of General Procedure $\mathbf{H}, \mathbf{J}$ and $\mathbf{K}$, involving a $\mathrm{SiO}_{2}$ filtration between $\mathbf{J}$ and $\mathbf{K}$, with 2-iodo-4-methoxyaniline ( $2.0 \mathrm{~g}, 8.03 \mathrm{mmol})$, NaHMDS (8.83 mL, 2.0 M solution in THF, $17.7 \mathrm{mmol}$ ), $\mathrm{Boc}_{2} \mathrm{O}$ (1.93 g, $8.83 \mathrm{mmol})$, THF (16 mL), $\mathrm{Pd}\left(\mathrm{PPh}_{3}\right)_{2} \mathrm{Cl}_{2}$ (281 mg, $\left.0.40 \mathrm{mmol}\right), \mathrm{CuI}(76.2 \mathrm{mg}$, $0.40 \mathrm{mmol})$, TMS-acetylene (1.71 mL, $12.1 \mathrm{mmol}^{2}, \mathrm{NEt}_{3}(1.68 \mathrm{~mL}, 12.1 \mathrm{mmol})$ and DMF $(40 \mathrm{~mL}), \mathrm{K}_{2} \mathrm{CO}_{3}(1.11 \mathrm{~g}, 8.03 \mathrm{mmol})$ and $\mathrm{MeOH}(160 \mathrm{~mL})$. Flash column chromatography $\left(\mathrm{SiO}_{2}, 0-10 \% \mathrm{Et}_{2} \mathrm{O}\right.$ in petrol) afforded terminal alkyne $\mathbf{3 0}$ as a brown solid (1.43 g, $72 \%$ yield over 3 steps).

m.p.: $57-59{ }^{\circ} \mathrm{C}$ (crystalized from $\mathrm{Et}_{2} \mathrm{O} /$ petrol); ${ }^{\mathbf{1}} \mathbf{H}-\mathbf{N M R}\left(400 \mathrm{MHz}, \mathrm{CDCl}_{3}\right.$ ): 7.85 $(1 \mathrm{H}, \mathrm{d}, J 2.5, \mathrm{C}(1) H), 7.35(1 \mathrm{H}, \mathrm{d}, J 8.5, \mathrm{C}(3) H), 7.31(1 \mathrm{H}$, br. s, NH), $6.54(1 \mathrm{H}, \mathrm{dd}, J$ 8.5 and 2.5, $\mathrm{C}(2) H), 3.86\left(3 \mathrm{H}, \mathrm{s}, \mathrm{OCH}_{3}\right), 3.45(1 \mathrm{H}, \mathrm{s}, \mathrm{CCH}), 1.56\left(9 \mathrm{H}, \mathrm{s}, \mathrm{C}\left(\mathrm{CH}_{3}\right)_{3}\right)$; ${ }^{13}$ C-NMR (100 MHz, $\left.\mathrm{CDCl}_{3}\right)$ : 161.1, 152.3, 141.8, 133.1, 109.1, 102.4, 101.8, 82.9, 80.9, 79.5, 55.4, 28.3; LRMS (ESI+, m/z): $270.1\left([\mathrm{M}+\mathrm{Na}]^{+}, 100 \%\right) ;$ HRMS (ESI+, $\mathrm{m} / \mathrm{z}): \mathrm{C}_{14} \mathrm{H}_{17} \mathrm{NNaO}_{3}\left([\mathrm{M}+\mathrm{Na}]^{+}\right)$requires 270.11006 , found 270.11003 ; IR $v_{\max }$ (film) $/ \mathrm{cm}^{-1}$ : 3406, 3283, 3126, 2979, 2936, 2837, 2099, 1731, 1613, 1579, 1523, 1469, 1435, 1426, 1393, 1368, 1309, 1288, 1256, 1233, 1212, 1155, 1108, 1049, 1036.

Data is consistent with the literature. ${ }^{6}$ 


\section{3,6-Dibromobenzene-1,2-diamine, 14}<smiles>Nc1c(Br)ccc(Br)c1N</smiles>

Under a $\mathrm{N}_{2(\mathrm{~g})}$ atmosphere at $0{ }^{\circ} \mathrm{C}$, to a solution of 4,7-dibromo-2,1,3-benzothiadiazole $(5.0 \mathrm{~g}, 17.0 \mathrm{mmol})$ in $\mathrm{EtOH}(600 \mathrm{~mL})$ was added portionwise sodium borohydride (11.6 g, $306 \mathrm{mmol})$. The reaction was then stirred at $0{ }^{\circ} \mathrm{C}$ for 10 minutes, before allowing to warm to room temperature and stir for 16 hours. The reaction was then cooled to $0{ }^{\circ} \mathrm{C}$, and quenched with $\mathrm{H}_{2} \mathrm{O}(150 \mathrm{~mL})$, and then concentrated in vacuo to remove the $\mathrm{EtOH}$. The resulting aqueous solution was extracted with $\mathrm{Et}_{2} \mathrm{O}(2 \times 100$ $\mathrm{mL})$, then the combined organic layers were washed with brine $(100 \mathrm{~mL})$, dried over $\mathrm{MgSO}_{4}$ and concentrated in vacuo to afford the crude di-aniline $\mathbf{1 4}$ as a cream solid (3.94 g, $87 \%$ yield). No further purification was necessary.

m.p.: $98-100{ }^{\circ} \mathrm{C}$ (crystalized from EtOAc/petrol); ${ }^{1} \mathbf{H}-\mathbf{N M R}\left(400 \mathrm{MHz}, \mathrm{CDCl}_{3}\right): 6.84$ $(2 \mathrm{H}, \mathrm{s}, \mathrm{ArH}), 3.71$ (4H, br. s, $\left.\mathrm{NH}_{2}\right) ;{ }^{13} \mathbf{C}-\mathbf{N M R}\left(100 \mathrm{MHz}, \mathrm{CDCl}_{3}\right): 133.7,123.3$, 109.7; LRMS (ESI+, $\quad \mathrm{m} / \mathrm{z}): \quad 265.0 \quad\left(\left[\mathrm{M}\left({ }^{79} \mathrm{Br}^{79} \mathrm{Br}\right)+\mathrm{H}\right]^{+}, \quad 50 \%\right), \quad 267.0$ $\left(\left[\mathrm{M}\left({ }^{79} \mathrm{Br}{ }^{81} \mathrm{Br}\right)+\mathrm{H}\right]^{+}, 100 \%\right), 269.0\left(\left[\mathrm{M}\left({ }^{81} \mathrm{Br}{ }^{81} \mathrm{Br}\right)+\mathrm{H}\right]^{+}, 50 \%\right)$.

Data is consistent with the literature. ${ }^{8}$ 


\section{Di-tert-butyl (3,6-bis((trimethylsilyl)ethynyl)-1,2-phenylene)dicarbamate, 12p}

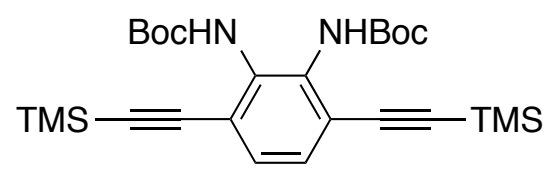

A flask containing compound 14 (4.04 g, $15.2 \mathrm{mmol}), \mathrm{CuI}(290 \mathrm{mg}, 1.52 \mathrm{mmol})$ and NaI (9.1 g, $60.8 \mathrm{mmol})$ was evacuated and backfilled with $\mathrm{N}_{2(\mathrm{~g})}$ three times. Then was added sequentially DMEDA $(0.327 \mathrm{~mL}, 3.04 \mathrm{mmol})$ and 1,4-dioxane $(19 \mathrm{~mL})$. The reaction was then heated at $100{ }^{\circ} \mathrm{C}$ for 40 hours. Once cooled to room temperature, the mixture was partitioned between $\mathrm{Et}_{2} \mathrm{O}(100 \mathrm{~mL})$ and sat. $\mathrm{NH}_{4} \mathrm{Cl}_{(\mathrm{aq})}$ $(50 \mathrm{~mL})$. The organic layers were then washed with brine $(50 \mathrm{~mL})$, dried over $\mathrm{MgSO}_{4}$, and concentrated in vacuo to afford a mixture of the di-iodo compound and a bromo-iodo compound. The inseparable crude mixture was then taken on to the Boc protection step according to General Procedure $\mathbf{H}$ with NaHMDS $(33.4 \mathrm{~mL}, 2.0 \mathrm{M}$ solution in THF, $66.8 \mathrm{mmol}), \mathrm{Boc}_{2} \mathrm{O}(7.30 \mathrm{~g}, 33.4 \mathrm{mmol})$ and THF $(30 \mathrm{~mL})$. Flash column chromatography $\left(\mathrm{SiO}_{2}, 5-10 \% \mathrm{Et}_{2} \mathrm{O}\right.$ in petrol) afforded $4.92 \mathrm{~g}$ of a mixture of the di-iodo and bromo-iodo compounds, which were taken on to the sonagashira step, as described by General Procedure $\mathbf{J}$ with $\mathrm{Pd}\left(\mathrm{PPh}_{3}\right)_{2} \mathrm{Cl}_{2}$ (625 mg, $\left.0.89 \mathrm{mmol}\right)$, CuI (170 mg, $0.89 \mathrm{mmol}$ ), TMS-acetylene (3.75 mL, $26.3 \mathrm{mmol}^{\text {), NEt }} \mathrm{N}_{3}(3.67 \mathrm{~mL}, 26.3$ $\mathrm{mmol})$ and DMF $(30 \mathrm{~mL})$. Flash column chromatography $\left(\mathrm{SiO}_{2}, 5-10 \% \mathrm{Et}_{2} \mathrm{O}\right.$ in petrol) afforded dialkyne 12p as a brown oil (3.46 g, $42 \%$ yield over 3 steps).

${ }^{1}$ H-NMR $\left(400 \mathrm{MHz}, \mathrm{CDCl}_{3}\right): 7.28(2 \mathrm{H}, \mathrm{s}, \mathrm{ArH}), 7.12(2 \mathrm{H}, \mathrm{br} . \mathrm{s}, \mathrm{NH}), 1.51(18 \mathrm{H}, \mathrm{s}$, $\left.\mathrm{C}\left(\mathrm{CH}_{3}\right)_{3}\right), 0.27\left(18 \mathrm{H}, \mathrm{s}, \mathrm{Si}\left(\mathrm{CH}_{3}\right)_{3}\right) ;{ }^{13} \mathrm{C}-\mathrm{NMR}\left(100 \mathrm{MHz}, \mathrm{CDCl}_{3}\right):$ 153.4, 134.1, 129.4, 101.8, 101.0, 80.5, 77.3, 28.3, -0.1; LRMS (ESI+, m/z): $523.2\left([\mathrm{M}+\mathrm{Na}]^{+}\right.$, $100 \%)$; HRMS $(\mathrm{ESI}+, \mathrm{m} / \mathrm{z}): \mathrm{C}_{26} \mathrm{H}_{40} \mathrm{~N}_{2} \mathrm{NaO}_{4} \mathrm{Si}_{2}\left([\mathrm{M}+\mathrm{Na}]^{+}\right)$requires 523.24188, found 523.24155; IR $v_{\max }($ film $) / \mathrm{cm}^{-1}: 3331,2966,2901,2159,1721,1599,1548,1507$, $1485,1427,1392,1367,1338,1271,1248,1160,1092,1061,1006$. 


\section{Di-tert-butyl (3,6-diethynyl-1,2-phenylene)dicarbamate, 3p}

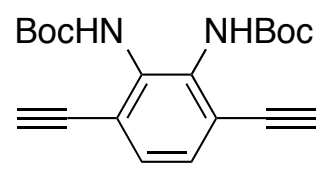

Compound 3p was prepared according to General Procedure $\mathbf{K}$ with dialkyne 12p (1.93 g, $3.85 \mathrm{mmol}), \mathrm{K}_{2} \mathrm{CO}_{3}(1.07 \mathrm{~g}, 7.71 \mathrm{mmol})$ and $\mathrm{MeOH}(40 \mathrm{~mL})$. Flash column chromatography $\left(\mathrm{SiO}_{2}, 20-30 \% \mathrm{Et}_{2} \mathrm{O}\right.$ in petrol) afforded dialkyne $\mathbf{3 p}$ as a pale brown solid (1.11 g, $81 \%$ yield).

m.p.: $159-161{ }^{\circ} \mathrm{C}$ (crystalized from $\mathrm{Et}_{2} \mathrm{O} /$ petrol); ${ }^{1} \mathbf{H}-\mathbf{N M R}\left(400 \mathrm{MHz}, \mathrm{CDCl}_{3}\right): 7.36$ $(2 \mathrm{H}, \mathrm{s}, \mathrm{Ar} H), 7.09\left(2 \mathrm{H}\right.$, br. s, NH), $3.43(2 \mathrm{H}, \mathrm{s}, \mathrm{CCH}), 1.53\left(18 \mathrm{H}, \mathrm{s}, \mathrm{C}\left(\mathrm{CH}_{3}\right)_{3}\right) ;{ }^{13} \mathrm{C}-$ NMR $\left(100 \mathrm{MHz}, \mathrm{CDCl}_{3}\right): 153.5,134.4,130.1,120.8,84.0,81.0,79.8,28.2$; LRMS $\left(\right.$ ESI+, m/z): $735.3\left([2 \mathrm{M}+\mathrm{Na}]^{+}, \quad 100 \%\right) ; \quad$ HRMS (ESI+, m/z): $\mathrm{C}_{20} \mathrm{H}_{24} \mathrm{~N}_{2} \mathrm{NaO}_{4}$ $\left([\mathrm{M}+\mathrm{Na}]^{+}\right)$requires 379.16283 , found 379.16264; IR $v_{\max }($ film $) / \mathrm{cm}^{-1}: 3294,2977$, 2931, 1710, 1599, 1555, 1508, 1459, 1393, 1363, 1287, 1271, 1245, 1161, 1085, 1056, 1030. 


\section{Trimethyl((2-nitrophenyl)ethynyl)silane, $12 y$}<smiles>CC(C)(C)C#Cc1ccccc1[N+](=O)[O-]</smiles>

Compound 12y was prepared according to General Procedure $\mathbf{J}$ with 2-iodonitrobenzene (1.00 g, $4.0 \mathrm{mmol}), \mathrm{Pd}\left(\mathrm{PPh}_{3}\right)_{2} \mathrm{Cl}_{2}(140 \mathrm{mg}, 0.20 \mathrm{mmol}), \mathrm{CuI}(38 \mathrm{mg}$, $0.20 \mathrm{mmol})$, TMS-acetylene $(0.853 \mathrm{~mL}, 6.0 \mathrm{mmol}), \mathrm{NEt}_{3}(0.800 \mathrm{~mL}, 6.0 \mathrm{mmol})$ and DMF $(20 \mathrm{~mL})$. Flash column chromatography $\left(\mathrm{SiO}_{2}, 5-10 \% \mathrm{Et}_{2} \mathrm{O}\right.$ in petrol) afforded aryl alkyne $\mathbf{1 2 y}$ as a brown oil (765 $\mathrm{mg}, 87 \%$ yield).

${ }^{1}$ H-NMR $\left(400 \mathrm{MHz}, \mathrm{CDCl}_{3}\right): 8.03(1 \mathrm{H}, \mathrm{ddd}, J$ 8.0, 1.5 and 0.5, C(1)H), $7.68(1 \mathrm{H}$, ddd, $J 7.5,1.5$ and $0.5, \mathrm{C}(4) H), 7.58(1 \mathrm{H}, \mathrm{td}, J 7.5$ and 1.5, C(3)H), $7.47(1 \mathrm{H}$, ddd, $J$ 8.0, 7.5 and 1.5, C(2)H), $0.31\left(9 \mathrm{H}, \mathrm{s}, \mathrm{Si}\left(\mathrm{CH}_{3}\right)_{3}\right) ;{ }^{13} \mathrm{C}-\mathbf{N M R}\left(100 \mathrm{MHz}, \mathrm{CDCl}_{3}\right): 150.1$, 135.1, 132.6, 128.8, 124.5, 118.4, 103.7, 99.3, -0.4; LRMS (ESI+, m/z): 242.1 $\left([\mathrm{M}+\mathrm{Na}]^{+}, 100 \%\right) ;$ HRMS $(\mathrm{ESI}+, \mathrm{m} / \mathrm{z}): \mathrm{C}_{11} \mathrm{H}_{14} \mathrm{NO}_{2} \mathrm{Si}\left([\mathrm{M}+\mathrm{H}]^{+}\right)$requires 220.07883, found 220.07884 .

Data is consistent with the literature. ${ }^{9}$

\section{1-Ethynyl-2-nitrobenzene, $3 y$}<smiles>C#Cc1ccccc1[N+](=O)[O-]</smiles>

Compound 3y was prepared according to General Procedure $\mathbf{K}$ with alkyne 12y (731 $\mathrm{mg}, 3.33 \mathrm{mmol}), \mathrm{K}_{2} \mathrm{CO}_{3}$ (461 mg, $3.33 \mathrm{mmol}$ ), and $\mathrm{MeOH}(40 \mathrm{~mL})$. Flash column chromatography $\left(\mathrm{SiO}_{2}, 5 \% \mathrm{Et}_{2} \mathrm{O}\right.$ in petrol) afforded terminal alkyne $\mathbf{3 y}$ as a brown solid (389 $\mathrm{mg}, 79 \%$ yield).

m.p.: $80-82{ }^{\circ} \mathrm{C}$ (crystalized from $\mathrm{Et}_{2} \mathrm{O} /$ petrol); ${ }^{1} \mathbf{H}-\mathbf{N M R}\left(400 \mathrm{MHz}, \mathrm{CDCl}_{3}\right): 8.08$ $(1 \mathrm{H}$, ddd, $J$ 8.0, 1.5 and $0.5, \mathrm{C}(1) H), 7.73(1 \mathrm{H}, \mathrm{dd}, J 7.5$ and $1.5, \mathrm{C}(4) H), 7.63(1 \mathrm{H}, \mathrm{td}$, $J 7.5$ and $1.5, \mathrm{C}(3) H), 7.54(1 \mathrm{H}$, dddd, $J 8.0,7.5,1.5$ and $0.5, \mathrm{C}(2) H), 3.54(1 \mathrm{H}, \mathrm{s}$, $\mathrm{CCH}) ;{ }^{13} \mathrm{C}-\mathrm{NMR}\left(100 \mathrm{MHz}, \mathrm{CDCl}_{3}\right): 150.2,135.5,132.9,129.4,124.6,117.4,85.2$, 78.5; HRMS (EI/FI, m/z): $\mathrm{C}_{8} \mathrm{H}_{5} \mathrm{NO}_{2}\left([\mathrm{M}]^{+}, 100 \%\right)$ requires 147.0320, found 147.0319 .

Data is consistent with the literature. ${ }^{10}$ 


\section{((4-Methoxy-2-nitrophenyl)ethynyl)trimethylsilane, $12 \mathrm{z}$}<smiles>COc1ccc(C#CC(C)(C)C)c([N+](=O)[O-])c1</smiles>

Compound $12 \mathrm{z}$ was prepared according to General Procedure $\mathbf{J}$ with 1-iodo-4methoxy-2-nitrobenzene $(2.00 \mathrm{~g}, 7.16 \mathrm{mmol}), \mathrm{Pd}\left(\mathrm{PPh}_{3}\right)_{2} \mathrm{Cl}_{2}(251 \mathrm{mg}, 0.36 \mathrm{mmol})$, $\mathrm{CuI}$ (69 mg, $0.36 \mathrm{mmol})$, TMS-acetylene (1.54 mL, $10.8 \mathrm{mmol}), \mathrm{NEt}_{3}$ (1.50 mL, 10.8 mmol) and DMF $(20 \mathrm{~mL})$. Flash column chromatography $\left(\mathrm{SiO}_{2}, 5-20 \% \mathrm{Et}_{2} \mathrm{O}\right.$ in petrol) afforded aryl alkyne $\mathbf{1 2 z}$ as a brown solid (1.36 g, 76\% yield).

m.p.: $68-70{ }^{\circ} \mathrm{C}$ (crystalized from $\mathrm{Et}_{2} \mathrm{O} /$ petrol); ${ }^{1} \mathbf{H}-\mathbf{N M R}\left(400 \mathrm{MHz}, \mathrm{CDCl}_{3}\right.$ ): 7.57 $(1 \mathrm{H}, \mathrm{d}, J 8.5, \mathrm{C}(3) H), 7.53(1 \mathrm{H}, \mathrm{d}, J 2.5, \mathrm{C}(1) H), 7.10(1 \mathrm{H}, \mathrm{dd}, J 8.5$ and $2.5, \mathrm{C}(2) H)$, $3.91\left(3 \mathrm{H}, \mathrm{s}, \mathrm{OCH}_{3}\right), 0.29\left(9 \mathrm{H}, \mathrm{s}, \mathrm{Si}\left(\mathrm{CH}_{3}\right)_{3}\right) ;{ }^{13} \mathbf{C}-\mathbf{N M R}\left(100 \mathrm{MHz}, \mathrm{CDCl}_{3}\right): 159.6$, 151.1, 136.0, 119.5, 110.5, 109.2, 101.4, 99.5, 56.0, -0.3; LRMS (ESI+, m/z): 272.1 $\left([\mathrm{M}+\mathrm{Na}]^{+}, 100 \%\right)$.

Data is consistent with the literature. ${ }^{11}$

\section{1-Ethynyl-4-methoxy-2-nitrobenzene, $3 z$}<smiles>C#Cc1ccc(OC)cc1[N+](=O)[O-]</smiles>

Compound $\mathbf{3 z}$ was prepared according to General Procedure $\mathbf{K}$ with alkyne $12 z$ (1.28 g, $5.13 \mathrm{mmol}), \mathrm{K}_{2} \mathrm{CO}_{3}(709 \mathrm{mg}, 5.13 \mathrm{mmol})$ and $\mathrm{MeOH}(50 \mathrm{~mL})$. Flash column chromatography $\left(\mathrm{SiO}_{2}, 5-20 \% \mathrm{Et}_{2} \mathrm{O}\right.$ in petrol) afforded terminal alkyne $\mathbf{3 z}$ as a brown solid (858 mg, $94 \%$ yield).

m.p.: $83-85{ }^{\circ} \mathrm{C}$ (crystalized from $\mathrm{Et}_{2} \mathrm{O} /$ petrol); ${ }^{1} \mathbf{H}-\mathbf{N M R}\left(400 \mathrm{MHz}, \mathrm{CDCl}_{3}\right.$ ): 7.62 $(1 \mathrm{H}, \mathrm{d}, J 8.5, \mathrm{C}(3) H), 7.57(1 \mathrm{H}, \mathrm{d}, J 2.5, \mathrm{C}(1) H), 7.14(1 \mathrm{H}, \mathrm{dd}, J 8.5$ and 2.5, C(2)H), $3.92\left(3 \mathrm{H}, \mathrm{s}, \mathrm{OCH}_{3}\right), 3.43(1 \mathrm{H}, \mathrm{s}, \mathrm{CCH}) ;{ }^{13} \mathbf{C}-\mathbf{N M R}\left(100 \mathrm{MHz}, \mathrm{CDCl}_{3}\right): 159.9,151.2$, 136.4, 119.5, 109.5, 109.4, 83.2, 78.6, 56.0; LRMS (ESI+, m/z): $200.1\left([\mathrm{M}+\mathrm{Na}]^{+}\right.$, $100 \%)$.

Data is consistent with the literature. ${ }^{12}$ 


\section{2-Methyleneoctanal, 15}<smiles>C=C(C=O)CCCCCC</smiles>

To a solution of octanal $(1.6 \mathrm{~mL}, 10 \mathrm{mmol})$ and formaldehyde $\left(37 \%\right.$ in $\mathrm{H}_{2} \mathrm{O}, 0.7 \mathrm{~mL}$, $10 \mathrm{mmol})$ in ${ }^{i} \mathrm{PrOH}(1.0 \mathrm{~mL})$ was added pyrrolidine $(83 \mu \mathrm{L}, 1.0 \mathrm{mmol})$ and propionic acid $(75 \mu \mathrm{L}, 1.0 \mathrm{mmol})$. The reaction mixture was heated at $45^{\circ} \mathrm{C}$ for 4 hours, then concentrated in vacuo. Flash column chromatography $\left(\mathrm{SiO}_{2}, 5 \% \mathrm{Et}_{2} \mathrm{O}\right.$ in petrol) afforded aldehyde 15 (793 mg, 56\%) as a colourless oil.

${ }^{1} \mathbf{H}-\mathbf{N M R}\left(400 \mathrm{MHz}, \mathrm{CDCl}_{3}\right): 9.56(1 \mathrm{H}, \mathrm{s}, \mathrm{CHO}), 6.27\left(1 \mathrm{H}, \mathrm{s}, \mathrm{C}=\mathrm{CH}_{\mathrm{A}} \mathrm{H}_{\mathrm{B}}\right), 6.01(1 \mathrm{H}$, $\left.\mathrm{s}, \mathrm{C}=\mathrm{CH}_{\mathrm{A}} H_{\mathrm{B}}\right), 2.26\left(2 \mathrm{H}, \mathrm{t}, J 7.5, \mathrm{C}(1) H_{2}\right), 1.50-1.43\left(2 \mathrm{H}, \mathrm{m}, \mathrm{C}(2) H_{2}\right), 1.37-1.26(6 \mathrm{H}$, m, C(3) $\left.H_{2}, \mathrm{C}(4) H_{2}, \mathrm{C}(5) H_{2}\right), 0.90\left(3 \mathrm{H}, \mathrm{t}, J 7.5, \mathrm{CH}_{3}\right) ;{ }^{13} \mathbf{C}-\mathbf{N M R}\left(100 \mathrm{MHz}, \mathrm{CDCl}_{3}\right)$ : 192.9, 150.5, 133.9, 31.6, 29.0, 27.8, 27.7, 22.6, 14.1; LRMS (ESI+, m/z): 195.1 $\left([\mathrm{M}+\mathrm{MeOH}+\mathrm{Na}]^{+}, 100 \%\right)$.

Data is consistent with the literature. ${ }^{13}$

\section{2-((Ethylthio)methyl)octanal, 2u}<smiles>CCCCC(C=O)CCCC</smiles>

To a solution of aldehyde $15(3.48 \mathrm{~g}, 24.8 \mathrm{mmol})$ in $\mathrm{CH}_{2} \mathrm{Cl}_{2}(7.0 \mathrm{~mL})$ was added EtSH $(2.69 \mathrm{~mL}, 37.2 \mathrm{mmol})$ and $\mathrm{NEt}_{3}(0.35 \mathrm{~mL}, 2.5 \mathrm{mmol})$. The resulting solution was stirred at room temperature for 16 hours. The solution was concentrated in vacuo, and Flash column chromatography $\left(\mathrm{SiO}_{2}, 5-10 \% \mathrm{Et}_{2} \mathrm{O}\right.$ in petrol) afforded aldehyde $\mathbf{2 u}$ as a colourless oil (3.06 g, 61\% yield).

${ }^{1}$ H-NMR (400 MHz, $\left.\mathrm{CDCl}_{3}\right): 9.66(1 \mathrm{H}, \mathrm{d}, J 2.5, \mathrm{CHO}), 2.82(1 \mathrm{H}, \mathrm{dd}, J 13.0$ and 8.0, $\left.\mathrm{C}(1) H_{\mathrm{A}} \mathrm{H}_{\mathrm{B}}\right), 2.68\left(1 \mathrm{H}, \mathrm{dd}, J 13.0\right.$ and $\left.6.0, \mathrm{C}(1) \mathrm{H}_{\mathrm{A}} H_{\mathrm{B}}\right), 2.56\left(2 \mathrm{H}, \mathrm{q}, J 7.5, \mathrm{SCH}_{2} \mathrm{CH}_{3}\right)$, 2.56-2.49 (1H, m, C(2)H), 1.76-1.55 (2H, m, C(3)H $)$, 1.36-1.25 (8H, m, $\left.\left(\mathrm{CH}_{2}\right)_{4}\right)$, $1.27\left(3 \mathrm{H}, \mathrm{t}, J\right.$ 7.5, $\left.\mathrm{SCH}_{2} \mathrm{CH}_{3}\right), 0.89\left(3 \mathrm{H}, \mathrm{t}, J 7.0,\left(\mathrm{CH}_{2}\right)_{4} \mathrm{CH}_{3}\right) ;{ }^{13} \mathbf{C}-\mathbf{N M R}(100 \mathrm{MHz}$, $\left.\mathrm{CDCl}_{3}\right): 203.7,51.6,31.6,30.5,29.3,28.8,26.71,26.57,22.6,14.6,14.0$; LRMS $(\mathrm{ESI}+, \mathrm{m} / \mathrm{z}): 257.1\left([\mathrm{M}+\mathrm{MeOH}+\mathrm{Na}]^{+}, 100 \%\right) ; \quad$ HRMS $(\mathrm{ESI}+, \mathrm{m} / \mathrm{z}): \mathrm{C}_{12} \mathrm{H}_{26} \mathrm{O}_{2} \mathrm{NaS}$ $\left([\mathrm{M}+\mathrm{MeOH}+\mathrm{Na}]^{+}\right)$requires 257.15457 , found $257.15471 ;$ IR $v_{\max }($ film $) / \mathrm{cm}^{-1}: 2957$, 2916, 2856, 2714, 1725, 1456, 1377, 1264, 1193, 1111, 1052. 


\section{References}

1. Chu, L.; Yue, X.; Qing, F.-L. Org. Lett. 2010, 12, 1644

2. Soto, S.; Vaz, E.; Dell'Aversana, C.; Álvarez, R. Altucci, L.; de Lera, Á. R. Org. Biomol. Chem. 2012, 10, 2101

3. Costa, M.; Della Cà, N.; Gabriele, B.; Massera, C.; Salerno, G.; Soliani, M. J. Org. Chem. 2004, 69, 2469

4. Ishizaki, M.; Zyo, M.; Kasama, Y.; Niimi, Y.; Hoshino, O.; Nishitani, K.; Hara, H. Heterocycles 2003, 60, 2259

5. Lee, W.-I.; Jung, J.-W.; Sim, J.; An, H.; Suh, Y.-G. Tetrahedron 2013, 69, 7211

6. Zehr, P. S.; Kayali, R.; Peña-Cabrera, E.; Robles-Resendiz, O.; VillanuevaRendon, A. D.; Söderberg, B. C. G. Tetrahedron 2008, 64, 5336

7. Racys, D. T.; Warrilow, C. E.; Pimlott, S. L.; Sutherland, A. Org. Lett. 2015, 17, 4782

8. Fukuzumi, M.; Nakanishi, W.; Ishikawa, T.; Kumamoto, T. Helv. Chim. Acta 2014, 97, 1453

9. Yamane, Y.; Liu, Z.; Hamasaki, A.; Ishida, T.; Haruta, M.; Yokoyama, T.; Tokunaga, M. Org. Lett. 2009, 11, 5162

10. Jadhav, A. M.; Bhunia, S.; Liao, H.-Y.; Liu, R.-S. J. Am. Chem. Soc. 2011, 133, 1769

11. Söderberg, B. C. G.; Gorugantula, S. P.; Howerton, C. R.; Petersen, J. L.; Dantale, S. W. Tetrahedron 2009, 65, 7357

12. Yamakawa, T.; Ideue, E.; Iwaki, Y.; Sato, A.; Tokuyama, H.; Shimokawa, J.; Fukuyama, T. Tetrahedron 2011, 67, 6547

13. Nakatsuji, Y.; Nakamura, T.; Yonetani, M.; Yuya, H.; Okahara, M. J. Am. Chem. Soc. 1988, 110, 531 


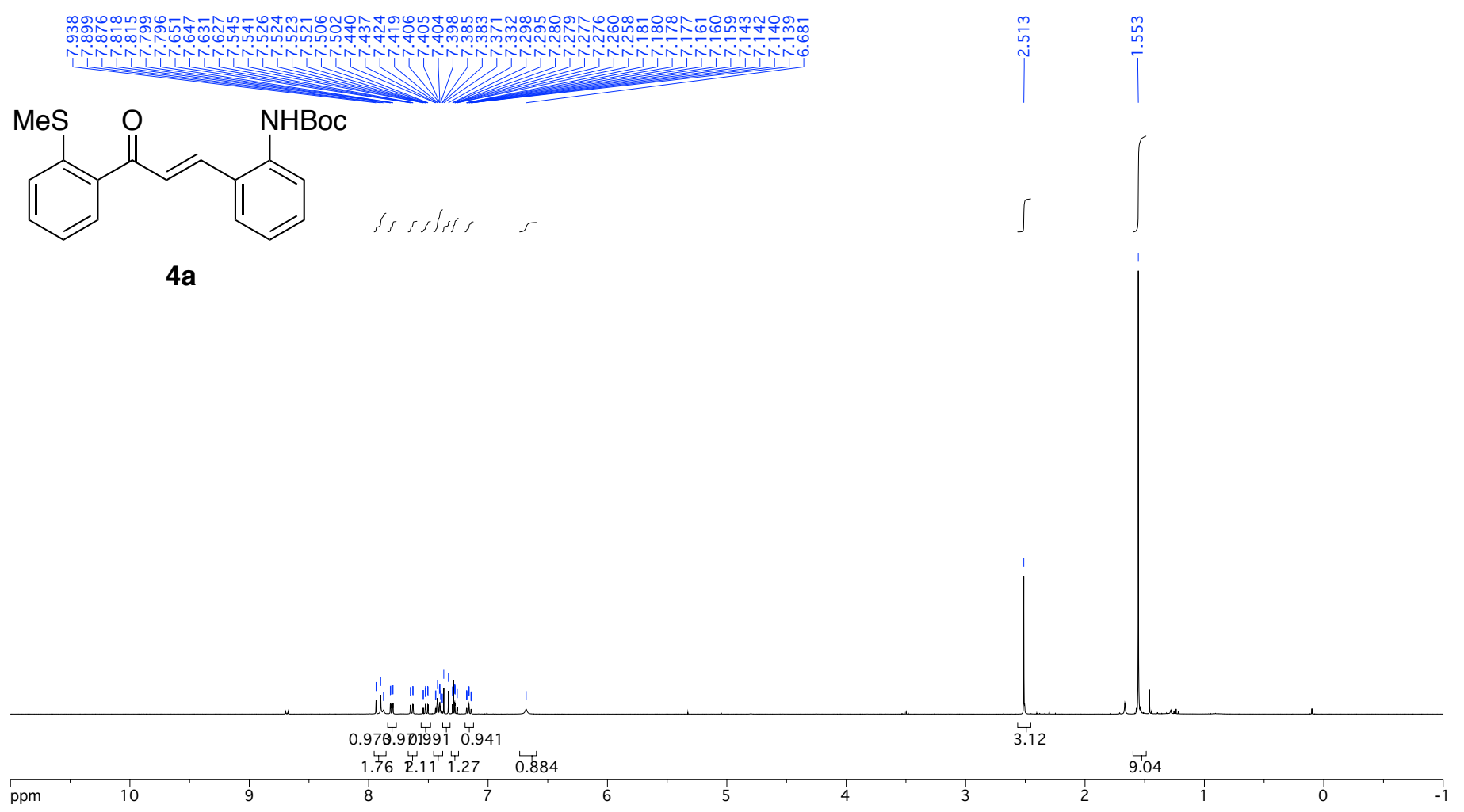

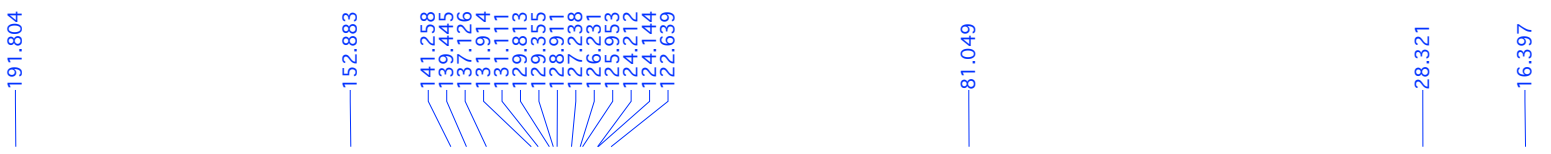

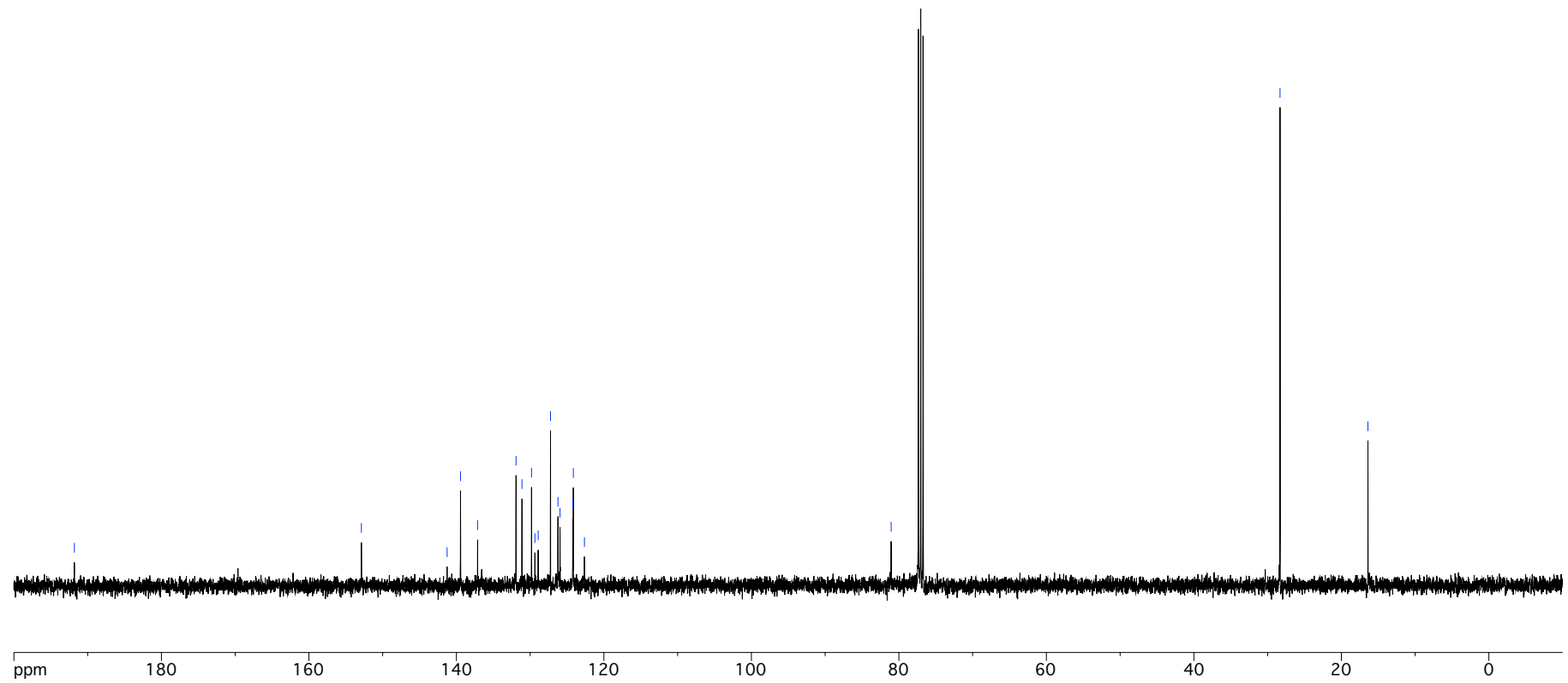



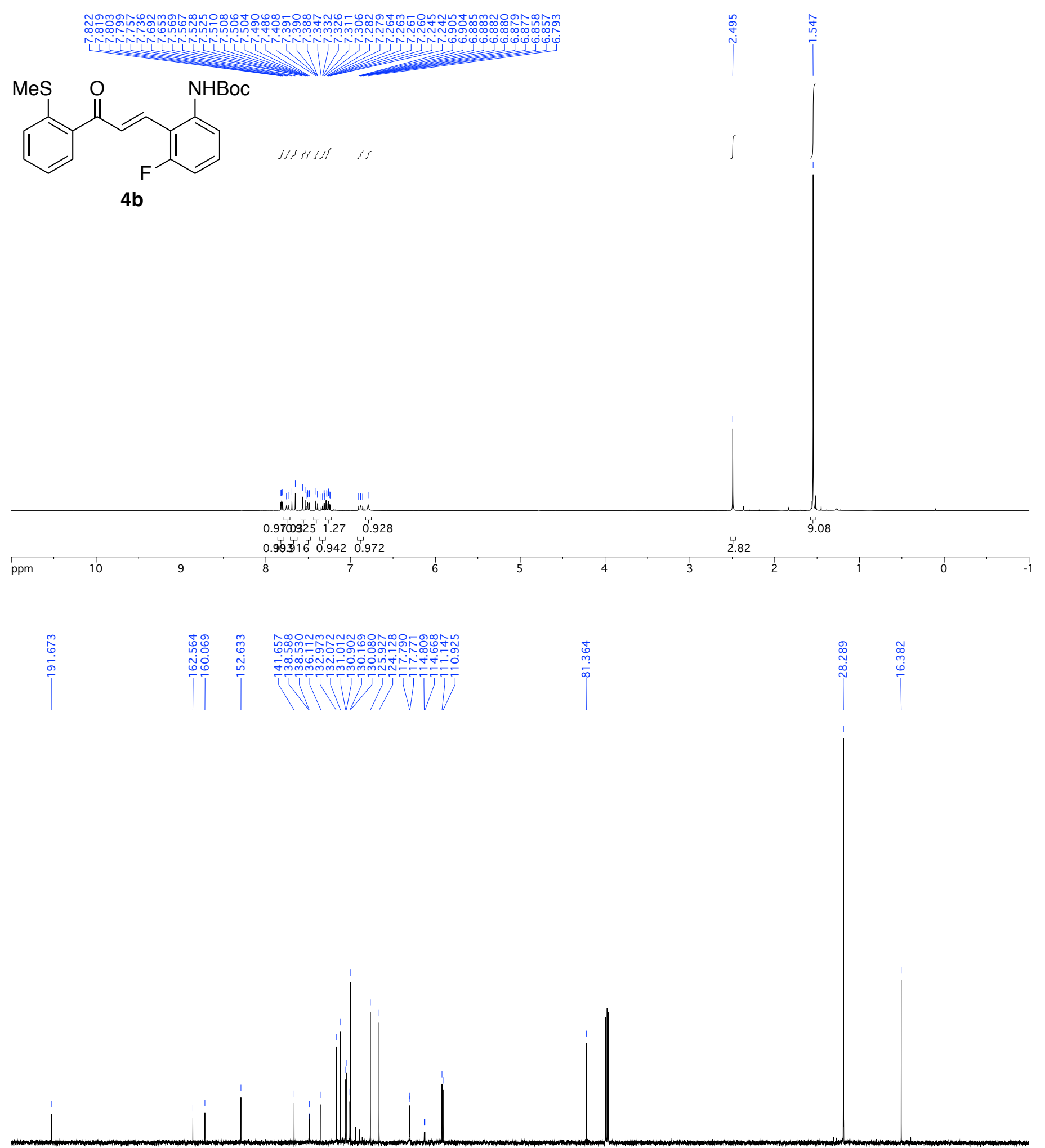

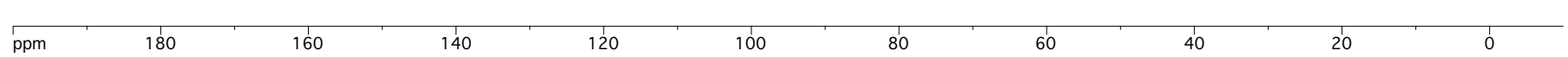




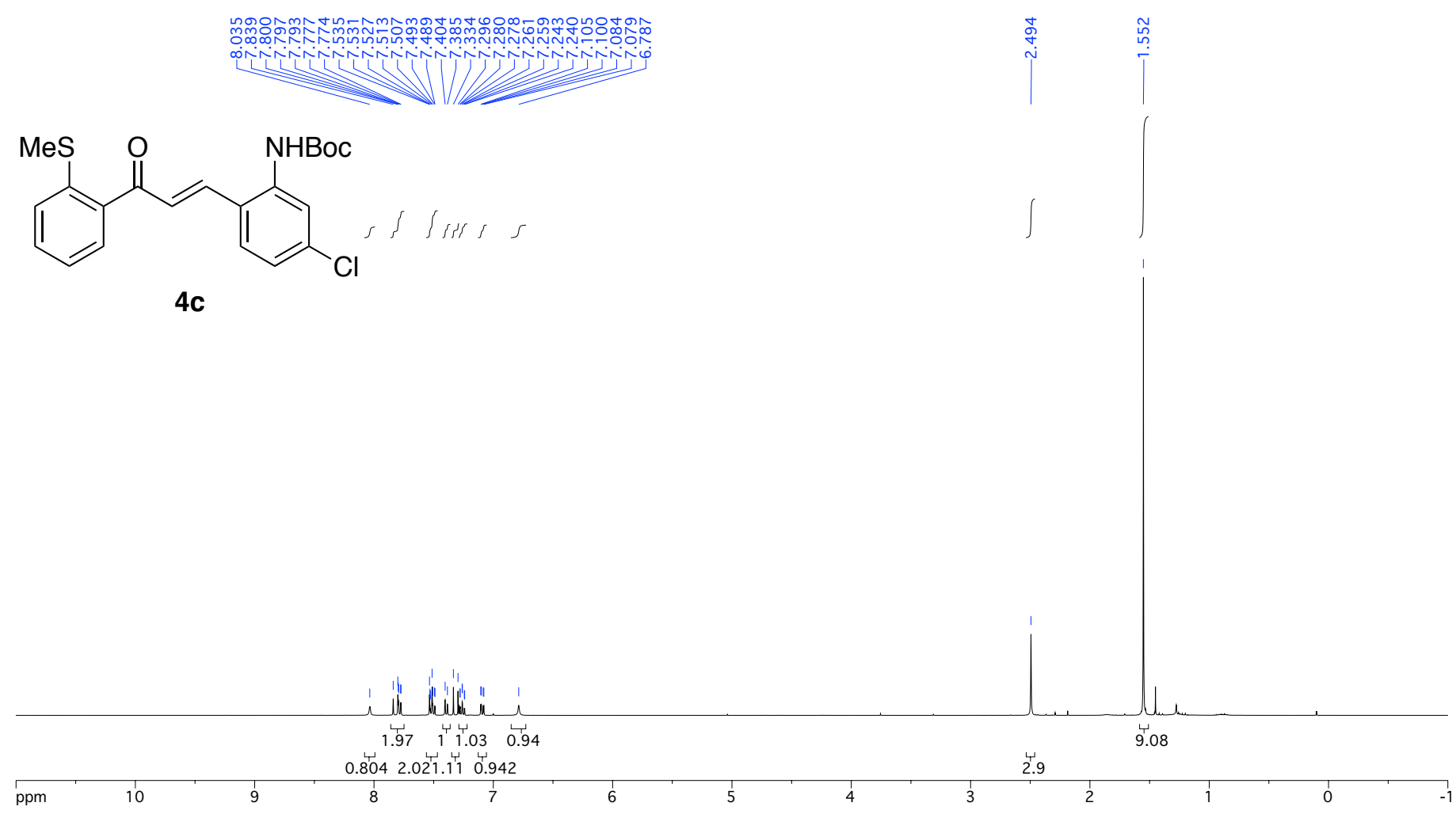

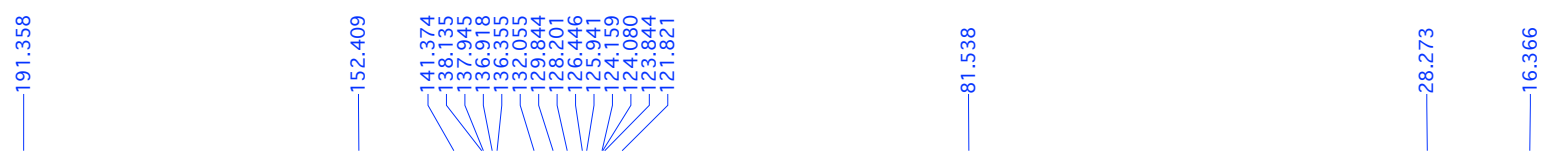

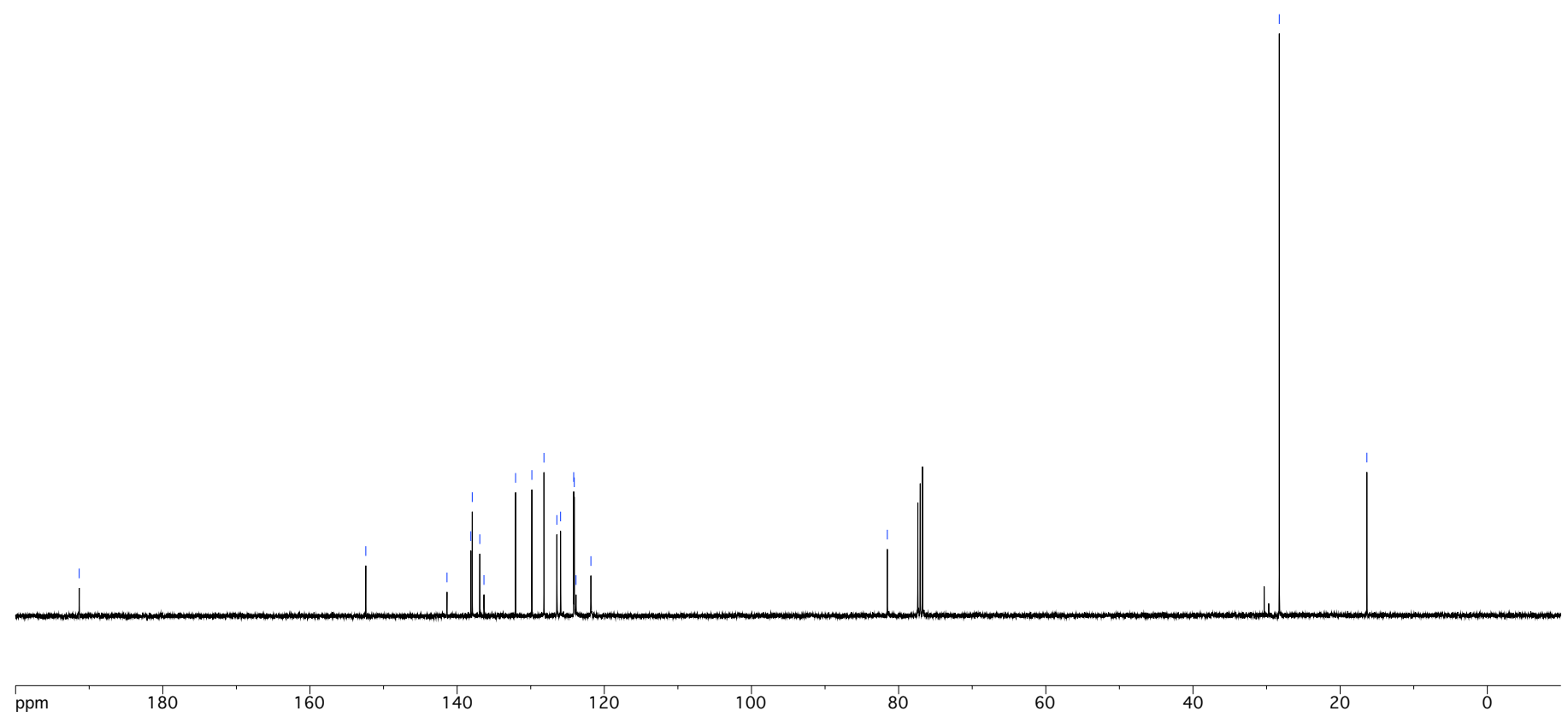



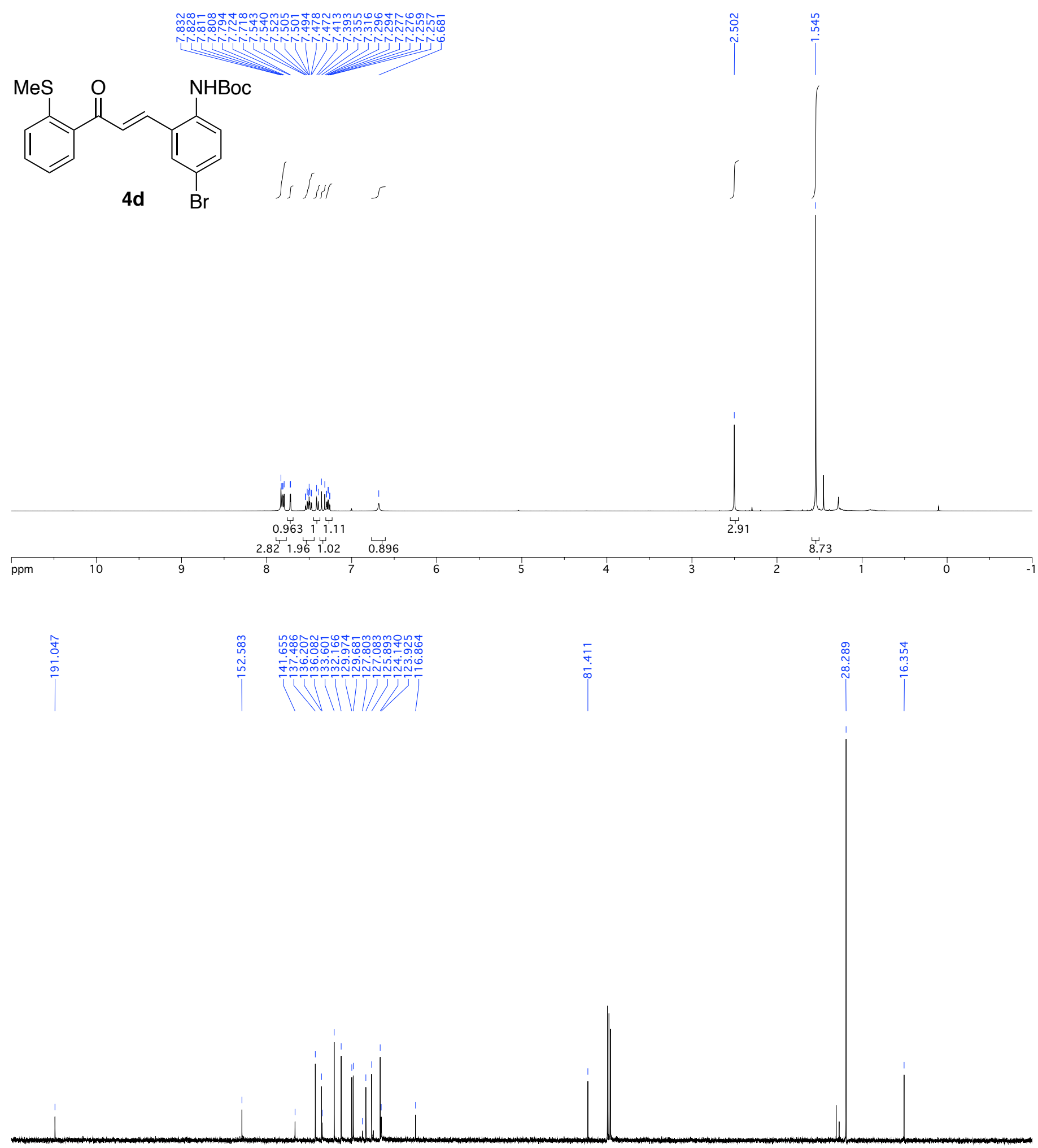

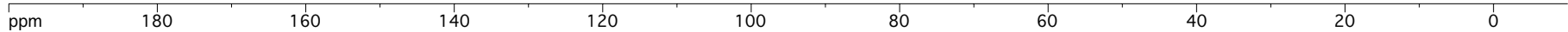



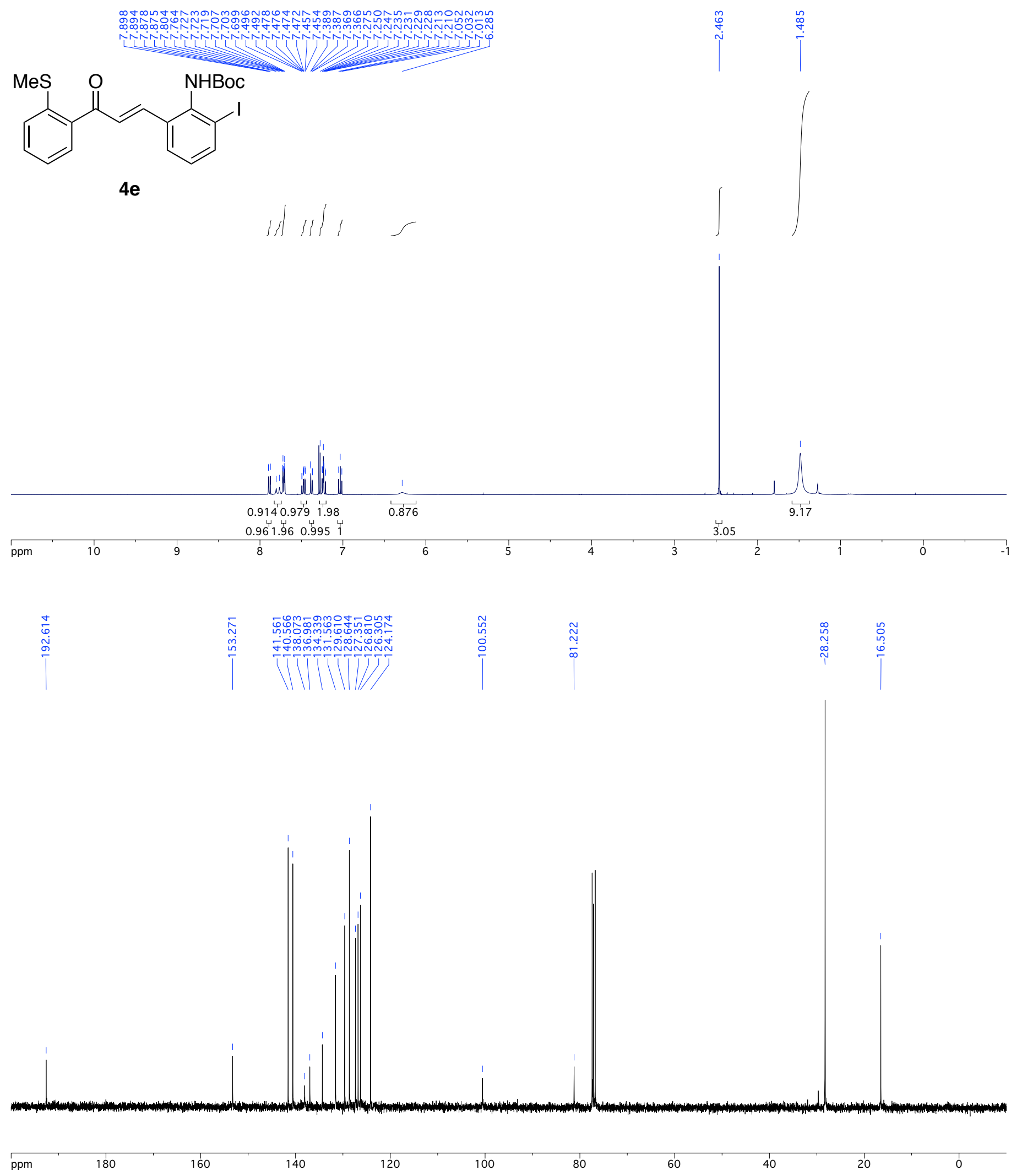

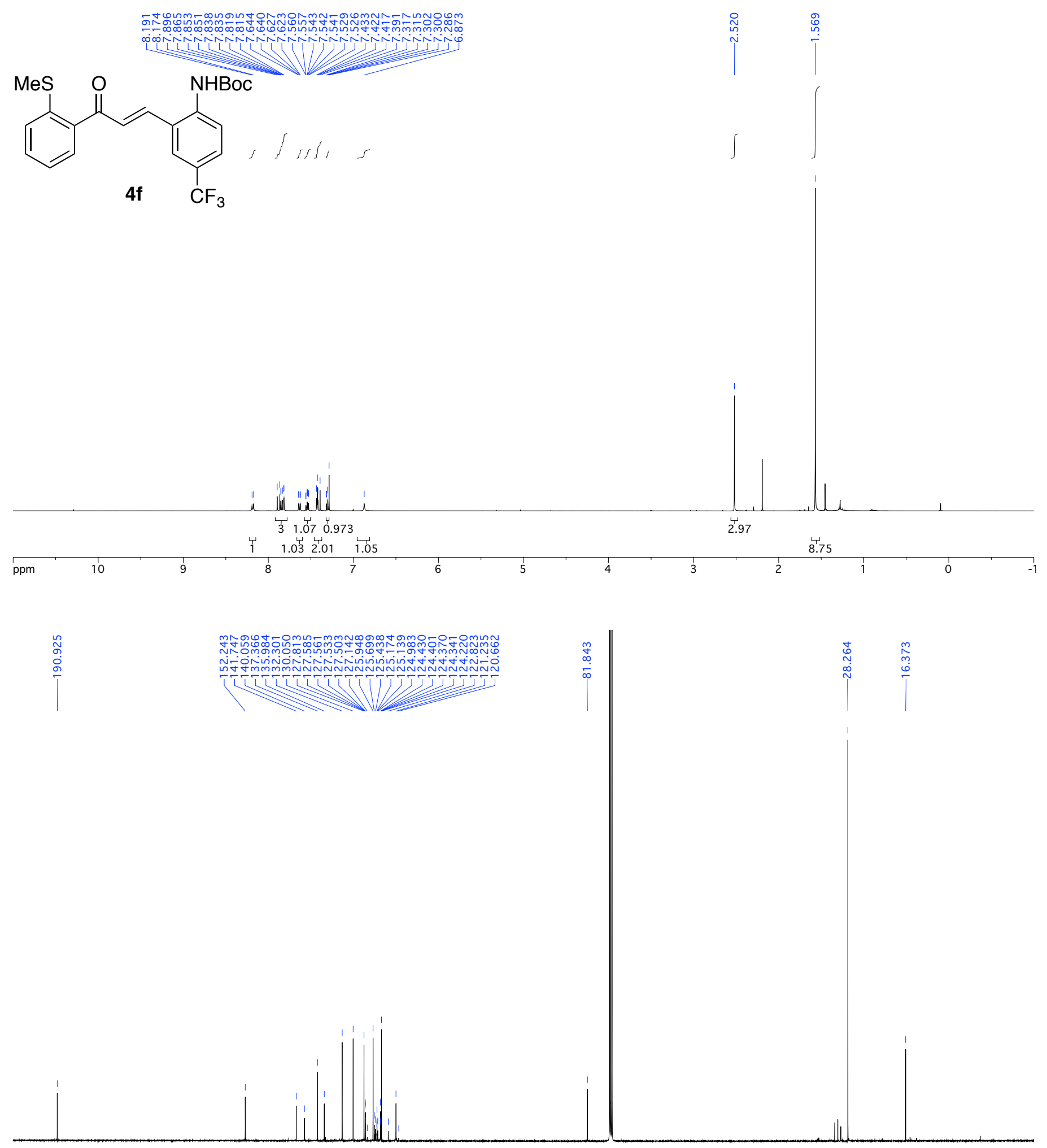

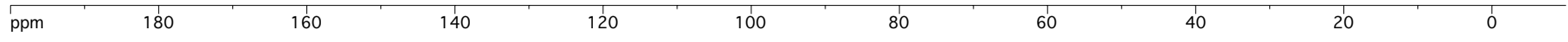



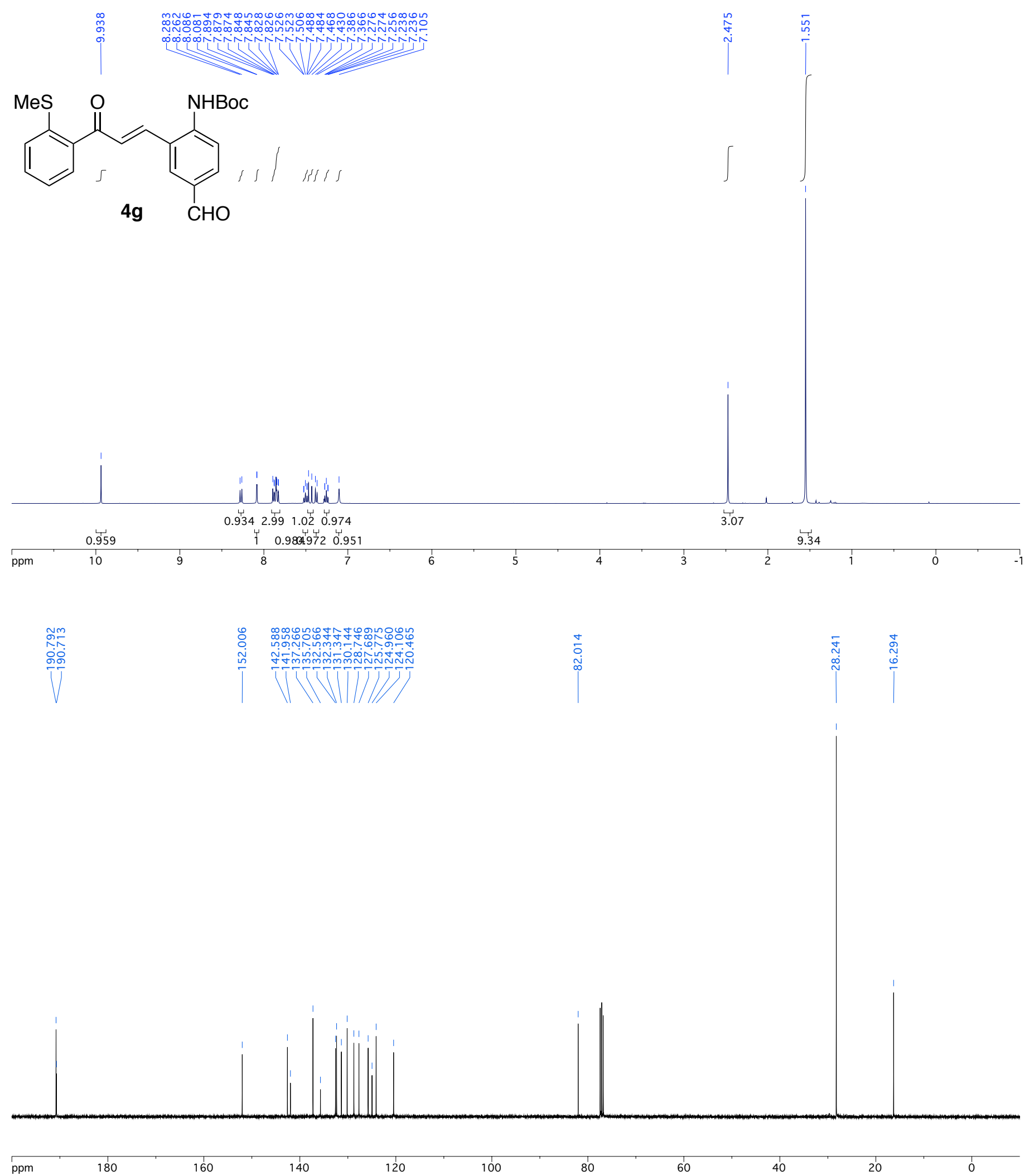

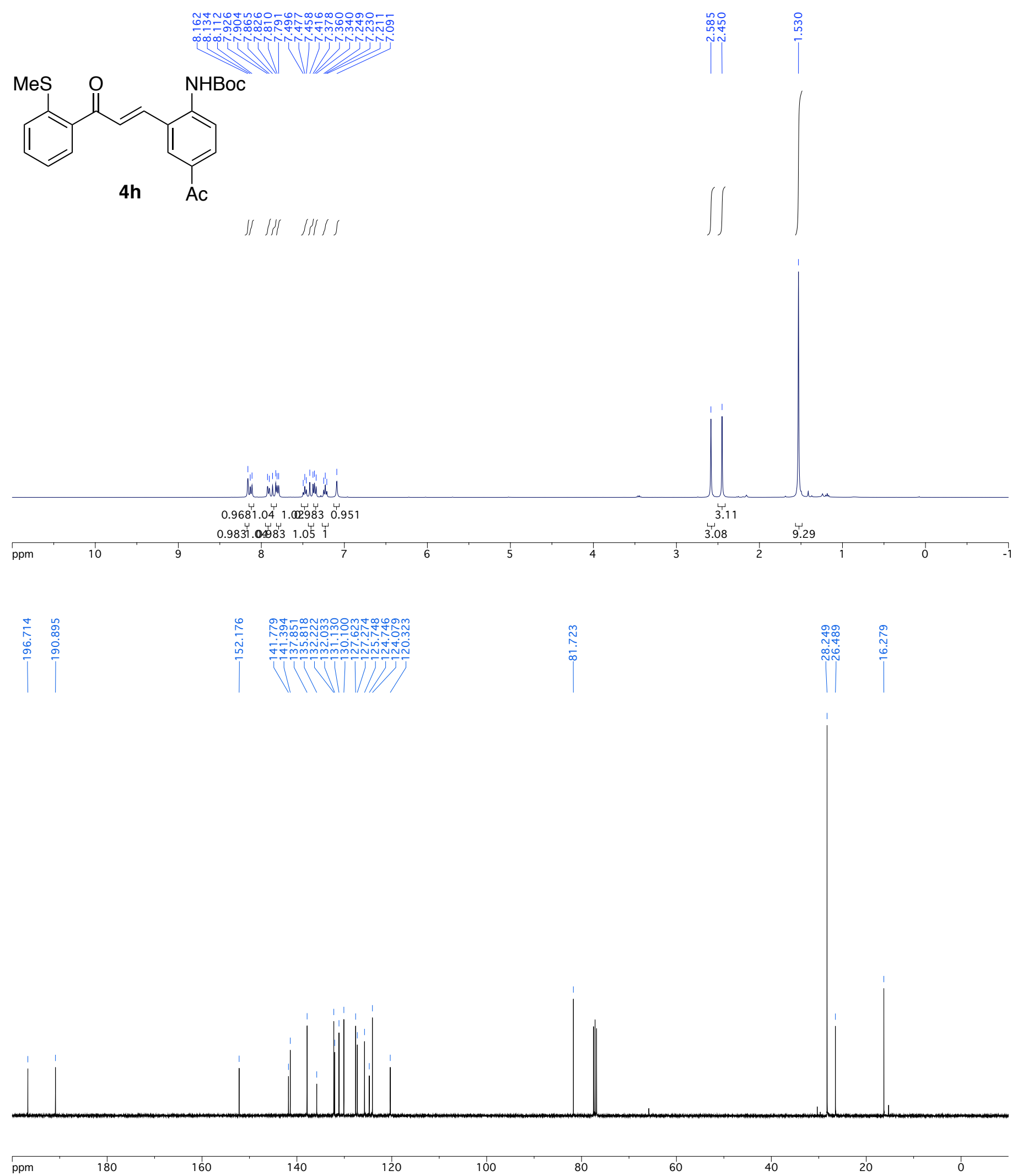

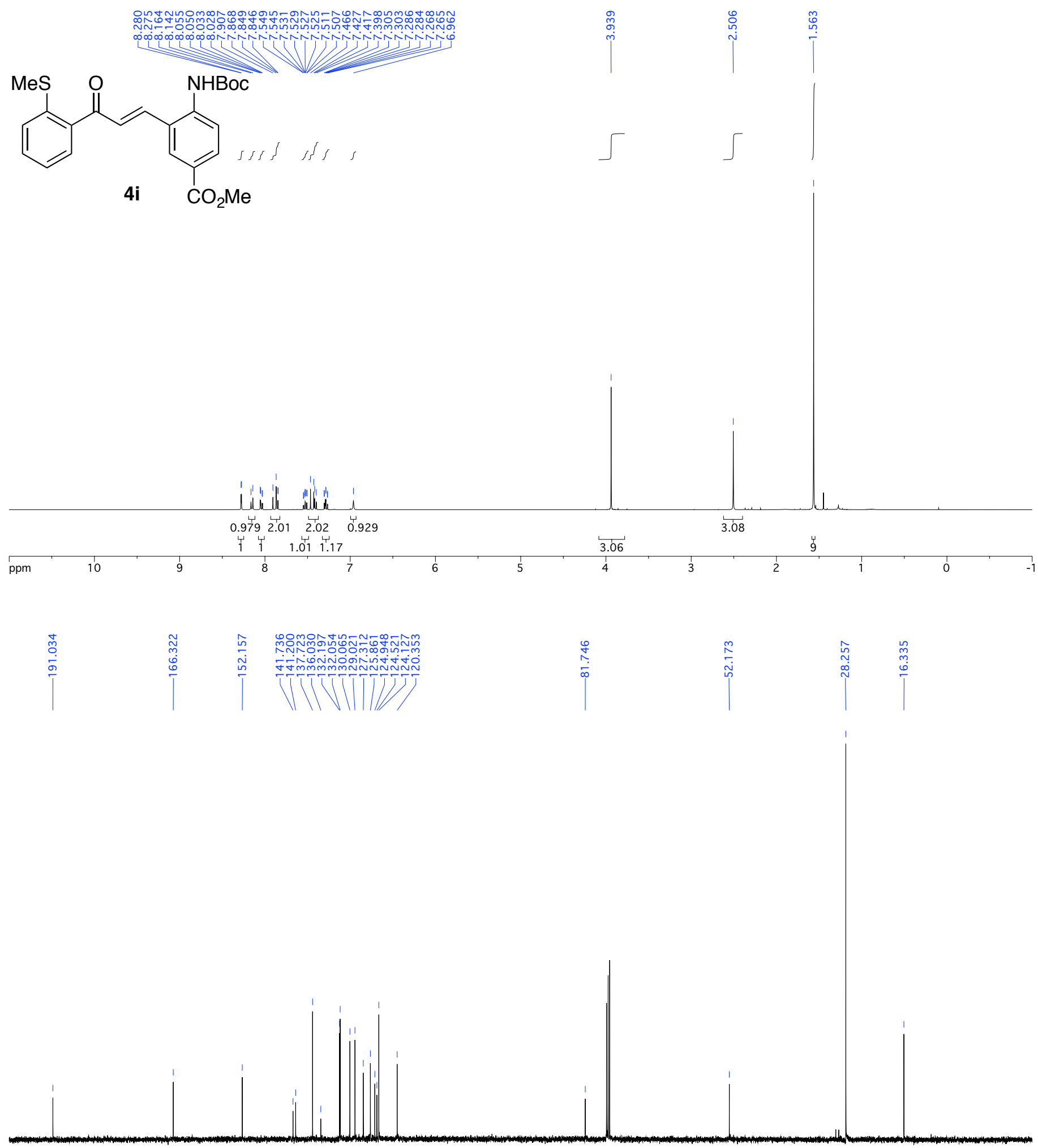

ppm

180

160

140

120

100

80

60

40

20 


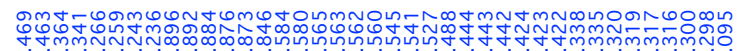

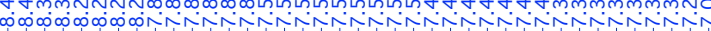
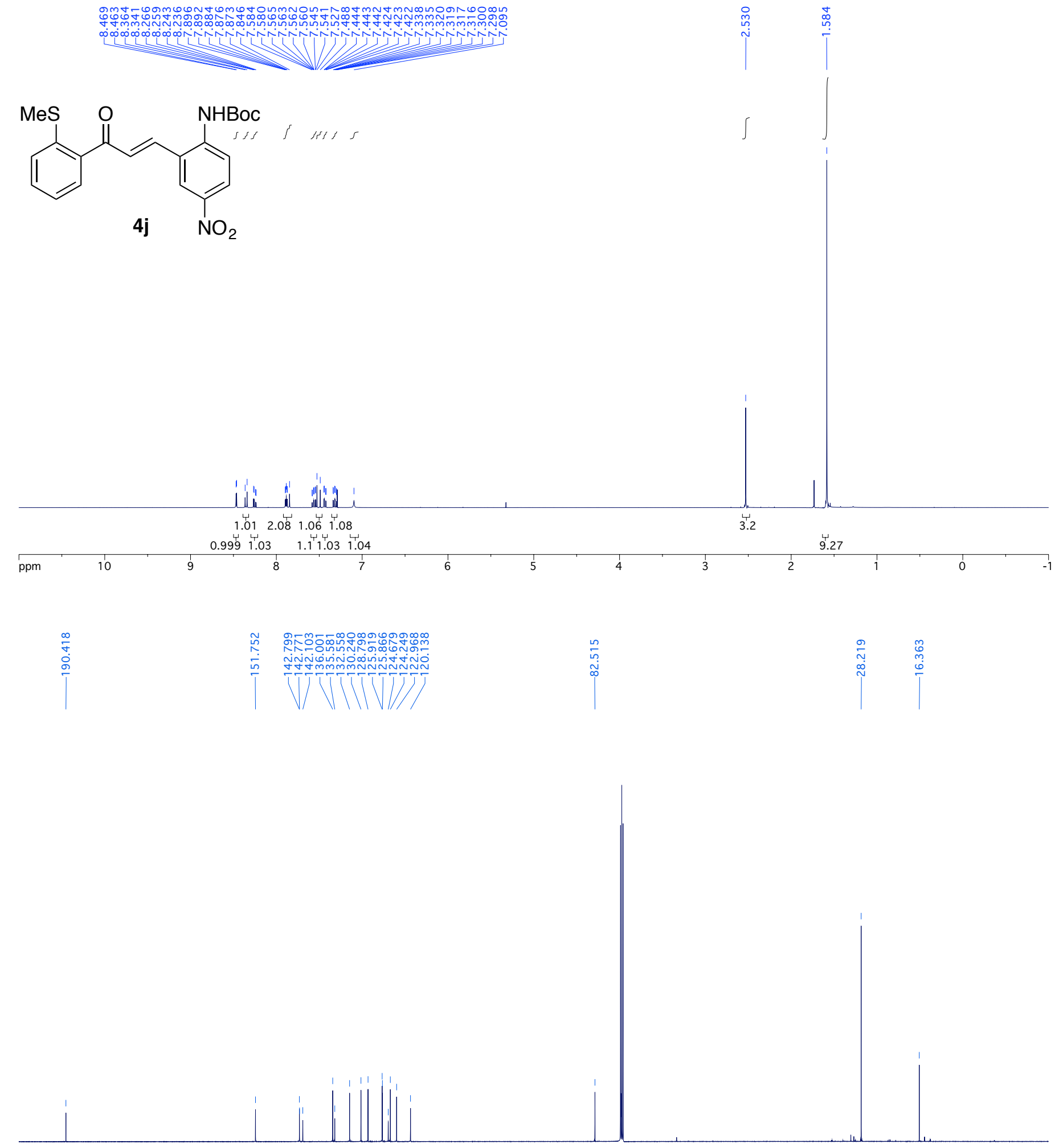

\begin{tabular}{lllllllll}
\hline ppm & 180 & 160 & 140 & 120 & 100 & 80 & 60 & 1
\end{tabular}




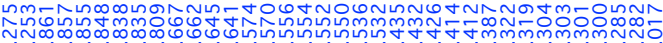

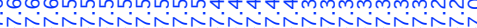

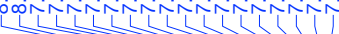

(1)

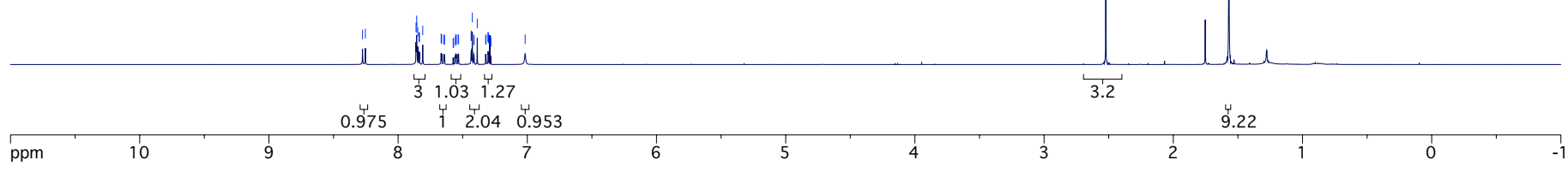

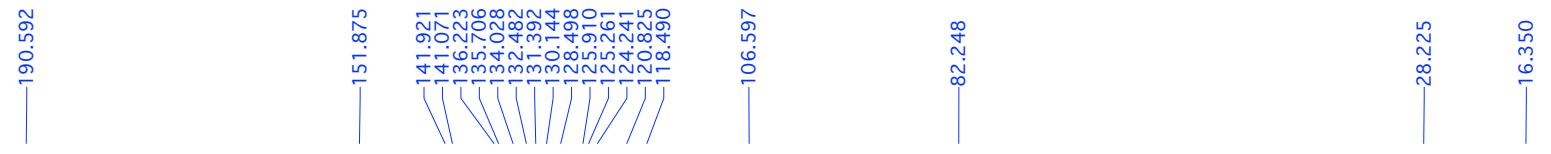

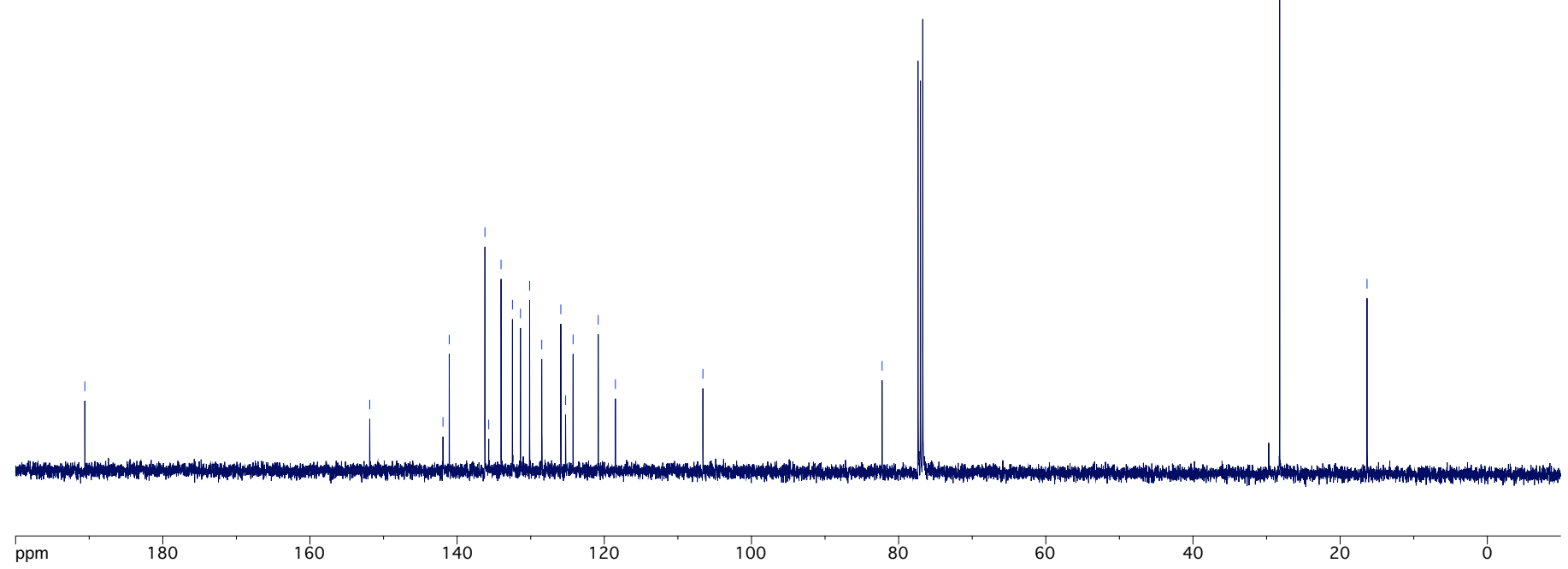



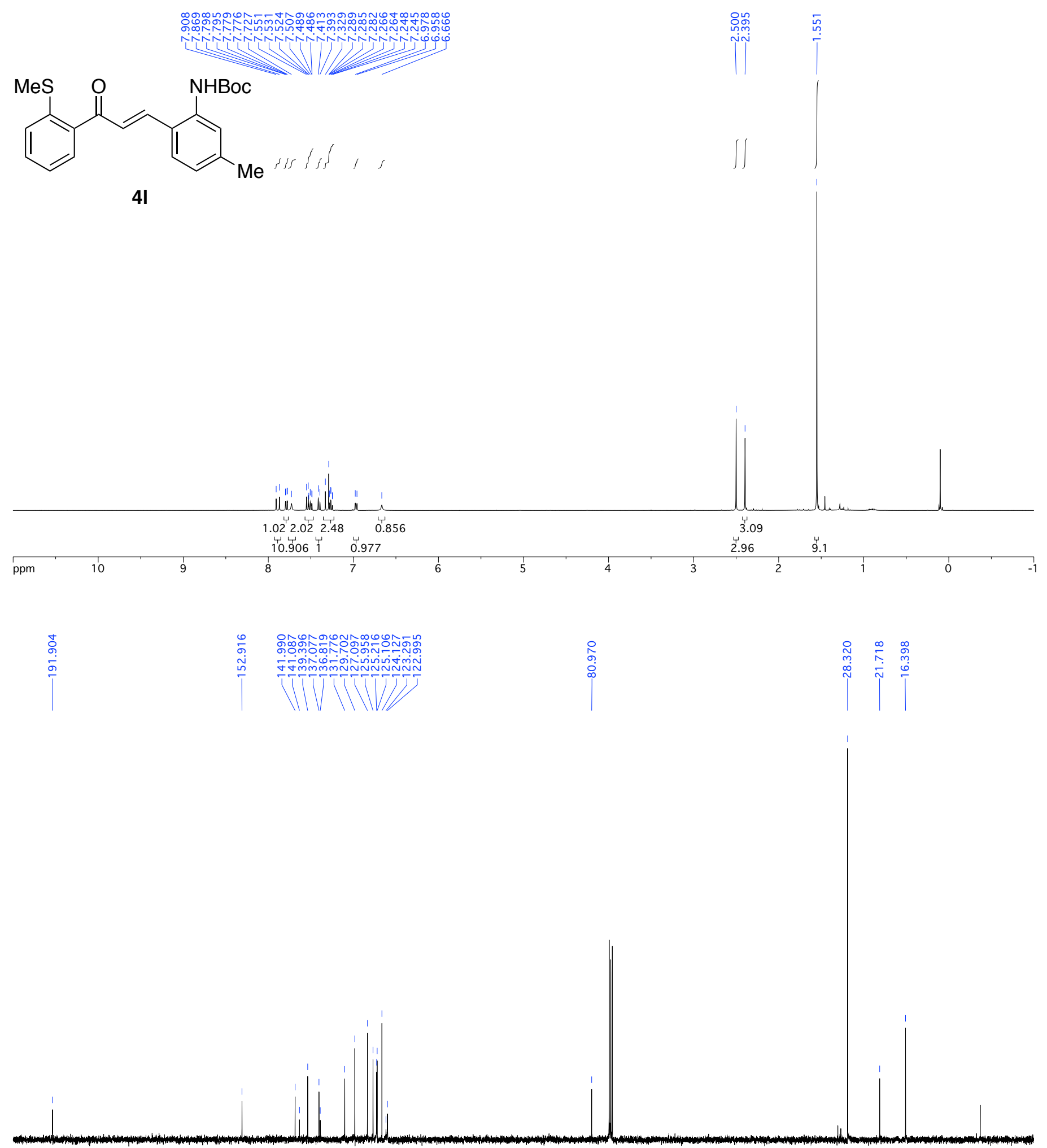

ppm

180

160

140

120

100

80

60

40

20 


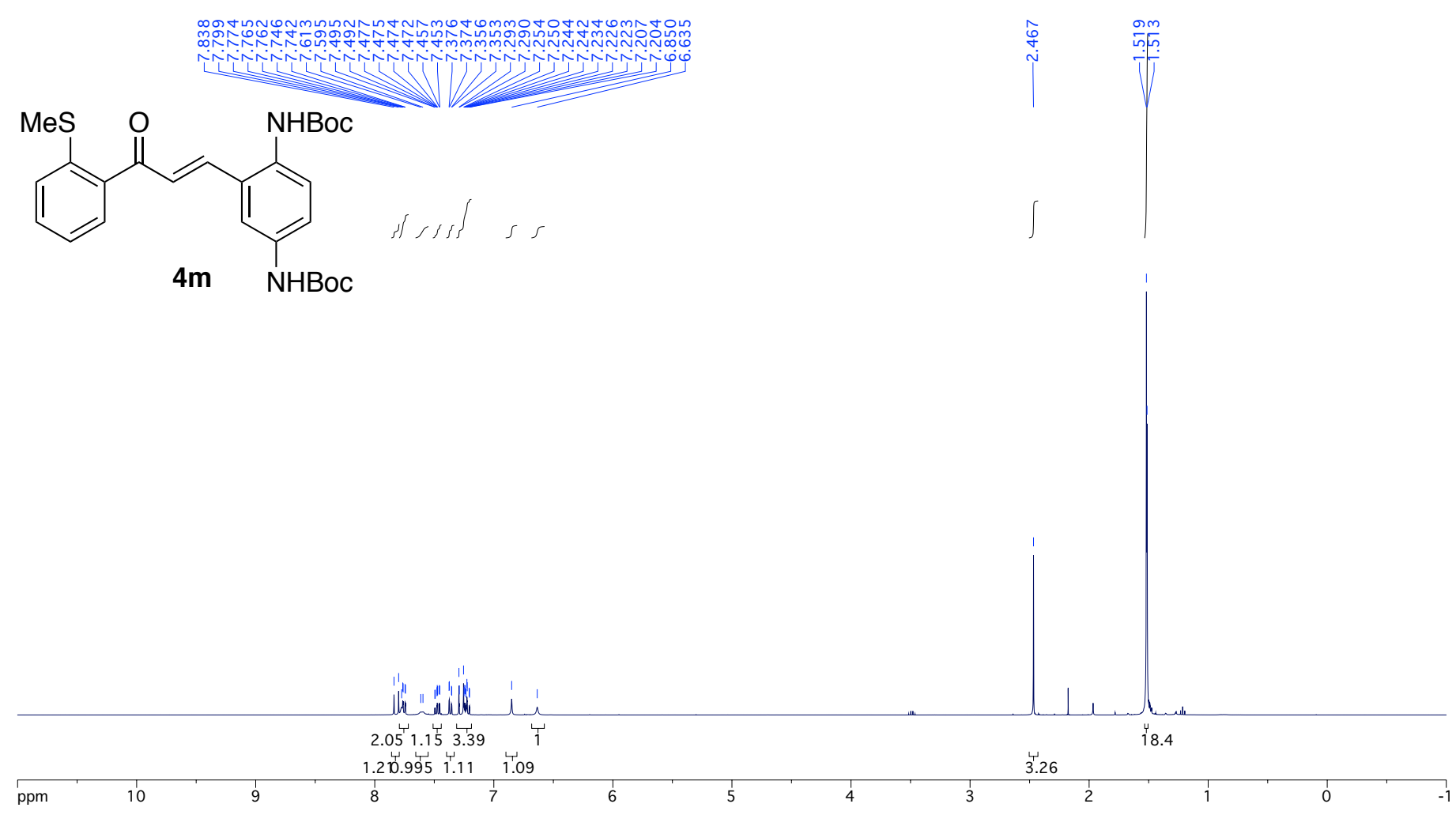

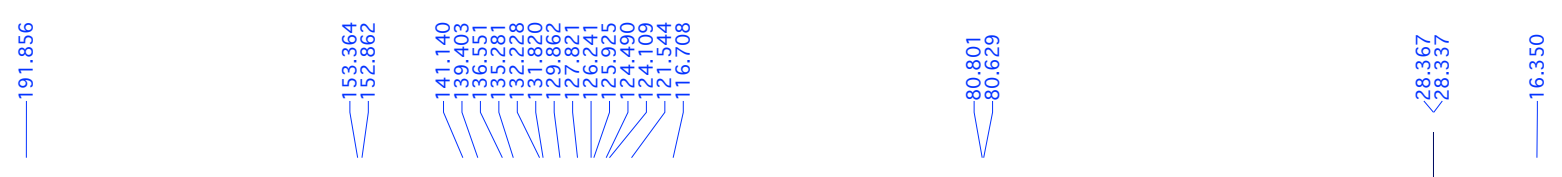

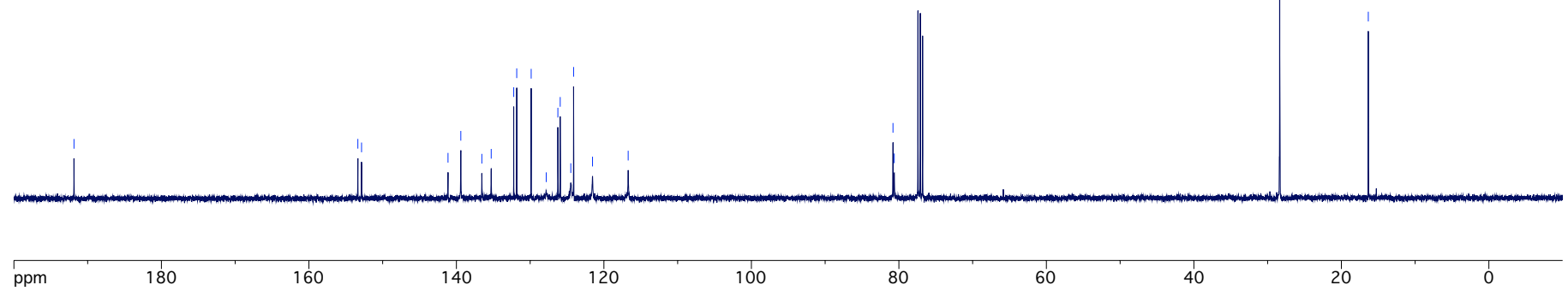


<smiles>CC(C)(C)Oc1ccccc1C(=O)/C=C/c1cccc(O)c1NS(C)(=O)=O</smiles>

$4 n$

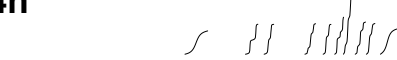

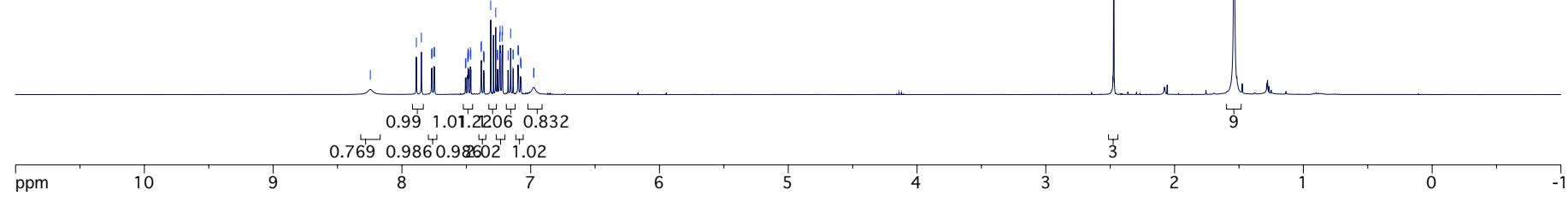

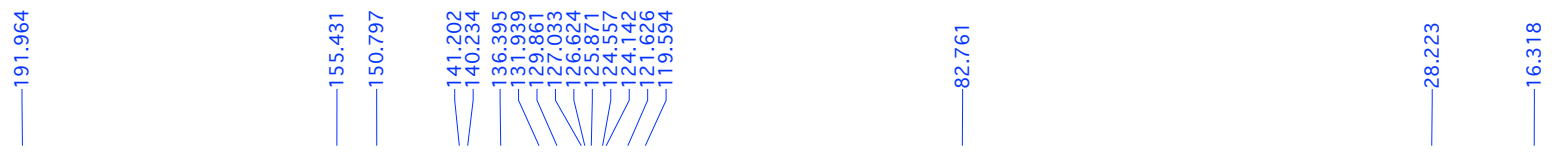

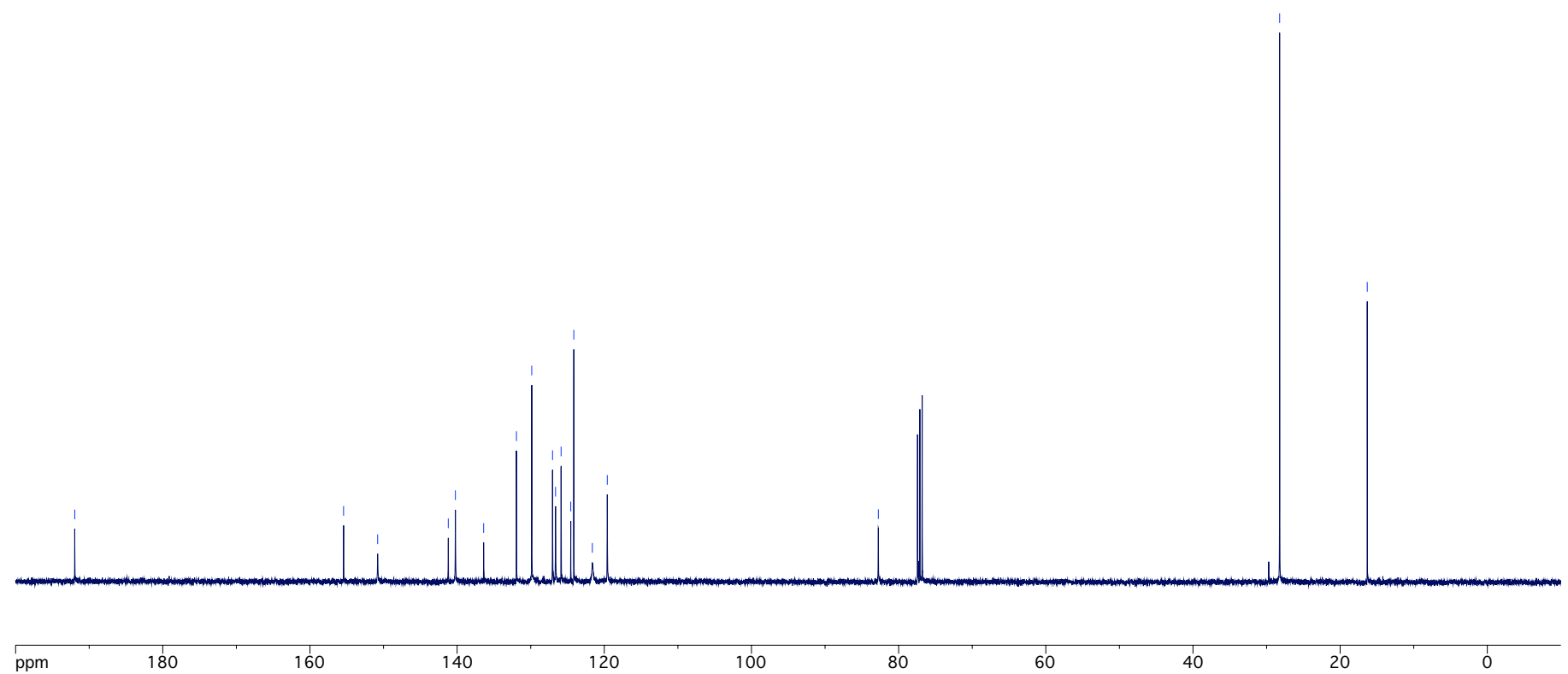



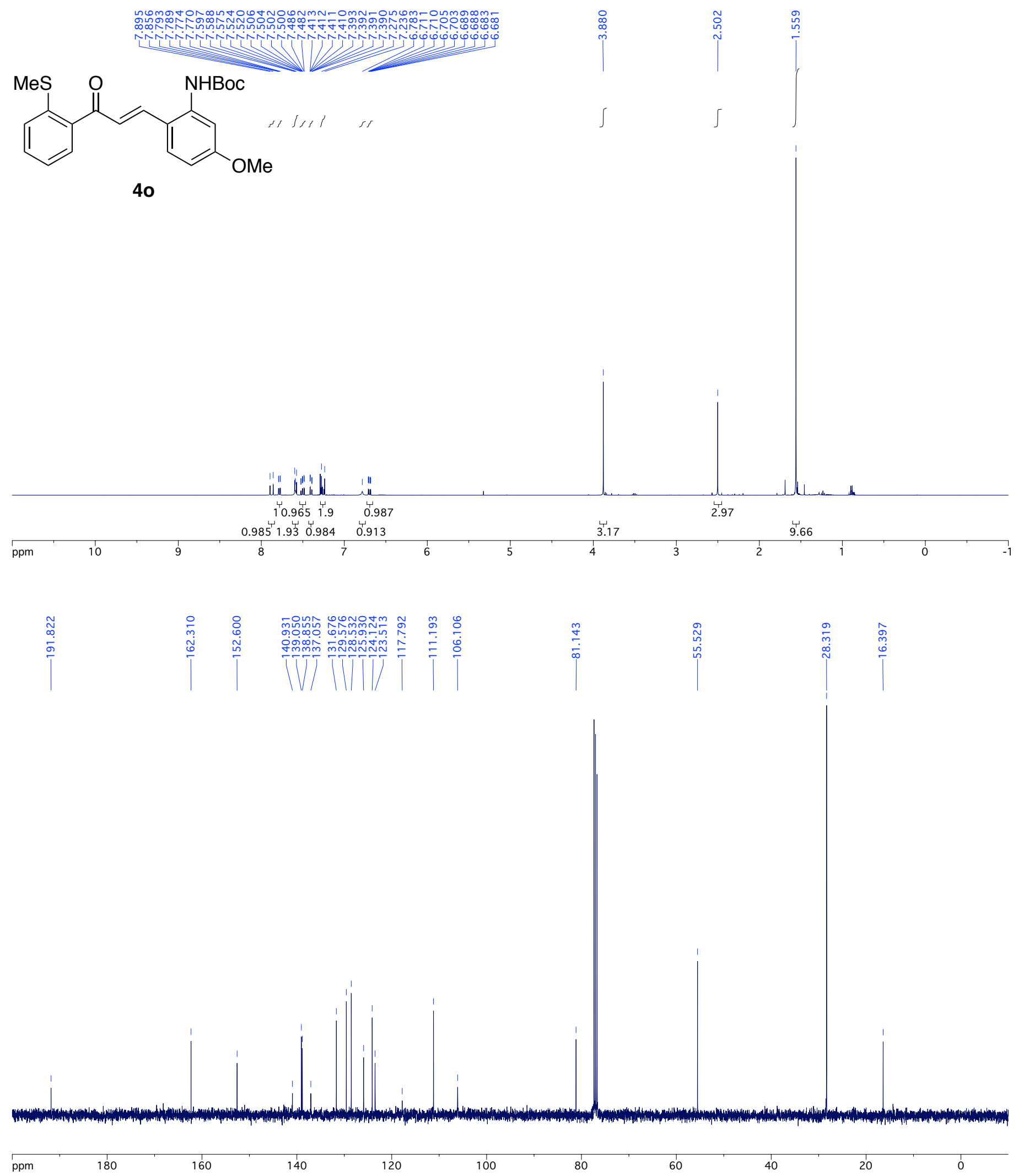

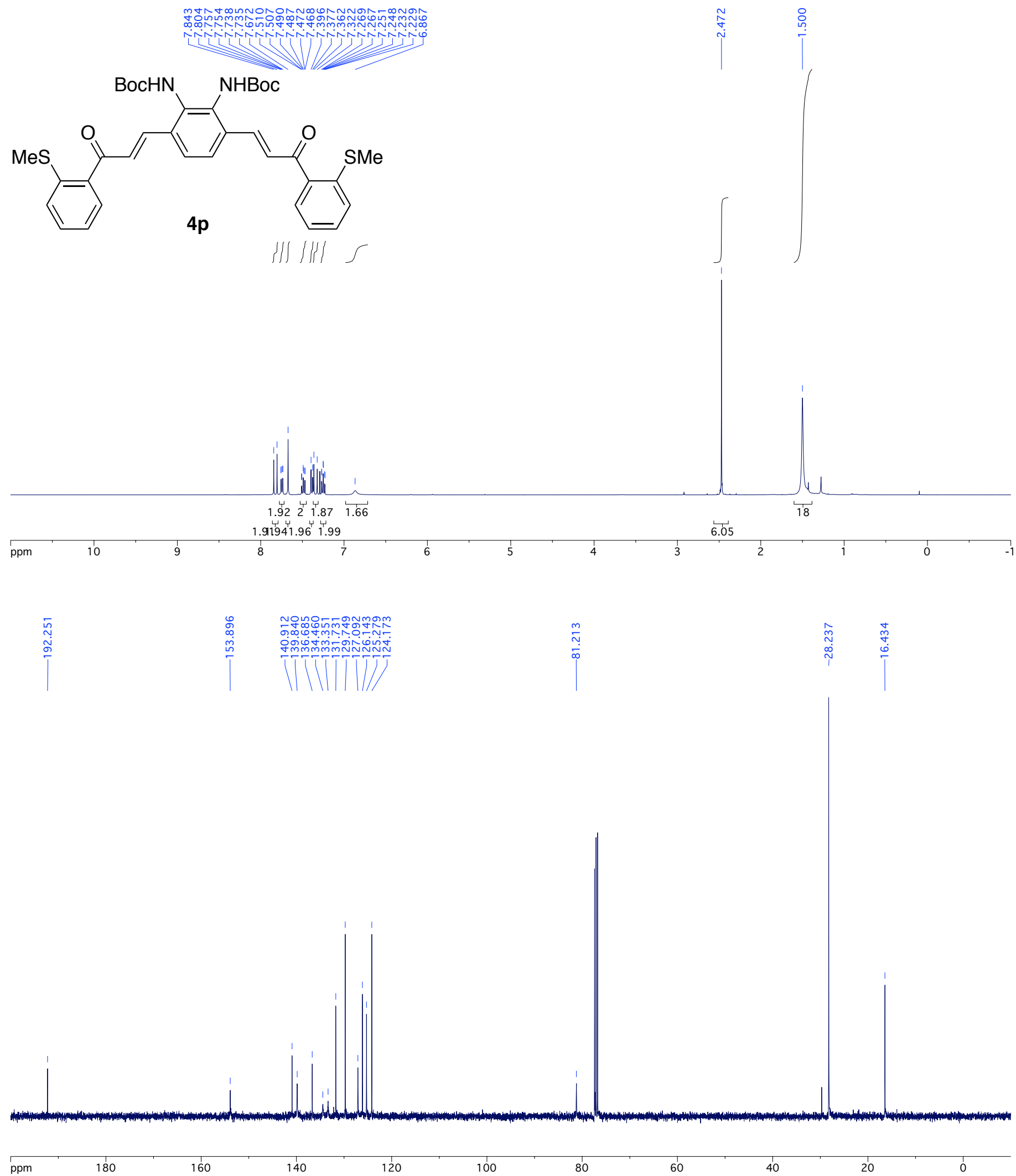

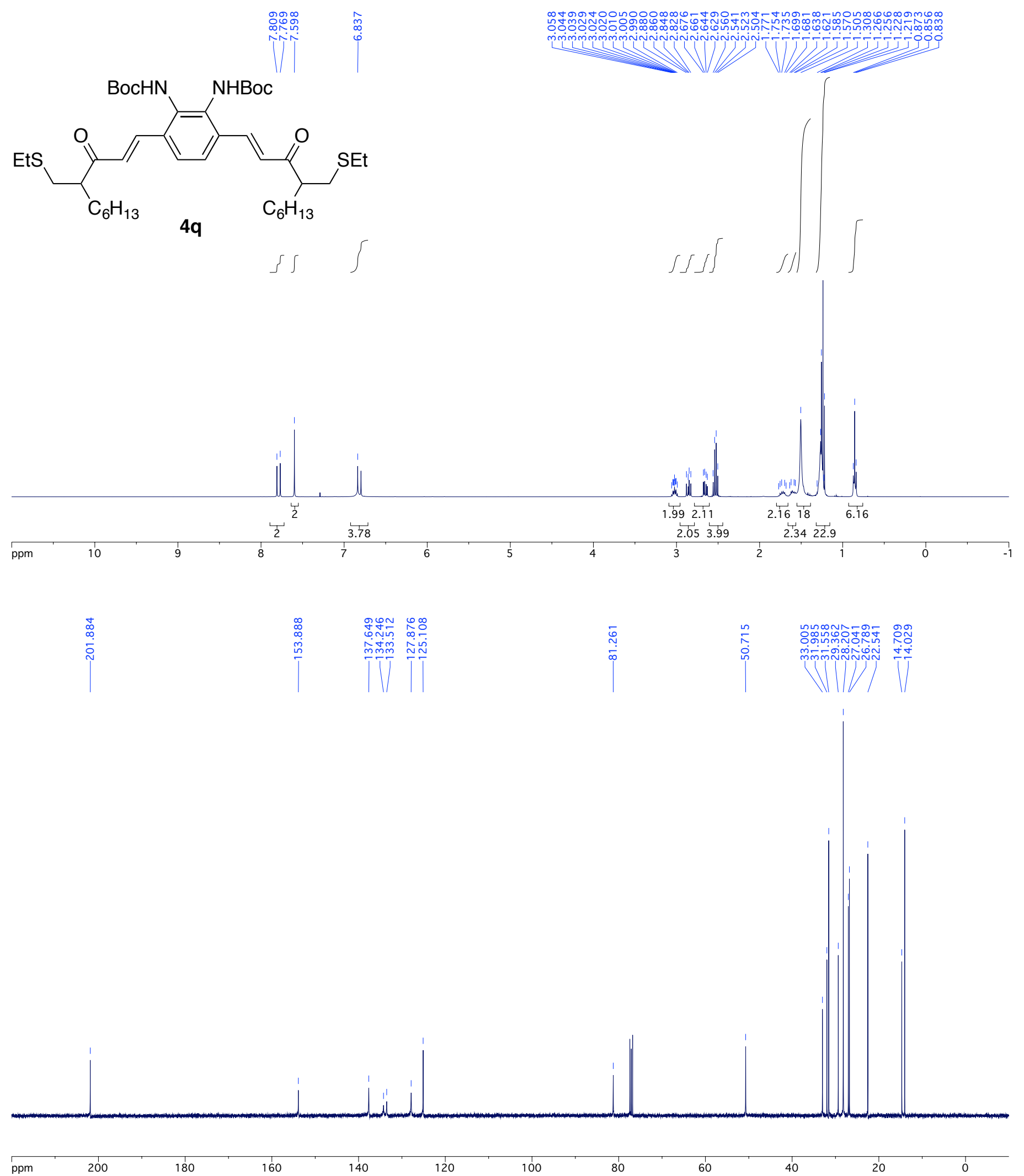

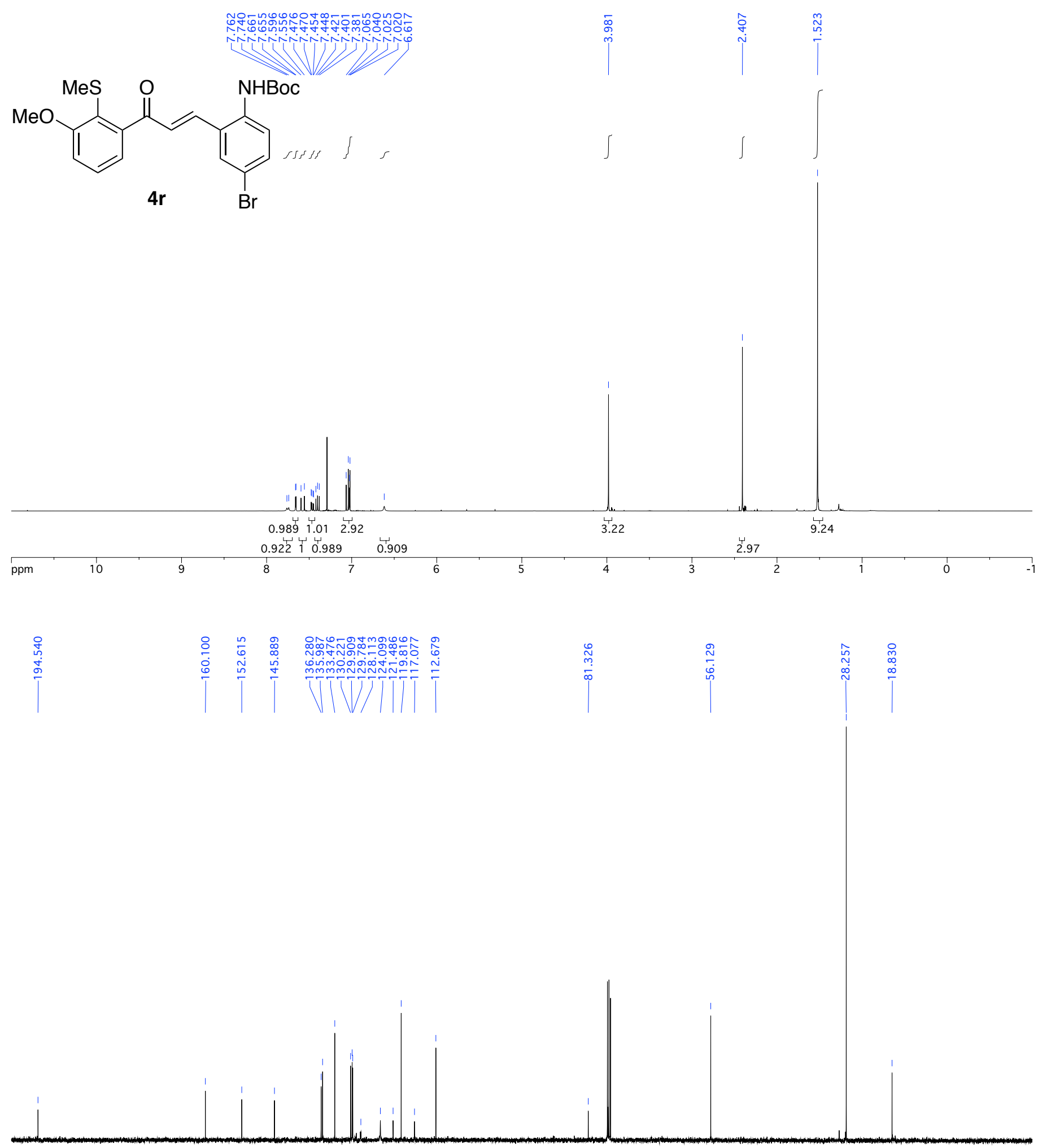

ppm

160

140

120

100

80

60

40

20 

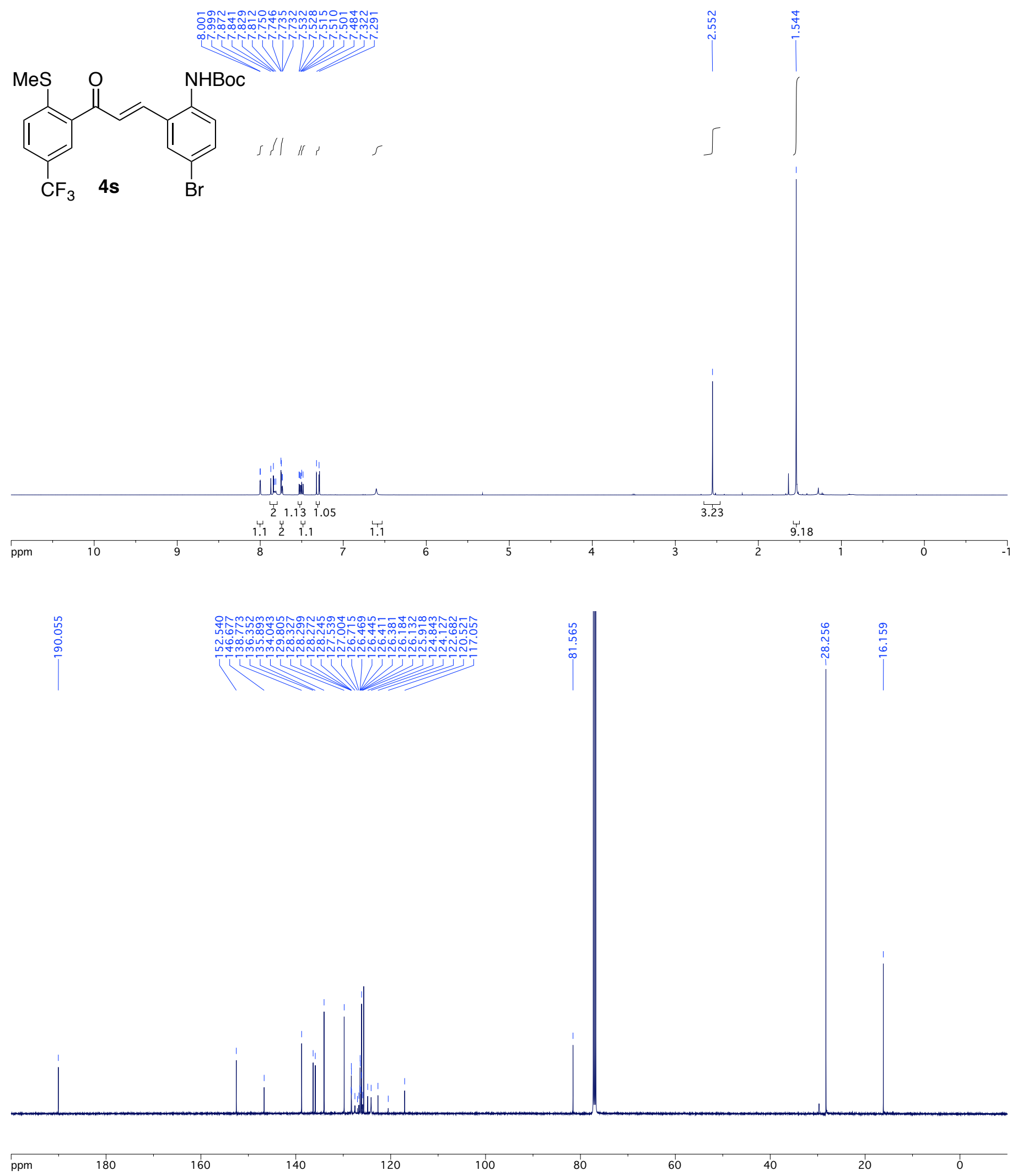


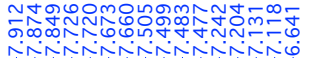
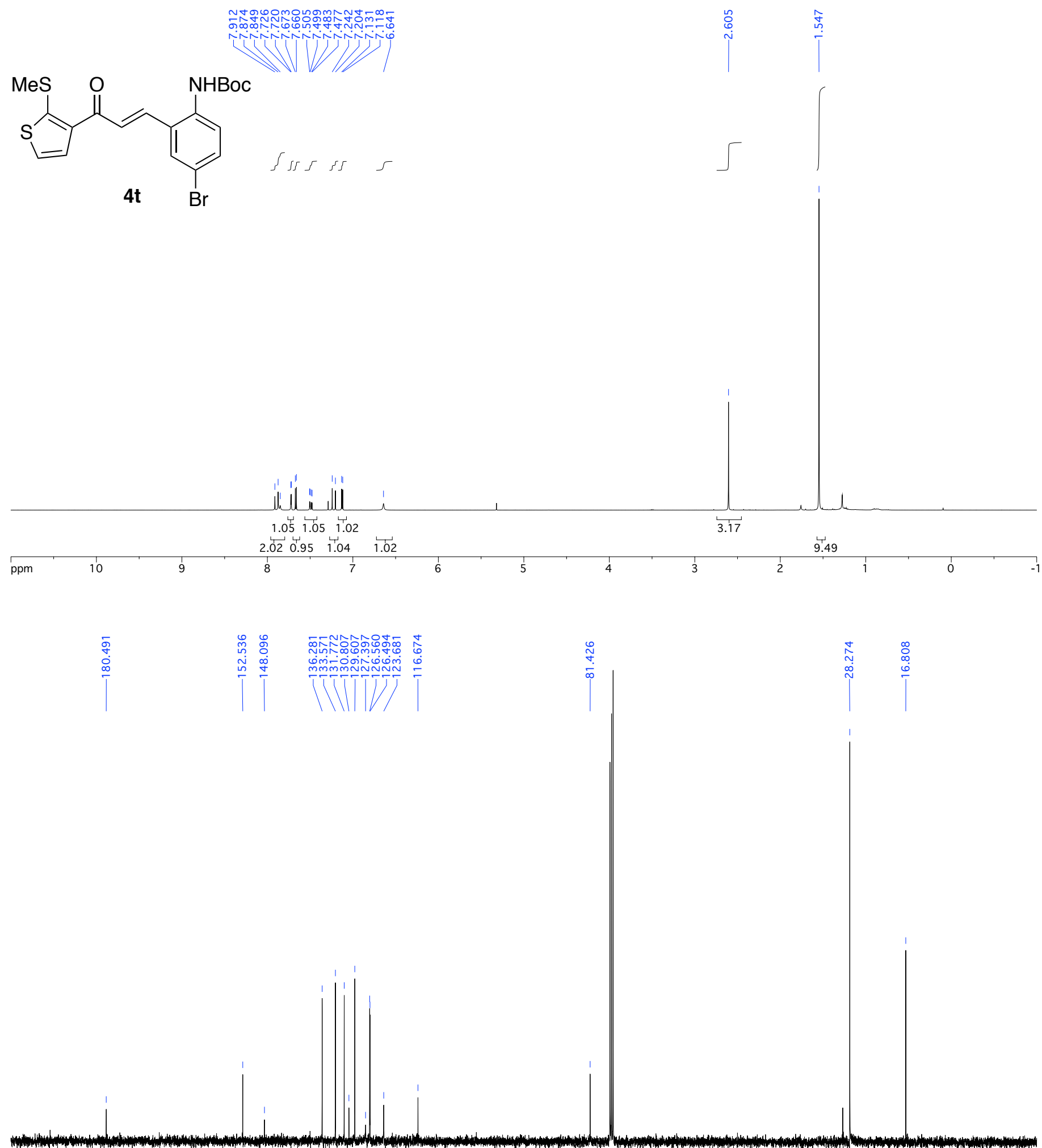

ppm

180

160

140

120

100

80

60

40

20 
<smiles>CCCCC(C(=O)C=CC=Cc1cc(Br)ccc1NC(=O)OCc1ccccc1)C(C)(C)C</smiles>

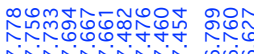

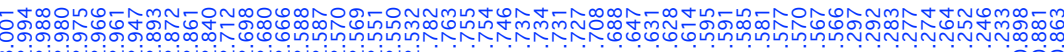

נS

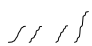

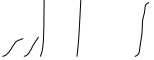

"II

$1.1^{4} 10.976$ 0.9740 .972
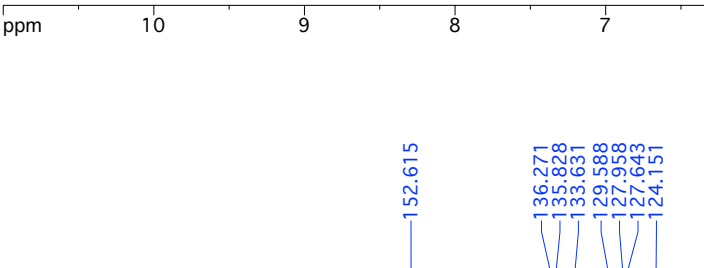

$\underset{\bar{c}}{\overline{\bar{c}}}$

$\stackrel{\substack{5 \\ \stackrel{m}{m}}}{i m}$

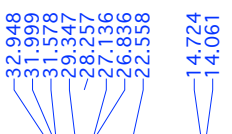

1.2511 .2

1.03 2 1.041 .07 1. 149.33

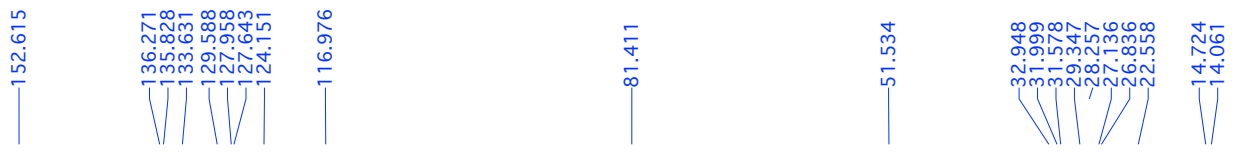

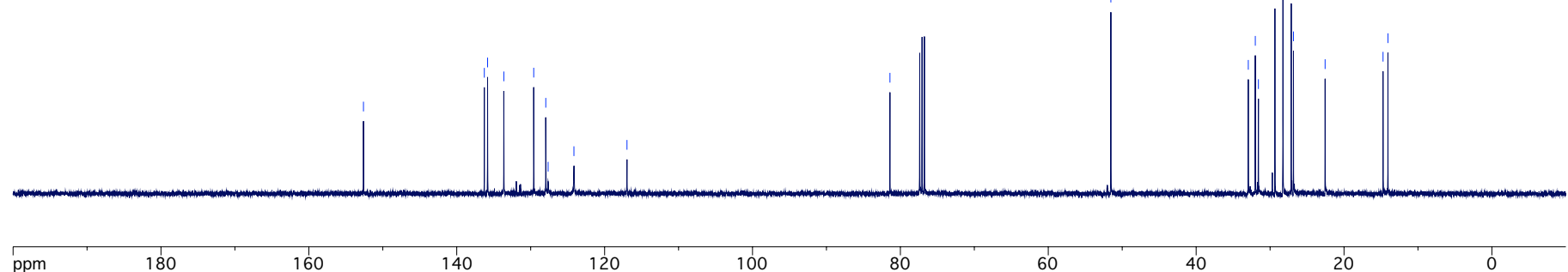




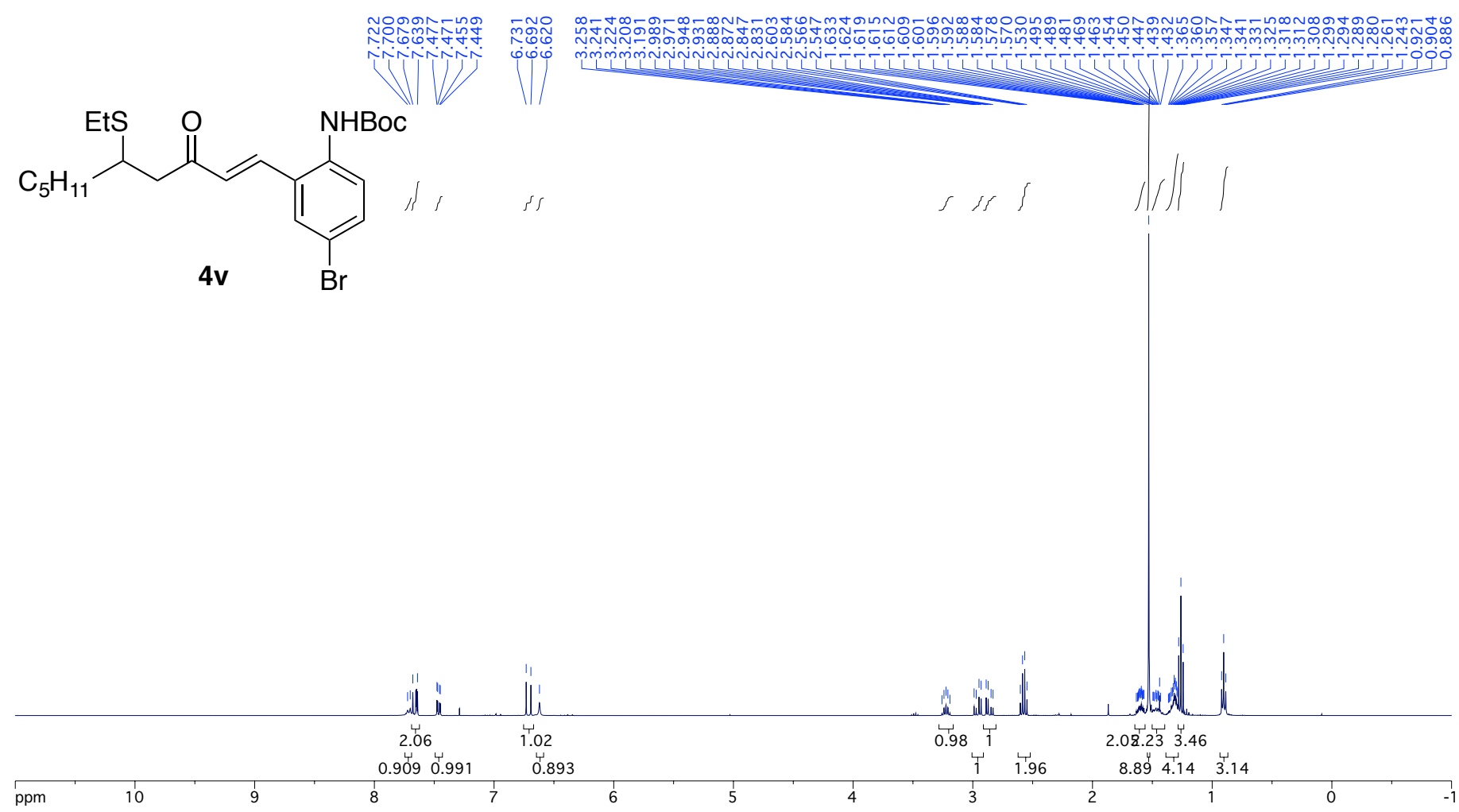

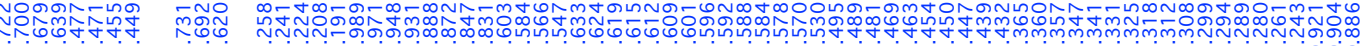

年

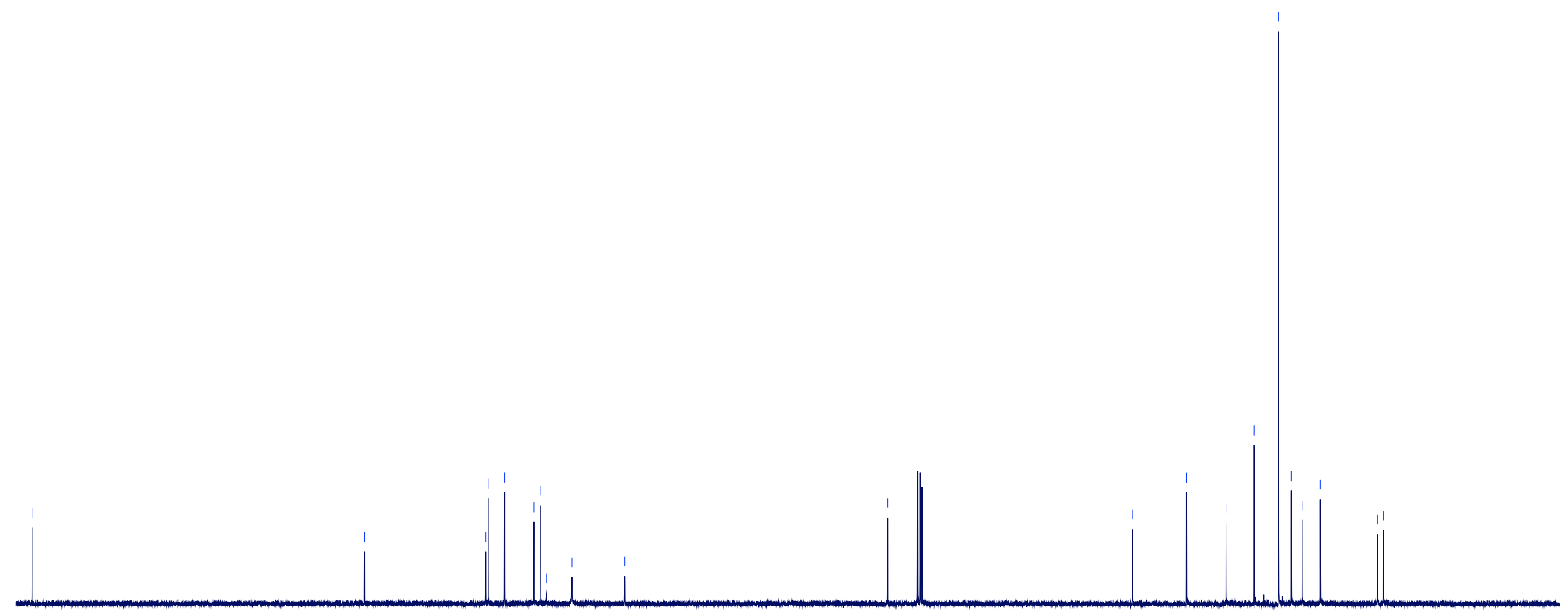

ppm

180

160

140

120

100

80

60

40

20 

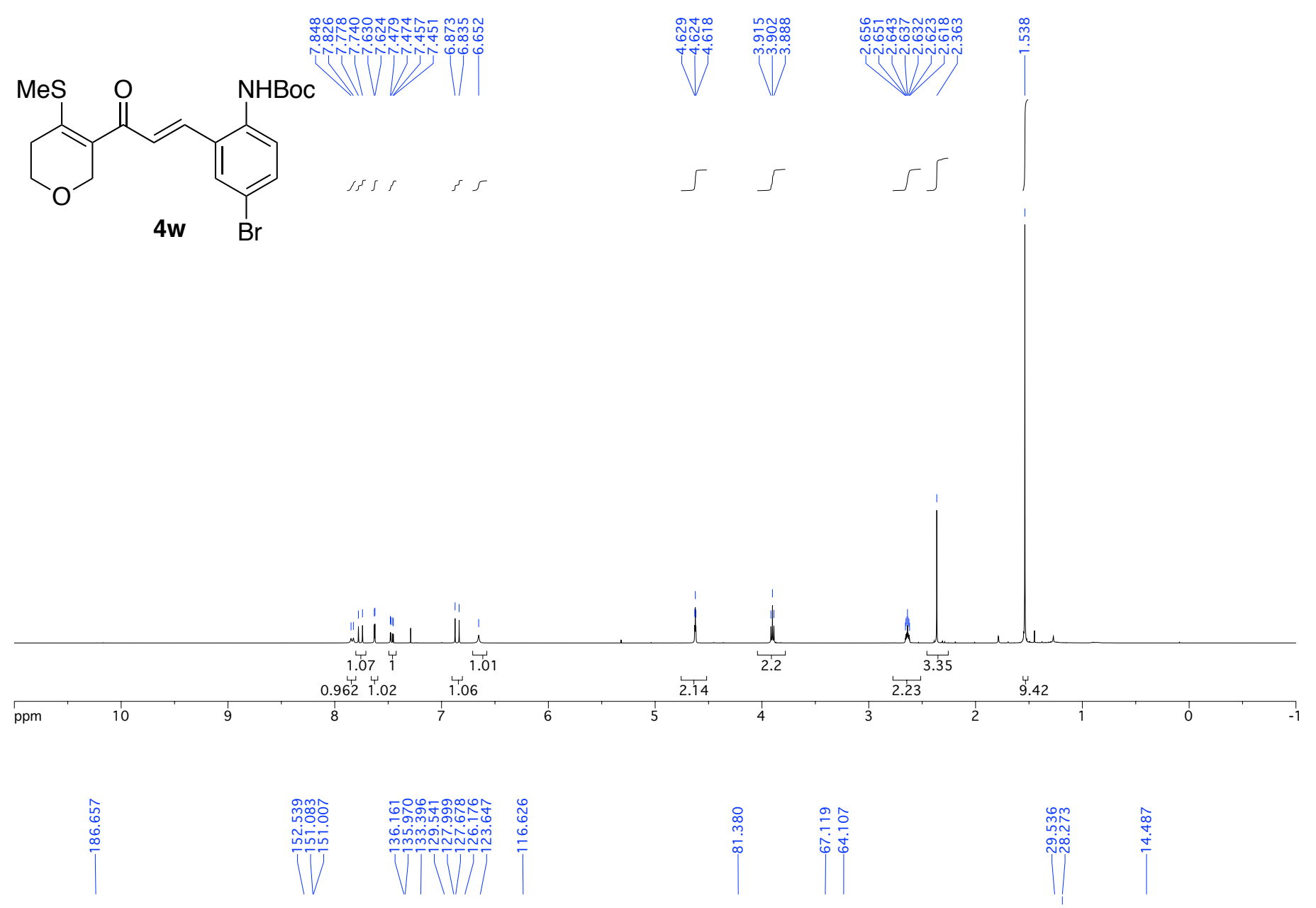

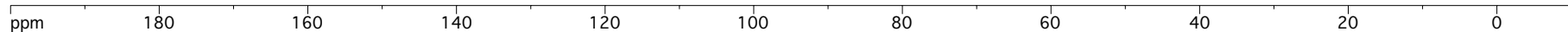



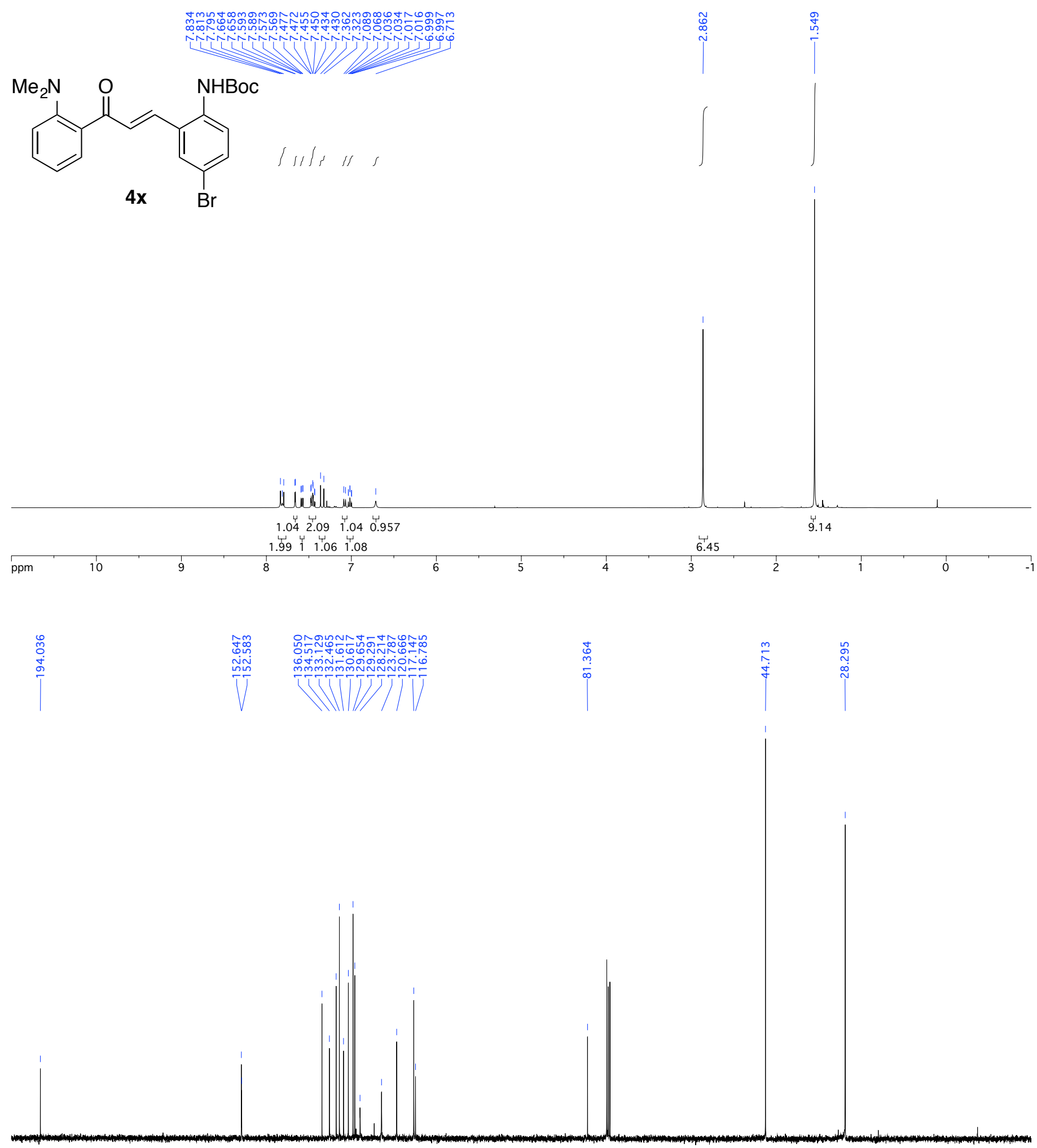

ppm

180

160

140

120

100

80

60

40

20 


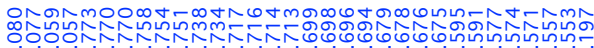

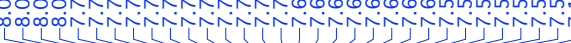

(n)

$4 y$

(1) Biblil

0.8180 .988 i $0.884^{4} 2.01^{4} \mathrm{a}^{4} .9710^{4} 0.975$

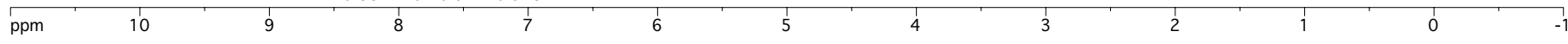

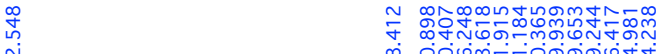

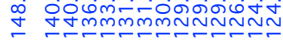

TTTTTJT)

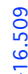

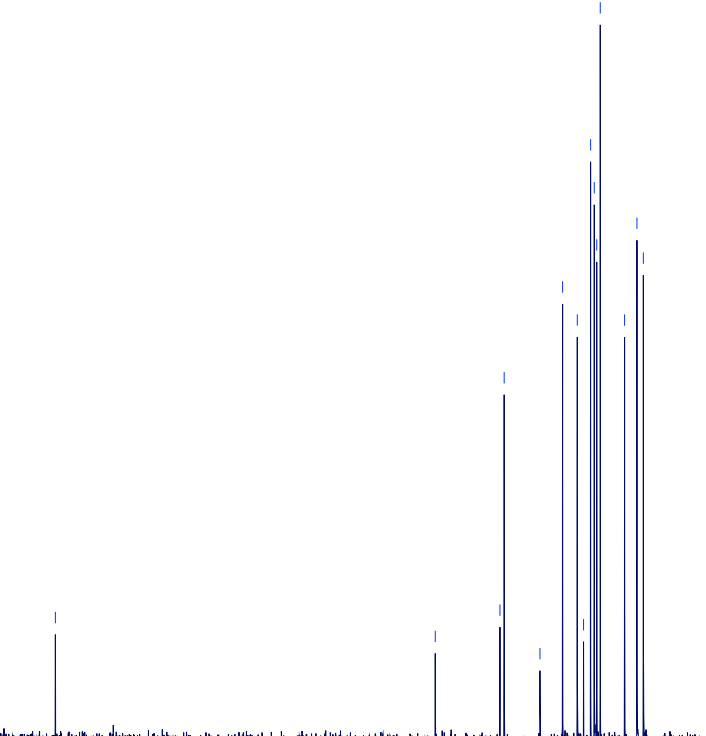

ppm

180

160

140

120

100

80

60

40

20 

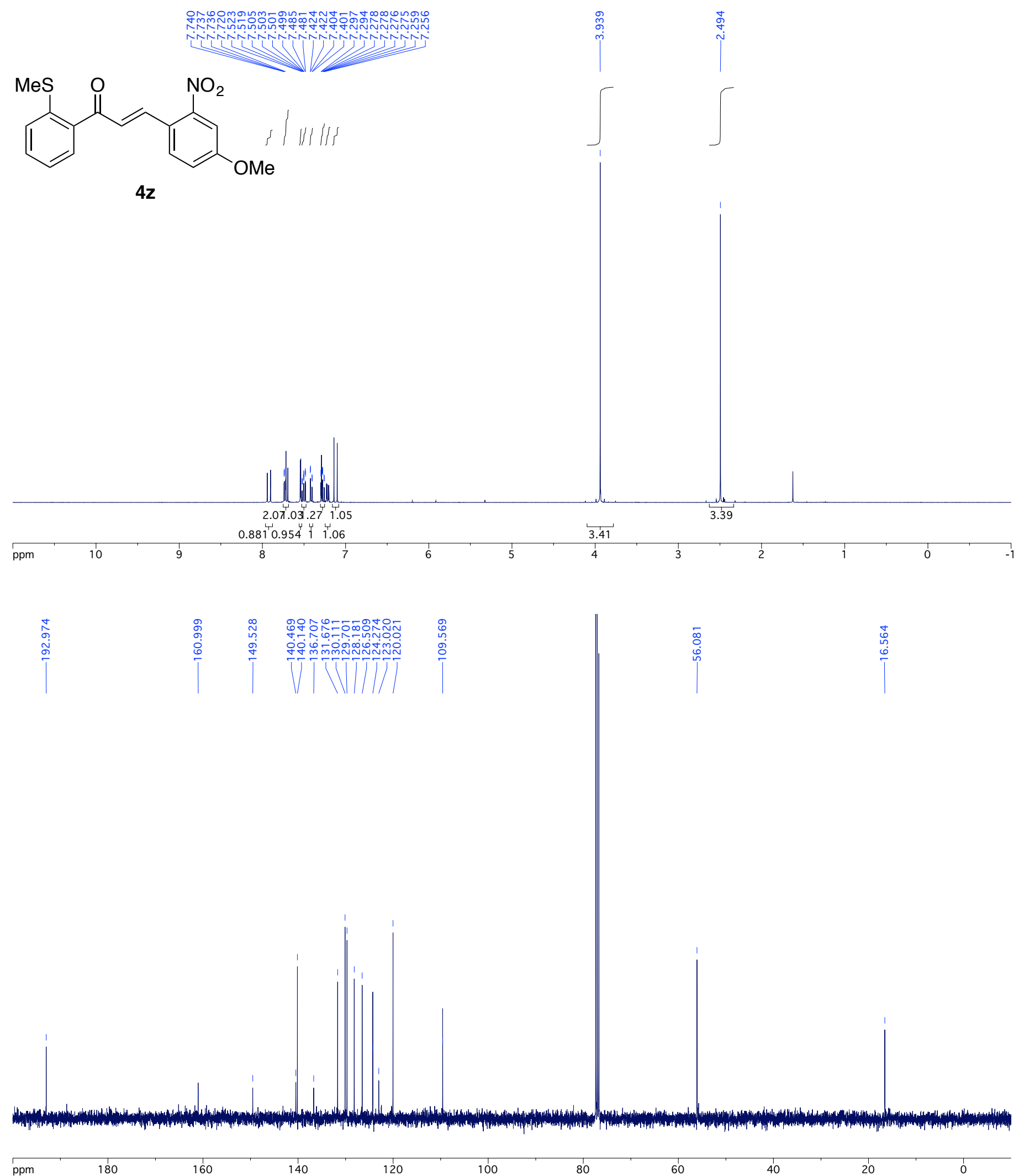


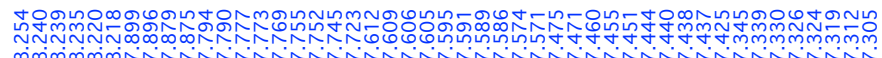

Pestifl

$5 a$
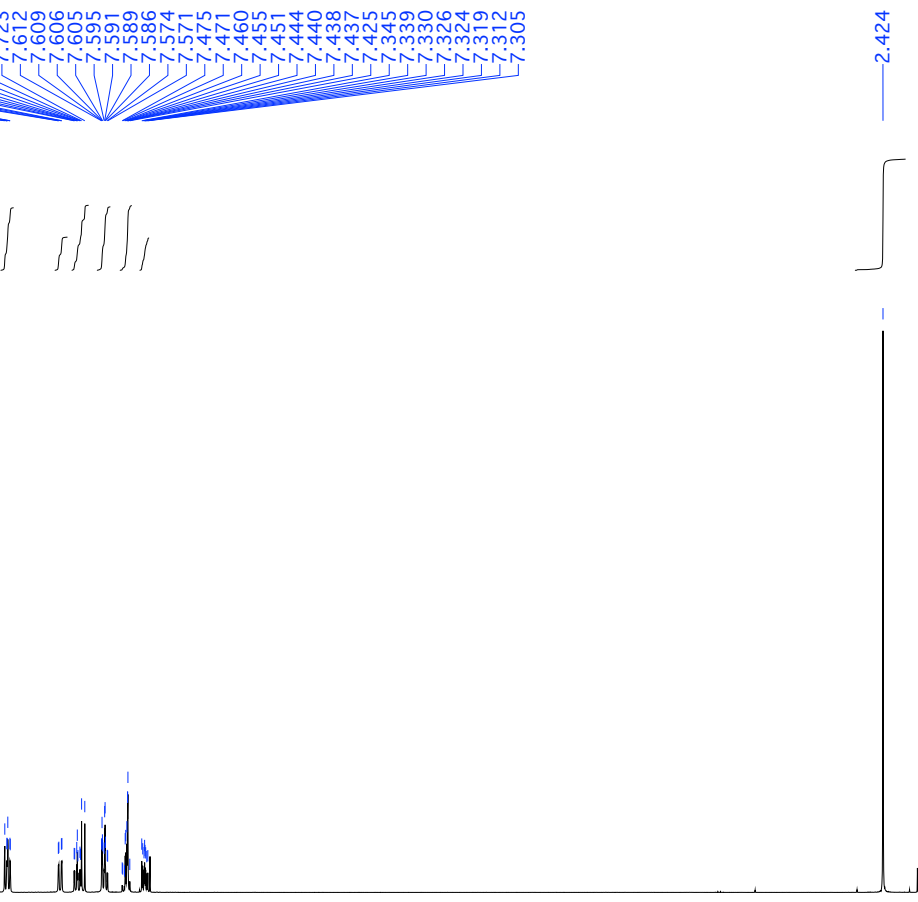

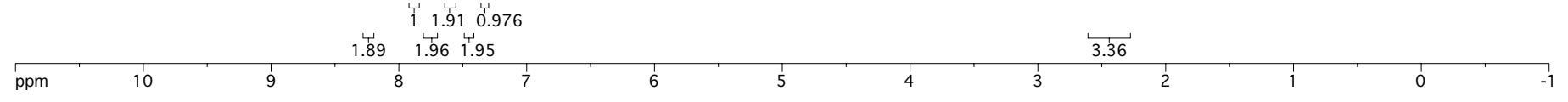

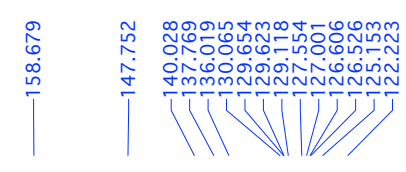

$\infty$
0
0
6
$\dot{6}$

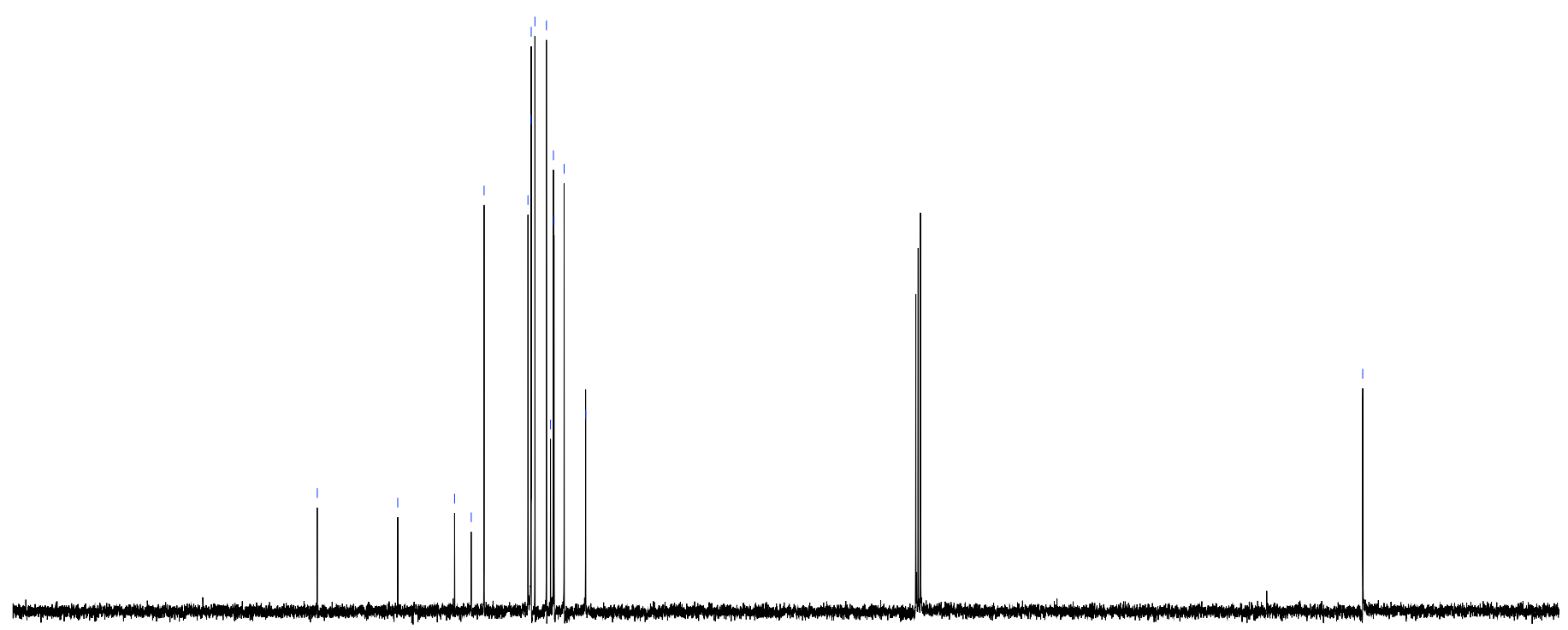

ppm

180

160

140

120

100

80

60

40

20 

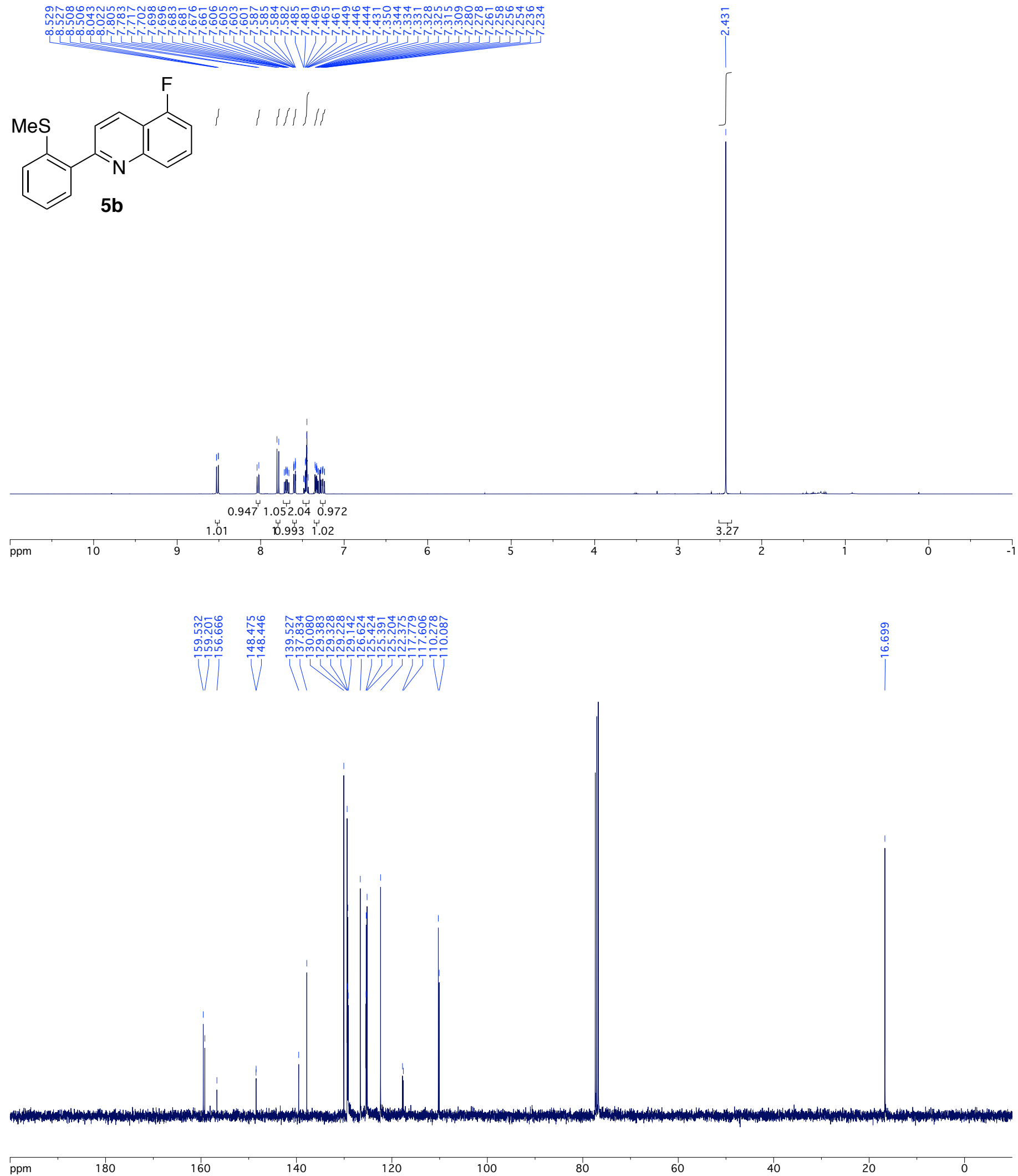

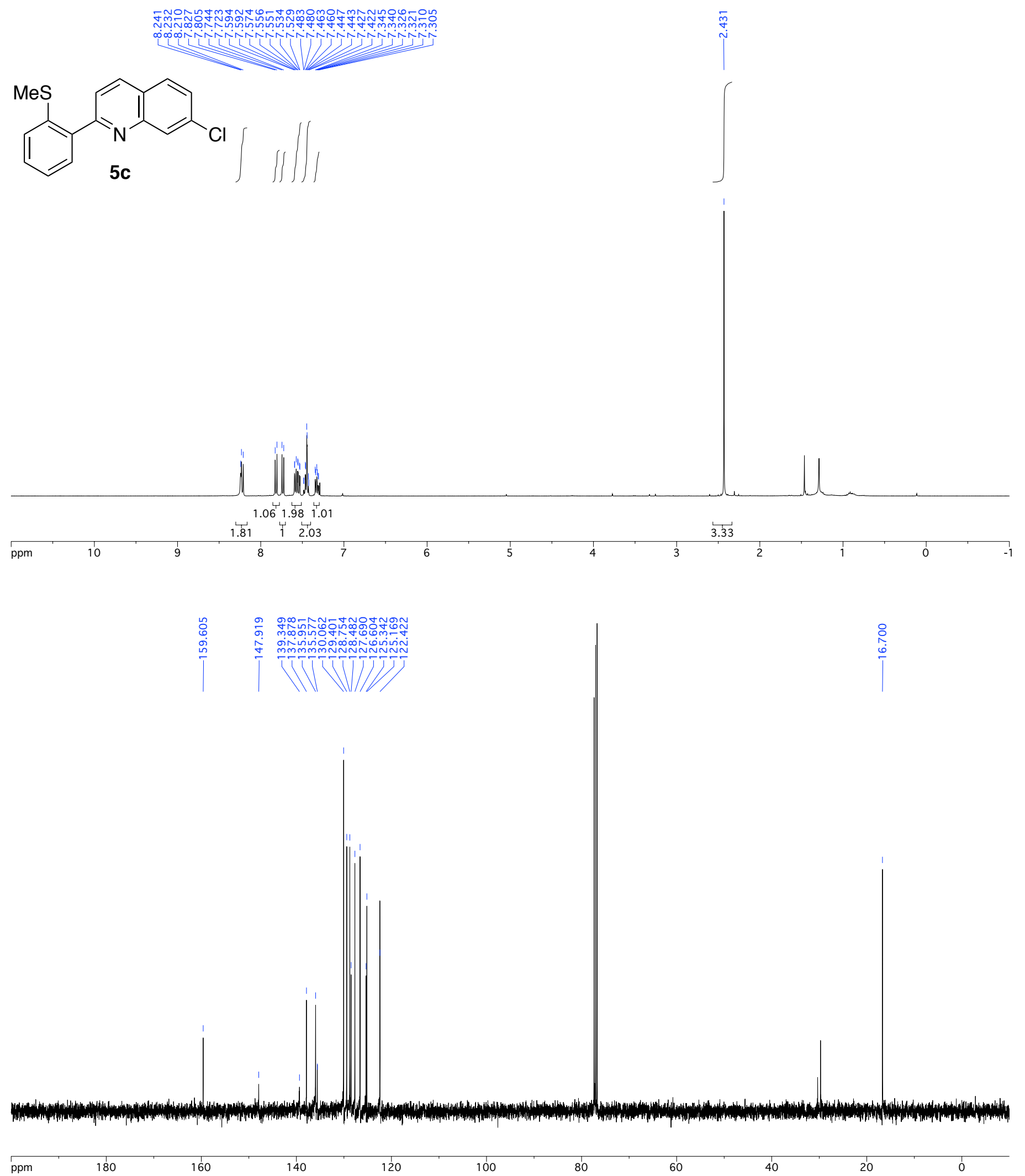


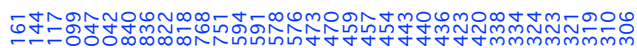
-
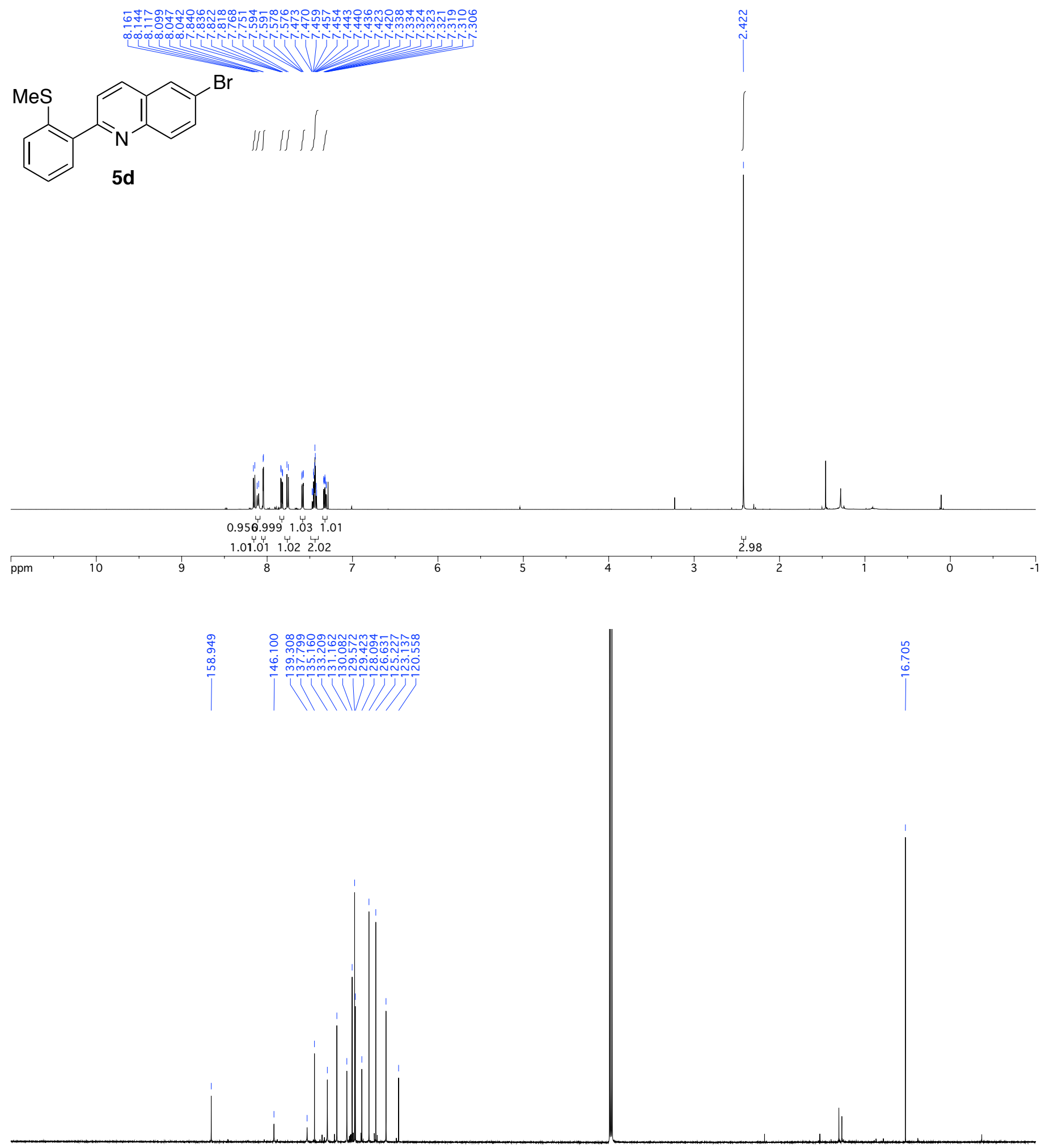

ppm

180

160

140

120

100

80

60

40

20 
นمN mom

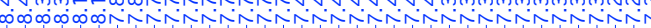

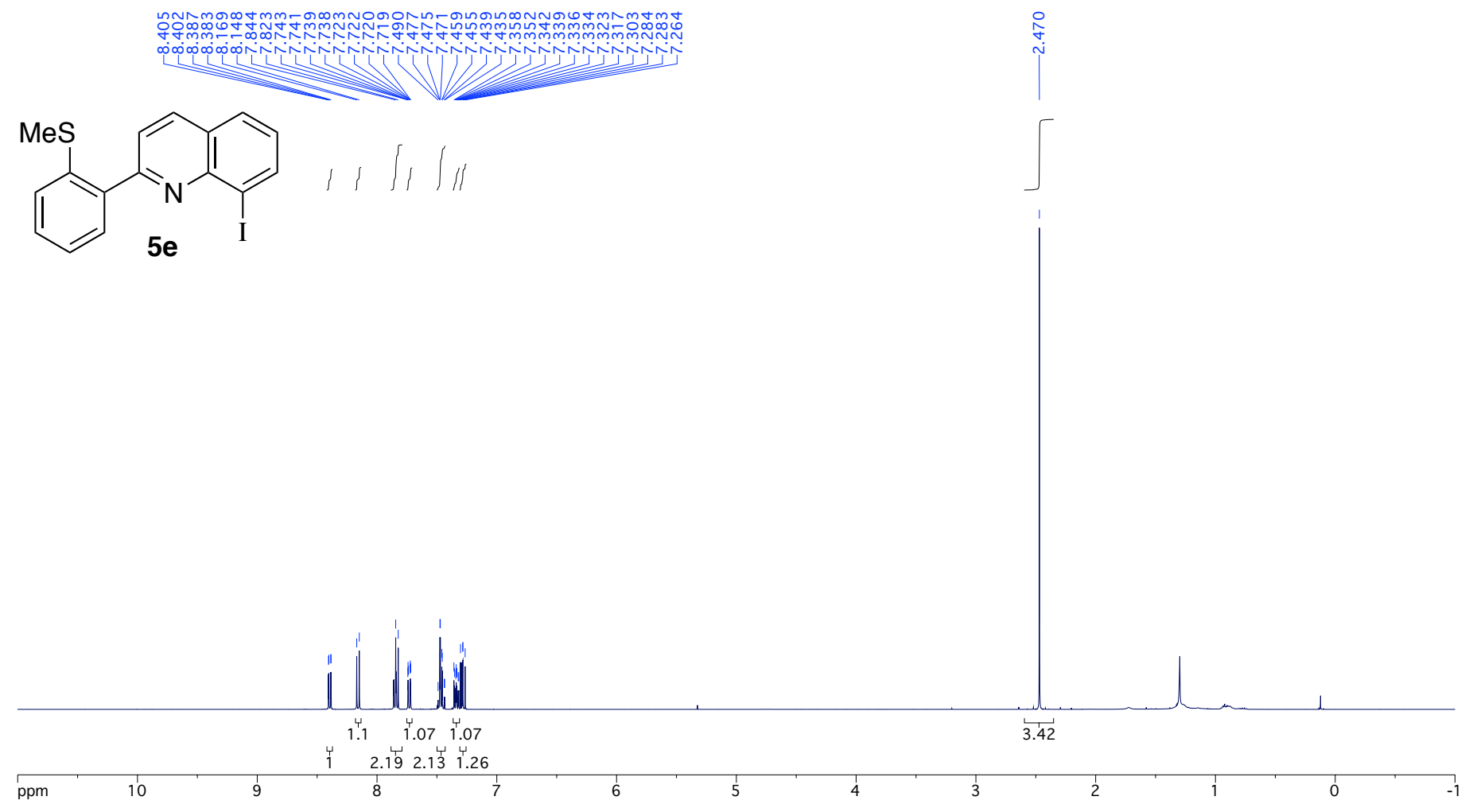

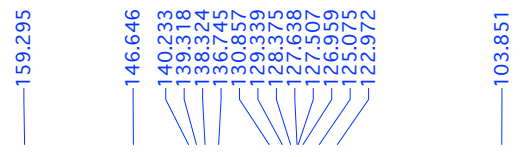
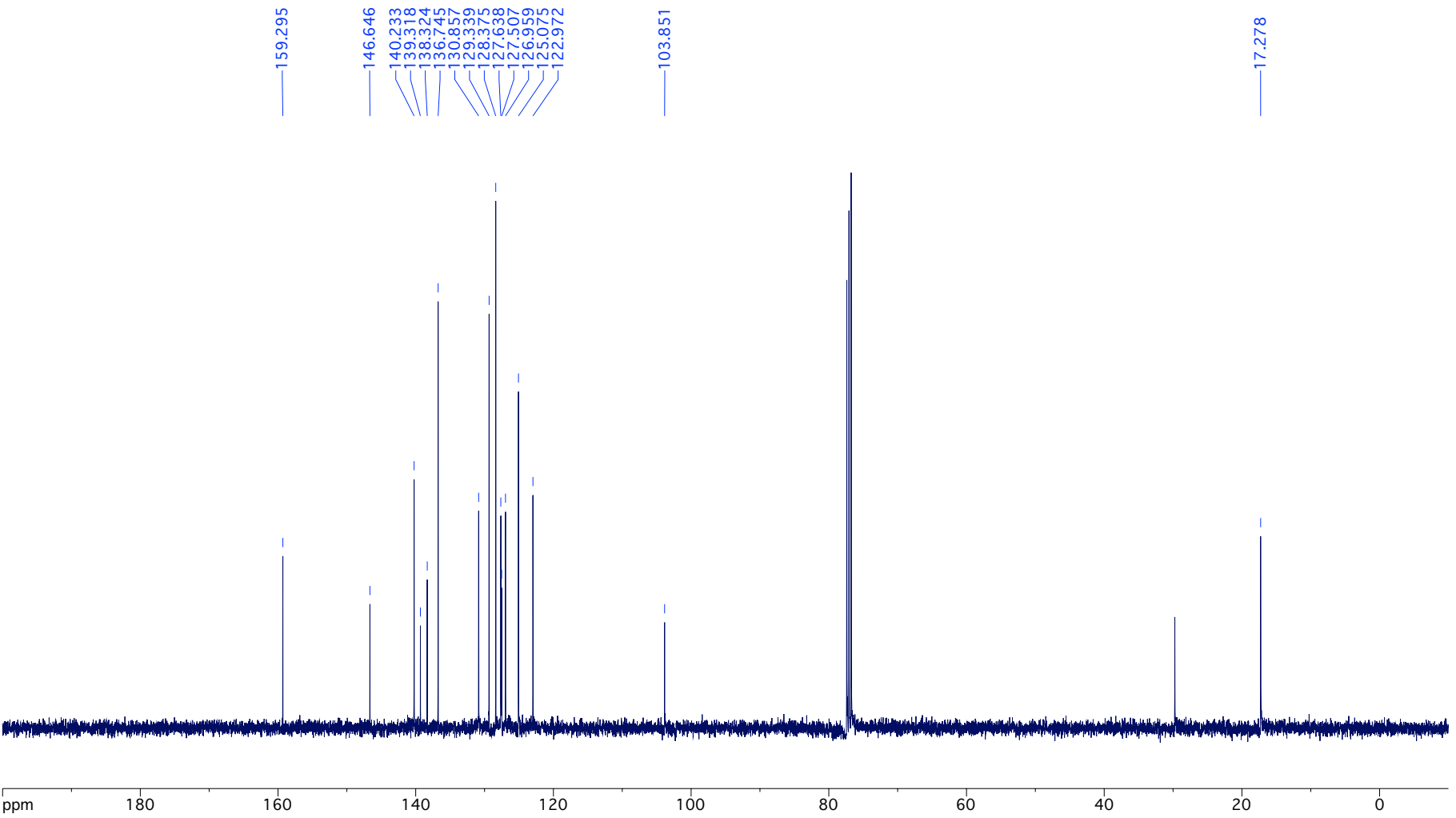

ppm

180

160

140

120

80 


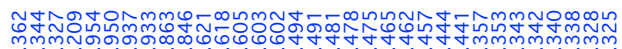
mем
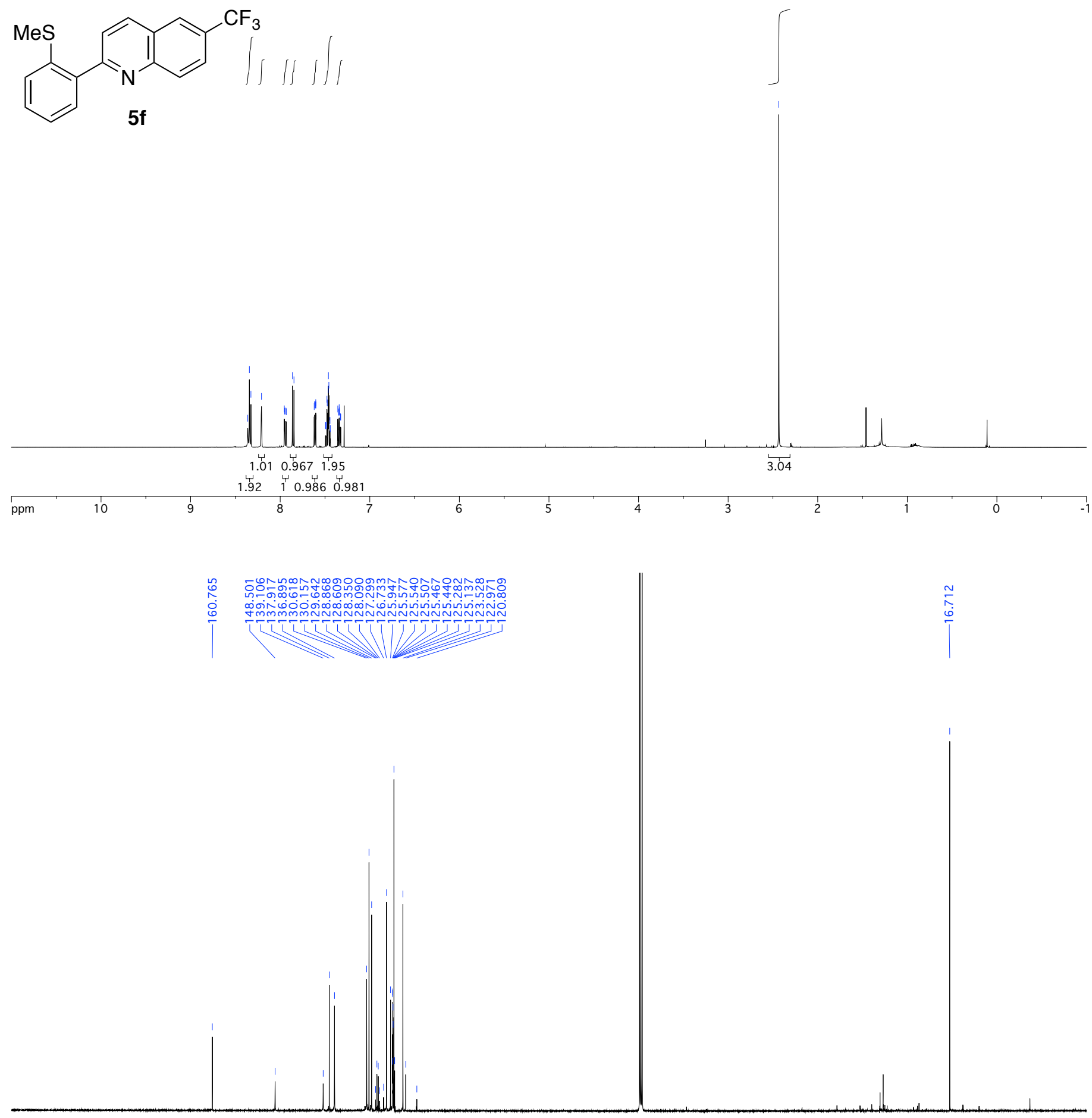

ppm

180

160

140

120

100

80

60

40

20 

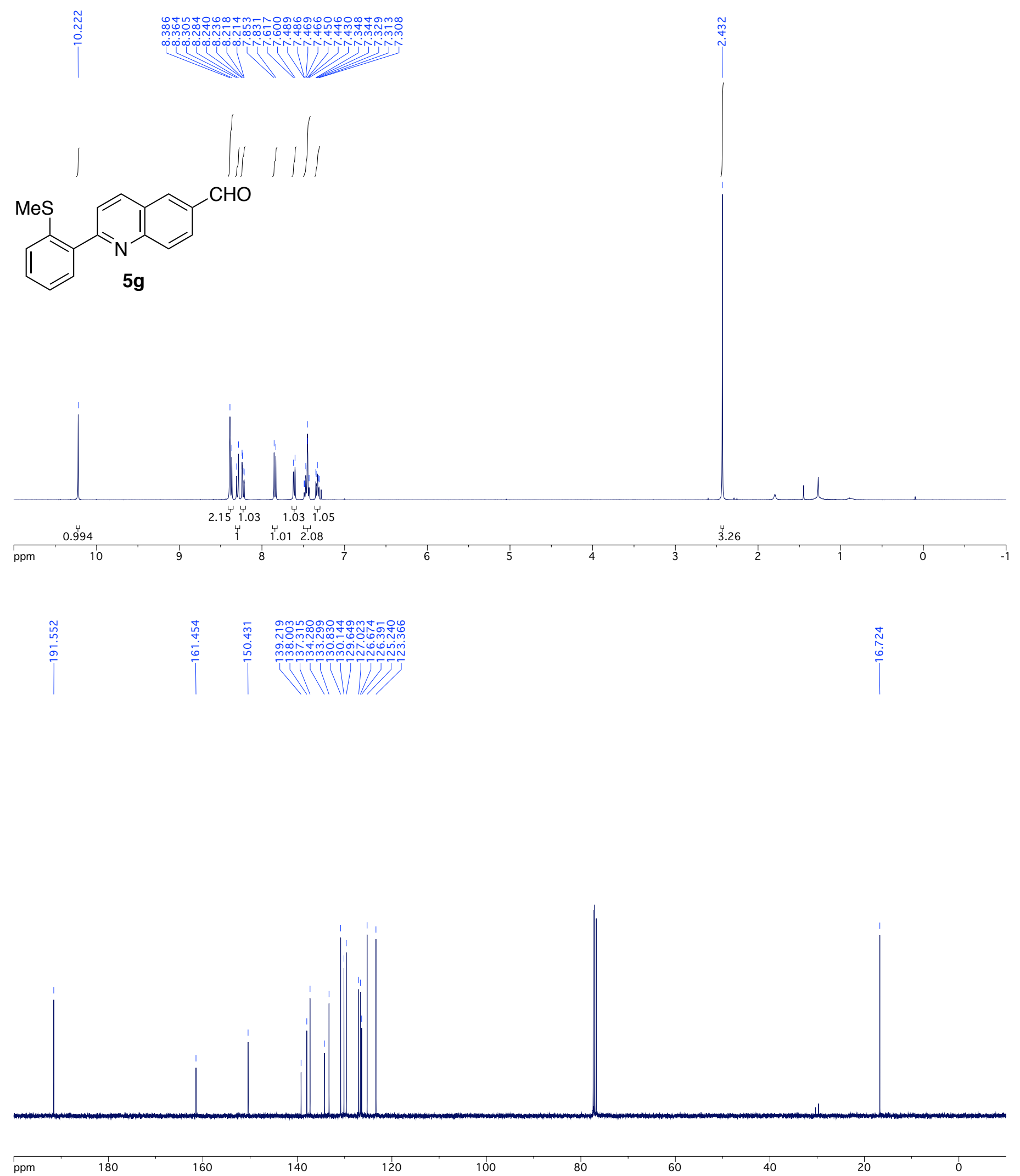

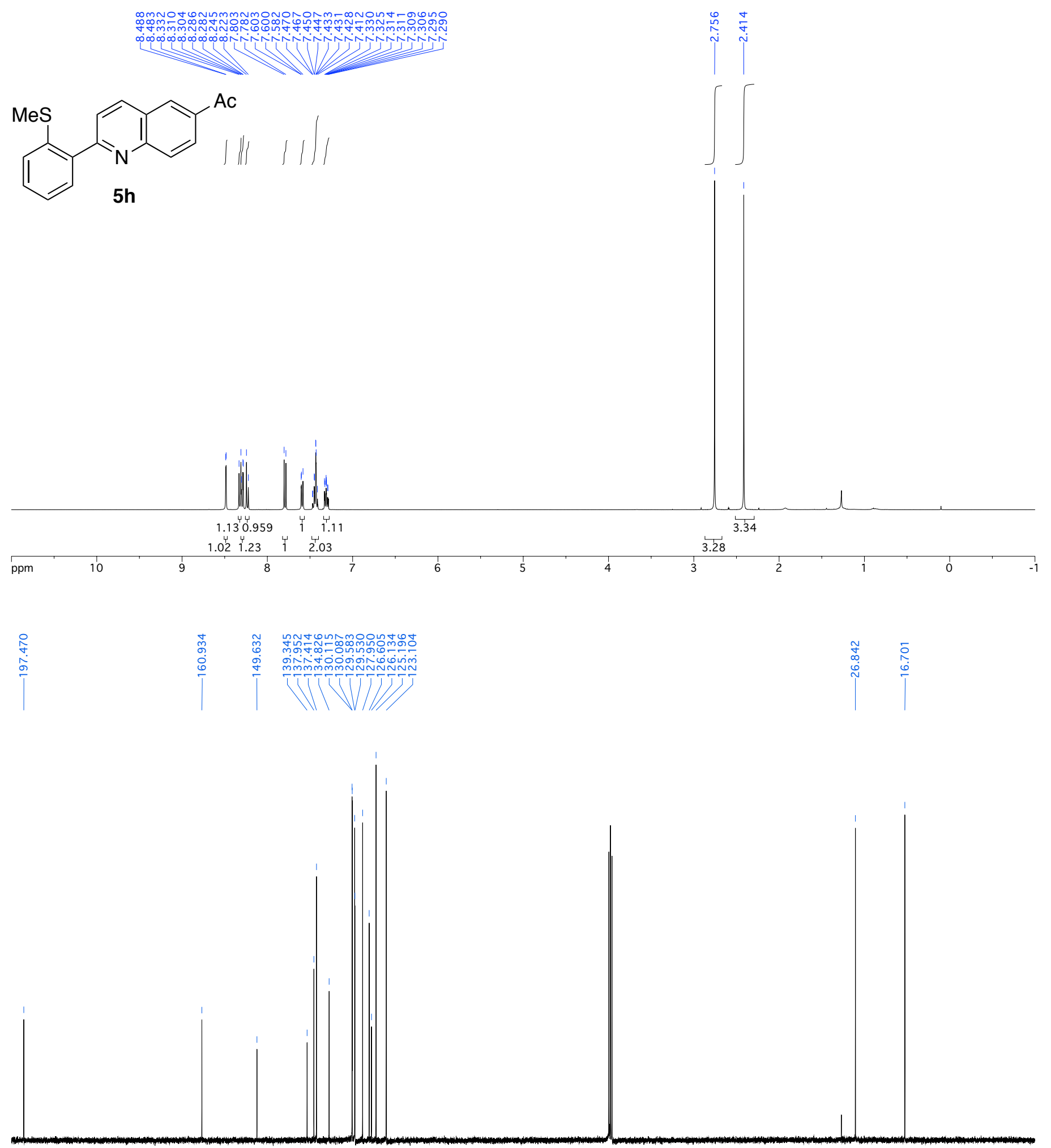

ppm

180

160

140

120

100

80

60

40

20 

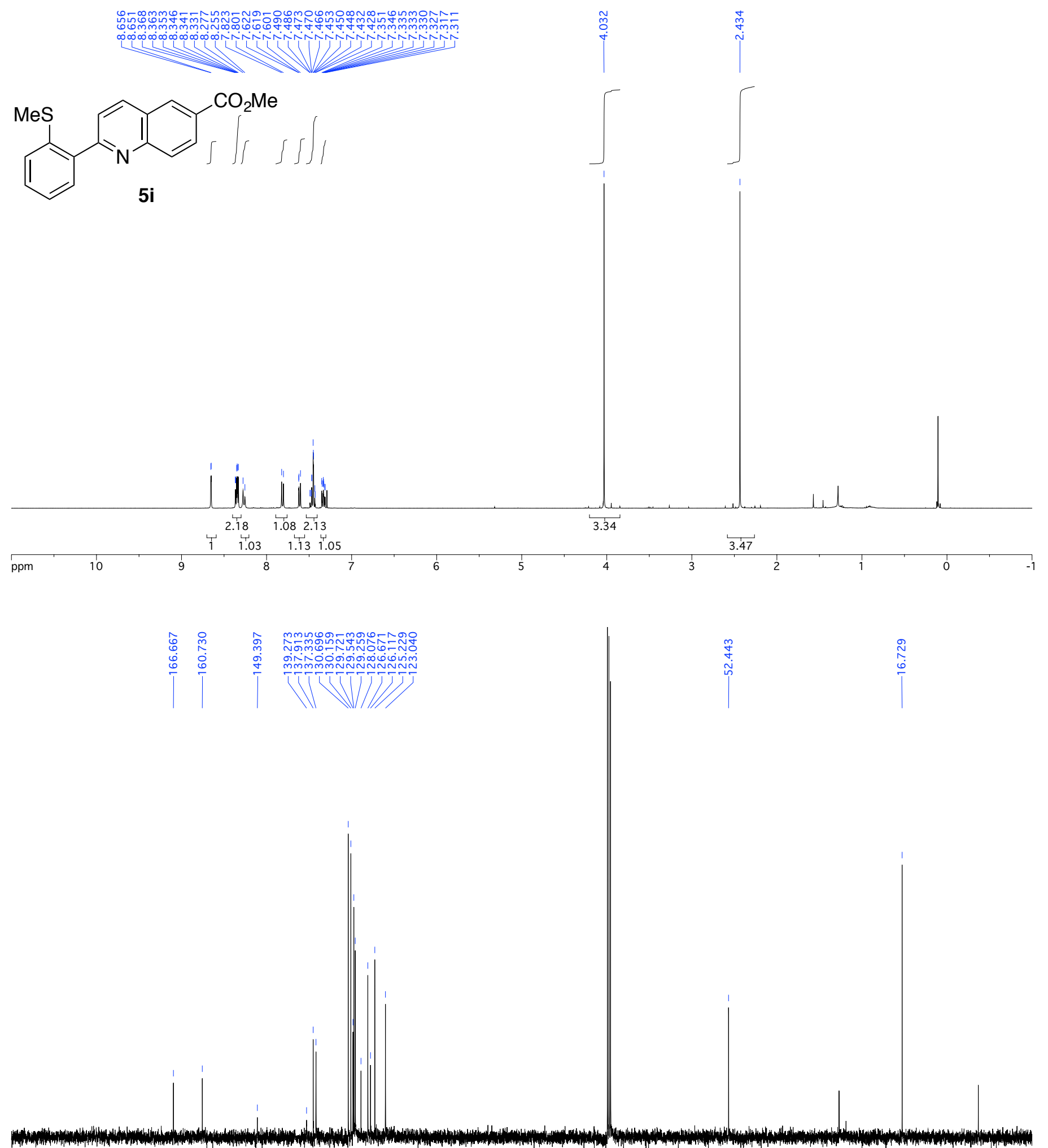

ppm

180

160

140

120

100

80

60

40

20 


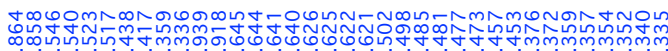

NiNiNiNi

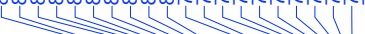

$\left.\iiint\right\} \int 1 / 1$<smiles>CS(=O)(=O)c1ccccc1-c1ccc2cc([N+](=O)[O-])ccc2n1</smiles>
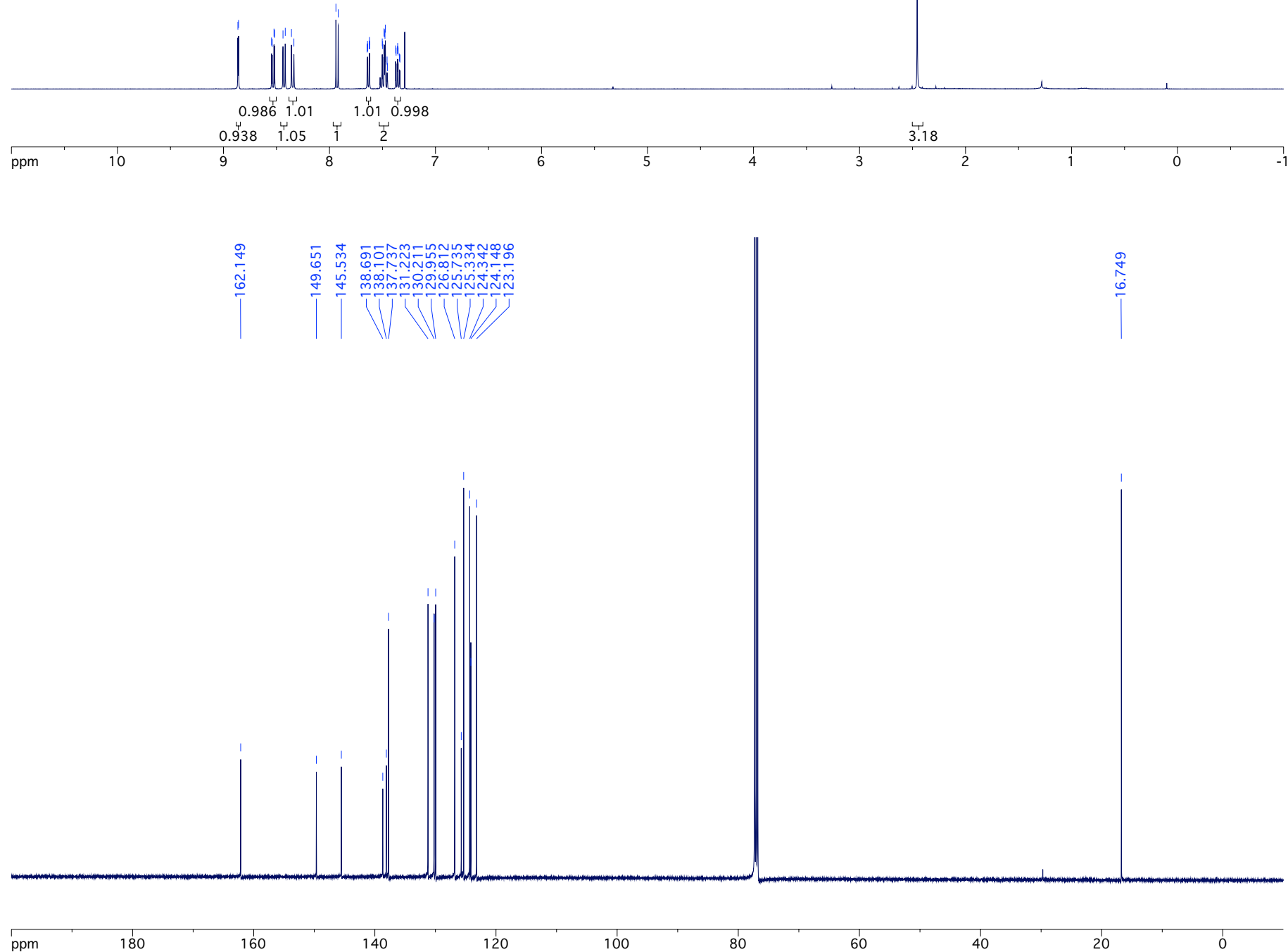


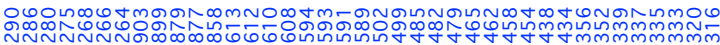
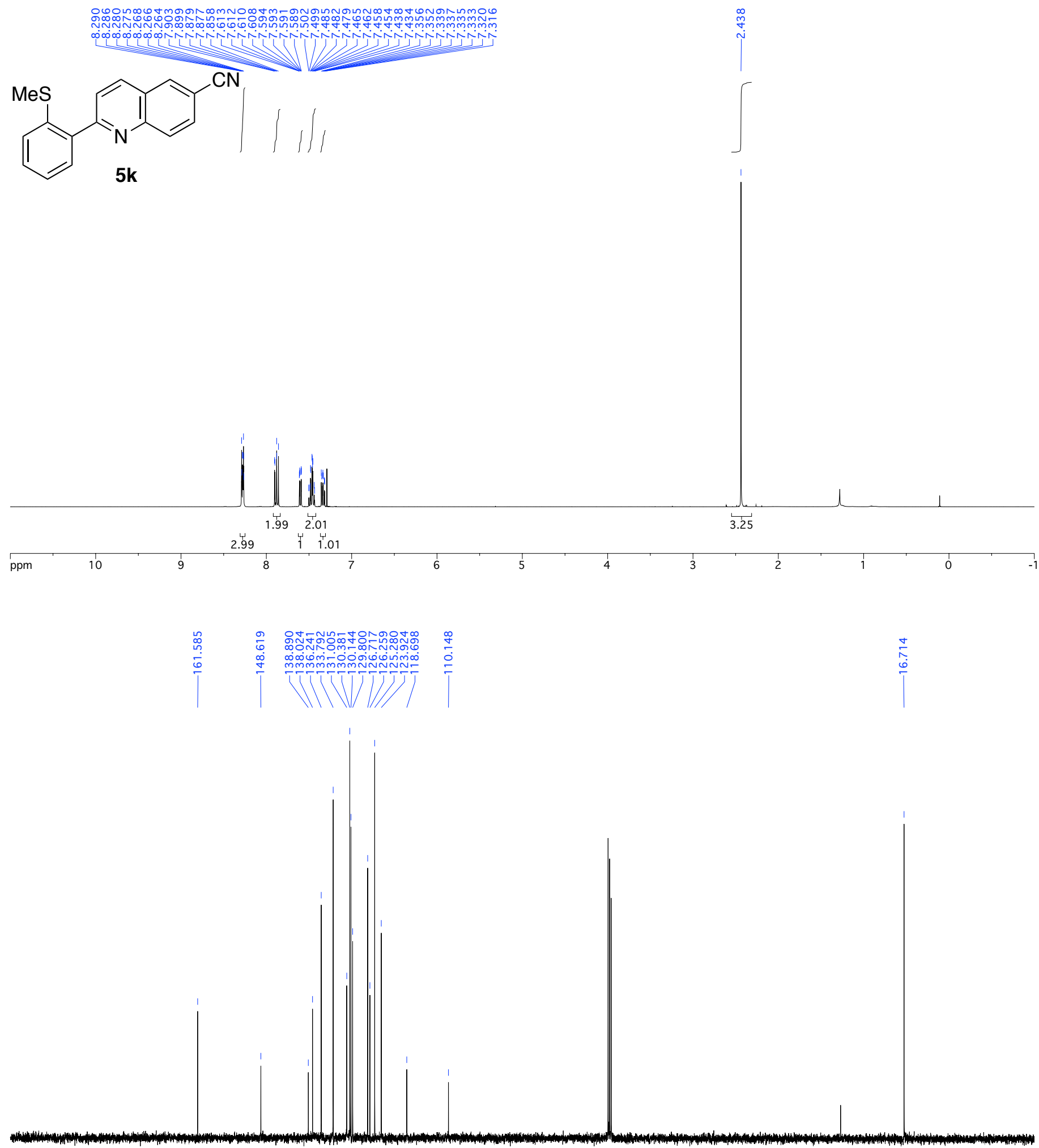

ppm

180

160

140

120

100

80

60

40

20 

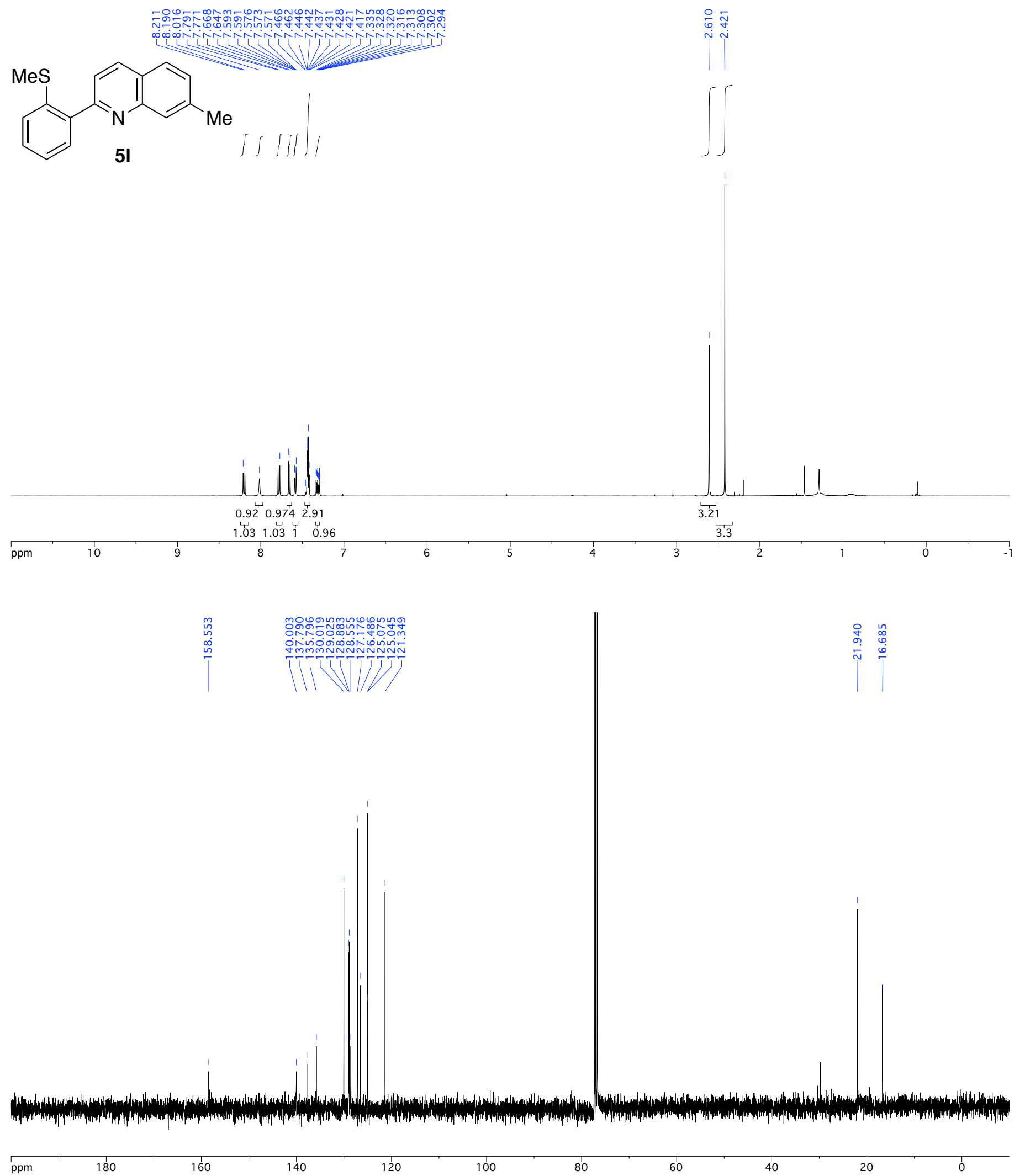
<smiles>CSc1ccccc1-c1ccc2cc(N)ccc2n1</smiles>
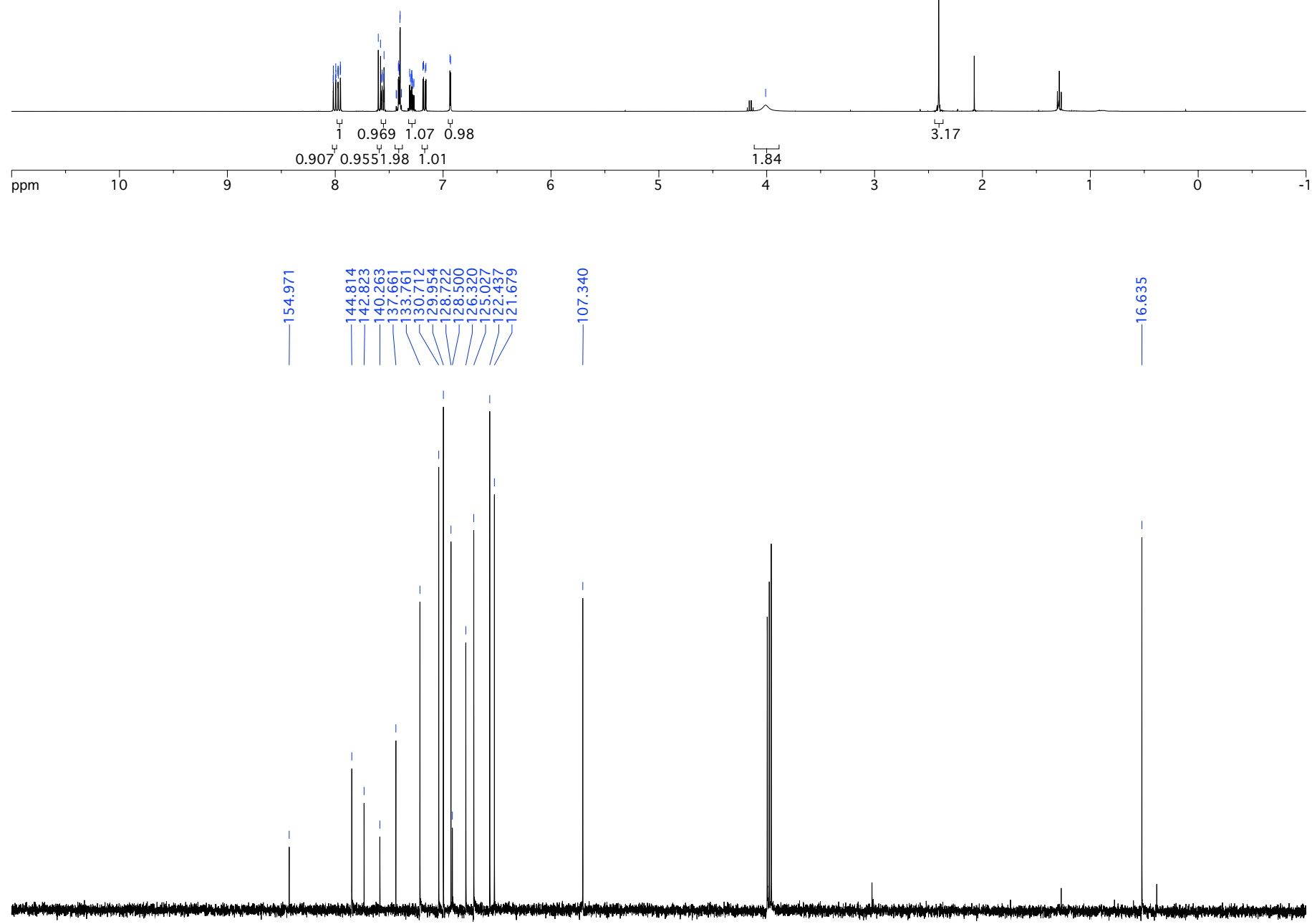

ppm

180

160

140

120

100

80

60

40

20 


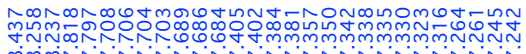

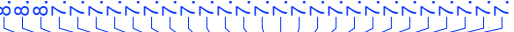

5n OH

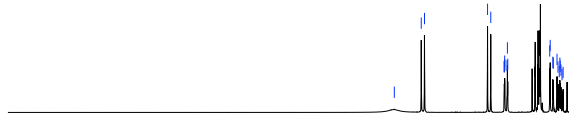

$1.12 \quad 1.08^{4} 1.066^{4}$ $\begin{array}{lrrr}0.806 & 1.09^{4} & 3.27 & 4 \\ 0.07\end{array}$ 3.43

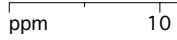

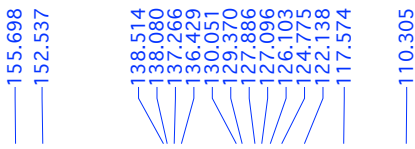

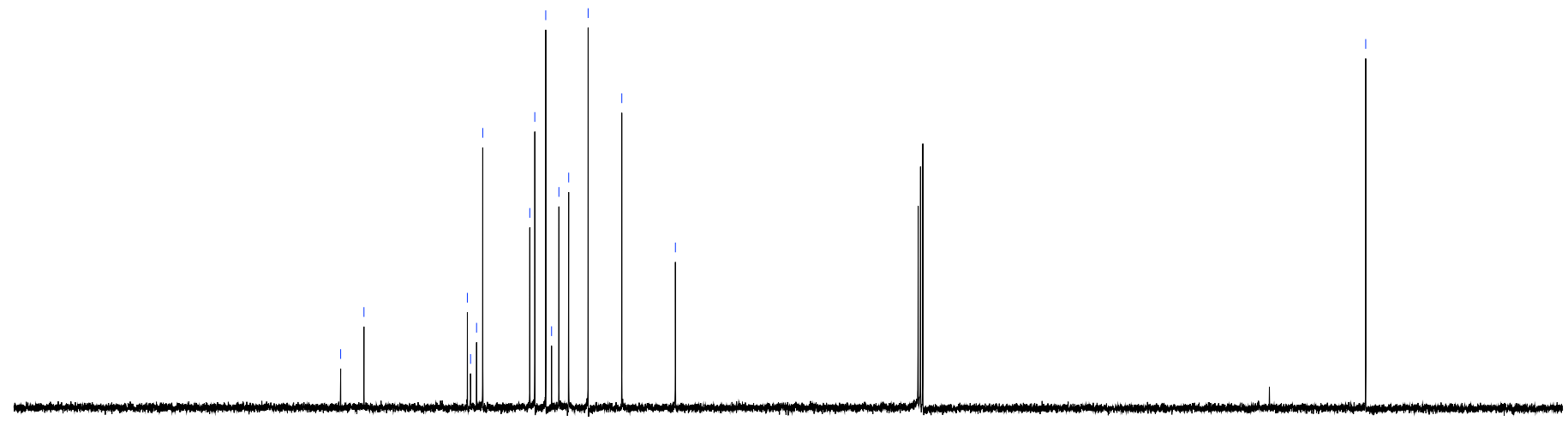

ppm

180

160

140

120

100

80

60

40

20 

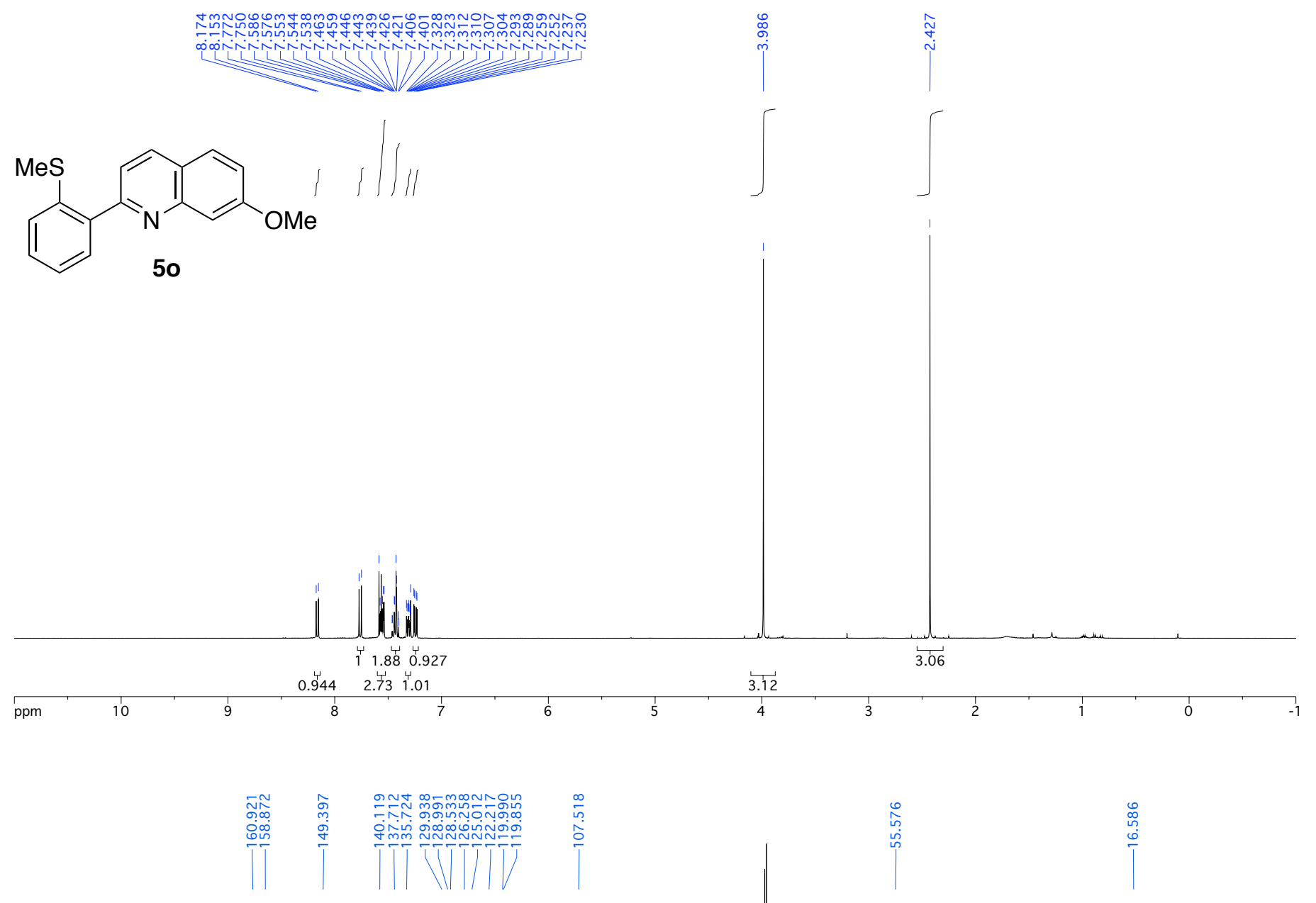

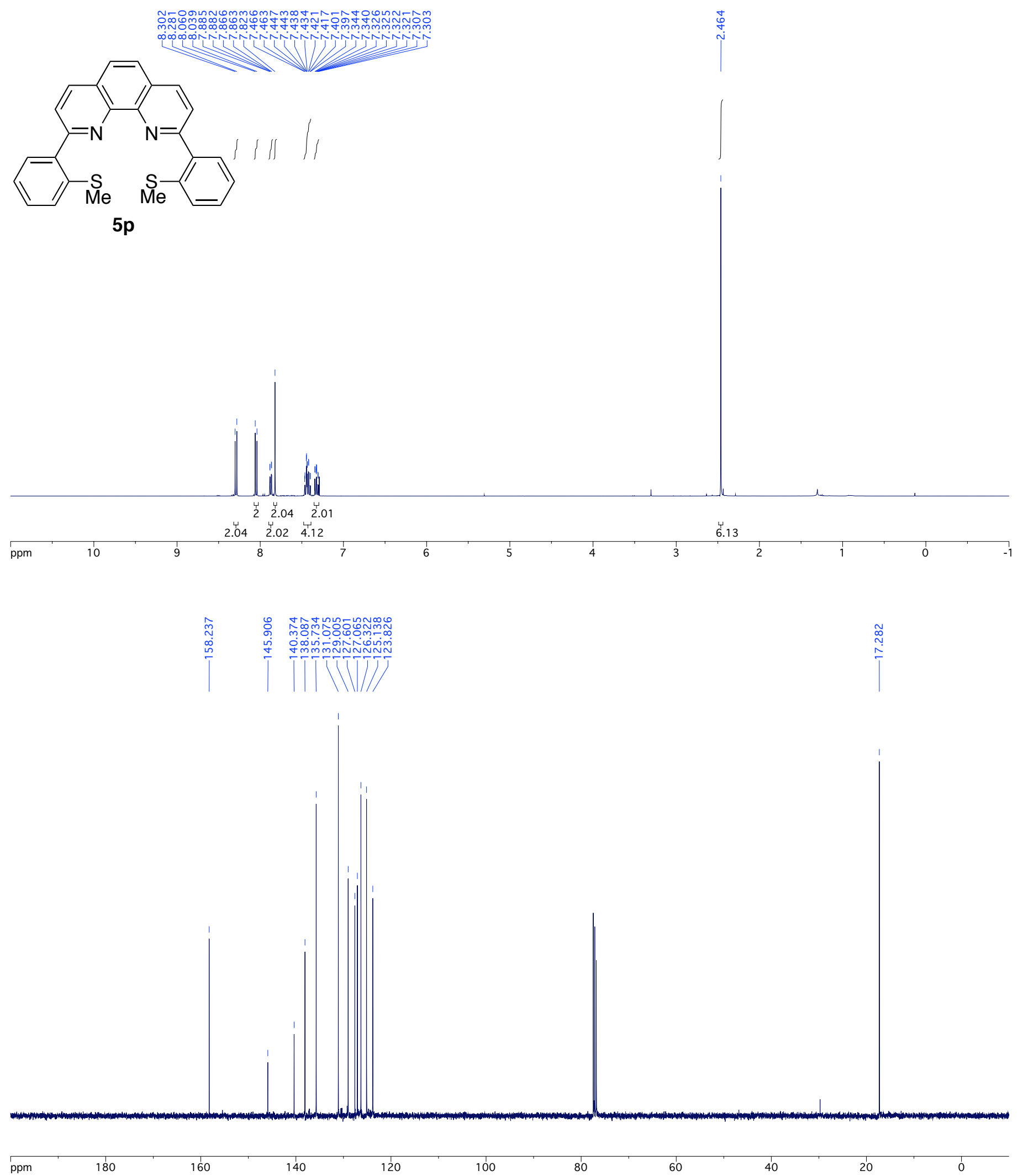


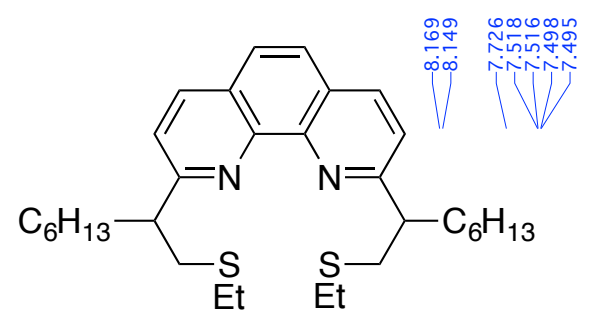

$5 q$

бㅇำ
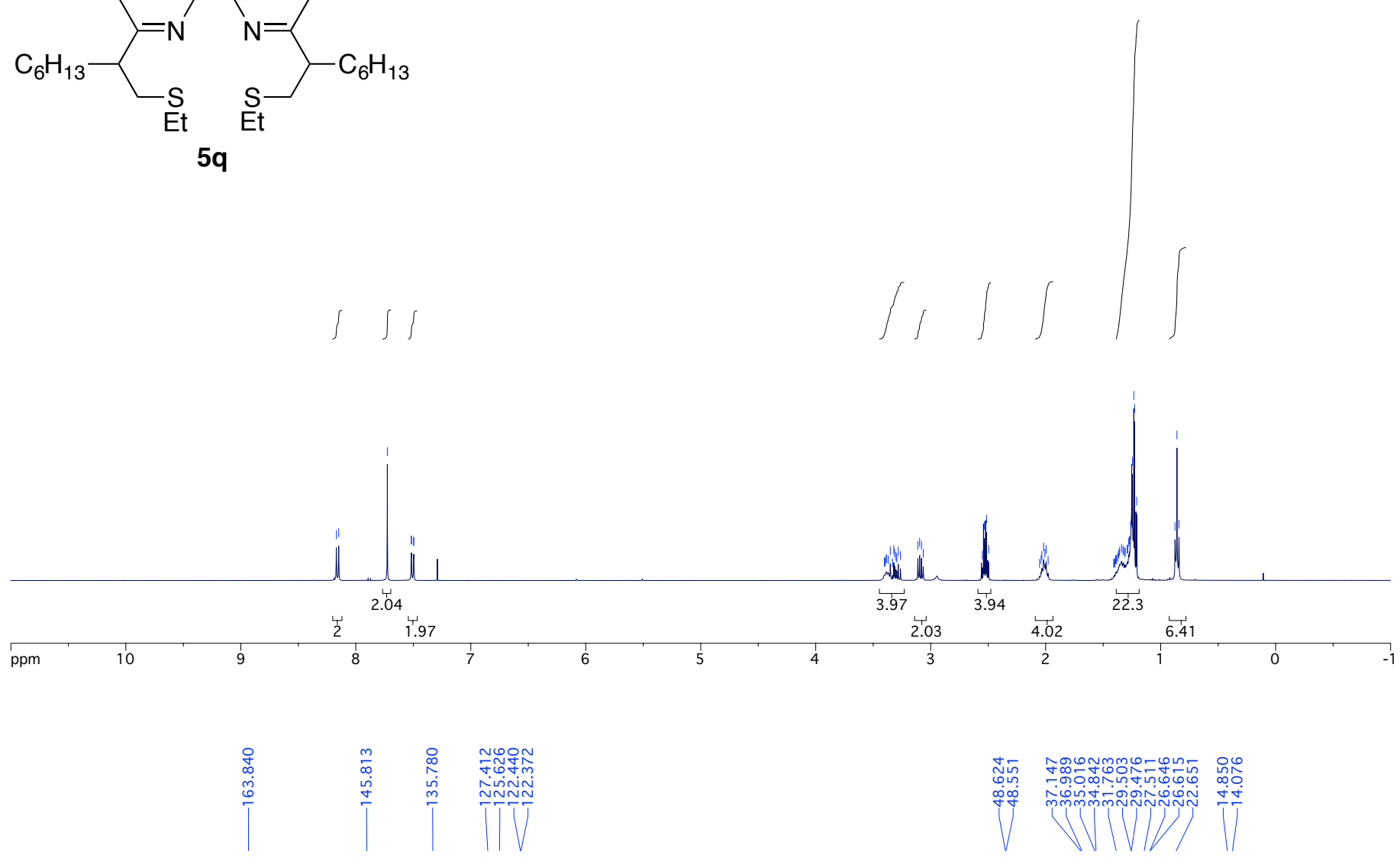

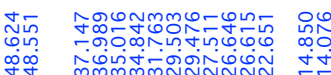

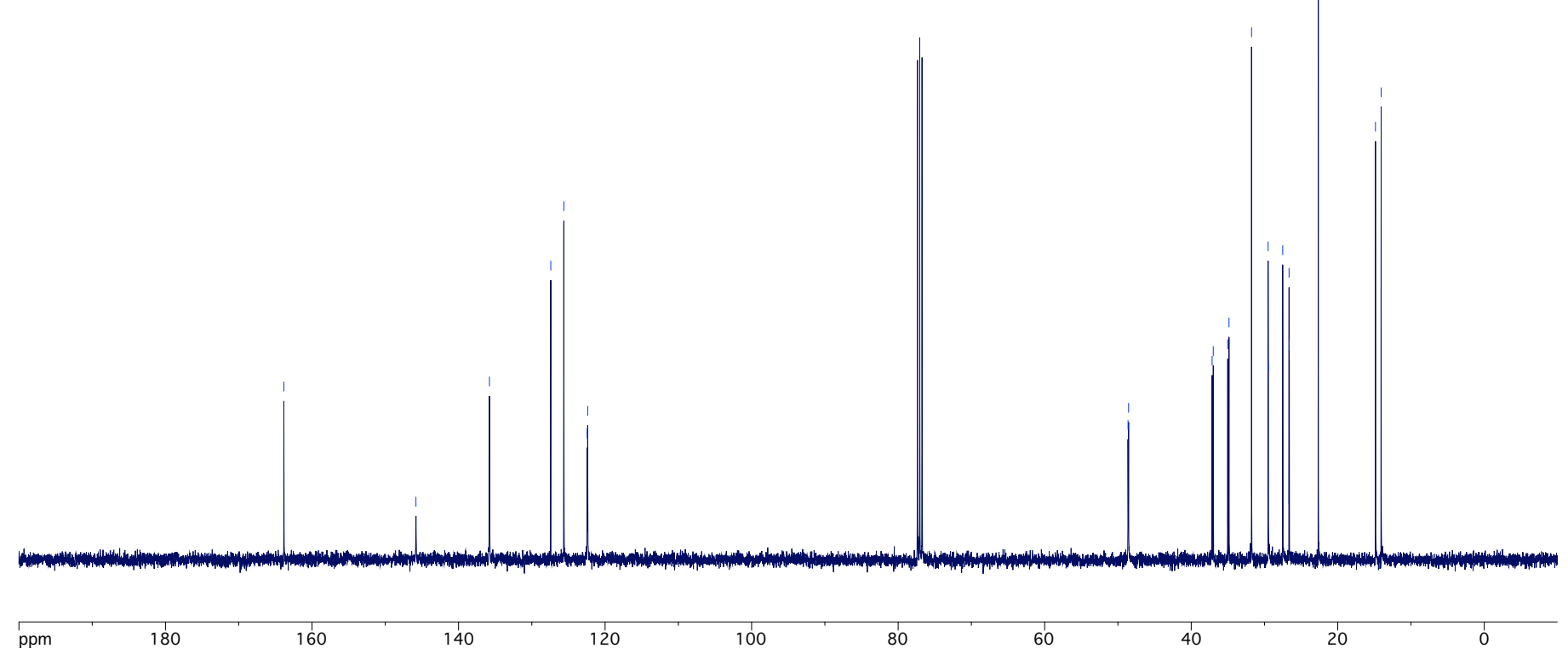



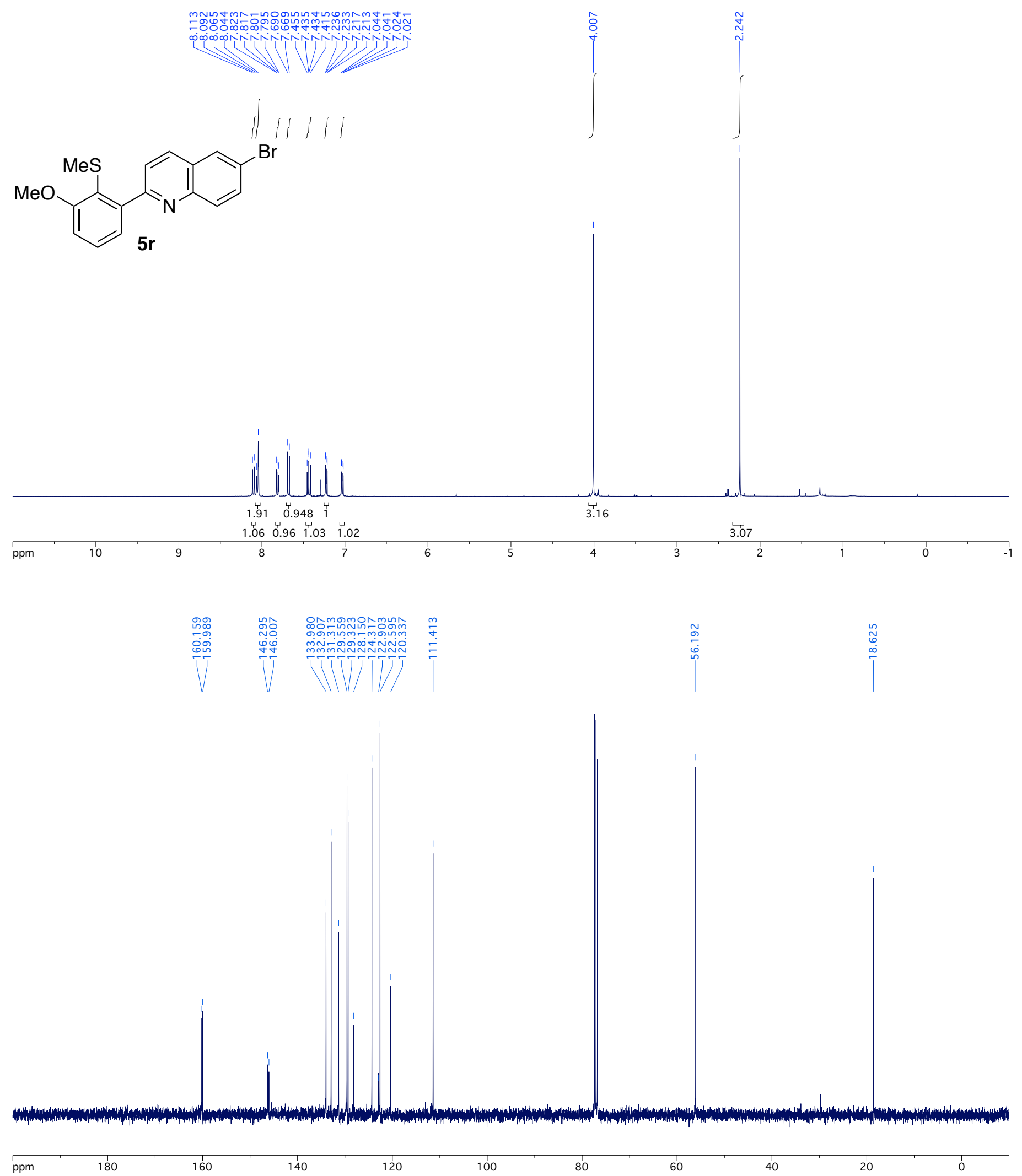

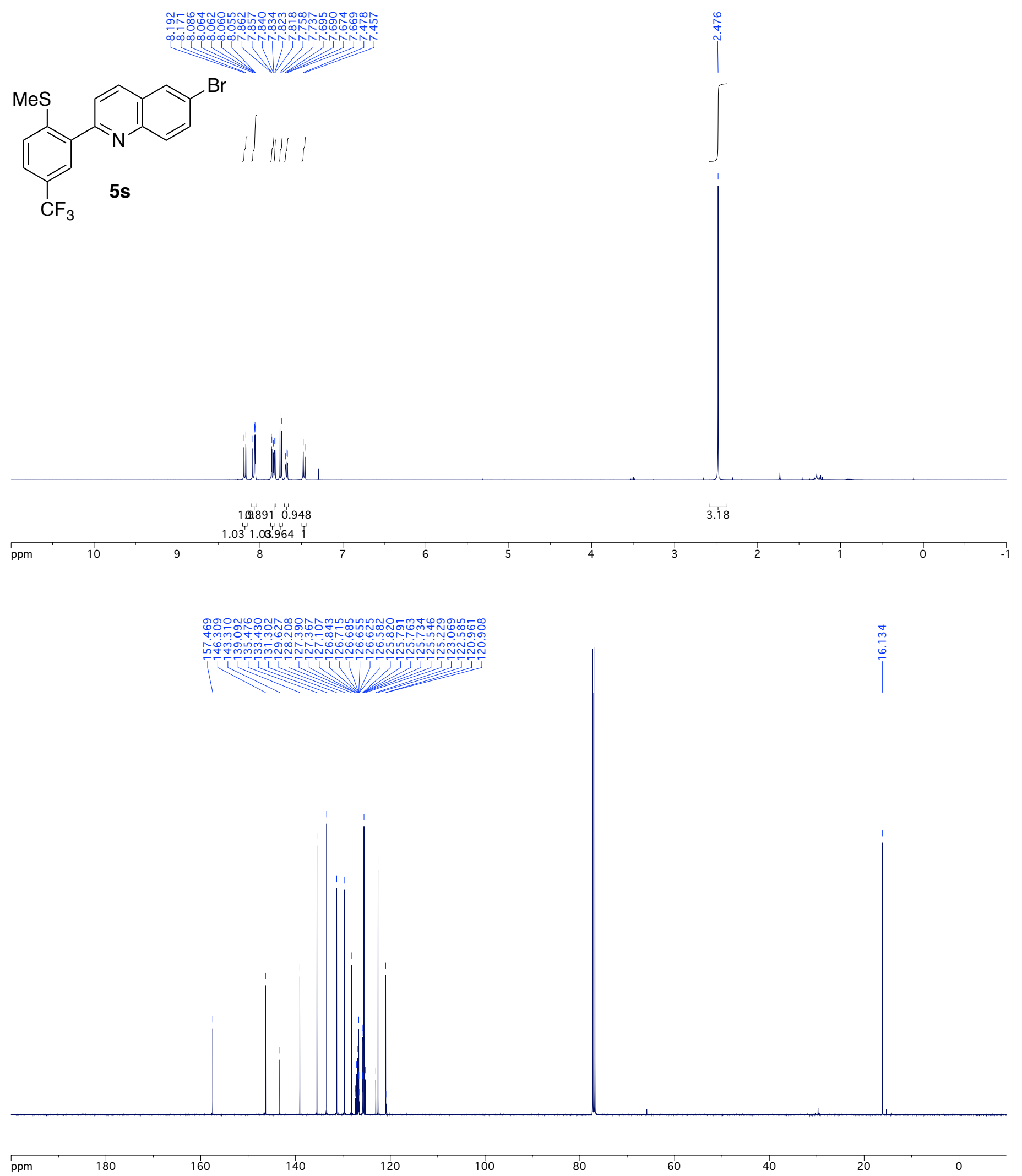

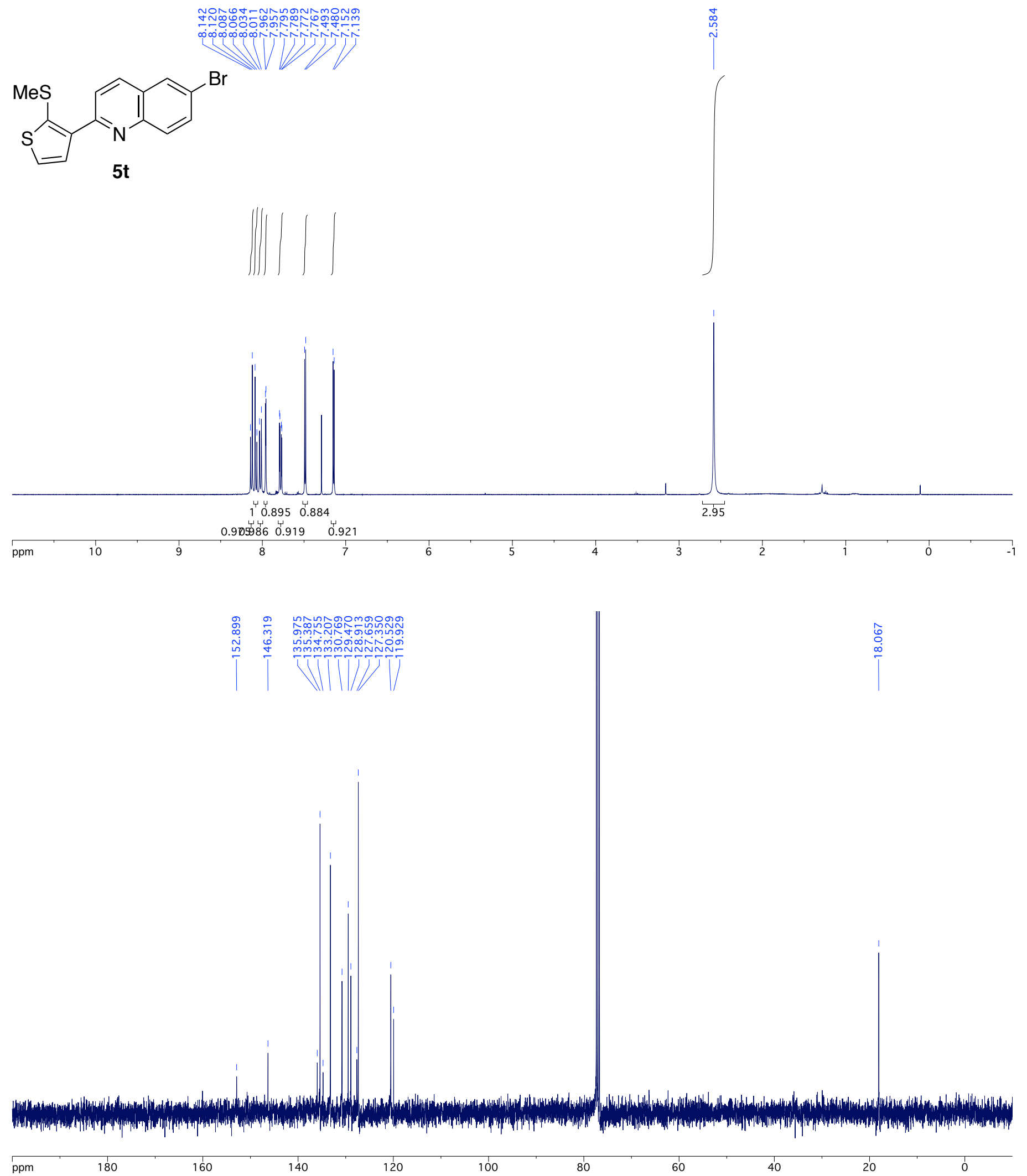
<smiles>CCCCCCC(CCSCC)c1ccc2cc(Br)ccc2n1</smiles>

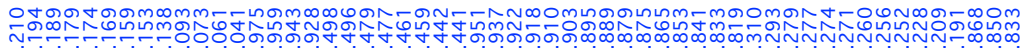
: L
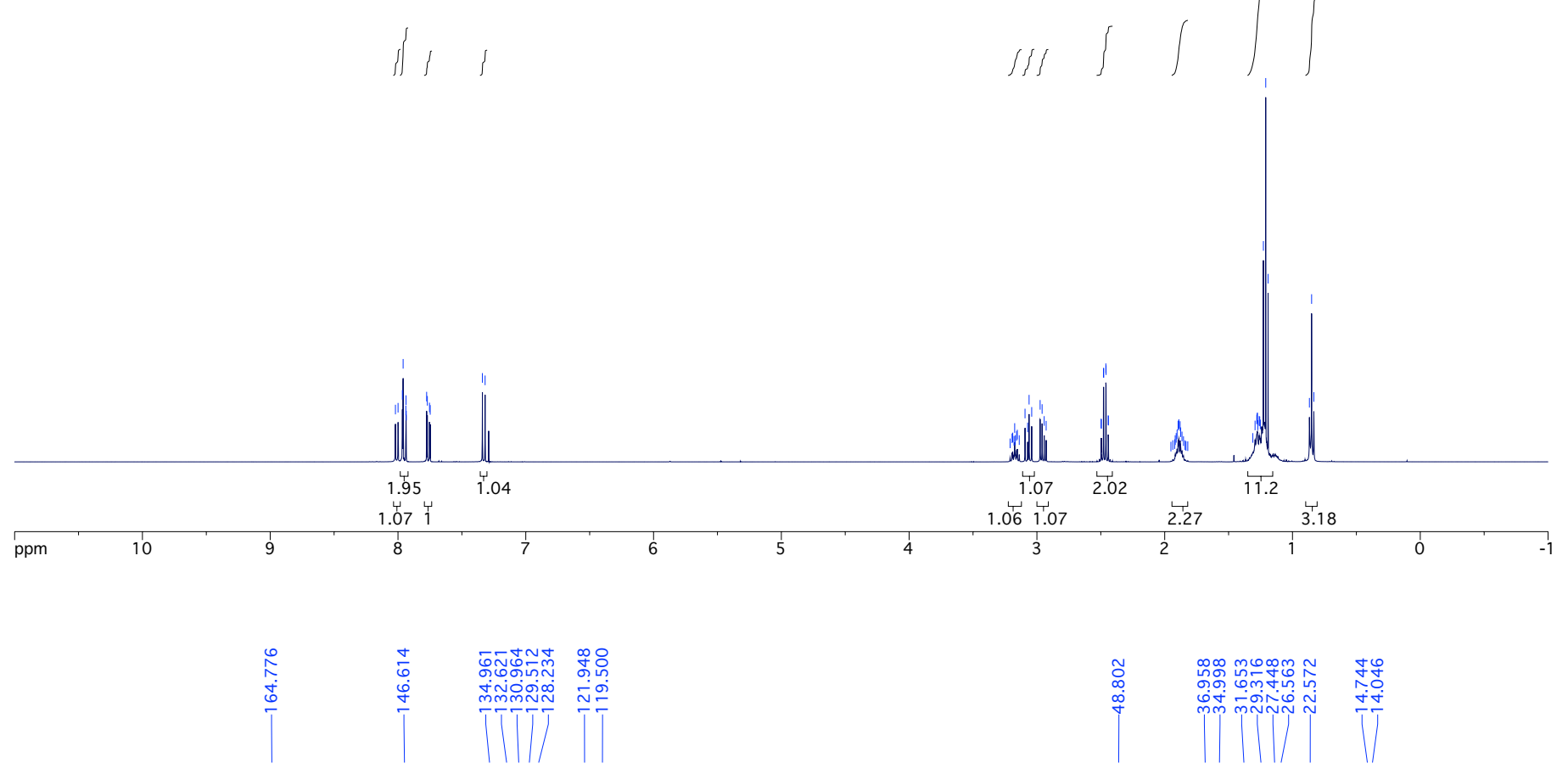

180

160

140

120

100

80

60

40

20 
<smiles>CCSC(Cc1ccc2cc(Br)ccc2n1)[Hg]Sc1ccccc1</smiles>

5v
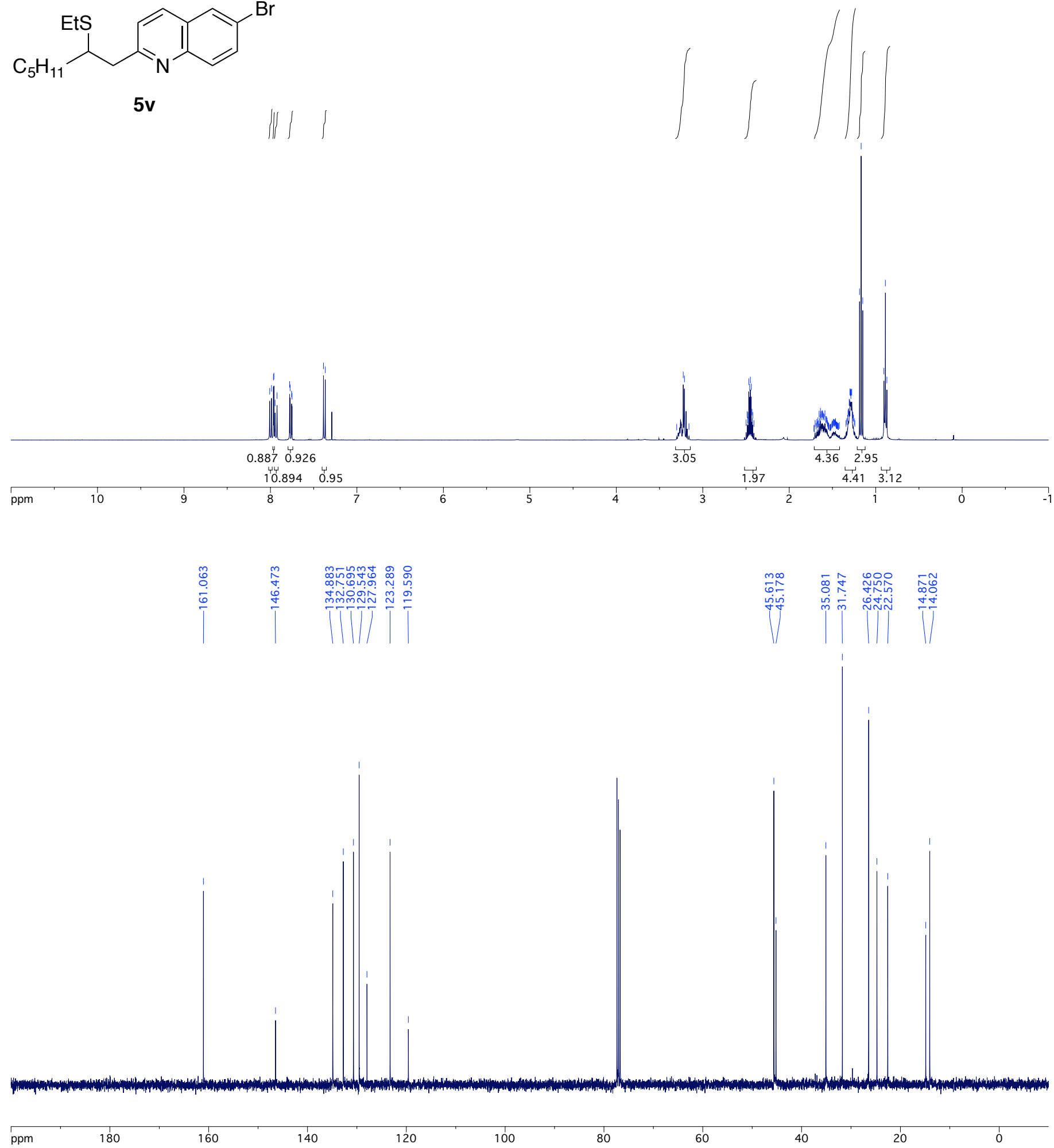

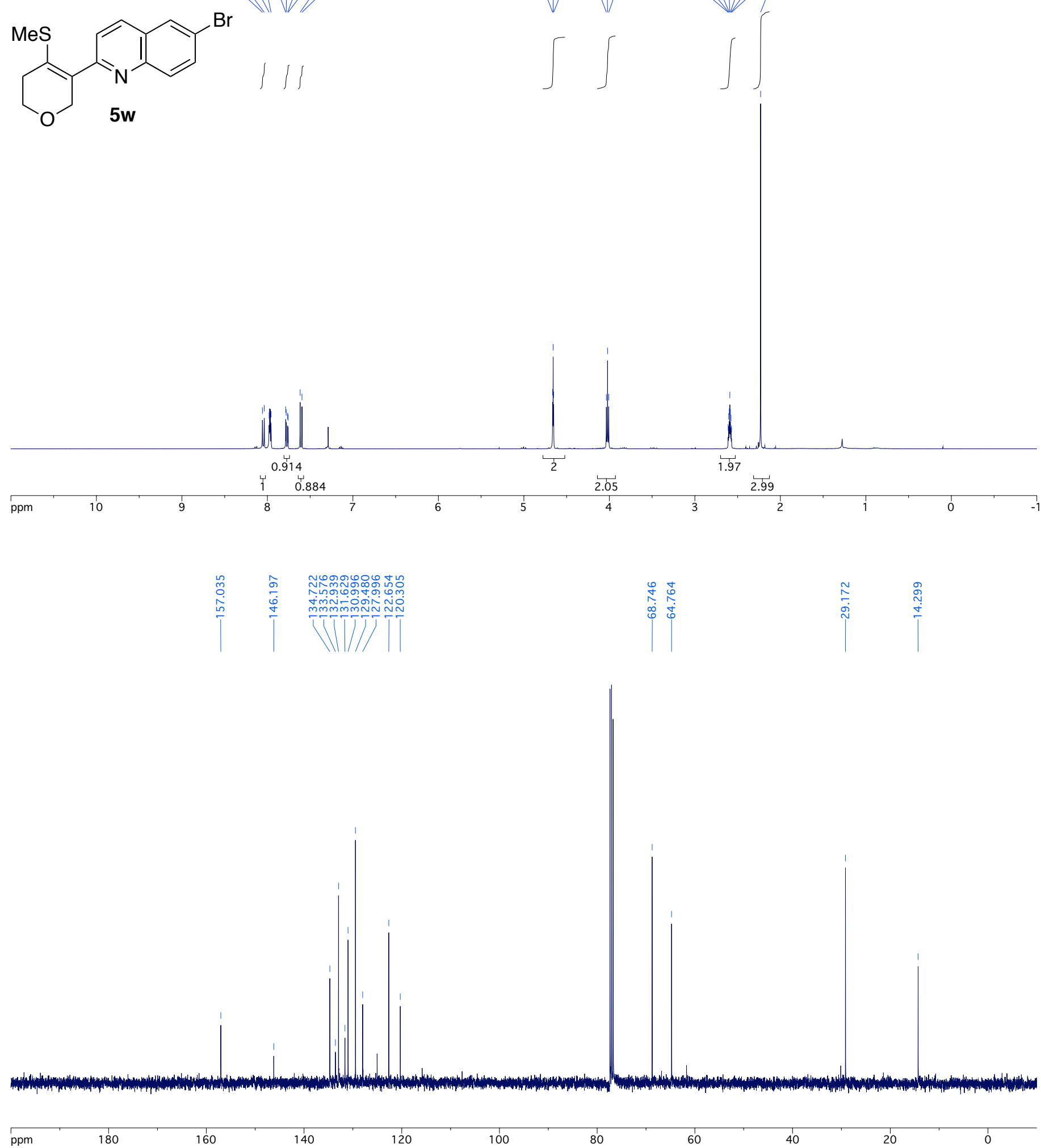

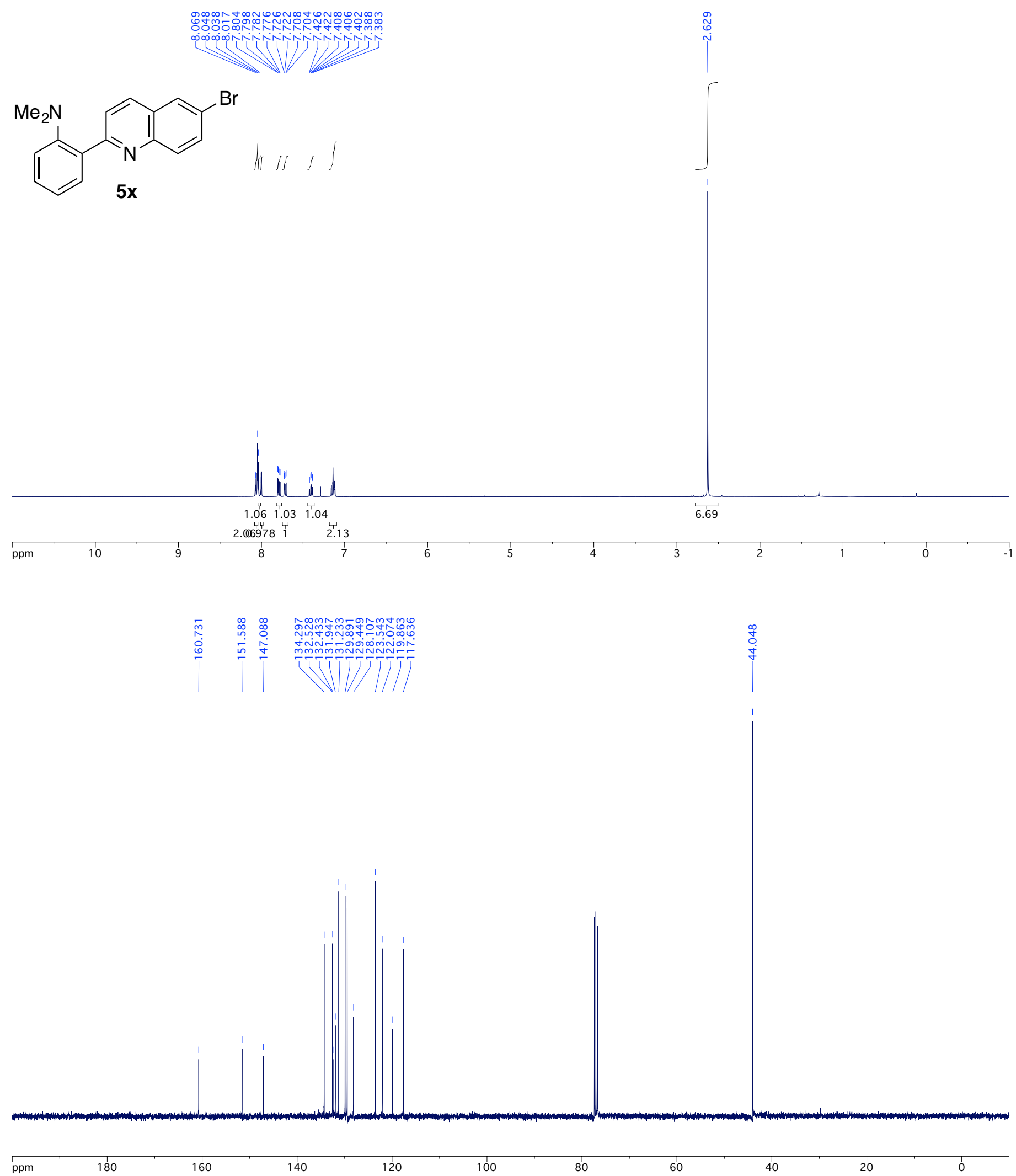


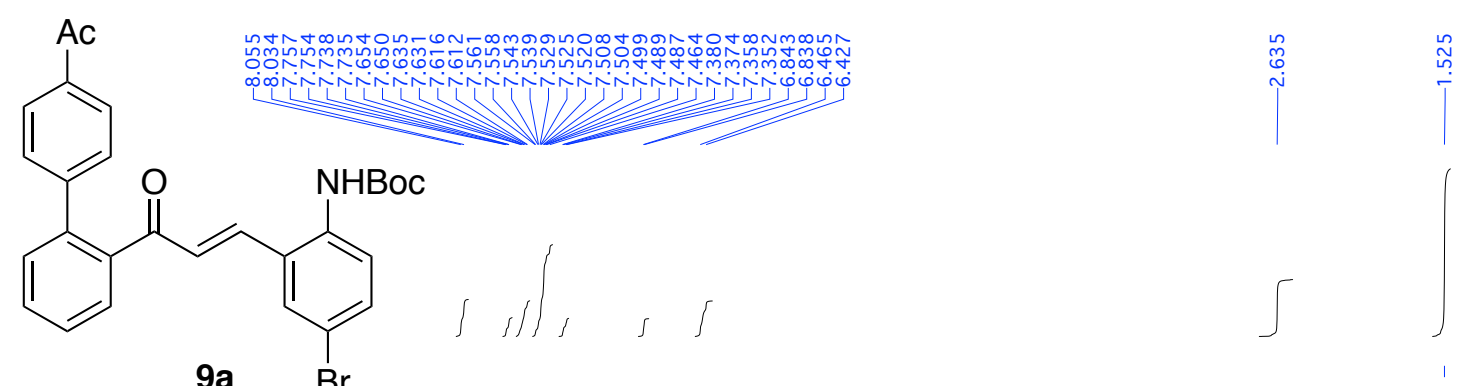

9a $\mathrm{Br}$
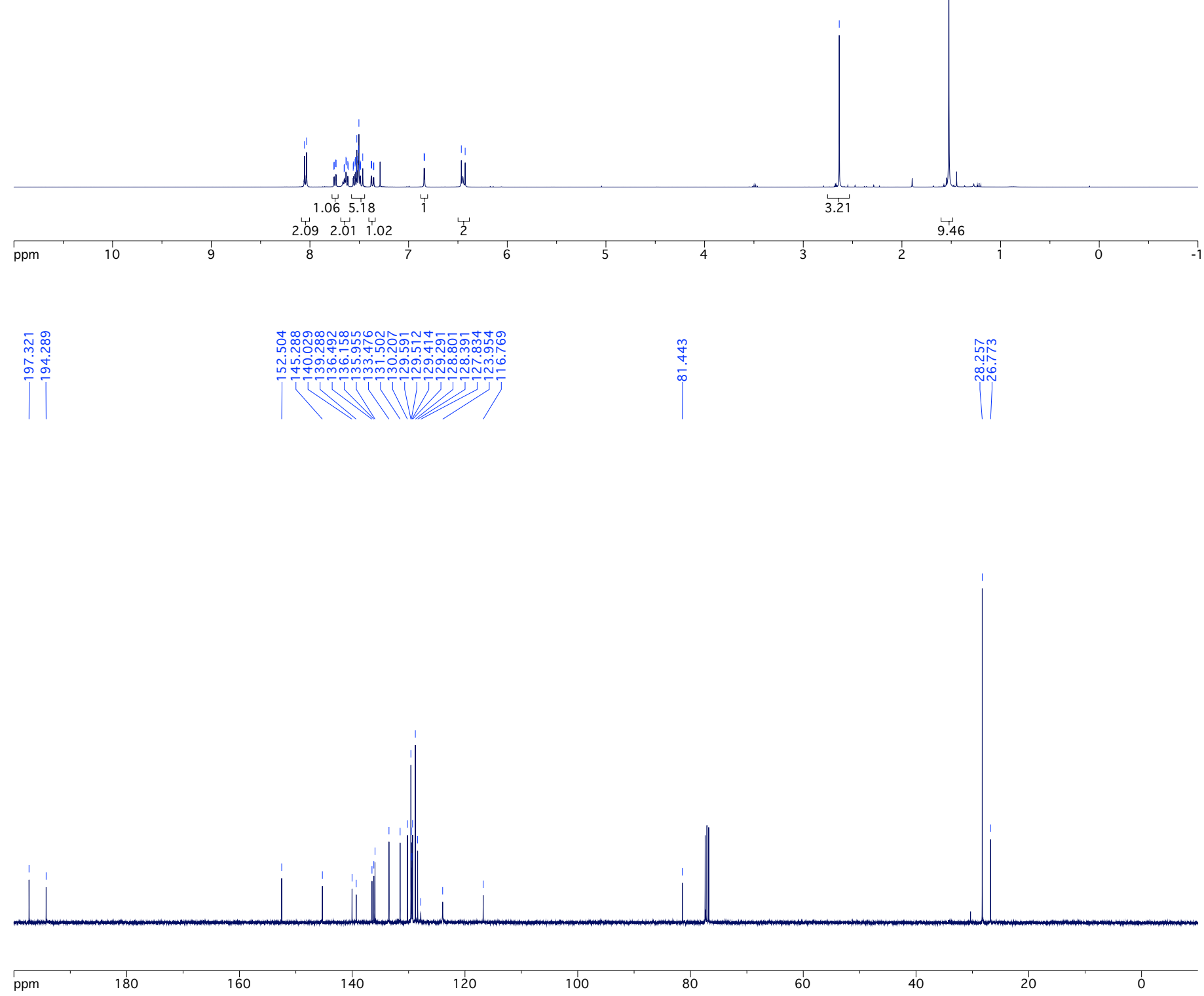

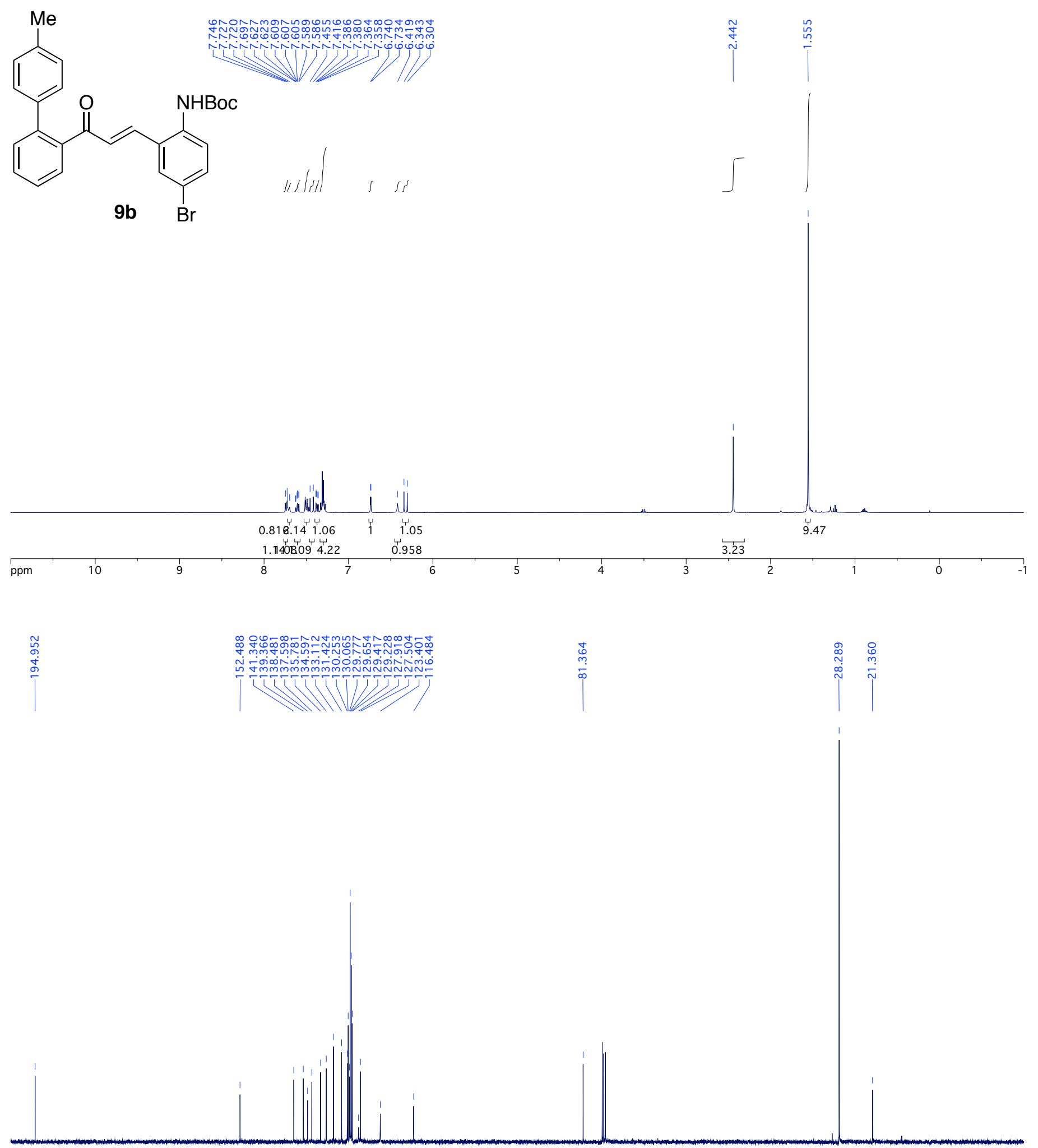

ppm

$180 \quad 160$

140

120

100

80

60

40

20 

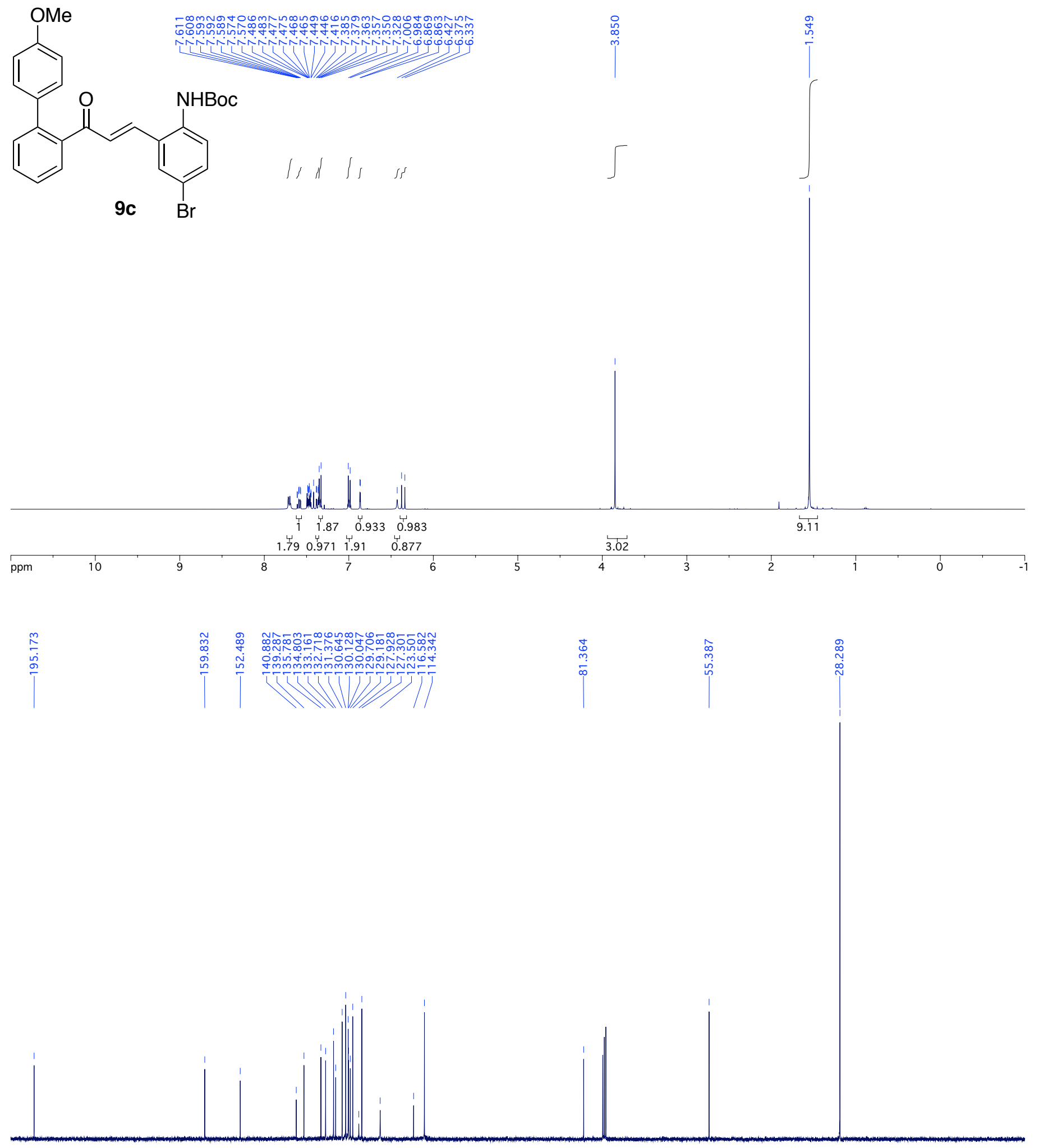

ppm

180

160

140

120

100

80

60

40

20 

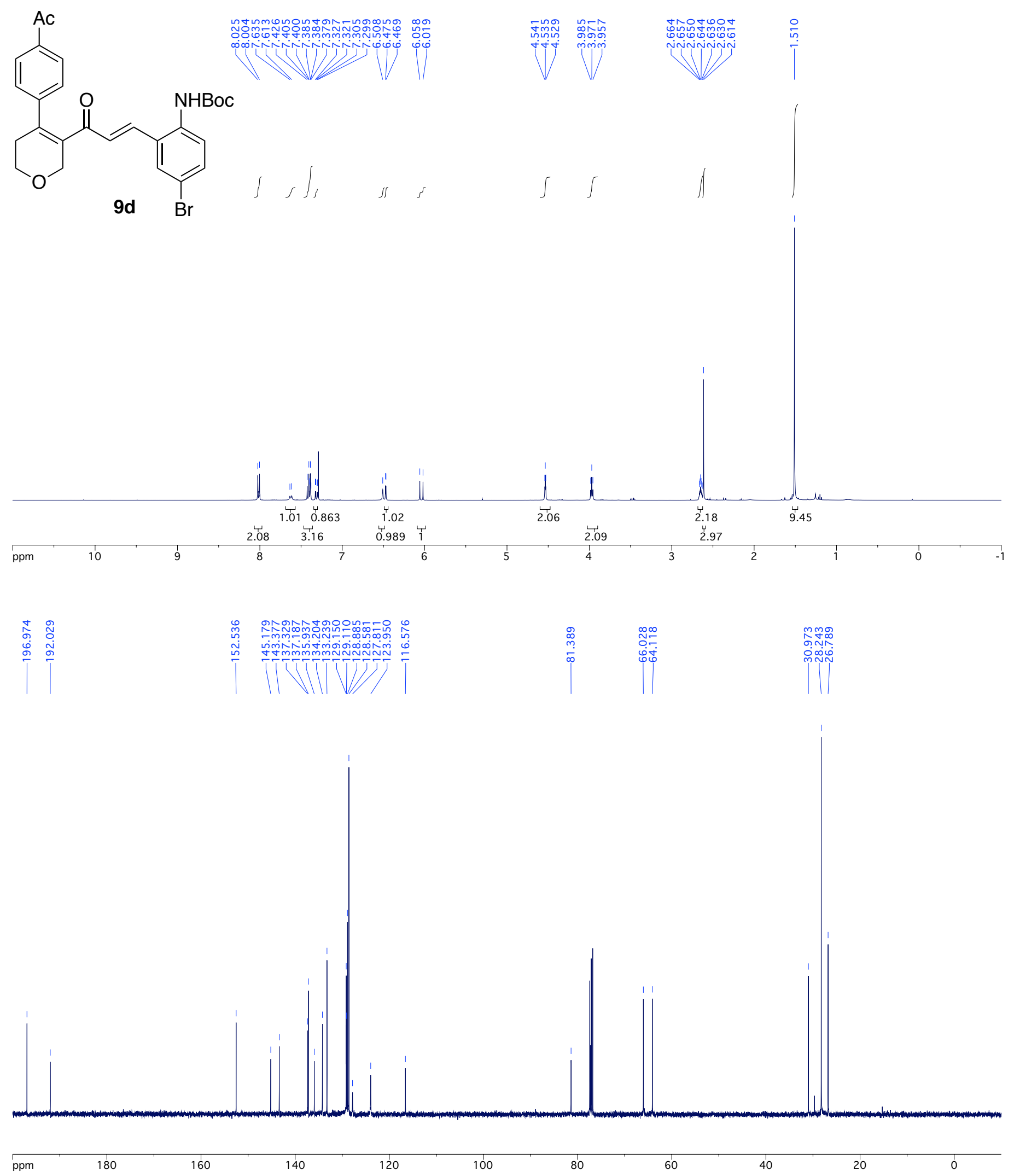

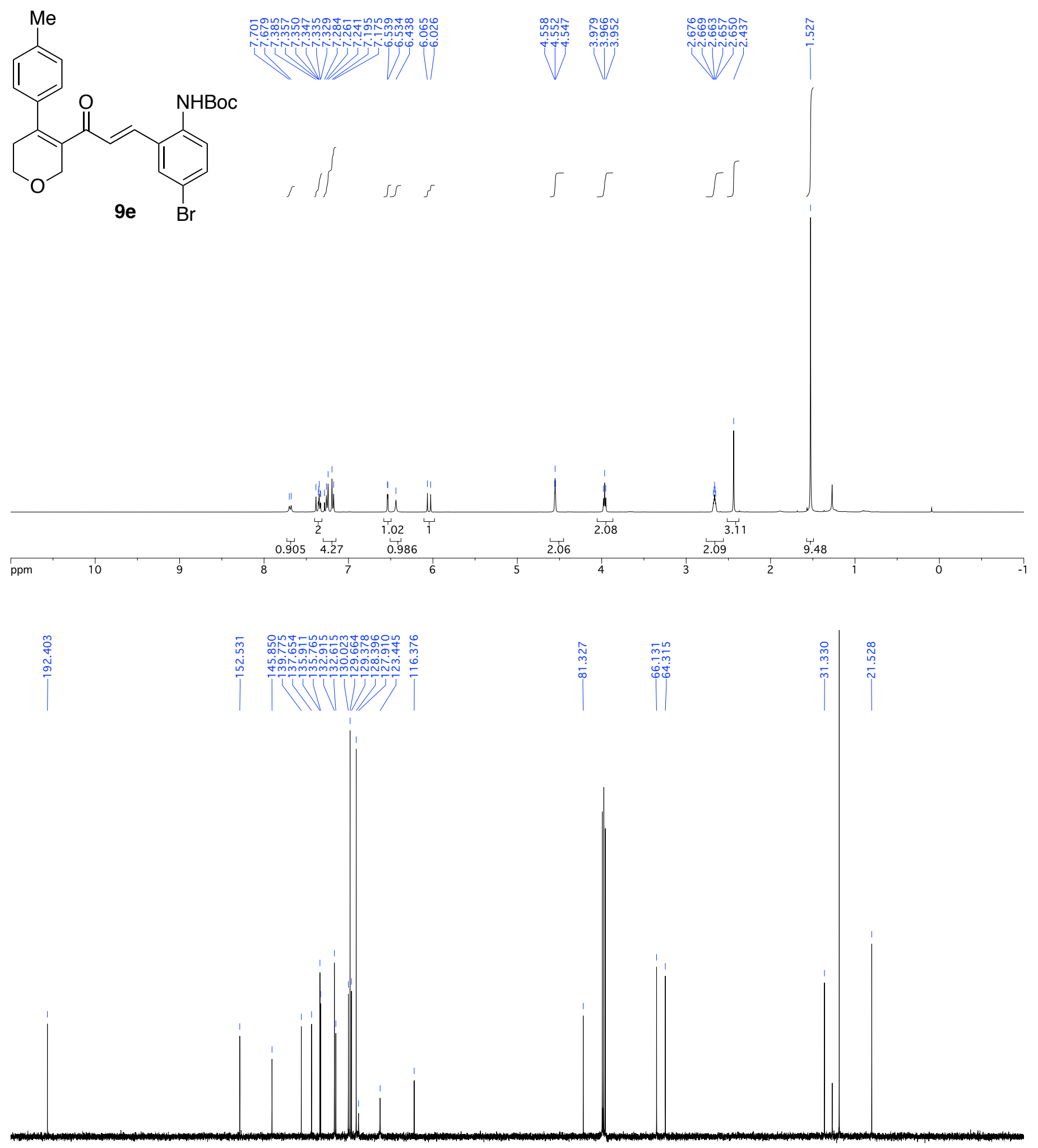

ppm

180

160

140

120

100

80

60

40

20 

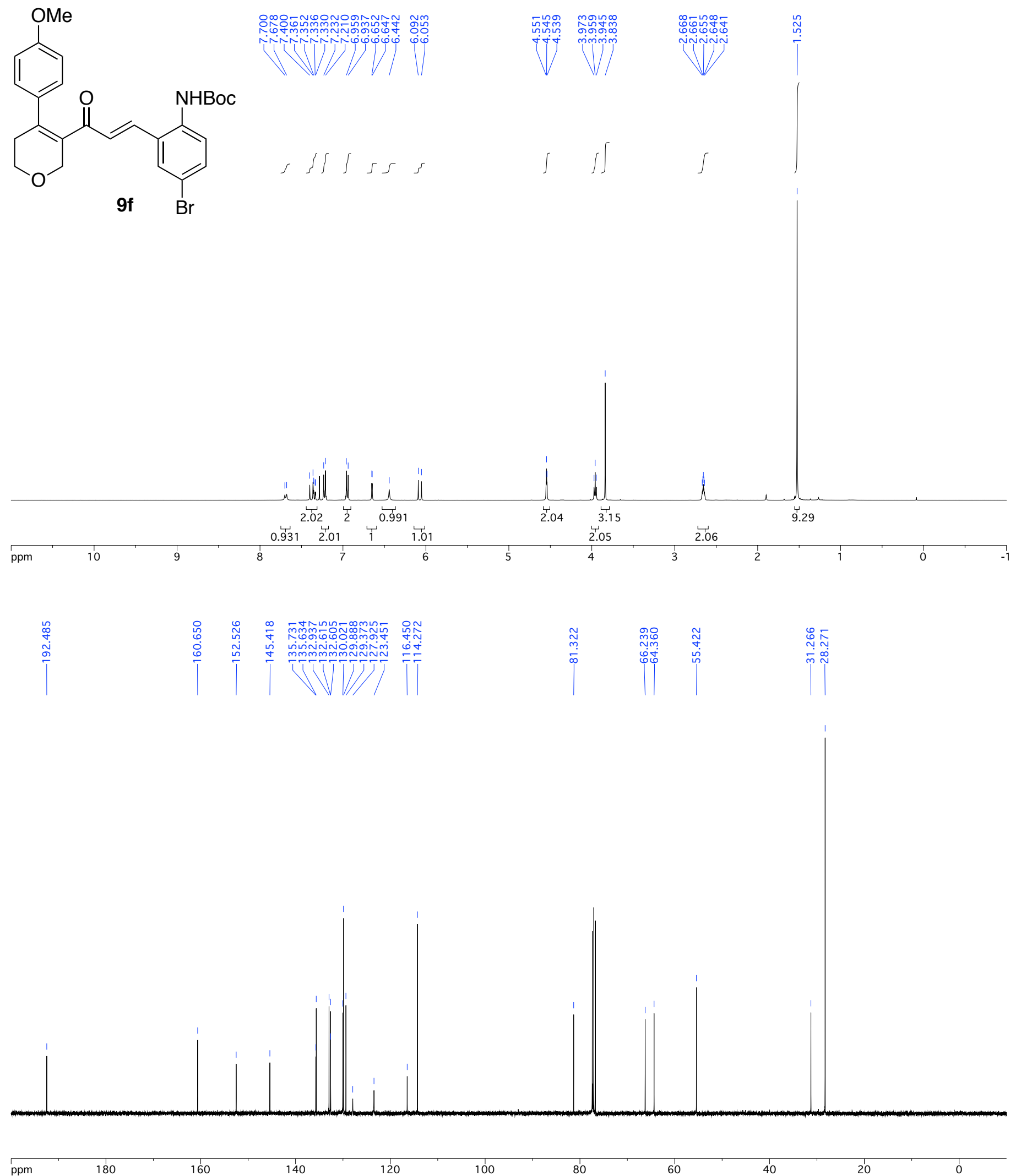

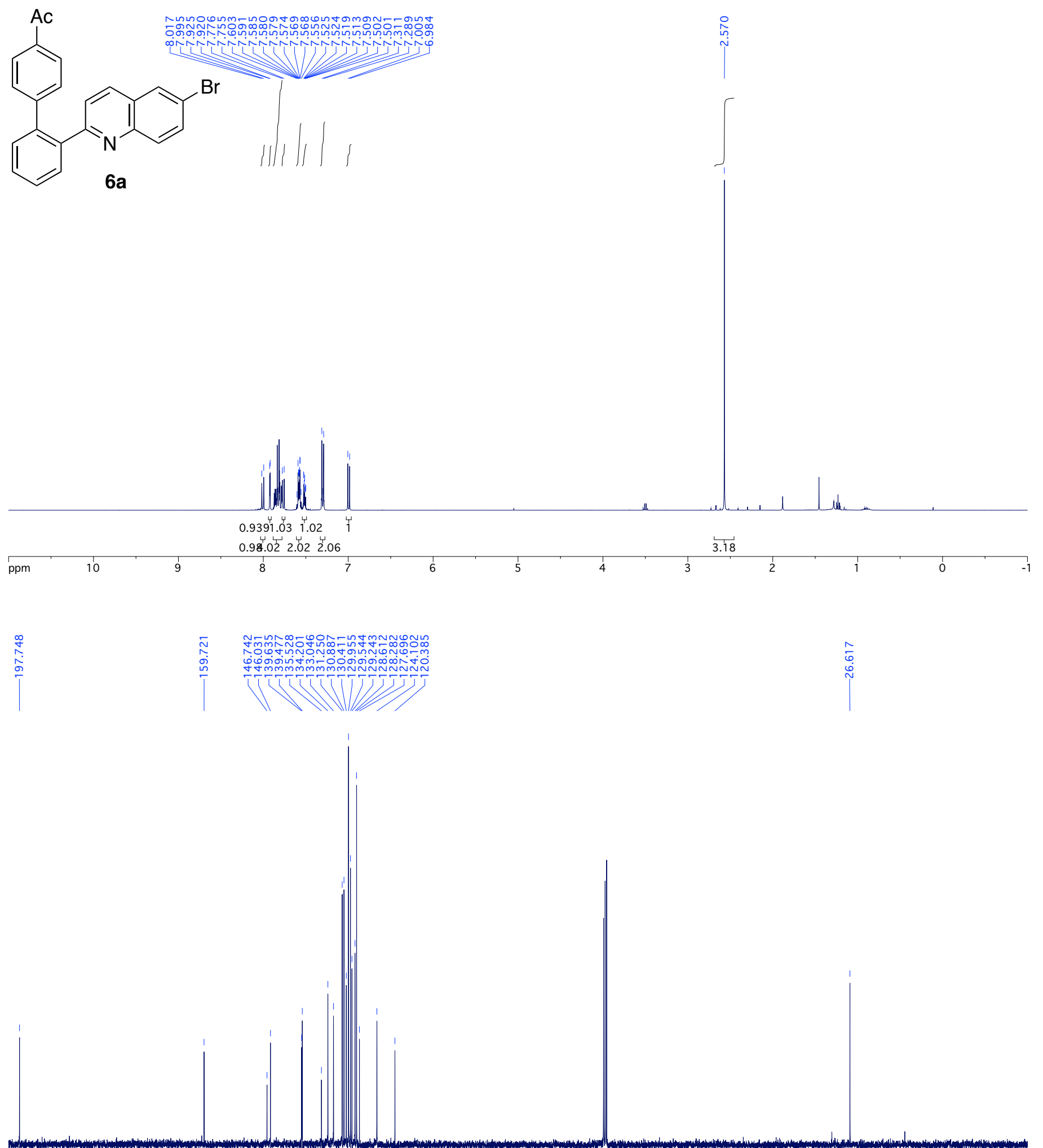

ppm

180

140

120

100

80

60

40

20 

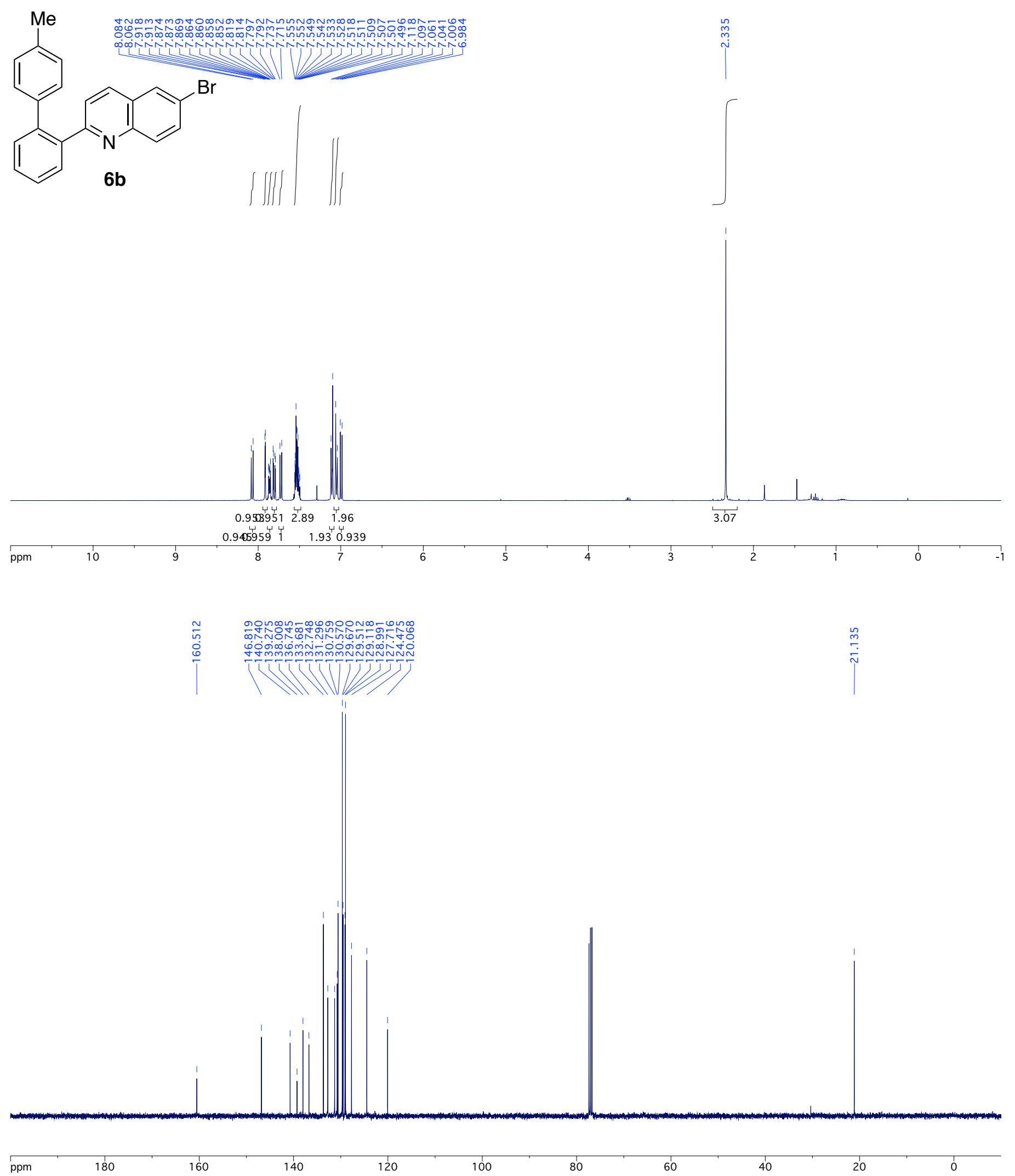

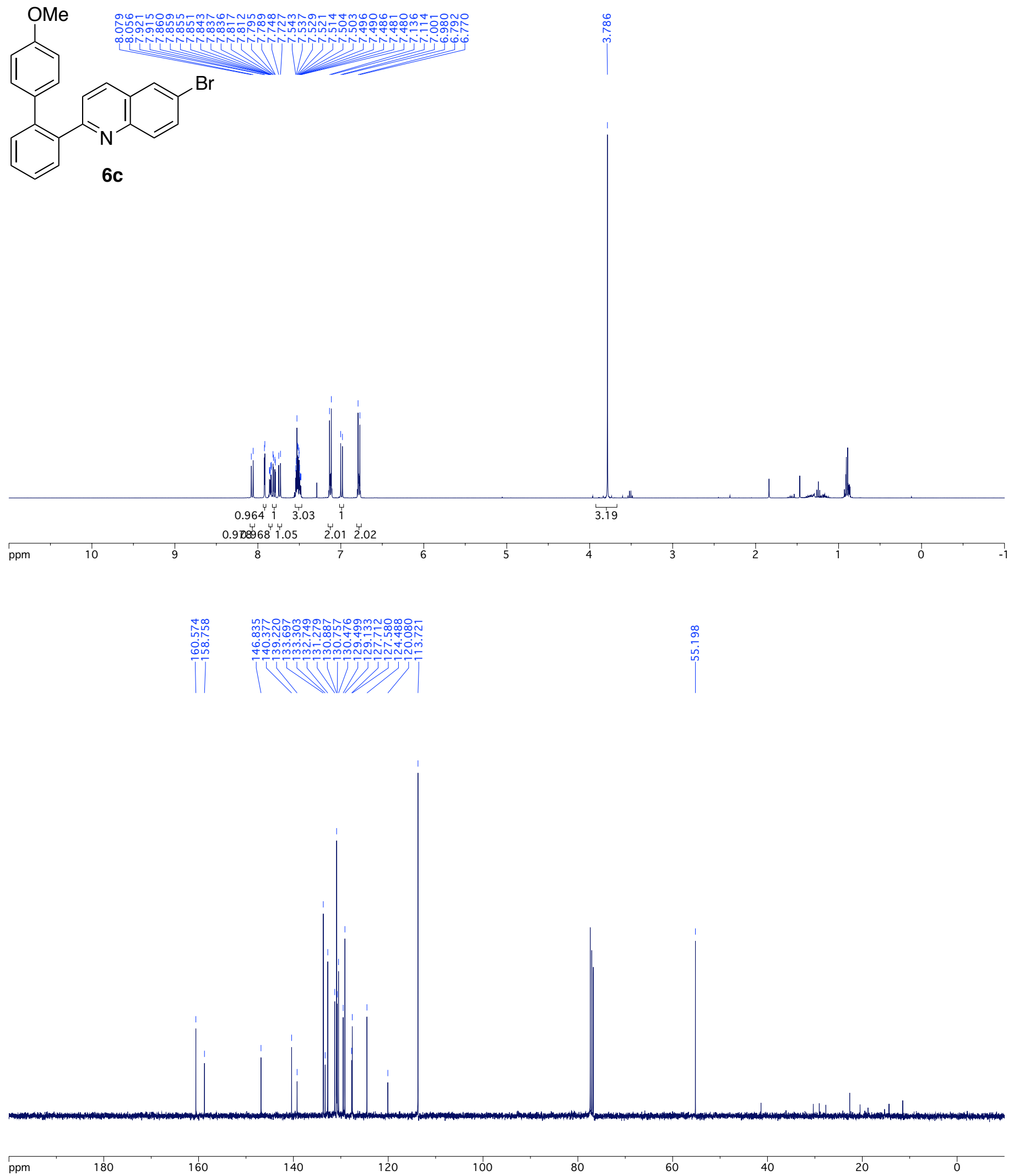

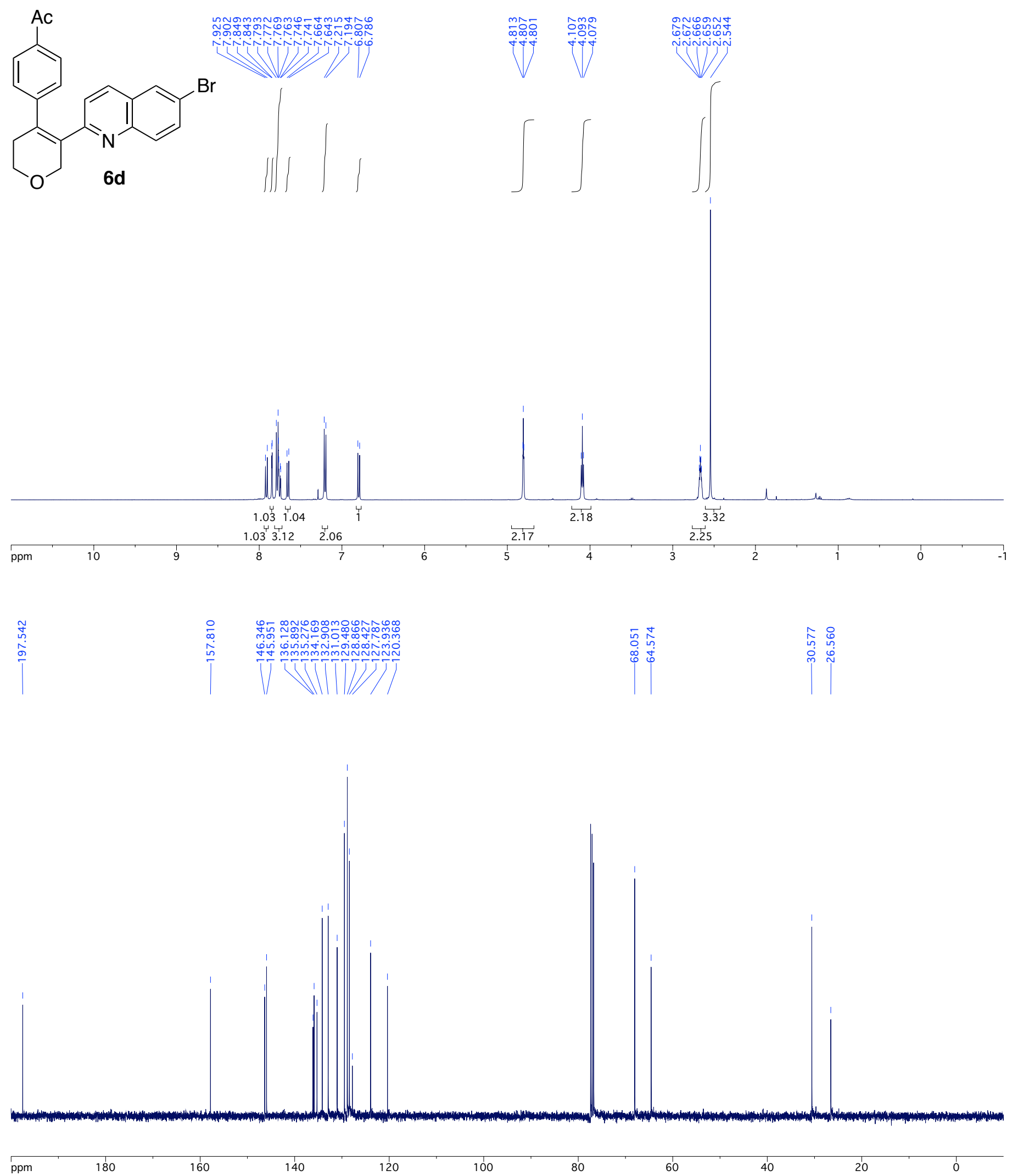

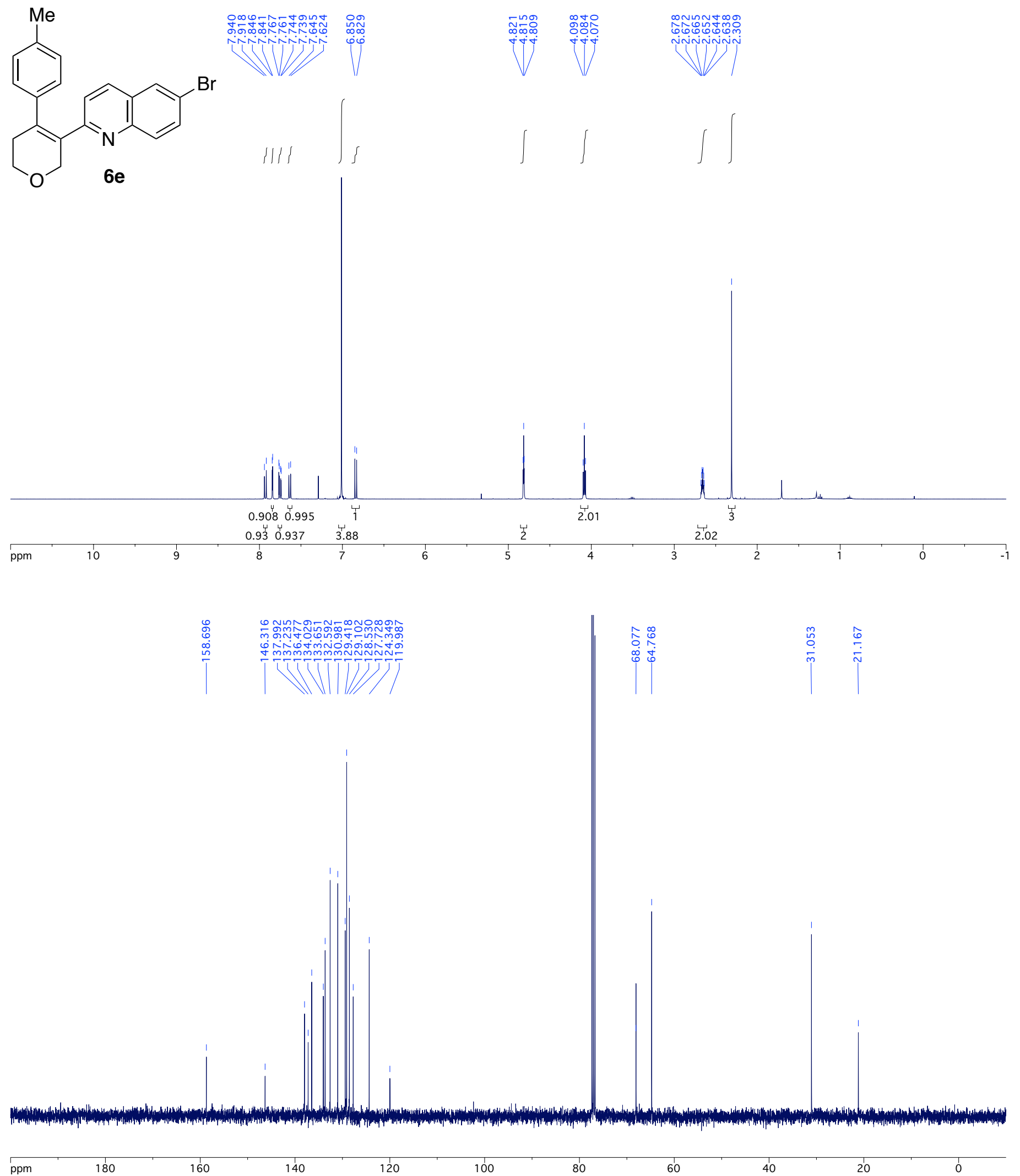

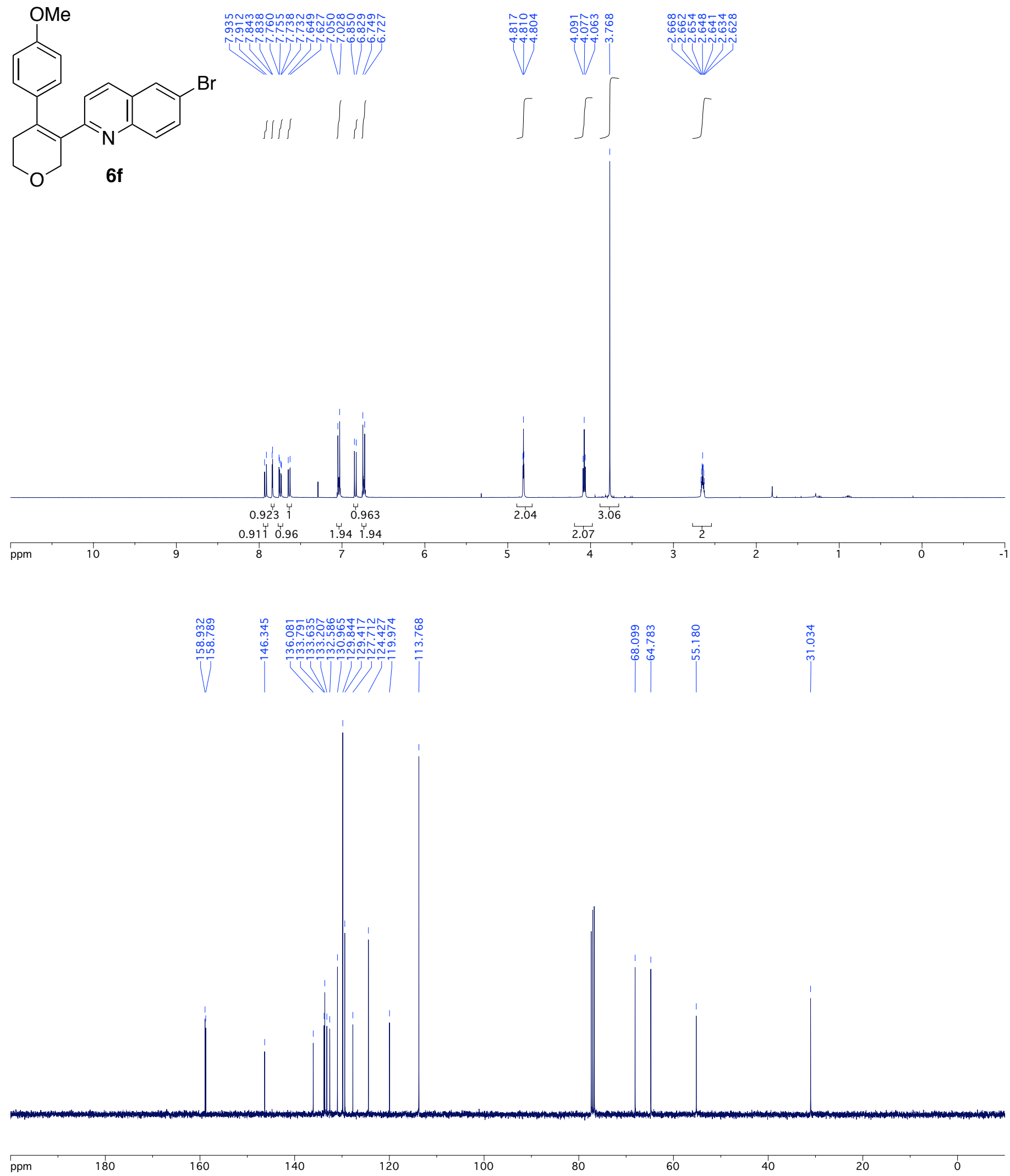

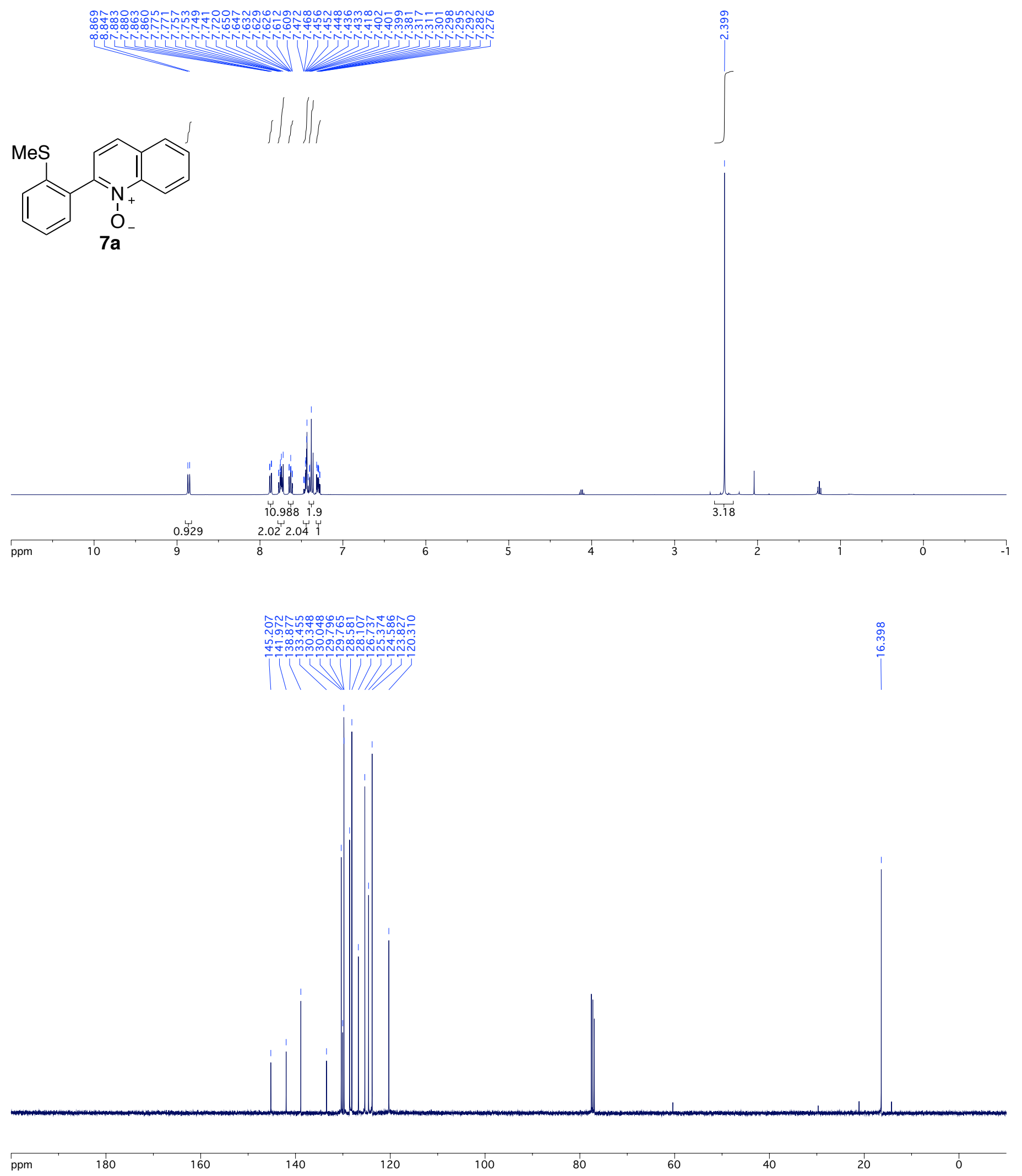

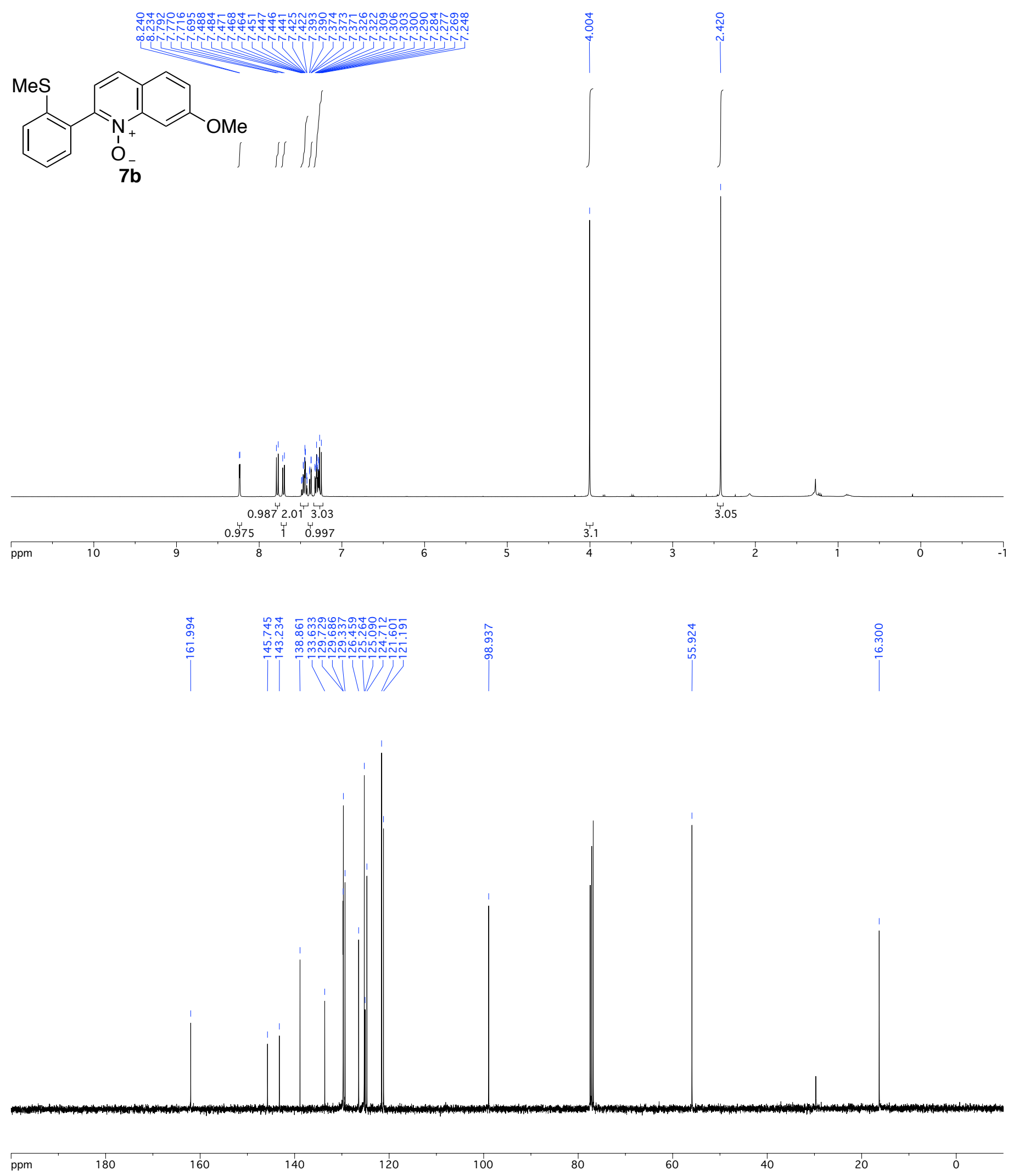


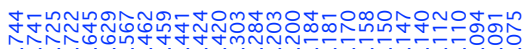

1<smiles>CCC(CC(=O)c1ccccc1S(C)(=O)=O)c1ccccc1NC(=O)O</smiles>

$10 a$

$0 a$

(a)

, 0.4871 .490 .931

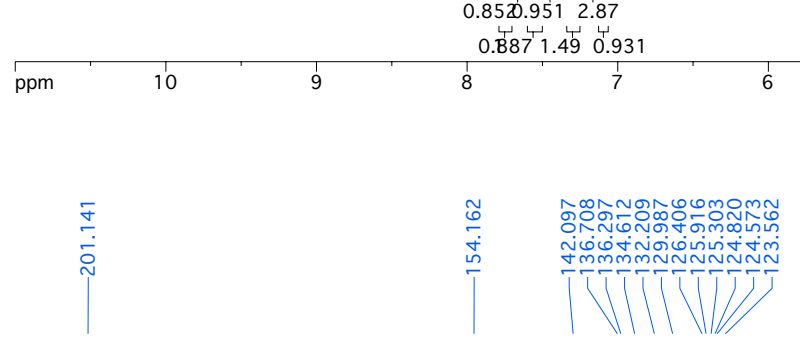

0.970 .968 0.987

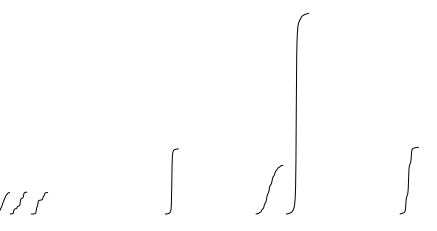

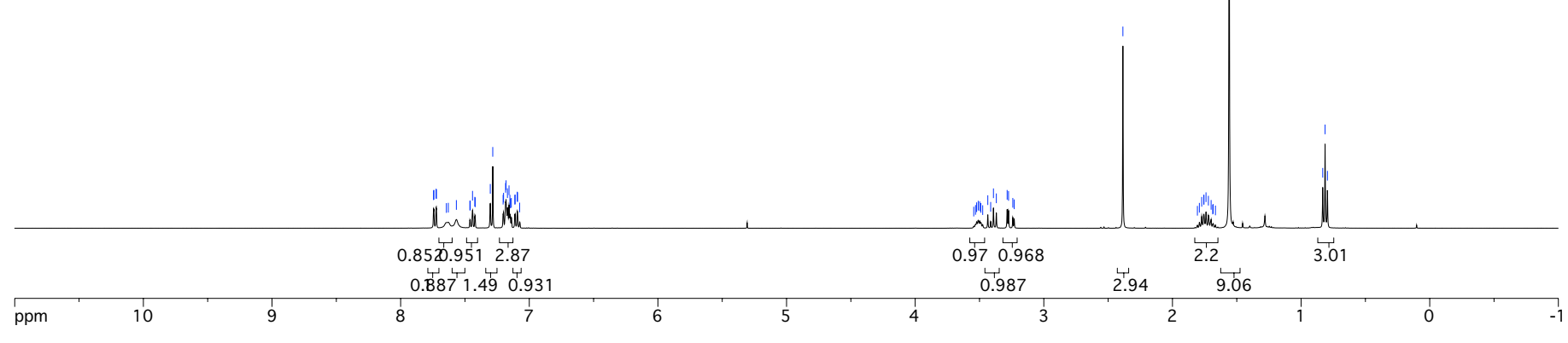

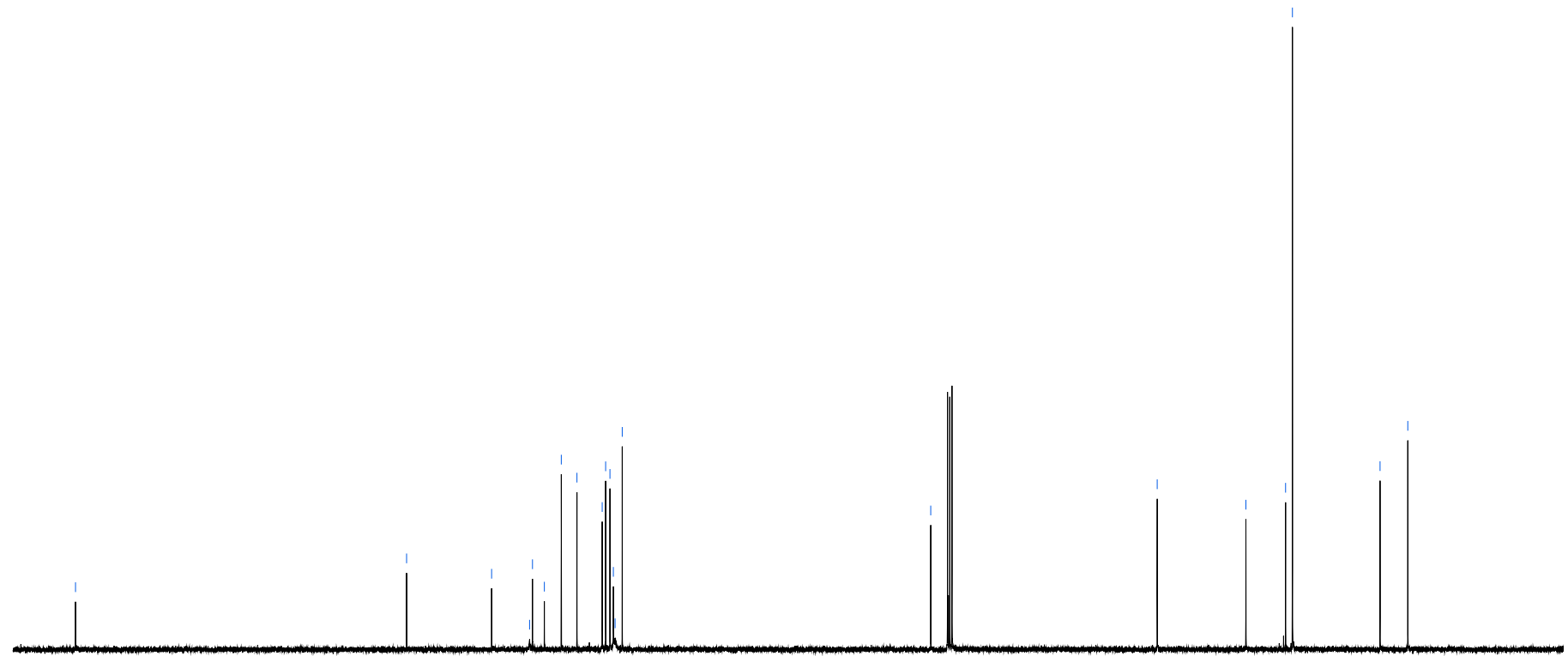

ppm 200

180

160

140

120

100

80

60

40

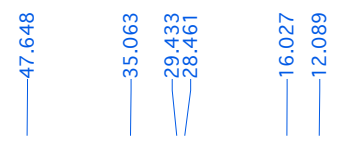

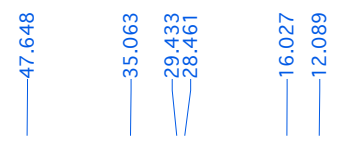


<smiles>CCCCC(=O)Nc1ccccc1C(CC(=O)c1ccccc1S(C)(=O)=O)c1ccc(C)cc1</smiles>

$10 b$
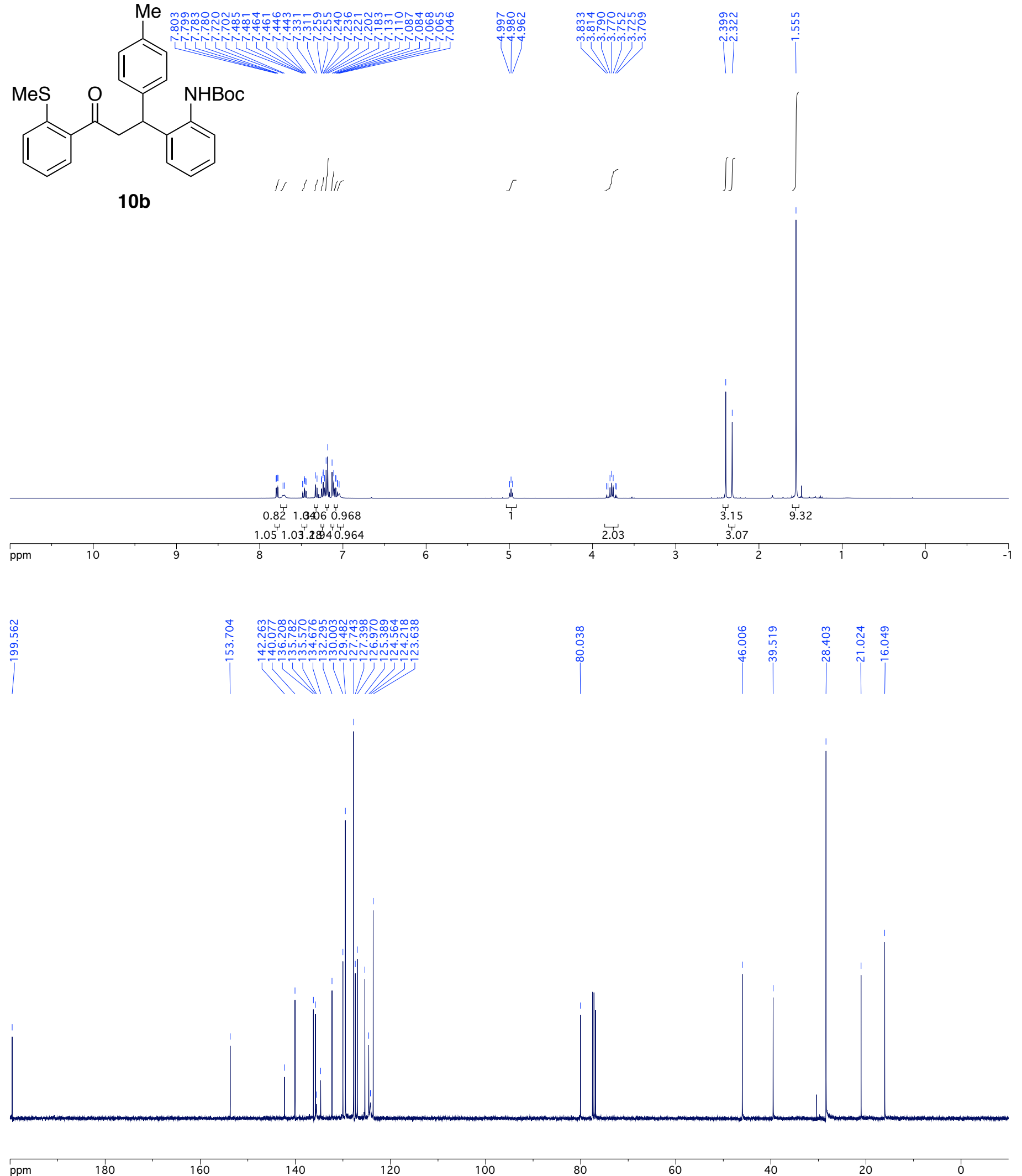


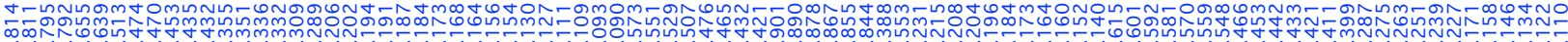
等

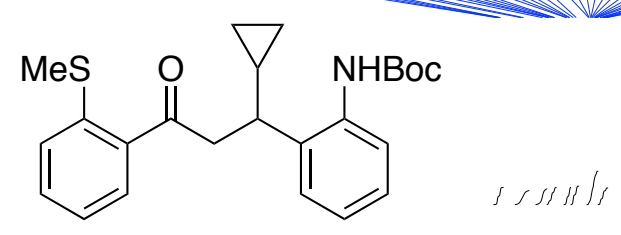

$10 \mathrm{c}$

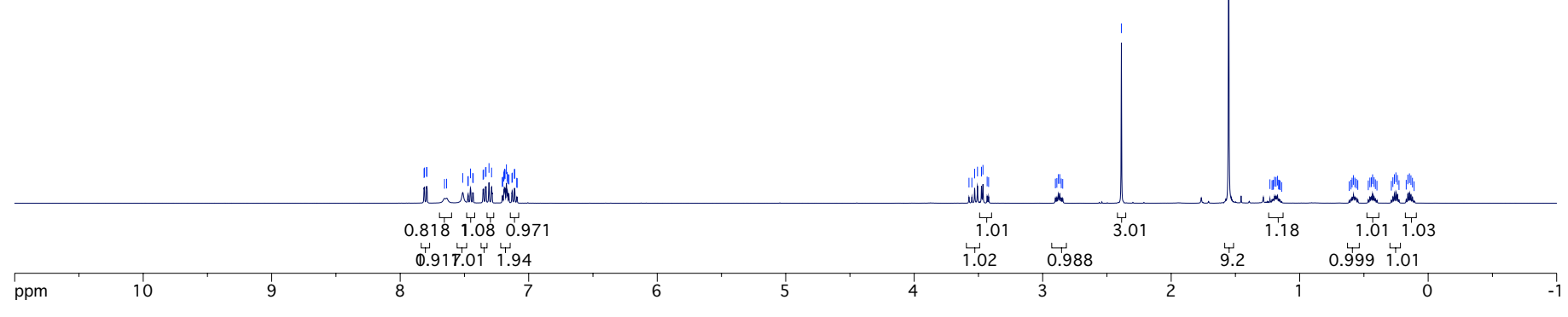

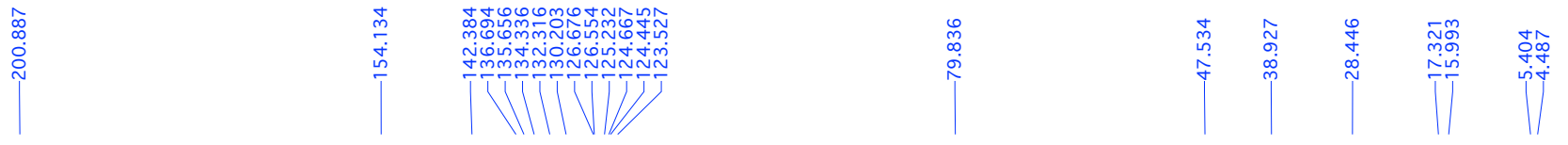

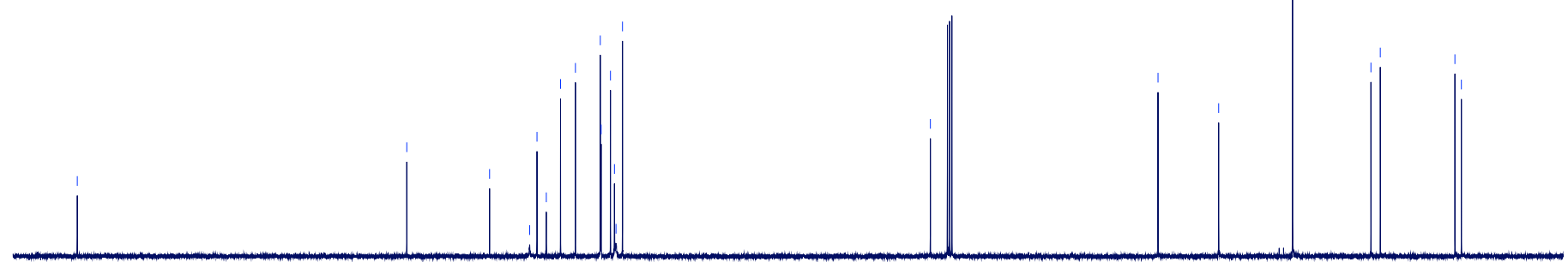

ppm 200

180

160

140

120

100

80

60

40

20

0 

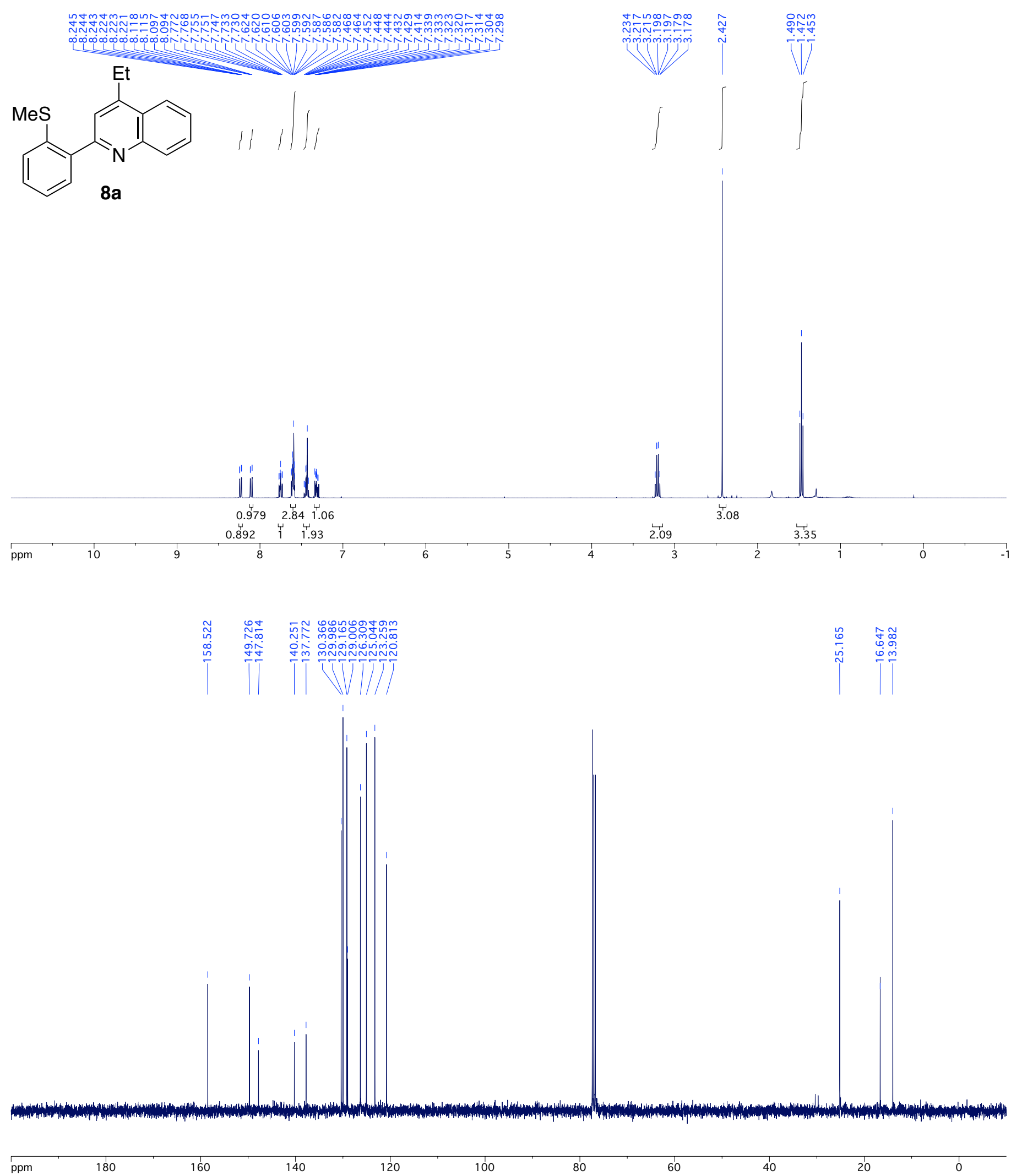

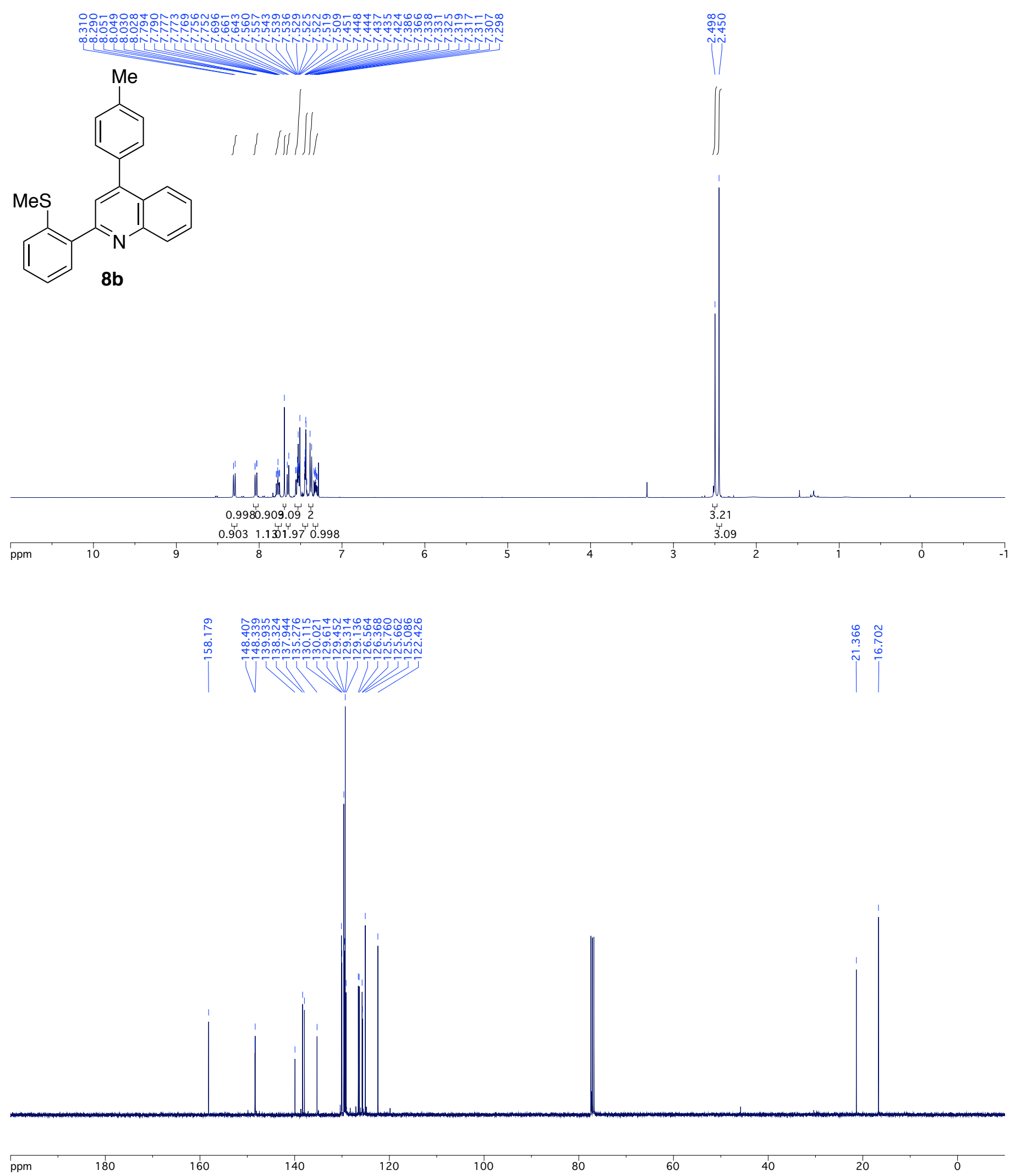

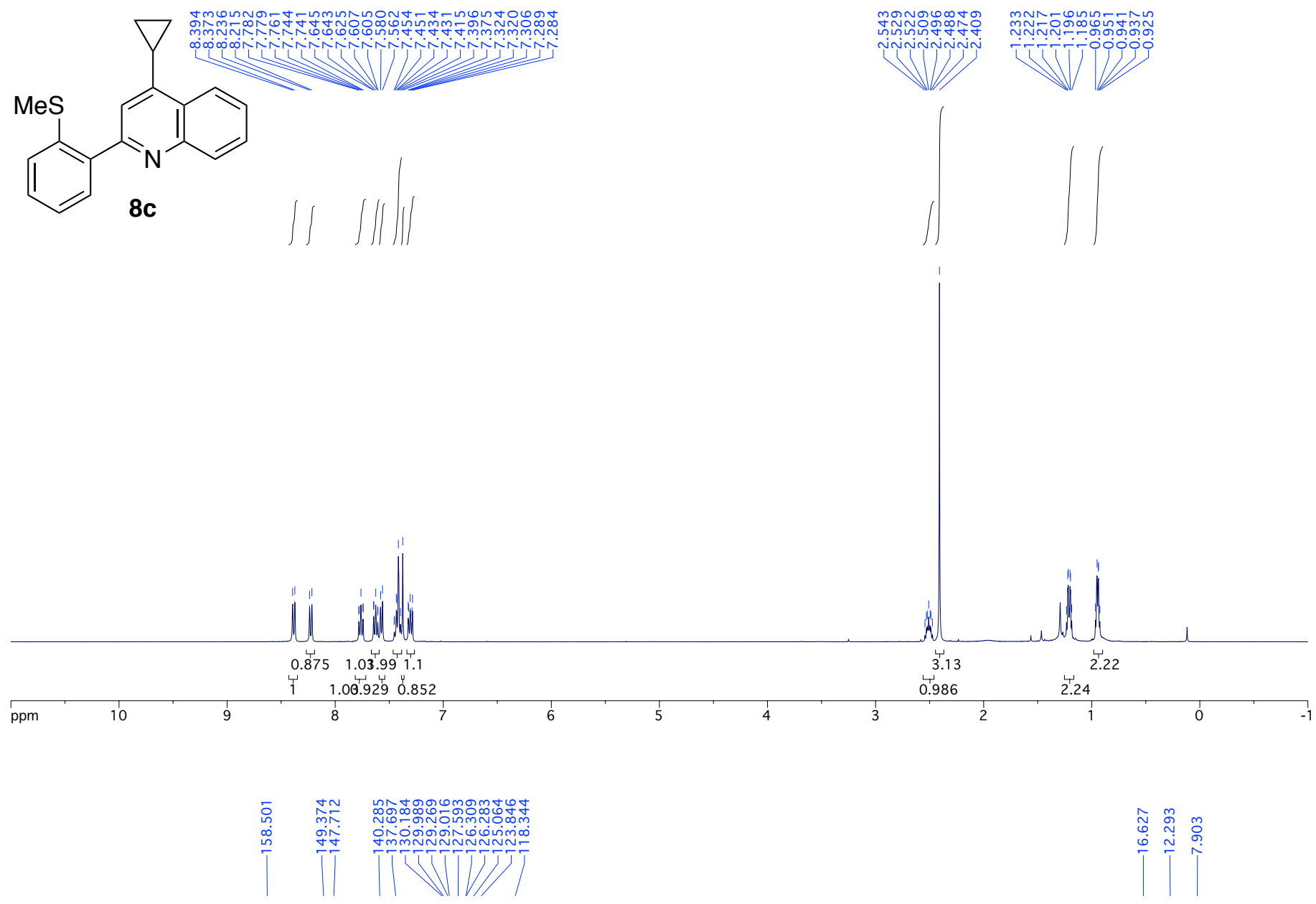

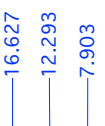

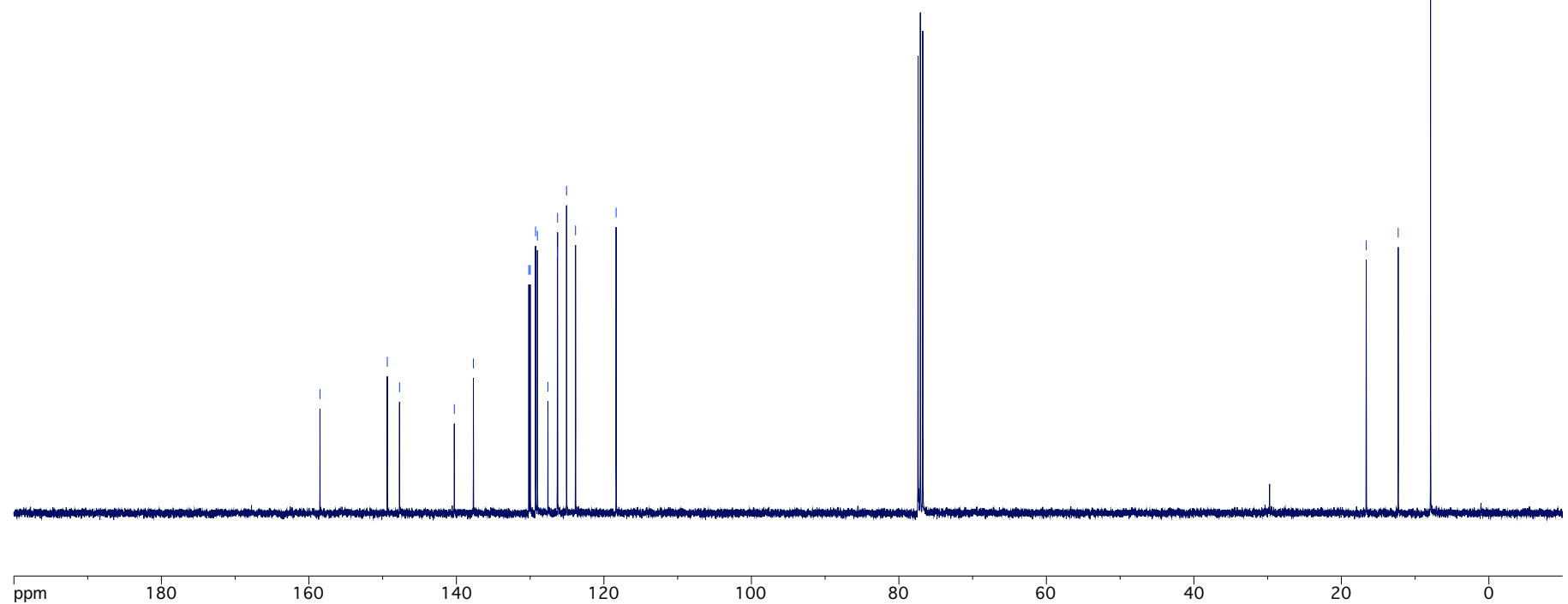


I

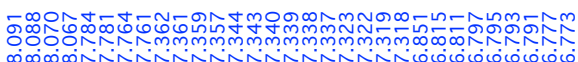

$\ldots+\cdots$

$11 a$

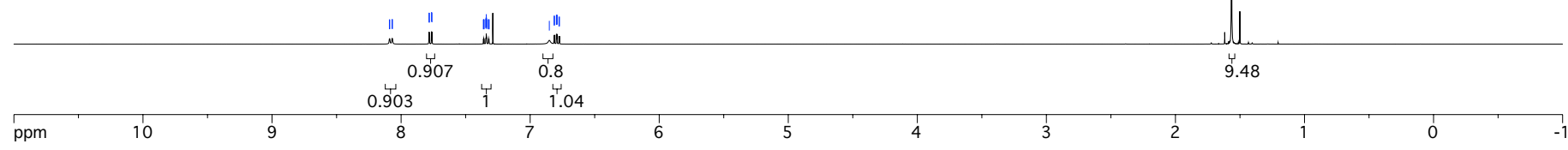

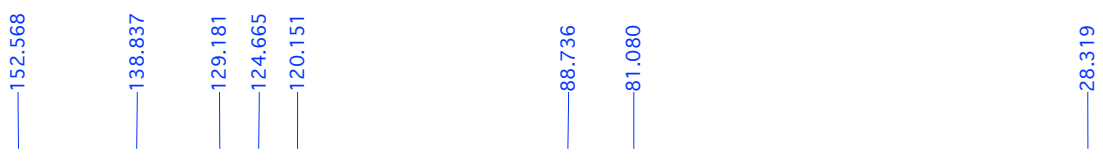

ppm

180

160

140

120

100

80

60

40

20 

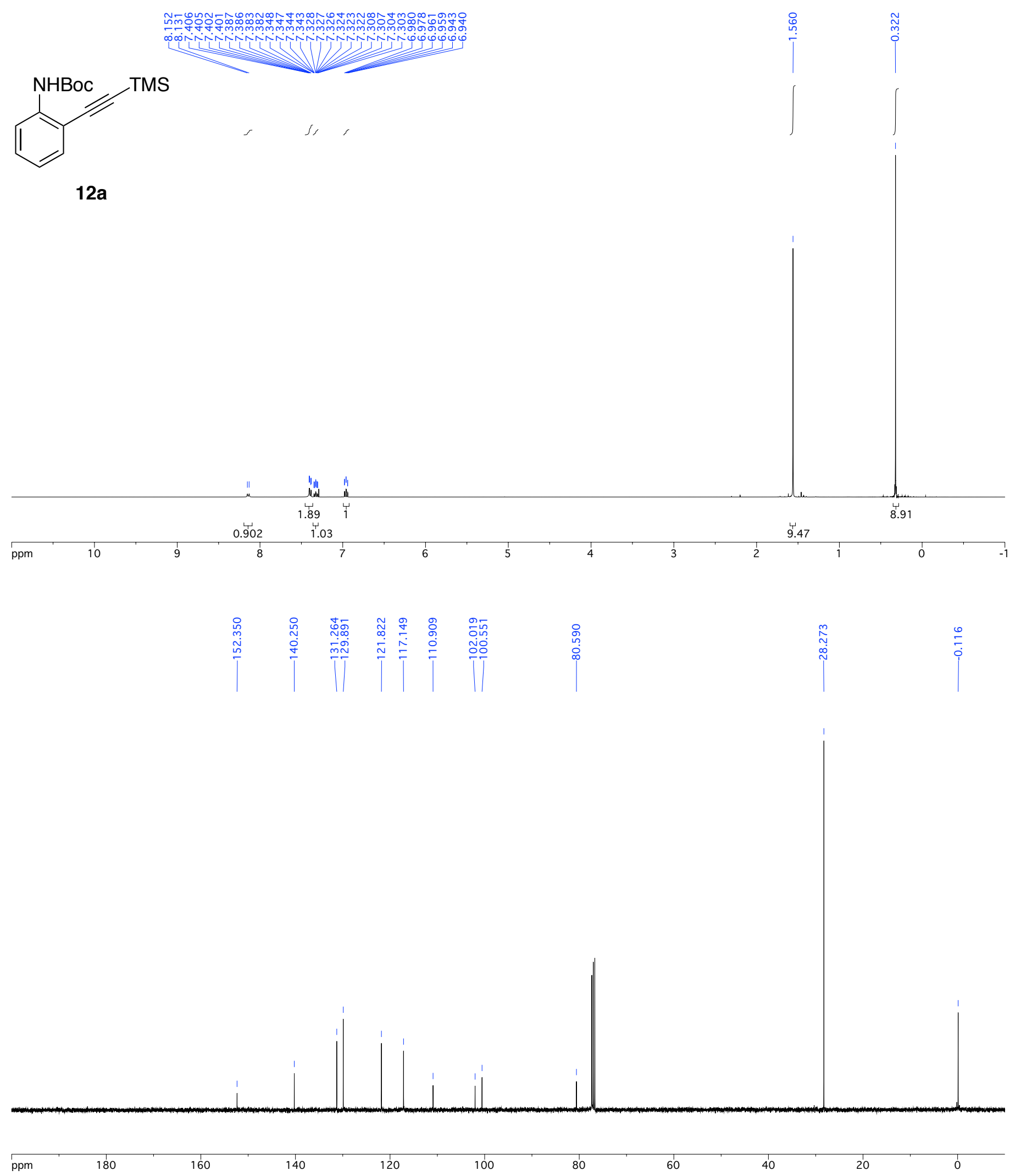

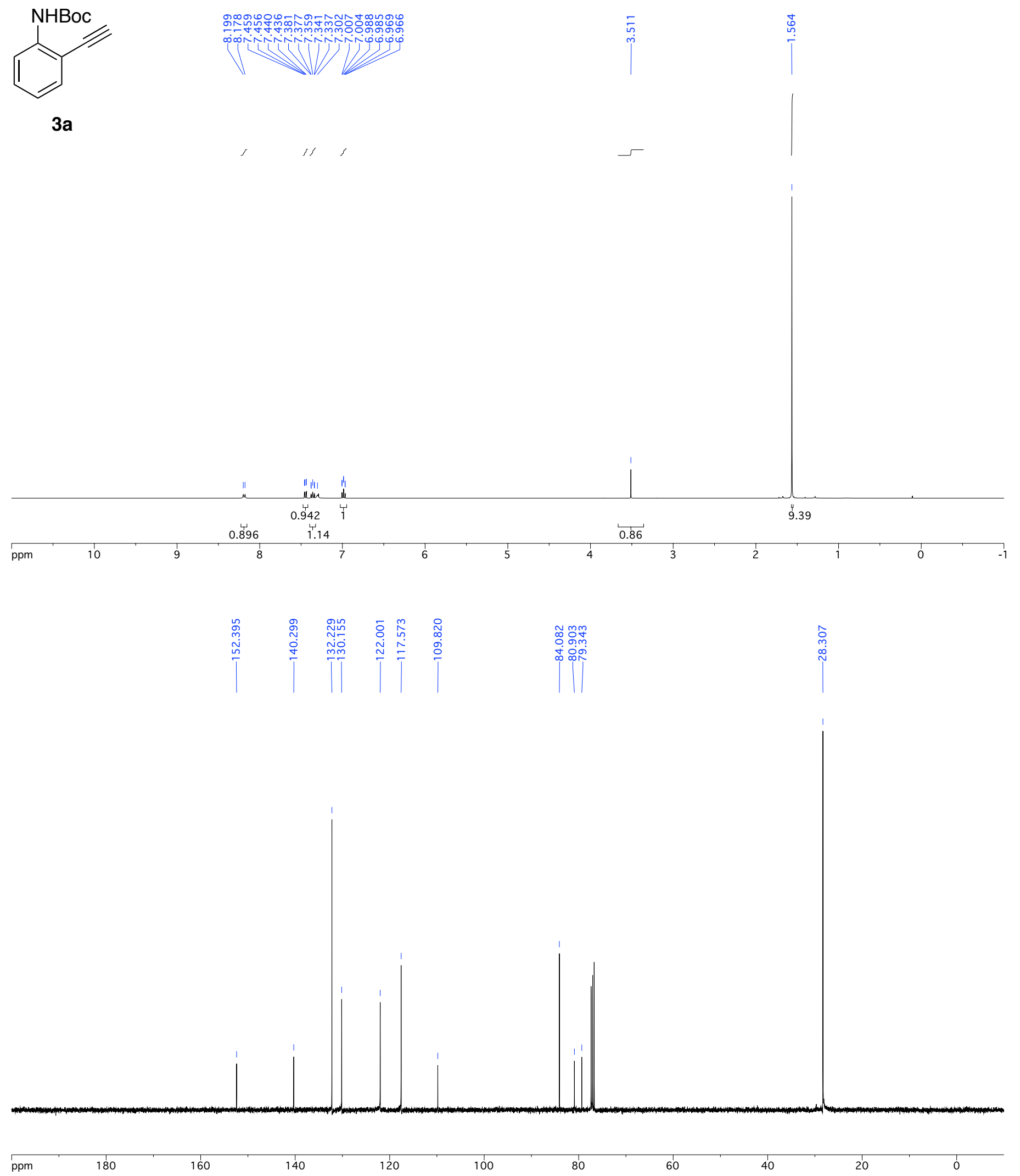


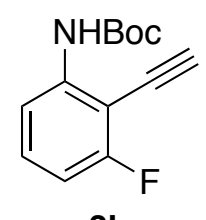

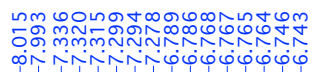

$\checkmark v$ W

$3 b$

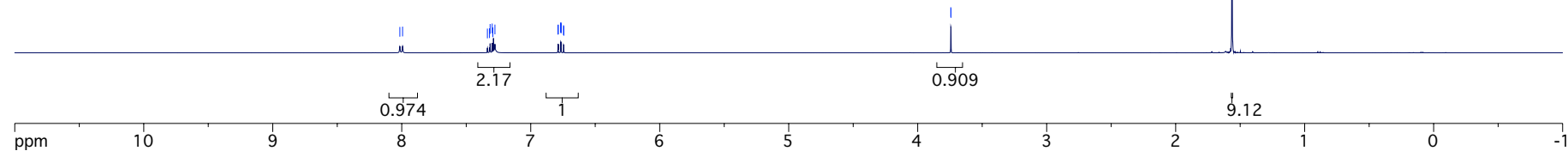

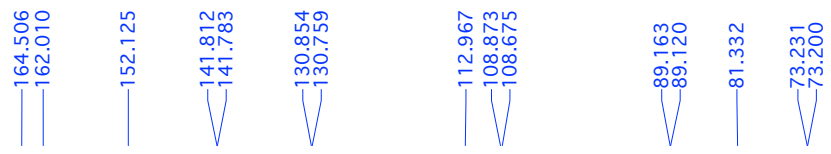

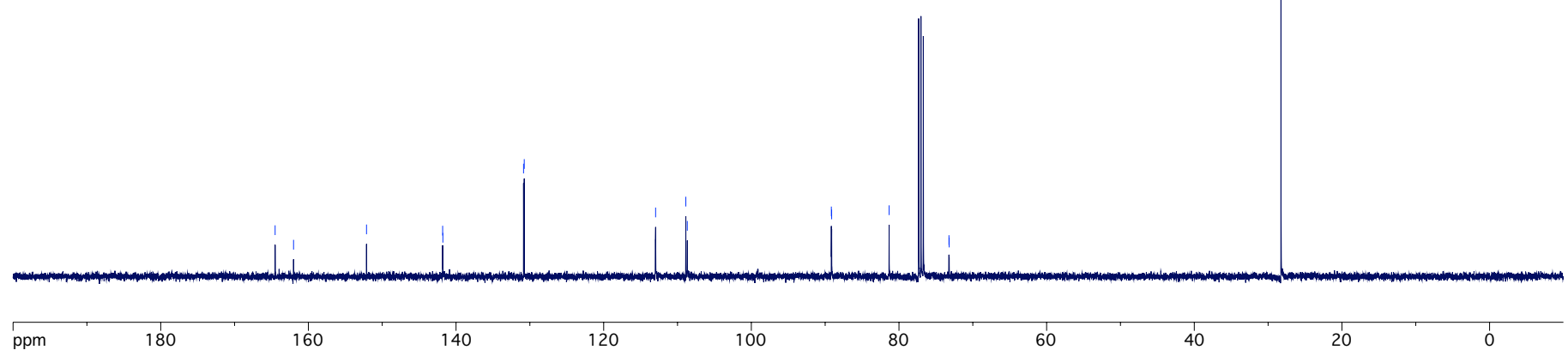



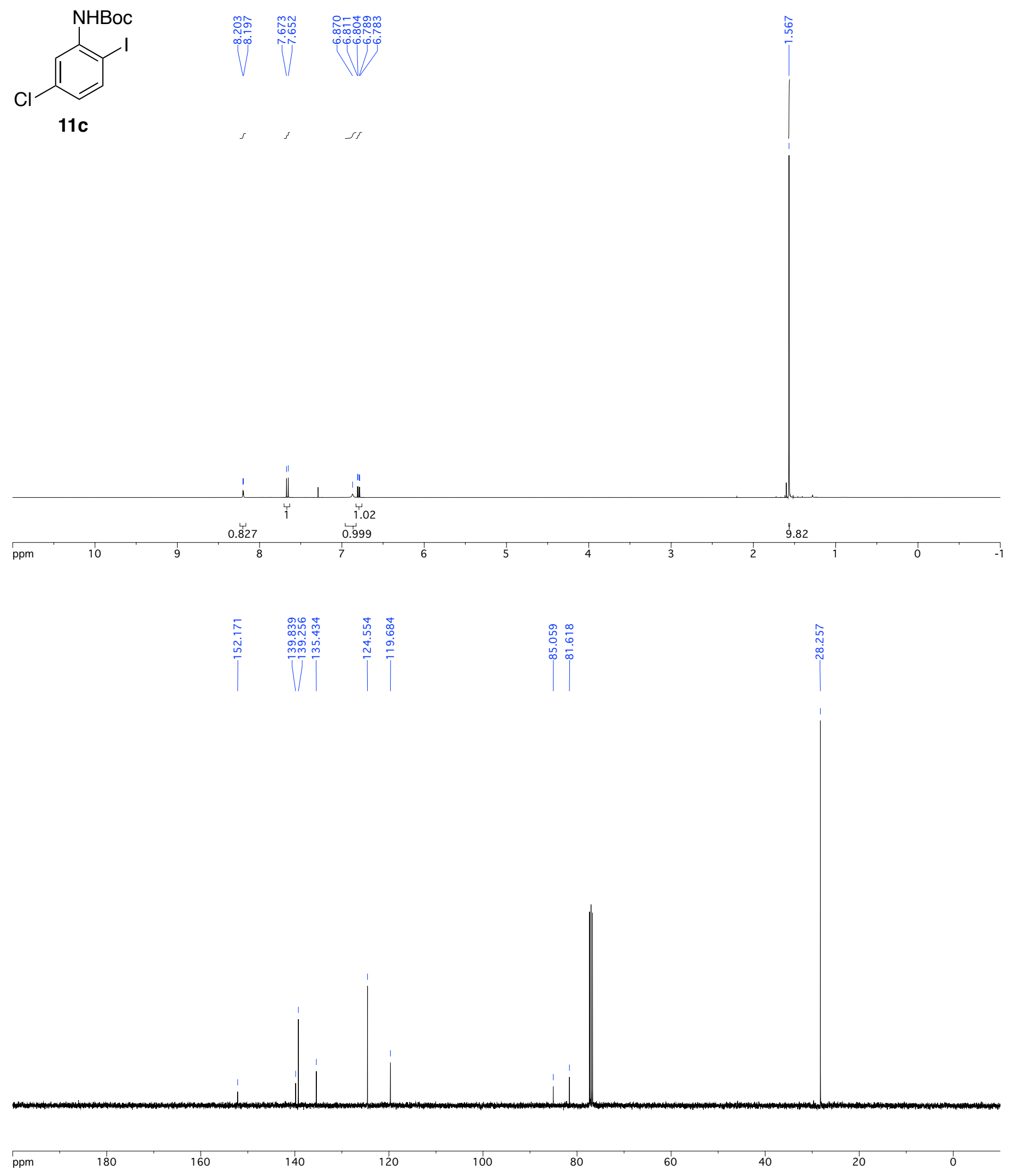

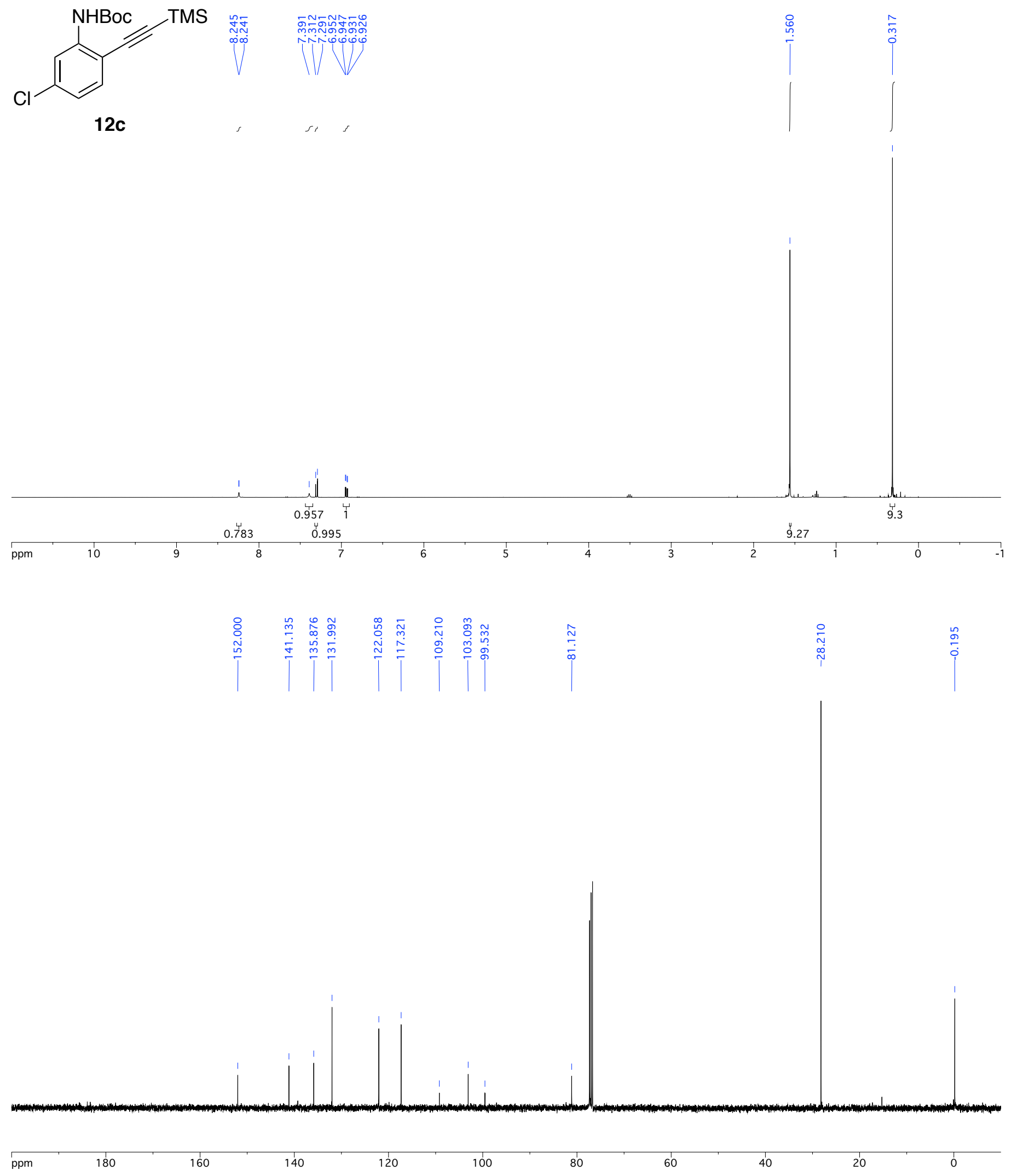

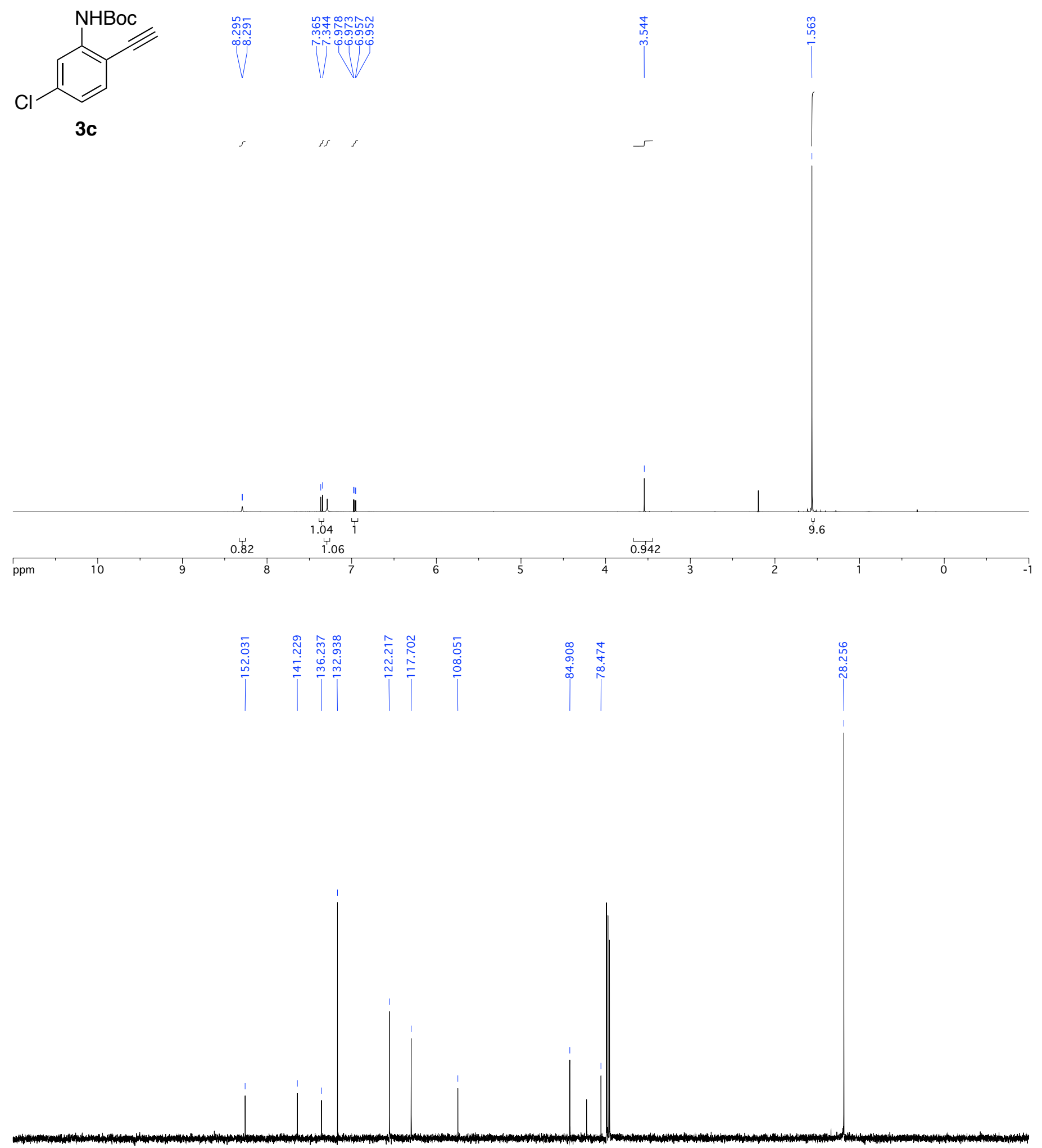

ppm

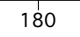

160

140

120

100

80

60

40

20 


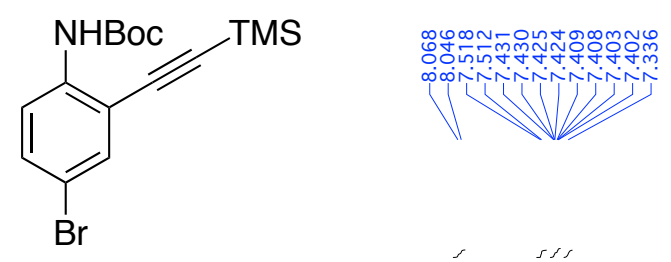

12d
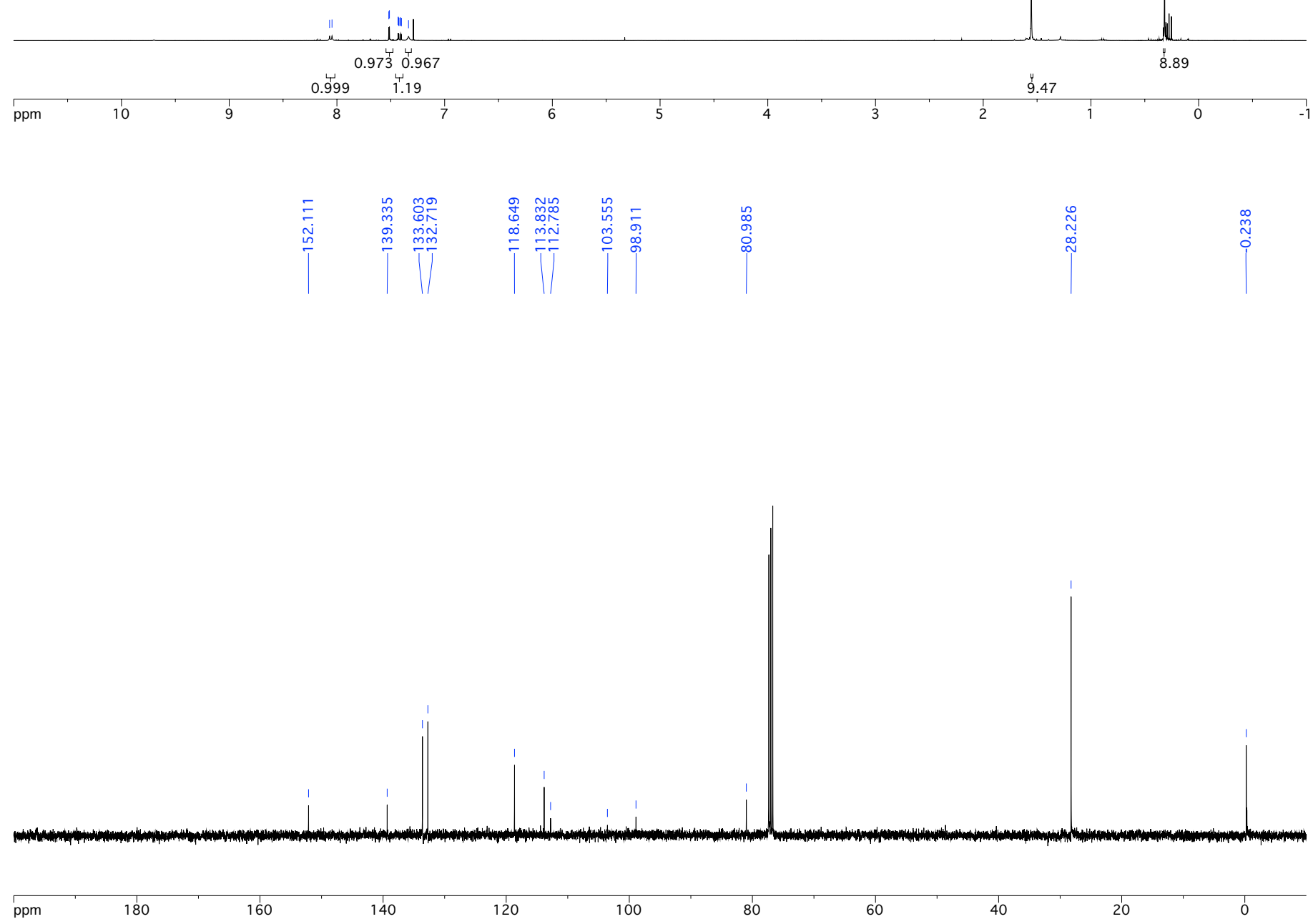


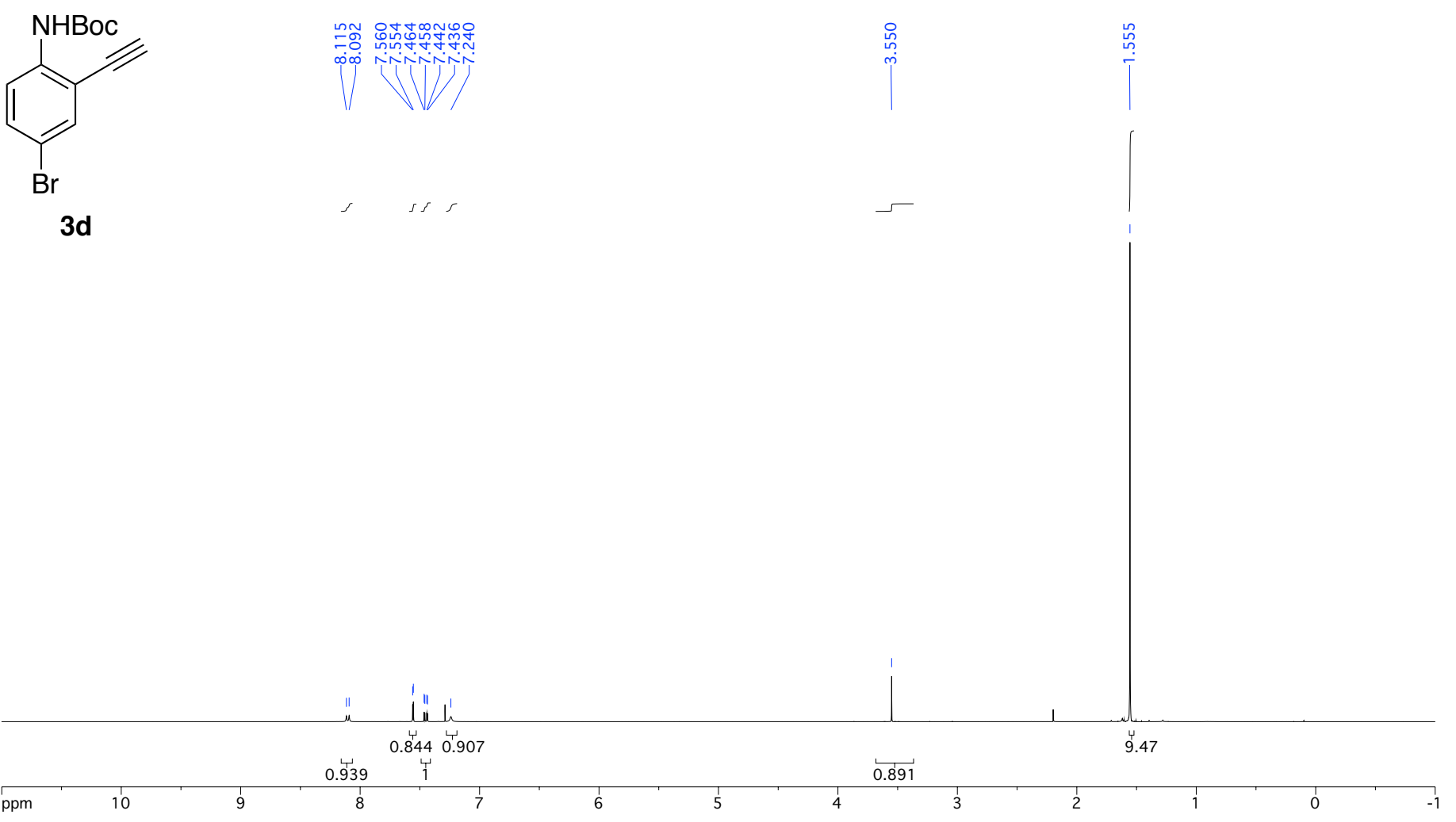

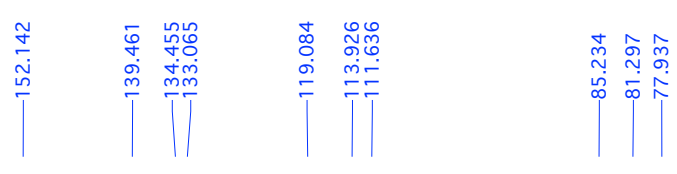




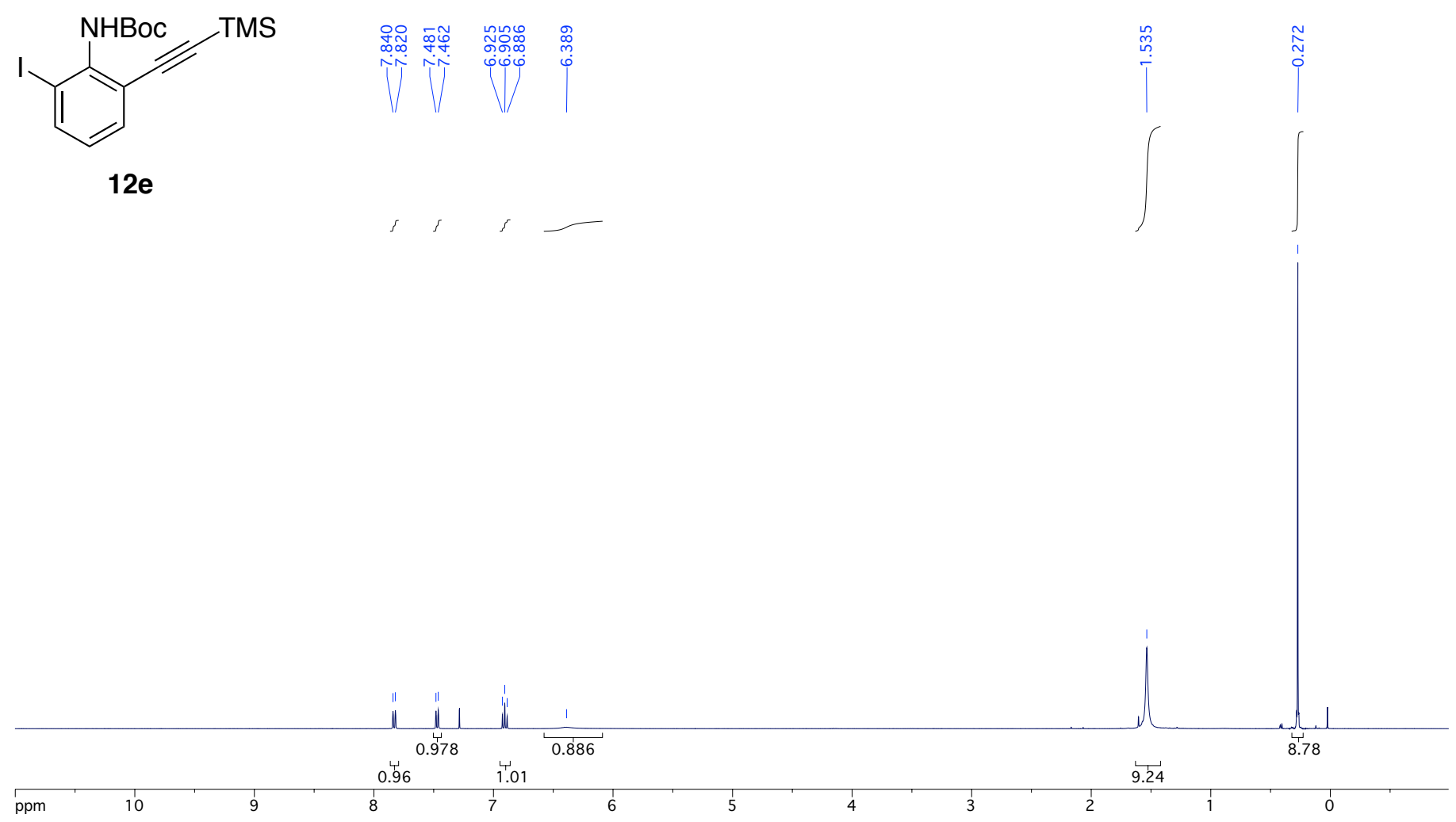

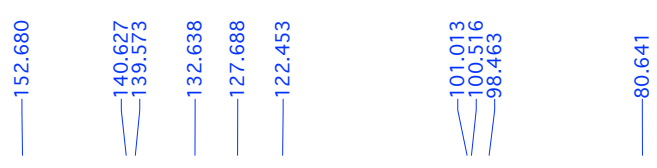
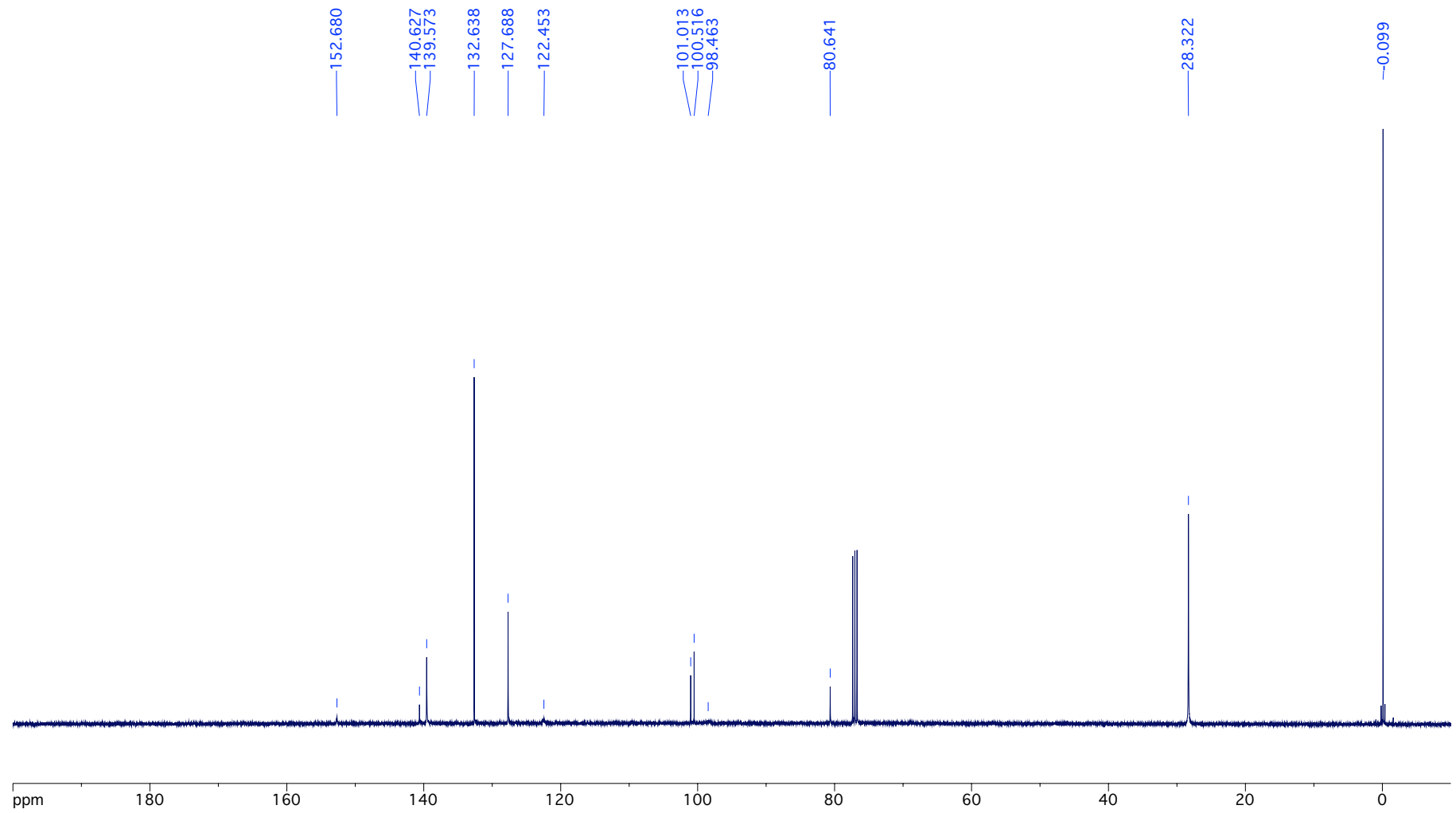

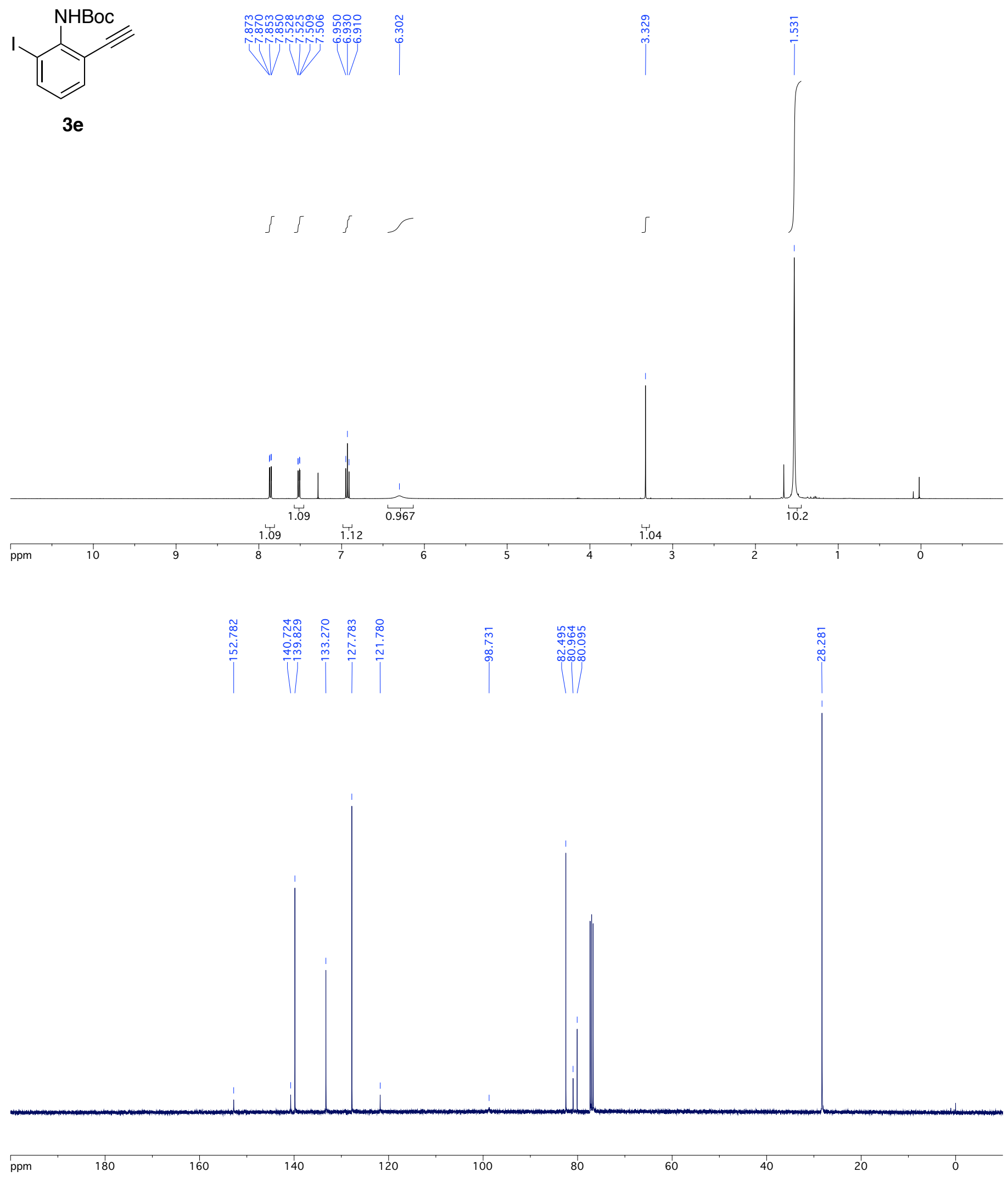
IF

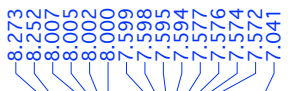

$11 f$

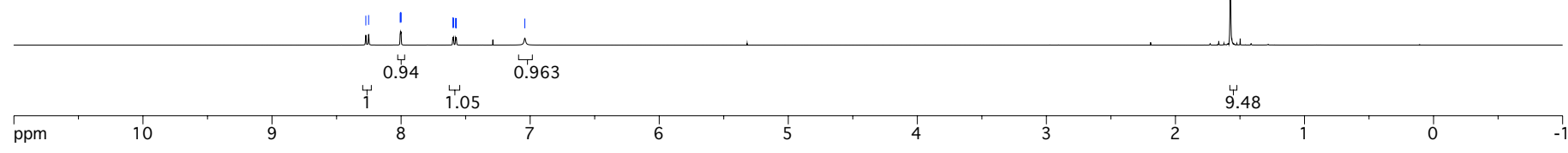

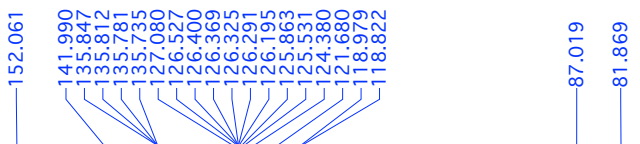

160

140

120

100

80

60

40

20 

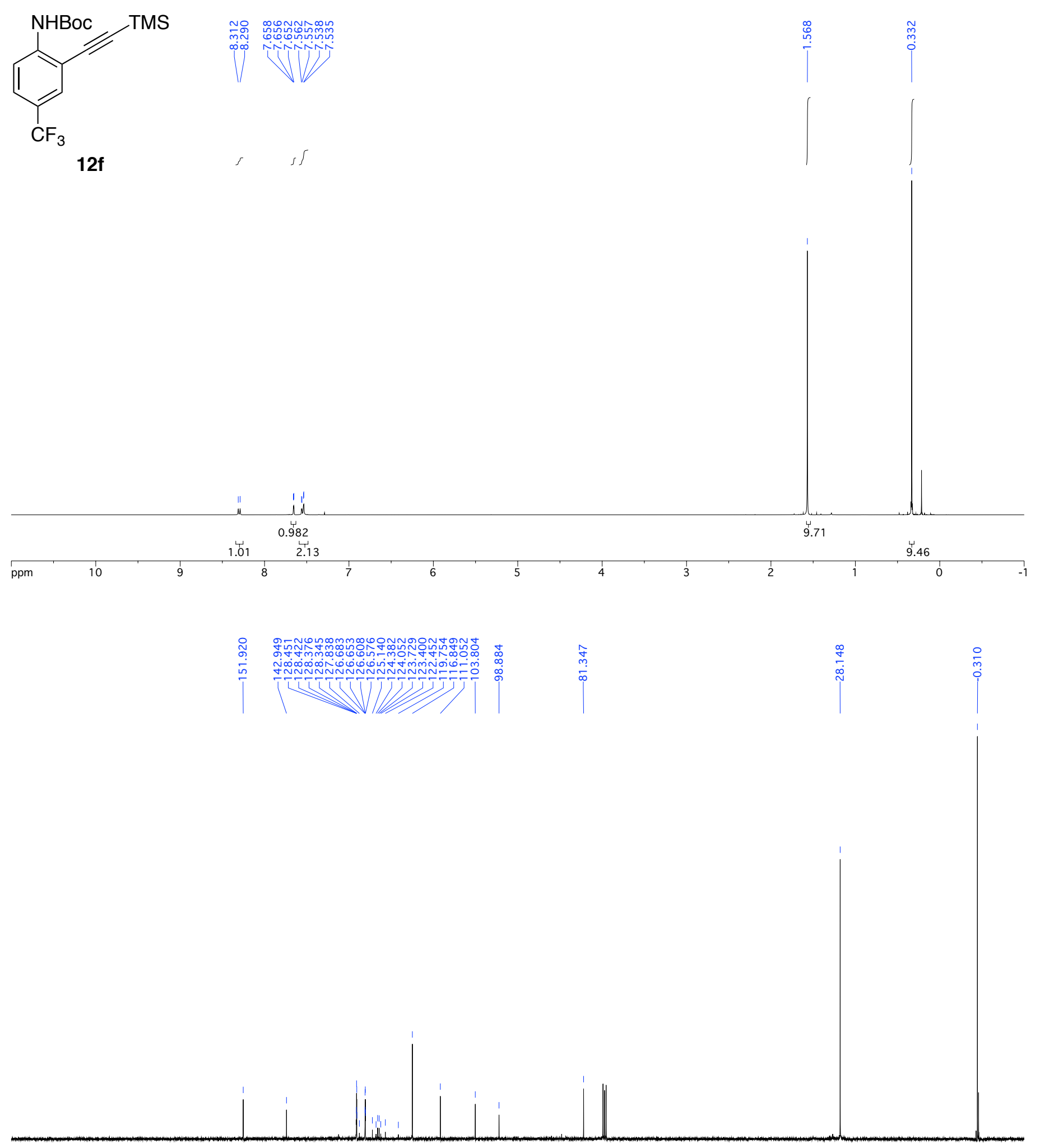

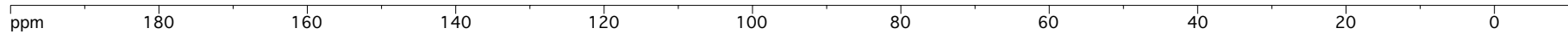



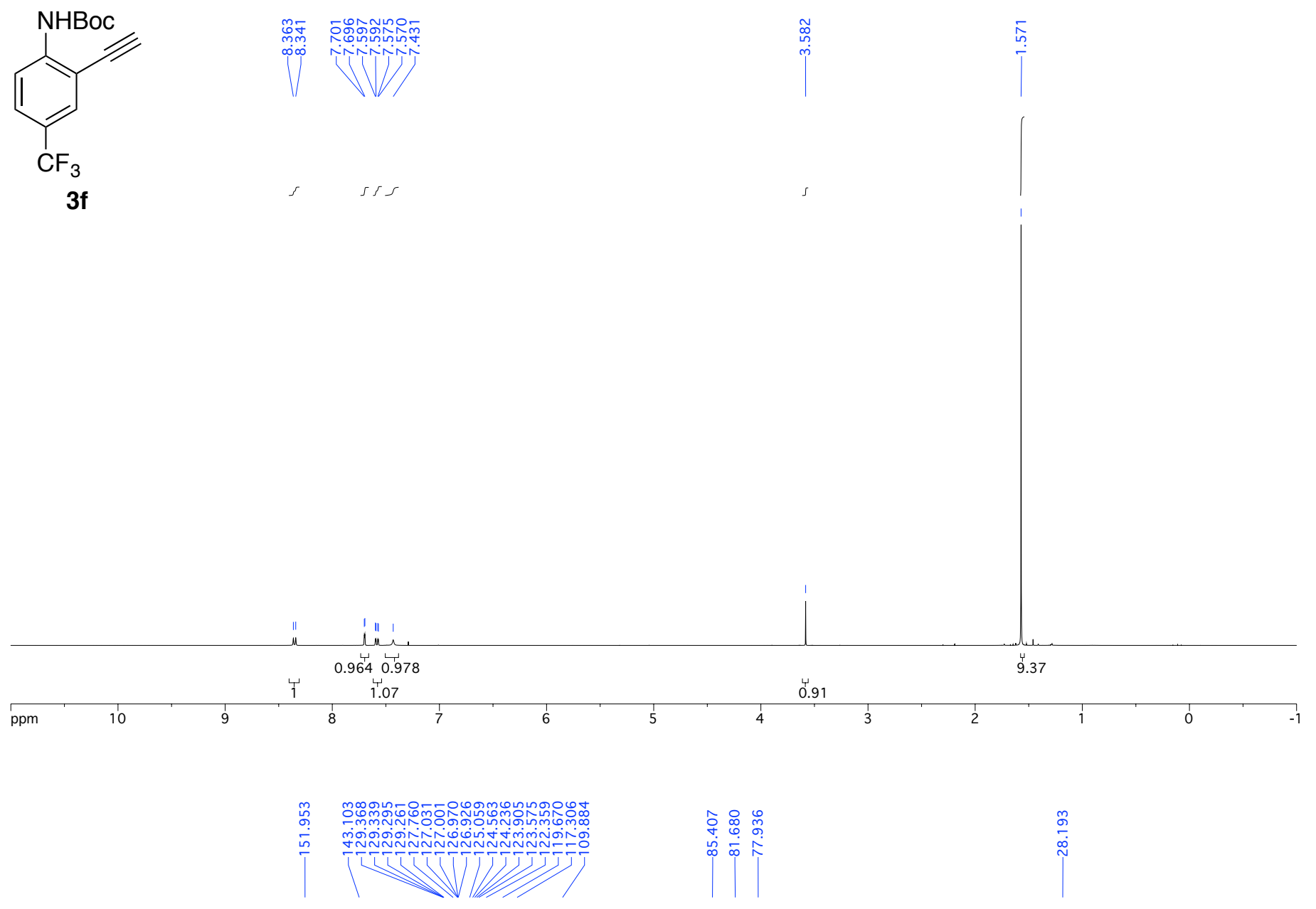

ppm

$180+160$

160

140

120

$100 \quad 80$

60

40

20 

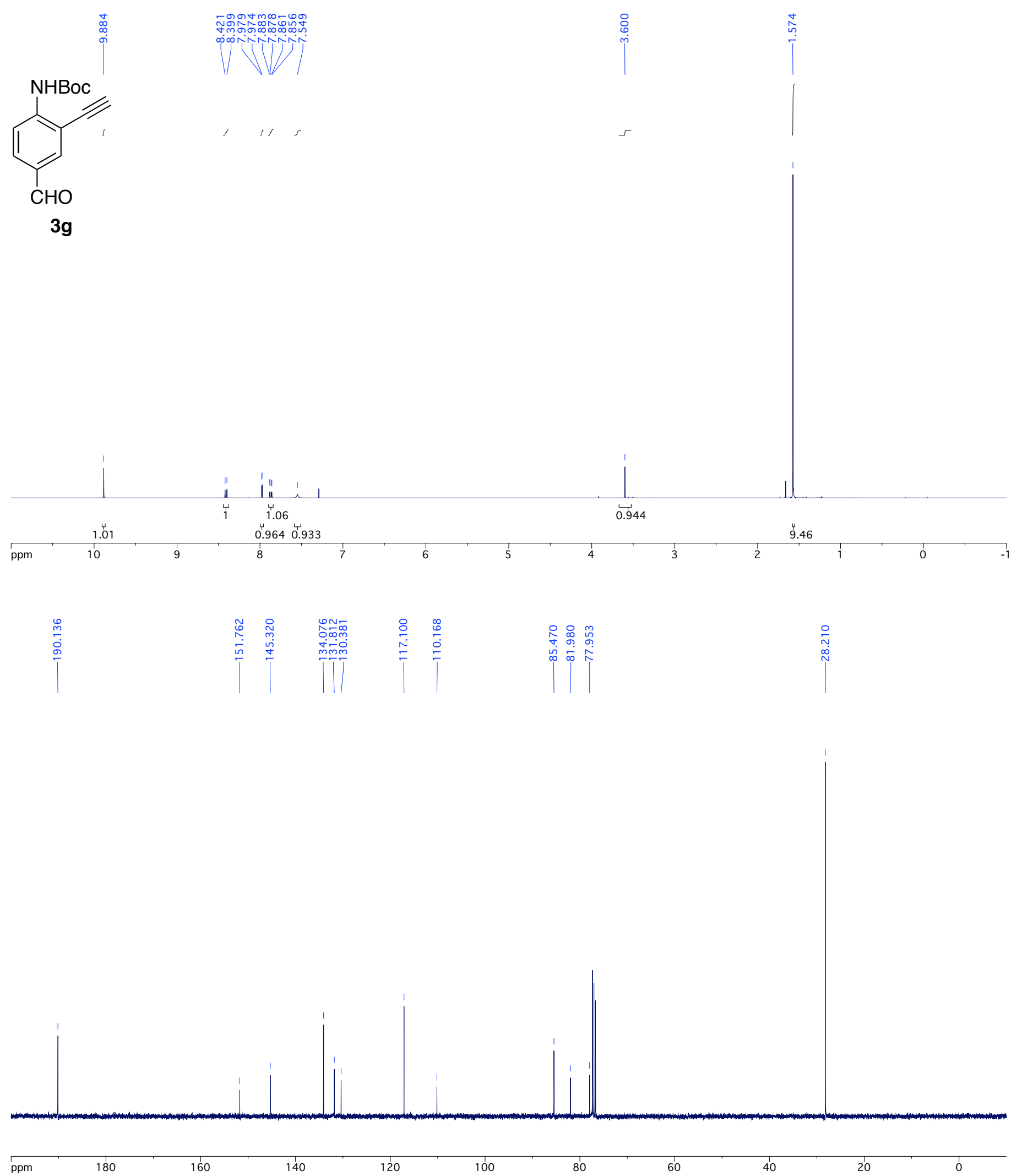

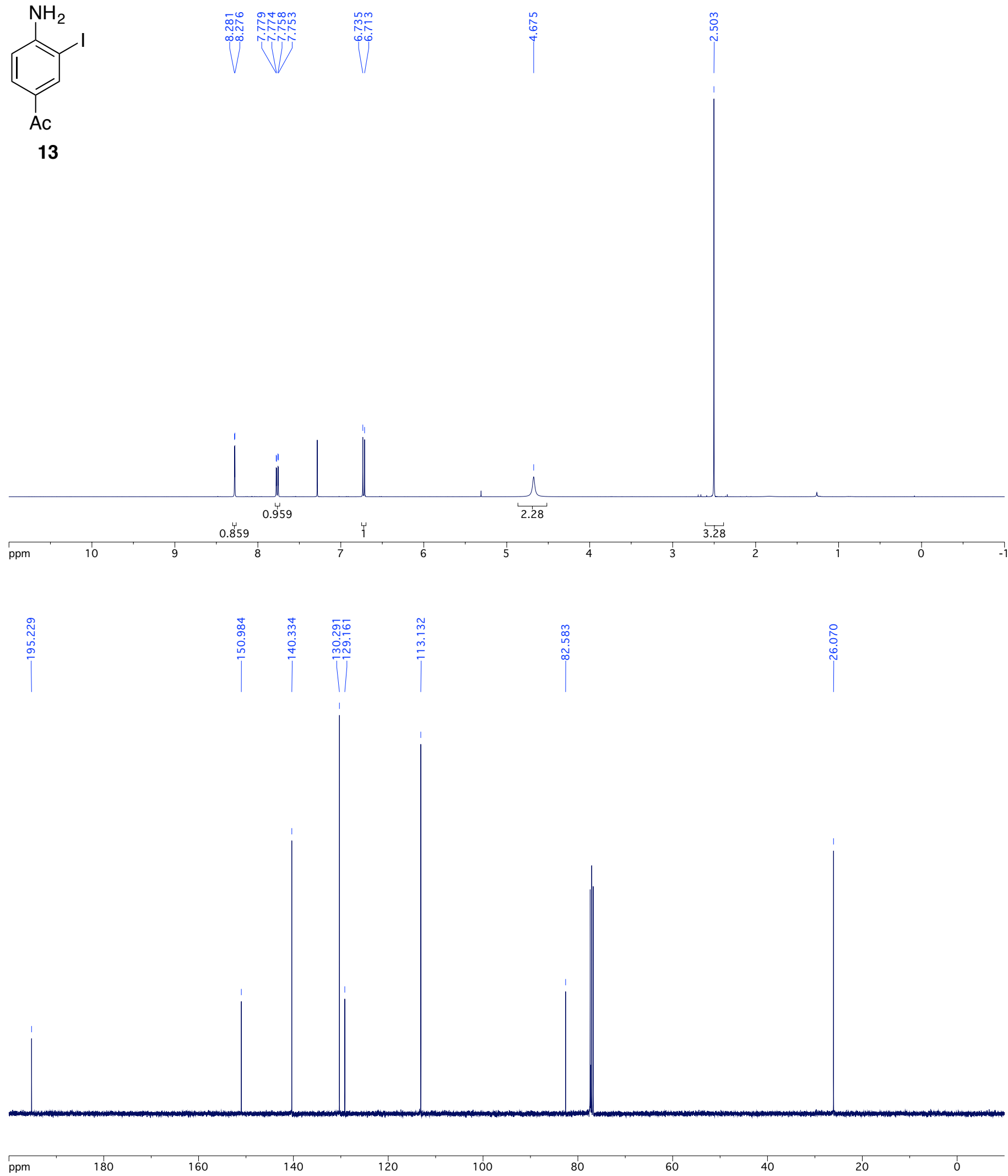
Ac
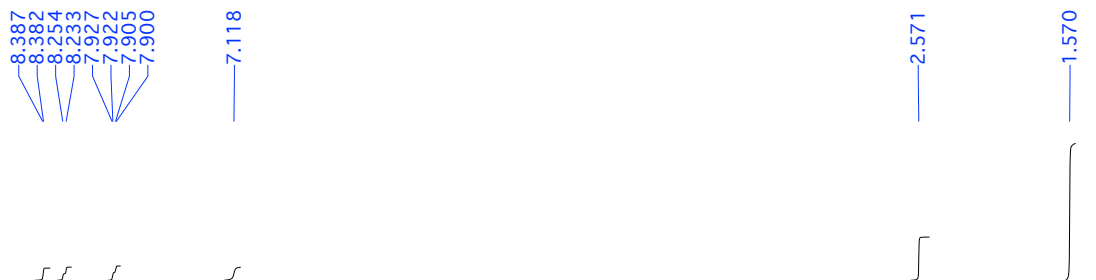

$11 \mathrm{~h}$
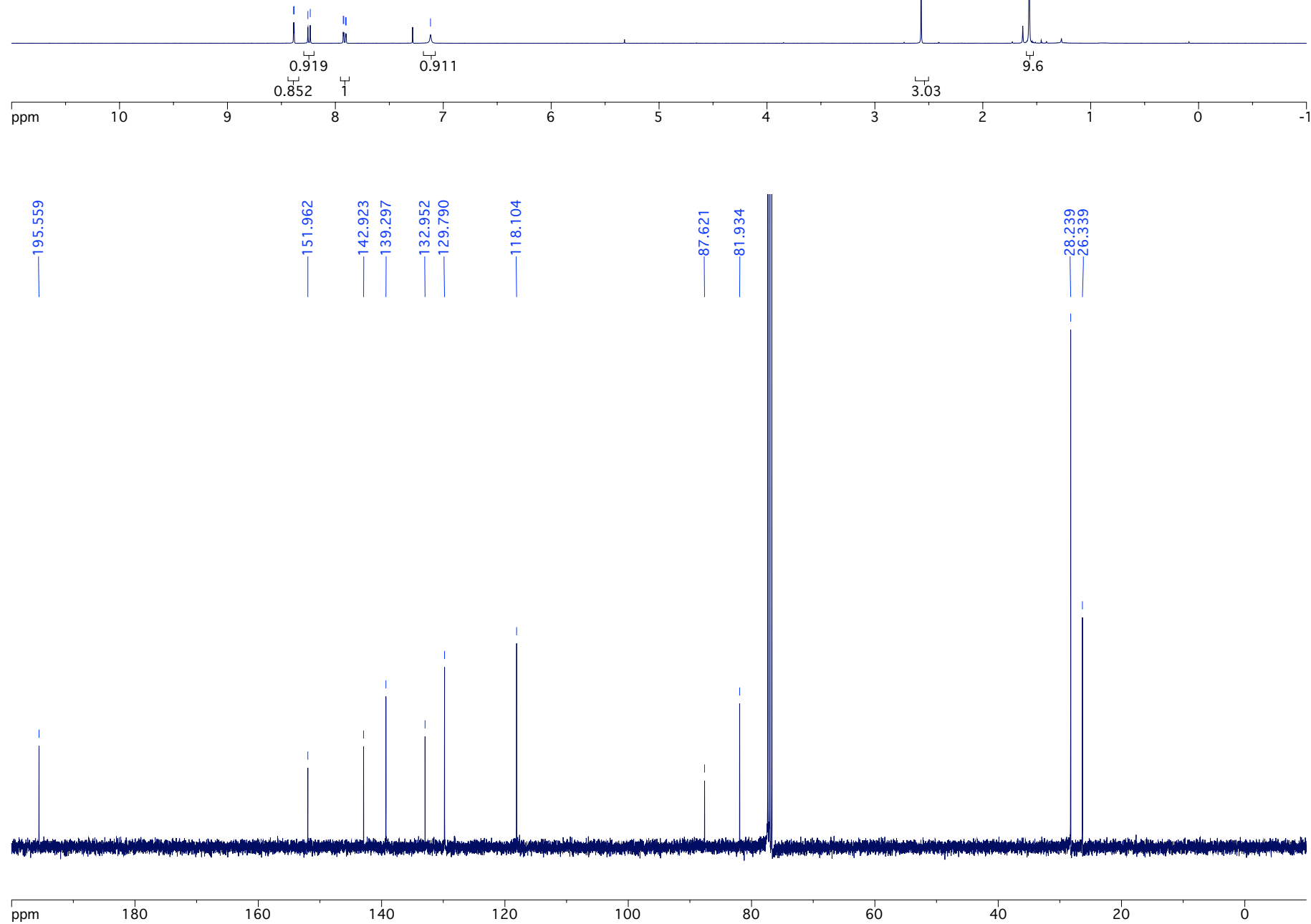

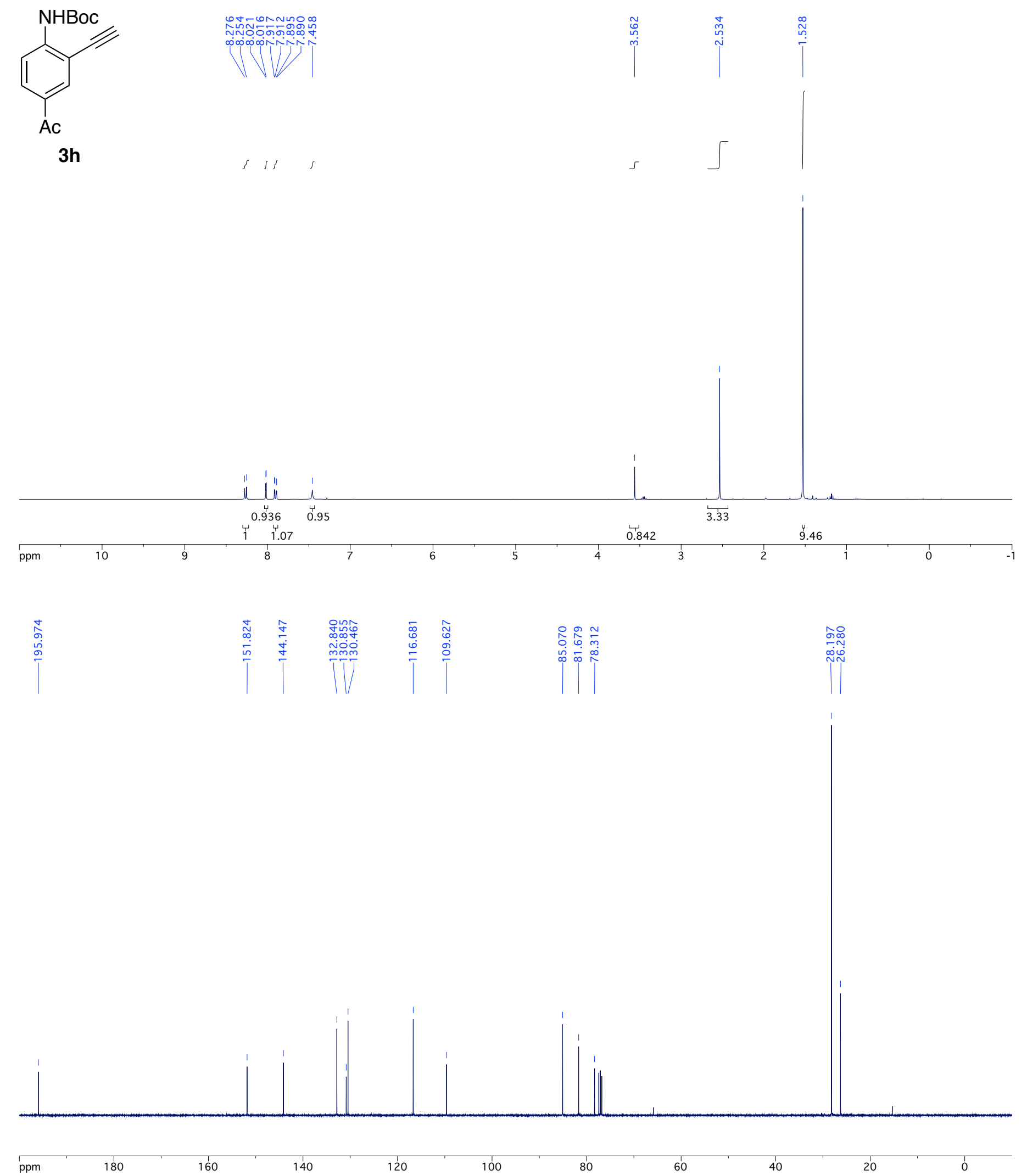

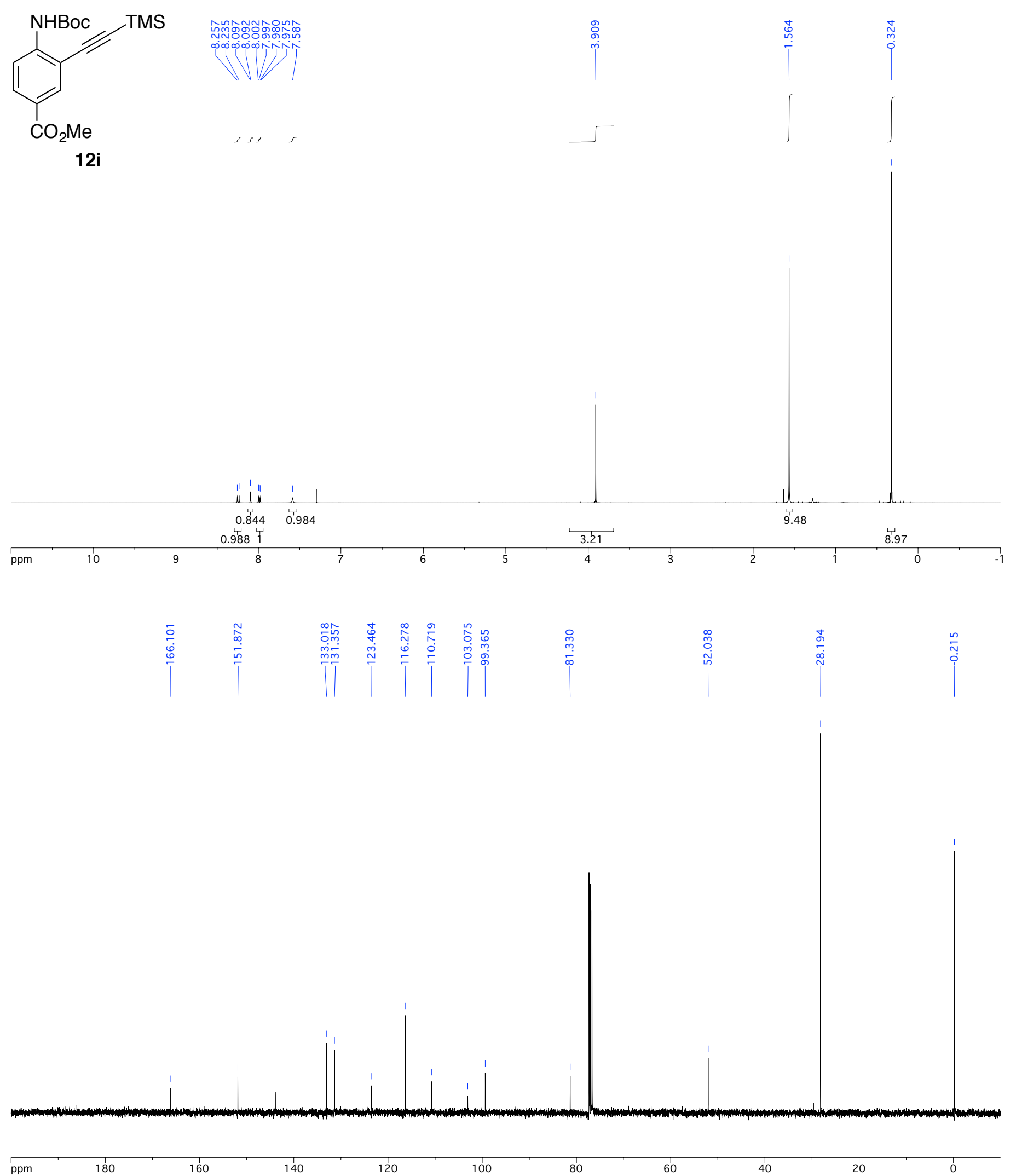

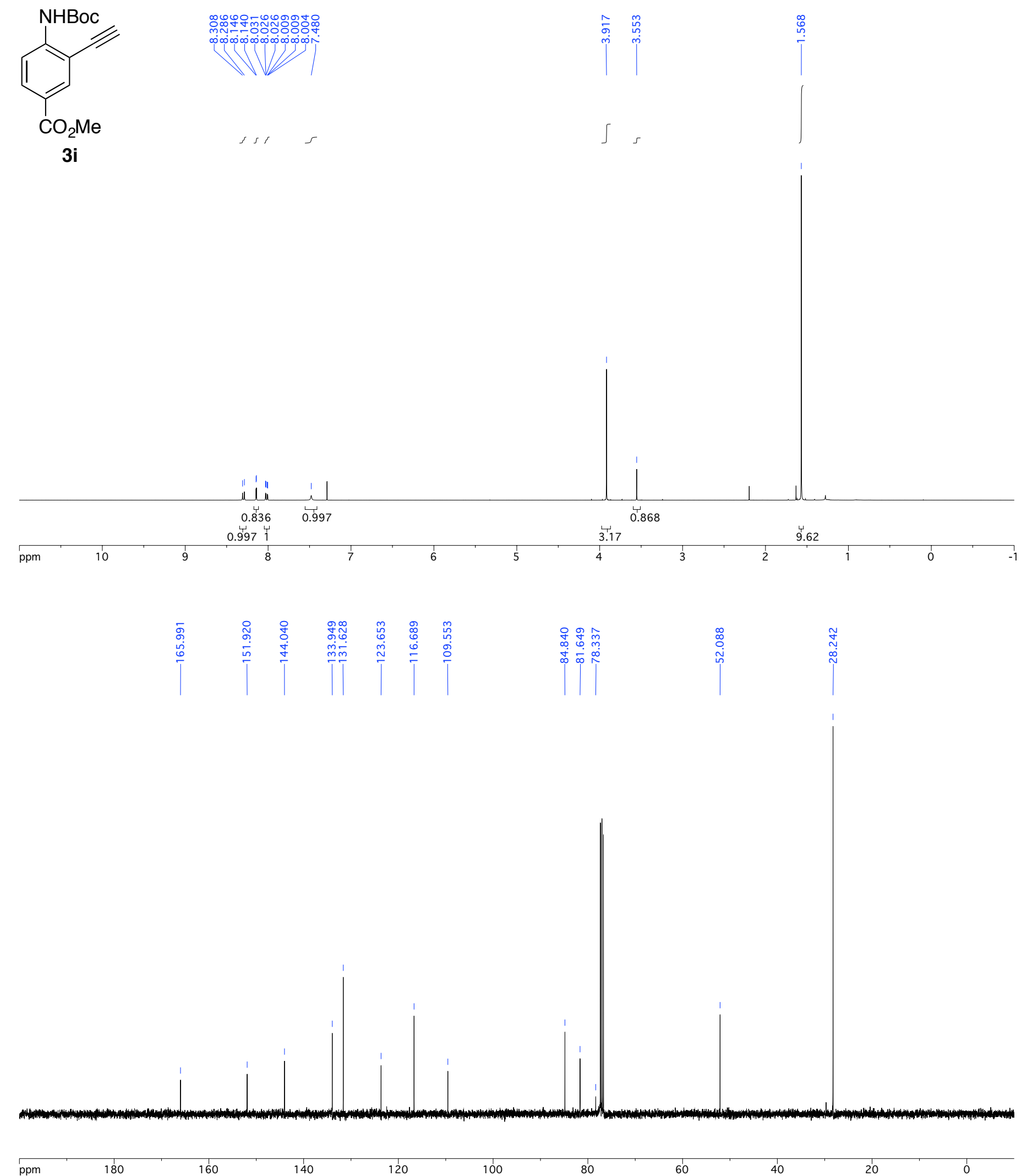

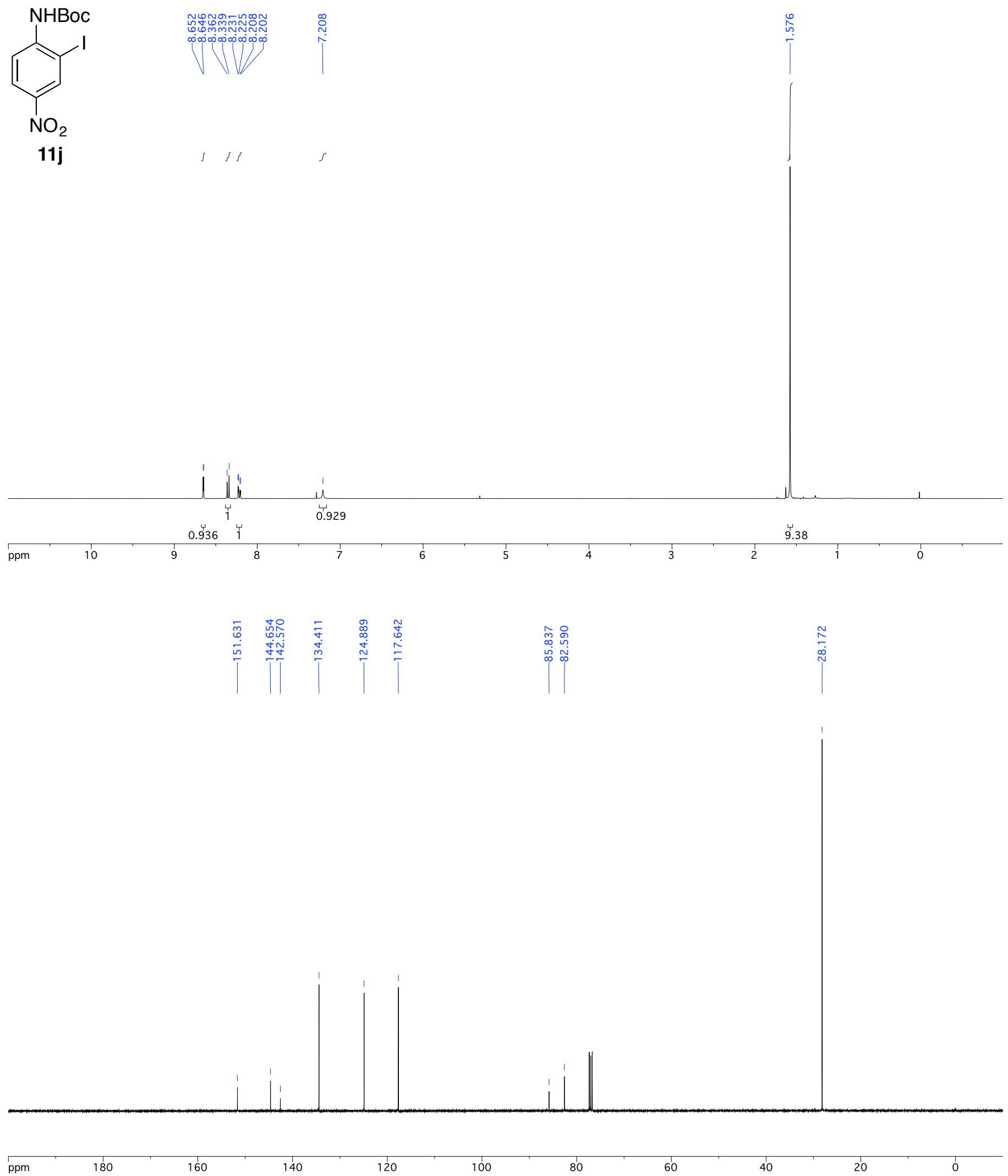


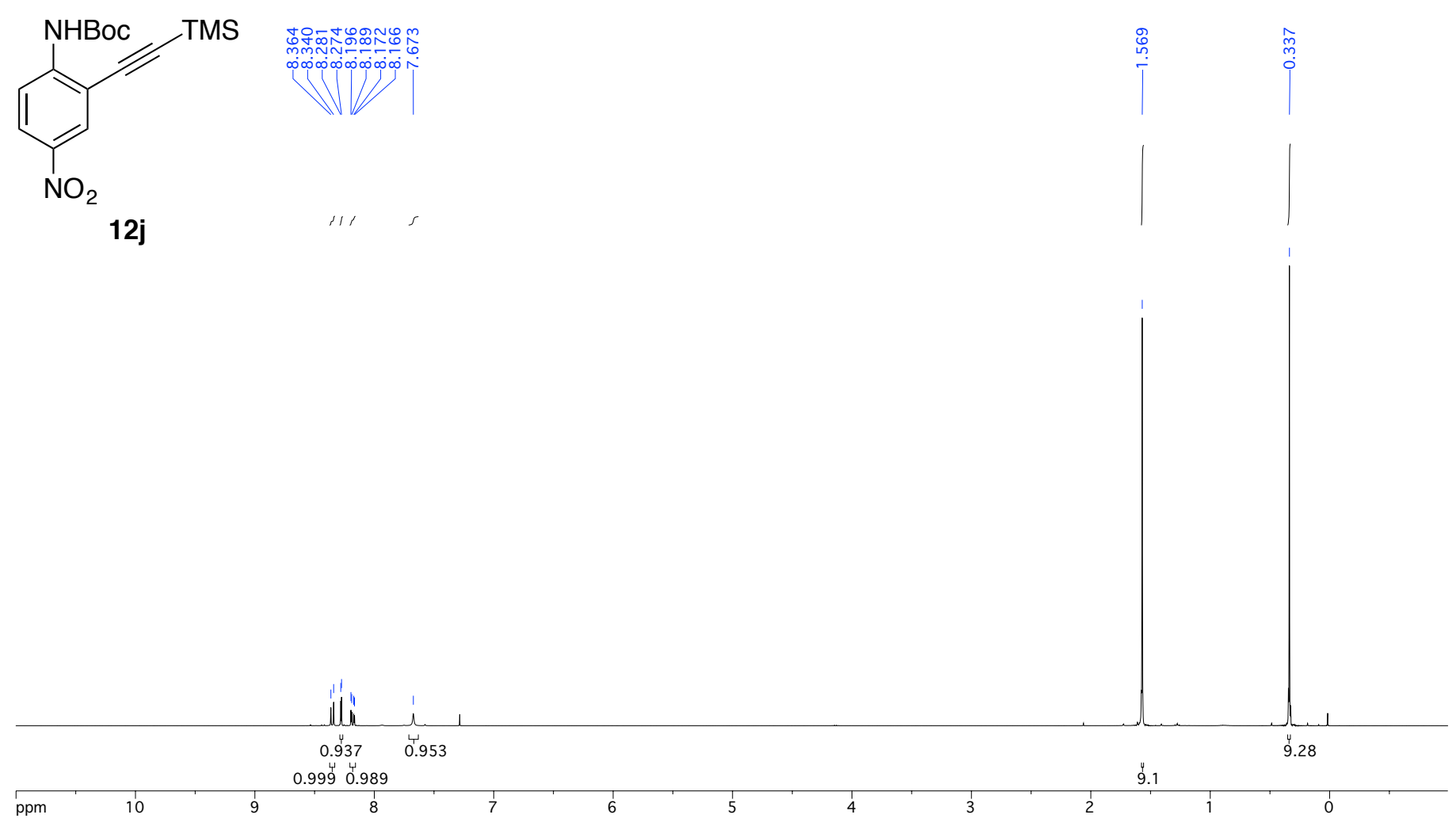

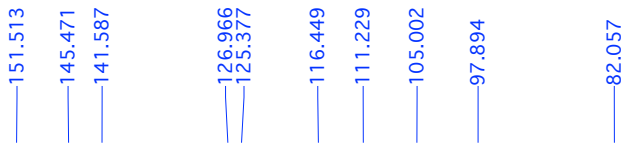

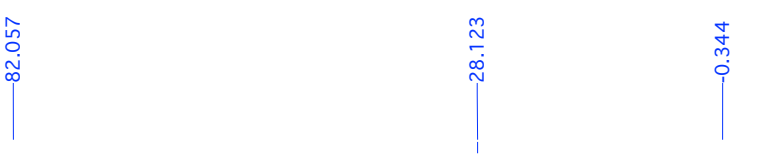

ppm

180

160

140

120

100

80

60

40

20 

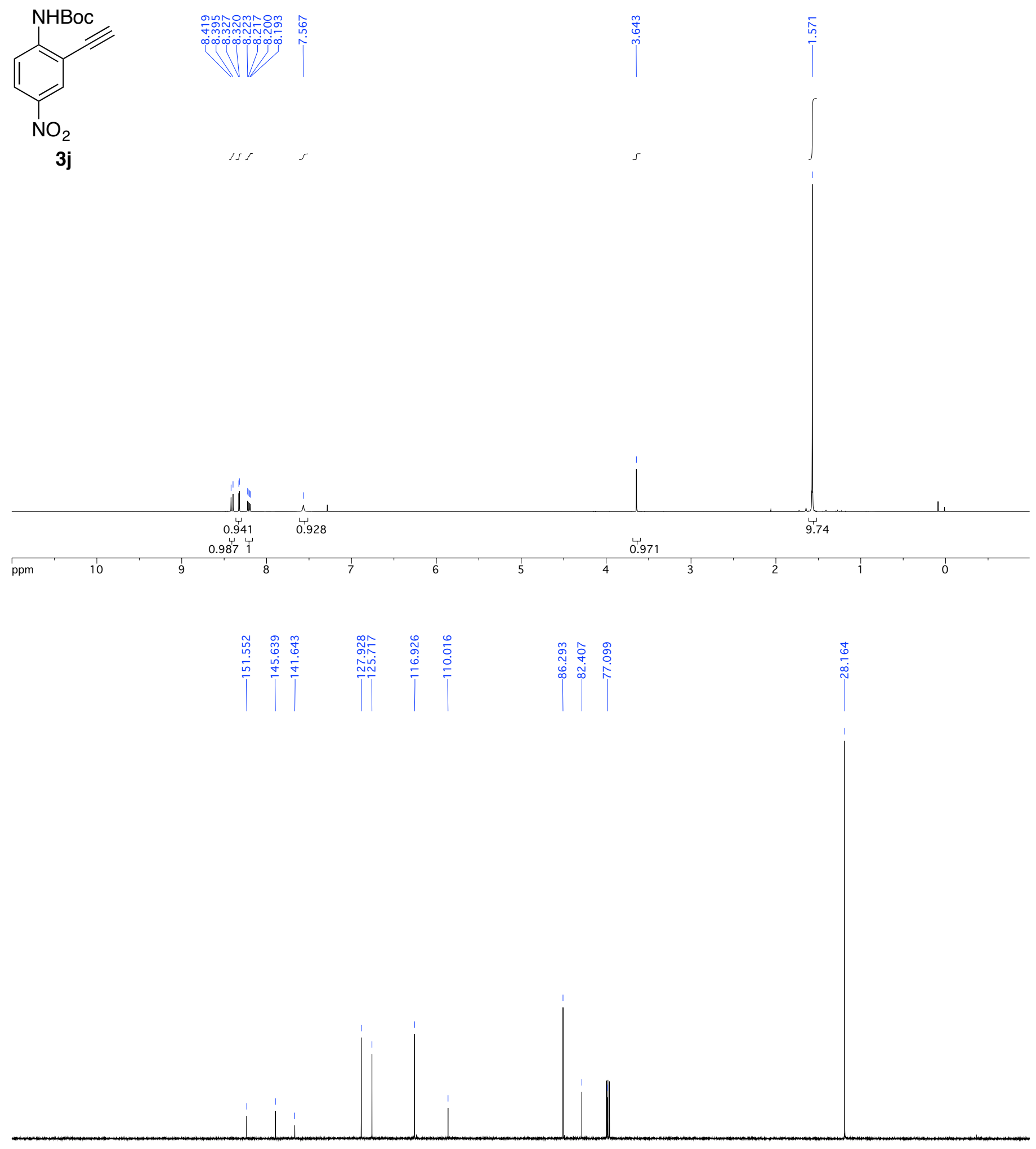

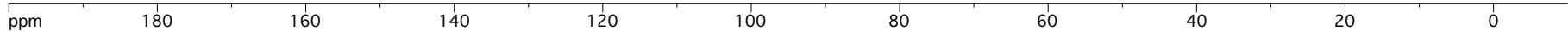



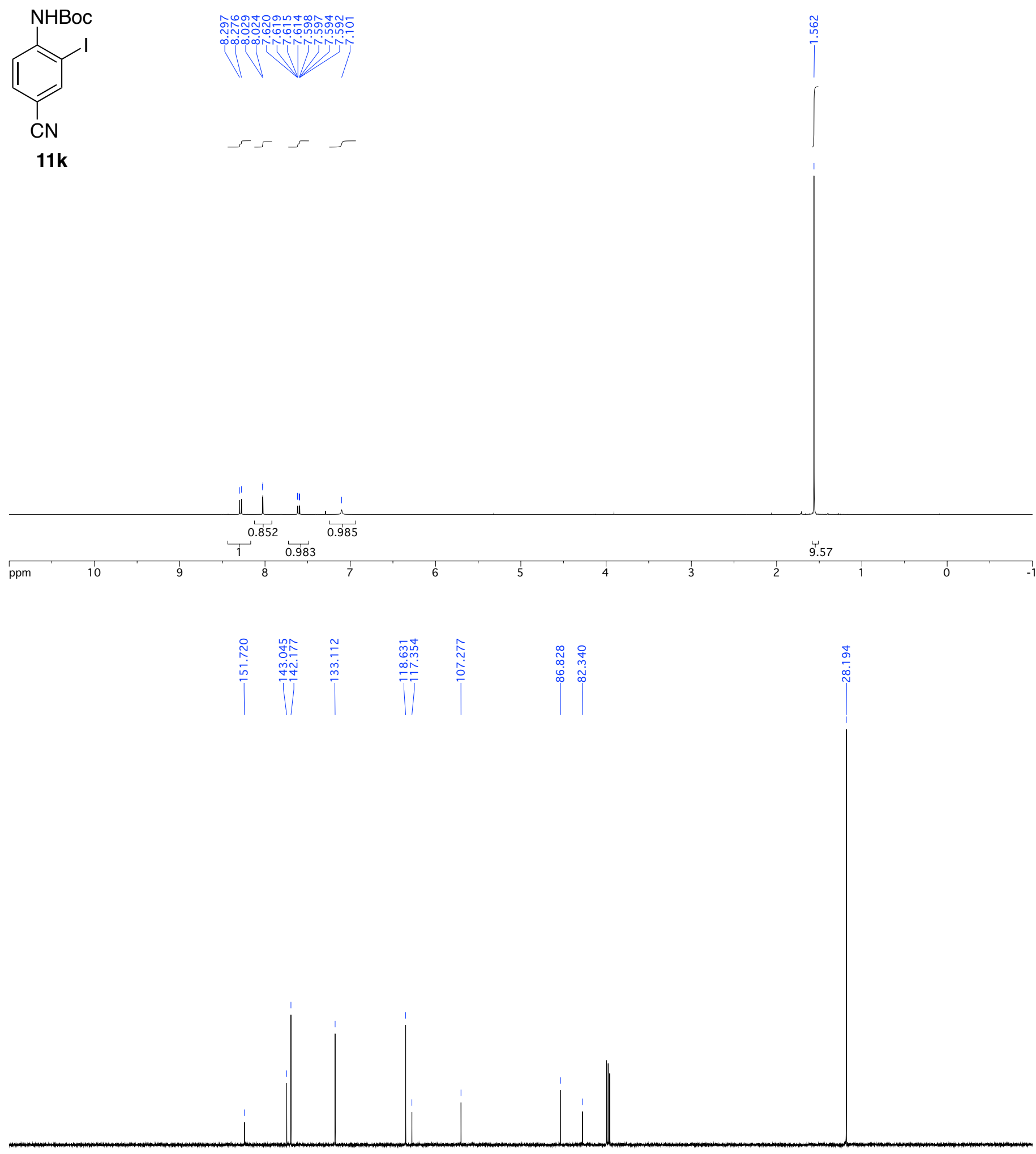

ppm

$180 \quad 160$

140

120

100

80

60

40

20 


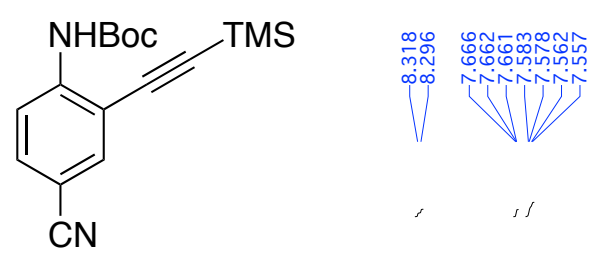

12k
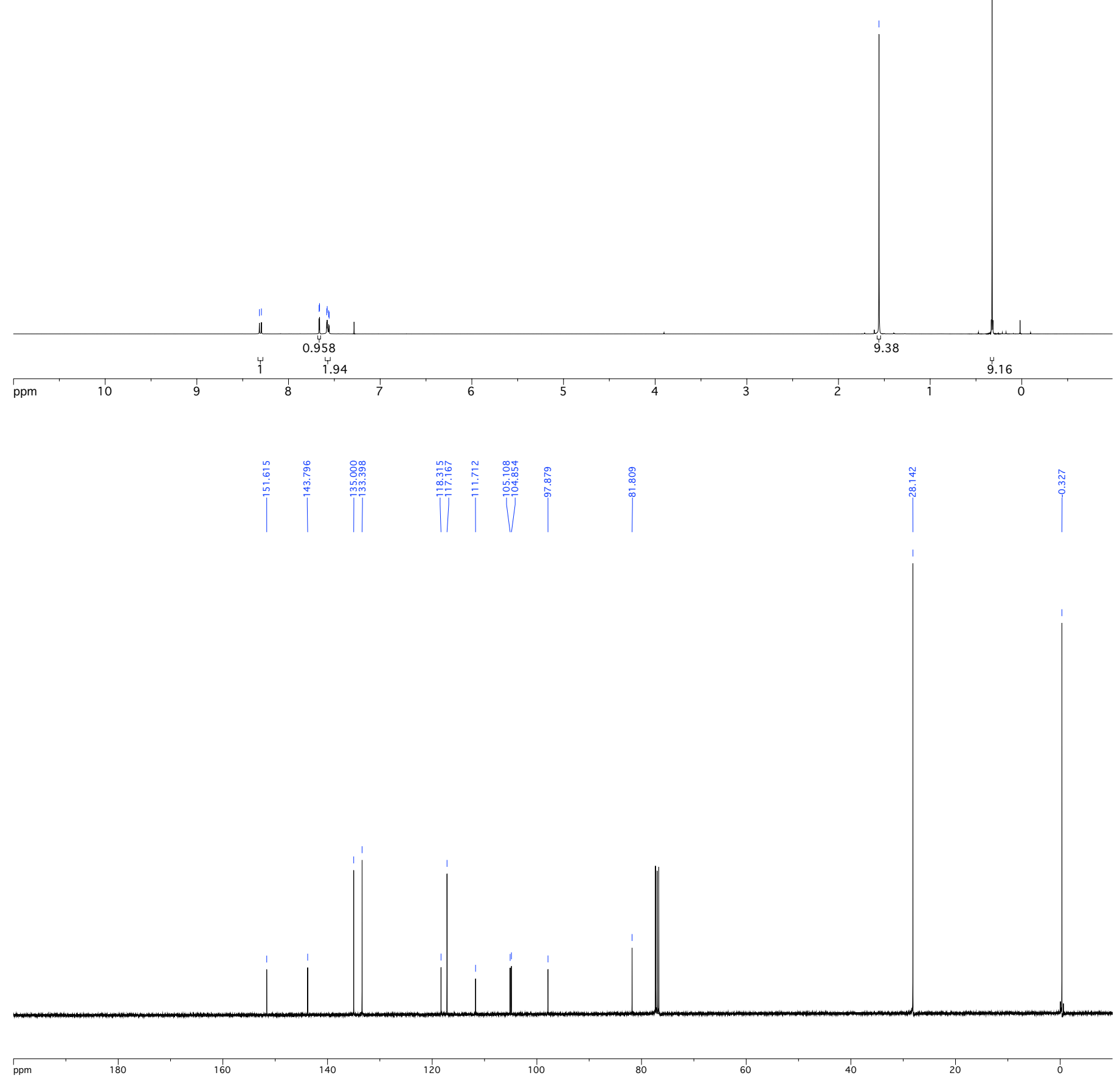


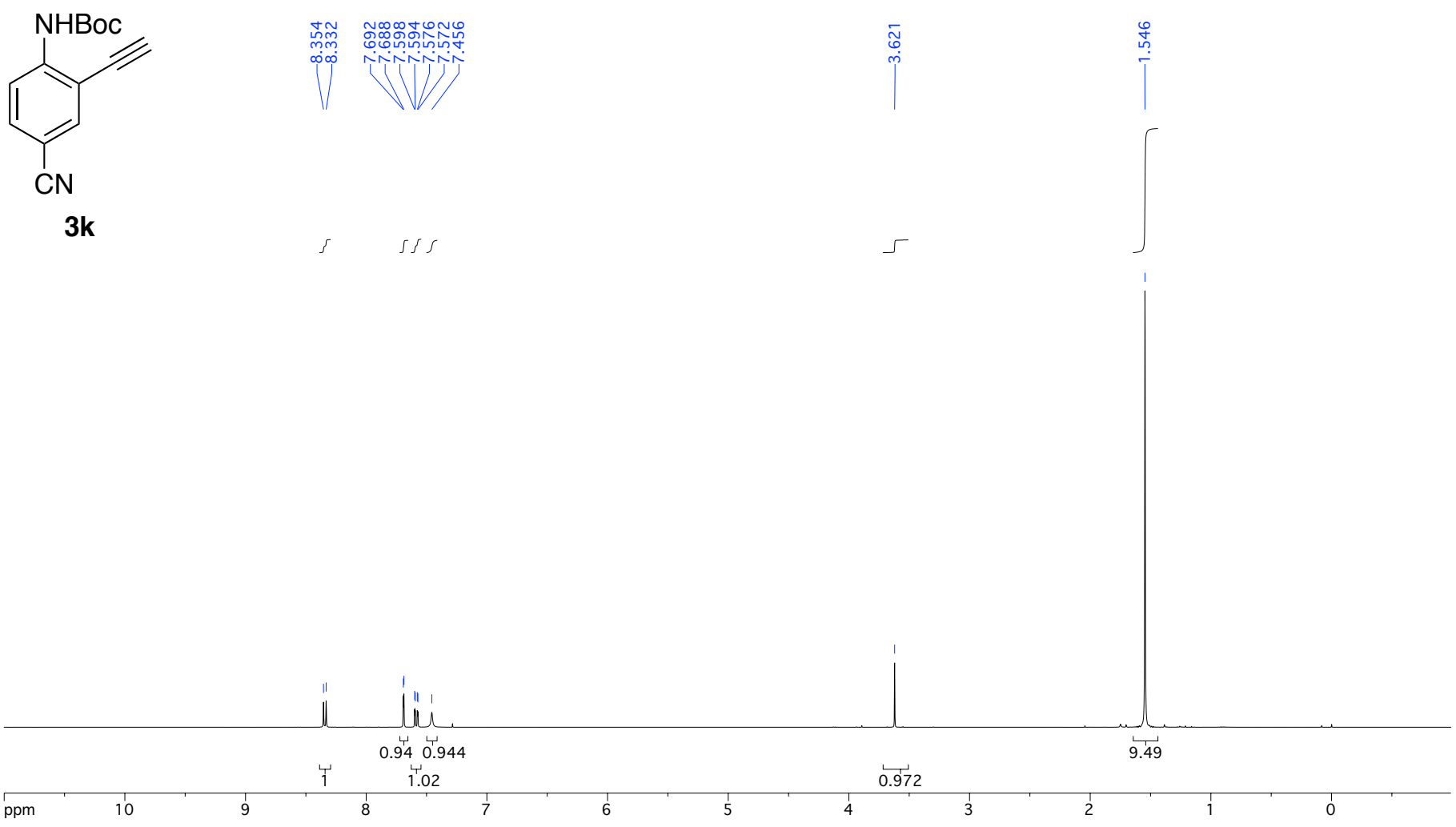

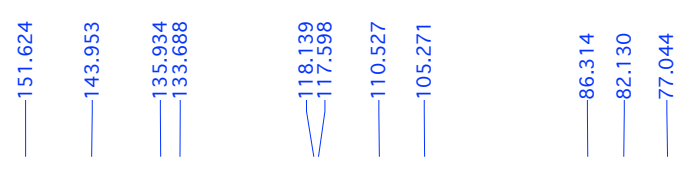


<smiles>Cc1ccc(I)c(NC(=O)OCc2ccccc2)c1</smiles>

111
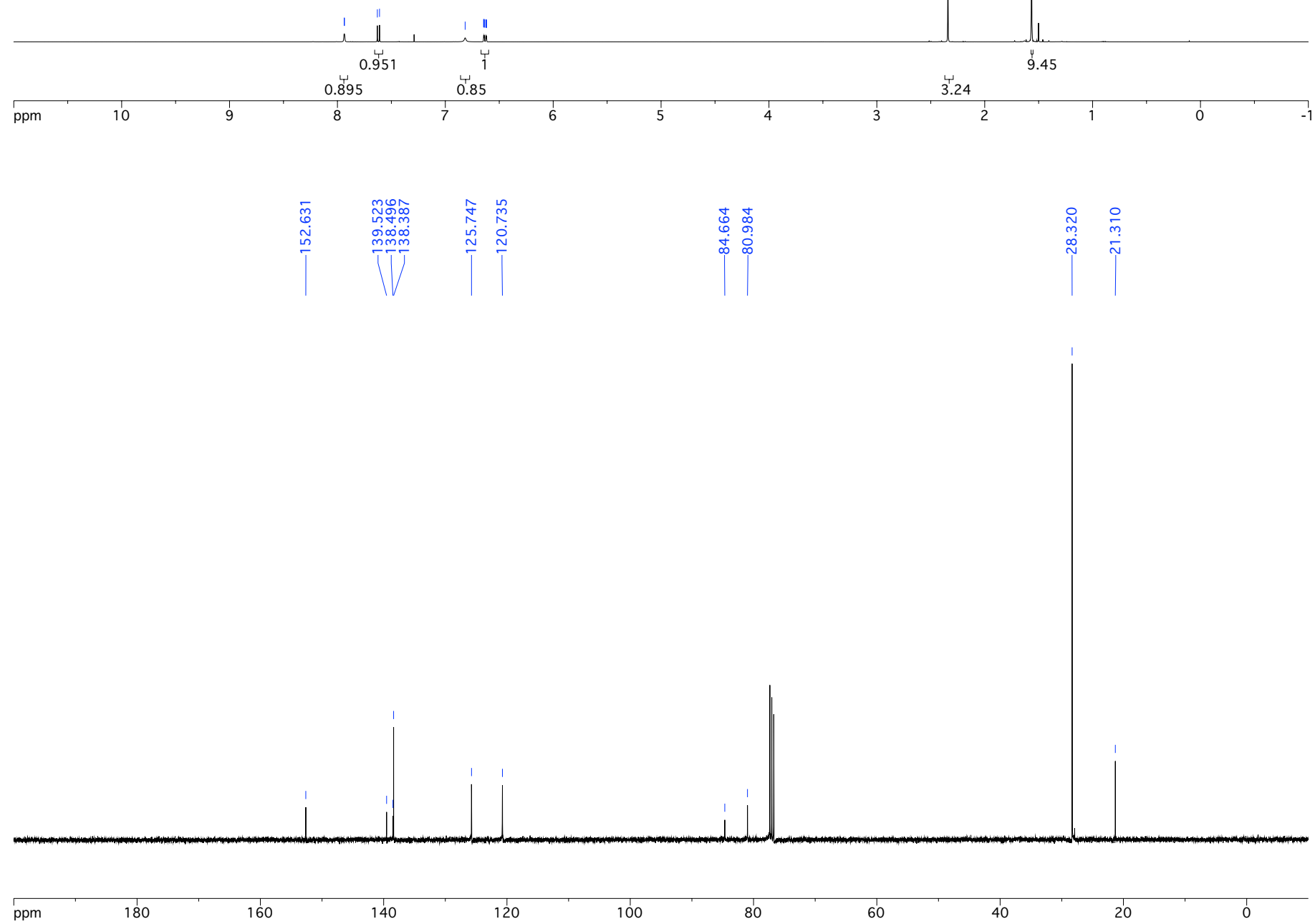


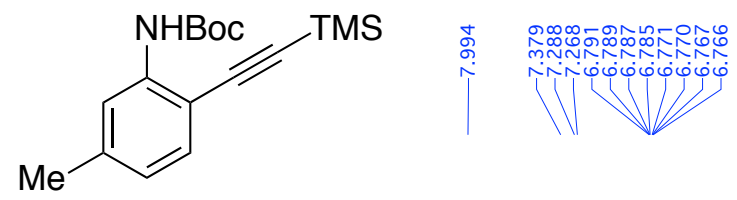

12I
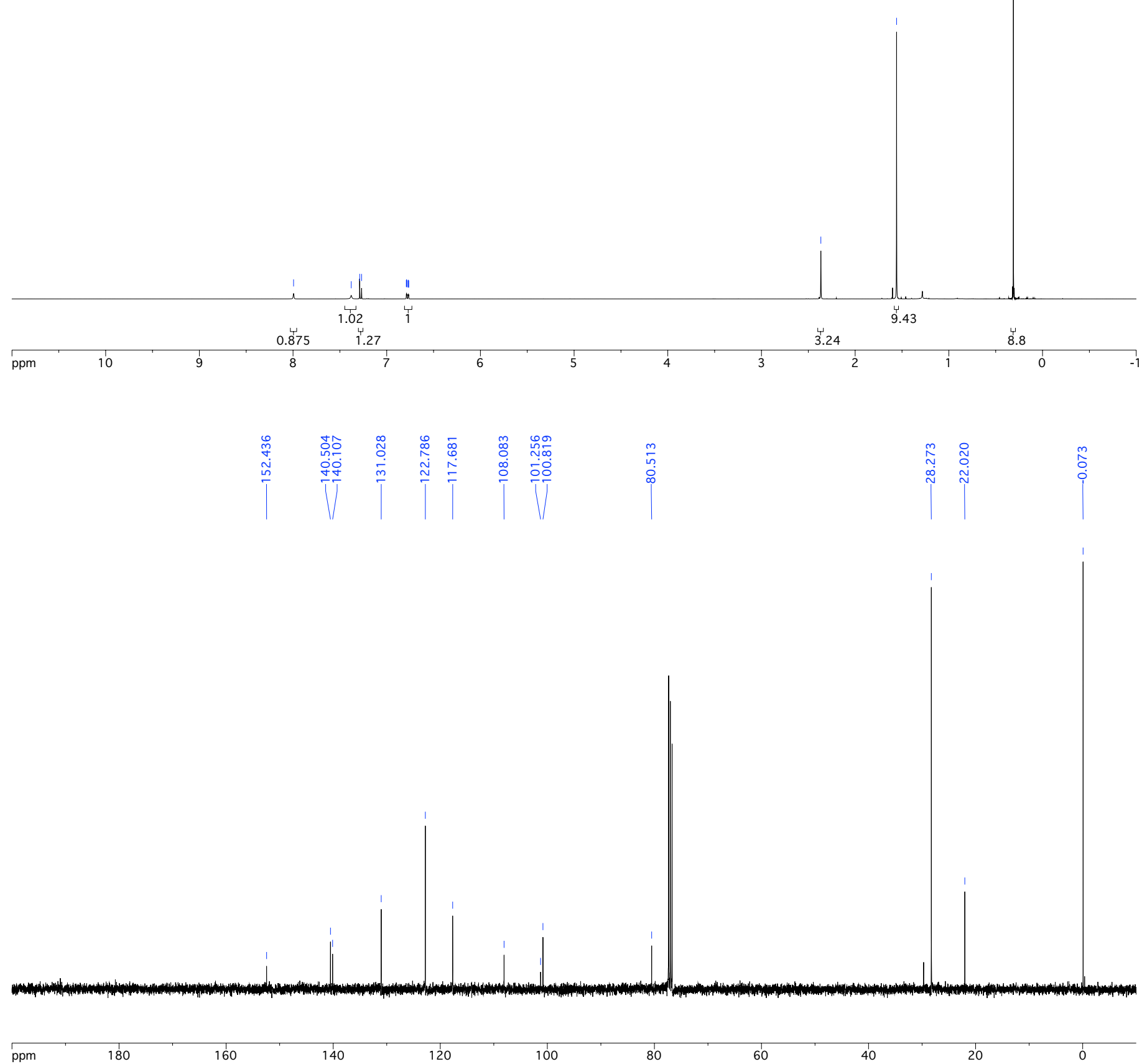

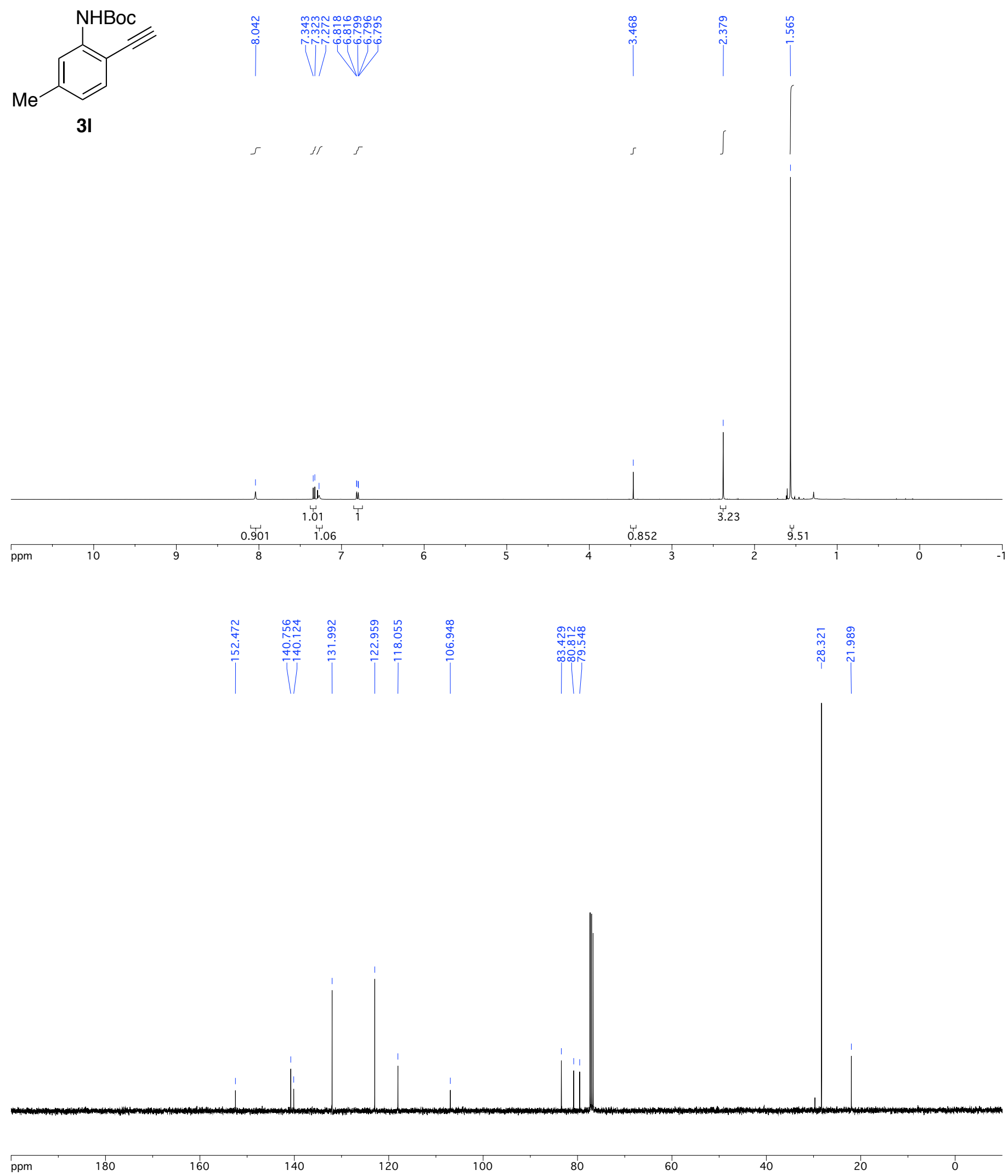
NHBoc

I

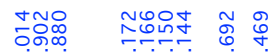

NiN Viñ

NHBoc

$11 \mathrm{~m}$

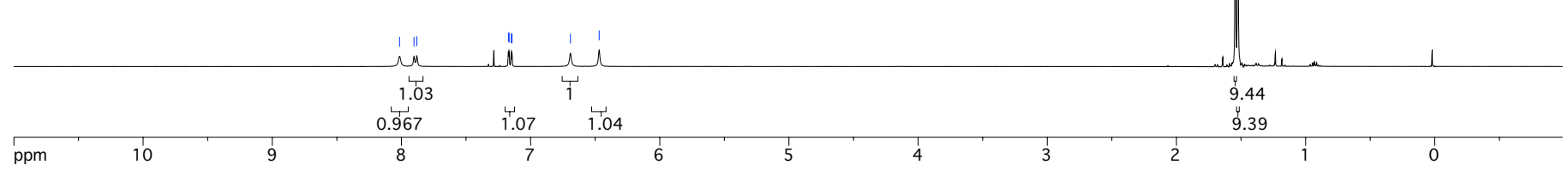

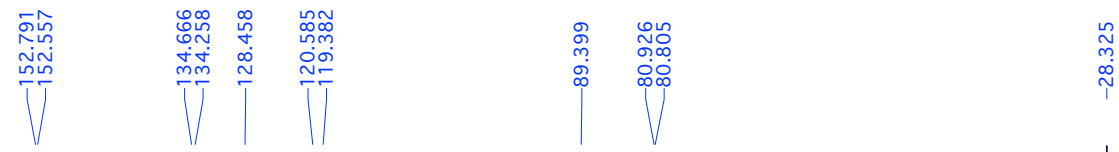

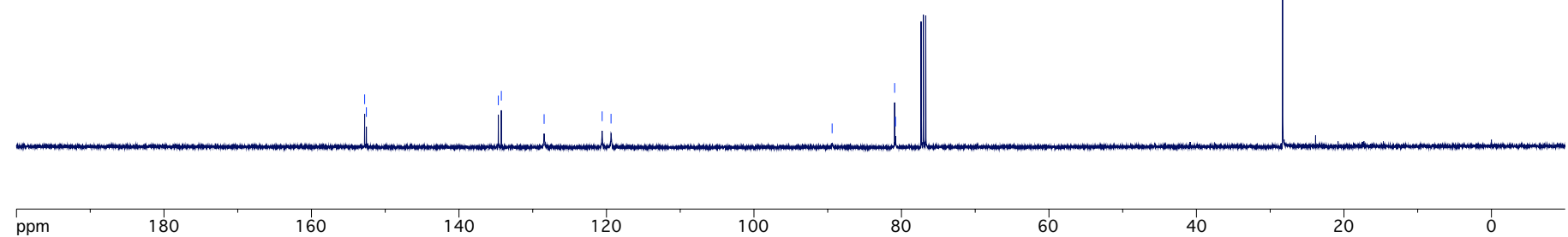



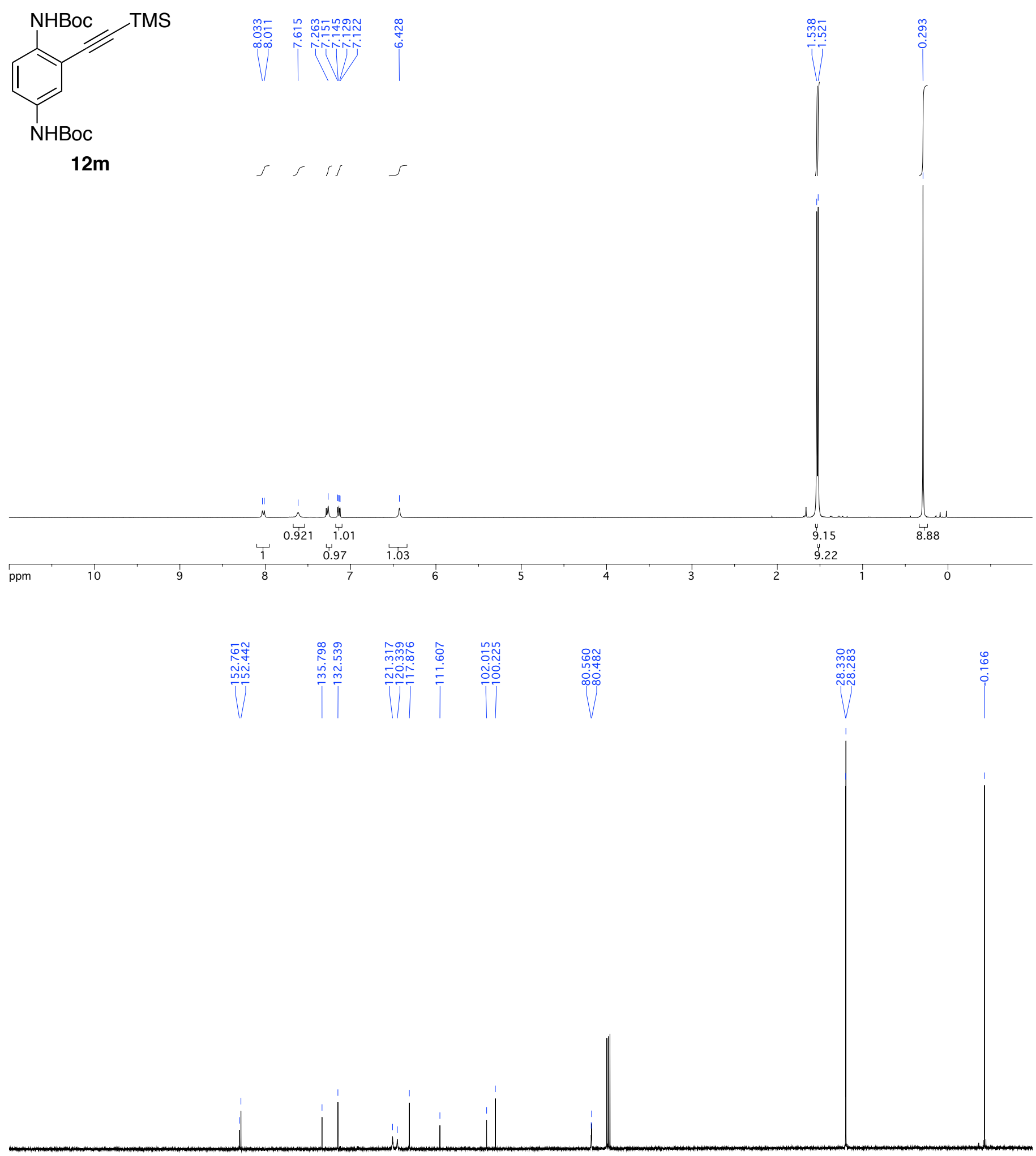

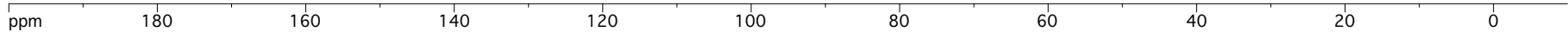




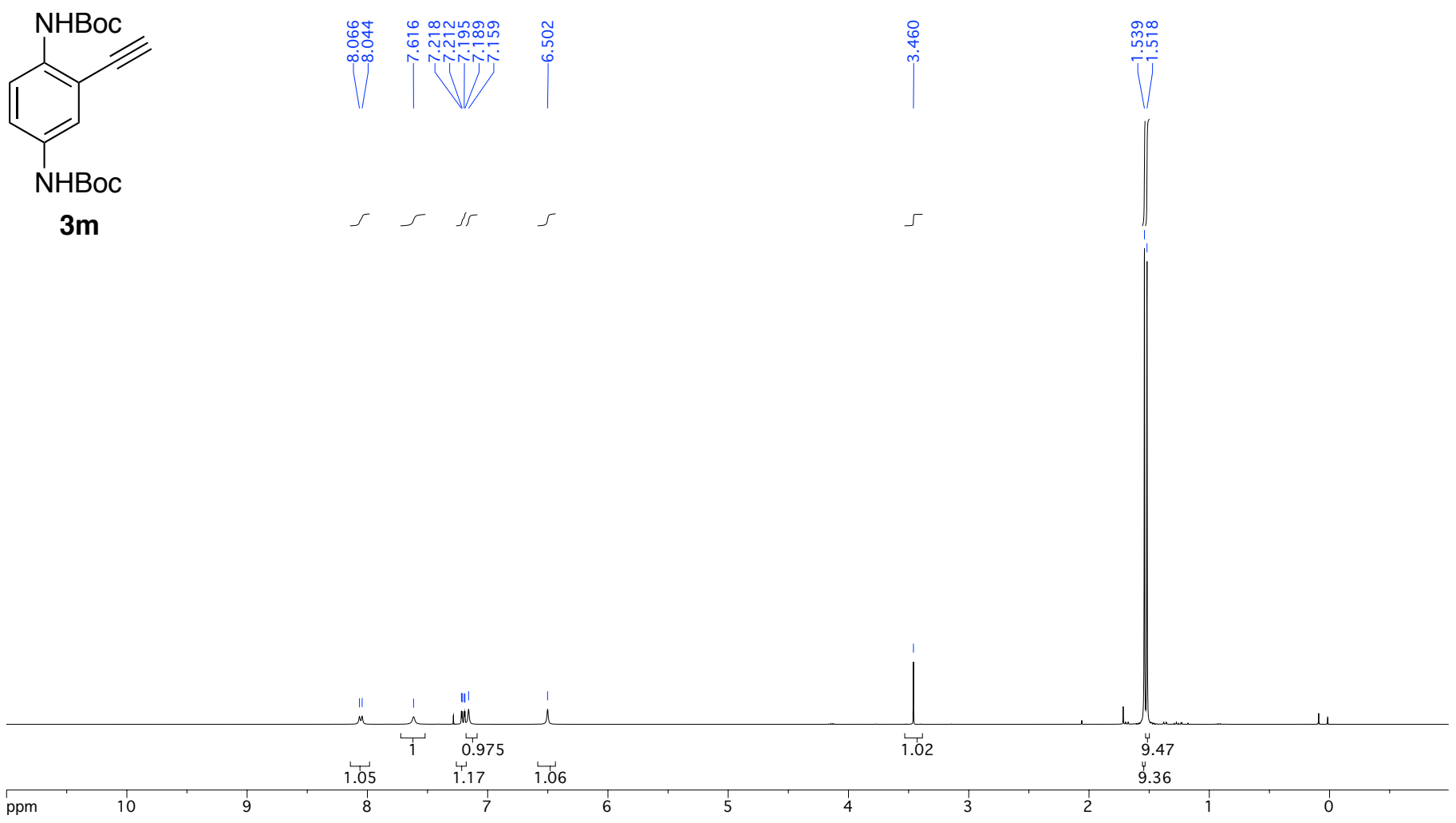

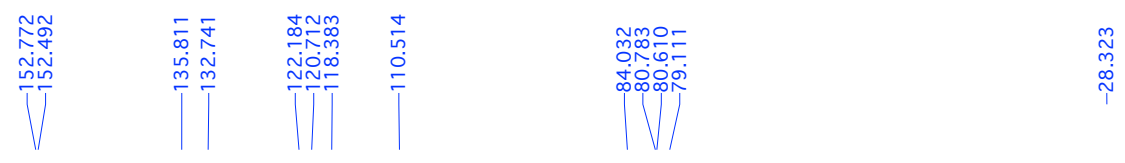

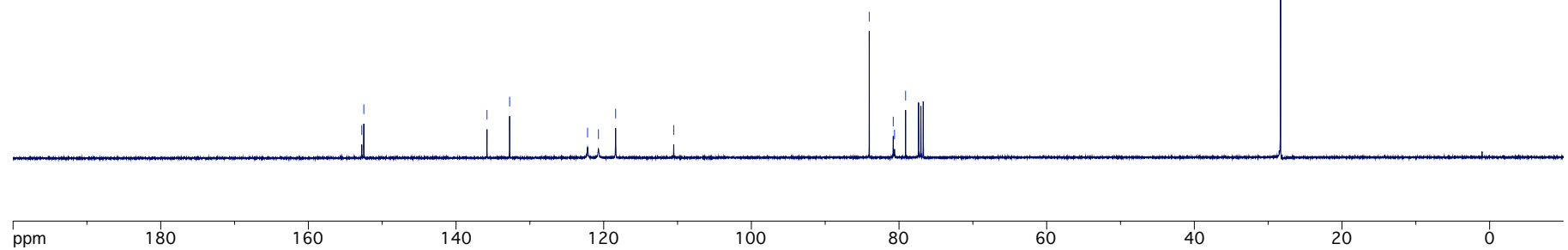




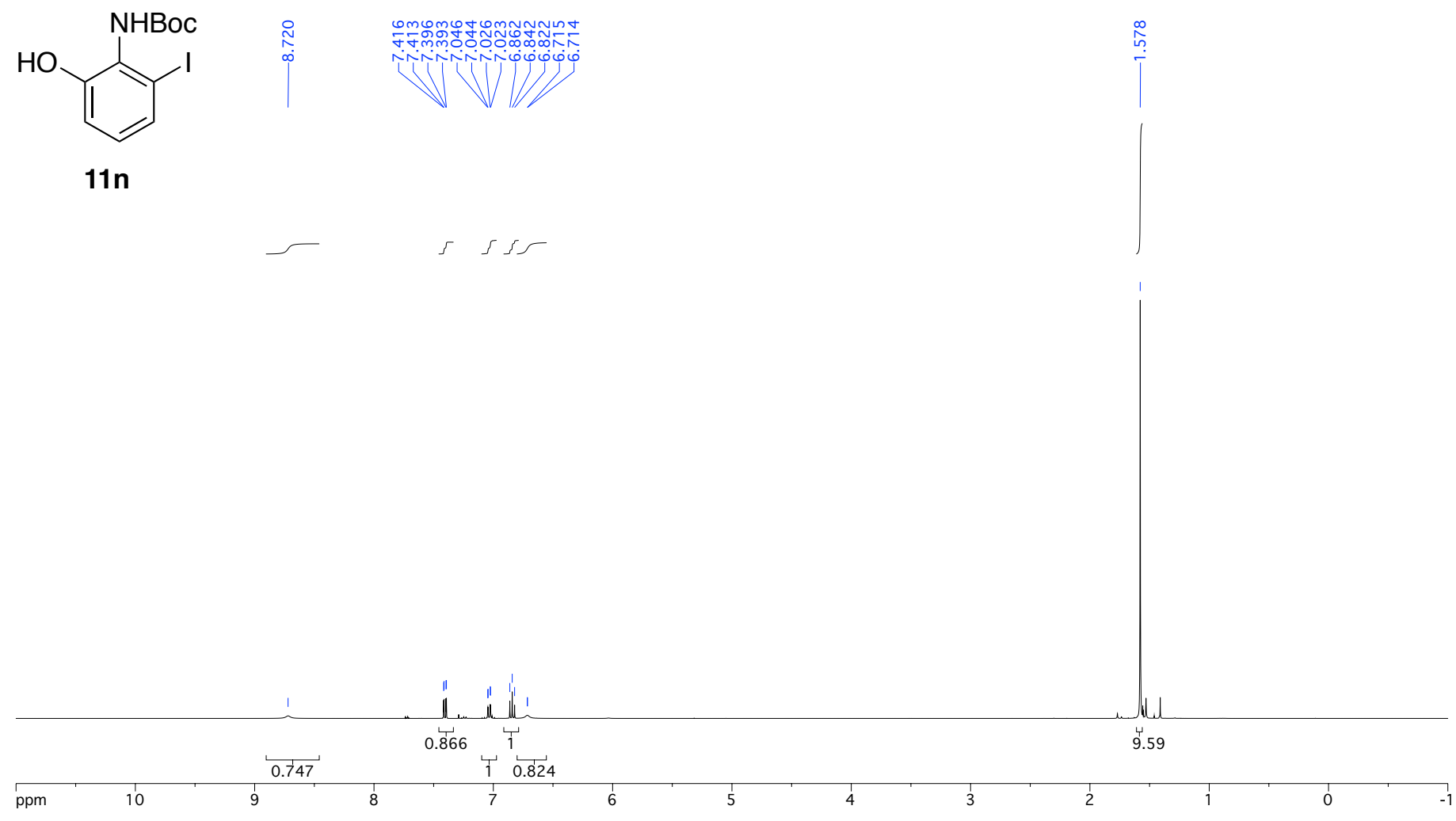

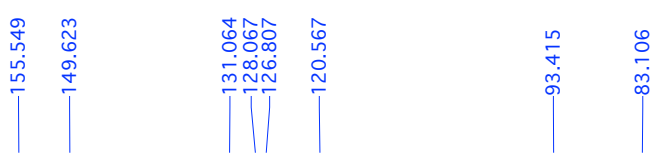

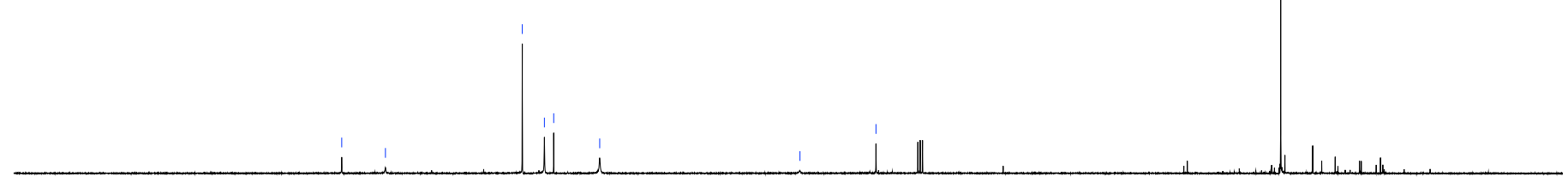

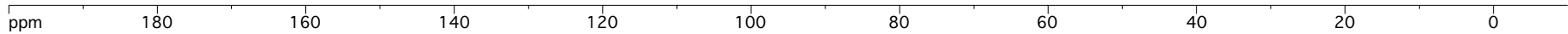



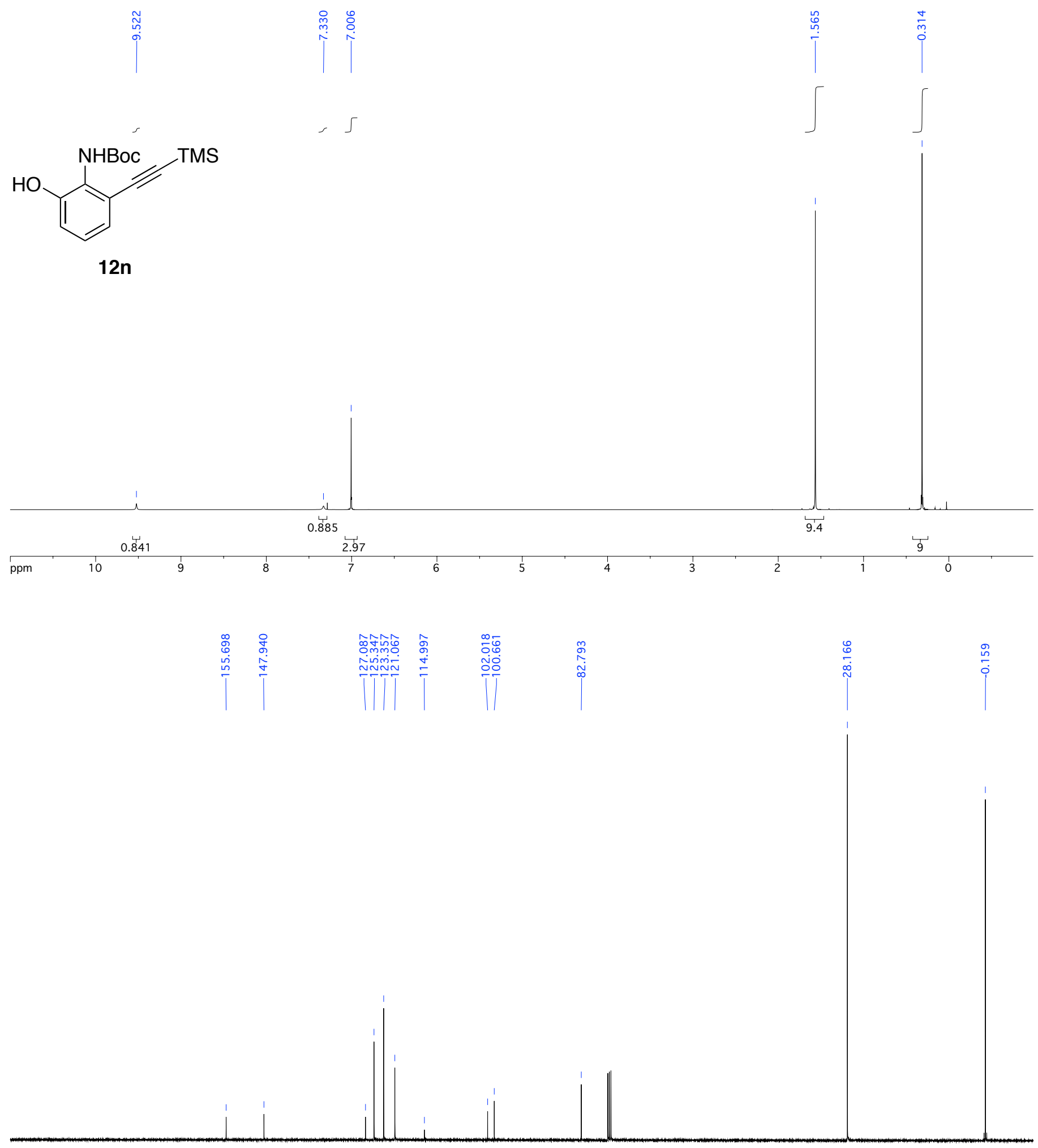

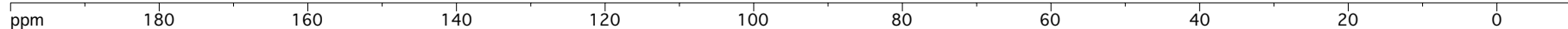




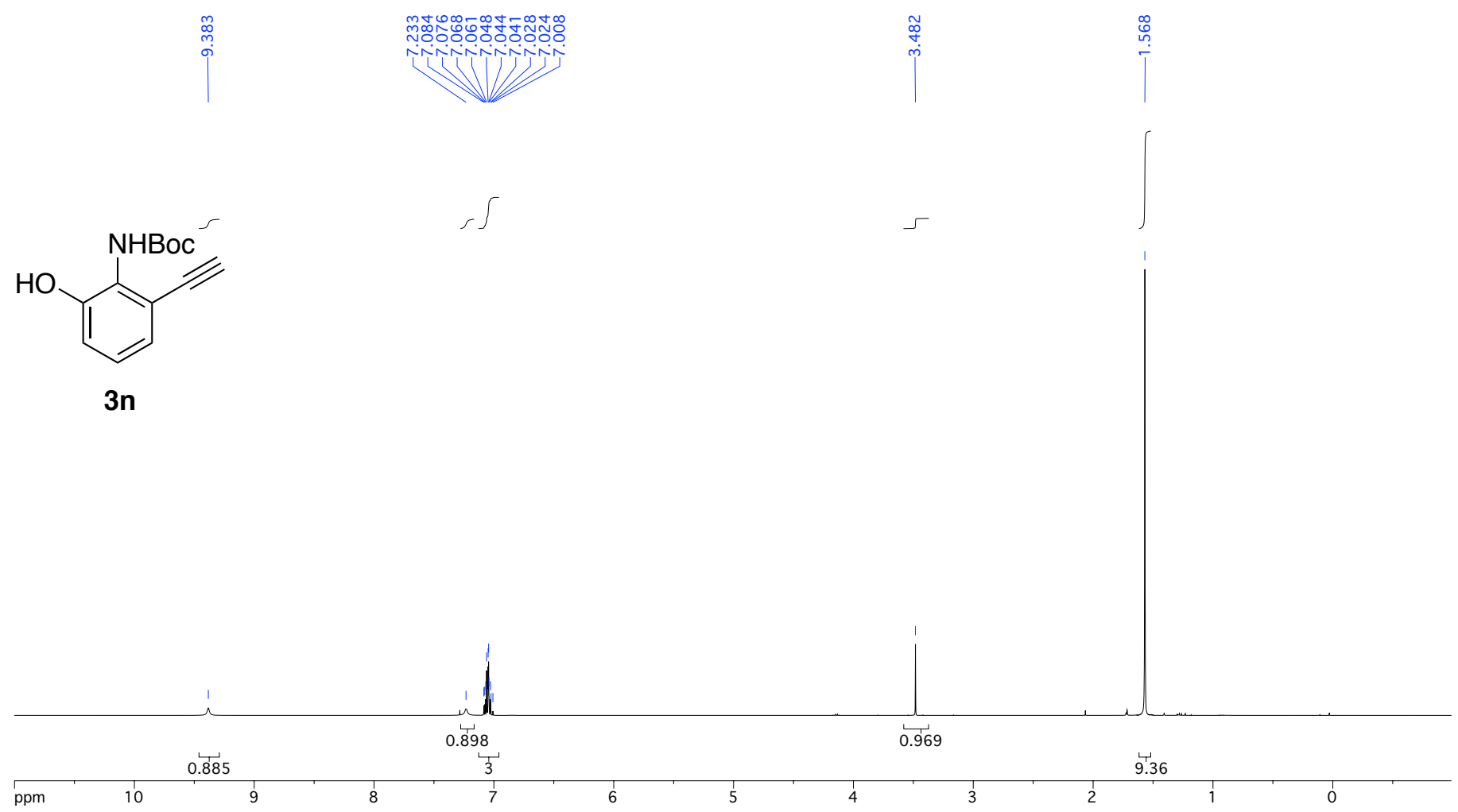

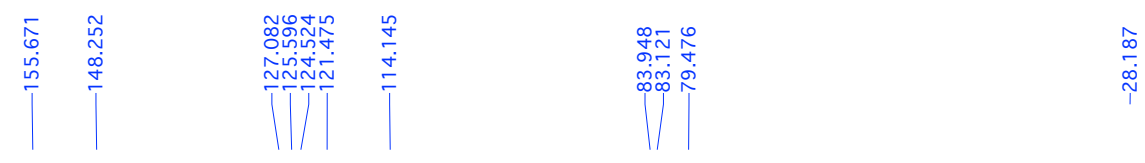

ppm

$180 \quad 160$

$160 \quad 140$

120

100

80

60

40

20 

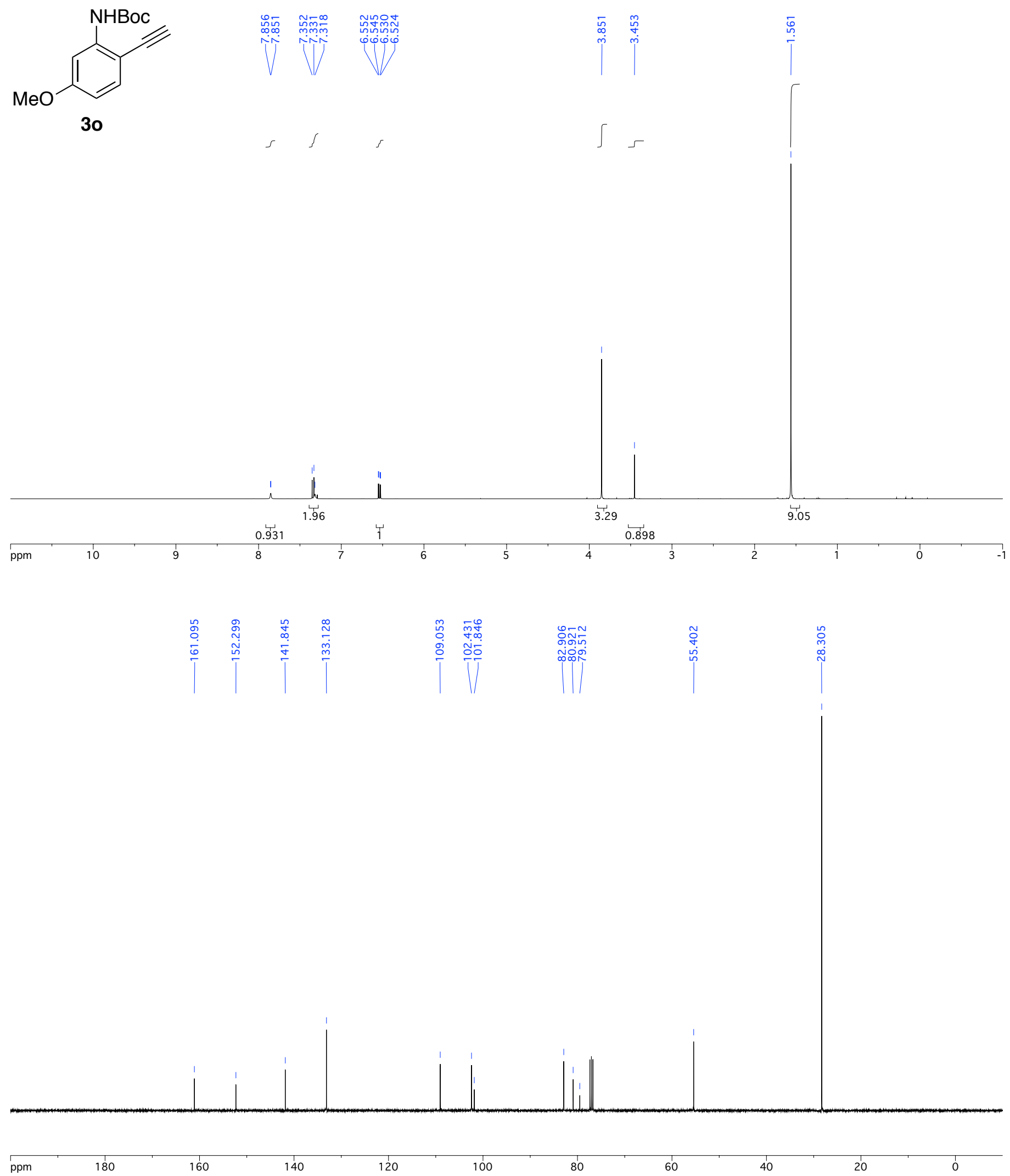

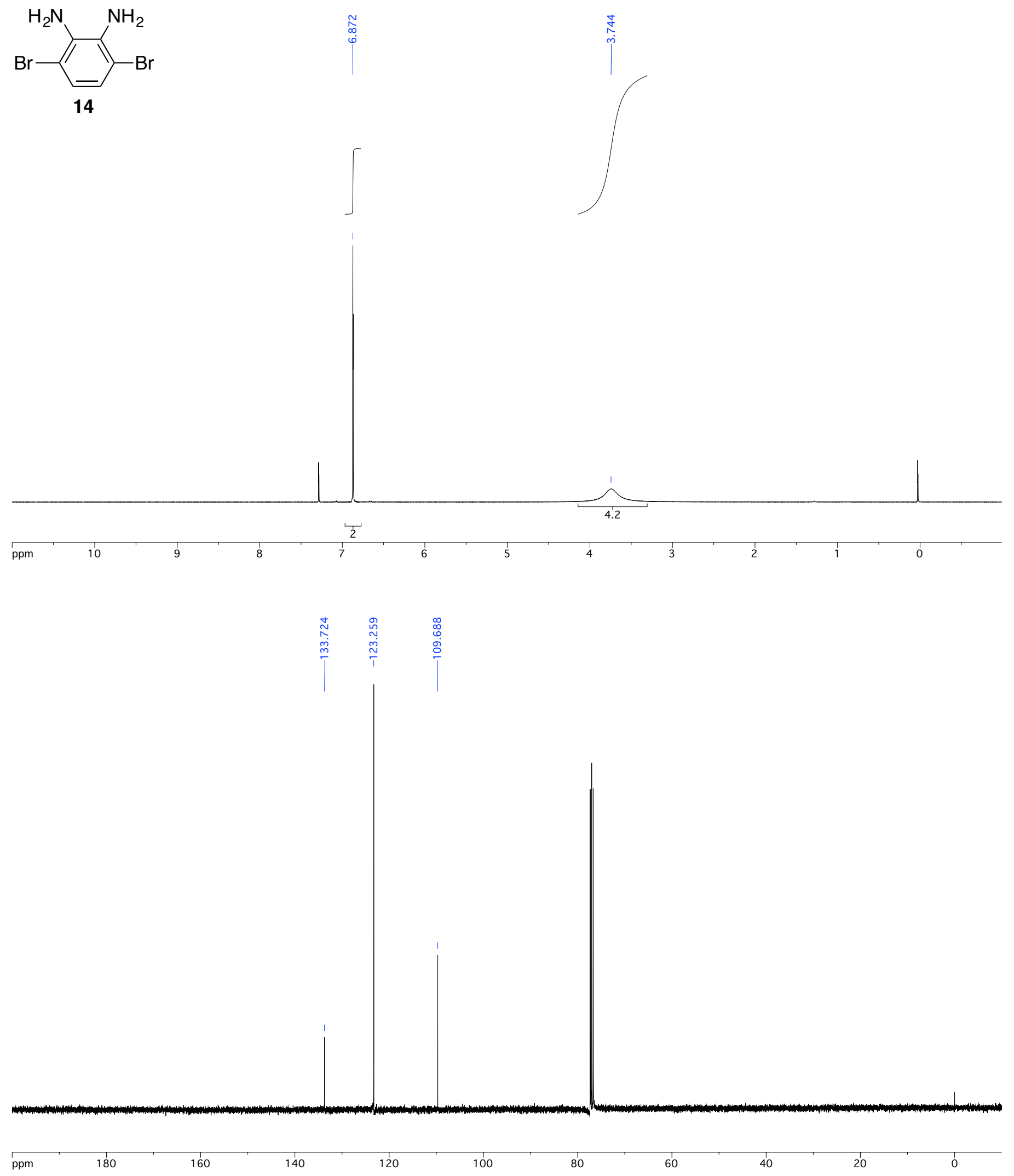

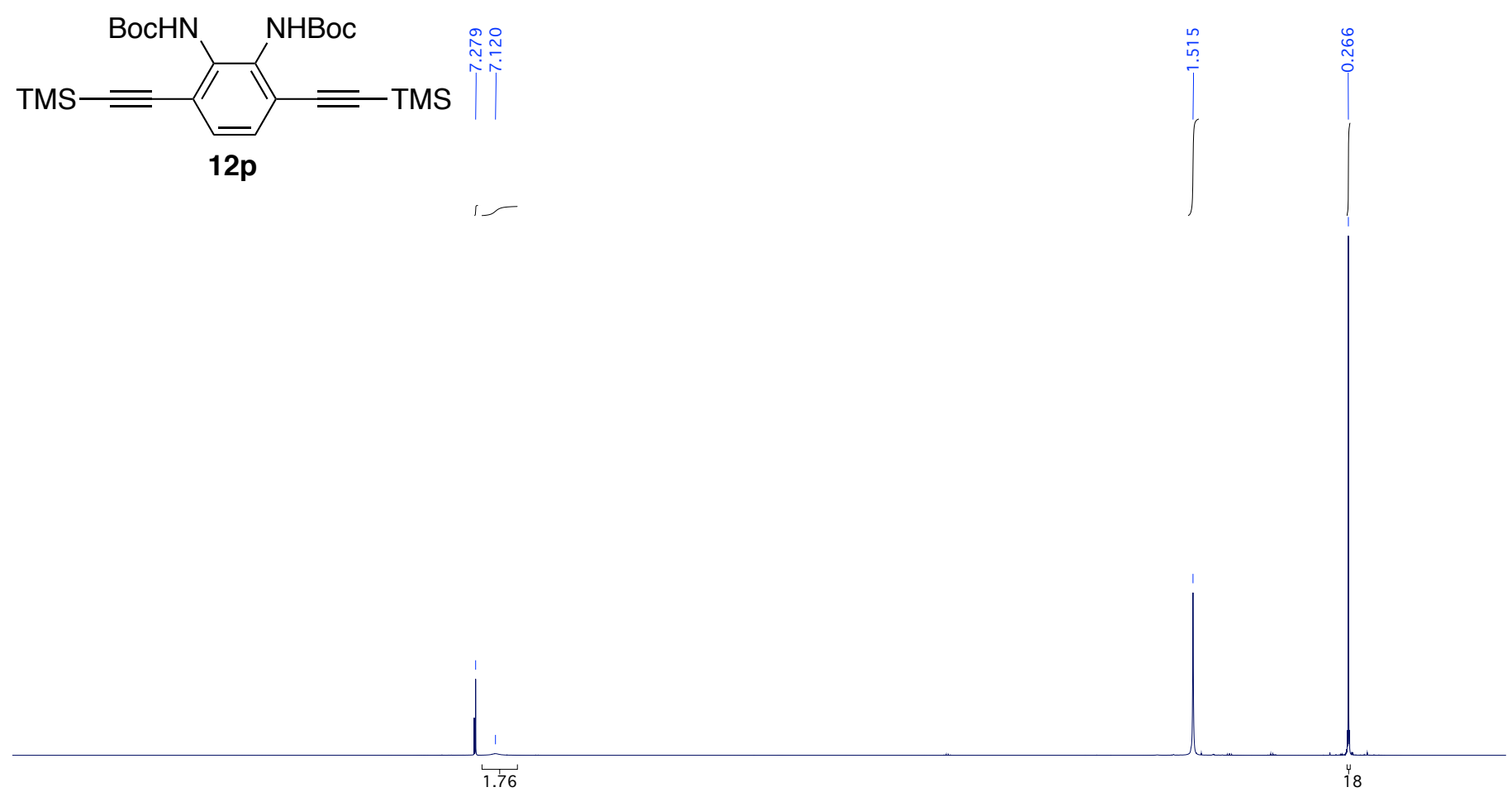

$\stackrel{4}{18.7}$

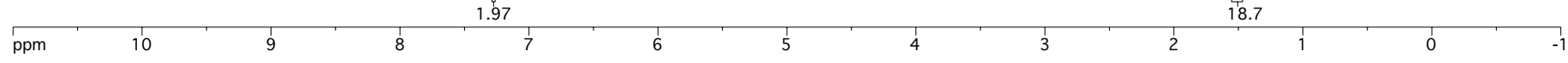

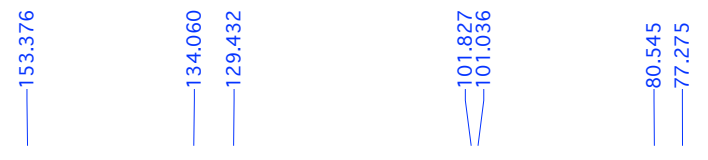

$\stackrel{\substack{N \\ \stackrel{\infty}{\infty}}}{\sim}$
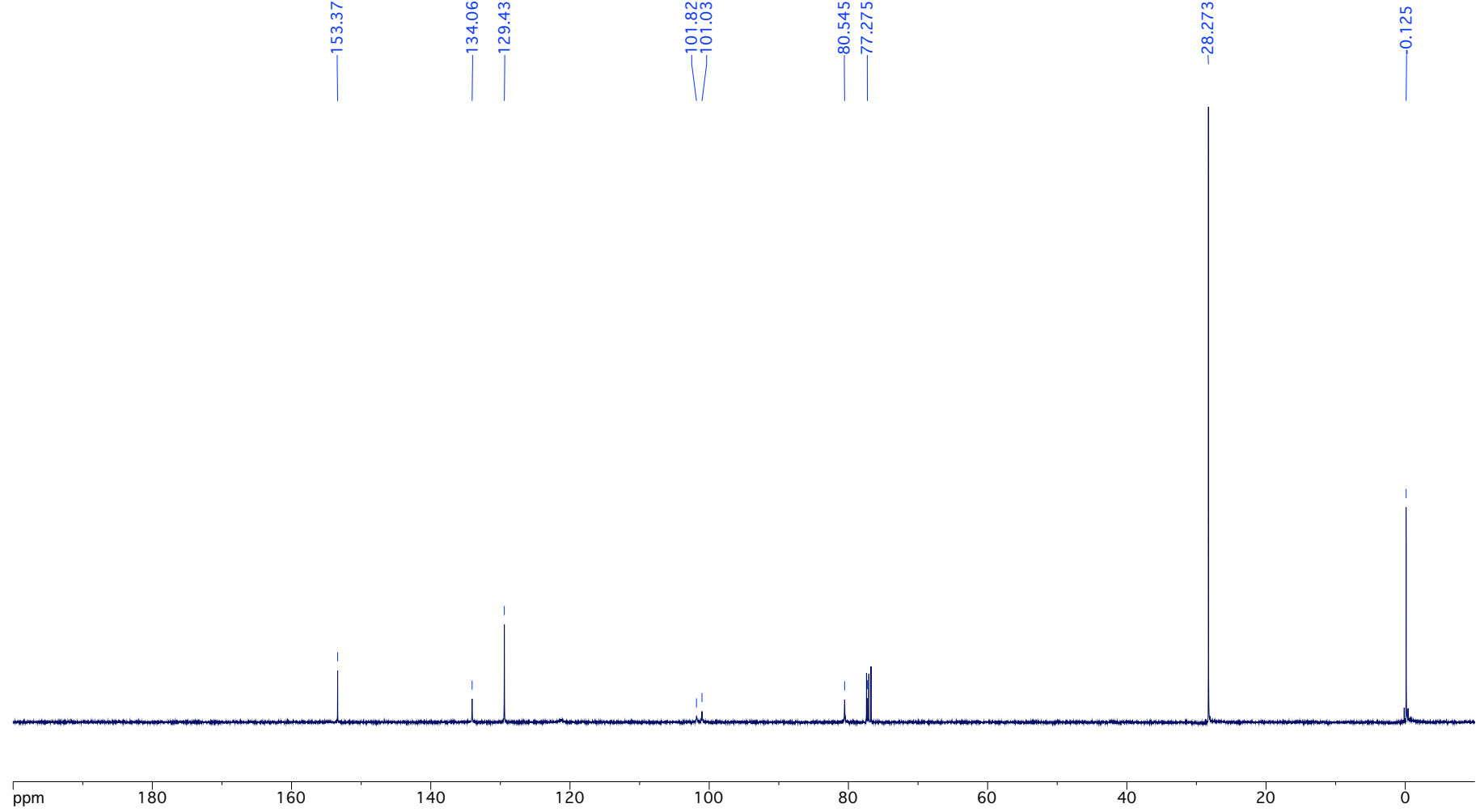

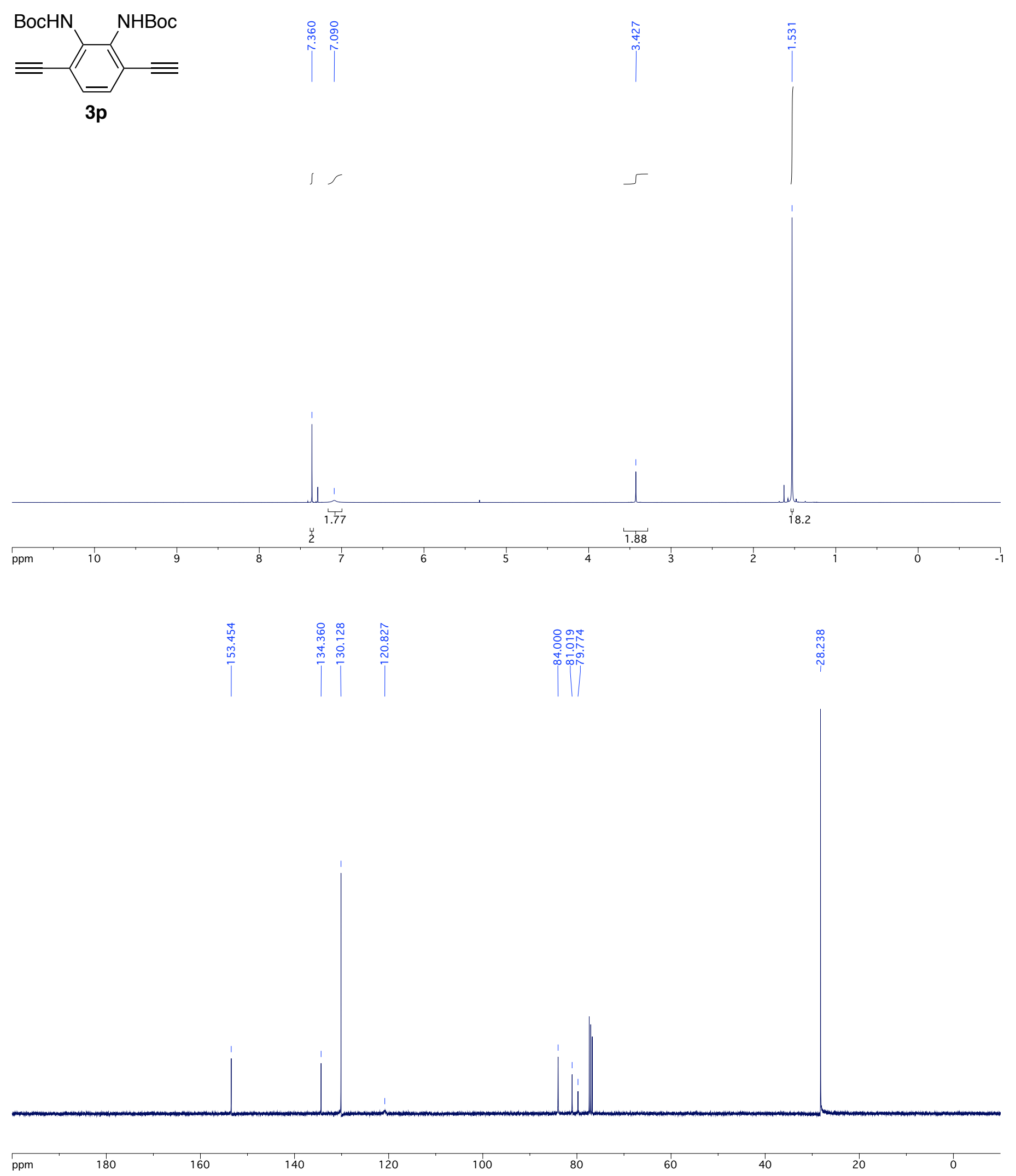

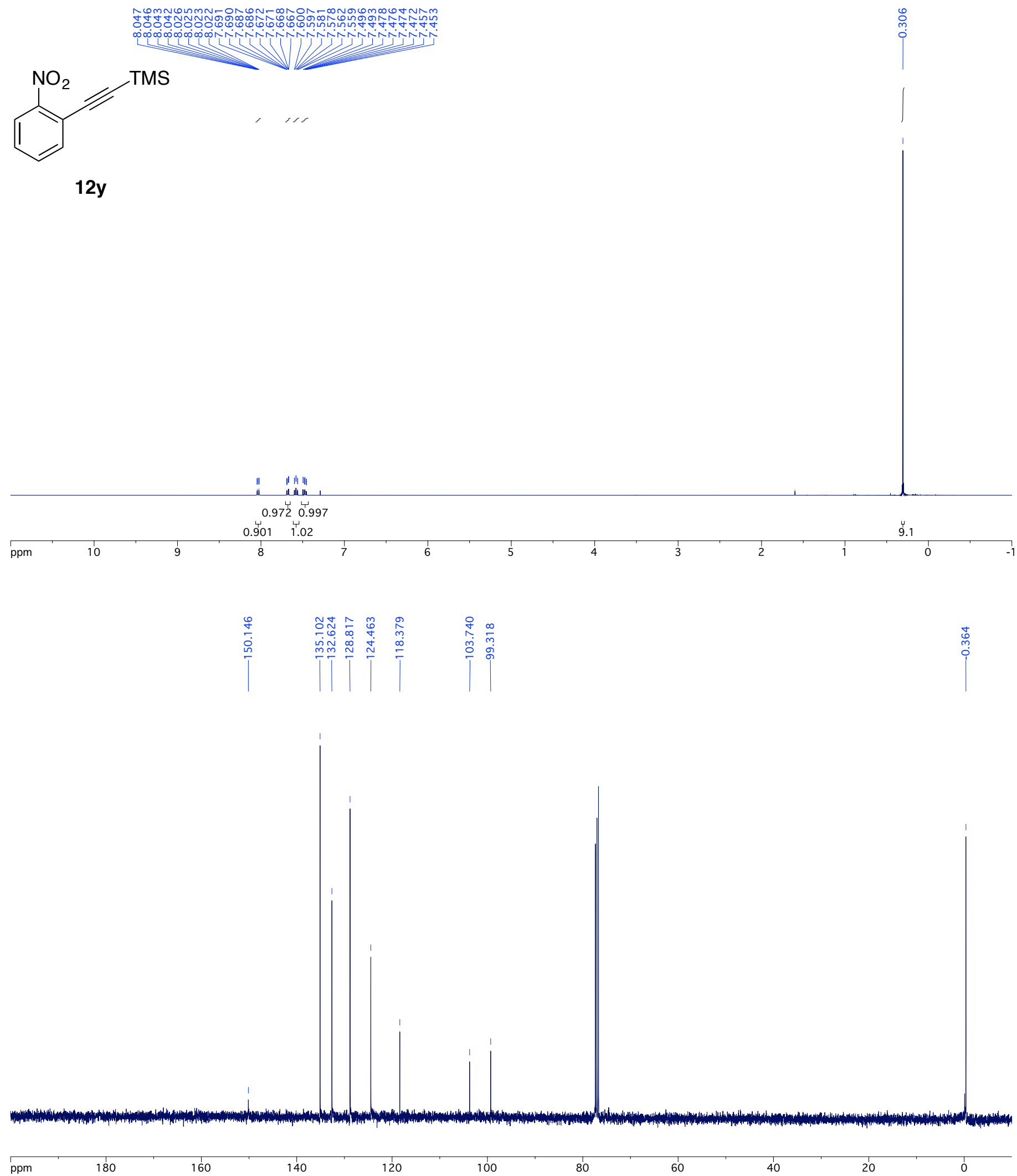

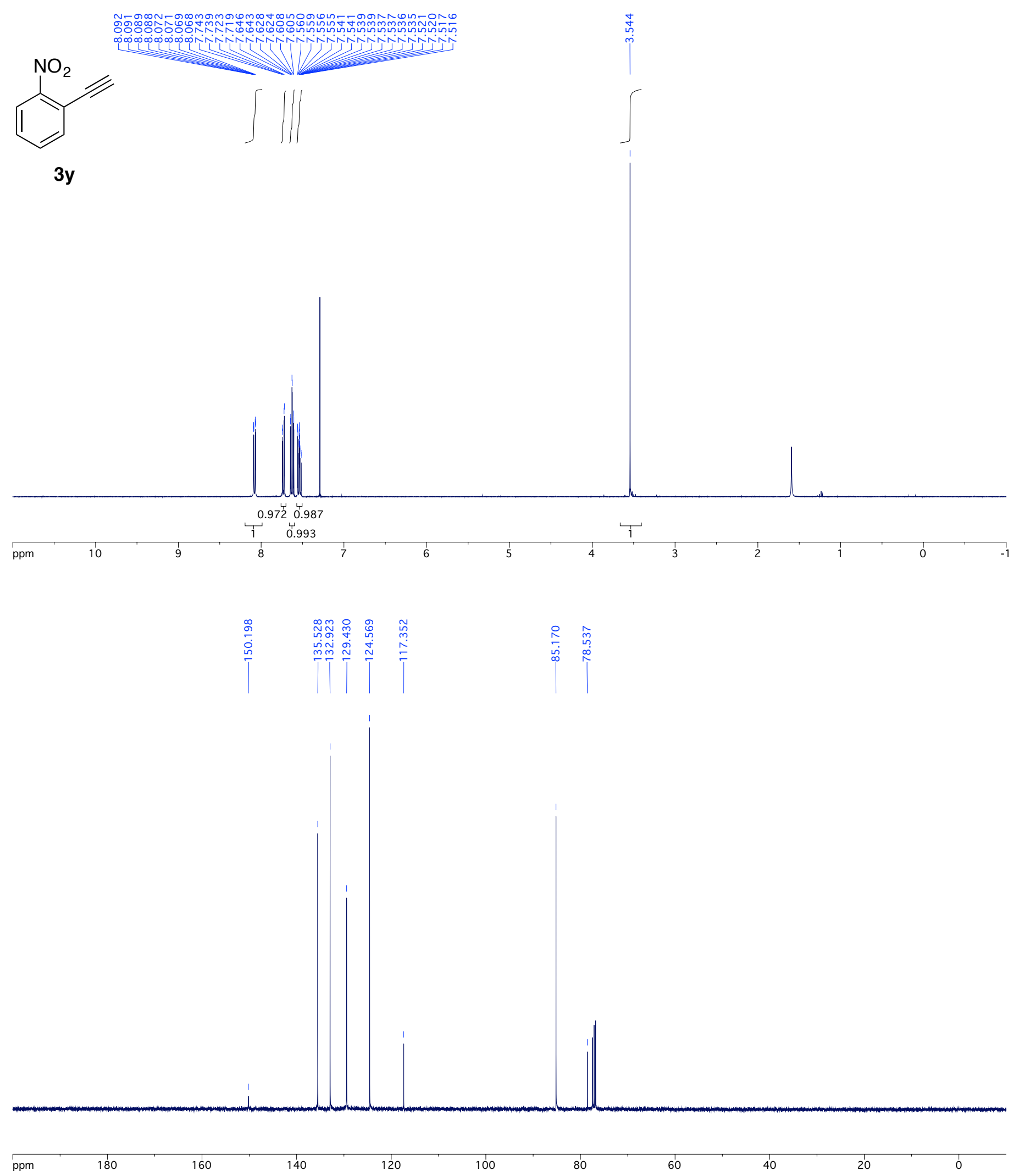

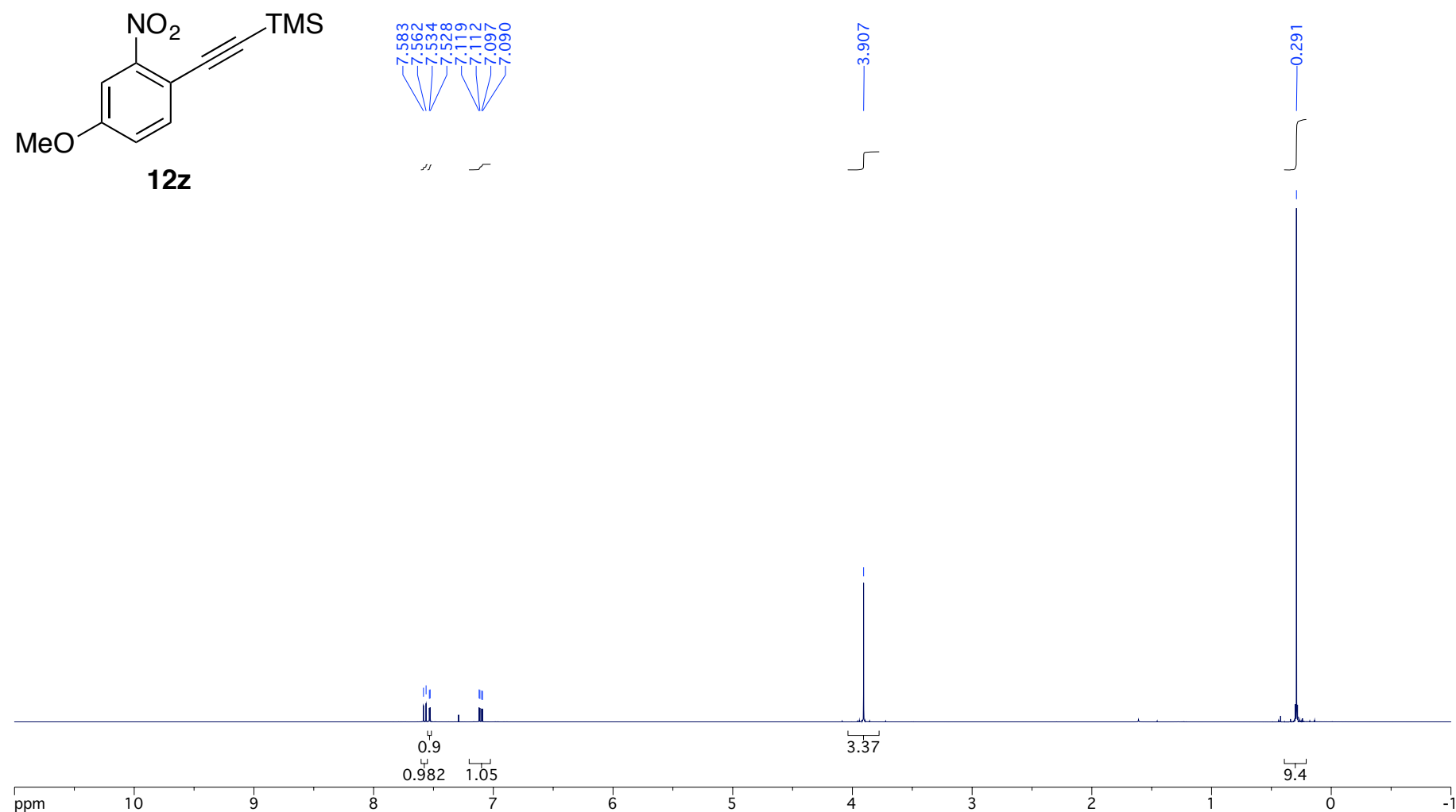

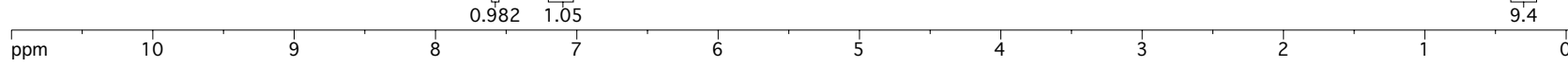
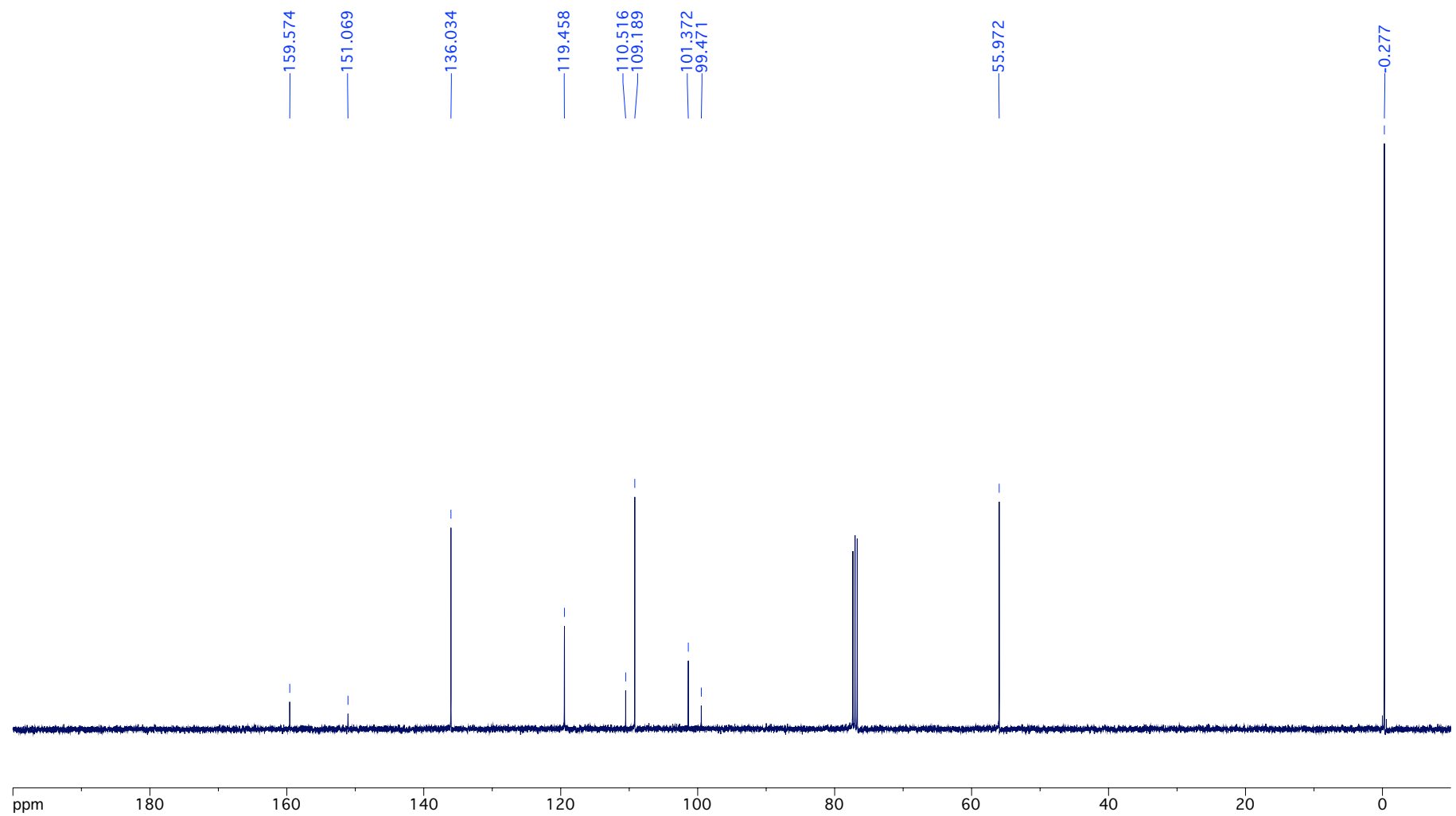

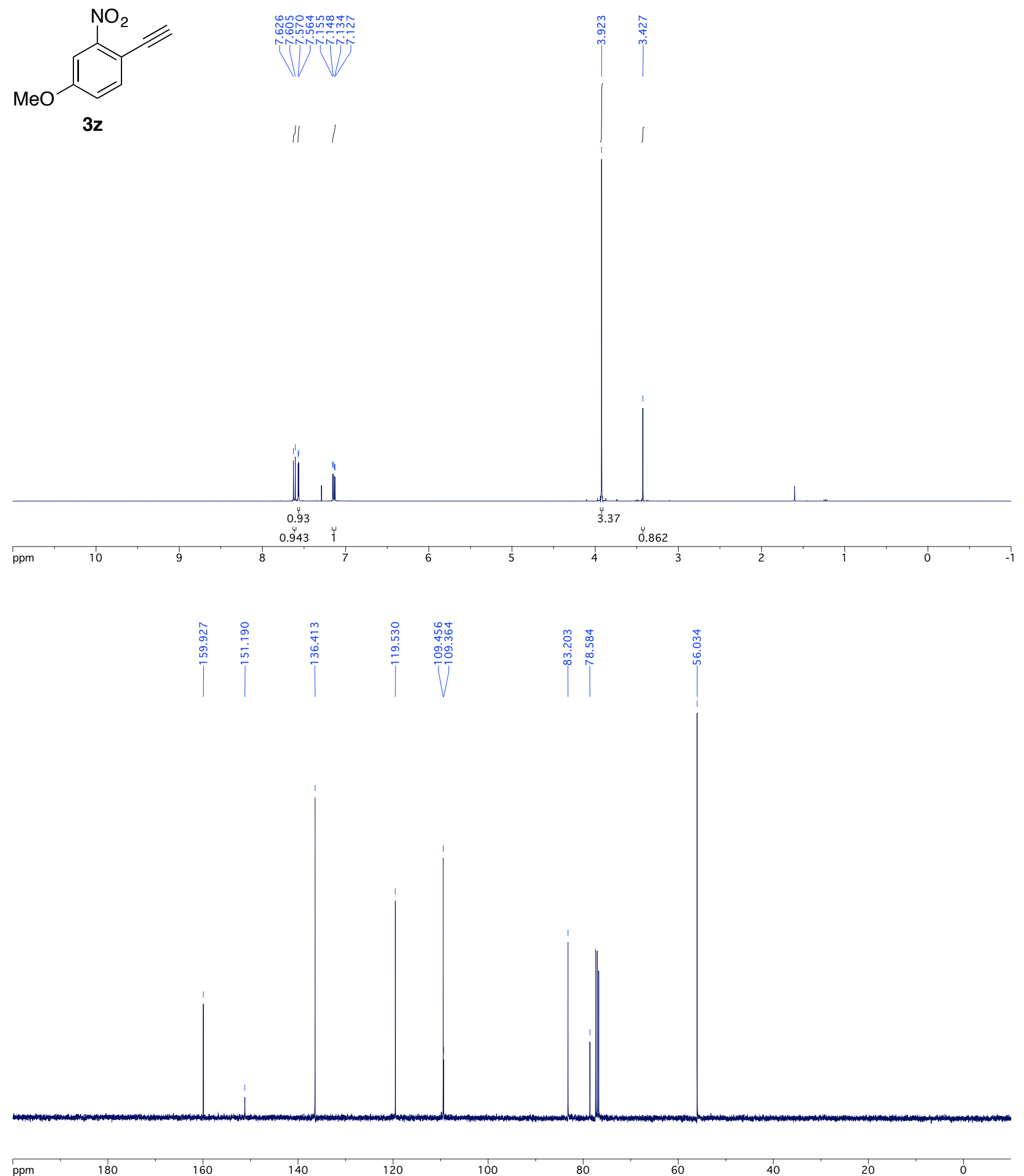
$\underbrace{1}$

กำ

ịisio

15
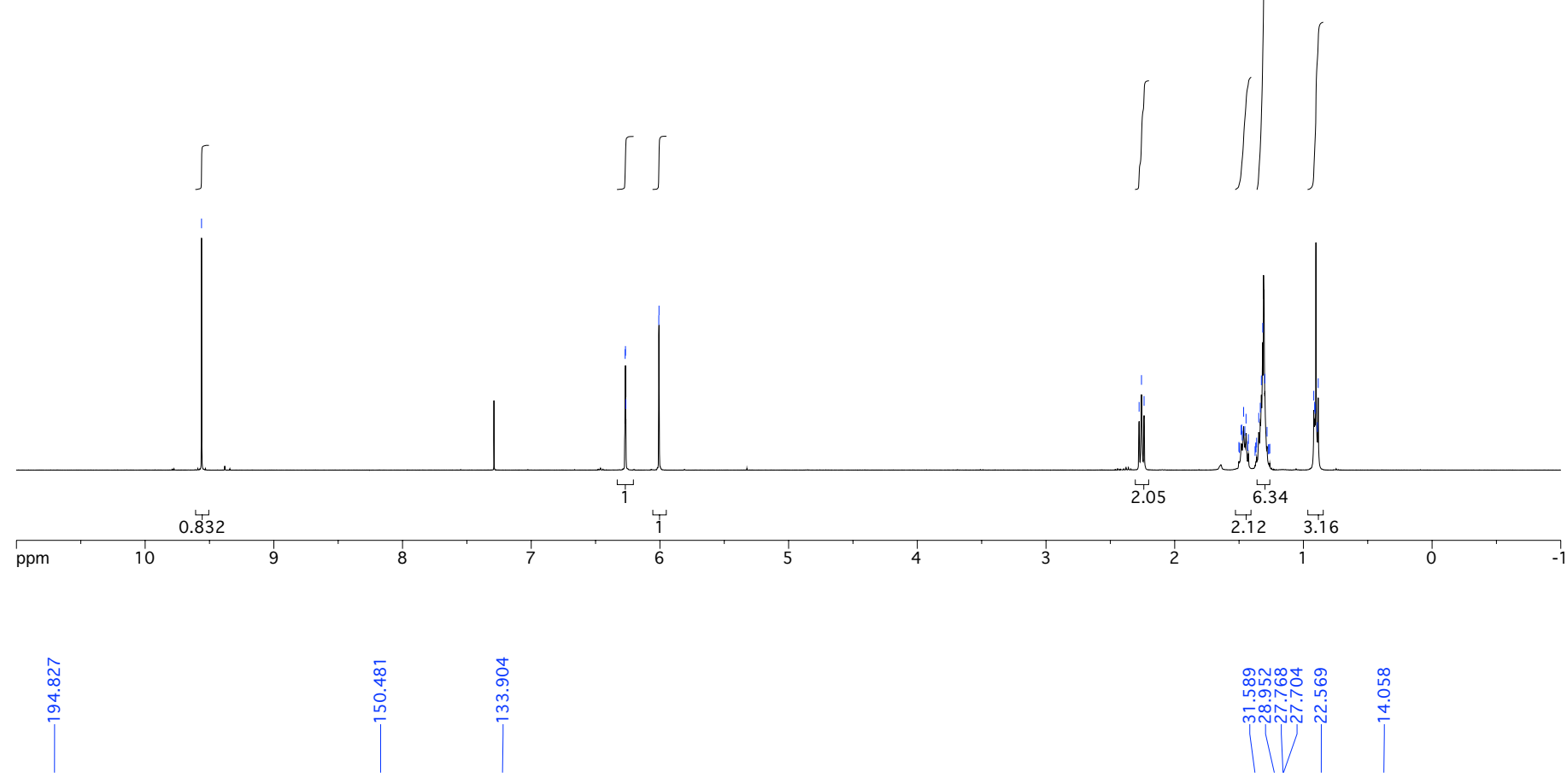

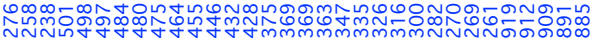

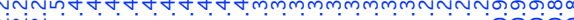

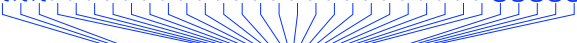

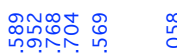

mîn în

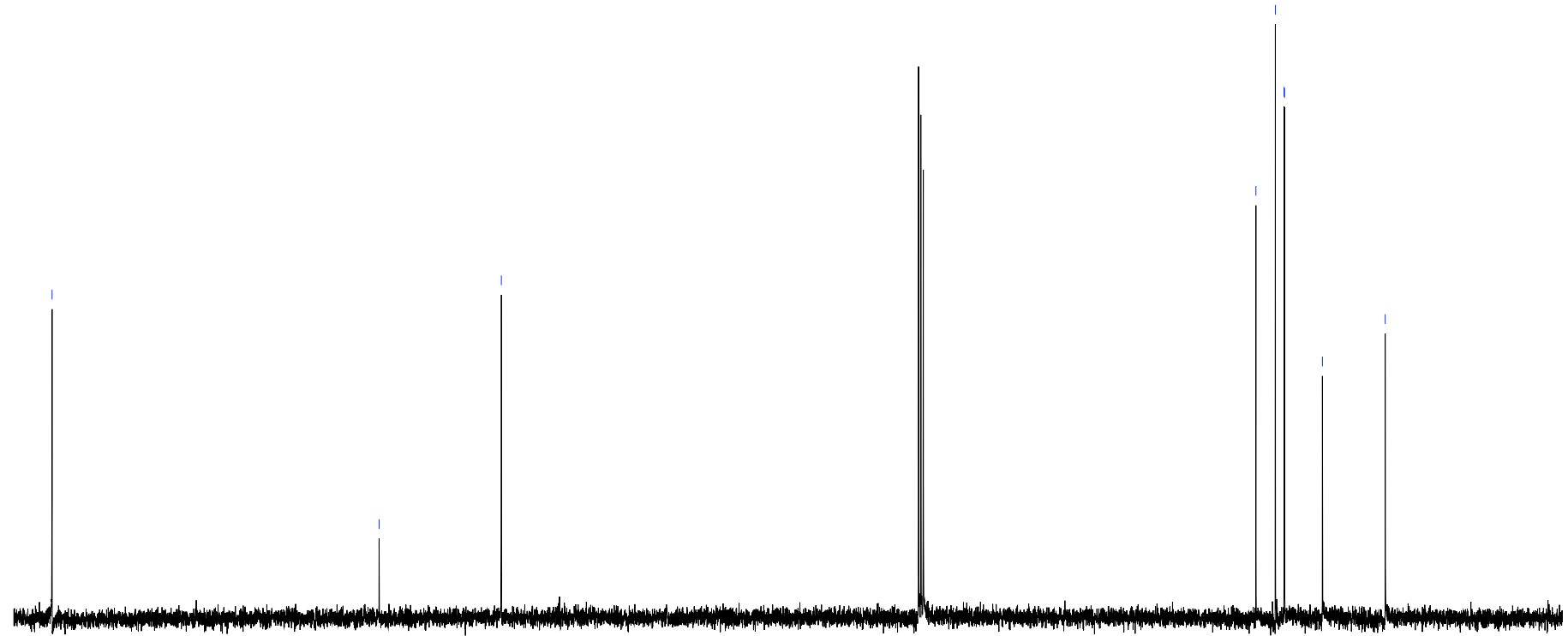

ppm

180

160

140

120

100

80

60

40 


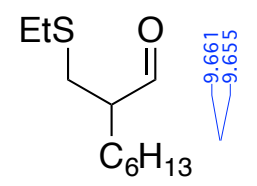

$2 u$

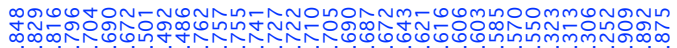

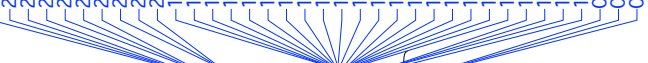

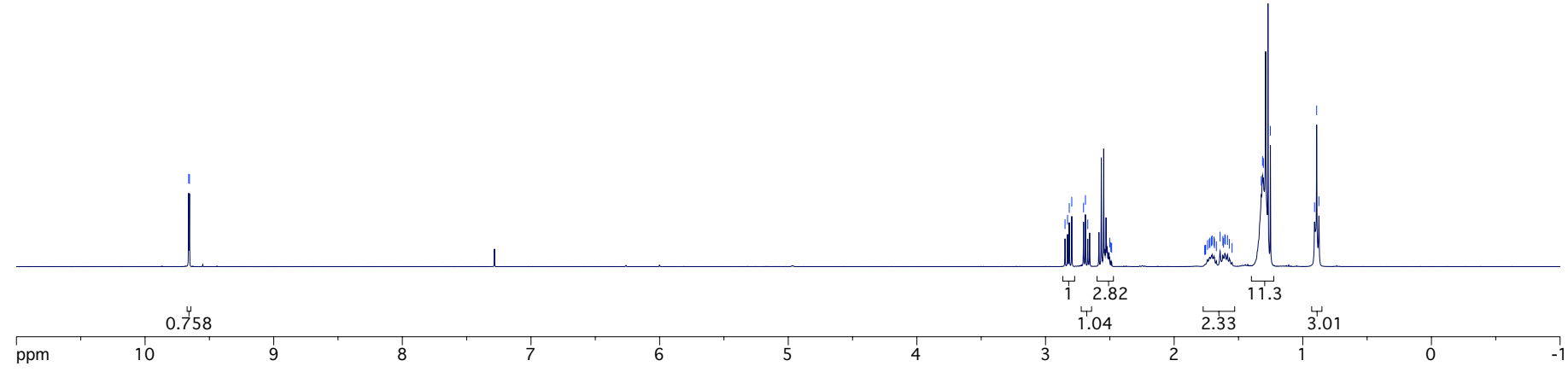
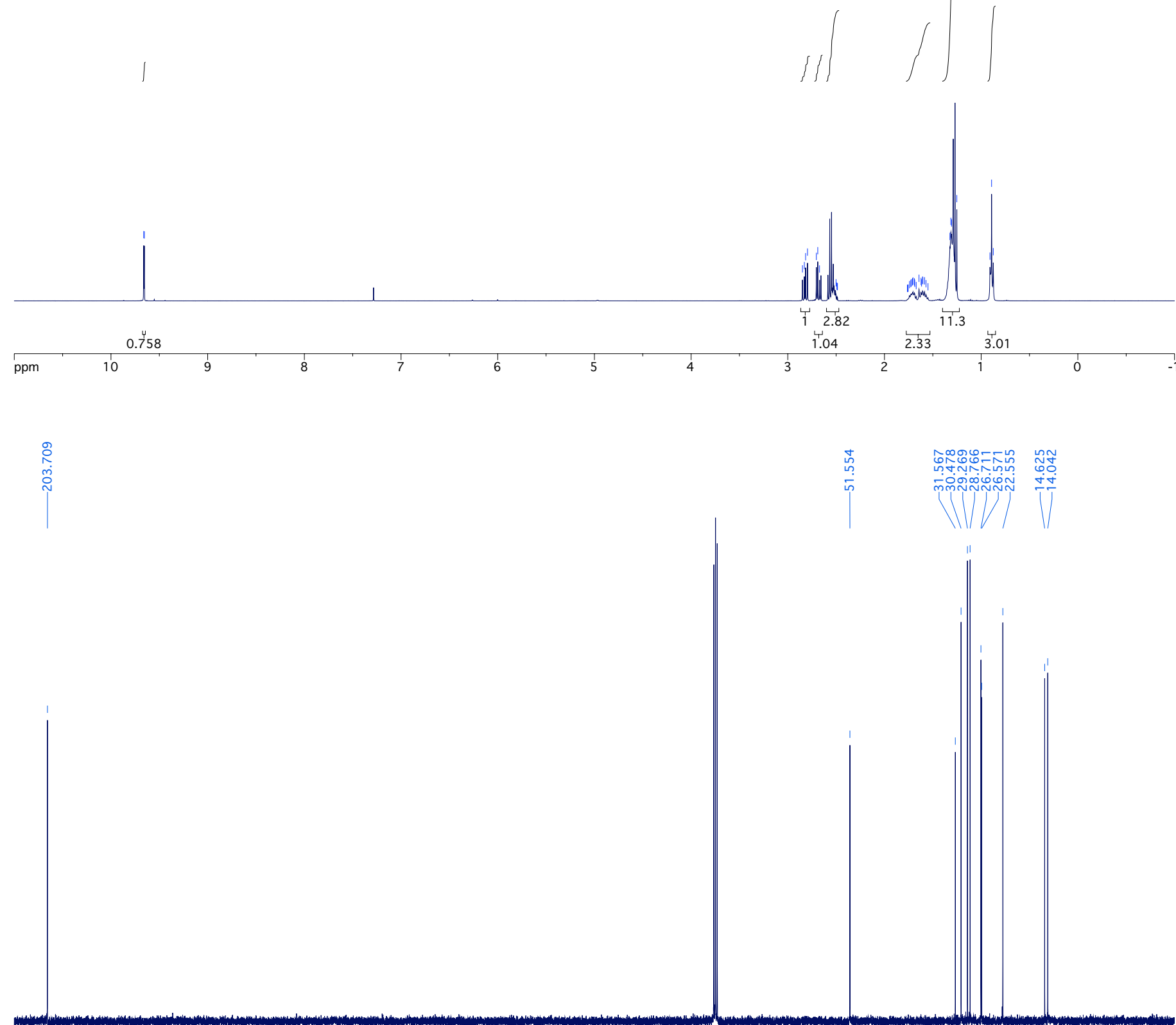

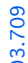

ppm 200

180

160

140

120

100

80

60

40

20 